\title{
Metallic Fuel Benchmark Simulations Based on the X430 Experiments
}

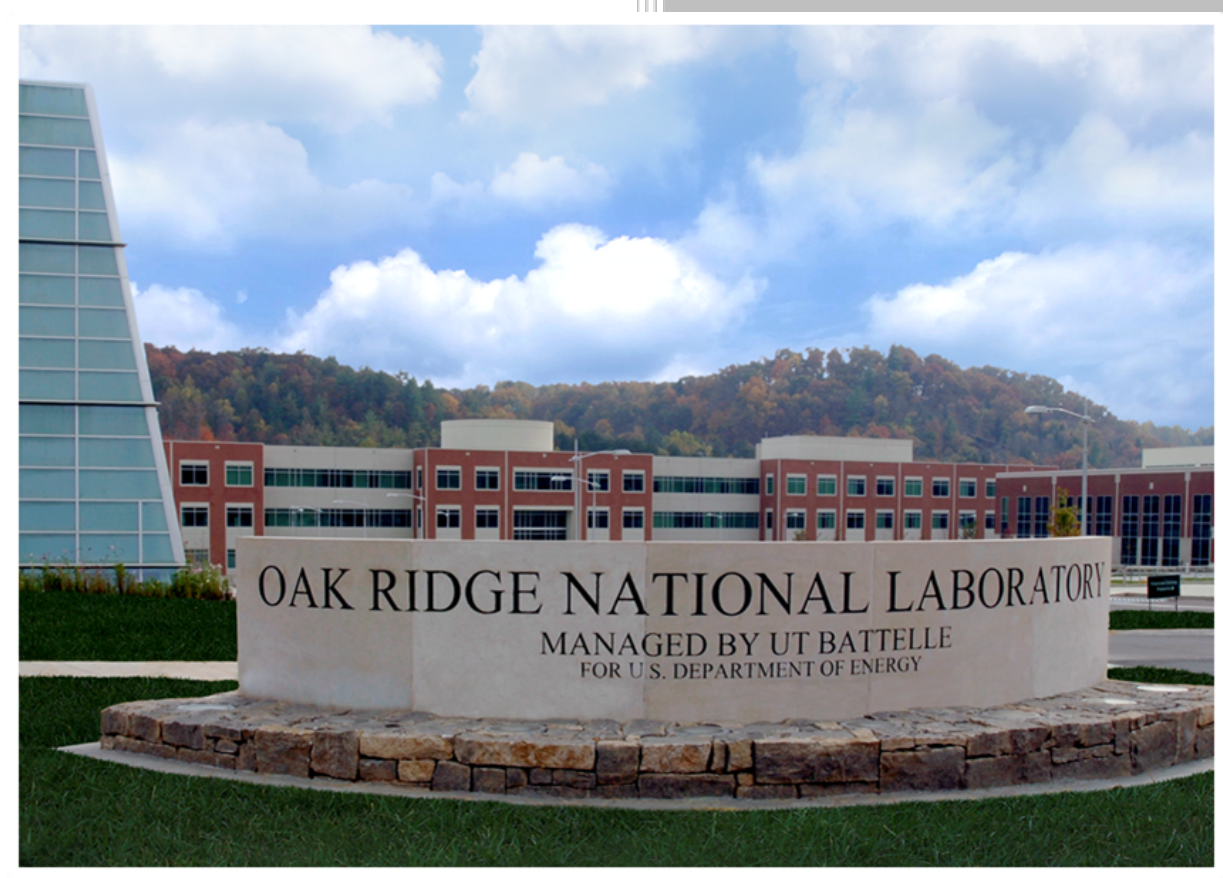

Approved for public release. Distribution is unlimited.

Ian Greenquist Jeffrey J. Powers

July 2020 


\title{
DOCUMENT AVAILABILITY
}

Reports produced after January 1, 1996, are generally available free via US Department of Energy (DOE) SciTech Connect.

Website www.osti.gov

Reports produced before January 1, 1996, may be purchased by members of the public from the following source:

\author{
National Technical Information Service \\ 5285 Port Royal Road \\ Springfield, VA 22161 \\ Telephone 703-605-6000 (1-800-553-6847) \\ TDD 703-487-4639 \\ Fax 703-605-6900 \\ E-mail info@ntis.gov \\ Website http://classic.ntis.gov/
}

Reports are available to DOE employees, DOE contractors, Energy Technology Data Exchange representatives, and International Nuclear Information System representatives from the following source:

Office of Scientific and Technical Information

PO Box 62

Oak Ridge, TN 37831

Telephone 865-576-8401

Fax 865-576-5728

E-mail reports@osti.gov

Website http://www.osti.gov/contact.html

This report was prepared as an account of work sponsored by an agency of the United States Government. Neither the United States Government nor any agency thereof, nor any of their employees, makes any warranty, express or implied, or assumes any legal liability or responsibility for the accuracy, completeness, or usefulness of any information, apparatus, product, or process disclosed, or represents that its use would not infringe privately owned rights. Reference herein to any specific commercial product, process, or service by trade name, trademark, manufacturer, or otherwise, does not necessarily constitute or imply its endorsement, recommendation, or favoring by the United States Government or any agency thereof. The views and opinions of authors expressed herein do not necessarily state or reflect those of the United States Government or any agency thereof. 
Reactor and Nuclear Systems Division

METALLIC FUEL BENCHMARK SIMULATIONS BASED ON THE X430 EXPERIMENTS

\author{
Ian Greenquist \\ Jeffrey J. Powers
}

July 2020

Prepared by

OAK RIDGE NATIONAL LABORATORY

Oak Ridge, TN 37831-6283

managed by

UT-BATTELLE, LLC

for the

US DEPARTMENT OF ENERGY

under contract DE-AC05-00OR22725 



\section{CONTENTS}

LIST OF FIGURES

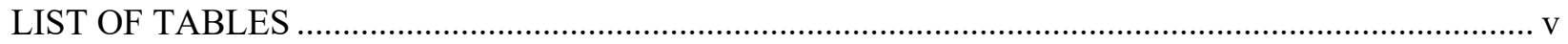

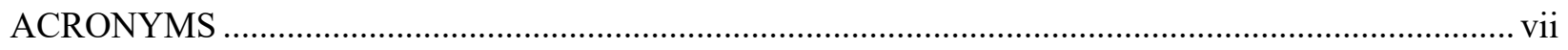

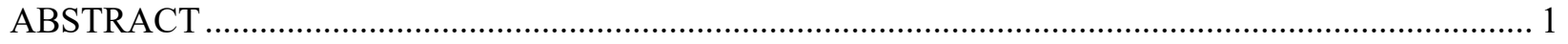

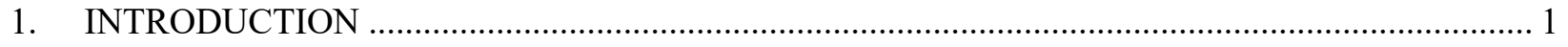

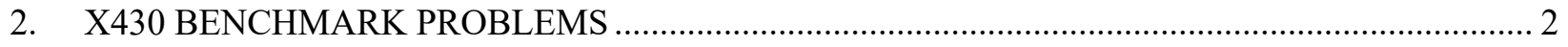

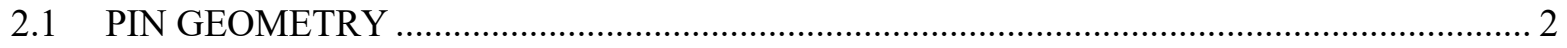

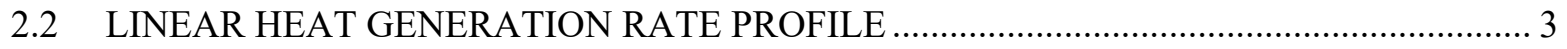

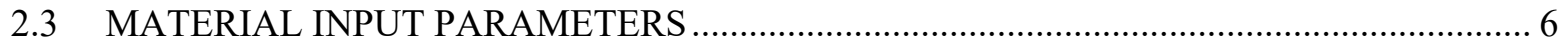

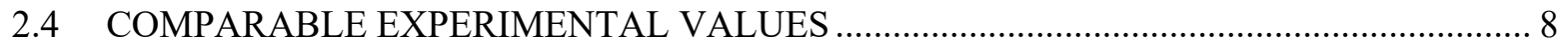

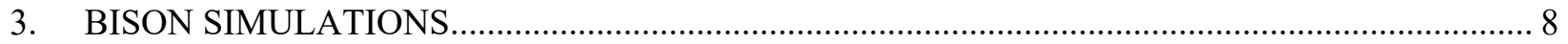

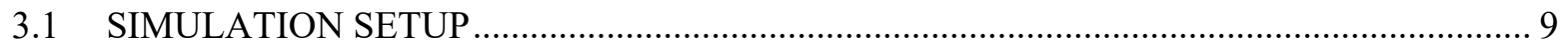

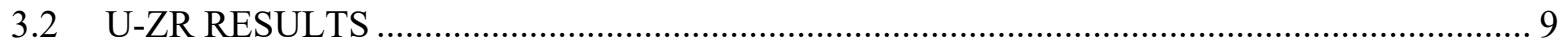

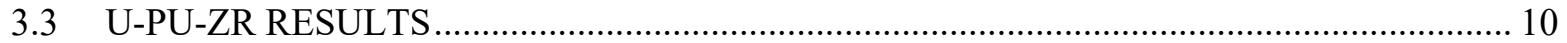

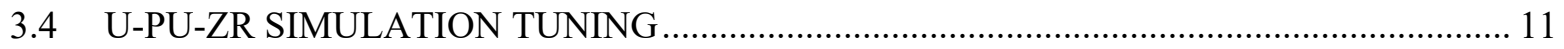

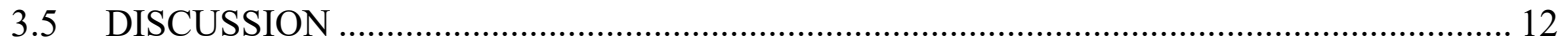

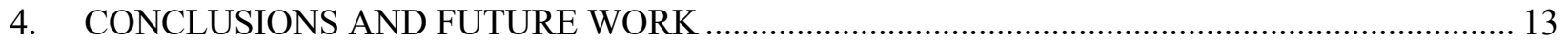

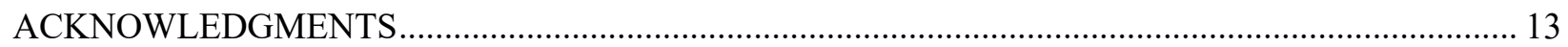

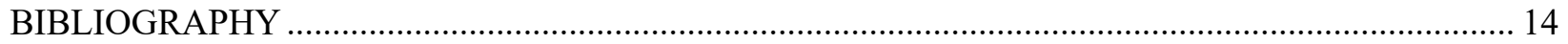

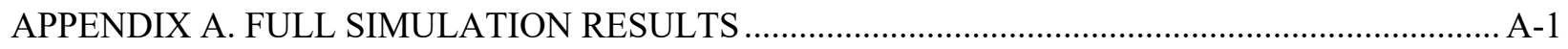

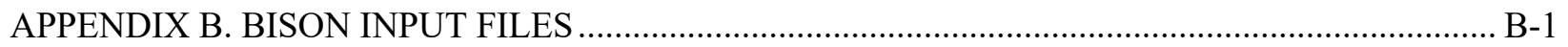





\section{LIST OF FIGURES}

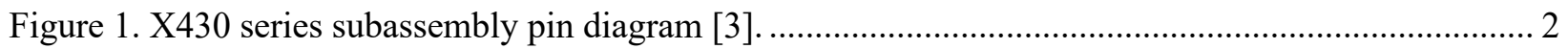

Figure 2. Fuel pin geometry for the X430 experiments (not to scale). .................................................. 3

Figure 3. LHGR and coolant inlet mass flux used for the benchmark problem. ....................................... 5

Figure 4. Mesh results of the three simulations of the X430 experiment series. ...................................... 9

Figure 5. Simulation results of the U-Zr pin (red) compared with experimental results for pin

T651 (black). Shaded background denotes the experiment.

Figure 6. Simulation results of the U-Pu-Zr pin (red) compared with experimental results of pin T654 (black). Shaded background denotes the experiment.

Figure 7. Simulation results of the tuned U-Pu-Zr pin (red) compared with experimental results of pin T654 (black). Shaded background denotes the experiment.

\section{LIST OF TABLES}

Table 1. Experiment and cycle information for the X430 experiment.

Table 2. Axial peaking factors for pins T651 (U-Zr) and T654 (U-Pu-Zr) at several axial positions and times. The peaking factor of each pin was derived by linearly interpolating these points.

Table 3. Physical parameters and modeling assumptions for $\mathrm{U}-\mathrm{Zr}$ fuel slug. ....................................... 6

Table 4. Physical parameters and modeling assumptions for $\mathrm{U}-\mathrm{Pu}-\mathrm{Zr}$ fuel slug.................................... 6

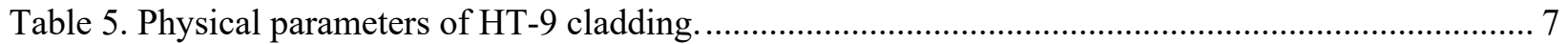

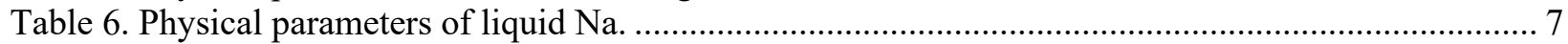





\section{ACRONYMS}

$\begin{array}{ll}\text { BOC } & \text { beginning of cycle } \\ \text { CTE } & \text { coefficient of thermal expansion } \\ \text { EBR-II } & \begin{array}{l}\text { Experimental Breeder Reactor II } \\ \text { effective full-power day }\end{array} \\ \text { EFPD } & \text { end of cycle } \\ \text { EOC } & \text { fission gas release } \\ \text { FGR } & \text { Integral Fast Reactor } \\ \text { IFR } & \text { Idaho National Laboratory } \\ \text { INL } & \text { linear heat generation rate } \\ \text { LHGR } & \text { Oak Ridge National Laboratory } \\ \text { ORNL } & \text { postirradiation examination }\end{array}$





\begin{abstract}
To gauge the accuracy of current BISON metallic fuel performance models and identify models that require further development, Oak Ridge National Laboratory has developed benchmark problems based on two pins of the X430 experiment. These benchmarks recreate the experimental conditions of the X430 series of experiments with as much accuracy as possible given the available data. The benchmarks were implemented in the BISON nuclear fuel performance code and the simulation results were compared with the experimental results to quantify the simulation accuracy.

Findings in this work were consistent with previous benchmark problems. The temperature profiles, burnup, and fission gas behavior were all as accurate as can reasonably be expected. The cladding radial growth was accurate enough to not affect the results of other measurements. The fuel axial growth was accurate for $\mathrm{U}-\mathrm{Zr}$ fuel but not for $\mathrm{U}-\mathrm{Pu}-\mathrm{Zr}$ fuel, which appears to be caused by a lack of U-Pu-Zr data. Swelling correlations have not been developed for U-Pu-Zr fuel because there were not enough data on this fuel type, so BISON used the U-Zr correlations. Finally, recommendations were made for near-term BISON development.
\end{abstract}

\title{
1. INTRODUCTION
}

To guide the development of metallic fuel performance models in BISON, Oak Ridge National Laboratory (ORNL) is developing metallic fuel benchmark problems based on past experiments. As part of that process, this work presents two benchmarks based on pins of the X430 series of experiments.

A benchmark problem is a simulation designed to recreate an experiment so that the simulation results can be compared against experimental results and designed to quantify the accuracy of the computational models. Previously, ORNL created a benchmark problem for uranium-19 wt $\%$ plutonium-10 wt $\%$ zirconium (U-Pu-Zr) fuel pins in the Integral Fast Reactor (IFR)-1 experiment [1,2]. In this current work, two models were developed based on two pins in the X430 series of experiments [3]. One pin was U-Pu$\mathrm{Zr}$ like in the IFR-1 benchmark. The other pin was uranium-10 wt $\%$ zirconium (U-Zr). By developing BISON models of two fuel types in the same experiment, the results from the two different fuels could be directly compared.

The X430 experiment series was irradiated in Experimental Breeder Reactor II (EBR-II). The series was designed to test the behavior of large-diameter fuel for the IFR [3] and was irradiated in three separate experiments. The X430 experiment ran from 1987 to early 1988, at which time the subassembly was removed and examined. Some pins were replaced so they could undergo destructive examination. The subassembly was rebuilt and placed back in EBR-II for the X430A experiment, which ran from late 1988 to early 1990. Again, some pins were removed and replaced. The subassembly was irradiated a third time in the X430B experiment from 1990 to 1992 . Of the 37 pins, 14 remained in place for all three experiments, as shown in Figure 1. Nine were U-Zr pins on the outer edge of the subassembly. Five were interior U-Pu-Zr pins. 


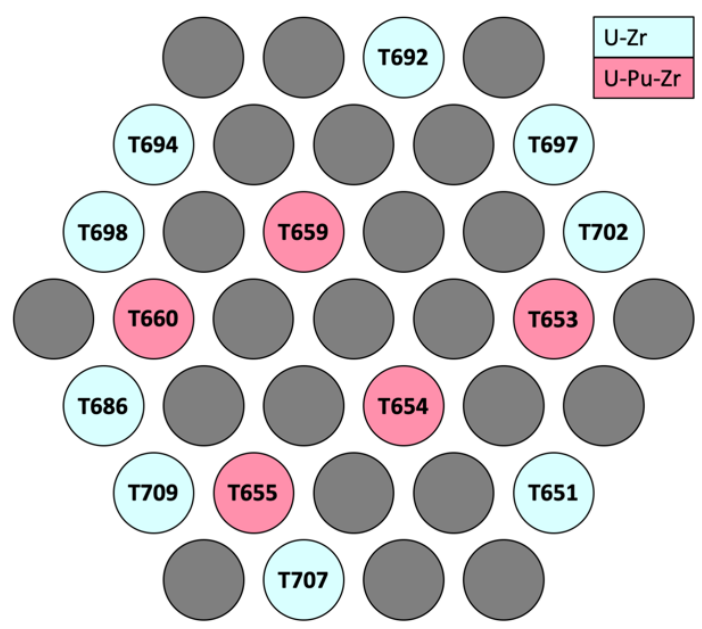

Figure 1. X430 series subassembly pin diagram [3]. The pins that stayed in the same position for all three experiments are labeled and colored according to fuel composition. The gray circles represent pins that were moved or replaced between experiments.

BISON is a fuel performance code under active development led by Idaho National Laboratory (INL). As such, this work is not meant to validate BISON metallic fuel models. The purpose of this work is to evaluate the models as they stand and point to areas in which improvements can be made in the near future. If any models are changed as a result of further development, these benchmark problems can be used to quantify any improvements and test new or modified models as they are developed.

\section{X430 BENCHMARK PROBLEMS}

The X430 report [3] includes pin-specific measurements for all the pins in the X430 experiment series. As such, specific pins could be simulated with reasonable accuracy. Two pins were chosen to be used as the basis for benchmark problems in this work: T651 (U-Zr) and T654 (U-Pu-Zr), both of which are identified in Figure 1. Once both benchmark problems were developed and simulated, their results could be directly compared to see how fuel composition affects BISON predictions.

\subsection{PIN GEOMETRY}

As part of the same subassembly, the two benchmark problems had identical dimensional design specifications. The fuel slugs were $343 \mathrm{~mm}$ long with a $5.72 \mathrm{~mm}$ diameter. The pins were $756 \mathrm{~mm}$ long, with the fuel slugs inside HT-9 stainless steel cladding [4] with a $6.55 \mathrm{~mm}$ inner diameter and a $7.37 \mathrm{~mm}$ outer diameter. The full dimensions are shown in Figure 2. Each pin was wrapped in a $1.4 \mathrm{~mm}$ diameter HT-9 helical wire, which acted as a spacer for the pins and a turbulence generator for the coolant. 


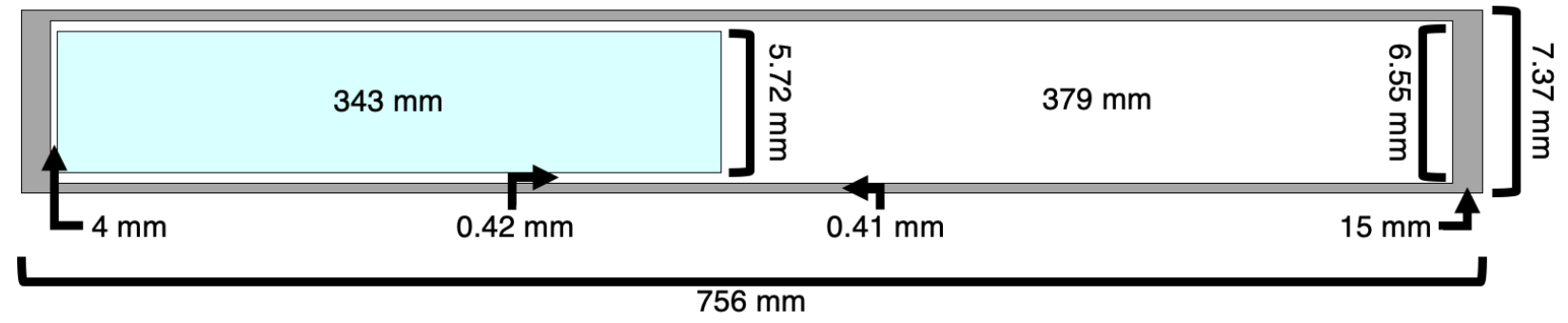

Figure 2. Fuel pin geometry for the X430 experiments (not to scale).

The largest source of uncertainty in the fuel dimensions was in the end plugs, which could have several different configurations. The bottom typically had a latching mechanism to hold it in place in the subassembly [5,6]. The tops could be rounded [7] or have features to facilitate handling during loading and hot cell examinations [5, 6]. However, BISON's internal mesh generator uses flat endcaps [8] by default. Since the X430 report [3] does not specify the pin end plug geometry and dimensions, this work assumed $15 \mathrm{~mm}$ thick flat endcaps. Since they affect the interior volume of the cladding, the endcaps could affect the calculated plenum pressure, but they were not expected to significantly impact the simulation results. Quantitative assessments confirmed that reasonable variations in end plug modeling choices in BISON did not significantly impact most simulation results currently being assessed, though this may need to be investigated further in the future if analyses try to assess performance or behavior of welds around the end plug.

\subsection{LINEAR HEAT GENERATION RATE PROFILE}

Among the most important input parameters for a fuel performance simulation was the linear heat generation rate (LHGR) of the fuel pins. The LHGR comprises two components: the average LHGR, $\overline{L H G R}(t)$, and the axial peaking factor, $p(t, z)$ :

$$
\operatorname{LHGR}(t, z)=\overline{\operatorname{LHGR}}(t) p(t, z)
$$

where $t$ is the time in seconds, and $z$ is the axial position along the pin in meters. The peaking factor represents the deviation from the average LHGR and so has the requirement

$$
\frac{1}{L} \int_{z_{0}}^{z_{0}+\mathrm{L}} p(z, t) d z=1
$$

where $z_{0}$ is the axial position of the pin bottom, and $L$ is the pin length. $\overline{L H G R}(t)$ was approximated using the power history information available in the X430 report [3]. Each of the three experiments was irradiated through multiple EBR-II operating cycles. For each cycle, the X430 report [3] recorded the subassembly power. Since the X430 report did not calculate individual pin LHGRs, the subassembly power was divided by 37 to calculate the average pin power, then divided by the fuel slug length of $0.3429 \mathrm{~m}$ to determine the average pin LHGR [W $\mathrm{m}^{-1}$ ]. This may cause significant error in the pinspecific simulations.

The length of each cycle was determined by dividing the total subassembly energy of each cycle (MW days) by an average operating power of EBR-II of $61.8 \mathrm{MW}$, which was estimated based on the EBR-II run data included in the X430 report [3]. This yielded the number of effective full-power days (EFPD).

Along with the LHGR, the coolant inlet mass flux was a necessary parameter for BISON simulations. This was calculated as 


$$
j_{c}(t)=\frac{V(t) \rho}{A}
$$

where $j_{c}$ is the coolant mass flux, $V \dot{(t)}$ is the coolant volumetric flow rate in the subassembly, $\rho$ is the coolant density, and $A$ is the cross-sectional area of the coolant channel. The coolant volumetric flow rate was recorded in the X430 report [3] as $3.56 \times 10^{-3} \mathrm{~m}^{3} \mathrm{~s}^{-1}$. The Na density was calculated according to Fink and Leibowitz [9] at an inlet temperature of $371^{\circ} \mathrm{C}$ [10] as $864.4 \mathrm{~kg} \mathrm{~m}^{-3}$. The area was approximated by subtracting the pin and spacing wire cross-sectional areas from the internal dimensions of the hexagonal subassembly [6]. This resulted in a coolant area of $1,118 \mathrm{~mm}^{2}$. The cycle time, average LHGR, and coolant mass flux for each EBR-II cycle are shown in

Table 1.

Table 1. Experiment and cycle information for the $\mathrm{X} 430$ experiment.

\begin{tabular}{|c|c|c|c|c|}
\hline Experiment & $\begin{array}{l}\text { EBR-II } \\
\text { cycle }\end{array}$ & $\begin{array}{l}\text { Cycle length } \\
\text { (EFPD) }\end{array}$ & $\begin{array}{l}\text { LHGR } \\
\left(\mathrm{W} \mathrm{m} \mathrm{m}^{-1}\right)\end{array}$ & $\begin{array}{c}\text { Coolant flux } \\
\left(\mathrm{kg} \mathrm{m}^{-2} \mathrm{~s}^{-1}\right)\end{array}$ \\
\hline \multirow{7}{*}{$\mathrm{X} 430$} & $144 \mathrm{~A}$ & 94.90 & $44,225.3$ & $2,699.1$ \\
\hline & $145 \mathrm{~A}$ & 64.90 & $43,106.1$ & $2,724.0$ \\
\hline & $146 \mathrm{~A}$ & 7.07 & $41,403.6$ & $2,697.2$ \\
\hline & $146 \mathrm{~B}$ & 79.63 & $41,119.9$ & $2,781.0$ \\
\hline & $147 \mathrm{~A}$ & 49.55 & $38,881.4$ & $2,721.1$ \\
\hline & $148 \mathrm{~A}$ & 7.65 & $38,353.3$ & $2,696.9$ \\
\hline & 148B & 74.64 & $39,472.5$ & $2,785.4$ \\
\hline \multirow{8}{*}{$\mathrm{X} 430 \mathrm{~A}$} & $150 \mathrm{~A}$ & 1.94 & $33,490.2$ & $2,793.7$ \\
\hline & $150 \mathrm{~B}$ & 77.25 & $36,863.6$ & $2,803.5$ \\
\hline & $151 \mathrm{~A}$ & 30.28 & $37,123.7$ & $2,814.2$ \\
\hline & $151 \mathrm{~B}$ & 18.79 & $32,717.8$ & $2,799.6$ \\
\hline & $152 \mathrm{~A}$ & 0.83 & $38,534.6$ & $2,840.1$ \\
\hline & $152 \mathrm{~B}$ & 5.81 & $38,432.1$ & $2,839.6$ \\
\hline & $152 \mathrm{C}$ & 34.50 & $36,784.8$ & $2,873.7$ \\
\hline & $152 \mathrm{D}$ & 9.37 & $36,036.0$ & $2,855.7$ \\
\hline \multirow{14}{*}{$\mathrm{X} 430 \mathrm{~B}$} & $155 \mathrm{~A}$ & 44.76 & $35,153.3$ & $2,826.4$ \\
\hline & $155 \mathrm{~B}$ & 20.73 & $35,153.3$ & $2,826.4$ \\
\hline & $156 \mathrm{~B}$ & 95.45 & $35,271.5$ & $2,788.4$ \\
\hline & $157 \mathrm{~A}$ & 1.47 & $33,663.6$ & $2,780.6$ \\
\hline & $157 \mathrm{C}$ & 4.17 & $34,459.7$ & $2,771.8$ \\
\hline & $157 \mathrm{D}$ & 21.91 & $34,640.9$ & $2,781.5$ \\
\hline & $157 \mathrm{H}$ & 2.85 & $34,428.1$ & $2,817.1$ \\
\hline & $157 \mathrm{~J}$ & 32.01 & $34,026.2$ & $2,807.4$ \\
\hline & $158 \mathrm{~A}$ & 53.43 & $33,624.2$ & $2,777.1$ \\
\hline & $158 \mathrm{~B}$ & 0.31 & $33,624.2$ & $2,777.1$ \\
\hline & $160 \mathrm{~A}$ & 53.75 & $33,718.8$ & $2,746.4$ \\
\hline & 160B & 5.83 & $34,057.7$ & $2,765.9$ \\
\hline & $160 \mathrm{C}$ & 4.56 & $34,057.7$ & $2,765.9$ \\
\hline & $161 \mathrm{~A}$ & 31.28 & $34,215.3$ & $2,777.1$ \\
\hline
\end{tabular}


$\overline{L H G R}(t)$ and $j_{c}(t)$ were constant during each cycle with a 1 hour transition time placed between cycles. This was more physically accurate and less stressful for the numerical solver in BISON than instantaneous jumps from one set of values to another. There was also a 1 hour startup period at the beginning of each experiment to go from zero power to operating power and coolant temperature. Three hours were added to the end of each experiment for a power ramp-down and cooldown. In the first hour, the average LHGR ramped down to zero power. In the second hour, the coolant inlet temperature reduced to a postirradiation examination (PIE) temperature of $305 \mathrm{~K}$ [3]. The third hour let the system reach thermal equilibrium so that PIE measurement conditions could be achieved. Figure 3 shows $\overline{L H G R}(t)$ and $j_{c}(t)$ for the entire benchmark problem.
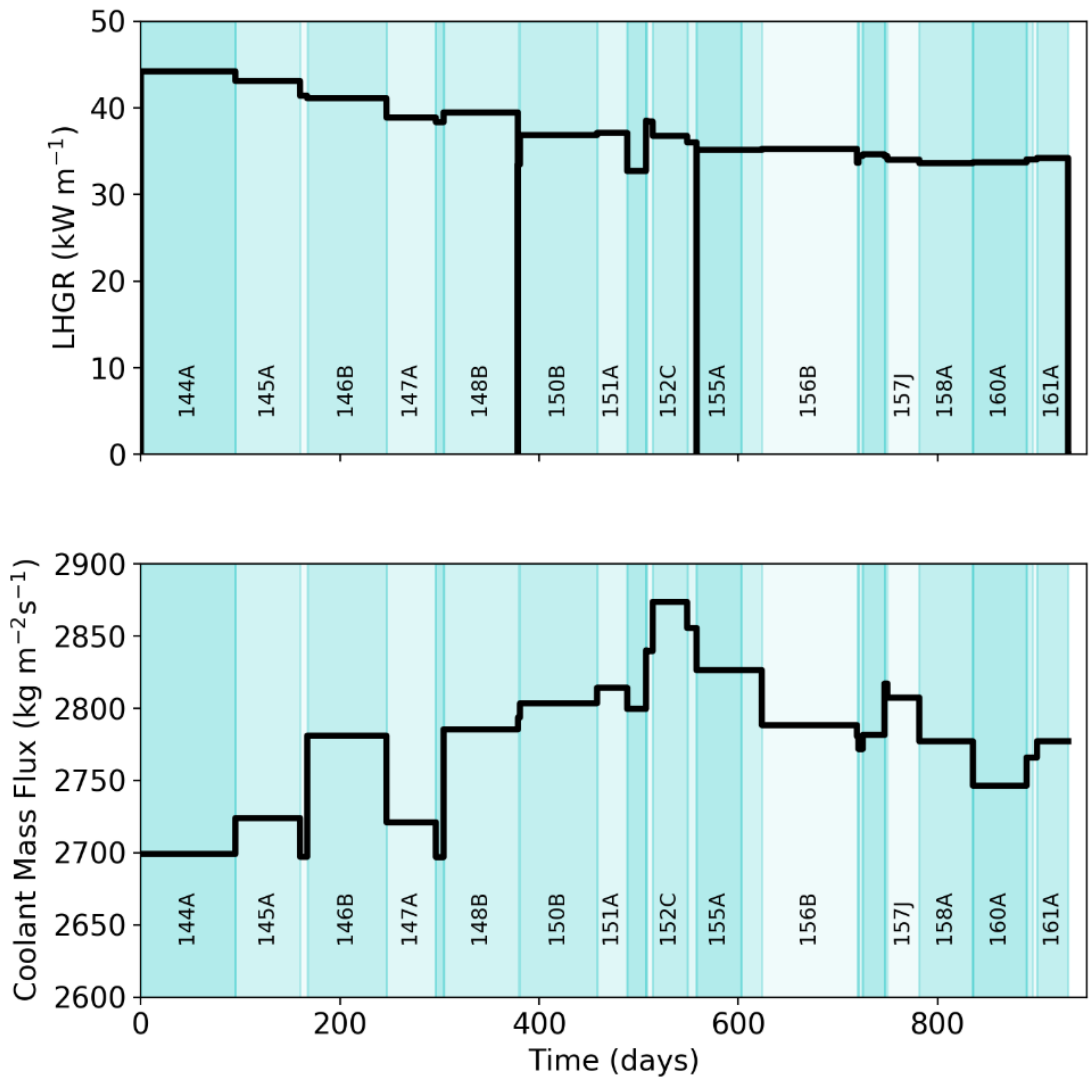

Figure 3. LHGR and coolant inlet mass flux used for the benchmark problem. Several cycles are labeled for reference.

The X430 report [3] did not contain enough pin-specific information to calculate $\overline{L H G R}(t)$ and $j_{c}(t)$ for individual pins, so the information in Table 1 and Figure 3 was derived based on the assumption that the power was evenly distributed radially among all the pins within this subassembly. However, there were enough pin-specific measurements to make separate $p(t, z)$ functions. At the beginning of cycle (BOC) of the first cycle of each experiment and the end of cycle (EOC) of the last cycle of X430B, the X430 report [3] calculated the LHGR for each pin at multiple axial positions. These were converted into peaking factors by approximating an average LHGR at each time. Table 2 shows the peaking factors for both pins at the times and positions from the X430 report [3]. $p(t, z)$ was calculated by linearly interpolating between the axial points at each time, then linearly interpolating between times. This formulation satisfies Eq. (2). 
Table 2. Axial peaking factors for pins T651 (U-Zr) and T654 (U-Pu-Zr) at several axial positions and times. The peaking factor of each pin was derived by linearly interpolating these points.

\begin{tabular}{c|cc|cc|cc|cc}
\hline $\boldsymbol{\Delta z}$ & \multicolumn{2}{|c|}{ X430 BOC } & \multicolumn{2}{c|}{ X430A BOC } & \multicolumn{2}{c|}{ X430B BOC } & \multicolumn{2}{c}{ X430B EOC } \\
$(\mathbf{m m})$ & $\mathbf{U}-\mathbf{Z r}$ & $\mathbf{U - P u}-\mathbf{Z r}$ & $\mathbf{U - Z r}$ & $\mathbf{U - P u}-\mathbf{Z r}$ & $\mathbf{U - Z r}$ & $\mathbf{U - P u}-\mathbf{Z r}$ & $\mathbf{U - Z r}$ & $\mathbf{U - P u - Z r}$ \\
\hline 0.0 & 0.914 & 0.888 & 0.940 & 0.906 & 0.935 & 0.896 & 0.915 & 0.895 \\
3.4 & 0.923 & 0.898 & 0.945 & 0.914 & 0.942 & 0.905 & 0.922 & 0.903 \\
34.3 & 1.003 & 0.988 & 0.997 & 0.986 & 1.001 & 0.985 & 0.983 & 0.975 \\
68.6 & 1.061 & 1.063 & 1.048 & 1.049 & 1.050 & 1.044 & 1.043 & 1.037 \\
102.9 & 1.104 & 1.115 & 1.089 & 1.101 & 1.089 & 1.094 & 1.074 & 1.088 \\
137.2 & 1.119 & 1.130 & 1.099 & 1.111 & 1.099 & 1.114 & 1.094 & 1.109 \\
171.5 & 1.104 & 1.115 & 1.089 & 1.101 & 1.089 & 1.104 & 1.094 & 1.098 \\
205.7 & 1.068 & 1.078 & 1.058 & 1.069 & 1.060 & 1.074 & 1.064 & 1.078 \\
240.0 & 1.003 & 1.003 & 1.007 & 1.018 & 1.001 & 1.014 & 1.023 & 1.016 \\
274.3 & 0.915 & 0.913 & 0.925 & 0.924 & 0.932 & 0.935 & 0.942 & 0.944 \\
308.6 & 0.814 & 0.808 & 0.843 & 0.831 & 0.844 & 0.835 & 0.851 & 0.842 \\
342.9 & 0.705 & 0.681 & 0.750 & 0.716 & 0.736 & 0.706 & 0.750 & 0.729 \\
\hline
\end{tabular}

\subsection{MATERIAL INPUT PARAMETERS}

Many of the material-specific models, such as fission gas release (FGR) and swelling, were calculated internally by BISON without the need for material-specific inputs. However, some parameters were required from the user. Tables 3-6 give the necessary material-specific values for $\mathrm{U}-\mathrm{Zr}$ fuel, $\mathrm{U}-\mathrm{Pu}-\mathrm{Zr}$ fuel, HT-9 cladding, and liquid Na coolant. If taken directly from the literature, then references are included. Values that were not taken from the literature are explained in more detail as follows.

Table 3. Physical parameters and modeling assumptions for $\mathrm{U}-\mathrm{Zr}$ fuel slug.

\begin{tabular}{llc}
\hline Parameter & Value & Reference \\
\hline Anisotropic swelling factor & 0.5 & \\
Atom fraction of Zr & 0.225 & {$[11]$} \\
$\mathrm{CTE}^{*}$ & $16.6 \times 10^{-6} \mathrm{~K}^{-1}$ & {$[11]$} \\
Density & $16,310 \mathrm{~kg} \mathrm{~m}^{-3}$ & {$[11]$} \\
Energy per fission & $3.2 \times 10^{-11} \mathrm{~J}$ & {$[12]$} \\
Fast flux factor & $5.67 \times 10^{14} \mathrm{n} \mathrm{J}^{-1} \mathrm{~m}^{-1}$ & \\
Friction coefficient & 0.2 & \\
\hline
\end{tabular}

*coefficient of thermal expansion

Table 4. Physical parameters and modeling assumptions for U-Pu-Zr fuel slug.

\begin{tabular}{llc}
\hline Parameter & Value & Reference \\
\hline Anisotropic swelling factor & 0.5 & \\
Atom fraction of $\mathrm{Pu}$ & 0.160 & {$[11]$} \\
Atom fraction of $\mathrm{Zr}$ & 0.226 & {$[11]$} \\
$\mathrm{CTE}$ & $17.3 \times 10^{-6} \mathrm{~K}^{-1}$ & {$[11]$} \\
Density & $15,800 \mathrm{~kg} \mathrm{~m}^{-3}$ & {$[11]$} \\
Energy per fission & $3.2 \times 10^{-11} \mathrm{~J}$ & {$[12]$} \\
Fast flux factor & $5.76 \times 10^{14} \mathrm{n} \mathrm{J}^{-1} \mathrm{~m}^{-1}$ & \\
Friction coefficient & 0.2 & \\
\hline
\end{tabular}


Table 5. Physical parameters of HT-9 cladding.

\begin{tabular}{llc}
\hline Parameter & Value & Reference \\
\hline CTE & $1.28 \times 10^{-5} \mathrm{~K}^{-1}$ & {$[4]$} \\
Density & $7,770.3 \mathrm{~kg} \mathrm{~m}^{-3}$ & {$[4]$} \\
Poisson's ratio & 0.2668 & {$[4]$} \\
Young's modulus & $1.726 \times 10^{11} \mathrm{~Pa}$ & {$[4]$} \\
\hline
\end{tabular}

Table 6. Physical parameters of liquid Na.

\begin{tabular}{llc}
\hline Parameter & Value & Reference \\
\hline Coolant inlet temperature & $644.15 \mathrm{~K}$ & {$[3]$} \\
Coolant pressure & $347,702.6 \mathrm{~Pa}$ & {$[10]$} \\
Gap thermal conductivity & $63.9 \mathrm{~W} \mathrm{~m}^{-1} \mathrm{~K}^{-1}$ & {$[9]$} \\
Plenum initial pressure & $91,193 \mathrm{~Pa}^{-1}$ & \\
\hline
\end{tabular}

The thermal expansion model needs more discussion before continuing since the same issue was found in both the metallic fuel and HT-9 cladding. The metallic fuels handbooks [4, 11] provided nonlinear thermal expansion equations, but BISON used a linear thermal expansion model:

$$
\frac{\Delta L}{L}=\alpha\left(T-T_{0}\right),
$$

where $L$ is the original length, $\Delta L$ is the displacement, $T_{0}$ is the stress-free temperature (the temperature corresponding to $L$ ), $T$ is the current temperature, and $\alpha$ is the CTE. To use the BISON model, linear fits were applied to the nonlinear equations. The CTEs given in Tables 3-5 were the slopes of those fits. Then, to maintain the room temperature dimensions of the fuel, every material's stress-free temperature was set to $295 \mathrm{~K}$.

Table 3 lists the parameters for the U-Zr fuel in pin T651. Most values were taken from the literature. The anisotropic swelling factor is a fitting parameter that determines how much swelling occurs in the radial and axial directions. Swelling factor values of $-1,0$, and 1 correspond to $100 \%$ axial swelling, isotropic swelling, and $100 \%$ radial swelling, respectively. Since swelling occurs preferentially in the radial direction [7], a value of 0.5 was assumed.

The fast flux factor is the ratio of fast neutron flux $\left(\mathrm{n} \mathrm{m}^{-2} \mathrm{~s}^{-1}\right)$ to LHGR $\left(\mathrm{W} \mathrm{m}^{-1}\right)$. To determine the factor, the final fast neutron fluence of pin T651 $\left(5.72 \times 10^{27} \mathrm{n} \mathrm{m}^{-2}\right)$ was taken from the X430 report [3] and divided by the time integral of $\overline{L H G R}(t)\left(3.04 \times 10^{12} \mathrm{~J} \mathrm{~m}^{-1}\right)$. The fast neutron flux at any time and axial position is the fast flux factor multiplied by $\operatorname{LHGR}(t, z)$.

The friction coefficient is another fitting parameter that controls the fuel/cladding friction behavior of BISON's contact model. The value was not known exactly for the current fuels, but previous work showed that the simulation results are insensitive to the value [2]. A value of 0.2 was assumed for this work.

Table 4 lists the parameters for the U-Pu-Zr fuel pin T654. The anisotropic swelling factor and friction coefficient were assumed to be the same values as for $\mathrm{U}-\mathrm{Zr}$ fuel. The fast flux factor was calculated in a similar manner as $\mathrm{U}-\mathrm{Zr}$ fuel but with the T654 fast neutron fluence $\left(1.75 \times 10^{27} \mathrm{n} \mathrm{m}^{-2}\right)$. 
Table 5 lists the parameters for the HT-9 cladding. All values are taken from Hofman et al. [4]. Table 6 lists the input parameters for the liquid $\mathrm{Na}$ used as a coolant and bonding agent between the fuel and cladding. The plenum initial pressure was unknown, so a value of $0.9 \mathrm{~atm}$ was assumed based upon the pin assembly having been performed in a glove box that was kept pressurized to slightly below the air pressure (nominally atmospheric or slightly sub-atmospheric pressure) in the surrounding room.

BISON also required inputs to control the FGR model. The values used in this work were provided by Casagranda [13] for U-Pu-Zr fuel. FGR is discussed further in Section 2.4.

\subsection{COMPARABLE EXPERIMENTAL VALUES}

To directly compare the BISON simulation results with the experimental results, it was important to identify experimental measurements and calculations that could be replicated by BISON. The pin diameter and fuel slug width were measured at hot cell temperatures during nondestructive examinations at the end of each experiment. The coolant outlet temperature was measured during each cycle and used to approximate a peak cladding temperature. The burnup was also calculated at the end of each cycle. These five measurements could be directly replicated by BISON.

Additionally, the U-Pu-Zr pins underwent destructive examination at the end of experiment X430B to measure plenum pressure and calculate FGR. Since plenum pressure was the directly measured value and comparisons have already been made between BISON and experimental FGR values [2], this work compared the simulated and experimental plenum pressure at the end of experiment $\mathrm{X} 430 \mathrm{~B}$ for the U-Pu$\mathrm{Zr}$ pin. Plenum pressure was not measured for any of the U-Zr pins in X430.

\section{BISON SIMULATIONS}

Three simulations were performed. All simulations were performed using the latest BISON version of as of April 3, 2020: git commit 575b0 8bb2d8b20f1ade 75 cb8cff 094844 b5e481e. Raw simulation outputs are provided in Appendix A. The U-Zr pin and the U-Pu-Zr pin were both simulated using the parameters listed in Section 2. The input files developed and used are provided in Appendix B. Then, two parameters of the U-Pu-Zr model were tuned to better match the experimental dimensional changes. This is explained in more detail in Section 3.4. Side-by-side comparisons of the mesh results are shown in Figure 4. At each time shown, the three pins are shown side by side. The temperature can be seen in the color of the mesh, and the displacements are seen through mesh deformation. 


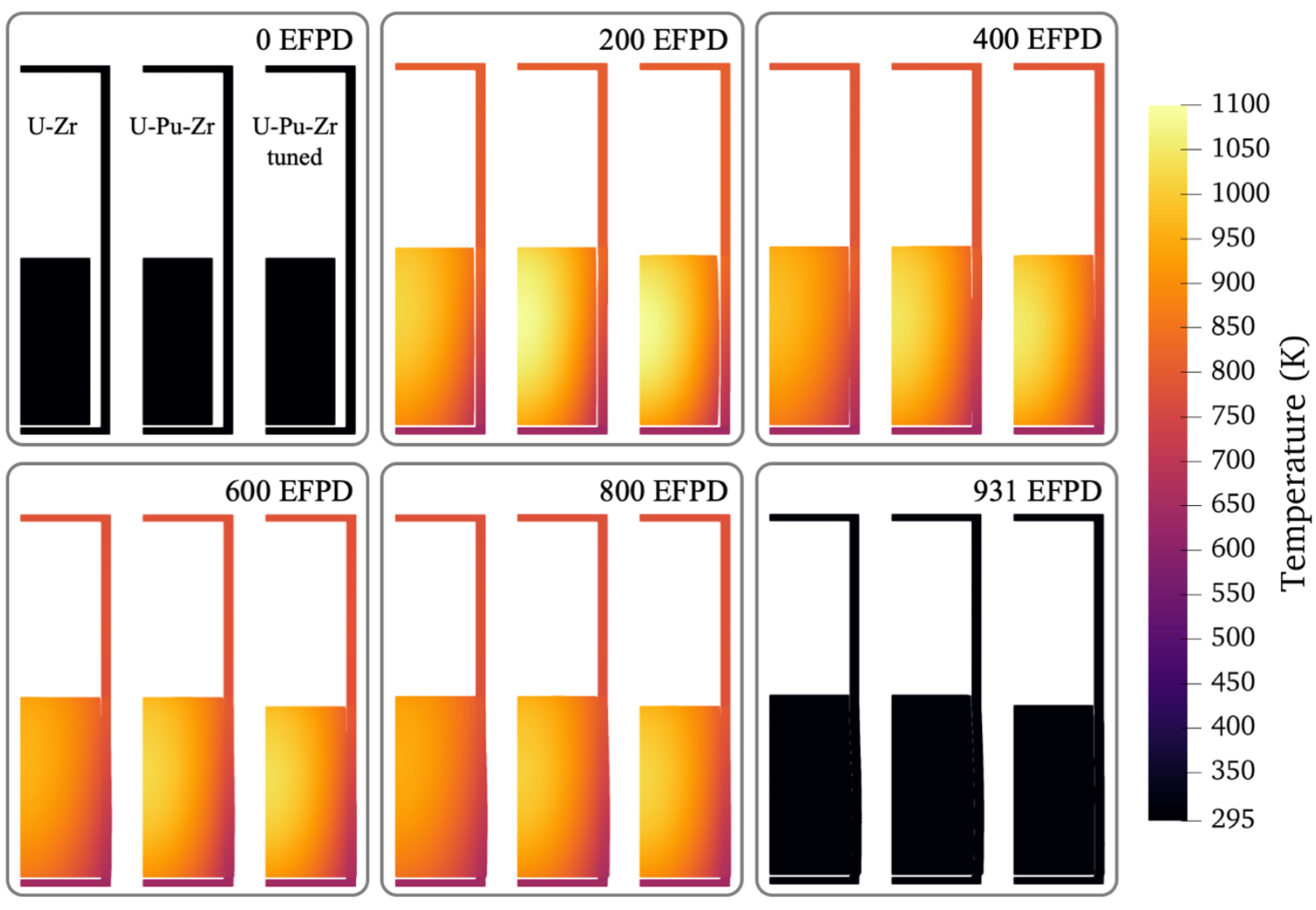

Figure 4. Mesh results of the three simulations of the X430 experiment series. The axial direction was compressed by a factor of 50 to make the results easier to view. The three simulations were compared side by side at six different times. At each time, the left mesh was the U-Zr pin, the center mesh was the U-Pu-Zr pin, and the right mesh was the tuned U-Pu-Zr pin.

\subsection{SIMULATION SETUP}

The simulation used a 2D R-Z axisymmetric mesh, which was more computationally efficient than a full 3D mesh. Two mesh blocks interacted: the fuel slug and the cladding. The plenum was the space between the blocks. The primary differential equation variables of the simulation were temperature, radial displacement, and axial displacement. Every other quantity was determined via auxiliary variables or postprocessing.

The initial conditions were a uniform temperature of $295 \mathrm{~K}$ and zero displacements. The temperature boundary conditions included zero-flux at the centerline and heat convection models that transported heat from the fuel slug to the cladding and coolant. The radial displacement boundary condition was zero displacement at the centerline. The axial displacement boundary conditions were zero displacement at the bottom edge of each mesh block.

\subsection{U-ZR RESULTS}

Five comparisons between the U-Zr simulation results and pin T651's experimental results are shown in Figure 5. The burnup was very accurate. The coolant outlet temperature and peak cladding temperature both had an offset bias throughout the simulation. On average, the bias was $35 \mathrm{~K}$. The cladding radial growth was accurate during experiments X430 and X430A, but predictions were significantly higher than 
experimental data by the end of X430B. The X430B radial growth was $0.6 \%(0.02 \mathrm{~mm})$ at X430B EOC, and the simulated growth was $2.7 \%(0.10 \mathrm{~mm})$ at the same point.
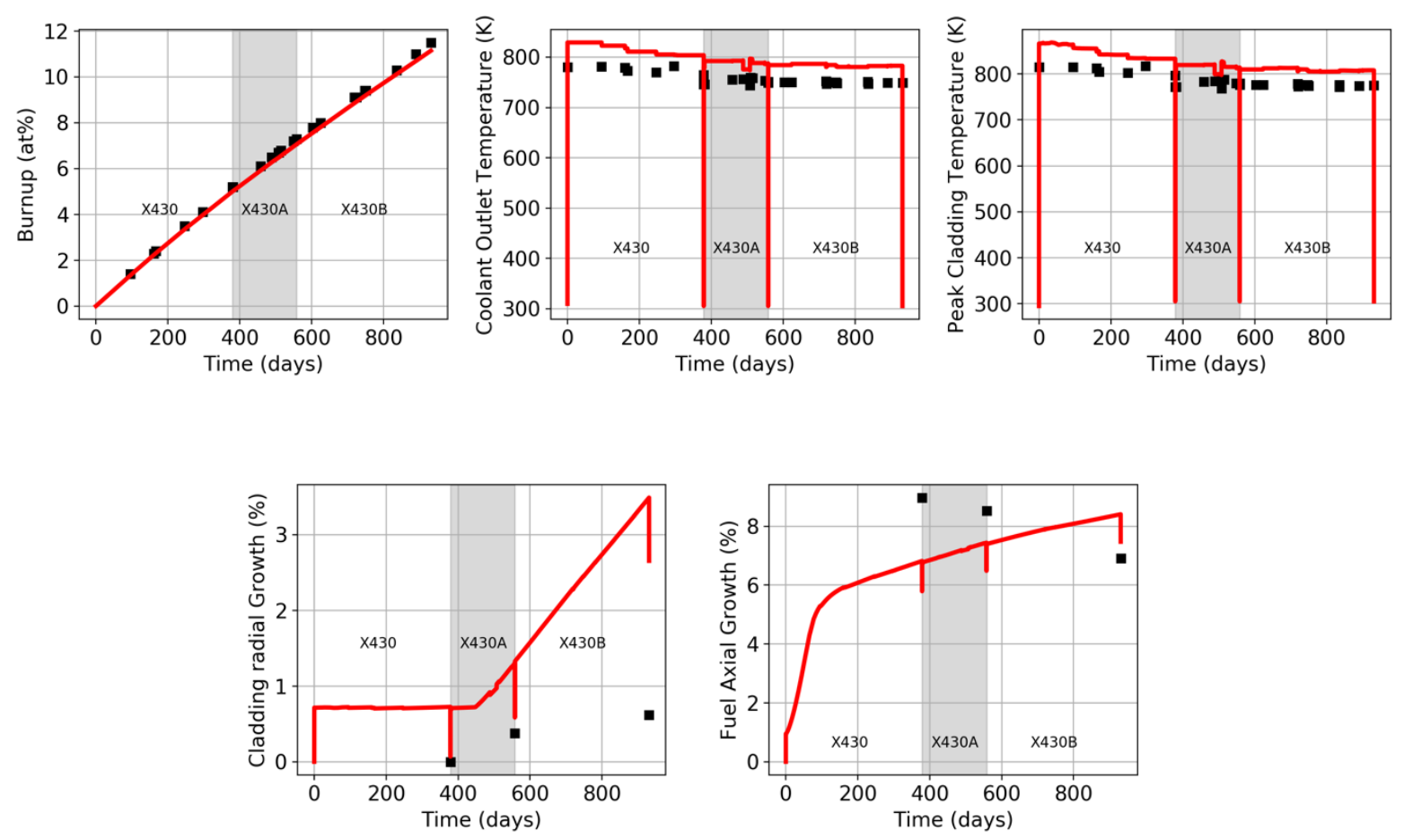

Figure 5. Simulation results of the U-Zr pin (red) compared with experimental results for pin T651 (black). Shaded background denotes the experiment.

According to the X430 report [3], all the pins had their peak axial growth after experiment X430. During experiments X430A and X430B, the slugs maintained their length or shrunk axially, as reflected in Figure 5. However, BISON predicted positive fuel growth throughout. The simulation under-predicted fuel growth in experiments X430 and X430A but was reasonably accurate by experiment X430B. The final experimental axial growth was $6.9 \%(24 \mathrm{~mm})$, and the simulated growth was $7.4 \%(26 \mathrm{~mm})$.

\subsection{U-PU-ZR RESULTS}

Six comparisons between the U-Pu-Zr simulation and the experimental results of pin $\mathrm{T} 654$ are shown in Figure 6 . Once again, burnup was very accurate. The coolant outlet temperature and peak cladding temperature were also very accurate; no bias showed in this pin between simulation and experimental results. On average, the simulated coolant and cladding temperatures were 4 and $3 \mathrm{~K}$ cooler than the respective experimental temperatures. 

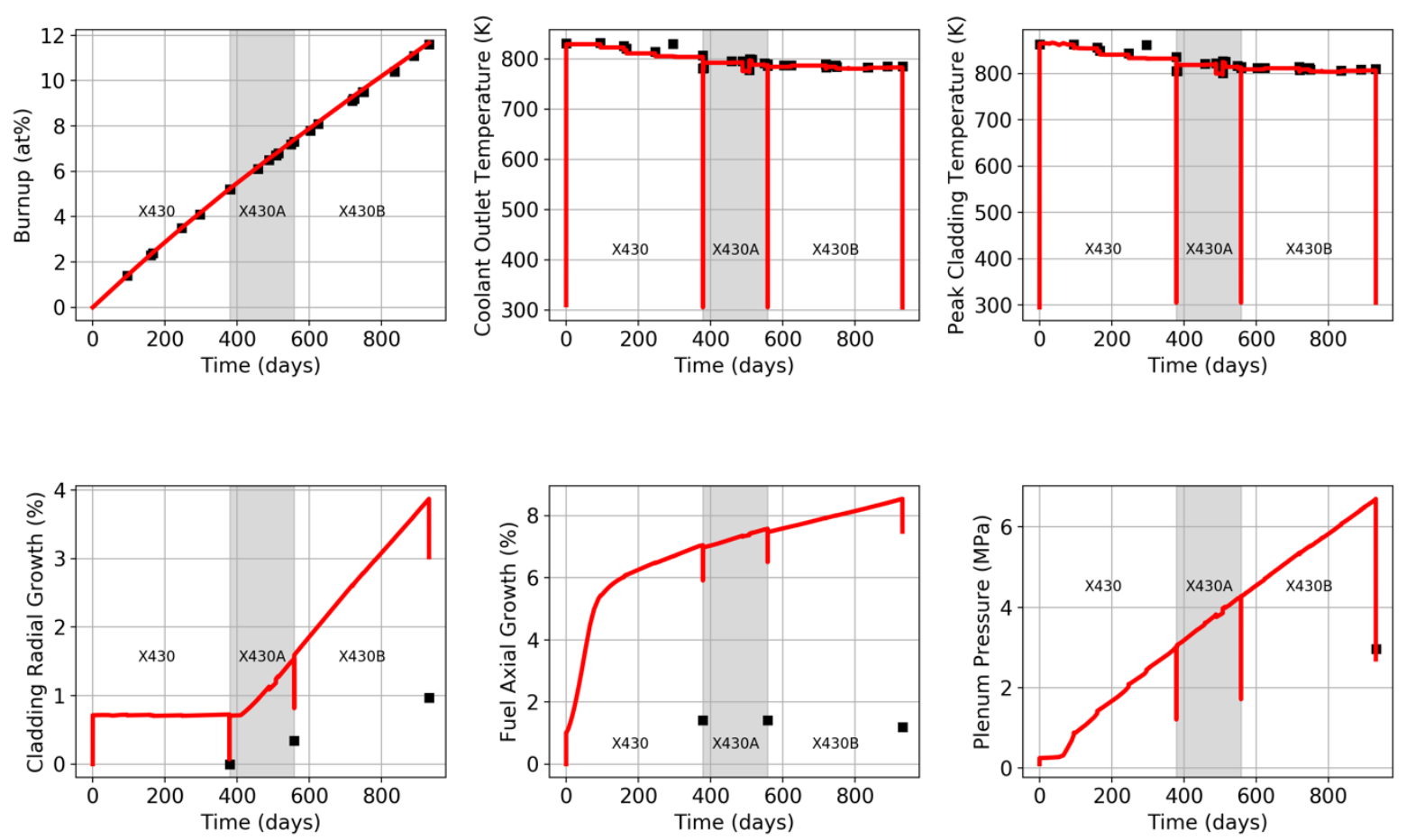

Figure 6. Simulation results of the U-Pu-Zr pin (red) compared with experimental results of pin T654 (black). Shaded background denotes the experiment.

The simulated and experimental cladding radial growths were once again very different. The experimental radial growth was $1.0 \%(0.04 \mathrm{~mm})$, and the simulated radial growth was $3.0 \%(0.11 \mathrm{~mm})$. The simulated fuel axial growth was about the same as for the U-Zr simulation: 7.5\% (26 mm). However, the experimental fuel axial growth was much smaller in U-Pu-Zr pins at only $1.2 \%(4 \mathrm{~mm})$.

The simulated plenum pressure was a relatively good match. The experimental pressure was $3.0 \mathrm{MPa}$, and the simulated pressure was $2.7 \mathrm{MPa}$.

\subsection{U-PU-ZR SIMULATION TUNING}

It was worth investigating whether the U-Pu-Zr simulation could be tuned to bring the cladding radial growth and fuel axial growth into better agreement with the experimental results without affecting the accuracy of the temperature, burnup, and plenum pressure. To do this, two parameters were chosen: the anisotropy factor and a coefficient applied to the gaseous and solid swelling of U-Pu-Zr. This tuning is meant to help isolate models that may be contributing to the inaccuracy of the U-Pu- $\mathrm{Zr}$ simulation. It is not meant to provide parameters that can be used to improve other simulations.

The parameter values that gave the best fit to the experimental data were an anisotropy factor of 0.948 and a swelling multiplier of 0.324 . In other words, the overall swelling was reduced by $68 \%$, but $97 \%$ of the swelling occurred in the radial direction.

The results of the tuned U-Pu-Zr simulation are shown in Figure 7. The burnup and temperatures were not affected. The cladding radial growth was much more accurate during the entire simulation, reaching $1.0 \%$. The fuel axial growth was still continuously increasing rather than peaking at X430, but the final 
value was more accurate at $1.3 \%$. The plenum pressure was slightly lower $(2.5 \mathrm{MPa})$, probably as a result of the reduced fuel volume.
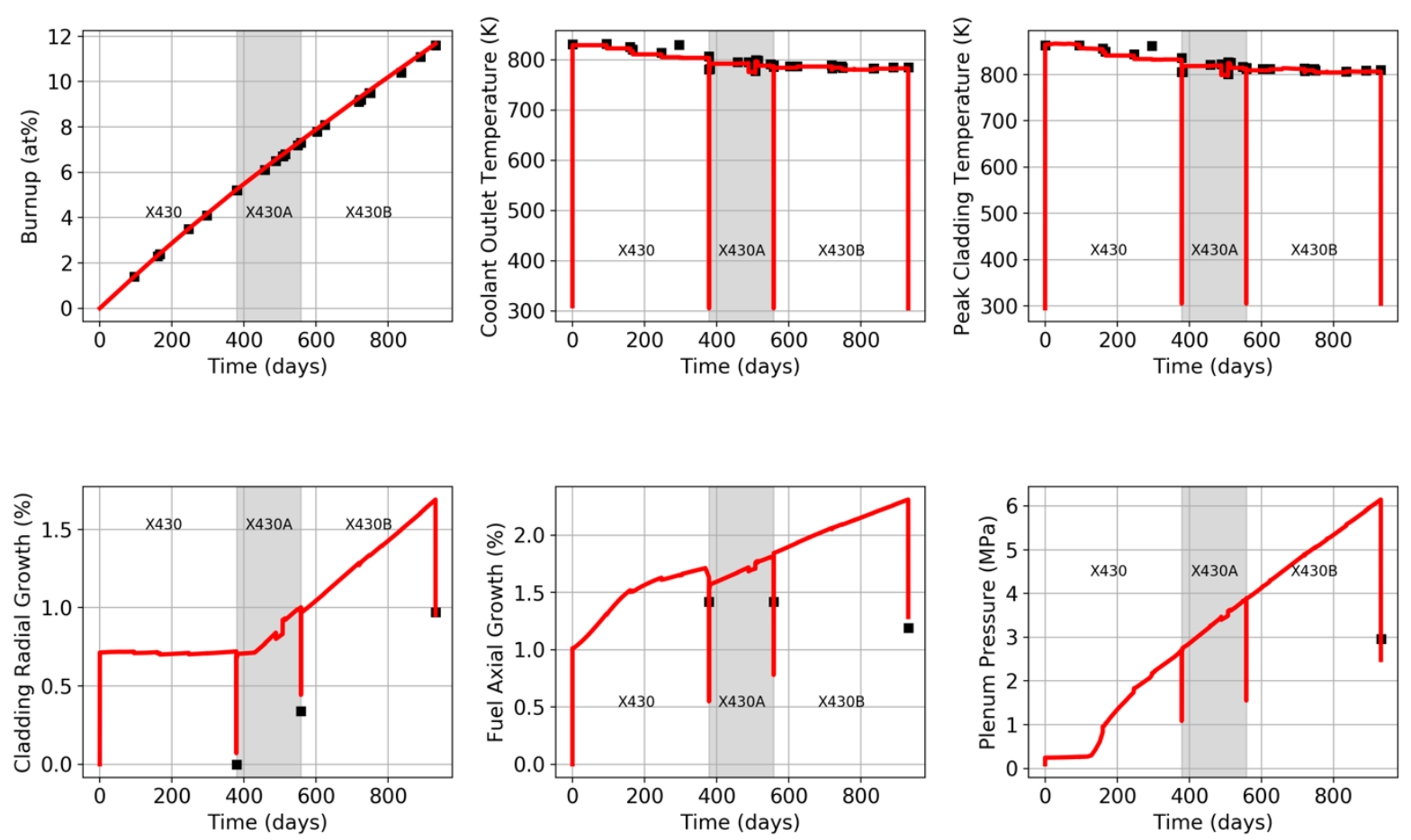

Figure 7. Simulation results of the tuned U-Pu-Zr pin (red) compared with experimental results of pin T654 (black). Shaded background denotes the experiment.

\subsection{DISCUSSION}

The U-Pu-Zr temperature profile was as accurate as could be expected. On the other hand, the U-Zr temperature was consistently too high. The U-Zr temperature bias did not appear to be a problem with the LHGR function; if the fuel were producing less fission heat than BISON assumed, then the simulated burnup would be inaccurate. The more likely cause is that the edges of the subassembly had higher coolant flow. The flat-to-flat distance of the experimental subassembly was $56.4 \mathrm{~mm}$ [6], but a tightly packed hexagon of fuel pins with wire spacers of the dimensions in Section 2.1 would have a flat-to-flat distance of $55.9 \mathrm{~mm}$. Therefore, each edge had up to $0.3 \mathrm{~mm}$ of additional coolant channel. Combine this extra space with the fact that edge pins have additional coolant flow even in a tightly packed subassembly, and an edge pin has a $20 \%$ larger coolant channel. The coolant channel model in BISON could be adjusted to increase the flow by increasing the rod pitch from 8.788 to $9.645 \mathrm{~mm}$. Doing so decreased the temperatures by $53 \mathrm{~K}$ so that they were $18 \mathrm{~K}$ colder than the experiment temperatures. This somewhat dramatic drop in temperature showed that the benchmark coolant channel boundary condition can be modified to account for edge pin coolant flow, but it will be more complicated than a simple linear scaling.

Swelling and growth behavior of the U-Zr and U-Pu-Zr simulations were very similar despite the different compositions and different experimental behaviors. This is problematic because U-Pu-Zr is known to exhibit less axial elongation and more radial growth than U-Zr [14]. However, this appears to be due to a lack of experimental data more than a bug or problem with code development. The BISON 
models were based on correlations developed as part of the IFR program [4, 8]. Those correlations did not distinguish between $\mathrm{U}-\mathrm{Zr}$ and $\mathrm{U}-\mathrm{Pu}-\mathrm{Zr}$ because there were not enough data to make separate correlations [4]. Since no US fast reactor has been in use since the IFR program ended, very little new data have been collected since then.

The margin of error of experimental fuel axial growth can be rather large [15]. Metallic fuels often developed a "fluff" on the top of the slug that made accurate measurements from neutron radiography difficult [16]. This could contribute to the axial shrinkage in the U-Zr pin experimental data either through measurement error or as more fuel is consumed by fluff. It could also contribute to the differences between simulated and experimental results.

The U-Pu-Zr growth behavior was significantly improved by reducing the overall swelling by $68 \%$ and increasing the swelling anisotropy. However, it is not recommended to use these adjustments in other simulations because it has not been established whether these adjustments will improve other $\mathrm{U}-\mathrm{Pu}-\mathrm{Zr}$ simulations. More work is needed to generate generalized swelling parameters for U-Pu-Zr fuel.

Compared with the IFR-1 benchmark [2], which also used U-Pu-Zr fuel, the temperature profiles in this work were more accurate. This was likely due partly to better experimental data or better documentation of the experimental inputs, operating conditions, and PIE results. In the IFR-1 benchmark, complete datasets were unavailable for individual pins, so averages were used. Also, the coolant mass flux and peaking factors could not be adjusted over time. These factors compounded, resulting in a temperature bias of more than $40 \mathrm{~K}$. Interestingly, BISON underpredicted cladding radial growth for IFR-1 while overpredicting it here for $\mathrm{X} 430$. This suggests that the tuning parameters developed here might not improve the accuracy of other simulations since decreasing the cladding radial growth of the IFR-1 benchmark problem would increase the error.

\section{CONCLUSIONS AND FUTURE WORK}

BISON accurately predicted burnup and plenum pressure for two pins in the X430 experiment. It also accurately predicted the temperature profiles of interior fuel pins, but corrections must be as accurate for edge pins. The relative error of BISON cladding radial growth predictions was quite large, but it was less than $0.1 \mathrm{~mm}$ in absolute terms. This amount of error does not appear to impact the simulation results discussed in this work, so it cannot be recommended as a priority for immediate improvement. However, the cladding radial growth likely has a significant effect on cladding stress and cladding failure probability. To quantify these effects, additional benchmark problems based on experiments that measured cladding stress will be needed.

The BISON prediction of fuel axial growth was reasonably accurate for $\mathrm{U}-\mathrm{Zr}$ fuel but significantly inaccurate for $\mathrm{U}-\mathrm{Pu}-\mathrm{Zr}$ fuel. BISON did not appear to distinguish between $\mathrm{U}-\mathrm{Zr}$ and $\mathrm{U}-\mathrm{Pu}-\mathrm{Zr}$ in its swelling models. The necessary correlations to do so have not been calculated, and there might not be enough experimental data to do so. Future work should focus on determining what improvements can be made given the existing data and in gathering more data to develop better correlations.

\section{ACKNOWLEDGMENTS}

This work was funded by the US Department of Energy Office of Nuclear Energy Versatile Test Reactor project. The authors wish to thank Stephen Novascone, Al Casagranda, and Adam Zabriskie of the INL BISON development team and Alexander Lindsay of the INL MOOSE development team for their valuable input in BISON fuel performance models in metallic fuels. Additional thanks to Aaron Oaks of Argonne National Laboratory, Christopher Mathews of Los Alamos National Laboratory, and Doug 
Crawford and Doug Porter of INL for providing access to EBR-II documents and databases. Doug

Crawford and Doug Porter also gave input and suggestions based upon their own personal knowledge.

\section{BIBLIOGRAPHY}

[1] K. M. Cunningham, J. J. Powers and R. A. Lefebvre, "Modeling the IFR-1 Experiment: A BISON Metallic Fuel Benchmark," Oak Ridge National Laboratory ORNL/TM-2019/1270, Oak Ridge, Tennessee, 2020.

[2] I. Greenquist, K. M. Cunningham, J. Hu and J. J. Powers, "A Metallic Fuel Performance Benchmark Problem Based on the IFR-1 Experiment," Oak Ridge National Laboratory ORNL/TM-2020/1534, Oak Ridge, Tennessee, 2020.

[3] S. L. Hayes, D. C. Crawford and R. G. Pahl, "Test design description and postirradiation examination of the HT9 advanced driver fuel test (X430)," Argonne National Laboratory ANL-IFR-225, Idaho Falls, Idaho, 1994.

[4] G. L. Hofman, M. C. Billone, J. F. Koenig, J. M. Kramer, L. D. B. Lambert, L. Leibowitz, Y. Orechwa, D. R. Pedersen, D. L. Porter, H. Tsai and A. E. Wright, "Metallic Fuels Handbook," Argonne National Laboratory ANL-NSE-3, Argonne, Illinois, 1989.

[5] D. Olander, "Nuclear fuels - Present and future," Journal of Nuclear Materials, vol. 389, no. 1, pp. $1-22,2009$.

[6] E. Hutter, O. S. Seim and A. Smaardyk, "Instrumented Subassembly System and In-Core Instrument Test Facility of EBR-II," Argonne National Laboratory CONF-690910-, Argonne, Illinois, 1970.

[7] Y. I. Chang, "Technical Rationale for metal fuel in fast reactors," Nuclear Engineering and Technoogy, vol. 39, no. 3, pp. 161-170, 2007.

[8] Idaho National Laboratory, "BISON Manual," Idaho National Laboratory, [Online]. Available: https://mooseframework.org/bison/. [Accessed 27 March 2020].

[9] J. K. Fink and L. Leibowitz, "Thermodynamic and transport properties of sodium liquid and vapor," Argonne National Laboratory ANL/RE-95/2, Argonne, Illinois, 1995.

[10] E. Snyder, "Report of EBR-II Operations: Run 146 and 147," Argonne National Laboratory ANLEBR.R146 ANLEBR.R147, Idaho Falls, Idaho, 1988.

[11] D. E. Janney, "Metallic Fuels Handbook," Idaho National Laboratory INL/EXT-15-36520, Idaho Falls, Idaho, 2018.

[12] J. K. Shultis and R. E. Faw, Fundamentals of Nuclear Science and Engineering, Boca Raton, Florida: CRC Press, 2008.

[13] A. Casagranda, BISON Metallic Fuel/VTR Training, presented at Oak Ridge, Tennessee, Tennessee: Idaho National Laboratory, January 2020.

[14] W. J. Carmack, D. L. Porter, Y. I. Chang, S. L. Hayes, M. K. Meyer, D. E. Burkes, C. B. Lee, T. Mizuno, F. Delage and J. Somers, "Metallic fuels for advanced reactors," Journal of Nuclear Materials, vol. 392, no. 2, pp. 139-150, 2009.

[15] T. R. Wright and D. L. Porter, "Utilization of Several Post-Irradiation Measurement Techniques to Determine Axial Growth in EBR-II Fuel," Transactions of the American Nuclear Society, vol. 121, pp. 678-679, 2019.

[16] L. Capriotti and J. Harp, "Status PIE Report on Legacy EBR-II and FFTF Metallic Fuel Experiments," Idaho National Laboratory INL/EXT-19-55847-Rev000, Idaho Falls, Idaho, 2019. 
APPENDIX A. FULL SIMULATION RESULTS 

PIN T651 FULL RESULTS

\begin{tabular}{|c|c|c|c|c|c|c|c|c|c|c|c|c|c|c|c|c|}
\hline Time (s) & $\begin{array}{c}\text { Coolant outlet } \\
\text { temperature }(\mathrm{K})\end{array}$ & $\begin{array}{l}\text { Peak cladding } \\
\text { temperature (K) }\end{array}$ & $\begin{array}{c}\text { Peak fuel } \\
\text { temperature }(K)\end{array}$ & $\begin{array}{c}\text { Average cladding } \\
\text { internal } \\
\text { temperature (K) }\end{array}$ & $\begin{array}{c}\text { Peak cumulative } \\
\text { damage factor }\end{array}$ & $\begin{array}{l}\text { Cladding interior } \\
\text { volume }\left(\mathrm{m}^{3}\right)\end{array}$ & $\begin{array}{l}\text { Fission gas } \\
\text { release (\%) }\end{array}$ & $\begin{array}{l}\text { Fission gas } \\
\text { produced } \\
\text { (moles) }\end{array}$ & $\begin{array}{l}\text { Fission gas } \\
\text { released (moles) }\end{array}$ & $\begin{array}{l}\text { Fuel volume } \\
\left(\mathrm{m}^{3}\right)\end{array}$ & $\begin{array}{l}\text { Plenum gas } \\
\text { volume }\left(\mathrm{m}^{3}\right)\end{array}$ & $\begin{array}{l}\text { Peak cladding } \\
\text { radial growth } \\
\text { (m) }\end{array}$ & $\begin{array}{l}\text { Peak fuel axial } \\
\text { growth }(m)\end{array}$ & $\begin{array}{c}\text { Peak burnup } \\
\text { (at\%) }\end{array}$ & $\begin{array}{l}\text { Peak cladding } \\
\text { hoop strain }\end{array}$ & $\begin{array}{c}\text { Plenum pressure } \\
\quad(\mathrm{Pa})\end{array}$ \\
\hline 0 & & 295 & 295 & 295 & 0 & $2.45 E-05$ & & 0 & 0 & $8.80 \mathrm{E}-06$ & $1.57 \mathrm{E}-05$ & 0 & 0 & 0 & 0 & \\
\hline 100 & 307.664386 & \begin{tabular}{|l|}
308.726431 \\
\end{tabular} & \begin{tabular}{|l|}
314.56793 \\
\end{tabular} & \begin{tabular}{|l|}
307.620231 \\
\end{tabular} & 0 & $2.45 \mathrm{E}-05$ & 0 & $3.30 \mathrm{E}-10$ & 0 & $8.80 \mathrm{E}-06$ & $1.57 \mathrm{E}-05$ & $5.90 \mathrm{E}-07$ & $9.07 E-05$ & $2.25 \mathrm{E}-09$ & \begin{tabular}{ll|}
0.00016015 \\
\end{tabular} & 95069.9375 \\
\hline 225 & \begin{tabular}{|l|l|}
323.519746 \\
\end{tabular} & 325.916282 & \begin{tabular}{|l|l|}
338.750723 \\
\end{tabular} & 323.416899 & 0 & $2.45 \mathrm{E}-05$ & 0 & $1.67 \mathrm{E}-09$ & 0 & $8.81 \mathrm{E}-06$ & $1.57 \mathrm{E}-05$ & $1.37 \mathrm{E}-06$ & 0.00020348 & $\begin{array}{ll}1.14 \mathrm{E}-08 \\
\end{array}$ & $\begin{array}{ll}0.00037205 \\
\end{array}$ & 99914.5699 \\
\hline 381.25 & 343.380123 & $\begin{array}{l}347.416257 \\
\end{array}$ & 368.553316 & \begin{tabular}{|l|}
343.190372 \\
\end{tabular} & 0 & $2.45 \mathrm{E}-05$ & 0 & $4.79 \mathrm{E}-09$ & 0 & $8.82 \mathrm{E}-06$ & $1.57 \mathrm{E}-05$ & $2.35 \mathrm{E}-06$ & 0.00034351 & $3.28 \mathrm{E}-08$ & \begin{tabular}{|l|l|}
0.00063881 \\
\end{tabular} & 105972.416 \\
\hline 576.5625 & \begin{tabular}{|l|}
368.27283 \\
\end{tabular} & \begin{tabular}{|l|}
374.322073 \\
\end{tabular} & \begin{tabular}{|l|l|}
405.17448 \\
\end{tabular} & \begin{tabular}{|l|l|}
367.950715 \\
\end{tabular} & \begin{tabular}{|l|l|}
$2.72 E-67$ \\
\end{tabular} & $2.45 \mathrm{E}-05$ & 0 & $1.10 \mathrm{E}-08$ & 0 & $8.84 \mathrm{E}-06$ & $1.57 \mathrm{E}-05$ & $3.59 \mathrm{E}-06$ & 0.00051709 & $\begin{array}{ll}7.49 \mathrm{E}-08 \\
\end{array}$ & \begin{tabular}{|l|}
0.00097319 \\
\end{tabular} & 113549.334 \\
\hline 820.703125 & 399.496533 & $\begin{array}{l}408.016856 \\
\end{array}$ & \begin{tabular}{|l|l|}
450.033914 \\
\end{tabular} & \begin{tabular}{|c|}
398.969487 \\
\end{tabular} & $5.27 E-58$ & $2.46 \mathrm{E}-05$ & 0 & $2.22 \mathrm{E}-08$ & 0 & $8.85 \mathrm{E}-06$ & $1.57 \mathrm{E}-05$ & $5.13 \mathrm{E}-06$ & 0.00073191 & $1.52 \mathrm{E}-07$ & \begin{tabular}{ll|}
0.00139262 \\
\end{tabular} & 123026.583 \\
\hline \begin{tabular}{|l|}
1125.87891 \\
\end{tabular} & \begin{tabular}{|l|l|}
438.69497 \\
\end{tabular} & \begin{tabular}{|l|l|}
450.259778 \\
\end{tabular} & 504.814055 & 437.851242 & $5.32 E-49$ & $2.46 \mathrm{E}-05$ & 0 & $4.18 \mathrm{E}-08$ & 0 & $8.87 \mathrm{E}-06$ & $1.57 \mathrm{E}-05$ & 7.07E-06 & 0.00099741 & $2.86 \mathrm{E}-07$ & \begin{tabular}{|l|l|}
0.00191915 \\
\end{tabular} & 134882.859 \\
\hline 1507.34863 & 487.948765 & 503.279574 & 571.528373 & 486.626966 & $4.24 E-40$ & $2.47 \mathrm{E}-05$ & 0 & $7.49 \mathrm{E}-08$ & 0 & $8.90 \mathrm{E}-06$ & $1.58 \mathrm{E}-05$ & $9.51 \mathrm{E}-06$ & 0.00132518 & $5.12 \mathrm{E}-07$ & 0.0025808 & 149718.467 \\
\hline \begin{tabular}{|l|l|}
1984.18579 \\
\end{tabular} & 549.885009 & 569.987335 & \begin{tabular}{|l|l|}
652.686438 \\
\end{tabular} & 547.878479 & $2.32 E-31$ & $2.47 \mathrm{E}-05$ & 0 & $1.30 \mathrm{E}-07$ & 0 & $8.93 \mathrm{E}-06$ & $1.58 \mathrm{E}-05$ & $1.26 \mathrm{E}-05$ & 0.00172973 & $8.88 \mathrm{E}-07$ & 0.00341357 & 168288.842 \\
\hline 2580.23224 & 627.795717 & $\begin{array}{l}654.09879 \\
\end{array}$ & \begin{tabular}{|l|l|}
751.48133 \\
\end{tabular} & \begin{tabular}{l|l|l}
624.899785 \\
\end{tabular} & $5.29 \mathrm{E}-23$ & $2.48 \mathrm{E}-05$ & 0 & $2.20 \mathrm{E}-07$ & 0 & $8.97 \mathrm{E}-06$ & $1.58 \mathrm{E}-05$ & $1.65 \mathrm{E}-05$ & 0.00222846 & $1.50 \mathrm{E}-06$ & \begin{tabular}{ll|}
0.00446356 \\
\end{tabular} & 191545.12 \\
\hline \begin{tabular}{|l|l|}
3325.2903 \\
\end{tabular} & 725.734468 & $\begin{array}{ll}760.667229 \\
\end{array}$ & 871.949986 & 721.893221 & 4.17E-15 & $2.49 \mathrm{E}-05$ & 0 & $3.65 \mathrm{E}-07$ & 0 & $9.02 \mathrm{E}-06$ & $1.59 \mathrm{E}-05$ & $2.14 \mathrm{E}-05$ & \begin{tabular}{ll|}
0.00284404 \\
\end{tabular} & $2.49 \mathrm{E}-06$ & \begin{tabular}{ll|}
0.00578982 \\
\end{tabular} & 220681.41 \\
\hline 3600 & 761.937266 & 800.106795 & 915.756788 & \begin{tabular}{|l|l|}
757.844007 \\
\end{tabular} & $3.63 \mathrm{E}-13$ & $2.49 \mathrm{E}-05$ & 0 & $4.27 \mathrm{E}-07$ & 0 & $9.04 \mathrm{E}-06$ & $1.59 \mathrm{E}-05$ & $2.32 E-05$ & 0.00307114 & $2.92 E-06$ & \begin{tabular}{|l|l|}
0.00628135 \\
\end{tabular} & 231438.647 \\
\hline $\begin{array}{l}4345.05806 \\
\end{array}$ & 761.937266 & 800.112068 & \begin{tabular}{|l|l|}
915.757668 \\
\end{tabular} & \begin{tabular}{|l|l|}
757.848361 \\
\end{tabular} & $1.34 E-12$ & $2.49 \mathrm{E}-05$ & 0 & $\begin{array}{ll}6.04 \mathrm{E}-07 \\
\end{array}$ & 0 & $9.04 \mathrm{E}-06$ & $1.59 \mathrm{E}-05$ & $2.32 \mathrm{E}-05$ & 0.00307093 & $4.13 \mathrm{E}-06$ & 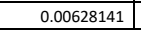 & 231440.144 \\
\hline 5276.38063 & 761.937266 & 800.112464 & 915.755698 & 757.848578 & $2.55 E-12$ & $2.49 \mathrm{E}-05$ & 0 & $8.25 \mathrm{E}-07$ & 0 & $9.04 \mathrm{E}-06$ & $1.59 \mathrm{E}-05$ & $2.32 E-05$ & 0.00307087 & $5.64 \mathrm{E}-06$ & $\begin{array}{l}0.00628142 \\
\end{array}$ & 231440.513 \\
\hline 6440.53385 & 761.937266 & 800.112559 & 915.754896 & 757.848844 & $4.07 E-12$ & $2.49 \mathrm{E}-05$ & 0 & $1.10 \mathrm{E}-06$ & 0 & $9.04 \mathrm{E}-06$ & $1.59 \mathrm{E}-05$ & $2.32 E-05$ & 0.00307096 & $7.53 \mathrm{E}-06$ & 0.00628143 & 231441.054 \\
\hline 7895.72538 & 761.937266 & 800.112275 & 915.75501 & 757.849177 & $5.97 E-12$ & $2.49 \mathrm{E}-05$ & 0 & $1.45 \mathrm{E}-06$ & 0 & $9.04 \mathrm{E}-06$ & $1.59 \mathrm{E}-05$ & $2.32 E-05$ & 0.0030712 & $\begin{array}{l}9.89 \mathrm{E}-06 \\
\end{array}$ & 0.00628144 & 231441.802 \\
\hline 9714.71478 & 761.937266 & 800.112817 & 915.757391 & 757.849641 & $8.34 \mathrm{E}-12$ & $2.49 \mathrm{E}-05$ & 0 & $1.88 \mathrm{E}-06$ & 0 & $9.04 \mathrm{E}-06$ & $1.59 \mathrm{E}-05$ & $2.32 \mathrm{E}-05$ & 0.00307163 & $1.28 \mathrm{E}-05$ & 0.00628146 & 231441.933 \\
\hline 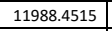 & 761.937266 & 800.11218 & 915.759322 & \begin{tabular}{|l|l|}
757.850211 \\
\end{tabular} & \begin{tabular}{|l|l|}
$1.13 E-11$ \\
\end{tabular} & $2.49 \mathrm{E}-05$ & 0 & $2.42 \mathrm{E}-06$ & 0 & $9.04 \mathrm{E}-06$ & $1.59 \mathrm{E}-05$ & $2.32 \mathrm{E}-05$ & 0.00307227 & $1.65 \mathrm{E}-05$ & 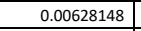 & 231443.003 \\
\hline 14830.6225 & 761.937266 & 800.111092 & 915.762733 & 757.850963 & $1.50 \mathrm{E}-11$ & $2.49 \mathrm{E}-05$ & 0 & $3.09 \mathrm{E}-06$ & 0 & $9.04 \mathrm{E}-06$ & $1.59 \mathrm{E}-05$ & $2.32 E-05$ & 0.00307317 & $2.12 E-05$ & 0.00628151 & 231444.431 \\
\hline 18383.3362 & 761.937266 & 800.109464 & 915.767991 & \begin{tabular}{|l|l|}
757.851963 \\
\end{tabular} & $1.96 E-11$ & $2.49 \mathrm{E}-05$ & 0 & $3.94 \mathrm{E}-06$ & 0 & $9.04 \mathrm{E}-06$ & $1.59 \mathrm{E}-05$ & $2.32 E-05$ & 0.0030744 & $2.69 \mathrm{E}-05$ & 0.00628154 & 231446.349 \\
\hline 22824.2283 & 761.937266 & 800.107224 & 915.775627 & 757.853301 & $2.54 E-11$ & $2.49 \mathrm{E}-05$ & 0 & $4.99 \mathrm{E}-06$ & 0 & $9.04 \mathrm{E}-06$ & $1.59 \mathrm{E}-05$ & $2.32 E-05$ & 0.00307604 & $3.41 \mathrm{E}-05$ & 0.00628158 & 231448.918 \\
\hline 28375.3434 & 761.937266 & 800.104263 & 915.786421 & \begin{tabular}{|l|}
757.8551 \\
\end{tabular} & $3.25 E-11$ & $2.49 \mathrm{E}-05$ & 0 & $6.31 \mathrm{E}-06$ & 0 & $9.04 \mathrm{E}-06$ & $1.59 \mathrm{E}-05$ & $2.32 \mathrm{E}-05$ & 0.00307822 & $4.31 \mathrm{E}-05$ & 0.00628163 & 231452.361 \\
\hline 35314.2373 & 761.937266 & 800.100435 & 915.801512 & 757.857526 & $4.15 E-11$ & $2.49 \mathrm{E}-05$ & 0 & $7.96 \mathrm{E}-06$ & 0 & $9.04 \mathrm{E}-06$ & $1.59 \mathrm{E}-05$ & $2.32 E-05$ & 0.00308112 & $5.44 \mathrm{E}-05$ & 0.00628169 & 231456.981 \\
\hline 43987.8547 & 761.937266 & 800.095548 & 915.822525 & 757.86081 & $5.26 \mathrm{E}-11$ & $2.49 \mathrm{E}-05$ & 0 & $1.00 \mathrm{E}-05$ & 0 & $9.04 \mathrm{E}-06$ & $1.59 \mathrm{E}-05$ & $2.32 \mathrm{E}-05$ & 0.00308497 & $6.85 \mathrm{E}-05$ & 0.00628176 & 231463.192 \\
\hline 54829.8764 & 761.937266 & 800.089371 & 915.851755 & 757.865263 & \begin{tabular}{|c|}
$6.64 E-11$ \\
\end{tabular} & $2.49 \mathrm{E}-05$ & 0 & $1.26 \mathrm{E}-05$ & 0 & $9.04 \mathrm{E}-06$ & $1.59 \mathrm{E}-05$ & $2.32 E-05$ & 0.00309009 & $8.61 E-05$ & 0.00628186 & 231471.563 \\
\hline 68382.4035 & 761.937266 & 800.081656 & 915.892412 & 757.871319 & $8.36 E-11$ & $2.49 \mathrm{E}-05$ & 0 & $1.58 \mathrm{E}-05$ & 0 & $9.04 \mathrm{E}-06$ & $1.59 \mathrm{E}-05$ & $2.32 E-05$ & 0.00309693 & 0.00010807 & 0.00628198 & 231482.872 \\
\hline 85323.0625 & 761.937266 & 800.072193 & 915.948981 & 757.879572 & $1.05 E-10$ & $2.49 \mathrm{E}-05$ & 0 & $1.98 \mathrm{E}-05$ & 0 & $9.04 \mathrm{E}-06$ & $1.59 \mathrm{E}-05$ & $2.32 \mathrm{E}-05$ & 0.00310608 & 0.00013557 & 0.00628214 & 231498.188 \\
\hline 106498.886 & 761.937266 & 800.060917 & 916.027717 & 757.890849 & $1.31 E-10$ & $2.49 \mathrm{E}-05$ & 0 & $2.49 \mathrm{E}-05$ & 0 & $9.04 \mathrm{E}-06$ & $1.59 \mathrm{E}-05$ & $2.32 E-05$ & 0.00311837 & 0.00016994 & 0.00628234 & 231518.981 \\
\hline 132968.666 & 761.937266 & 800.012407 & 916.128344 & \begin{tabular}{|l|l|}
757.905106 \\
\end{tabular} & $1.64 E-10$ & $2.49 \mathrm{E}-05$ & 0 & $3.11 \mathrm{E}-05$ & 0 & $9.04 \mathrm{E}-06$ & $1.59 \mathrm{E}-05$ & $2.32 E-05$ & 0.00313486 & 0.0002129 & 0.00628248 & 231579.036 \\
\hline 166055.89 & 761.937266 & 799.993227 & 916.277839 & 757.925953 & $2.04 E-10$ & $2.49 \mathrm{E}-05$ & 0 & $3.90 \mathrm{E}-05$ & 0 & $9.05 \mathrm{E}-06$ & $1.59 \mathrm{E}-05$ & $2.32 E-05$ & 0.00315716 & 0.00026661 & 0.00628281 & 231629.204 \\
\hline \begin{tabular}{|l|}
207414.921 \\
\end{tabular} & 761.937266 & 799.97926 & 916.486283 & 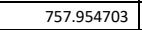 & $2.53 E-10$ & $2.49 \mathrm{E}-05$ & 0 & $4.88 \mathrm{E}-05$ & 0 & $9.05 \mathrm{E}-06$ & $1.59 \mathrm{E}-05$ & $2.32 E-05$ & 0.00318732 & 0.00033374 & \begin{tabular}{|c|}
0.0628326 \\
\end{tabular} & 231697.979 \\
\hline 259113.709 & 761.937266 & 799.975046 & 916.777446 & 757.994315 & $3.13 E-10$ & $2.49 \mathrm{E}-05$ & 0 & $6.11 \mathrm{E}-05$ & 0 & $9.06 \mathrm{E}-06$ & $1.58 \mathrm{E}-05$ & $2.32 E-05$ & 0.00322821 & 0.00041766 & 0.0062839 & 231792.353 \\
\hline 323737.195 & 761.937266 & 799.997175 & 917.183456 & 758.049068 & $3.86 \mathrm{E}-10$ & $2.49 \mathrm{E}-05$ & 0 & $7.64 \mathrm{E}-05$ & 0 & $9.06 \mathrm{E}-06$ & $1.58 \mathrm{E}-05$ & $2.32 E-05$ & 0.00328379 & 0.00052255 & 0.00628482 & 231922.164 \\
\hline 404516.551 & 761.937266 & 800.097161 & 917.750535 & 758.125415 & $4.75 E-10$ & $2.49 \mathrm{E}-05$ & 0 & $9.56 \mathrm{E}-05$ & 0 & $9.07 \mathrm{E}-06$ & $1.58 \mathrm{E}-05$ & $2.32 E-05$ & 0.00335952 & 0.00065366 & 0.00628623 & 232101.282 \\
\hline 505490.747 & 761.937266 & 800.368071 & 918.545691 & 758.232468 & $5.84 \mathrm{E}-10$ & $2.49 \mathrm{E}-05$ & 0 & 0.00011959 & 0 & $9.09 \mathrm{E}-06$ & $1.58 \mathrm{E}-05$ & $2.32 \mathrm{E}-05$ & 0.00346292 & 0.00081756 & 0.00628853 & 232349.154 \\
\hline 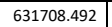 & 761.937266 & 800.447653 & 919.660464 & \begin{tabular}{|l|}
758.380895 \\
\end{tabular} & $7.16 E-10$ & $2.49 \mathrm{E}-05$ & 0 & 0.00014956 & 0 & $9.11 \mathrm{E}-06$ & $1.58 \mathrm{E}-05$ & $2.32 E-05$ & 0.00360438 & 0.00102243 & 0.00629084 & 232692.462 \\
\hline 789480.673 & 761.937266 & 800.322076 & 921.229365 & 758.588972 & $8.71 E-10$ & $2.49 \mathrm{E}-05$ & 0 & 0.00018702 & 0 & $9.14 \mathrm{E}-06$ & $1.58 \mathrm{E}-05$ & $2.32 E-05$ & 0.00379829 & 0.00127851 & 0.00629337 & 233169.971 \\
\hline 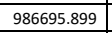 & 761.937266 & 801.506693 & 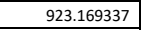 & 758.906462 & $1.02 E-09$ & $2.49 \mathrm{E}-05$ & 0 & 0.00023385 & 0 & $9.18 \mathrm{E}-06$ & $1.57 \mathrm{E}-05$ & $2.33 E-05$ & 0.00406431 & 0.00159862 & 0.0062894 & 233843.301 \\
\hline 1233214.93 & 761.937266 & 800.099733 & 925.176923 & 758.890161 & \begin{tabular}{l|l|}
$1.19 E-09$ \\
\end{tabular} & $2.49 \mathrm{E}-05$ & 0 & 0.00029238 & 0 & $9.23 \mathrm{E}-06$ & $1.57 \mathrm{E}-05$ & $2.32 \mathrm{E}-05$ & 0.00442422 & 0.00199875 & 0.00629387 & 234639.694 \\
\hline 1541363.72 & 761.937266 & 799.93368 & 927.999061 & 758.779953 & $1.40 \mathrm{E}-09$ & $2.49 \mathrm{E}-05$ & 0 & 0.00036555 & 0 & $9.31 \mathrm{E}-06$ & $1.56 \mathrm{E}-05$ & $2.33 \mathrm{E}-05$ & 0.0049168 & 0.00249892 & 0.00630002 & 235728.019 \\
\hline 1926549.71 & 761.937266 & 800.62031 & 931.968256 & 7558.69557 & \begin{tabular}{|c|}
$1.60 \mathrm{E}-09$ \\
\end{tabular} & $2.49 \mathrm{E}-05$ & 0 & 0.00045701 & 0 & $9.41 \mathrm{E}-06$ & $1.55 \mathrm{E}-05$ & $2.33 E-05$ & 0.00559282 & 0.00312413 & 0.00630178 & 237284.184 \\
\hline \begin{tabular}{|l|l|}
2408032.2 \\
\end{tabular} & 761.937266 & 801.413193 & 937.564383 & 758.544274 & $1.80 \mathrm{E}-09$ & $2.49 \mathrm{E}-05$ & 0 & 0.00057133 & 0 & $9.56 \mathrm{E}-06$ & $1.54 \mathrm{E}-05$ & $2.33 E-05$ & 0.00651832 & 0.00390564 & 0.00630595 & 239485.513 \\
\hline 3009885.31 & 761.937266 & 799.293945 & 945.513081 & 758.241071 & $2.08 \mathrm{E}-09$ & $2.49 \mathrm{E}-05$ & 0 & 0.00071423 & 0 & $9.76 \mathrm{E}-06$ & $1.51 \mathrm{E}-05$ & $2.33 \mathrm{E}-05$ & 0.00777887 & 0.00488253 & 0.00630957 & 242615.977 \\
\hline 3762201.69 & 761.937266 & 801.063059 & 956.67972 & 757.947336 & $2.93 E-09$ & $2.49 \mathrm{E}-05$ & 0 & 0.00089286 & 0 & $1.00 \mathrm{E}-05$ & $1.49 \mathrm{E}-05$ & $2.33 E-05$ & 0.00949038 & 0.00610364 & 0.00631633 & 247221.378 \\
\hline 4702597.17 & 761.937266 & 802.174079 & \begin{tabular}{|c|}
972.24509 \\
\end{tabular} & $\begin{array}{l}756.501321 \\
\end{array}$ & $2.94 \mathrm{E}-09$ & $2.49 \mathrm{E}-05$ & 0 & 0.00111614 & 0 & $1.05 \mathrm{E}-05$ & $1.45 \mathrm{E}-05$ & $2.33 E-05$ & 0.01176845 & 0.00763003 & 0.00627189 & 253654.73 \\
\hline 5702597.17 & 761.937266 & 800.896843 & 987.816975 & \begin{tabular}{|l|l|}
755.450589 \\
\end{tabular} & $2.95 E-09$ & $2.49 \mathrm{E}-05$ & 19.6523767 & 0.00135358 & 0.00026601 & $1.09 \mathrm{E}-05$ & $1.40 \mathrm{E}-05$ & $2.33 E-05$ & 0.01421281 & 0.00925317 & 0.00623906 & 261088.677 \\
\hline 6702597.17 & 761.937266 & 801.887494 & 997.387273 & \begin{tabular}{|l|l|}
756.74728 \\
\end{tabular} & $3.00 \mathrm{E}-09$ & $2.49 \mathrm{E}-05$ & 44.0882291 & 0.00159101 & 0.00070145 & $1.12 \mathrm{E}-05$ & $1.37 \mathrm{E}-05$ & $2.33 E-05$ & 0.0161813 & 0.0108763 & 0.00631955 & 390258.291 \\
\hline 7702597.17 & 761.937266 & 797.428062 & 1003.00893 & 756.643653 & $3.94 \mathrm{E}-09$ & $2.49 \mathrm{E}-05$ & 55.2015531 & 0.00182845 & 0.00100933 & $1.14 \mathrm{E}-05$ & $1.35 \mathrm{E}-05$ & $2.34 \mathrm{E}-05$ & 0.01750768 & 0.01249944 & 0.00634877 & 600132.069 \\
\hline 8203212 & 761.937266 & 797.567918 & 1004.61275 & 756.621718 & $4.67 \mathrm{E}-09$ & $2.49 \mathrm{E}-05$ & 58.1876039 & 0.00194731 & 0.00113309 & $1.15 \mathrm{E}-05$ & $1.34 \mathrm{E}-05$ & $2.35 E-05$ & 0.01796328 & 0.013312 & 0.0063582 & 748086.921 \\
\hline 8206812 & 757.872748 & & 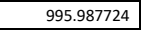 & 752.925032 & & $2.49 \mathrm{E}-05$ & 58.1949386 & 0.00194816 & 0.00113373 & $1.15 \mathrm{E}-05$ & $1.34 \mathrm{E}-05$ & $2.33 E-05$ & 0.01793191 & 0.01331777 & 0.00630028 & 802288.656 \\
\hline 8707426.83 & 757.872748 & 792.172201 & 996.338603 & 752.878316 & $5.06 \mathrm{E}-09$ & $2.49 \mathrm{E}-05$ & 60.1442509 & 0.00206401 & 0.00124138 & $1.16 \mathrm{E}-05$ & $1.34 \mathrm{E}-05$ & $2.33 E-05$ & 0.01820483 & 0.01410977 & 0.00630411 & 804844.636 \\
\hline 9333195.37 & 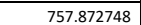 & 794.051783 & 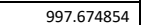 & 752.875748 & $5.57 \mathrm{E}-09$ & \begin{tabular}{|l|l|}
$2.49 E-05$ \\
\end{tabular} & 61.9798785 & 0.00220883 & 0.00136903 & $\begin{array}{ll}1.16 E-05 \\
\end{array}$ & $1.33 \mathrm{E}-05$ & \begin{tabular}{|l|l|}
$2.33 E-05$ \\
\end{tabular} & 0.0186384 & 0.01509978 & \begin{tabular}{|c|}
00631106 \\
\end{tabular} & 859761.135 \\
\hline
\end{tabular}




\begin{tabular}{|c|c|c|c|c|c|c|c|c|c|c|c|c|c|c|c|c|}
\hline Time (s) & $\begin{array}{l}\text { Coolant outlet } \\
\text { temperature (K) }\end{array}$ & $\begin{array}{l}\text { Peak cladding } \\
\text { temperature (K) }\end{array}$ & $\begin{array}{c}\text { Peak fuel } \\
\text { temperature (K) }\end{array}$ & \begin{tabular}{|c|} 
Average cladding \\
internal \\
temperature (K) \\
\end{tabular} & $\begin{array}{l}\text { Peak cumulative } \\
\text { damage factor }\end{array}$ & $\begin{array}{c}\text { Cladding interior } \\
\text { volume }\left(\mathrm{m}^{3}\right)\end{array}$ & $\begin{array}{l}\text { Fission gas } \\
\text { release (\%) }\end{array}$ & $\begin{array}{c}\begin{array}{c}\text { Fission gas } \\
\text { produced } \\
\text { (moles) }\end{array} \\
\end{array}$ & $\begin{array}{c}\text { Fission gas } \\
\text { released (moles) }\end{array}$ & $\begin{array}{c}\text { Fuel volume } \\
\left(\mathrm{m}^{3}\right)\end{array}$ & $\begin{array}{l}\text { Plenum gas } \\
\text { volume }\left(m^{3}\right)\end{array}$ & $\begin{array}{c}\text { Peak cladding } \\
\text { radial growth } \\
\text { (m) }\end{array}$ & $\begin{array}{l}\text { Peak fuel axial } \\
\text { growth (m) }\end{array}$ & $\begin{array}{c}\text { Peak burnup } \\
\text { (ato) }\end{array}$ & $\begin{array}{l}\text { Peak cladding } \\
\text { hoop strain }\end{array}$ & \begin{tabular}{|c} 
Plenum pressure \\
(Pa)
\end{tabular} \\
\hline 10115406 & 757.872748 & 791.774686 & 998.938093 & \begin{tabular}{|l|}
752.785972 \\
\end{tabular} & $6.24 \mathrm{E}-09$ & $2.49 \mathrm{E}-05$ & 63.2526286 & 0.00238986 & 0.00151165 & $1.17 \mathrm{E}-05$ & $1.32 \mathrm{E}-05$ & $2.33 \mathrm{E}-05$ & 0.01909755 & 0.01633728 & 0.00632004 & 924894.097 \\
\hline 11093169.4 & 757.872748 & 791.597036 & 999.962767 & \begin{tabular}{|l|l|}
752.700368 \\
\end{tabular} & $7.11 \mathrm{E}-09$ & $2.49 E-05$ & 64.5022451 & 0.00261614 & 0.00168747 & $1.18 \mathrm{E}-05$ & $1.31 E-05$ & $2.34 \mathrm{E}-05$ & 0.01956368 & 0.01788416 & 0.00633129 & 997800.878 \\
\hline 12093169.4 & 757.872748 & 791.457541 & 1000.52884 & 752.643878 & $8.06 \mathrm{E}-09$ & $2.49 \mathrm{E}-05$ & 65.5748534 & 0.00284756 & 0.00186729 & $1.18 \mathrm{E}-05$ & $1.31 E-05$ & $2.34 \mathrm{E}-05$ & 0.01994026 & 0.01946622 & 0.00634398 & 1086102.77 \\
\hline 13093169.4 & 757.872748 & 792.314917 & 1000.79694 & \begin{tabular}{|l|l|}
752.701031 \\
\end{tabular} & $9.12 \mathrm{E}-09$ & $2.49 E-05$ & 66.4862201 & 0.00307899 & 0.00204711 & 1.19E- 05 & $1.30 \mathrm{E}-05$ & $2.35 E-05$ & 0.02025332 & 0.02104828 & 0.00635729 & 1176229.15 \\
\hline 13814423 & 757.872748 & 791.994846 & 1000.86484 & \begin{tabular}{|l|l|}
752.668844 \\
\end{tabular} & $9.95 \mathrm{E}-09$ & $2.49 \mathrm{E}-05$ & 67.062878 & 0.00324591 & 0.0021768 & 1.19E- 05 & $1.30 \mathrm{E}-05$ & $2.35 E-05$ & 0.02044355 & 0.02218934 & 0.00636838 & 1265072.34 \\
\hline 13818023 & 754.438177 & 787.19797 & 989.131475 & 749.19701 & $9.95 \mathrm{E}-09$ & $2.49 \mathrm{E}-05$ & 67.0655537 & 0.00324673 & 0.00217744 & $1.19 \mathrm{E}-05$ & $1.30 \mathrm{E}-05$ & $2.33 E-05$ & 0.02040368 & 0.02219493 & 0.0063155 & 1321169.59 \\
\hline 14428975 & 754.438177 & 787.244587 & 989.050306 & 749.181237 & $1.03 \mathrm{E}-08$ & $2.49 E-05$ & 67.4925204 & 0.00338253 & 0.00228296 & $1.19 \mathrm{E}-05$ & $1.30 E-05$ & $2.33 E-05$ & 0.02051167 & 0.02312332 & 0.00632185 & 1322882.43 \\
\hline 14432575 & 750.349057 & 782.82645 & 985.009182 & 745.865165 & $1.03 E-08$ & $2.49 E-05$ & 67.4949264 & 0.00338333 & 0.00228358 & 1.19E- 05 & $1.30 \mathrm{E}-05$ & $2.31 E-05$ & 0.02049383 & 0.02312877 & 0.00626869 & 1367502.18 \\
\hline 14437075 & 750.349057 & 782.826429 & 985.009143 & 745.865137 & $1.03 \mathrm{E}-08$ & $2.49 \mathrm{E}-05$ & 67.497922 & 0.00338432 & $\int_{0.0228435}$ & $1.19 \mathrm{E}-05$ & $1.30 \mathrm{E}-05$ & $2.31 E-05$ & $\sum_{0}^{0.0204}$ & 0.023135 & $\begin{array}{l}0.00626875 \\
\end{array}$ & 1367797.79 \\
\hline 14442700 & 750.349057 & $\begin{array}{ll}782.827578 \\
\end{array}$ & 985.008722 & 745.865027 & $1.03 E-08$ & $2.49 \mathrm{E}-05$ & 67.501664 & 0.00338557 & 0.00228531 & 1.19E- 05 & $1.30 \mathrm{E}-05$ & $2.31 E-05$ & 0.02049575 & 0.02314405 & 0.00626882 & 1368176.64 \\
\hline 14449731.3 & 750.349057 & 782.828854 & 985.008047 & 745.86489 & $1.03 \mathrm{E}-08$ & $2.49 E-05$ & 67.5063377 & 0.00338712 & 0.00228652 & 1.19E-05 & $1.30 \mathrm{E}-05$ & $2.31 E-05$ & 0.02049698 & 0.02315466 & 0.00626892 & 1368650.26 \\
\hline 14458520.3 & 750.349057 & 782.830413 & 985.007132 & 745.864721 & $1.04 E-08$ & $2.49 E-05$ & 67.5121738 & 0.00338906 & 0.00228803 & $1.19 \mathrm{E}-05$ & $1.30 \mathrm{E}-05$ & $2.31 E-05$ & 0.02049851 & 0.02316792 & 0.00626903 & 1369242.31 \\
\hline 14469506.6 & 750.349057 & 782.832377 & 985.005955 & \begin{tabular}{|l|}
745.864509 \\
\end{tabular} & $1.04 \mathrm{E}-08$ & $2.49 \mathrm{E}-05$ & 67.5194595 & 0.00339148 & 0.00228991 & $1.19 \mathrm{E}-05$ & $1.30 \mathrm{E}-05$ & $2.31 E-05$ & 0.0205004 & 0.0231845 & 0.00626918 & 1369982.4 \\
\hline 14483239.6 & 750.349057 & 782.834883 & 985.004472 & 745.864245 & $1.04 \mathrm{E}-08$ & $2.49 E-05$ & 67.528552 & 0.00339452 & 0.00229227 & $1.19 \mathrm{E}-05$ & $1.30 \mathrm{E}-05$ & $2.31 E-05$ & 0.02050277 & 0.02320523 & 0.00626936 & 1370907.54 \\
\hline 14500405.7 & 750.349057 & 782.838101 & 985.002613 & 745.863916 & $1.04 \mathrm{E}-08$ & $2.49 E-05$ & 67.5398948 & 0.00339831 & 0.00229521 & $1.19 E-05$ & $1.30 \mathrm{E}-05$ & $2.31 E-05$ & 0.02050574 & 0.02323113 & 0.00626959 & 1372064.03 \\
\hline 14521863.4 & 750.349057 & 782.842262 & 985.000292 & 745.863504 & $1.04 \mathrm{E}-08$ & $2.49 E-05$ & 67.5540378 & 0.00340304 & 0.00229889 & 1.19E- 05 & $1.30 \mathrm{E}-05$ & $2.31 E-05$ & 0.02050944 & 0.02326352 & 0.00626987 & 1373509.71 \\
\hline 14548685.5 & 750.349057 & 782.854479 & 984.993789 & 745.862351 & $1.04 \mathrm{E}-08$ & $2.49 \mathrm{E}-05$ & 67.5716613 & 0.00340896 & 0.00230349 & $1.19 \mathrm{E}-05$ & $1.30 \mathrm{E}-05$ & $2.31 E-05$ & 0.02051406 & 0.023304 & $\underbrace{0.0062}_{0}$ & 1375380.46 \\
\hline 14582213.1 & 750.349057 & 782.863457 & 984.98929 & 745.861549 & $1.04 \mathrm{E}-08$ & $2.49 E-05$ & 67.5936048 & 0.00341637 & 0.00230924 & $1.19 E-05$ & $1.30 \mathrm{E}-05$ & $2.31 E-05$ & 0.02051987 & 0.0233546 & 0.00627068 & 1377656.13 \\
\hline 14624122.6 & 750.349057 & 782.87449 & 984.98413 & 745.860628 & $1.04 E-08$ & $2.49 E-05$ & 67.6209007 & 0.00342562 & 0.00231643 & 1.19E-05 & $1.30 \mathrm{E}-05$ & $2.31 E-05$ & 0.02052716 & 0.02341784 & 0.00627123 & 1380501.55 \\
\hline 14676509.5 & 750.349057 & 782.890897 & 984.977002 & 745.859367 & $1.04 \mathrm{E}-08$ & $2.49 E-05$ & 67.654814 & 0.00343718 & 0.00232542 & $1.19 \mathrm{E}-05$ & $1.30 \mathrm{E}-05$ & $2.31 E-05$ & 0.02053628 & 0.0234969 & 0.00627193 & 1384058.9 \\
\hline 14741993.1 & 750.349057 & 782.913588 & 984.968025 & 745.857789 & $1.05 E-08$ & $2.49 E-05$ & 67.696886 & 0.00345164 & 0.00233665 & 1.19E-05 & $1.30 \mathrm{E}-05$ & $2.31 E-05$ & 0.0205477 & 0.02359573 & 0.00627281 & 1388506.78 \\
\hline 14823847.6 & 750.349057 & 782.944678 & 984.957021 & 745.855864 & $1.05 E-08$ & $2.49 E-05$ & 67.748983 & 0.00346971 & 0.00235069 & $1.19 \mathrm{E}-05$ & $1.30 \mathrm{E}-05$ & $2.31 E-05$ & 0.02056202 & 0.02371926 & 0.0062739 & 1394068.83 \\
\hline 14926165.8 & 750.349057 & 782.987044 & 984.943312 & 745.853474 & $1.05 E-08$ & $2.49 E-05$ & 67.8133461 & 0.0034923 & 0.00236824 & $1.19 E-05$ & $1.30 \mathrm{E}-05$ & $2.31 E-05$ & 0.02057994 & 0.02387368 & 0.00627527 & 1401025.25 \\
\hline 15054063.5 & 750.349057 & 782.966235 & 984.926169 & 745.850052 & $1.06 E-08$ & $2.49 E-05$ & 67.8926385 & 0.00352053 & 0.00239018 & $1.19 \mathrm{E}-05$ & $1.30 \mathrm{E}-05$ & $2.32 \mathrm{E}-05$ & 0.02060261 & 0.02406669 & 0.006277 & 1409727.34 \\
\hline 15213935.6 & 750.349057 & 782.890773 & 984.904852 & 745.845482 & $1.06 E-08$ & $2.49 E-05$ & 67.9899832 & 0.00355583 & 0.00241761 & $1.19 \mathrm{E}-05$ & $1.30 \mathrm{E}-05$ & $2.32 E-05$ & 0.02063151 & 0.02430797 & 0.00627915 & 1420617.21 \\
\hline 15413775.7 & 750.349057 & 782.781988 & 984.87825 & $\begin{array}{l}745.839628 \\
\end{array}$ & $1.07 \mathrm{E}-08$ & $2.49 E-05$ & 68.1089799 & 0.00359994 & 0.00245189 & 1.19E-05 & $1.30 \mathrm{E}-05$ & $2.32 E-05$ & 0.02066827 & 0.02460956 & 0.00628183 & 1434247.88 \\
\hline 15663575.9 & 750.349057 & 782.628564 & 984.845086 & 745.832135 & $1.08 \mathrm{E}-08$ & $2.49 E-05$ & 68.2536862 & 0.00365509 & 0.00249473 & $1.19 \mathrm{E}-05$ & $1.30 \mathrm{E}-05$ & $2.32 E-05$ & 0.02071524 & 0.02498655 & 0.00628519 & 1451313.85 \\
\hline 15975826.1 & 750.349057 & 782.456702 & 984.803767 & 745.822743 & $1.09 \mathrm{E}-08$ & $2.49 E-05$ & 68.4285422 & 0.00372402 & 0.0025483 & $1.19 \mathrm{E}-05$ & $1.30 \mathrm{E}-05$ & $2.32 E-05$ & 0.02077531 & 0.02545778 & 0.00628937 & 1472687.07 \\
\hline 16366138.9 & 750.349057 & 782.284461 & 984.752282 & 745.811192 & $\begin{array}{l}1.11 \mathrm{E}-08 \\
\end{array}$ & $2.49 \mathrm{E}-05$ & 68.6382149 & 0.00381019 & 0.00261525 & $1.20 \mathrm{E}-05$ & $1.29 E-05$ & $2.32 E-05$ & 0.02085211 & 0.02604683 & 0.00629462 & 1499462.64 \\
\hline 16854029.9 & 750.349057 & 782.158649 & 984.688116 & 745.797219 & $1.13 \mathrm{E}-08$ & $2.49 E-05$ & 68.8873364 & 0.0039179 & 0.00269894 & $1.20 \mathrm{E}-05$ & $1.29 E-05$ & $2.32 E-05$ & 0.02094961 & 0.02678314 & 0.00630125 & 1533014.13 \\
\hline 17463893.6 & 750.349057 & 782.022529 & 984.607999 & 745.779804 & $1.16 \mathrm{E}-08$ & $2.49 E-05$ & 69.1801162 & 0.00405254 & 0.00280355 & $1.20 \mathrm{E}-05$ & $1.29 E-05$ & $2.33 E-05$ & 0.0210721 & 0.02770352 & 0.00630965 & 1575059.82 \\
\hline 18226223.3 & 750.349057 & 782.022045 & 984.50846 & 745.759671 & $1.19 \mathrm{E}-08$ & $2.49 E-05$ & 69.5198248 & 0.00422083 & 0.00293431 & $1.20 \mathrm{E}-05$ & $1.29 E-05$ & $2.33 E-05$ & 0.02122449 & 0.028854 & 0.00632034 & 1627766.2 \\
\hline 19179135.3 & 750.349057 & 782.248142 & 984.420086 & 745.7357777 & $1.24 \mathrm{E}-08$ & $2.49 E-05$ & 69.9081737 & 0.0044312 & 0.00309777 & $1.20 \mathrm{E}-05$ & $1.29 \mathrm{E}-05$ & $2.34 E-05$ & $\begin{array}{l}0.0214132 \\
\end{array}$ & 0.0302921 & 0.00633399 & 1693861.19 \\
\hline 20179135.3 & 750.349057 & 782.475616 & 984.320572 & 745.71182 & $1.29 \mathrm{E}-08$ & $2.49 E-05$ & 70.2779431 & 0.00465196 & 0.0032693 & $1.21 \mathrm{E}-05$ & $1.28 E-05$ & $2.34 \mathrm{E}-05$ & 0.02160578 & 0.03180126 & 0.00634923 & 1776063.91 \\
\hline 21179135.3 & 750.349057 & 782.091694 & 984.214318 & 745.68464 & $1.34 E-08$ & $\begin{array}{l}2.49 E-05 \\
\end{array}$ & 70.6142069 & 0.00487273 & 0.00344084 & $1.21 \mathrm{E}-05$ & $1.28 \mathrm{E}-05$ & $2.35 E-05$ & 0.0217927 & 0.03331042 & 0.0063653 & 1862386.75 \\
\hline 21312419 & 750.349057 & 782.074077 & 984.203765 & 745.681271 & $1.35 E-08$ & $2.49 E-05$ & 70.656738 & 0.00490215 & 0.0034637 & $1.21 \mathrm{E}-05$ & $1.28 E-05$ & $2.35 E-05$ & 0.02181708 & 0.03351157 & 0.00637067 & 1945774.34 \\
\hline 21314262.2 & 748.525721 & 779.379724 & 976.53732 & 743.730988 & $1.35 \mathrm{E}-08$ & $2.49 \mathrm{E}-05$ & 70.6573145 & 0.00490255 & 0.00346401 & $1.21 \mathrm{E}-05$ & $1.28 E-05$ & $2.34 E-05$ & 0.02179075 & 0.03351431 & 0.00634022 & 1951516.02 \\
\hline 21316019 & 746.750018 & 776.776042 & 969.165527 & 741.84272 & $1.35 \mathrm{E}-08$ & $2.49 E-05$ & 70.6578487 & 0.00490292 & 0.0034643 & $1.21 \mathrm{E}-05$ & $1.28 E-05$ & $2.33 E-05$ & 0.02176628 & 0.03351686 & 0.00631026 & 1946530.95 \\
\hline 21317862.2 & 746.750018 & 776.776064 & 969.165404 & 741.842694 & $1.35 \mathrm{E}-08$ & $2.49 \mathrm{E}-05$ & 70.6584013 & 0.00490331 & 0.0034646 & $1.21 \mathrm{E}-05$ & $1.28 \mathrm{E}-05$ & $2.33 E-05$ & 0.02176806 & 0.03351949 & 0.00631028 & 1946668.88 \\
\hline 21320166.2 & 746.750018 & 776.774571 & 969.166047 & 741.84263 & $1.35 \mathrm{E}-08$ & $2.49 E-05$ & 70.6590919 & 0.00490379 & 0.00346497 & $1.21 E-05$ & $1.28 E-05$ & $2.33 E-05$ & 0.0217693 & 0.03352277 & 0.00631032 & 1946818.16 \\
\hline 21323046.2 & 746.750018 & 776.773536 & 969.16633 & 741.842566 & $1.35 \mathrm{E}-08$ & $2.49 E-05$ & 70.659955 & 0.00490439 & 0.00346544 & $1.21 \mathrm{E}-05$ & $1.28 \mathrm{E}-05$ & $2.33 E-05$ & 0.0217703 & 0.03352688 & 0.00631036 & $\begin{array}{r}1947005.05 \\
\end{array}$ \\
\hline 21326646.2 & 746.750018 & 776.772698 & 969.166326 & 741.842495 & $1.35 E-08$ & $2.49 \mathrm{E}-05$ & 70.6610335 & 0.00490514 & 0.00346602 & $1.21 \mathrm{E}-05$ & $1.28 \mathrm{E}-05$ & $2.33 E-05$ & 0.02177119 & 0.03353202 & 0.00631042 & 1947238.84 \\
\hline 21331146.2 & 746.750018 & 776.771936 & 969.166092 & 741.842412 & $1.35 E-08$ & $2.49 E-05$ & 70.6623813 & 0.00490608 & 0.00346675 & $1.21 \mathrm{E}-05$ & $1.28 \mathrm{E}-05$ & $2.33 E-05$ & 0.02177209 & 0.03353844 & 0.00631048 & 1947531.16 \\
\hline 21336771.2 & 746.750018 & 776.771163 & 969.165658 & 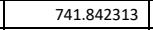 & $1.35 E-08$ & $2.49 E-05$ & 70.6640652 & 0.00490726 & 0.00346767 & $1.21 \mathrm{E}-05$ & $1.28 E-05$ & $2.33 E-05$ & 0.02177308 & 0.03354647 & 0.00631057 & 1947896.62 \\
\hline 21343802.5 & 746.750018 & 776.770305 & 969.16504 & 741.84219 & $1.35 \mathrm{E}-08$ & $2.49 \mathrm{E}-05$ & 70.666169 & 0.00490872 & 0.00346881 & $1.21 \mathrm{E}-05$ & $1.28 E-05$ & $2.33 E-05$ & 0.02177426 & 0.0335565 & 0.00631067 & 1948353.47 \\
\hline 21352591.5 & 746.750018 & 776.7693 & 969.164229 & 741.842039 & $1.35 \mathrm{E}-08$ & $2.49 E-05$ & 70.668797 & 0.00491056 & 0.00347023 & $1.21 \mathrm{E}-05$ & $1.28 E-05$ & $2.33 E-05$ & 0.02177569 & 0.03356904 & 0.0063108 & 1948924.57 \\
\hline 21363577.8 & 746.750018 & 776.76809 & 969.163198 & \begin{tabular}{|c|}
741.84185 \\
\end{tabular} & $1.35 \mathrm{E}-08$ & $2.49 E-05$ & 70.6720792 & 0.00491285 & 0.00347201 & $1.21 \mathrm{E}-05$ & $1.28 \mathrm{E}-05$ & $2.33 E-05$ & 0.02177748 & 0.03358472 & 0.00631097 & 1949638.46 \\
\hline 21377310.8 & 746.750018 & 776.766623 & 969.161903 & 741.841615 & $1.35 \mathrm{E}-08$ & $2.49 E-05$ & 70.6761776 & 0.00491572 & 0.00347424 & $1.21 \mathrm{E}-05$ & $1.28 \mathrm{E}-05$ & $2.33 E-05$ & 0.02177971 & 0.03360432 & 0.00631117 & 1950530.86 \\
\hline 21394476.9 & 746.750018 & 776.764848 & 969.160283 & 741.841322 & $1.35 \mathrm{E}-08$ & $2.49 \mathrm{E}-05$ & 70.681294 & 0.0049193 & 0.00347703 & $1.21 \mathrm{E}-05$ & $1.28 \mathrm{E}-05$ & $2.33 E-05$ & 0.02178249 & 0.03362882 & 0.00631143 & 1951646.4 \\
\hline 21415934.6 & 746.750018 & 776.762717 & 969.158258 & 741.840955 & 1.35E-08 & $2.49 E-05$ & 70.687679 & 0.00492378 & 0.00348051 & $1.21 E-05$ & $1.28 \mathrm{E}-05$ & $2.33 E-05$ & 0.02178597 & 0.03365944 & 0.00631175 & 1953040.9 \\
\hline 21442756.7 & 746.750018 & 776.760189 & 969.155727 & \begin{tabular}{|l|l|l} 
\\
\end{tabular} & $11.36 E-08$ & $2.49 E-05$ & 70.6956438 & 0.00492938 & 0.00348486 & $1.21 \mathrm{E}-05$ & $1.28 \mathrm{E}-05$ & $2.33 E-05$ & 0.02179031 & 0.03369771 & 0.00631215 & $\begin{array}{l}1954784.14 \\
\end{array}$ \\
\hline 21476284.3 & 746.750018 & 776.757238 & 969.152563 & & & $2.49 E-05$ & 70.7055746 & 0.00493638 & 0.0034903 & & & $2.33 E-05$ & & 0.03374555 & 0.00631266 & 1956963.36 \\
\hline 21518193.8 & 746.750018 & 776.753874 & 969.148608 & 741.83922 & $1.36 \mathrm{E}-08$ & $2.49 \mathrm{E}-05$ & 70.7179484 & 0.00494513 & 0.00349709 & $1.21 \mathrm{E}-05$ & $1.28 \mathrm{E}-05$ & $2.33 E-05$ & 0.02180254 & 0.03380536 & 0.00631328 & 1959687.67 \\
\hline 21570580.7 & 746.750018 & 776.750175 & 69.143667 & 741.838337 & $1.36 \mathrm{E}-08$ & $2.49 E-05$ & 70.7333543 & 0.00495606 & 0.00350559 & $1.21 \mathrm{E}-05$ & \begin{tabular}{|l|l|}
$1.28 E-05$ \\
\end{tabular} & $2.33 \mathrm{E}-05$ & 0.02181102 & 0.03388012 & 0.00631407 & 1963093.48 \\
\hline 21636064.3 & 746.750018 & 776.746441 & 969.137491 & 741.837221 & $1.36 \mathrm{E}-08$ & $2.49 E-05$ & 70.7525163 & 0.00496973 & 0.00351621 & $1.21 \mathrm{E}-05$ & $1.28 E-05$ & $2.33 \mathrm{E}-05$ & 0.02182163 & 0.03397356 & 0.00631506 & 1967351.38 \\
\hline
\end{tabular}




\begin{tabular}{|c|c|c|c|c|c|c|c|c|c|c|c|c|c|c|c|c|}
\hline Time (s) & $\begin{array}{c}\text { Coolant outlet } \\
\text { temperature (K) }\end{array}$ & $\begin{array}{c}\text { Peak cladding } \\
\text { temperature (K) }\end{array}$ & $\begin{array}{c}\text { Peak fuel } \\
\text { temperature }(K)\end{array}$ & $\begin{array}{c}\text { Average cladding } \\
\text { internal } \\
\text { temperature (K) }\end{array}$ & $\begin{array}{l}\text { Peak cumulative } \\
\text { damage factor }\end{array}$ & $\begin{array}{l}\text { Cladding interior } \\
\text { volume }\left(\mathrm{m}^{3}\right)\end{array}$ & $\begin{array}{l}\text { Fission gas } \\
\text { release (\%) }\end{array}$ & $\begin{array}{l}\text { Fission gas } \\
\text { produced } \\
\text { (moles) }\end{array}$ & $\begin{array}{c}\text { Fission gas } \\
\text { released (moles) }\end{array}$ & $\begin{array}{c}\text { Fuel volume } \\
\left(\mathrm{m}^{3}\right)\end{array}$ & $\begin{array}{l}\text { Plenum gas } \\
\text { volume }\left(m^{3}\right)\end{array}$ & $\begin{array}{l}\text { Peak cladding } \\
\text { radial growth } \\
\text { (m) }\end{array}$ & $\begin{array}{l}\text { Peak fuel axial } \\
\text { growth }(\mathrm{m})\end{array}$ & $\begin{array}{c}\text { Peak burnup } \\
\text { (at\%) }\end{array}$ & $\begin{array}{l}\text { Peak cladding } \\
\text { hoop strain }\end{array}$ & $\begin{array}{c}\text { Plenum pressure } \\
(\mathrm{Pa})\end{array}$ \\
\hline 21717918.8 & 746.750018 & 776.74306 & 969.129775 & 741.835826 & $1.36 \mathrm{E}-08$ & $2.49 \mathrm{E}-05$ & 70.7763211 & 0.00498682 & 0.00352949 & $1.21 \mathrm{E}-05$ & $1.28 \mathrm{E}-05$ & $2.33 \mathrm{E}-05$ & 0.02183489 & 0.03409037 & 0.00631629 & 1972674.77 \\
\hline 21820237 & \begin{tabular}{|l|l|}
746.750018 \\
\end{tabular} & 776.740772 & \begin{tabular}{|l|l|}
969.120133 \\
\end{tabular} & 741.834099 & $\begin{array}{ll}1.37 \mathrm{E}-08 \\
\end{array}$ & $2.49 \mathrm{E}-05$ & 70.8058487 & 0.00500818 & 0.00354608 & $1.21 \mathrm{E}-05$ & $1.28 \mathrm{E}-05$ & $2.33 E-05$ & 0.02185146 & 0.03423638 & 0.00631783 & 1979330.67 \\
\hline 21948134.7 & 746.750018 & 776.740961 & \begin{tabular}{|l|l|}
969.108087 \\
\end{tabular} & 741.831963 & $1.37 \mathrm{E}-08$ & $2.49 \mathrm{E}-05$ & 70.8424058 & 0.00503488 & 0.00356683 & $1.21 \mathrm{E}-05$ & $1.28 \mathrm{E}-05$ & $2.33 \mathrm{E}-05$ & 0.02187217 & 0.03441889 & 0.00631977 & 1987653.12 \\
\hline 22108006.8 & \begin{tabular}{|l|l|}
746.750018 \\
\end{tabular} & 776.760204 & \begin{tabular}{l|l|}
969.074322 \\
\end{tabular} & 741.826059 & $\begin{array}{ll}1.37 \mathrm{E}-08 \\
\end{array}$ & $2.49 \mathrm{E}-05$ & 70.8875607 & 0.00506825 & 0.00359276 & $1.21 \mathrm{E}-05$ & $1.28 \mathrm{E}-05$ & $2.33 \mathrm{E}-05$ & 0.02189799 & 0.03464703 & 0.00632223 & 1998585.6 \\
\hline 22307846.9 & 746.750018 & 776.790277 & 969.050878 & 741.822041 & $1.38 \mathrm{E}-08$ & $2.49 \mathrm{E}-05$ & 70.9431748 & 0.00510996 & 0.00362517 & $1.21 \mathrm{E}-05$ & $1.28 \mathrm{E}-05$ & $2.33 \mathrm{E}-05$ & 0.02193032 & 0.0349322 & 0.00632528 & 2011736.64 \\
\hline 22557647.1 & 746.750018 & 776.84825 & 969.021611 & 741.817129 & $1.39 \mathrm{E}-08$ & $2.49 \mathrm{E}-05$ & 71.0114285 & 0.00516211 & 0.00366569 & $1.21 \mathrm{E}-05$ & $1.28 \mathrm{E}-05$ & $2.33 \mathrm{E}-05$ & 0.02197073 & 0.03528867 & 0.00632916 & 2028187.87 \\
\hline 22869897.3 & 746.750018 & 776.952732 & 968.985083 & 741.811175 & $1.40 \mathrm{E}-08$ & $2.49 \mathrm{E}-05$ & 71.0948307 & 0.00522729 & 0.00371633 & $1.21 \mathrm{E}-05$ & $1.28 \mathrm{E}-05$ & $2.33 \mathrm{E}-05$ & 0.02202122 & 0.03573425 & 0.00633406 & 2048771.39 \\
\hline 23260210.1 & \begin{tabular}{|l|l|}
746.750018 \\
\end{tabular} & 777.005511 & \begin{tabular}{|l|l|}
968.939513 \\
\end{tabular} & 741.803336 & $\begin{array}{ll}1.41 \mathrm{E}-08 \\
\end{array}$ & $2.49 \mathrm{E}-05$ & 71.1962034 & 0.00530877 & 0.00377964 & $1.21 \mathrm{E}-05$ & $1.28 \mathrm{E}-05$ & $2.34 \mathrm{E}-05$ & 0.02208429 & 0.03629123 & $\begin{array}{ll}0.00634024 \\
\end{array}$ & 2074529.3 \\
\hline 23748101.1 & 746.750018 & 776.836396 & \begin{tabular}{|l|l|}
968.882801 \\
\end{tabular} & 741.792368 & $1.43 \mathrm{E}-08$ & $2.49 \mathrm{E}-05$ & 71.3186259 & 0.00541061 & 0.00385877 & $1.21 \mathrm{E}-05$ & $1.28 \mathrm{E}-05$ & $2.34 \mathrm{E}-05$ & 0.02216307 & 0.03698745 & 0.00634803 & 2106769.62 \\
\hline 24357964.8 & 746.750018 & 776.625866 & 968.81236 & 741.778723 & $1.45 E-08$ & $2.49 \mathrm{E}-05$ & 71.4653219 & 0.00553792 & 0.00395769 & $1.21 \mathrm{E}-05$ & $1.28 \mathrm{E}-05$ & $2.34 \mathrm{E}-05$ & 0.02226144 & 0.03785773 & 0.00635788 & 2147142.71 \\
\hline 25120294.5 & 746.750018 & 776.561968 & \begin{tabular}{|l|l|}
968.724771 \\
\end{tabular} & 741.850914 & $1.48 \mathrm{E}-08$ & $2.49 \mathrm{E}-05$ & 71.6394723 & 0.00569705 & 0.00408134 & $1.22 \mathrm{E}-05$ & $1.27 \mathrm{E}-05$ & $2.35 E-05$ & 0.022385 & 0.03894558 & 0.00637053 & 2198034.98 \\
\hline 25596874 & \begin{tabular}{|l|l|}
746.750018 \\
\end{tabular} & 776.552081 & 968.670149 & 741.840016 & $1.49 \mathrm{E}-08$ & $2.49 \mathrm{E}-05$ & 71.7434871 & 0.00579654 & 0.00415864 & $1.22 \mathrm{E}-05$ & $1.27 \mathrm{E}-05$ & $2.35 \mathrm{E}-05$ & 0.02246165 & 0.03962566 & 0.00638 & 2259655.39 \\
\hline 25600474 & 746.260781 & 775.669269 & 965.316762 & 741.214322 & $1.49 \mathrm{E}-08$ & $2.49 \mathrm{E}-05$ & 71.744254 & 0.00579728 & 0.00415922 & $1.22 \mathrm{E}-05$ & $1.27 \mathrm{E}-05$ & $2.35 E-05$ & 0.02245125 & 0.03963076 & 0.00637211 & 2295025.26 \\
\hline 26077053.5 & 746.260781 & 775.661752 & 965.264207 & 741.204096 & $1.51 \mathrm{E}-08$ & $2.49 \mathrm{E}-05$ & 71.843391 & 0.00589541 & 0.00423547 & $1.22 \mathrm{E}-05$ & $1.27 \mathrm{E}-05$ & $2.35 E-05$ & 0.02252783 & 0.04030161 & 0.0063789 & 2297104.19 \\
\hline 26261755 & 746.260781 & 775.659447 & 965.243487 & 741.199942 & $1.52 \mathrm{E}-08$ & $2.49 \mathrm{E}-05$ & 71.8809305 & 0.00593345 & 0.00426502 & $1.22 \mathrm{E}-05$ & $1.27 \mathrm{E}-05$ & $2.35 E-05$ & 0.02255719 & 0.0405616 & 0.00638323 & 2334729.6 \\
\hline 26265355 & 745.89865 & 776.094503 & 971.503058 & 741.436241 & $1.52 \mathrm{E}-08$ & $2.49 \mathrm{E}-05$ & 71.881668 & 0.0059342 & 0.0042656 & $1.22 \mathrm{E}-05$ & $1.27 \mathrm{E}-05$ & $2.35 \mathrm{E}-05$ & 0.02257723 & 0.04056674 & 0.00638614 & 2350226.12 \\
\hline 26450056.5 & 745.89865 & 776.092376 & 971.479778 & 741.431738 & $1.52 \mathrm{E}-08$ & $2.49 \mathrm{E}-05$ & 71.9197941 & 0.00597334 & 0.00429601 & $1.22 \mathrm{E}-05$ & $1.27 \mathrm{E}-05$ & $2.35 \mathrm{E}-05$ & 0.02260604 & 0.04083432 & 0.00638891 & 2351245.48 \\
\hline 26680933.3 & 745.89865 & 776.089911 & 971.452083 & 741.425903 & $1.53 \mathrm{E}-08$ & $2.49 \mathrm{E}-05$ & 71.9667548 & 0.00602227 & 0.00433403 & $1.22 \mathrm{E}-05$ & $1.27 \mathrm{E}-05$ & $2.35 \mathrm{E}-05$ & 0.02264377 & 0.04116879 & 0.00639304 & 2366912.51 \\
\hline 26969529.3 & 745.89865 & 776.087868 & 971.417401 & 741.418841 & $1.54 \mathrm{E}-08$ & $2.49 \mathrm{E}-05$ & 72.0243933 & 0.00608343 & 0.00438155 & $1.22 \mathrm{E}-05$ & $1.27 \mathrm{E}-05$ & $2.35 \mathrm{E}-05$ & 0.02269097 & 0.04158688 & 0.00639824 & 2386514.81 \\
\hline 27330274.4 & 745.89865 & 776.086394 & 971.374087 & 741.410093 & $1.56 \mathrm{E}-08$ & $2.49 \mathrm{E}-05$ & 72.094832 & 0.00615988 & 0.00444095 & $1.22 \mathrm{E}-05$ & $1.27 \mathrm{E}-05$ & $2.36 \mathrm{E}-05$ & 0.02275009 & 0.04210949 & 0.00640477 & 2411046.76 \\
\hline 27781205.7 & 745.89865 & 776.086772 & 971.331467 & 741.399011 & $1.58 \mathrm{E}-08$ & $2.49 \mathrm{E}-05$ & 72.1804593 & 0.00625544 & 0.0045152 & $1.22 \mathrm{E}-05$ & $1.27 \mathrm{E}-05$ & $2.36 \mathrm{E}-05$ & 0.02282455 & 0.04276276 & 0.00641301 & 2441764.78 \\
\hline 28344869.8 & 745.89865 & 776.076053 & 971.2825 & 741.385279 & $1.60 \mathrm{E}-08$ & $2.49 \mathrm{E}-05$ & 72.2838833 & 0.00637489 & 0.00460802 & $1.22 \mathrm{E}-05$ & $1.27 \mathrm{E}-05$ & $2.36 \mathrm{E}-05$ & 0.02291819 & 0.04357934 & 0.00642344 & 2480242.26 \\
\hline 29049450 & 745.89865 & 776.05807 & 971.217141 & 741.367915 & $1.63 \mathrm{E}-08$ & $2.49 \mathrm{E}-05$ & 72.4078376 & 0.0065242 & 0.00472403 & $1.23 \mathrm{E}-05$ & $1.27 \mathrm{E}-05$ & $2.37 E-05$ & 0.02303534 & 0.04460006 & 0.00643666 & 2528452.27 \\
\hline 29930175.2 & 745.89865 & 776.03977 & 971.129982 & 741.349819 & $1.67 \mathrm{E}-08$ & $2.49 \mathrm{E}-05$ & 72.5550237 & 0.00671084 & 0.00486906 & $1.23 \mathrm{E}-05$ & $1.26 \mathrm{E}-05$ & $2.37 E-05$ & 0.02318185 & 0.04587597 & 0.00645347 & 2588908.04 \\
\hline 30930175.2 & 745.89865 & 776.02257 & \begin{tabular}{|l|}
971.025059 \\
\end{tabular} & 741.327754 & $1.72 \mathrm{E}-08$ & $2.49 \mathrm{E}-05$ & 72.7125215 & 0.00692276 & 0.00503372 & $1.23 \mathrm{E}-05$ & $1.26 \mathrm{E}-05$ & $2.38 \mathrm{E}-05$ & 0.0233479 & 0.04732467 & 0.00647329 & 2664267.25 \\
\hline 31930175.2 & 745.89865 & 776.011519 & \begin{tabular}{|c|}
90.916241 \\
\end{tabular} & 741.305115 & $1.77 \mathrm{E}-08$ & $2.49 \mathrm{E}-05$ & 72.8606631 & 0.00713468 & 0.00519838 & $1.23 \mathrm{E}-05$ & $1.26 \mathrm{E}-05$ & $2.39 \mathrm{E}-05$ & 0.02351364 & 0.04877337 & 0.00649404 & 2749493.36 \\
\hline 32714598 & 745.89865 & 776.004036 & 970.831616 & 741.289418 & $1.81 \mathrm{E}-08$ & $2.49 \mathrm{E}-05$ & 72.9708498 & 0.00730092 & 0.00532754 & $1.23 \mathrm{E}-05$ & $1.26 \mathrm{E}-05$ & $2.39 \mathrm{E}-05$ & 0.0236435 & 0.04990976 & 0.00651171 & 2833954.42 \\
\hline 32718198 & 644.15 & 644.166545 & 644.152511 & 644.150781 & $1.81 \mathrm{E}-08$ & $2.48 E-05$ & 72.9710968 & 0.0073013 & 0.00532784 & $1.22 \mathrm{E}-05$ & $1.26 \mathrm{E}-05$ & $1.86 \mathrm{E}-05$ & 0.02233897 & 0.04991237 & 0.00511744 & 2509753.83 \\
\hline 32721798 & 305 & 305.051632 & 305.004328 & 305.00224 & $1.81 \mathrm{E}-08$ & $2.45 E-05$ & 72.9710968 & 0.0073013 & 0.00532784 & $1.20 \mathrm{E}-05$ & $1.25 \mathrm{E}-05$ & $2.41 \mathrm{E}-06$ & 0.02028851 & 0.04991237 & 0.00071222 & 1199833.43 \\
\hline 32725398 & 305 & 305.051632 & 305.004328 & 305.00224 & $1.81 \mathrm{E}-08$ & $2.45 E-05$ & 72.9710968 & 0.0073013 & 0.00532784 & $1.20 \mathrm{E}-05$ & $1.25 \mathrm{E}-05$ & $2.41 \mathrm{E}-06$ & 0.02028851 & 0.04991237 & 0.00071222 & 1199833.43 \\
\hline 32728998 & 730.114707 & 755.520902 & 926.306421 & 726.262716 & $\begin{array}{ll}1.81 \mathrm{E}-08 \\
\end{array}$ & $2.49 \mathrm{E}-05$ & 72.9713064 & 0.00730162 & 0.00532809 & $1.23 \mathrm{E}-05$ & $1.26 \mathrm{E}-05$ & $2.30 \mathrm{E}-05$ & 0.02341412 & 0.04991458 & 0.00627401 & 2837631.43 \\
\hline 32732598 & 730.114707 & 755.524374 & 926.310271 & 726.266384 & $1.81 \mathrm{E}-08$ & $2.49 \mathrm{E}-05$ & 72.9717256 & 0.00730227 & 0.00532859 & $1.23 \mathrm{E}-05$ & $1.26 \mathrm{E}-05$ & $2.30 \mathrm{E}-05$ & 0.02341156 & 0.04991901 & 0.00627406 & 2837776.65 \\
\hline 32737098 & 730.114707 & 755.524129 & \begin{tabular}{|l|}
926.308646 \\
\end{tabular} & 726.266347 & $1.81 E-08$ & $2.49 \mathrm{E}-05$ & 72.9722495 & 0.00730308 & 0.00532922 & $1.23 \mathrm{E}-05$ & $1.26 \mathrm{E}-05$ & $2.30 \mathrm{E}-05$ & 0.02341032 & 0.04992454 & 0.00627414 & 2838036.49 \\
\hline 32742723 & 730.114707 & 755.524126 & 926.308637 & 726.266348 & $1.81 \mathrm{E}-08$ & $2.49 \mathrm{E}-05$ & 72.9729041 & 0.00730409 & 0.00533001 & $1.23 \mathrm{E}-05$ & $1.26 \mathrm{E}-05$ & $2.30 \mathrm{E}-05$ & 0.02341012 & 0.04993145 & 0.00627424 & 2838338.05 \\
\hline 32749754.3 & 730.114707 & 755.523992 & 926.307477 & 726.266275 & $1.81 \mathrm{E}-08$ & $2.49 \mathrm{E}-05$ & 72.9737221 & 0.00730536 & 0.00533099 & $1.23 \mathrm{E}-05$ & $1.26 \mathrm{E}-05$ & $2.30 \mathrm{E}-05$ & 0.02341066 & 0.0499401 & 0.00627436 & 2838738.51 \\
\hline 32758543.3 & 730.114707 & 755.523922 & 926.306535 & 726.266171 & $1.81 \mathrm{E}-08$ & $2.49 \mathrm{E}-05$ & 72.9747443 & 0.00730694 & 0.00533222 & $1.23 \mathrm{E}-05$ & $1.26 \mathrm{E}-05$ & $2.30 \mathrm{E}-05$ & 0.02341175 & 0.0499509 & 0.00627452 & 2839238.77 \\
\hline 32769529.6 & 730.114707 & 755.523885 & 926.305613 & 726.266033 & $1.81 \mathrm{E}-08$ & $2.49 \mathrm{E}-05$ & 72.9760214 & 0.00730891 & 0.00533375 & $1.23 \mathrm{E}-05$ & $1.26 \mathrm{E}-05$ & $2.30 \mathrm{E}-05$ & 0.02341329 & 0.0499644 & 0.00627471 & 2839864.09 \\
\hline 32783262.6 & 730.114707 & 755.523861 & 926.304571 & 726.265856 & $1.81 \mathrm{E}-08$ & $2.49 \mathrm{E}-05$ & 72.9776168 & 0.00731138 & 0.00533567 & $1.23 \mathrm{E}-05$ & $1.26 \mathrm{E}-05$ & $2.30 \mathrm{E}-05$ & 0.02341529 & 0.04998128 & 0.00627496 & 2840645.78 \\
\hline 32800428.7 & 730.114707 & 755.52384 & 926.303309 & 726.265634 & $1.81 \mathrm{E}-08$ & $2.49 \mathrm{E}-05$ & 72.9796095 & 0.00731447 & 0.00533807 & $1.23 \mathrm{E}-05$ & $1.26 \mathrm{E}-05$ & $2.30 \mathrm{E}-05$ & 0.02341781 & 0.05000238 & 0.00627526 & 2841622.94 \\
\hline 32821886.4 & 730.114707 & 755.523819 & 926.301743 & 726.265355 & $1.81 \mathrm{E}-08$ & $2.49 \mathrm{E}-05$ & 72.982098 & 0.00731832 & 0.00534107 & $1.23 \mathrm{E}-05$ & $1.26 \mathrm{E}-05$ & $2.30 \mathrm{E}-05$ & 0.02342096 & 0.05002876 & 0.00627564 & 2842844.44 \\
\hline 32848708.5 & 730.114707 & 755.523798 & \begin{tabular}{|l|}
926.29979 \\
\end{tabular} & 726.265005 & $1.81 \mathrm{E}-08$ & $2.49 \mathrm{E}-05$ & 72.985205 & 0.00732315 & 0.00534481 & $1.23 \mathrm{E}-05$ & $1.26 \mathrm{E}-05$ & $2.30 \mathrm{E}-05$ & 0.02342491 & 0.05006172 & 0.00627612 & 2844371.39 \\
\hline 32882236.1 & 730.114707 & 755.523779 & \begin{tabular}{|c|}
926.297349 \\
\end{tabular} & 726.264567 & $1.81 \mathrm{E}-08$ & $2.49 \mathrm{E}-05$ & 72.989083 & 0.00732918 & 0.0053495 & $1.23 \mathrm{E}-05$ & $1.26 \mathrm{E}-05$ & $2.30 \mathrm{E}-05$ & 0.02342984 & 0.05010293 & 0.00627672 & 2846280.21 \\
\hline 32896765 & 729.812123 & 755.21347 & 926.108736 & 726.028475 & $1.81 \mathrm{E}-08$ & $2.49 \mathrm{E}-05$ & 72.9907615 & 0.00733179 & 0.00535153 & $1.23 \mathrm{E}-05$ & $1.26 \mathrm{E}-05$ & $2.30 \mathrm{E}-05$ & 0.02343095 & 0.05012079 & 0.00627316 & 2847829.91 \\
\hline 32900365 & 738.505875 & 766.536618 & 951.298421 & 734.336528 & $1.81 \mathrm{E}-08$ & $2.49 \mathrm{E}-05$ & 72.9911981 & 0.00733247 & 0.00535206 & $1.23 \mathrm{E}-05$ & $1.26 \mathrm{E}-05$ & $2.35 \mathrm{E}-05$ & 0.02352203 & 0.05012544 & 0.0064062 & 2882046.45 \\
\hline 32914893.9 & 738.505875 & 766.536717 & 951.298585 & 734.3366 & $1.81 \mathrm{E}-08$ & $2.49 \mathrm{E}-05$ & 72.993044 & 0.00733534 & 0.00535429 & $1.23 \mathrm{E}-05$ & $1.26 \mathrm{E}-05$ & $2.35 \mathrm{E}-05$ & 0.02352029 & 0.0501451 & 0.00640646 & 2882302.6 \\
\hline 32933055.1 & 738.505875 & 766.536261 & $\begin{array}{l}951.2904903 \\
\end{array}$ & $\begin{array}{r}734.3364007 \\
\end{array}$ & $\begin{array}{l}1.011-0.0 \\
1.81 E-08\end{array}$ & $\begin{array}{l}2.459-05 \\
2.49-05\end{array}$ & 72.9953493 & 0.007333894 & 0.000535708 & $\begin{array}{l}1.250-035 \\
1.23 \mathrm{E}-05\end{array}$ & $\begin{array}{l}1.200-05 \\
1.26 \mathrm{E}-05\end{array}$ & 2 & 0.02352241 & 0.05014967 & 0.00640681 & 28835555.39 \\
\hline 32955756.6 & 738.505875 & 766.536065 & 951.292633 & 734.336061 & $1.81 \mathrm{E}-08$ & $2.49 \mathrm{E}-05$ & 72.9982277 & 0.00734343 & 0.00536057 & $1.23 \mathrm{E}-05$ & $1.26 \mathrm{E}-05$ & $2.35 \mathrm{E}-05$ & 0.02352582 & 0.05020038 & 0.00640726 & 2884894.4 \\
\hline 32984133.4 & 738.505875 & 766.535884 & 951.290201 & 734.335606 & $1.81 \mathrm{E}-08$ & $2.49 \mathrm{E}-05$ & 73.0018208 & 0.00734905 & 0.00536494 & $1.23 \mathrm{E}-05$ & $1.26 \mathrm{E}-05$ & $2.35 \mathrm{E}-05$ & 0.02353021 & 0.05023877 & 0.00640782 & 2886693.33 \\
\hline 33019604.4 & 738.505875 & 766.535673 & 951.287224 & 734.335034 & $1.81 \mathrm{E}-08$ & $2.49 \mathrm{E}-05$ & 73.0063044 & 0.00735607 & 0.00537039 & $1.23 \mathrm{E}-05$ & $1.26 \mathrm{E}-05$ & $2.35 E-05$ & 0.02353573 & 0.05028677 & 0.00640853 & 2888942.2 \\
\hline 33063943.2 & 738.505875 & 766.53542 & 951.283513 & 734.334316 & $1.82 \mathrm{E}-08$ & $2.49 \mathrm{E}-05$ & 73.011897 & 0.00736484 & 0.00537721 & $1.23 \mathrm{E}-05$ & $1.26 \mathrm{E}-05$ & $2.35 \mathrm{E}-05$ & 0.02354263 & 0.05034675 & 0.00640941 & 2891753.56 \\
\hline 33119366.7 & 738.505875 & 766.5350999 & 951.278877 & 734.333584 & $1.82 \mathrm{E}-08$ & $2.49 \mathrm{E}-05$ & 73.0188689 & 0.00737581 & 0.00538573 & $1.23 \mathrm{E}-05$ & $1.26 \mathrm{E}-05$ & $2.35 \mathrm{E}-05$ & 0.02355125 & 0.05042174 & 0.00641051 & 2895268.86 \\
\hline 33188646.1 & 738.505875 & 766.53471 & 951.273083 & 734.332755 & $1.82 \mathrm{E}-08$ & $2.49 \mathrm{E}-05$ & 73.0275548 & 0.00738952 & 0.00539639 & $1.23 \mathrm{E}-05$ & $1.26 \mathrm{E}-05$ & $2.35 \mathrm{E}-05$ & 0.02356204 & 0.05051547 & 0.00641189 & 2899664.05 \\
\hline 33275245.3 & 738.505875 & 766.534256 & 951.265815 & 734.331706 & $1.82 \mathrm{E}-08$ & $2.49 \mathrm{E}-05$ & 73.0383669 & 0.00740666 & 0.0054097 & $1.23 \mathrm{E}-05$ & $1.26 \mathrm{E}-05$ & $2.35 \mathrm{E}-05$ & 0.02357551 & 0.05063263 & 0.00641362 & 2905159.06 \\
\hline 33383494.3 & 738.505875 & 766.533736 & 951.256717 & 734.330375 & $1.82 \mathrm{E}-08$ & $2.49 \mathrm{E}-05$ & 73.0518118 & 0.00742809 & 0.00542635 & $1.23 \mathrm{E}-05$ & $1.26 \mathrm{E}-05$ & $2.35 E-05$ & 0.02359236 & 0.05077909 & 0.00641578 & 2912029.42 \\
\hline 33518805.6 & 738.505875 & 766.53316 & 951.245351 & 734.328679 & $1.82 \mathrm{E}-08$ & $2.49 \mathrm{E}-05$ & 73.0685093 & 0.00745487 & 0.00544716 & $1.23 \mathrm{E}-05$ & $1.26 E-05$ & $2.36 \mathrm{E}-05$ & 0.02361341 & 0.05096216 & 0.00641849 & 2920619.88 \\
\hline
\end{tabular}




\begin{tabular}{|c|c|c|c|c|c|c|c|c|c|c|c|c|c|c|c|c|}
\hline Time (s) & $\begin{array}{c}\text { Coolant outlet } \\
\text { temperature }(\mathrm{K})\end{array}$ & $\begin{array}{l}\text { Peak cladding } \\
\text { temperature (K) }\end{array}$ & $\begin{array}{c}\text { Peak fuel } \\
\text { temperature (K) }\end{array}$ & $\begin{array}{l}\text { Average cladding } \\
\text { internal } \\
\text { temperature (K) }\end{array}$ & $\begin{array}{c}\text { Peak cumulative } \\
\text { damage factor }\end{array}$ & $\begin{array}{l}\text { Cladding interior } \\
\text { volume }\left(\mathrm{m}^{3}\right)\end{array}$ & $\begin{array}{l}\text { Fission gas } \\
\text { release (\%) }\end{array}$ & $\begin{array}{l}\text { Fission gas } \\
\text { produced } \\
\text { (moles) }\end{array}$ & $\begin{array}{c}\text { Fission gas } \\
\text { released (moles) }\end{array}$ & $\begin{array}{l}\text { Fuel volume } \\
\left(\mathrm{m}^{3}\right)\end{array}$ & $\begin{array}{l}\text { Plenum gas } \\
\text { volume }\left(m^{3}\right)\end{array}$ & $\begin{array}{l}\text { Peak cladding } \\
\text { radial growth } \\
\text { (m) }\end{array}$ & $\begin{array}{l}\text { Peak fuel axial } \\
\text { growth }(m)\end{array}$ & $\begin{array}{c}\text { Peak burnup } \\
\text { (at\%) }\end{array}$ & $\begin{array}{l}\text { Peak cladding } \\
\text { hoop strain }\end{array}$ & $\begin{array}{c}\text { Plenum pressure } \\
(\mathrm{Pa})\end{array}$ \\
\hline 33687944.7 & 738.505875 & $\begin{array}{l}766.531944 \\
\end{array}$ & \begin{tabular}{|l|l|}
951.213491 \\
\end{tabular} & 734.324274 & $\begin{array}{ll}1.83 \mathrm{E}-08 \\
\end{array}$ & $2.49 \mathrm{E}-05$ & \begin{tabular}{|l|}
73.0892132 \\
\end{tabular} & 0.00748834 & 0.00547317 & $1.24 \mathrm{E}-05$ & $1.26 \mathrm{E}-05$ & $2.36 \mathrm{E}-05$ & 0.02363966 & 0.051191 & $\begin{array}{l}0.00642193 \\
\end{array}$ & 2932148.44 \\
\hline 33899368.5 & 738.505875 & 766.531372 & 951.191372 & 734.321259 & $1.83 \mathrm{E}-08$ & $2.49 \mathrm{E}-05$ & 73.1148343 & 0.00753018 & 0.00550568 & $1.24 \mathrm{E}-05$ & $1.26 \mathrm{E}-05$ & $2.36 \mathrm{E}-05$ & 0.02367252 & 0.05147704 & 0.00642621 & 2945784.67 \\
\hline 34163648.3 & 738.505875 & 766.531002 & \begin{tabular}{|l|l|}
951.163761 \\
\end{tabular} & 734.31742 & $1.83 \mathrm{E}-08$ & $2.49 \mathrm{E}-05$ & $\begin{array}{l}73.146463 \\
\end{array}$ & 0.00758249 & 0.00554632 & $1.24 \mathrm{E}-05$ & $1.25 \mathrm{E}-05$ & $2.36 \mathrm{E}-05$ & 0.02371359 & 0.0518346 & 0.00643158 & 2962842.33 \\
\hline 34493998.1 & 738.505875 & 766.530966 & \begin{tabular}{|l|l|}
951.129307 \\
\end{tabular} & 734.311941 & $1.84 \mathrm{E}-08$ & $2.49 \mathrm{E}-05$ & 73.1853904 & 0.00764787 & 0.00559712 & $1.24 \mathrm{E}-05$ & $1.25 \mathrm{E}-05$ & $2.36 \mathrm{E}-05$ & 0.0237649 & 0.05228155 & 0.00643834 & 2984181.39 \\
\hline 34906935.3 & 738.505875 & 766.530506 & 951.086323 & 734.304819 & $1.85 \mathrm{E}-08$ & $2.49 \mathrm{E}-05$ & 73.2331237 & 0.00772959 & 0.00566062 & $1.24 \mathrm{E}-05$ & $1.25 \mathrm{E}-05$ & $2.36 \mathrm{E}-05$ & 0.02382901 & 0.05284023 & 0.00644685 & 3010884.96 \\
\hline 35423106.8 & 738.505875 & 766.524329 & $\begin{array}{l}951.032766 \\
\end{array}$ & 734.341457 & $1.86 \mathrm{E}-08$ & $2.49 \mathrm{E}-05$ & 73.2913894 & 0.00783175 & 0.00574 & $1.24 \mathrm{E}-05$ & $1.25 \mathrm{E}-05$ & $2.37 \mathrm{E}-05$ & 0.02390933 & 0.05353858 & 0.00645759 & 3044500.37 \\
\hline 36068321.2 & 738.505875 & 766.511101 & 950.966042 & 734.329222 & $1.87 \mathrm{E}-08$ & $2.49 \mathrm{E}-05$ & 73.3621183 & 0.00795945 & 0.00583922 & $1.24 \mathrm{E}-05$ & $1.25 \mathrm{E}-05$ & $2.37 \mathrm{E}-05$ & 0.02400936 & 0.05441153 & 0.00647117 & 3086359.14 \\
\hline 36874839.2 & 738.505875 & 766.500008 & $\begin{array}{l}950.882845 \\
\end{array}$ & 734.313163 & $1.88 \mathrm{E}-08$ & $2.49 \mathrm{E}-05$ & \begin{tabular}{|l|l|}
73.4474006 \\
\end{tabular} & 0.00811907 & 0.00596324 & $1.24 \mathrm{E}-05$ & $1.25 \mathrm{E}-05$ & $2.38 E-05$ & 0.0241343 & 0.05550271 & 0.0064884 & 3138797.4 \\
\hline 37874839.2 & 738.505875 & 766.499907 & 950.788277 & 734.292479 & $1.90 \mathrm{E}-08$ & $2.49 \mathrm{E}-05$ & 73.5485965 & 0.00831698 & 0.00611702 & $1.24 \mathrm{E}-05$ & $1.25 \mathrm{E}-05$ & $2.38 \mathrm{E}-05$ & 0.02432509 & 0.05685566 & 0.00651068 & 3204474.96 \\
\hline 38874839.2 & 738.505875 & 766.491404 & 950.679752 & 734.273486 & $1.92 \mathrm{E}-08$ & $2.49 \mathrm{E}-05$ & \begin{tabular}{|l|}
73.6450881 \\
\end{tabular} & 0.00851489 & 0.0062708 & $1.25 \mathrm{E}-05$ & $1.25 \mathrm{E}-05$ & $2.41 \mathrm{E}-05$ & 0.0244675 & 0.05820861 & 0.00671205 & 3284312.86 \\
\hline 39574695 & $\begin{array}{l}738.144449 \\
\end{array}$ & 766.105673 & $\begin{array}{l}950.38635 \\
\end{array}$ & 733.979691 & $\begin{array}{ll}1.94 \mathrm{E}-08 \\
\end{array}$ & $2.49 \mathrm{E}-05$ & \begin{tabular}{|l|l|}
73.7099928 \\
\end{tabular} & 0.0086534 & 0.00637842 & $1.25 \mathrm{E}-05$ & $1.24 \mathrm{E}-05$ & $2.54 \mathrm{E}-05$ & 0.02456805 & 0.05915547 & 0.00713667 & 3361319.76 \\
\hline 39578295 & 738.81262 & 766.976263 & 952.302886 & 734.618272 & $1.94 \mathrm{E}-08$ & $2.49 \mathrm{E}-05$ & 73.7103225 & 0.00865412 & 0.00637898 & $1.25 \mathrm{E}-05$ & $1.24 \mathrm{E}-05$ & $2.55 \mathrm{E}-05$ & 0.02457427 & 0.05916036 & 0.00716141 & 3417047.2 \\
\hline 40278150.8 & 738.81262 & 766.962261 & \begin{tabular}{|c|}
952.229827 \\
\end{tabular} & 734.60666 & $\begin{array}{ll}1.96 \mathrm{E}-08 \\
\end{array}$ & $2.49 \mathrm{E}-05$ & \begin{tabular}{|l|l|}
73.7736083 \\
\end{tabular} & 0.00879361 & 0.00648736 & $1.25 \mathrm{E}-05$ & $1.24 \mathrm{E}-05$ & $2.69 \mathrm{E}-05$ & 0.02467819 & 0.06011391 & 0.00761784 & 3420053.41 \\
\hline 41152970.5 & 738.81262 & 768.111861 & 952.02256 & 734.648589 & $1.98 \mathrm{E}-08$ & $2.49 \mathrm{E}-05$ & 73.8499472 & 0.00896796 & 0.00662284 & $1.25 \mathrm{E}-05$ & $1.24 \mathrm{E}-05$ & $2.88 \mathrm{E}-05$ & 0.02480894 & 0.06130585 & 0.00822915 & 3476635.49 \\
\hline 41221690 & 738.81262 & 767.982437 & 952.001788 & 734.646809 & $\begin{array}{l}1.98 \mathrm{E}-08 \\
\end{array}$ & $2.49 \mathrm{E}-05$ & 73.8558182 & 0.00898166 & 0.00663348 & $1.25 \mathrm{E}-05$ & $1.24 \mathrm{E}-05$ & $2.90 \mathrm{E}-05$ & 0.02481814 & 0.06139948 & 0.00827773 & 3543418.52 \\
\hline 41290409.4 & 738.81262 & 767.854184 & 951.978606 & 734.645034 & $1.98 \mathrm{E}-08$ & $2.49 E-05$ & 73.8616714 & 0.00899536 & 0.00664412 & $1.25 \mathrm{E}-05$ & $1.24 \mathrm{E}-05$ & $2.92 E-05$ & 0.02482749 & 0.06149311 & 0.00832616 & 3548857.72 \\
\hline 41376308.8 & 738.81262 & 767.68823 & 951.948727 & 734.642768 & $\begin{array}{l}1.98 \mathrm{E}-08 \\
\end{array}$ & $2.49 \mathrm{E}-05$ & 73.8689629 & 0.00901248 & 0.00665742 & $1.25 \mathrm{E}-05$ & $1.24 \mathrm{E}-05$ & $2.93 \mathrm{E}-05$ & 0.02483984 & 0.06161015 & 0.00838712 & 3554359.52 \\
\hline 41483683 & 738.81262 & 767.493218 & 951.911438 & 734.63998 & $1.99 \mathrm{E}-08$ & $2.49 \mathrm{E}-05$ & 73.8780384 & 0.00903388 & 0.00667405 & $1.25 \mathrm{E}-05$ & $1.24 \mathrm{E}-05$ & $2.96 \mathrm{E}-05$ & 0.02485555 & 0.06175645 & 0.00846389 & 3561236.22 \\
\hline 41617900.7 & 738.81262 & 767.267745 & 951.865094 & 734.636584 & $\begin{array}{l}1.99 \mathrm{E}-08 \\
\end{array}$ & $2.49 \mathrm{E}-05$ & 73.8893224 & 0.00906063 & 0.00669484 & $1.25 \mathrm{E}-05$ & $1.24 \mathrm{E}-05$ & $2.99 \mathrm{E}-05$ & 0.02487534 & 0.06193932 & 0.00856077 & 3569833.9 \\
\hline 41785672.9 & 738.81262 & 767.014464 & 951.807153 & 734.632477 & $2.28 \mathrm{E}-08$ & $2.49 \mathrm{E}-05$ & 73.9033341 & 0.00909407 & 0.00672082 & $1.25 \mathrm{E}-05$ & $1.24 \mathrm{E}-05$ & $3.03 E-05$ & 0.02490045 & 0.06216791 & 0.00868321 & 3580577.79 \\
\hline 41995388.1 & 738.81262 & 766.933049 & 951.735376 & 734.627561 & $3.48 \mathrm{E}-08$ & $2.49 \mathrm{E}-05$ & 73.9207045 & 0.00913587 & 0.0067533 & $1.25 \mathrm{E}-05$ & $1.24 \mathrm{E}-05$ & $3.07 E-05$ & 0.02493205 & 0.06245364 & 0.00883825 & 3593998.81 \\
\hline 42194062 & 739.310003 & 767.440227 & 951.969224 & 735.010075 & $5.21 \mathrm{E}-08$ & $2.49 \mathrm{E}-05$ & 73.9370144 & 0.00917546 & 0.00678406 & $1.25 \mathrm{E}-05$ & $1.24 \mathrm{E}-05$ & $3.12 \mathrm{E}-05$ & 0.02496341 & 0.06272433 & 0.00899009 & 3612400.45 \\
\hline 42197662 & 727.940399 & 752.65564 & 919.149236 & 724.155418 & $5.21 \mathrm{E}-08$ & $2.49 \mathrm{E}-05$ & 73.9372912 & 0.00917614 & 0.00678459 & $1.25 \mathrm{E}-05$ & $1.24 \mathrm{E}-05$ & $3.00 \mathrm{E}-05$ & 0.02486344 & 0.06272895 & 0.0086203 & 3574364.01 \\
\hline 42396335.9 & 727.940399 & 752.654131 & 919.136955 & 724.151077 & $5.26 \mathrm{E}-08$ & $2.49 \mathrm{E}-05$ & 73.951547 & 0.00921104 & 0.0068117 & $1.25 \mathrm{E}-05$ & $1.24 \mathrm{E}-05$ & $3.03 \mathrm{E}-05$ & 0.02491348 & 0.06296752 & 0.00872455 & 3575322.5 \\
\hline 42644678.4 & \begin{tabular}{|l|l|}
727.940399 \\
\end{tabular} & 752.651768 & 919.109357 & 724.147042 & $5.37 \mathrm{E}-08$ & $2.49 \mathrm{E}-05$ & 73.9692157 & 0.00925466 & 0.0068456 & $1.25 \mathrm{E}-05$ & $1.24 \mathrm{E}-05$ & $3.08 E-05$ & 0.02494755 & 0.06326572 & 0.00888996 & 3589145.62 \\
\hline 42893020.8 & $\begin{array}{l}727.940399 \\
\end{array}$ & 752.648758 & 919.05484 & 724.143482 & $5.58 \mathrm{E}-08$ & $2.49 \mathrm{E}-05$ & 73.9867185 & 0.00929828 & 0.00687949 & $1.25 \mathrm{E}-05$ & $1.24 \mathrm{E}-05$ & $3.13 \mathrm{E}-05$ & 0.02497362 & 0.06356393 & 0.00905711 & 3606088.83 \\
\hline 43203448.9 & \begin{tabular}{|l|l|}
727.940399 \\
\end{tabular} & 752.645749 & 918.977697 & 724.138991 & \begin{tabular}{|c|c|}
$6.09 \mathrm{E}-08$ \\
\end{tabular} & $2.49 \mathrm{E}-05$ & 74.0083674 & 0.00935281 & 0.00692186 & $1.25 \mathrm{E}-05$ & $1.24 \mathrm{E}-05$ & $3.20 \mathrm{E}-05$ & 0.02501006 & 0.06393669 & 0.00926955 & 3623163.6 \\
\hline 43513876.9 & 727.940399 & 752.643455 & 918.899409 & 724.134586 & 7.01E-08 & $2.49 \mathrm{E}-05$ & 74.0297654 & 0.00940734 & 0.00696423 & $1.25 \mathrm{E}-05$ & $1.24 \mathrm{E}-05$ & $3.27 \mathrm{E}-05$ & 0.02504809 & 0.06430945 & 0.0094841 & 3644374.49 \\
\hline 43820808 & 726.737506 & 751.408976 & 918.088277 & 723.194957 & $8.43 \mathrm{E}-08$ & $2.49 \mathrm{E}-05$ & $\begin{array}{l}74.0506799 \\
\end{array}$ & 0.00946125 & 0.00700612 & $1.25 \mathrm{E}-05$ & $1.24 \mathrm{E}-05$ & $3.33 E-05$ & 0.02508203 & 0.06467801 & 0.00969031 & 3661003.74 \\
\hline 43824408 & 741.534973 & 770.70979 & 961.158465 & 737.357601 & $1.09 \mathrm{E}-07$ & $2.50 \mathrm{E}-05$ & 74.0509454 & 0.00946194 & 0.00700666 & $1.26 \mathrm{E}-05$ & $1.24 \mathrm{E}-05$ & $3.50 \mathrm{E}-05$ & 0.02520791 & 0.06468272 & 0.0102113 & 3752607.56 \\
\hline 43895709 & 741.552252 & 770.727682 & 961.161717 & 737.37002 & $7.05 E-07$ & $2.50 \mathrm{E}-05$ & 74.0566254 & 0.00947669 & 0.00701812 & $1.26 \mathrm{E}-05$ & $1.24 \mathrm{E}-05$ & $3.53 \mathrm{E}-05$ & 0.02521281 & 0.06478356 & 0.01029755 & 3753152.8 \\
\hline 43899309 & 741.29119 & 770.386901 & 960.410987 & 737.120036 & $7.32 \mathrm{E}-07$ & $2.50 \mathrm{E}-05$ & 74.0569113 & 0.00947744 & 0.0070187 & $1.26 \mathrm{E}-05$ & $1.24 \mathrm{E}-05$ & $3.53 \mathrm{E}-05$ & 0.02521136 & 0.06478864 & 0.01029304 & 3757579.01 \\
\hline 43903809 & 741.29119 & 770.386924 & 960.408698 & 737.119951 & $\begin{array}{l}7.68 \mathrm{E}-07 \\
\end{array}$ & $2.50 \mathrm{E}-05$ & 74.0572682 & 0.00947836 & 0.00701942 & $1.26 \mathrm{E}-05$ & $1.24 \mathrm{E}-05$ & $3.53 \mathrm{E}-05$ & 0.02521252 & 0.06479499 & 0.0102968 & 3757882.14 \\
\hline 43909434 & \begin{tabular}{|l|l|}
741.29119 \\
\end{tabular} & 770.38694 & 960.405894 & 737.119848 & $8.12 \mathrm{E}-07$ & $2.50 \mathrm{E}-05$ & 74.0577142 & 0.00947952 & 0.00702032 & $1.26 \mathrm{E}-05$ & $1.24 \mathrm{E}-05$ & $3.53 \mathrm{E}-05$ & 0.025214 & 0.06480292 & 0.0103014 & 3758258.94 \\
\hline 43916465.3 & 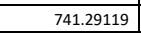 & 770.386975 & 960.400059 & 737.119719 & $8.67 \mathrm{E}-07$ & $2.50 \mathrm{E}-05$ & 74.0582715 & 0.00948098 & 0.00702145 & $1.26 \mathrm{E}-05$ & $1.24 \mathrm{E}-05$ & $3.53 \mathrm{E}-05$ & 0.02521581 & 0.06481284 & 0.01030703 & 3758732.06 \\
\hline 43925254.3 & 741.29119 & 770.387008 & 960.391459 & 737.119558 & $9.36 \mathrm{E}-07$ & $2.50 \mathrm{E}-05$ & 74.058968 & 0.00948279 & 0.00702286 & $1.26 \mathrm{E}-05$ & $1.24 \mathrm{E}-05$ & $3.53 \mathrm{E}-05$ & 0.02521806 & 0.06482524 & 0.01031399 & 3759321.83 \\
\hline 43936240.6 & 741.29119 & 770.387035 & 960.38039 & 737.119357 & $1.02 E-06$ & $2.50 \mathrm{E}-05$ & 74.0598382 & 0.00948506 & 0.00702462 & $1.26 \mathrm{E}-05$ & $1.24 \mathrm{E}-05$ & $3.54 \mathrm{E}-05$ & 0.02522088 & 0.06484073 & 0.01032271 & 3760058.51 \\
\hline 43949973.6 & 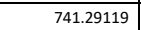 & 770.387077 & 960.363212 & 737.119107 & $1.13 \mathrm{E}-06$ & $2.50 \mathrm{E}-05$ & 74.0609253 & 0.00948789 & 0.00702682 & $1.26 \mathrm{E}-05$ & $1.24 \mathrm{E}-05$ & $3.54 \mathrm{E}-05$ & 0.02522431 & 0.06486011 & 0.01033368 & 3760979.87 \\
\hline 43967139.7 & 741.29119 & 770.387127 & 960.340684 & 737.118799 & $1.27 \mathrm{E}-06$ & $2.50 \mathrm{E}-05$ & 74.0622833 & 0.00949143 & 0.00702957 & $1.26 \mathrm{E}-05$ & $1.24 \mathrm{E}-05$ & $3.54 \mathrm{E}-05$ & 0.02522851 & 0.06488432 & 0.0103475 & 3762132.45 \\
\hline 43988597.4 & 741.29119 & 770.387128 & 960.316989 & 737.118421 & $1.45 \mathrm{E}-06$ & $2.50 \mathrm{E}-05$ & 74.0639794 & 0.00949586 & 0.00703301 & $1.26 \mathrm{E}-05$ & $1.24 \mathrm{E}-05$ & $3.55 \mathrm{E}-05$ & 0.02523345 & 0.06491458 & 0.01036491 & 3763565.18 \\
\hline 44015419.5 & 741.29119 & 770.38713 & 960.288118 & 737.117955 & $1.67 \mathrm{E}-06$ & $2.50 \mathrm{E}-05$ & 74.0660973 & 0.00950139 & 0.00703731 & $1.26 \mathrm{E}-05$ & $1.24 \mathrm{E}-05$ & $3.56 \mathrm{E}-05$ & 0.02523936 & 0.06495242 & 0.01038681 & 3765356.25 \\
\hline 44048947.1 & \begin{tabular}{|l|l|}
741.29119 \\
\end{tabular} & 770.387097 & 960.259144 & 737.11738 & $1.94 \mathrm{E}-06$ & $2.50 \mathrm{E}-05$ & 74.0687412 & 0.00950831 & 0.00704269 & $1.26 \mathrm{E}-05$ & $1.24 \mathrm{E}-05$ & $3.57 \mathrm{E}-05$ & 0.02524636 & 0.06499971 & 0.01041435 & 3767594.09 \\
\hline 44090856.6 & 741.29119 & 770.386895 & 960.238121 & 737.116674 & $2.30 \mathrm{E}-06$ & $2.50 \mathrm{E}-05$ & 74.0720406 & 0.00951696 & 0.00704941 & $1.26 \mathrm{E}-05$ & $1.24 \mathrm{E}-05$ & $3.58 \mathrm{E}-05$ & 0.02525409 & 0.06505882 & 0.01044895 & 3770374.91 \\
\hline 44143243.5 & \begin{tabular}{|l|l|}
741.29119 \\
\end{tabular} & 770.386573 & 960.219995 & 737.115802 & $2.74 \mathrm{E}-06$ & $2.50 \mathrm{E}-05$ & 74.0761565 & 0.00952777 & 0.0070578 & $1.26 \mathrm{E}-05$ & $1.24 \mathrm{E}-05$ & $3.59 \mathrm{E}-05$ & 0.02526308 & 0.06513272 & 0.01049242 & 3773845.71 \\
\hline 44208727.1 & 741.29119 & 770.386173 & 960.199714 & 737.114732 & $3.31 E-06$ & $2.50 \mathrm{E}-05$ & 74.0812883 & 0.00954128 & 0.0070683 & $1.26 \mathrm{E}-05$ & $1.24 \mathrm{E}-05$ & $3.61 \mathrm{E}-05$ & 0.02527409 & 0.06522508 & 0.010547 & 3778182.82 \\
\hline 44290581.6 & 741.29119 & 770.385537 & 960.185094 & 737.113418 & $4.05 E-06$ & $2.50 \mathrm{E}-05$ & 74.0876825 & 0.00955817 & 0.00708143 & $1.26 \mathrm{E}-05$ & $1.24 \mathrm{E}-05$ & $3.63 \mathrm{E}-05$ & 0.02528685 & 0.06534054 & 0.01061552 & 3783591.01 \\
\hline 44372436.1 & \begin{tabular}{|l|l|}
741.29119 \\
\end{tabular} & 770.384987 & 960.166454 & 737.112114 & $4.80 \mathrm{E}-06$ & $2.50 \mathrm{E}-05$ & 74.0940543 & 0.00957506 & 0.00709455 & $1.26 \mathrm{E}-05$ & $1.24 \mathrm{E}-05$ & $3.65 \mathrm{E}-05$ & 0.02529993 & 0.065456 & 0.01068431 & 3790297.54 \\
\hline 44401212 & 740.12978 & 769.192658 & 959.45876 & 736.207686 & 5.04E-06 & $2.50 \mathrm{E}-05$ & 74.0962889 & 0.00958099 & 0.00709916 & $1.26 \mathrm{E}-05$ & $1.24 \mathrm{E}-05$ & $3.65 \mathrm{E}-05$ & 0.02530089 & 0.06549658 & 0.01070111 & 3792365.79 \\
\hline 44404812 & 735.985891 & 763.770708 & 947.422262 & 732.232369 & $5.05 \mathrm{E}-06$ & $2.50 \mathrm{E}-05$ & 74.0965623 & 0.00958172 & 0.00709973 & $1.26 \mathrm{E}-05$ & $1.24 \mathrm{E}-05$ & $3.61 \mathrm{E}-05$ & 0.02526745 & 0.06550155 & 0.01056764 & 3774431.71 \\
\hline 44433587.9 & 735.985891 & 763.771275 & 947.368369 & 732.231898 & $5.11 \mathrm{E}-06$ & $2.50 \mathrm{E}-05$ & 74.0986982 & 0.0095874 & 0.00710414 & $1.26 \mathrm{E}-05$ & $1.24 \mathrm{E}-05$ & $3.61 \mathrm{E}-05$ & 0.02528035 & 0.0655404 & 0.01058329 & 3774838.18 \\
\hline 44469557.7 & 735.985891 & 763.771833 & 947.332873 & 732.231429 & $5.19 \mathrm{E}-06$ & $2.50 \mathrm{E}-05$ & 74.1013646 & 0.00959451 & 0.00710966 & $1.26 \mathrm{E}-05$ & $1.24 \mathrm{E}-05$ & $3.62 \mathrm{E}-05$ & 0.02529091 & 0.06558896 & 0.01061125 & 3777158.58 \\
\hline 44514520 & 735.985891 & 763.772138 & 947.31175 & 732.230828 & $5.29 \mathrm{E}-06$ & $2.50 \mathrm{E}-05$ & 74.104692 & 0.00960339 & 0.00711656 & $1.26 \mathrm{E}-05$ & $1.24 \mathrm{E}-05$ & $3.63 \mathrm{E}-05$ & 0.02530098 & 0.06564967 & 0.01064738 & 3780033.07 \\
\hline 44570722.8 & 735.985891 & 763.771503 & 947.312216 & 732.230004 & $5.42 E-06$ & $2.50 \mathrm{E}-05$ & 74.1088426 & 0.00961449 & 0.00712519 & $1.26 \mathrm{E}-05$ & $1.24 \mathrm{E}-05$ & $3.65 \mathrm{E}-05$ & 0.02530743 & 0.06572554 & 0.01069277 & 3783549.33 \\
\hline 44640976.4 & 735.985891 & 763.770512 & 947.326647 & 732.228973 & $5.60 \mathrm{E}-06$ & $2.50 \mathrm{E}-05$ & 74.1140174 & 0.00962836 & 0.00713597 & $1.26 \mathrm{E}-05$ & $1.24 \mathrm{E}-05$ & $3.66 \mathrm{E}-05$ & 0.0253144 & 0.06582039 & 0.01074966 & 3787922.77 \\
\hline 44728793.3 & 735985891 & 763.769467 & 947.336217 & 732,227712 & $5.83 \mathrm{E}-06$ & $2.50 \mathrm{E}-05$ & 74,120465 & 0.0096457 & 0.00714944 & $126 \mathrm{E}-05$ & $1.24 E-05$ & $3.68 \mathrm{E}-05$ & 0.02532414 & 0.06593895 & 0.01082097 & 3793400.34 \\
\hline
\end{tabular}




\begin{tabular}{|c|c|c|c|c|c|c|c|c|c|c|c|c|c|c|c|c|}
\hline Time (s) & $\begin{array}{c}\text { Coolant outlet } \\
\text { temperature (K) }\end{array}$ & $\begin{array}{c}\text { Peak cladding } \\
\text { temperature (K) }\end{array}$ & $\begin{array}{c}\text { Peak fuel } \\
\text { temperature }(K)\end{array}$ & $\begin{array}{c}\text { Average cladding } \\
\text { internal } \\
\text { temperature (K) }\end{array}$ & $\begin{array}{l}\text { Peak cumulative } \\
\text { damage factor }\end{array}$ & $\begin{array}{l}\text { Cladding interior } \\
\text { volume }\left(\mathrm{m}^{3}\right)\end{array}$ & $\begin{array}{l}\text { Fission gas } \\
\text { release (\%) }\end{array}$ & $\begin{array}{l}\text { Fission gas } \\
\text { produced } \\
\text { (moles) }\end{array}$ & $\begin{array}{c}\text { Fission gas } \\
\text { released (moles) }\end{array}$ & $\begin{array}{c}\text { Fuel volume } \\
\left(\mathrm{m}^{3}\right)\end{array}$ & $\begin{array}{l}\text { Plenum gas } \\
\text { volume }\left(m^{3}\right)\end{array}$ & $\begin{array}{l}\text { Peak cladding } \\
\text { radial growth } \\
\text { (m) }\end{array}$ & $\begin{array}{l}\text { Peak fuel axial } \\
\text { growth }(\mathrm{m})\end{array}$ & $\begin{array}{c}\text { Peak burnup } \\
\text { (at\%) }\end{array}$ & $\begin{array}{l}\text { Peak cladding } \\
\text { hoop strain }\end{array}$ & $\begin{array}{c}\text { Plenum pressure } \\
(\mathrm{Pa})\end{array}$ \\
\hline 44838564.5 & 735.985891 & 763.76845 & 947.335053 & 732.225844 & $6.15 \mathrm{E}-06$ & $2.50 \mathrm{E}-05$ & 74.128492 & 0.00966738 & 0.00716629 & $1.26 \mathrm{E}-05$ & $1.24 \mathrm{E}-05$ & $3.71 \mathrm{E}-05$ & 0.02533778 & 0.06608714 & 0.01091036 & 3800262.82 \\
\hline $\begin{array}{l}49975778.5 \\
\end{array}$ & 735.985891 & 763.767458 & \begin{tabular}{|l|l|}
947.319074 \\
\end{tabular} & 732.22295 & $\begin{array}{ll}6.58 \mathrm{E}-06 \\
\end{array}$ & $2.50 \mathrm{E}-05$ & 74.1384752 & 0.00969448 & 0.00718734 & $1.26 \mathrm{E}-05$ & $1.24 \mathrm{E}-05$ & $3.75 \mathrm{E}-05$ & 0.02535583 & 0.06627239 & 0.01102246 & 3808854.61 \\
\hline 45147296 & 735.985891 & 763.766374 & 947.29356 & 732.219423 & $7.18 \mathrm{E}-06$ & $2.50 \mathrm{E}-05$ & 74.150876 & 0.00972835 & 0.00721366 & $1.26 \mathrm{E}-05$ & $1.24 \mathrm{E}-05$ & $3.79 \mathrm{E}-05$ & 0.02537894 & 0.06650395 & 0.01116306 & 3819598.37 \\
\hline \begin{tabular}{|l|}
45361692.9 \\
\end{tabular} & 735.985891 & 763.765263 & \begin{tabular}{l|l|}
947.257142 \\
\end{tabular} & 732.214976 & $8.04 \mathrm{E}-06$ & $2.50 \mathrm{E}-05$ & 74.166256 & 0.0097707 & 0.00724656 & $1.26 \mathrm{E}-05$ & $1.24 \mathrm{E}-05$ & $3.84 \mathrm{E}-05$ & 0.02540837 & 0.0667934 & 0.01133943 & 3833035.7 \\
\hline 45629689 & 735.985891 & 763.764147 & \begin{tabular}{|l|l|}
947.208849 \\
\end{tabular} & 732.208933 & $9.28 \mathrm{E}-06$ & $2.50 \mathrm{E}-05$ & 74.1852947 & 0.00982362 & 0.00728768 & $1.26 \mathrm{E}-05$ & $1.24 \mathrm{E}-05$ & $3.91 \mathrm{E}-05$ & 0.02544531 & 0.06715521 & 0.01156079 & 3849834.26 \\
\hline \begin{tabular}{|l|}
45897685.1 \\
\end{tabular} & 735.985891 & 763.763176 & $\begin{array}{l}947.16206 \\
\end{array}$ & 732.202989 & $1.07 \mathrm{E}-05$ & $2.50 \mathrm{E}-05$ & 74.2041293 & 0.00987655 & 0.00732881 & $1.26 \mathrm{E}-05$ & $1.24 \mathrm{E}-05$ & $3.98 \mathrm{E}-05$ & 0.02548201 & 0.06751702 & 0.01178305 & 3870673.95 \\
\hline 46165681.2 & 735.985891 & 763.761317 & \begin{tabular}{|l|l|}
947.115687 \\
\end{tabular} & 732.19712 & $1.24 \mathrm{E}-05$ & $2.50 \mathrm{E}-05$ & 74.2227631 & 0.00992948 & 0.00736993 & $1.26 \mathrm{E}-05$ & $1.24 \mathrm{E}-05$ & $4.05 E-05$ & 0.02551859 & 0.06787883 & 0.01200616 & 3891515.57 \\
\hline \begin{tabular}{ll|}
46433677.3 \\
\end{tabular} & 735.985891 & 763.759708 & \begin{tabular}{l|l|}
947.072066 \\
\end{tabular} & 732.191408 & $1.43 \mathrm{E}-05$ & $2.50 \mathrm{E}-05$ & 74.2411993 & 0.0099824 & 0.00741105 & $1.26 \mathrm{E}-05$ & $1.24 \mathrm{E}-05$ & $4.12 \mathrm{E}-05$ & 0.02555448 & 0.06824064 & 0.01223009 & 3912351.19 \\
\hline 46701673.4 & 735.985891 & 763.754973 & 947.030402 & 732.185842 & $1.65 \mathrm{E}-05$ & $2.50 \mathrm{E}-05$ & 74.2594411 & 0.01003533 & 0.00745218 & $1.26 \mathrm{E}-05$ & $1.24 \mathrm{E}-05$ & $4.18 \mathrm{E}-05$ & 0.02558964 & 0.06860245 & 0.01245481 & 3933181.55 \\
\hline 46969669.5 & 735.985891 & 763.7486 & 946.991303 & 732.180383 & $1.89 \mathrm{E}-05$ & $2.50 \mathrm{E}-05$ & 74.2774914 & 0.01008825 & 0.0074933 & $1.26 \mathrm{E}-05$ & $1.24 \mathrm{E}-05$ & $4.25 \mathrm{E}-05$ & 0.02562444 & 0.06896426 & 0.01268028 & 3954004.35 \\
\hline 47237665.5 & 735.985891 & 763.74253 & 946.951111 & 732.175024 & $2.16 \mathrm{E}-05$ & $2.50 \mathrm{E}-05$ & 74.2953534 & 0.01014118 & 0.00753443 & $1.26 \mathrm{E}-05$ & $1.24 \mathrm{E}-05$ & $4.32 \mathrm{E}-05$ & 0.02565957 & 0.06932607 & 0.01290644 & 3974837.8 \\
\hline 47385472 & 736.569009 & 764.337615 & $\begin{array}{l}947.281982 \\
\end{array}$ & 732.625891 & $2.33 \mathrm{E}-05$ & $2.50 \mathrm{E}-05$ & 74.3051252 & 0.01017037 & 0.00755711 & $1.26 \mathrm{E}-05$ & $1.24 \mathrm{E}-05$ & $4.36 \mathrm{E}-05$ & 0.02568069 & 0.06952562 & 0.01303523 & 3997794.79 \\
\hline 47389072 & 734.674123 & 761.863547 & \begin{tabular}{|l|l|}
941.78243 \\
\end{tabular} & 730.811689 & $2.33 \mathrm{E}-05$ & $2.50 \mathrm{E}-05$ & 74.30536 & 0.01017108 & 0.00755765 & $1.26 \mathrm{E}-05$ & $1.24 \mathrm{E}-05$ & $4.34 \mathrm{E}-05$ & 0.0256662 & 0.06953043 & 0.01297592 & 3999203.33 \\
\hline 47536878.5 & 734.674123 & 761.860625 & 941.756776 & 730.808867 & $2.42 \mathrm{E}-05$ & $2.50 \mathrm{E}-05$ & 74.3148773 & 0.01019967 & 0.00757987 & $1.26 \mathrm{E}-05$ & $1.24 \mathrm{E}-05$ & $4.38 \mathrm{E}-05$ & 0.02568825 & 0.06972591 & 0.01309376 & 3999820.44 \\
\hline 47721636.5 & 734.674123 & 761.856981 & 941.730092 & 730.805565 & $2.55 \mathrm{E}-05$ & $2.50 \mathrm{E}-05$ & 74.3266992 & 0.01023542 & 0.00760765 & $1.26 \mathrm{E}-05$ & $1.24 \mathrm{E}-05$ & $4.43 \mathrm{E}-05$ & 0.02570913 & 0.06997027 & 0.01324656 & 4011102.39 \\
\hline 47952584.1 & 734.674123 & 761.852782 & 941.700021 & 730.801442 & $2.74 \mathrm{E}-05$ & $2.50 \mathrm{E}-05$ & 74.3413609 & 0.0102801 & 0.00764236 & $1.26 \mathrm{E}-05$ & $1.24 \mathrm{E}-05$ & $4.48 E-05$ & 0.02573721 & 0.07027572 & 0.01343822 & 4025236.02 \\
\hline 48198548 & 735.6194 & 762.817995 & 942.24029 & 731.531944 & $2.98 \mathrm{E}-05$ & $2.50 \mathrm{E}-05$ & 74.3568363 & 0.01032768 & 0.00767934 & $1.26 \mathrm{E}-05$ & $1.24 \mathrm{E}-05$ & $4.55 \mathrm{E}-05$ & 0.02577106 & 0.07060102 & 0.01364908 & 4046755.48 \\
\hline 48199031.2 & 723.263894 & 746.737432 & 906.12223 & 719.731084 & $2.98 \mathrm{E}-05$ & $2.50 \mathrm{E}-05$ & 74.3568646 & 0.01032777 & 0.00767941 & $1.26 \mathrm{E}-05$ & $1.24 \mathrm{E}-05$ & $4.42 \mathrm{E}-05$ & 0.02565349 & 0.07060162 & 0.01326097 & 4000312.43 \\
\hline 48199514.4 & 710.930879 & 730.708219 & 868.982721 & 707.952933 & $2.98 \mathrm{E}-05$ & $2.50 \mathrm{E}-05$ & 74.3568887 & 0.01032785 & 0.00767946 & $1.26 \mathrm{E}-05$ & $1.24 \mathrm{E}-05$ & $4.29 \mathrm{E}-05$ & 0.0255359 & 0.07060213 & 0.01286761 & 3935632.57 \\
\hline 48200118.3 & 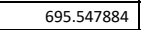 & 710.741933 & 821.012802 & 693.263309 & $2.98 \mathrm{E}-05$ & $2.50 \mathrm{E}-05$ & 74.3569132 & 0.01032792 & 0.00767952 & $1.26 \mathrm{E}-05$ & $1.24 \mathrm{E}-05$ & $4.12 \mathrm{E}-05$ & 0.02538317 & 0.07060265 & 0.01235819 & 3854718.76 \\
\hline 48200873.3 & 676.374052 & 685.886305 & 758.300275 & 674.951269 & $2.98 \mathrm{E}-05$ & $2.49 \mathrm{E}-05$ & 74.3569349 & 0.01032799 & 0.00767958 & $1.26 \mathrm{E}-05$ & $1.24 \mathrm{E}-05$ & $3.90 \mathrm{E}-05$ & 0.02517139 & 0.0706031 & 0.01170971 & 3753288.48 \\
\hline 48201628.3 & 657.264812 & 6001.129958 & 692.071823 & 656.691901 & $2.98 \mathrm{E}-05$ & $2.49 \mathrm{E}-05$ & 74.3569467 & 0.01032803 & 0.0076796 & $1.25 \mathrm{E}-05$ & $1.24 \mathrm{E}-05$ & $3.70 \mathrm{E}-05$ & 0.02490285 & 0.07060335 & 0.01112793 & 3650411.07 \\
\hline 48202148 & 644.15 & 644.16477 & 644.152377 & 644.150703 & $2.98 \mathrm{E}-05$ & $2.49 \mathrm{E}-05$ & 74.3569491 & 0.01032803 & 0.00767961 & $1.25 \mathrm{E}-05$ & $1.24 \mathrm{E}-05$ & $3.65 \mathrm{E}-05$ & 0.02470597 & 0.0706034 & 0.01096485 & 3578781.25 \\
\hline 48202903 & 573.02509 & 573.079705 & 573.029716 & 573.027724 & $2.98 \mathrm{E}-05$ & $2.48 E-05$ & 74.3569491 & 0.01032803 & 0.00767961 & $1.25 \mathrm{E}-05$ & $1.23 \mathrm{E}-05$ & $3.29 \mathrm{E}-05$ & 0.02427197 & 0.0706034 & 0.009997 & 3190192.54 \\
\hline 48203846.7 & 484.118952 & 484.172439 & \begin{tabular}{|c|}
484.12347 \\
\end{tabular} & 484.121442 & $2.98 \mathrm{E}-05$ & $2.47 \mathrm{E}-05$ & 74.3569491 & 0.01032803 & 0.00767961 & $1.24 \mathrm{E}-05$ & $1.23 \mathrm{E}-05$ & $2.85 \mathrm{E}-05$ & 0.0237305 & 0.0706034 & 0.00881228 & 2702052.01 \\
\hline 48205026.3 & 372.98628 & 373.038793 & 372.99068 & 372.988612 & $2.98 \mathrm{E}-05$ & $2.46 \mathrm{E}-05$ & 74.3569491 & 0.01032803 & 0.00767961 & $1.24 \mathrm{E}-05$ & $1.23 \mathrm{E}-05$ & $2.31 E-05$ & 0.02305479 & 0.0706034 & 0.00735994 & 2088279.34 \\
\hline 48205748 & 305 & 305.051622 & 305.004316 & 305.002233 & $2.98 \mathrm{E}-05$ & $2.46 E-05$ & 74.3569491 & 0.01032803 & 0.00767961 & $1.23 \mathrm{E}-05$ & $1.22 \mathrm{E}-05$ & $1.98 \mathrm{E}-05$ & 0.02264203 & 0.0706034 & 0.00647257 & 1710899.12 \\
\hline 48206927.6 & 305 & 305.051622 & 305.004316 & 305.002233 & $2.98 \mathrm{E}-05$ & $2.46 \mathrm{E}-05$ & 74.3569491 & 0.01032803 & 0.00767961 & $1.23 \mathrm{E}-05$ & $1.22 \mathrm{E}-05$ & $1.98 \mathrm{E}-05$ & 0.02264203 & 0.0706034 & 0.00647257 & 1710899.12 \\
\hline 48208402.2 & 305 & 305.051622 & \begin{tabular}{|c|}
305.004316 \\
\end{tabular} & 305.002233 & $2.98 \mathrm{E}-05$ & $2.46 \mathrm{E}-05$ & 74.3569491 & 0.01032803 & 0.00767961 & $1.23 \mathrm{E}-05$ & $1.22 \mathrm{E}-05$ & $1.98 \mathrm{E}-05$ & 0.02264203 & 0.0706034 & 0.00647257 & 1710899.12 \\
\hline 48209348 & 305 & 305.051622 & 305.004316 & 305.002233 & $2.98 \mathrm{E}-05$ & $2.46 E-05$ & 74.3569491 & 0.01032803 & 0.00767961 & $1.23 \mathrm{E}-05$ & $1.22 \mathrm{E}-05$ & $1.98 \mathrm{E}-05$ & 0.02264203 & 0.0706034 & 0.00647257 & 1710899.12 \\
\hline 48210822.6 & 478.604011 & 489.256824 & 589.165528 & 477.369555 & $2.98 \mathrm{E}-05$ & $2.47 \mathrm{E}-05$ & 74.3569675 & 0.01032809 & 0.00767965 & $1.25 \mathrm{E}-05$ & $1.23 \mathrm{E}-05$ & $2.80 \mathrm{E}-05$ & 0.02401779 & 0.07060379 & 0.00870709 & 2672187.53 \\
\hline 48211305.7 & 536.135624 & 550.207304 & 672.561807 & 534.281512 & $2.98 \mathrm{E}-05$ & $2.48 E-05$ & 74.3569816 & 0.01032813 & 0.00767969 & $1.25 \mathrm{E}-05$ & $1.23 \mathrm{E}-05$ & $3.21 \mathrm{E}-05$ & 0.02441959 & 0.07060409 & 0.00988493 & 2985968.75 \\
\hline 48211788.9 & 593.934884 & 611.482411 & \begin{tabular}{|c|}
752.67364 \\
\end{tabular} & 591.416359 & $2.98 \mathrm{E}-05$ & $2.49 \mathrm{E}-05$ & 74.3569996 & 0.01032819 & 0.00767973 & $1.25 \mathrm{E}-05$ & $1.23 \mathrm{E}-05$ & $3.60 \mathrm{E}-05$ & 0.02480993 & 0.07060447 & 0.01098656 & 3299692.73 \\
\hline 48212392.9 & 666.484041 & 688.559294 & 849.529391 & 663.150745 & $2.98 \mathrm{E}-05$ & $2.49 \mathrm{E}-05$ & 74.3570277 & 0.01032828 & 0.0076798 & $1.26 \mathrm{E}-05$ & $1.23 \mathrm{E}-05$ & $4.09 \mathrm{E}-05$ & 0.02527446 & 0.07060506 & 0.01237704 & 3691804.4 \\
\hline 48212948 & 733.362872 & 759.869589 & 935.746573 & 729.375637 & $2.98 \mathrm{E}-05$ & $2.50 \mathrm{E}-05$ & 74.357059 & 0.01032837 & 0.00767987 & $1.26 \mathrm{E}-05$ & $1.24 \mathrm{E}-05$ & $4.57 \mathrm{E}-05$ & 0.02566414 & 0.07060572 & 0.01374214 & 4051726.46 \\
\hline 48213552 & 733.362872 & 759.873784 & \begin{tabular}{|c|}
935.751859 \\
\end{tabular} & 729.379588 & $2.98 \mathrm{E}-05$ & $2.50 \mathrm{E}-05$ & 74.3570959 & 0.01032849 & 0.00767996 & $1.26 \mathrm{E}-05$ & $1.24 \mathrm{E}-05$ & $4.59 \mathrm{E}-05$ & 0.02564988 & 0.0706065 & 0.01378931 & 4051788.69 \\
\hline 48214307 & 733.362872 & 759.873868 & 935.752001 & 729.379748 & $2.99 \mathrm{E}-05$ & $2.50 \mathrm{E}-05$ & 74.357142 & 0.01032863 & 0.00768007 & $1.26 \mathrm{E}-05$ & $1.24 \mathrm{E}-05$ & $4.60 \mathrm{E}-05$ & 0.02564058 & 0.07060748 & 0.0138206 & 4051831.83 \\
\hline 48215250.7 & 733.362872 & 759.873934 & 935.752229 & 729.379865 & $2.99 \mathrm{E}-05$ & $2.50 \mathrm{E}-05$ & 74.3571997 & 0.01032881 & 0.00768021 & $1.26 \mathrm{E}-05$ & $1.24 \mathrm{E}-05$ & $4.61 \mathrm{E}-05$ & 0.0256335 & 0.0706087 & 0.01384486 & 4051887 \\
\hline 48216430.3 & 733.362872 & 759.873981 & 935.752486 & 729.379953 & $3.00 \mathrm{E}-05$ & $2.50 \mathrm{E}-05$ & $\begin{array}{l}74.3572717 \\
\end{array}$ & 0.01032903 & 0.00768038 & $1.26 \mathrm{E}-05$ & $1.24 \mathrm{E}-05$ & $4.61 \mathrm{E}-05$ & 0.0256277 & 0.07061022 & 0.0138651 & 4051958.2 \\
\hline 48217904.9 & 733.362872 & 759.874009 & \begin{tabular}{|c|}
935.7527 \\
\end{tabular} & 729.380019 & $3.01 E-05$ & $2.50 \mathrm{E}-05$ & 74.3573618 & 0.01032931 & 0.0076806 & $1.26 \mathrm{E}-05$ & $1.24 \mathrm{E}-05$ & $4.62 \mathrm{E}-05$ & 0.02562282 & 0.07061212 & 0.01388273 & 4052048.03 \\
\hline 48219748.1 & 733.362872 & 759.874017 & 935.752823 & 729.380063 & $3.03 \mathrm{E}-05$ & $2.50 \mathrm{E}-05$ & 74.3574744 & 0.01032966 & 0.00768087 & $1.26 \mathrm{E}-05$ & $1.24 \mathrm{E}-05$ & $4.63 \mathrm{E}-05$ & 0.02561874 & 0.0706145 & 0.01389846 & 4052159.02 \\
\hline 48222052.1 & 733.362872 & 759.874004 & 935.752875 & 729.380086 & $3.05 \mathrm{E}-05$ & $2.50 \mathrm{E}-05$ & 74.3576151 & 0.01033009 & 0.00768121 & $1.26 \mathrm{E}-05$ & $1.24 \mathrm{E}-05$ & $4.63 \mathrm{E}-05$ & 0.02561549 & 0.07061747 & 0.0139119 & 4052299.07 \\
\hline 48224932.1 & 733.362872 & 759.873967 & 935.752765 & 729.380079 & $3.07 \mathrm{E}-05$ & $2.50 \mathrm{E}-05$ & 74.3577909 & 0.01033063 & 0.00768163 & $1.26 \mathrm{E}-05$ & $1.24 \mathrm{E}-05$ & $4.63 \mathrm{E}-05$ & 0.02561311 & 0.07062119 & 0.01392356 & 4052471.54 \\
\hline 48228532.1 & 733.362872 & 759.873905 & 935.752503 & 729.380045 & $3.11 \mathrm{E}-05$ & $2.50 \mathrm{E}-05$ & 74.3580107 & 0.01033131 & 0.00768216 & $1.26 \mathrm{E}-05$ & $1.24 \mathrm{E}-05$ & $4.64 \mathrm{E}-05$ & 0.02561158 & 0.07062583 & 0.01393325 & 4052686.72 \\
\hline 48233032.1 & 733.362872 & 759.873815 & 935.752103 & 729.379984 & $3.15 \mathrm{E}-05$ & $2.50 \mathrm{E}-05$ & 74.3582854 & 0.01033216 & 0.00768282 & $1.26 \mathrm{E}-05$ & $1.24 \mathrm{E}-05$ & $4.64 \mathrm{E}-05$ & 0.02561082 & 0.07063164 & 0.01394153 & 4052954.35 \\
\hline 48023303657.1 & 733.3628282 & $\begin{array}{l}759.08780013696 \\
\end{array}$ & 935.751518 & 729.379889 & $\begin{array}{l}3.150-05 \\
3.20 \mathrm{E}-05\end{array}$ & $2.50 E-05$ & 74.358202877 & 0.01033322 & 0.000768364 & $\begin{array}{l}1.200-035 \\
1.26 \mathrm{E}-05\end{array}$ & $\begin{array}{l}1.24-24-05 \\
1.24-05\end{array}$ & $\begin{array}{l}4.044-65 \\
4.64-05\end{array}$ & 0.02561078 & 0.07063889 & 0.01394897 & 4053289.33 \\
\hline 48245688.3 & 733.362872 & 759.873541 & 935.750731 & 729.379762 & $\begin{array}{ll}3.26 \mathrm{E}-05 \\
\end{array}$ & $2.50 \mathrm{E}-05$ & 74.3590578 & 0.01033455 & 0.00768467 & $\begin{array}{ll}1.26 \mathrm{E}-05 \\
\end{array}$ & $1.24 E-05$ & $4.64 E-05$ & 0.02561125 & 0.07064797 & 0.01395638 & 4053707.63 \\
\hline 48254477.4 & 733.362872 & 759.873345 & 935.749723 & 729.379599 & $3.34 \mathrm{E}-05$ & $2.50 \mathrm{E}-05$ & 74.359594 & 0.01033621 & 0.00768596 & $1.26 \mathrm{E}-05$ & $1.24 \mathrm{E}-05$ & $4.65 \mathrm{E}-05$ & 0.0256121 & 0.0706593 & 0.01396452 & 4054229.78 \\
\hline 48265463.7 & 733.362872 & 759.8731 & \begin{tabular}{|c|}
935.748443 \\
\end{tabular} & 729.379395 & $3.45 \mathrm{E}-05$ & $2.50 \mathrm{E}-05$ & 74.3602639 & 0.01033828 & 0.00768757 & $1.26 \mathrm{E}-05$ & $1.24 \mathrm{E}-05$ & $4.65 \mathrm{E}-05$ & 0.02561331 & 0.07067348 & 0.01397412 & 4054882.63 \\
\hline 48279196.6 & 733.362872 & 759.872793 & \begin{tabular}{|c|}
935.746869 \\
\end{tabular} & 729.379138 & $3.57 \mathrm{E}-05$ & $2.50 \mathrm{E}-05$ & 74.361101 & 0.01034088 & 0.00768959 & $1.26 \mathrm{E}-05$ & $1.24 \mathrm{E}-05$ & $4.65 \mathrm{E}-05$ & 0.02561486 & 0.0706912 & 0.01398585 & 4055698.82 \\
\hline 48296362.8 & 733.362872 & 759.872411 & 935.744906 & 729.378817 & $3.73 \mathrm{E}-05$ & $2.50 \mathrm{E}-05$ & 74.3621467 & 0.01034411 & 0.00769211 & $1.26 \mathrm{E}-05$ & $1.24 \mathrm{E}-05$ & $4.66 \mathrm{E}-05$ & 0.02561688 & 0.07071334 & 0.01400042 & 4056720.4 \\
\hline 48317820.4 & 733.362872 & 759.871936 & 935.742487 & 729.378416 & $3.93 \mathrm{E}-05$ & $2.50 \mathrm{E}-05$ & 74.363453 & 0.01034816 & 0.00769525 & $1.26 \mathrm{E}-05$ & $1.24 \mathrm{E}-05$ & $4.66 \mathrm{E}-05$ & 0.0256194 & 0.07074103 & 0.01401857 & 4057997.15 \\
\hline 48344642.5 & 733.362872 & 759.871345 & 935.739522 & 729.377914 & $4.19 \mathrm{E}-05$ & $2.50 \mathrm{E}-05$ & 74.3650844 & 0.01035323 & 0.00769919 & $1.26 \mathrm{E}-05$ & $1.24 \mathrm{E}-05$ & $4.67 \mathrm{E}-05$ & 0.02562261 & 0.07077563 & 0.01404125 & 4059593.61 \\
\hline 48378170.1 & 733.362872 & 759.870613 & 935.735896 & 729.377284 & $4.52 \mathrm{E}-05$ & $2.50 \mathrm{E}-05$ & 74.3671213 & 0.01035955 & 0.0077041 & $1.26 \mathrm{E}-05$ & $1.24 E-05$ & $4.68 \mathrm{E}-05$ & 0.02562671 & 0.07081889 & 0.01406959 & 4061590.82 \\
\hline 48420079.7 & 733.362872 & 759.869706 & 935.731456 & 729.376496 & $4.91 \mathrm{E}-05$ & $2.50 \mathrm{E}-05$ & 74.3696641 & 0.01036746 & 0.00771025 & $1.26 \mathrm{E}-05$ & $1.24 E-05$ & $4.69 \mathrm{E}-05$ & 0.02563188 & 0.07087296 & 0.01410501 & 4064088.56 \\
\hline
\end{tabular}




\begin{tabular}{|c|c|c|c|c|c|c|c|c|c|c|c|c|c|c|c|c|}
\hline Time (s) & $\begin{array}{c}\text { Coolant outlet } \\
\text { temperature (K) }\end{array}$ & $\begin{array}{l}\text { Peak cladding } \\
\text { temperature (K) }\end{array}$ & $\begin{array}{c}\text { Peak fuel } \\
\text { temperature (K) }\end{array}$ & $\begin{array}{c}\text { Average cladding } \\
\text { internal } \\
\text { temperature (K) }\end{array}$ & $\begin{array}{l}\text { Peak cumulative } \\
\text { damage factor }\end{array}$ & $\begin{array}{l}\text { Cladding interior } \\
\text { volume }\left(\mathrm{m}^{3}\right)\end{array}$ & $\begin{array}{l}\text { Fission gas } \\
\text { release (\%) }\end{array}$ & $\begin{array}{c}\text { Fission gas } \\
\text { produced } \\
\text { (moles) }\end{array}$ & $\begin{array}{c}\text { Fission gas } \\
\text { released (moles) }\end{array}$ & $\begin{array}{c}\text { Fuel volume } \\
\left(\mathrm{m}^{3}\right)\end{array}$ & $\begin{array}{l}\text { Plenum gas } \\
\text { volume }\left(m^{3}\right)\end{array}$ & $\begin{array}{l}\text { Peak cladding } \\
\text { radiag growth } \\
(\mathbf{m})\end{array}$ & $\begin{array}{l}\text { Peak fuel axial } \\
\text { growth }(m)\end{array}$ & $\begin{array}{c}\text { Peak burnup } \\
\text { (at\%) }\end{array}$ & $\begin{array}{l}\text { Peak cladding } \\
\text { hoop strain }\end{array}$ & $\begin{array}{c}\text { Plenum pressure } \\
(\mathrm{Pa})\end{array}$ \\
\hline 48472466.6 & 733.362872 & 759.868582 & 935.725952 & 729.3755 & $5.44 E-05$ & $2.50 \mathrm{E}-05$ & 74.3728371 & 0.01037735 & 0.00771793 & $1.26 \mathrm{E}-05$ & $1.24 \mathrm{E}-05$ & $\begin{array}{r}4.70 E-05 \\
\end{array}$ & 0.02563846 & 0.07094055 & 0.01414928 & 4067216.34 \\
\hline 48537950.2 & 733.362872 & 759.867169 & 935.71903 & 729.374272 & $6.08 \mathrm{E}-05$ & $2.50 \mathrm{E}-05$ & \begin{tabular}{|l|l|}
74.3767948 \\
\end{tabular} & 0.01038971 & 0.00772753 & $1.26 \mathrm{E}-05$ & $1.24 \mathrm{E}-05$ & $4.72 \mathrm{E}-05$ & 0.0256467 & 0.07102504 & 0.01420461 & 4071121.8 \\
\hline 48619804.7 & 733.362872 & 759.865427 & 935.710189 & 729.372763 & $6.86 \mathrm{E}-05$ & $2.50 \mathrm{E}-05$ & 74.3817287 & 0.01040516 & 0.00773954 & $1.26 \mathrm{E}-05$ & $1.24 \mathrm{E}-05$ & $4.74 \mathrm{E}-05$ & 0.0256564 & 0.07113064 & 0.01427378 & 4075998.18 \\
\hline 48722122.9 & 733.362872 & 759.863304 & 935.698931 & 729.370886 & $7.79 \mathrm{E}-05$ & $2.50 \mathrm{E}-05$ & \begin{tabular}{|l|l|}
74.3878756 \\
\end{tabular} & 0.01042447 & 0.00775454 & $1.27 \mathrm{E}-05$ & $1.24 \mathrm{E}-05$ & $4.77 \mathrm{E}-05$ & 0.02566866 & 0.07126265 & 0.01436021 & 4082097.4 \\
\hline 48850020.5 & 733.362872 & 759.860736 & 935.684507 & 729.368552 & $8.90 \mathrm{E}-05$ & $2.50 \mathrm{E}-05$ & $\begin{array}{l}74.3955272 \\
\end{array}$ & 0.01044861 & 0.0077733 & $1.27 \mathrm{E}-05$ & $1.24 \mathrm{E}-05$ & $4.80 \mathrm{E}-05$ & 0.02568422 & 0.07142766 & 0.01446818 & 4089725.86 \\
\hline 48977918.2 & 733.362872 & 759.858265 & 935.669858 & 729.366246 & $9.95 \mathrm{E}-05$ & $2.50 \mathrm{E}-05$ & 74.4031435 & 0.01047275 & 0.00779205 & $1.27 \mathrm{E}-05$ & $1.24 \mathrm{E}-05$ & $4.83 \mathrm{E}-05$ & $\begin{array}{ll}0.02570003 \\
\end{array}$ & 0.07159267 & 0.01457609 & 4099193.16 \\
\hline 49137790.4 & 733.362872 & 759.855284 & 935.65105 & 729.363395 & 0.00011195 & $2.50 \mathrm{E}-05$ & 74.4126147 & 0.01050292 & 0.0078155 & $1.27 \mathrm{E}-05$ & $1.24 \mathrm{E}-05$ & $4.87 \mathrm{E}-05$ & 0.02571937 & 0.07179894 & 0.01471082 & 4108727.55 \\
\hline 49297662.5 & 733.362872 & 759.852473 & 935.6318 & 729.360575 & 0.00012382 & $2.50 \mathrm{E}-05$ & 74.4220316 & 0.01053309 & 0.00783894 & $1.27 \mathrm{E}-05$ & $1.24 \mathrm{E}-05$ & $4.91 \mathrm{E}-05$ & $\begin{array}{ll}0.02573918 \\
\end{array}$ & 0.0720052 & 0.01484543 & 4120568.51 \\
\hline 49477534.6 & 733.362872 & 759.849734 & 935.612406 & 729.35764 & 0.00013515 & $2.50 \mathrm{E}-05$ & 74.4313948 & 0.01056326 & 0.00786238 & $1.27 \mathrm{E}-05$ & $1.24 \mathrm{E}-05$ & $4.95 \mathrm{E}-05$ & 0.02575813 & 0.07221146 & $\begin{array}{l}0.01497991 \\
\end{array}$ & 4132397.45 \\
\hline 49657374.7 & \begin{tabular}{ll|}
733.362872 \\
\end{tabular} & 759.846464 & \begin{tabular}{|l|l|}
935.588344 \\
\end{tabular} & 729.353822 & 0.00014863 & $2.50 \mathrm{E}-05$ & \begin{tabular}{|l|l}
74.4430238 \\
\end{tabular} & 0.01060098 & 0.00789169 & $1.27 \mathrm{E}-05$ & $1.24 \mathrm{E}-05$ & $5.01 \mathrm{E}-05$ & \begin{tabular}{l|l|}
0.02578174 \\
\end{tabular} & 0.07246929 & 0.01514782 & 4144309.77 \\
\hline 49907174.9 & 733.362872 & 759.84264 & 935.558833 & 729.34922 & 0.0001646 & $2.50 \mathrm{E}-05$ & 74.4574441 & 0.01064812 & 0.00792832 & $1.27 \mathrm{E}-05$ & $1.24 \mathrm{E}-05$ & $5.07 \mathrm{E}-05$ & 0.02581075 & 0.07279158 & 0.01535748 & 4159194.28 \\
\hline 50156975.1 & 733.362872 & 759.838995 & 935.530284 & 729.344779 & 0.00017985 & $2.50 \mathrm{E}-05$ & 74.4717374 & 0.01069527 & 0.00796495 & $1.27 \mathrm{E}-05$ & $1.24 \mathrm{E}-05$ & $5.13 \mathrm{E}-05$ & 0.02583874 & 0.07311387 & 0.01556698 & 4177658.07 \\
\hline 50406775.3 & 733.362872 & 759.83549 & 935.50287 & 729.34047 & 0.0001945 & $2.50 \mathrm{E}-05$ & $\begin{array}{l}74.4859051 \\
\end{array}$ & 0.01074241 & 0.00800158 & $1.27 \mathrm{E}-05$ & $1.24 \mathrm{E}-05$ & $5.20 \mathrm{E}-05$ & 0.02586627 & 0.07343615 & 0.01577632 & 4196113.59 \\
\hline 50656575.4 & 733.362872 & 759.832259 & 935.476404 & 729.336281 & 0.00020867 & $2.50 \mathrm{E}-05$ & \begin{tabular}{|l|l|}
74.4999491 \\
\end{tabular} & 0.01078956 & 0.00803822 & $1.27 \mathrm{E}-05$ & $1.24 \mathrm{E}-05$ & $5.26 \mathrm{E}-05$ & 0.02589386 & 0.07375844 & 0.01598553 & 4214576.94 \\
\hline 50906375.6 & 733.362872 & 759.229222 & 935.450569 & 729.33222 & 0.00022242 & $2.50 \mathrm{E}-05$ & 74.5138709 & 0.0108367 & 0.00807485 & $1.27 \mathrm{E}-05$ & $1.23 \mathrm{E}-05$ & $5.32 \mathrm{E}-05$ & 0.02592151 & 0.07408073 & 0.01619461 & 4233041.45 \\
\hline 51156175.8 & 733.362872 & 759.826346 & 935.425356 & 729.328292 & 0.00023584 & $2.50 \mathrm{E}-05$ & 74.527672 & 0.01088385 & 0.00811148 & $1.27 \mathrm{E}-05$ & $1.23 \mathrm{E}-05$ & $5.39 \mathrm{E}-05$ & 0.02594876 & 0.07440302 & 0.01640359 & 4251499.78 \\
\hline 51405976 & 733.362872 & 759.823831 & 935.400506 & 729.324273 & 0.00024897 & $2.51 E-05$ & $\begin{array}{r}74.5413541 \\
\end{array}$ & 0.01093099 & 0.00814811 & $1.27 \mathrm{E}-05$ & $1.23 \mathrm{E}-05$ & $5.45 E-05$ & 0.02597731 & 0.0747253 & 0.01661247 & 4269972.14 \\
\hline 51655776.2 & 733.362872 & 759.821449 & 935.37582 & 729.320071 & 0.00026186 & $2.51 \mathrm{E}-05$ & 74.5549188 & 0.01097814 & 0.00818474 & $1.27 \mathrm{E}-05$ & $1.23 \mathrm{E}-05$ & $5.52 \mathrm{E}-05$ & 0.026005 & 0.07504759 & 0.01682126 & 4288438.6 \\
\hline 51968026.4 & 733.362872 & 759.819011 & 935.345121 & 729.314979 & 0.00027918 & $2.51 E-05$ & 74.5717115 & 0.01103707 & 0.00823053 & $1.27 \mathrm{E}-05$ & $1.23 \mathrm{E}-05$ & $5.59 \mathrm{E}-05$ & 0.02604101 & 0.07545045 & 0.01708212 & 4307046.31 \\
\hline 52079977 & 733.362872 & 759.818061 & 935.33406 & 729.3132 & 0.00028582 & $2.51 \mathrm{E}-05$ & 74.5776886 & 0.0110582 & 0.00824695 & $1.27 E-05$ & $1.23 \mathrm{E}-05$ & $5.62 \mathrm{E}-05$ & 0.02605307 & 0.07559489 & 0.01717572 & 4329739.099 \\
\hline 52083577 & 733.362872 & 759.818018 & 935.333696 & 729.31314 & 0.00028604 & $2.51 E-05$ & 74.5778805 & 0.01105888 & 0.00824748 & $1.27 \mathrm{E}-05$ & $1.23 \mathrm{E}-05$ & $5.62 \mathrm{E}-05$ & 0.02605332 & 0.07559953 & 0.01717895 & 4337806.52 \\
\hline 52195527.6 & 733.362872 & 759.817175 & 935.322718 & 729.311376 & 0.00029269 & $2.51 \mathrm{E}-05$ & 74.5838341 & 0.01108001 & 0.00826389 & $1.27 \mathrm{E}-05$ & $1.23 \mathrm{E}-05$ & $5.65 \mathrm{E}-05$ & 0.0260657 & 0.07574397 & 0.01727221 & 4338282.04 \\
\hline 52335465.9 & 733.362872 & 759.816247 & 935.309045 & 729.309174 & 0.00030103 & $2.51 \mathrm{E}-05$ & 74.5912442 & 0.01110642 & 0.00828441 & $1.27 \mathrm{E}-05$ & $1.23 \mathrm{E}-05$ & $5.69 \mathrm{E}-05$ & 0.02608154 & 0.07592451 & 0.01738904 & 4346620.53 \\
\hline 52475404.1 & 733.362872 & 759.815555 & 935.295446 & 729.307012 & 0.00030938 & $2.51 \mathrm{E}-05$ & 74.5986191 & 0.01113283 & 0.00830494 & $1.27 \mathrm{E}-05$ & $1.23 \mathrm{E}-05$ & $5.72 \mathrm{E}-05$ & 0.0260979 & 0.07610506 & 0.01750586 & 4356982.93 \\
\hline 52650326.9 & 733.362872 & 759.814616 & 935.278479 & 729.304328 & 0.00031985 & $2.51 E-05$ & 74.6077888 & 0.01116584 & 0.00833059 & $1.27 \mathrm{E}-05$ & $1.23 \mathrm{E}-05$ & $5.77 \mathrm{E}-05$ & 0.02611748 & 0.07633074 & 0.01765184 & 4367406.23 \\
\hline 52868980.5 & 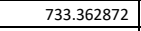 & 759.813551 & 935.257346 & 729.301038 & 0.00033299 & $2.51 \mathrm{E}-05$ & $\begin{array}{l}74.6191748 \\
\end{array}$ & 0.01120711 & 0.00836265 & $1.27 \mathrm{E}-05$ & $1.23 \mathrm{E}-05$ & $5.83 \mathrm{E}-05$ & $\begin{array}{l}0.026142 \\
\end{array}$ & 0.07661284 & 0.01783427 & 4380430.66 \\
\hline 53087634 & 733.362872 & 759.812781 & 935.236327 & 729.297799 & 0.00034619 & $2.51 E-05$ & 74.6304774 & 0.01124837 & 0.00839472 & $1.27 \mathrm{E}-05$ & $1.23 \mathrm{E}-05$ & $5.88 E-05$ & 0.02616631 & 0.07689494 & 0.01801667 & 4396611.68 \\
\hline 53360950.9 & 733.362872 & 759.807677 & 935.21034 & 729.293794 & 0.00036279 & $2.51 \mathrm{E}-05$ & 74.6444895 & 0.01129996 & 0.0084348 & $1.27 \mathrm{E}-05$ & $1.23 \mathrm{E}-05$ & $5.95 E-05$ & 0.02619565 & 0.07724757 & 0.01824459 & 4412880.93 \\
\hline 53634267.8 & 733.362872 & 759.802257 & 935.184834 & 729.289878 & 0.0003795 & $2.51 \mathrm{E}-05$ & 74.6583742 & 0.01135154 & 0.00847488 & $1.27 \mathrm{E}-05$ & $1.23 \mathrm{E}-05$ & $6.02 \mathrm{E}-05$ & $\begin{array}{l}0.0262241 \\
\end{array}$ & 0.0776002 & 0.01847246 & 4433073.37 \\
\hline 53874489 & 734.587462 & 761.052909 & 935.906909 & 730.238863 & 0.00039624 & $2.51 \mathrm{E}-05$ & 74.6704739 & 0.01139688 & 0.0085101 & $1.28 \mathrm{E}-05$ & $1.23 \mathrm{E}-05$ & $6.09 \mathrm{E}-05$ & 0.02625318 & 0.07791013 & 0.01868082 & 4458824.81 \\
\hline 53878089 & 734.893756 & 761.450282 & 936.782308 & 730.530396 & 0.00039651 & $2.51 E-05$ & 74.6706548 & 0.01139756 & 0.00851063 & $1.28 \mathrm{E}-05$ & $1.23 \mathrm{E}-05$ & $6.09 \mathrm{E}-05$ & 0.02625587 & 0.07791478 & 0.01869384 & 4477916.34 \\
\hline 54118310.2 & 734.893756 & 761.445034 & 936.760465 & 730.527046 & 0.0004163 & $2.51 \mathrm{E}-05$ & 74.6826973 & 0.01144305 & 0.00854598 & $1.28 \mathrm{E}-05$ & $1.23 \mathrm{E}-05$ & $6.15 \mathrm{E}-05$ & 0.02628153 & 0.07822575 & 0.01889553 & 4478631.74 \\
\hline 54418586.7 & 734.893756 & 761.43859 & 936.733725 & 730.522859 & 0.00044319 & $2.51 E-05$ & 74.6976165 & 0.01149991 & 0.00859016 & $1.28 \mathrm{E}-05$ & $1.23 \mathrm{E}-05$ & $6.23 \mathrm{E}-05$ & 0.02631435 & 0.078614466 & 0.0191467 & 4496603.29 \\
\hline 54718863.3 & 734.893756 & 761.432323 & 936.707388 & 730.518289 & 0.00047019 & $2.51 \mathrm{E}-05$ & 74.7123889 & 0.01155677 & 0.00863434 & $1.28 \mathrm{E}-05$ & $1.23 \mathrm{E}-05$ & $6.31 \mathrm{E}-05$ & 0.02634806 & 0.07900318 & 0.01939777 & 4518939.15 \\
\hline 55019139.8 & 734.893756 & 761.4262 & 936.681334 & 730.513933 & 0.00049728 & $2.51 E-05$ & 74.7270166 & 0.01161363 & 0.00867852 & $1.28 \mathrm{E}-05$ & $1.23 \mathrm{E}-05$ & $6.39 \mathrm{E}-05$ & 0.02638044 & 0.07939189 & 0.01964871 & 4541257.82 \\
\hline 55319416.3 & 734.893756 & 761.420326 & 936.655538 & 730.50793 & 0.00052446 & $2.51 \mathrm{E}-05$ & 74.7415018 & 0.01167049 & 0.0087227 & $1.28 \mathrm{E}-05$ & $1.23 \mathrm{E}-05$ & $6.46 \mathrm{E}-05$ & 0.02641237 & 0.0797806 & 0.01989953 & 4563563.01 \\
\hline 55619692.8 & 734.893756 & 762.303001 & 936.630044 & 730.581894 & 0.00055174 & $2.51 \mathrm{E}-05$ & 74.7558466 & 0.01172736 & 0.00876688 & $1.28 \mathrm{E}-05$ & $1.23 \mathrm{E}-05$ & $6.54 \mathrm{E}-05$ & 0.02644539 & 0.08016932 & 0.02015022 & 4586377.91 \\
\hline 55919969.4 & 734.893756 & 762.19889 & 936.604943 & 730.57594 & 0.00057909 & $2.51 \mathrm{E}-05$ & 74.7700529 & 0.01178422 & 0.00881107 & $1.28 \mathrm{E}-05$ & $1.23 \mathrm{E}-05$ & $6.62 \mathrm{E}-05$ & 0.02647758 & 0.08055803 & 0.02040079 & 4608691.38 \\
\hline 56220245.9 & 734.893756 & 762.109587 & 936.580203 & 730.570099 & 0.00060649 & $2.51 E-05$ & 74.7841222 & 0.01184108 & 0.00885525 & $1.28 \mathrm{E}-05$ & $1.23 \mathrm{E}-05$ & $6.70 \mathrm{E}-05$ & 0.02650929 & 0.08094674 & 0.02065122 & 4631004.34 \\
\hline 56520522.4 & 734.893756 & 762.040327 & 936.555845 & 730.564483 & 0.00063577 & $2.51 \mathrm{E}-05$ & 74.7980581 & 0.01189794 & 0.00889943 & $1.28 \mathrm{E}-05$ & $1.23 \mathrm{E}-05$ & $6.77 \mathrm{E}-05$ & 0.02653863 & 0.08133546 & 0.02090153 & 4653275.52 \\
\hline 56820798.9 & 734.893756 & 761.977389 & 936.531915 & 730.558882 & 0.00066803 & $2.51 E-05$ & 74.8118609 & 0.0119548 & 0.00894361 & $1.28 \mathrm{E}-05$ & $1.23 \mathrm{E}-05$ & $6.85 \mathrm{E}-05$ & 0.02656932 & 0.08172417 & 0.0211517 & 4675575.58 \\
\hline 57196144.6 & 734.893756 & 761.916011 & 936.502709 & 730.552073 & 0.0007087 & $2.51 \mathrm{E}-05$ & 74.8289309 & 0.01202588 & 0.00899884 & $1.28 \mathrm{E}-05$ & $1.23 \mathrm{E}-05$ & $6.95 \mathrm{E}-05$ & 0.02660749 & 0.08221006 & 0.0214642 & 4697998.86 \\
\hline 57571490.3 & 734.893756 & 761.871538 & 936.474127 & 730.545367 & 0.00074966 & $2.51 \mathrm{E}-05$ & 74.8458002 & 0.01209696 & 0.00905407 & $1.28 \mathrm{E}-05$ & $1.23 \mathrm{E}-05$ & $7.04 E-05$ & 0.02664622 & 0.08269595 & 0.02177651 & 4725878.18 \\
\hline 57946835.9 & $\begin{array}{l}734.893756 \\
\end{array}$ & 761.845248 & 936.446083 & 730.538808 & 0.00079088 & $2.51 \mathrm{E}-05$ & 74.8624725 & 0.01216804 & 0.00910929 & $1.28 \mathrm{E}-05$ & $1.23 \mathrm{E}-05$ & $7.14 \mathrm{E}-05$ & 0.02668451 & 0.08318184 & 0.0220886 & 4753755.27 \\
\hline 58322181.6 & $\begin{array}{l}734.893756 \\
\end{array}$ & 761.836667 & 936.41853 & 730.532403 & 0.00083231 & $2.52 \mathrm{E}-05$ & 74.8789512 & 0.01223911 & 0.00916452 & $1.28 \mathrm{E}-05$ & $1.23 \mathrm{E}-05$ & $7.24 \mathrm{E}-05$ & $\begin{array}{l}0.02672219 \\
\end{array}$ & 0.08366774 & 0.02240048 & 4781624.21 \\
\hline 58697527.2 & 734.893756 & 761.846132 & 936.39142 & 730.526082 & 0.00087392 & $2.52 \mathrm{E}-05$ & 74.8952395 & 0.01231019 & 0.00921975 & $1.29 \mathrm{E}-05$ & $1.23 \mathrm{E}-05$ & $7.33 \mathrm{E}-05$ & 0.02676007 & 0.08415363 & 0.02271212 & 4809499.05 \\
\hline 59072872.9 & 734.893756 & 761.875505 & 936.364705 & 730.519816 & 0.00091565 & $2.52 \mathrm{E}-05$ & 74.9113408 & 0.01238127 & 0.00927497 & $1.29 \mathrm{E}-05$ & $1.23 \mathrm{E}-05$ & $7.43 \mathrm{E}-05$ & 0.0267986 & 0.08463952 & 0.02302353 & 4837385.74 \\
\hline 59448218.5 & 734.893756 & 761.920518 & 936.33835 & 730.513692 & 0.00095746 & $2.52 \mathrm{E}-05$ & 74.9272584 & 0.01245235 & 0.0093302 & $1.29 \mathrm{E}-05$ & $1.23 \mathrm{E}-05$ & $7.53 \mathrm{E}-05$ & 0.02683494 & 0.08512541 & 0.02333471 & 4865242.07 \\
\hline 59823564.2 & 734.893756 & 761.991271 & 936.312369 & 730.507635 & 0.0009993 & $2.52 E-05$ & 74.9429952 & 0.01252342 & 0.00938543 & $1.29 \mathrm{E}-05$ & $1.23 \mathrm{E}-05$ & $7.62 E-05$ & 0.02687329 & 0.0856113 & 0.02364565 & 4893134.56 \\
\hline 60198909.8 & 734.893756 & 762.076536 & 936.286712 & 730.501695 & 0.00104112 & $2.52 \mathrm{E}-05$ & 74.9585544 & 0.0125945 & 0.00944066 & $1.29 \mathrm{E}-05$ & $1.23 \mathrm{E}-05$ & $7.72 \mathrm{E}-05$ & 0.02691041 & 0.08609719 & 0.02395635 & 4921006.45 \\
\hline 60391086.8 & 734.893756 & 762.124319 & 936.273647 & 730.498697 & 0.00106499 & $2.52 \mathrm{E}-05$ & 74.966453 & 0.01263089 & 0.00946893 & $1.29 \mathrm{E}-05$ & $1.23 \mathrm{E}-05$ & $7.77 \mathrm{E}-05$ & 0.02692883 & 0.08634597 & 0.02411542 & 4948567.54 \\
\hline 60631308 & 734.893756 & & 936.257464 & 730.494283 & 0.00109592 & $2.52 \mathrm{E}-05$ & 74.9762624 & 0.01267638 & 0.00950428 & $1.29 \mathrm{E}-05$ & $1.23 \mathrm{E}-05$ & $7.83 \mathrm{E}-05$ & 0.02695203 & 0.08665694 & 0.02431403 & 4962903.97 \\
\hline 60871529.3 & 734.893756 & 762.010798 & 936.241418 & 730.489961 & 0.00112691 & $2.52 \mathrm{E}-05$ & $\begin{array}{ll}74.9860016 \\
\end{array}$ & 0.01272187 & 0.00953962 & $1.29 \mathrm{E}-05$ & $1.23 \mathrm{E}-05$ & $7.89 \mathrm{E}-05$ & 0.02697464 & 0.08696791 & 0.02451257 & 4980727.14 \\
\hline 61111750.5 & 734.893756 & 761.971774 & 936.225517 & 730.485674 & 0.00115792 & $2.52 E-05$ & 74.9956715 & 0.01276736 & 0.00957497 & $1.29 \mathrm{E}-05$ & $1.23 \mathrm{E}-05$ & $7.95 \mathrm{E}-05$ & 0.02699781 & 0.08727888 & 0.02471101 & 4998554.11 \\
\hline 61351971.7 & 734.893756 & 761.909807 & 936.209762 & 730.481311 & 0.00118895 & $2.52 \mathrm{E}-05$ & 75.0052727 & 0.01281285 & 0.00961031 & $1.29 \mathrm{E}-05$ & $1.23 E-05$ & $8.02 E-05$ & 0.02702061 & 0.08758985 & 0.02490933 & 5016380.76 \\
\hline
\end{tabular}




\begin{tabular}{|c|c|c|c|c|c|c|c|c|c|c|c|c|c|c|c|c|}
\hline Time (s) & $\begin{array}{c}\text { Coolant outlet } \\
\text { temperature (K) }\end{array}$ & $\begin{array}{l}\text { Peak cladding } \\
\text { temperature (K) }\end{array}$ & $\begin{array}{c}\text { Peak fuel } \\
\text { temperature (K) }\end{array}$ & $\begin{array}{c}\text { Average cladding } \\
\text { internal } \\
\text { temperature (K) } \\
\end{array}$ & $\begin{array}{l}\text { Peak cumulative } \\
\text { damage factor }\end{array}$ & $\begin{array}{l}\text { Cladding interior } \\
\text { volume }\left(\mathrm{m}^{3}\right)\end{array}$ & $\begin{array}{l}\text { Fission gas } \\
\text { release (\%) }\end{array}$ & $\begin{array}{c}\text { Fission gas } \\
\text { produced } \\
\text { (moles) }\end{array}$ & $\begin{array}{c}\text { Fission gas } \\
\text { released (moles) }\end{array}$ & $\begin{array}{c}\text { Fuel volume } \\
\left(\mathrm{m}^{3}\right)\end{array}$ & $\begin{array}{l}\text { Plenum gas } \\
\text { volume }\left(m^{3}\right)\end{array}$ & $\begin{array}{l}\text { Peak cladding } \\
\text { radial growth } \\
\text { (m) }\end{array}$ & $\begin{array}{l}\text { Peak fuel axial } \\
\text { growth }(m)\end{array}$ & $\begin{array}{c}\text { Peak burnup } \\
\text { (at\%) }\end{array}$ & $\begin{array}{l}\text { Peak cladding } \\
\text { hoop strain }\end{array}$ & $\begin{array}{c}\text { Plenum pressure } \\
(\mathrm{Pa})\end{array}$ \\
\hline 61592192.9 & 734.893756 & 761.842184 & 936.194158 & 730.476931 & 0.00121999 & $2.52 E-05$ & 75.0148059 & 0.01285834 & 0.00964566 & $1.29 \mathrm{E}-05$ & $1.23 E-05$ & $8.08 \mathrm{E}-05$ & 0.02704388 & 0.08790082 & 0.02510756 & $\begin{array}{r}5034207.16 \\
\end{array}$ \\
\hline 61892469.5 & 734.893756 & 761.761017 & 936.174876 & 730.471472 & 0.00125875 & $2.52 \mathrm{E}-05$ & \begin{tabular}{|l|}
75.026628 \\
\end{tabular} & 0.0129152 & 0.00968984 & $1.29 \mathrm{E}-05$ & $1.23 \mathrm{E}-05$ & $8.15 \mathrm{E}-05$ & 0.02707315 & 0.08828954 & 0.02535516 & 5052139.78 \\
\hline 62125235 & 735.150157 & 761.965537 & 936.315713 & 730.666662 & 0.00128972 & $2.52 E-05$ & 75.0357208 & 0.01295928 & 0.00972409 & $1.29 \mathrm{E}-05$ & $1.23 \mathrm{E}-05$ & $8.21 \mathrm{E}-05$ & 0.02709674 & 0.08859085 & 0.02554884 & 5075656.56 \\
\hline 62128835 & 730.972873 & 756.547581 & \begin{tabular}{|l|}
924.37101 \\
\end{tabular} & 726.695099 & 0.00128983 & $2.52 \mathrm{E}-05$ & \begin{tabular}{|l|l|}
75.0358578 \\
\end{tabular} & 0.01295995 & 0.00972461 & $1.29 \mathrm{E}-05$ & $1.23 \mathrm{E}-05$ & $8.17 \mathrm{E}-05$ & $\begin{array}{ll}0.0270655 \\
\end{array}$ & 0.08859541 & 0.02541672 & 5065536.42 \\
\hline 62256058 & $\begin{array}{l}731.250458 \\
\end{array}$ & 756.775625 & 924.532542 & 726.908326 & 0.00129383 & $2.52 \mathrm{E}-05$ & \begin{tabular}{ll|}
75.0405761 \\
\end{tabular} & 0.01298294 & 0.00974247 & $1.29 \mathrm{E}-05$ & $1.23 \mathrm{E}-05$ & $8.20 \mathrm{E}-05$ & 0.02709487 & 0.08875259 & 0.0255066 & 5067622.7 \\
\hline 62259658 & $\begin{array}{l}733.325046 \\
\end{array}$ & 759.460834 & 930.462915 & 728.879339 & 0.00129405 & $2.52 \mathrm{E}-05$ & \begin{tabular}{|l|}
75.0407109 \\
\end{tabular} & 0.0129836 & 0.00974298 & $1.29 \mathrm{E}-05$ & $1.23 \mathrm{E}-05$ & $8.22 \mathrm{E}-05$ & 0.02711096 & 0.08875709 & 0.02557155 & 5089975.34 \\
\hline 62264158 & 733.325046 & 759.460724 & 930.462863 & 728.879354 & 0.00129435 & $2.52 E-05$ & 75.0408815 & 0.01298443 & 0.00974363 & $1.29 \mathrm{E}-05$ & $1.23 \mathrm{E}-05$ & $8.22 \mathrm{E}-05$ & 0.02710999 & 0.08876279 & 0.02558215 & 5090122.5 \\
\hline 62269783 & 733.325046 & 759.460335 & 930.462621 & 728.879279 & 0.00129473 & $2.52 E-05$ & 75.0410946 & 0.01298547 & 0.00974444 & $1.29 \mathrm{E}-05$ & $1.23 \mathrm{E}-05$ & $8.22 \mathrm{E}-05$ & 0.02710971 & 0.0887699 & 0.02558822 & 5090444.21 \\
\hline 62276814.3 & 733.325046 & 759.459661 & 930.462233 & 728.879174 & 0.0012952 & $2.52 E-05$ & 75.0413609 & 0.01298677 & 0.00974545 & $1.29 \mathrm{E}-05$ & $1.23 \mathrm{E}-05$ & $8.23 \mathrm{E}-05$ & 0.02710977 & 0.08877879 & 0.025594 & 5090846.97 \\
\hline 62285603.3 & $\begin{array}{l}733.325046 \\
\end{array}$ & 759.458546 & 930.461691 & 728.879039 & 0.00129579 & $2.52 \mathrm{E}-05$ & \begin{tabular}{|l|}
75.0416937 \\
\end{tabular} & 0.0129884 & 0.00974671 & $1.29 \mathrm{E}-05$ & $1.23 \mathrm{E}-05$ & $8.23 \mathrm{E}-05$ & 0.02711017 & 0.08878991 & 0.02560195 & 5091350.68 \\
\hline 62296589.6 & 733.325046 & 759.457233 & 930.461009 & 728.878872 & 0.00129653 & $2.52 \mathrm{E}-05$ & 75.0421097 & 0.01299043 & 0.00974829 & $1.29 \mathrm{E}-05$ & $1.23 \mathrm{E}-05$ & $8.23 \mathrm{E}-05$ & 0.02711076 & 0.0888038 & 0.02561094 & 5091982.76 \\
\hline 62310322.6 & 733.325046 & 759.45559 & 930.460183 & 728.878661 & 0.00129746 & $2.52 E-05$ & 75.0426294 & 0.01299297 & 0.00975027 & $1.29 \mathrm{E}-05$ & $1.23 \mathrm{E}-05$ & $8.24 E-05$ & 0.02711157 & 0.08882117 & 0.02562198 & 5092773.12 \\
\hline 62327488.7 & 733.325046 & 759.453349 & 930.459165 & 728.878394 & 0.00129863 & $2.52 \mathrm{E}-05$ & \begin{tabular}{ll|l|}
55.0432788 \\
\end{tabular} & 0.01299615 & 0.00975273 & $1.29 \mathrm{E}-05$ & $1.23 \mathrm{E}-05$ & $8.24 \mathrm{E}-05$ & 0.02711261 & 0.08884288 & 0.0256357 & 5093764.03 \\
\hline 62348946.4 & 733.325046 & 759.450511 & 930.457894 & 728.878059 & 0.00130009 & $2.52 \mathrm{E}-05$ & 75.04409 & 0.01300012 & 0.00975582 & $1.29 \mathrm{E}-05$ & $1.23 \mathrm{E}-05$ & $8.25 \mathrm{E}-05$ & 0.02711393 & 0.08887002 & 0.02565284 & 5095003.32 \\
\hline 62375768.5 & 733.325046 & 759.446769 & 930.456304 & 728.877638 & 0.00130192 & $2.52 E-05$ & 75.0451034 & 0.01300508 & 0.00975967 & $1.29 \mathrm{E}-05$ & $1.23 \mathrm{E}-05$ & $8.25 \mathrm{E}-05$ & 0.02711569 & 0.08890394 & 0.02567432 & 5096552.53 \\
\hline 62409296.1 & 733.325046 & 759.441894 & 930.454318 & 728.877107 & 0.00130423 & $2.52 E-05$ & 75.0463691 & 0.01301128 & 0.00976449 & $1.29 \mathrm{E}-05$ & $1.23 \mathrm{E}-05$ & $8.26 \mathrm{E}-05$ & 0.02711802 & 0.08894634 & 0.02570121 & 5098490.01 \\
\hline 62451205.6 & 733.325046 & 759.435652 & 930.451838 & 728.876441 & 0.00130713 & $2.52 E-05$ & 75.0479495 & 0.01301903 & 0.00977052 & $1.29 \mathrm{E}-05$ & $1.23 \mathrm{E}-05$ & $8.27 \mathrm{E}-05$ & 0.02712105 & 0.08899935 & 0.02573482 & 5100913.17 \\
\hline 62503592.5 & 733.325046 & 759.426864 & 930.448739 & 728.875593 & 0.00131079 & $2.52 \mathrm{E}-05$ & 75.0499223 & 0.01302873 & 0.00977805 & $1.29 \mathrm{E}-05$ & $1.23 \mathrm{E}-05$ & $8.28 \mathrm{E}-05$ & 0.02712509 & 0.0890656 & 0.02577683 & 5103949.71 \\
\hline 62569076.1 & 733.325046 & 759.415966 & 930.444867 & 728.874531 & 0.0013154 & $2.52 E-05$ & 75.0523842 & 0.01304084 & 0.00978746 & $1.29 \mathrm{E}-05$ & $1.23 \mathrm{E}-05$ & $8.30 \mathrm{E}-05$ & 0.02713033 & 0.08914842 & 0.02582935 & 5107745.98 \\
\hline 62620357 & 733.011845 & 759.085968 & 930.251173 & 728.630175 & 0.0013189 & $2.52 \mathrm{E}-05$ & 75.054309 & 0.01305033 & 0.00979483 & $1.29 \mathrm{E}-05$ & $1.23 \mathrm{E}-05$ & $8.31 \mathrm{E}-05$ & 0.02713349 & 0.08921328 & 0.02586829 & 5110801.06 \\
\hline 62623957 & 733.482458 & 759.695299 & 931.596199 & 729.07753 & 0.00131919 & $2.52 E-05$ & 75.0544444 & 0.013051 & 0.00979535 & $1.29 \mathrm{E}-05$ & $1.23 \mathrm{E}-05$ & $8.32 \mathrm{E}-05$ & 0.02713725 & 0.08921784 & 0.02588613 & 5117507.44 \\
\hline 62675237.9 & \begin{tabular}{|l|l|}
733.482458 \\
\end{tabular} & 759.687698 & 931.593198 & 729.076719 & 0.00132337 & $2.52 \mathrm{E}-05$ & 75.0563762 & 0.01306053 & 0.00980276 & $1.29 \mathrm{E}-05$ & $1.23 \mathrm{E}-05$ & $8.33 \mathrm{E}-05$ & $\begin{array}{l}0.0271405 \\
\end{array}$ & 0.08928304 & 0.02592911 & 5117818.33 \\
\hline 62739339 & 733.482458 & 759.676687 & 931.589351 & 729.07566 & 0.00132863 & $2.52 \mathrm{E}-05$ & 75.0587871 & 0.01307246 & 0.00981203 & $1.29 \mathrm{E}-05$ & $1.23 \mathrm{E}-05$ & $8.35 E-05$ & 0.0271459 & 0.08936454 & 0.02598096 & 5121557.13 \\
\hline 62819465.5 & 733.482458 & 759.661928 & 931.584531 & 729.074318 & 0.00133526 & $2.52 \mathrm{E}-05$ & 75.0617945 & 0.01308736 & 0.00982361 & $1.29 \mathrm{E}-05$ & $1.23 \mathrm{E}-05$ & $8.37 \mathrm{E}-05$ & 0.02715314 & 0.08946641 & 0.02604567 & 5126242.29 \\
\hline 62919623.5 & 733.482458 & 759.643883 & 931.578496 & 729.072641 & 0.00134362 & $2.52 \mathrm{E}-05$ & 75.0655441 & 0.01310598 & 0.00983808 & $1.29 \mathrm{E}-05$ & $1.23 \mathrm{E}-05$ & $8.39 \mathrm{E}-05$ & 0.02716235 & 0.08959375 & 0.02612653 & 5132097.86 \\
\hline 63044821 & \begin{tabular}{|l|l|}
733.42458 \\
\end{tabular} & 759.621586 & 931.570943 & 729.070538 & 0.00135418 & $2.52 \mathrm{E}-05$ & $\begin{array}{r}75.0702162 \\
\end{array}$ & 0.01312927 & 0.00985617 & $1.29 \mathrm{E}-05$ & $1.23 \mathrm{E}-05$ & $8.42 \mathrm{E}-05$ & 0.02717398 & 0.08975292 & 0.0262276 & 5139426.41 \\
\hline 63201317.9 & 733.482458 & 759.596894 & 931.561514 & 729.067942 & 0.00137046 & $2.52 E-05$ & 75.0760331 & 0.01315837 & 0.00987879 & $1.29 \mathrm{E}-05$ & $1.23 \mathrm{E}-05$ & $8.46 \mathrm{E}-05$ & 0.02718793 & 0.08995188 & 0.02635392 & 5148570.41 \\
\hline 63396939.1 & 733.482458 & 759.568188 & 931.549787 & 729.064698 & 0.00139324 & $2.52 \mathrm{E}-05$ & 75.0832681 & 0.01319476 & 0.00990705 & $1.29 \mathrm{E}-05$ & $1.23 \mathrm{E}-05$ & $8.51 \mathrm{E}-05$ & 0.02720541 & 0.09020059 & 0.0265118 & 5159998.16 \\
\hline 63641465.5 & 733.482458 & 759.534545 & 931.535271 & 729.060644 & 0.00142232 & $2.52 \mathrm{E}-05$ & 75.0922559 & 0.01324023 & 0.00994239 & $1.29 \mathrm{E}-05$ & $1.23 \mathrm{E}-05$ & $8.57 \mathrm{E}-05$ & 0.02722771 & 0.09051148 & $\begin{array}{l}0.0267091 \\
\end{array}$ & 5174301.47 \\
\hline 63885991.9 & 733.482458 & 759.50625 & 931.520918 & 729.056641 & 0.00146042 & $2.52 \mathrm{E}-05$ & 75.1011822 & 0.01328571 & 0.00997772 & $1.30 \mathrm{E}-05$ & $1.23 \mathrm{E}-05$ & $8.64 \mathrm{E}-05$ & 0.02724942 & 0.09082236 & 0.02690636 & 5192069.27 \\
\hline 64130518.3 & 733.482458 & 759.481995 & 931.50673 & 729.052681 & 0.00150166 & $2.52 \mathrm{E}-05$ & 75.1100476 & 0.01333119 & 0.01001306 & $1.30 \mathrm{E}-05$ & $1.23 \mathrm{E}-05$ & $8.70 \mathrm{E}-05$ & 0.02727102 & 0.09113325 & 0.02710357 & 5209840.8 \\
\hline 64436176.3 & 733.482458 & 759.457582 & 931.489244 & 729.047769 & 0.00155482 & $2.52 \mathrm{E}-05$ & 75.1210446 & 0.01338803 & 0.01005723 & $1.30 \mathrm{E}-05$ & $1.23 \mathrm{E}-05$ & $8.77 \mathrm{E}-05$ & 0.02729831 & 0.09152185 & 0.02734995 & 5227701.81 \\
\hline 64516928 & 732.345558 & 758.285412 & 930.792704 & 728.162369 & 0.00156692 & $2.52 E-05$ & 75.1239344 & 0.01340305 & 0.0100689 & $1.30 \mathrm{E}-05$ & $1.23 \mathrm{E}-05$ & $8.79 \mathrm{E}-05$ & 0.02730205 & 0.09162452 & 0.02740709 & 5243446.43 \\
\hline 64520528 & \begin{tabular}{|l}
731.799948 \\
\end{tabular} & 757.576998 & 929.216877 & 727.642578 & 0.00156736 & $2.52 \mathrm{E}-05$ & 75.1240626 & 0.01340372 & 0.01006942 & $1.30 \mathrm{E}-05$ & $1.23 \mathrm{E}-05$ & $8.79 \mathrm{E}-05$ & 0.0272985 & 0.09162908 & 0.02739222 & 5245542.75 \\
\hline 64601279.7 & 731.799948 & 757.572296 & 929.212273 & 727.64129 & 0.00157734 & $2.52 E-05$ & 75.1269279 & 0.01341864 & 0.01008101 & $1.30 \mathrm{E}-05$ & $1.23 \mathrm{E}-05$ & $8.81 \mathrm{E}-05$ & 0.02730646 & 0.09173111 & 0.02745502 & 5245918.66 \\
\hline 64702219.2 & 731.799948 & 757.567536 & 929.206673 & 727.639735 & 0.00159001 & $2.53 \mathrm{E}-05$ & 75.1305006 & 0.0134373 & 0.01009551 & $1.30 \mathrm{E}-05$ & $1.23 \mathrm{E}-05$ & $8.83 \mathrm{E}-05$ & 0.02731446 & 0.09185866 & 0.02753557 & 5251755.01 \\
\hline 64766586 & \begin{tabular}{|l|l|}
732.104937 \\
\end{tabular} & 757.877877 & 929.388888 & 727.875933 & 0.00159852 & $2.53 \mathrm{E}-05$ & 75.1327736 & 0.0134492 & 0.01010476 & $1.30 \mathrm{E}-05$ & $1.23 \mathrm{E}-05$ & $8.85 \mathrm{E}-05$ & 0.02732049 & 0.09193999 & 0.02758915 & 5260628.01 \\
\hline 64770186 & 731.070994 & 756.536894 & 926.406134 & 726.891655 & 0.00159885 & $2.53 \mathrm{E}-05$ & 75.1328999 & 0.01344986 & 0.01010527 & $1.30 \mathrm{E}-05$ & $1.23 \mathrm{E}-05$ & $8.84 \mathrm{E}-05$ & 0.02731334 & 0.09194451 & 0.0275579 & 5258246.76 \\
\hline 64834552.8 & 731.070994 & 756.534032 & 926.402482 & 726.890621 & 0.00160469 & $2.53 \mathrm{E}-05$ & 75.1351422 & 0.01346162 & 0.01011441 & $1.30 \mathrm{E}-05$ & $1.23 \mathrm{E}-05$ & $8.85 E-05$ & 0.02732165 & 0.09202489 & 0.02760595 & 5258603.69 \\
\hline 64915011.3 & \begin{tabular}{|l|l|}
731.070994 \\
\end{tabular} & 756.531499 & 926.398179 & 726.889421 & 0.00161213 & $2.53 \mathrm{E}-05$ & $\begin{array}{ll}75.1379396 \\
\end{array}$ & 0.01347632 & 0.01012583 & $1.30 \mathrm{E}-05$ & $1.23 \mathrm{E}-05$ & $8.87 \mathrm{E}-05$ & 0.02732791 & 0.09212537 & 0.02766901 & 5263199.83 \\
\hline 65015584.3 & 731.070994 & 756.528856 & 926.392825 & 726.887932 & 0.00162165 & $2.53 \mathrm{E}-05$ & 75.1414277 & 0.01349469 & 0.0101401 & $1.30 \mathrm{E}-05$ & $1.23 \mathrm{E}-05$ & $8.90 \mathrm{E}-05$ & 0.02733563 & 0.09225097 & 0.0277481 & 5268937.59 \\
\hline 65141300.7 & 731.070994 & 756.526368 & 926.386162 & 726.886066 & 0.00163388 & $2.53 \mathrm{E}-05$ & 75.1457746 & 0.01351766 & 0.01015795 & $1.30 \mathrm{E}-05$ & $1.23 \mathrm{E}-05$ & $8.93 \mathrm{E}-05$ & 0.02734578 & 0.09240796 & 0.02784698 & 5276116.63 \\
\hline 65298446.2 & 731.070994 & 756.508376 & 926.377877 & 726.883659 & 0.00164968 & $2.53 \mathrm{E}-05$ & 75.1511875 & 0.01354636 & 0.01018025 & $1.30 \mathrm{E}-05$ & $1.23 \mathrm{E}-05$ & $8.97 \mathrm{E}-05$ & 0.02735837 & 0.09260421 & 0.02797058 & 5285090.09 \\
\hline 65494878 & 731.070994 & 756.48954 & 926.367586 & 726.880578 & 0.00167021 & $2.53 \mathrm{E}-05$ & 75.1579214 & 0.01358225 & 0.01020813 & $1.30 \mathrm{E}-05$ & $1.23 \mathrm{E}-05$ & $9.01 \mathrm{E}-05$ & $\begin{array}{l}0.0273753 \\
\end{array}$ & 0.09284952 & 0.02812509 & 5296325.38 \\
\hline 65691309.8 & \begin{tabular}{|l|l|}
731.070994 \\
\end{tabular} & 756.4856 & 926.357357 & 726.877513 & 0.00169151 & $2.53 \mathrm{E}-05$ & $\begin{array}{l}75.1646198 \\
\end{array}$ & 0.01361813 & 0.01023602 & $1.30 \mathrm{E}-05$ & $1.23 \mathrm{E}-05$ & $9.06 \mathrm{E}-05$ & 0.0273925 & 0.09309482 & 0.02827961 & 5310306.91 \\
\hline 65887741.7 & 731.070994 & 756.481831 & 926.347189 & 726.874475 & 0.00171356 & $2.53 \mathrm{E}-05$ & 75.171283 & 0.01365401 & 0.0102639 & $1.30 \mathrm{E}-05$ & $1.23 \mathrm{E}-05$ & $9.11 \mathrm{E}-05$ & 0.02740937 & 0.09334013 & 0.02843412 & 5324288.54 \\
\hline 66133281.4 & 731.070994 & 756.477344 & 926.33457 & 726.870714 & 0.00174225 & $2.53 \mathrm{E}-05$ & 75.179563 & 0.01369887 & 0.01029875 & $1.30 \mathrm{E}-05$ & $1.23 \mathrm{E}-05$ & $9.17 \mathrm{E}-05$ & 0.02743018 & 0.09364676 & 0.02862723 & 5338333.35 \\
\hline 66440206.2 & 731.070994 & 756.47189 & 926.318928 & 726.866037 & 0.00177978 & $2.53 \mathrm{E}-05$ & 75.189837 & 0.01375494 & 0.01034232 & $1.30 \mathrm{E}-05$ & $1.23 \mathrm{E}-05$ & $9.25 \mathrm{E}-05$ & $\begin{array}{r}0.027457 \\
\end{array}$ & 0.09403005 & 0.02886857 & 5355901.58 \\
\hline 66823862.1 & 731.070994 & 756.46453 & 926.299563 & 726.860289 & 0.0018321 & $2.53 E-05$ & 75.2025622 & 0.01382502 & 0.01039677 & $1.30 \mathrm{E}-05$ & $1.23 \mathrm{E}-05$ & $9.34 \mathrm{E}-05$ & 0.02748975 & 0.09450917 & 0.02917017 & 5377851 \\
\hline 67207518 & 731.070994 & 756.455845 & 926.280366 & 726.854684 & 0.0018935 & $2.53 \mathrm{E}-05$ & 75.2151591 & 0.01389511 & 0.01045123 & $1.30 \mathrm{E}-05$ & $1.23 \mathrm{E}-05$ & $9.43 \mathrm{E}-05$ & 0.0275199 & 0.09498828 & 0.0294717 & 5405107.66 \\
\hline 67535546 & 732.026076 & 757.424693 & 926.846584 & 727.592639 & 0.0019561 & $2.53 \mathrm{E}-05$ & 75.2258292 & 0.01395503 & 0.01049779 & $1.30 \mathrm{E}-05$ & $1.23 \mathrm{E}-05$ & $9.52 \mathrm{E}-05$ & 0.02754341 & 0.09539793 & 0.02973634 & 5437556.96 \\
\hline 67539146 & 730.980605 & 756.072333 & & 726.59987 & 0.00195656 & $2.53 \mathrm{E}-05$ & 75.2259451 & 0.01395569 & 0.0104983 & $1.30 \mathrm{E}-05$ & $1.23 \mathrm{E}-05$ & $9.51 \mathrm{E}-05$ & 0.02753625 & 0.0954024 & 0.02970504 & \\
\hline 67867174 & \begin{tabular}{|l|l|}
730.98005 \\
\end{tabular} & $\begin{array}{l}756.063414 \\
\end{array}$ & 923.835633 & 726.595297 & 0.00200106 & $2.53 \mathrm{E}-05$ & 75.2363985 & 0.0140149 & 0.01054431 & $1.30 \mathrm{E}-05$ & $1.23 \mathrm{E}-05$ & $9.58 \mathrm{E}-05$ & 0.02756002 & 0.0958072 & 0.02995581 & 5453804.05 \\
\hline 68277209.1 & 730.980605 & 756.051721 & 923.816084 & 726.589662 & 0.00206043 & $2.53 \mathrm{E}-05$ & 75.2493417 & 0.01408892 & 0.01060182 & $1.30 \mathrm{E}-05$ & $1.23 \mathrm{E}-05$ & $9.68 \mathrm{E}-05$ & 0.02758864 & 0.09631321 & 0.03027285 & 5476829.49 \\
\hline 68687244.1 & 730.980605 & 756.050802 & 923.796733 & 726.583894 & 0.00212335 & $2.53 \mathrm{E}-05$ & 75.2621496 & 0.01416294 & 0.01065934 & $1.30 \mathrm{E}-05$ & $1.23 E-05$ & $9.78 E-05$ & 0.02761829 & 0.09681922 & 0.03058991 & 5505539.67 \\
\hline
\end{tabular}




\begin{tabular}{|c|c|c|c|c|c|c|c|c|c|c|c|c|c|c|c|c|}
\hline Time (s) & $\begin{array}{c}\text { Coolant outlet } \\
\text { temperature }(\mathrm{K})\end{array}$ & $\begin{array}{l}\text { Peak cladding } \\
\text { temperature (K) }\end{array}$ & $\begin{array}{c}\text { Peak fuel } \\
\text { temperature (K) }\end{array}$ & \begin{tabular}{|c|} 
Average cladding \\
internal \\
temperature (K) \\
\end{tabular} & $\begin{array}{c}\text { Peak cumulative } \\
\text { damage factor }\end{array}$ & $\begin{array}{l}\text { Cladding interior } \\
\text { volume }\left(\mathrm{m}^{3}\right)\end{array}$ & $\begin{array}{l}\text { Fission gas } \\
\text { release (\%) }\end{array}$ & $\begin{array}{l}\begin{array}{c}\text { Fission gas } \\
\text { produced } \\
\text { (moles) }\end{array} \\
\end{array}$ & $\begin{array}{c}\text { Fission gas } \\
\text { released (moles) }\end{array}$ & $\begin{array}{c}\text { Fuel volume } \\
\left(\mathrm{m}^{3}\right)\end{array}$ & $\begin{array}{l}\text { Plenum gas } \\
\text { volume }\left(m^{3}\right)\end{array}$ & $\begin{array}{l}\text { Peak cladding } \\
\text { radial growth } \\
\text { (m) }\end{array}$ & $\begin{array}{l}\text { Peak fuel axial } \\
\text { growth }(m)\end{array}$ & $\begin{array}{c}\text { Peak burnup } \\
\text { (at\%) }\end{array}$ & $\begin{array}{l}\text { Peak cladding } \\
\text { hoop strain }\end{array}$ & $\begin{array}{c}\text { Plenum pressure } \\
(\mathrm{Pa})\end{array}$ \\
\hline 69097279.1 & 730.980605 & 756.045698 & 923.777593 & 726.578076 & 0.00218959 & $2.53 \mathrm{E}-05$ & 75.2748244 & 0.01423696 & 0.01071685 & $1.30 \mathrm{E}-05$ & $1.23 \mathrm{E}-05$ & $9.88 \mathrm{E}-05$ & 0.02764837 & 0.09732523 & 0.03090688 & 5534250.34 \\
\hline 69507314.2 & 730.980605 & 756.049133 & 923.758666 & 726.572303 & 0.00225894 & $2.53 \mathrm{E}-05$ & 75.287368 & 0.01431098 & 0.01077436 & $1.31 E-05$ & $1.23 \mathrm{E}-05$ & $9.98 \mathrm{E}-05$ & 0.02767862 & 0.09783123 & 0.03122373 & 5562962.2 \\
\hline 69917349.2 & 730.980605 & 756.060827 & 923.739949 & 726.566567 & 0.00233119 & $2.53 \mathrm{E}-05$ & 75.2997825 & 0.014385 & 0.01083188 & $1.31 \mathrm{E}-05$ & $1.23 \mathrm{E}-05$ & 0.0001008 & 0.02770898 & 0.09833724 & 0.03154044 & 5591676.74 \\
\hline 70327384.2 & 730.980605 & 756.079387 & 923.721431 & 726.560884 & 0.00240613 & $2.53 \mathrm{E}-05$ & 75.3120699 & 0.01445902 & 0.01088939 & $1.31 E-05$ & $1.23 \mathrm{E}-05$ & 0.00010179 & 0.02773913 & 0.09884325 & 0.031857 & 5620377 \\
\hline 70737419.3 & 730.980605 & 756.105947 & 923.703098 & 726.555285 & $\begin{array}{l}0.00248354 \\
\end{array}$ & $2.53 \mathrm{E}-05$ & 75.3242322 & 0.01453304 & 0.0109469 & $1.31 \mathrm{E}-05$ & $1.23 \mathrm{E}-05$ & 0.00010278 & 0.02776918 & 0.09934926 & 0.0321734 & 5649092.54 \\
\hline 71147454.3 & 730.980605 & 756.139847 & 923.684933 & 726.549745 & 0.00256321 & $2.54 E-05$ & 75.3362712 & 0.01460706 & 0.01100442 & $1.31 E-05$ & $1.23 \mathrm{E}-05$ & 0.00010377 & 0.0277998 & 0.09985526 & 0.03248962 & 5677813.79 \\
\hline 71557489.3 & 730.980605 & 756.178323 & 923.666922 & 726.544233 & $\begin{array}{ll}0.00264492 \\
\end{array}$ & $2.54 E-05$ & 75.3481887 & 0.01468108 & 0.01106193 & $1.31 E-05$ & $1.23 \mathrm{E}-05$ & 0.00010476 & 0.02782938 & 0.10036127 & 0.03280566 & 5706515.65 \\
\hline 71967524.4 & 730.980605 & 756.219895 & 923.649048 & $\begin{array}{l}726.53876 \\
\end{array}$ & $\begin{array}{ll}0.00273357 \\
\end{array}$ & $2.54 \mathrm{E}-05$ & 75.3599868 & 0.0147551 & 0.01111944 & 1.31E-05 & $1.23 \mathrm{E}-05$ & 0.00010575 & 0.02785792 & 0.10086728 & 0.0331215 & 5735190.59 \\
\hline 72155534 & 730.980605 & 756.237083 & 923.640872 & 726.536279 & 0.00278033 & $2.54 E-05$ & 75.3653569 & 0.01478904 & 0.01114582 & $1.31 E-05$ & $1.23 \mathrm{E}-05$ & 0.0001062 & 0.02786989 & 0.10109929 & 0.03326635 & 5763643.64 \\
\hline 72159134 & 730.980605 & 756.237328 & 923.640707 & 726.53623 & 0.00278123 & $2.54 E-05$ & 75.3654595 & 0.01478969 & 0.01114632 & $1.31 E-05$ & $1.23 \mathrm{E}-05$ & 0.00010621 & 0.02787004 & 0.10110374 & 0.03326945 & 5776624.87 \\
\hline 72185697 & 731.958091 & 757.243224 & 924.23649 & 727.29572 & 0.00278897 & $2.54 \mathrm{E}-05$ & 75.3662162 & 0.01479449 & 0.01115005 & $1.31 E-05$ & $1.23 \mathrm{E}-05$ & 0.0001063 & 0.02787422 & 0.10113652 & 0.03329658 & 5782725.93 \\
\hline 72189297 & 732.206885 & 757.564853 & 924.942719 & 727.531258 & 0.00279013 & $2.54 E-05$ & 75.3663188 & 0.01479514 & 0.01115055 & $1.31 E-05$ & $1.23 \mathrm{E}-05$ & 0.00010633 & 0.02787612 & 0.10114097 & 0.03330753 & 5786384.62 \\
\hline 72193797 & 732.206885 & 757.564829 & 924.942585 & 727.531205 & 0.00279157 & $2.54 E-05$ & 75.3664473 & 0.01479595 & 0.01115118 & $1.31 E-05$ & $1.23 \mathrm{E}-05$ & 0.00010635 & 0.02787606 & 0.10114653 & 0.03331134 & 5786636.82 \\
\hline 72199422 & 732.206885 & 757.564801 & 924.942379 & 727.531134 & 0.00279338 & $2.54 E-05$ & 75.3666079 & 0.01479697 & 0.01115198 & $1.31 E-05$ & $1.23 E-05$ & 0.00010636 & 0.02787611 & 0.1011535 & 0.03331589 & 5786951 \\
\hline 72206453.3 & 732.206885 & 757.565393 & 924.942084 & 727.531041 & 0.00279564 & $2.54 \mathrm{E}-05$ & 75.3668086 & 0.01479824 & 0.01115296 & $1.31 E-05$ & $1.23 \mathrm{E}-05$ & 0.00010638 & 0.02787645 & 0.1011622 & 0.03332149 & 5787341.08 \\
\hline 72215242.3 & 732.206885 & 757.565412 & 924.941707 & 727.530925 & 0.00279846 & $2.54 E-05$ & 75.3670594 & 0.01479984 & 0.0111542 & $1.31 E-05$ & $1.23 \mathrm{E}-05$ & 0.0001064 & 0.02787668 & 0.10117307 & 0.03332836 & 5787834.44 \\
\hline$\overline{7}=2226288.6$ & 732.206885 & 757.5665 & 924.941228 & 727.53078 & 0.002802 & $2.54 E-05$ & 75.3673729 & 0.01480182 & 0.01115575 & $1.31 E-05$ & $1.23 E-05$ & 0.00010643 & 0.02787731 & 0.10118667 & 0.03333686 & 5788448.57 \\
\hline 72239961.6 & 732.206885 & 757.567622 & 924.940628 & 727.530598 & 0.00280642 & $2.54 E-05$ & 75.3677646 & 0.01480431 & 0.01115768 & $1.31 E-05$ & $1.23 \mathrm{E}-05$ & 0.00010646 & 0.02787812 & 0.10120366 & 0.03334749 & 5789218.98 \\
\hline 72257127.7 & 732.206885 & 757.56945 & 924.939878 & 727.530369 & 0.00281194 & $2.54 E-05$ & 75.3682541 & 0.01480742 & 0.01116009 & $1.31 \mathrm{E}-05$ & $1.23 E-05$ & 0.0001065 & 0.02787925 & 0.10122491 & 0.03336076 & 5790185.07 \\
\hline 72278585.4 & 732.206885 & 757.571807 & 924.938941 & 727.530084 & 0.00281885 & $2.54 E-05$ & 75.3688656 & 0.0148113 & 0.01116311 & $1.31 E-05$ & $1.23 \mathrm{E}-05$ & 0.00010655 & 0.02788069 & 0.10125146 & 0.03337735 & 5791393.21 \\
\hline 72305407.5 & 732.206885 & 757.574885 & 924.937769 & 727.529726 & 0.0028275 & $2.54 \mathrm{E}-05$ & 75.3696296 & 0.01481616 & 0.01116688 & $1.31 E-05$ & $1.23 \mathrm{E}-05$ & 0.00010662 & 0.02788255 & 0.10128466 & 0.03339808 & 5792904.95 \\
\hline 72338935.1 & 732.206885 & 757.578953 & 924.936307 & 727.52928 & 0.00283831 & $2.54 E-05$ & 75.3705838 & 0.01482223 & 0.0111716 & $1.31 E-05$ & $1.23 E-05$ & 0.0001067 & 0.02788496 & 0.10132615 & 0.033424 & 5794795.1 \\
\hline 72380844.6 & 732.206885 & 757.584117 & 924.934479 & 727.528722 & 0.0028528 & $2.54 \mathrm{E}-05$ & 75.37177555 & 0.01482981 & 0.01117749 & $1.31 E-05$ & $1.23 \mathrm{E}-05$ & 0.0001068 & 0.02788807 & 0.10137801 & 0.03345639 & 5797157.7 \\
\hline 72433231.5 & 732.206885 & 757.591316 & 924.932198 & 727.528025 & 0.00287347 & $2.54 \mathrm{E}-05$ & 75.3732635 & 0.0148393 & 0.01118486 & $1.31 \mathrm{E}-05$ & $1.23 \mathrm{E}-05$ & 0.00010693 & 0.02789198 & 0.10144284 & 0.03349687 & 5800117.42 \\
\hline 72498715.1 & 732.206885 & 757.599935 & 924.929349 & 727.527154 & 0.00289936 & $2.54 E-05$ & 75.3751207 & 0.01485115 & 0.01119407 & $1.31 E-05$ & $1.23 E-05$ & 0.00010709 & 0.02789682 & 0.10152388 & 0.03354746 & 5803811.81 \\
\hline 72580569.6 & 732.206885 & 757.610984 & 924.925794 & 727.526068 & 0.0029318 & $2.54 E-05$ & 75.3774381 & 0.01486597 & 0.01120559 & $1.31 E-05$ & $1.23 \mathrm{E}-05$ & 0.00010729 & 0.02790291 & 0.10162518 & 0.0336107 & 5808430.73 \\
\hline 72682887.8 & 732.206885 & 757.625132 & 924.921357 & 727.5247112 & 0.00297249 & $2.54 \mathrm{E}-05$ & 75.3803283 & 0.01488449 & 0.01121998 & $1.31 E-05$ & $1.23 \mathrm{E}-05$ & 0.00010753 & 0.02791052 & 0.1017518 & 0.03368973 & 5814206.58 \\
\hline 72810785.5 & 732.206885 & 757.643394 & 924.915822 & 727.523021 & 0.00302354 & $2.54 E-05$ & 75.383931 & 0.01490765 & 0.01123797 & $1.31 E-05$ & $1.23 \mathrm{E}-05$ & 0.00010784 & 0.02792011 & 0.10191008 & 0.03378849 & 5821425.49 \\
\hline 72970657.6 & 732.206885 & 757.666424 & 924.908923 & 727.520899 & 0.00308765 & $2.54 E-05$ & 75.3884187 & 0.01493659 & 0.01126046 & $1.31 E-05$ & $1.23 \mathrm{E}-05$ & 0.00010823 & 0.02793199 & 0.10210792 & 0.03391191 & 5830445.09 \\
\hline 73170497.7 & 732.206885 & 757.69747 & 924.900333 & 727.518244 & 0.00316822 & $2.54 E-05$ & 75.3940039 & 0.01497276 & 0.01128857 & $1.31 E-05$ & $1.23 E-05$ & 0.00010871 & 0.02794688 & 0.10235523 & 0.03406612 & 5841732.52 \\
\hline 73370337.9 & 732.206885 & 757.729527 & 924.891773 & 727.515597 & 0.00324922 & $2.54 \mathrm{E}-05$ & 75.3995621 & 0.01500894 & 0.01131668 & $1.31 E-05$ & $1.23 \mathrm{E}-05$ & 0.0001092 & 0.02796196 & 0.10260254 & 0.03422029 & 5855788.69 \\
\hline 73570178 & 732.206885 & 757.762475 & 924.883245 & 727.512963 & 0.00333781 & $2.54 E-05$ & 75.4050937 & 0.01504512 & 0.01134479 & $1.31 E-05$ & $1.23 \mathrm{E}-05$ & 0.00010968 & 0.027977 & 0.10284985 & 0.0343744 & 5869843.27 \\
\hline 73819978.2 & 732.206885 & 757.80284 & 924.872629 & 727.50968 & 0.00346186 & $2.54 E-05$ & 75.4119708 & 0.01509034 & 0.01137992 & $1.31 E-05$ & $1.23 \mathrm{E}-05$ & 0.00011028 & 0.02799531 & 0.10315898 & 0.03456693 & 5883942.8 \\
\hline 74132228.4 & 732.206885 & 757.826288 & 924.85942 & 727.505443 & 0.00361857 & $2.54 E-05$ & 75.4205095 & 0.01514687 & 0.01142384 & $1.31 E-05$ & $1.23 \mathrm{E}-05$ & 0.00011104 & 0.02801802 & 0.1035454 & 0.03480745 & 5901564.26 \\
\hline 74444478.6 & 732.206885 & 757.850435 & 924.84627 & 727.501223 & 0.00377684 & $2.54 \mathrm{E}-05$ & 75.4289846 & 0.01520339 & 0.01146776 & $1.32 \mathrm{E}-05$ & $1.23 \mathrm{E}-05$ & 0.00011179 & 0.02804007 & 0.10393182 & 0.03504786 & 5923498.9 \\
\hline 74834791.4 & 732.206885 & 757.89374 & 924.829933 & 727.496009 & 0.003977 & $2.54 E-05$ & 75.4394904 & 0.01527405 & 0.01152267 & $1.32 E-05$ & $1.23 \mathrm{E}-05$ & 0.00011274 & 0.02806864 & 0.10441485 & 0.03534819 & 5945520.31 \\
\hline 75225104.2 & 732.206885 & 757.883101 & 924.813682 & 727.490541 & 0.0041889 & $2.54 \mathrm{E}-05$ & 75.4498994 & 0.01534471 & 0.01157757 & $1.32 E-05$ & $1.23 \mathrm{E}-05$ & 0.00011368 & 0.02809732 & 0.10489787 & 0.0356484 & 5972965.49 \\
\hline 75615417 & 732.206885 & 757.79657 & 924.797498 & 727.484677 & 0.00442974 & $2.54 E-05$ & 75.4602129 & 0.01541537 & 0.01163247 & $1.32 E-05$ & $1.23 \mathrm{E}-05$ & 0.00011462 & 0.02812632 & 0.1053809 & 0.03594845 & 6000415.69 \\
\hline 76005729.8 & 732.206885 & 757.714052 & 924.781384 & 727.478695 & 0.00467325 & $2.54 \mathrm{E}-05$ & 75.4704324 & 0.01548602 & 0.01168737 & $1.32 E-05$ & $1.23 \mathrm{E}-05$ & 0.00011556 & 0.02815571 & 0.10586392 & 0.03624833 & 6027866.31 \\
\hline 76396042.6 & 732.206885 & 757.642664 & 924.765331 & 727.47281 & 0.00495542 & $2.54 E-05$ & 75.480559 & 0.01555668 & 0.01174227 & $1.32 E-05$ & $1.23 \mathrm{E}-05$ & 0.00011651 & 0.02818381 & 0.10634694 & 0.03654809 & 6055299.85 \\
\hline 76786355.3 & 732.206885 & 757.581243 & 924.749337 & 727.467028 & 0.00524303 & $2.55 E-05$ & 75.490594 & 0.01562734 & 0.01179717 & $1.32 E-05$ & $1.23 \mathrm{E}-05$ & 0.00011745 & 0.02821135 & 0.10682997 & 0.03684767 & 6082705.24 \\
\hline 76833647 & 731.581701 & 756.932548 & 924.365655 & 726.980371 & 0.00527433 & $2.55 E-05$ & 75.4918038 & 0.0156359 & 0.01180382 & $1.32 E-05$ & $1.23 \mathrm{E}-05$ & 0.00011755 & 0.02821306 & 0.10688849 & 0.03687954 & 6105890.47 \\
\hline 76837247 & 732.466704 & 758.076834 & 926.887967 & 727.81868 & 0.00527769 & $2.55 \mathrm{E}-05$ & 75.4918963 & 0.01563656 & 0.01180433 & $1.32 \mathrm{E}-05$ & $1.23 \mathrm{E}-05$ & 0.00011765 & 0.02821961 & 0.10689297 & 0.03691097 & 6116023.7 \\
\hline 76884538.7 & 732.466704 & 758.071606 & 926.886584 & 727.818037 & 0.0053223 & $2.55 E-05$ & 75.4931167 & 0.0156452 & 0.01181105 & $1.32 E-05$ & $1.23 \mathrm{E}-05$ & 0.00011778 & 0.02822085 & 0.10695208 & 0.03695259 & 6116293.13 \\
\hline 76943653.2 & 732.466704 & 758.063159 & 926.884575 & 727.817155 & 0.00537831 & $2.55 \mathrm{E}-05$ & 75.4946403 & 0.01565601 & 0.01181945 & $1.32 \mathrm{E}-05$ & $1.23 \mathrm{E}-05$ & 0.00011792 & 0.02822477 & 0.10702598 & 0.03700012 & 6119657.4 \\
\hline 77017546.5 & 732.466704 & 758.052388 & 926.881901 & 727.816044 & 0.00544845 & $2.55 E-05$ & 75.4965419 & 0.01566952 & 0.01182995 & $1.32 E-05$ & $1.23 \mathrm{E}-05$ & 0.00011811 & 0.02823024 & 0.10711834 & 0.0370583 & 6123860.93 \\
\hline 77109913 & 732.466704 & 758.037896 & 926.878373 & 727.814633 & 0.00554049 & $2.55 \mathrm{E}-05$ & 75.4989143 & 0.01568641 & 0.01184307 & $1.32 E-05$ & $1.23 \mathrm{E}-05$ & 0.00011833 & 0.02823737 & 0.10723379 & 0.03713047 & 6129134.38 \\
\hline 77225371.2 & 732.466704 & 758.020721 & 926.873792 & 727.81288 & 0.00566375 & $2.55 \mathrm{E}-05$ & 75.5018727 & 0.01570752 & 0.01185947 & $1.32 E-05$ & $1.23 \mathrm{E}-05$ & 0.00011862 & 0.02824623 & 0.10737811 & 0.0372204 & 6135724.27 \\
\hline 77340548 & 732.466704 & 758.004522 & 926.869131 & 727.811142 & 0.00578637 & $2.55 \mathrm{E}-05$ & 75.5048159 & 0.01572858 & 0.01187584 & $1.32 E-05$ & $1.23 \mathrm{E}-05$ & 0.0001189 & 0.02825503 & 0.10752208 & 0.03730998 & 6143929.22 \\
\hline 77344148 & 732.466704 & 758.004308 & 926.868982 & 727.811093 & 0.0057902 & $2.55 \mathrm{E}-05$ & 75.5049077 & 0.01572924 & 0.01187635 & $1.32 E-05$ & $1.23 \mathrm{E}-05$ & 0.00011891 & 0.02825519 & 0.10752658 & 0.037313 & 6152005.01 \\
\hline 77459324.8 & 732.466704 & 757.988553 & 926.864273 & 727.809357 & 0.00591248 & $2.55 \mathrm{E}-05$ & 75.5078428 & 0.0157503 & 0.01189271 & $1.32 \mathrm{E}-05$ & $1.23 E-05$ & 0.00011919 & 0.028264 & 0.10767055 & 0.03740229 & 6152371.84 \\
\hline 77603295.9 & 732.466704 & 757.970627 & 926.858347 & 727.807201 & 0.00606478 & $2.55 E-05$ & 75.5115006 & 0.01577663 & 0.01191317 & $1.32 \mathrm{E}-05$ & $1.23 \mathrm{E}-05$ & 0.00011954 & 0.02827488 & 0.10785051 & 0.03751411 & 6160574.16 \\
\hline 77738400 & 732.108004 & 757.585692 & 926.633919 & 727.526321 & 0.00619834 & $2.55 \mathrm{E}-05$ & 75.5149221 & 0.01580133 & 0.01193236 & $1.32 E-05$ & $1.23 \mathrm{E}-05$ & 0.00011986 & 0.02828435 & 0.10801938 & 0.03761626 & 6168525.95 \\
\hline 77742000 & 732.517917 & 758.115575 & 927.804175 & 727.91479 & 0.00620255 & $2.55 \mathrm{E}-05$ & 75.5150133 & 0.01580199 & 0.01193288 & $1.32 \mathrm{E}-05$ & $1.23 \mathrm{E}-05$ & 0.00011992 & 0.02828763 & 0.10802389 & 0.03763298 & 6181201.77 \\
\hline 77877104.1 & 732517917 & 758.100427 & 927.799181 & 727912778 & 0.00636191 & $2.55 E-05$ & 75.5184396 & 0.01582681 & 0.01195216 & $1.32 E-05$ & $1.23 E-05$ & 0.00012025 & 0.02829697 & 0.10819355 & 0.03774057 & 6181574.81 \\
\hline
\end{tabular}




\begin{tabular}{|c|c|c|c|c|c|c|c|c|c|c|c|c|c|c|c|c|}
\hline Time (s) & $\begin{array}{c}\text { Coolant outlet } \\
\text { temperature }(\mathrm{K})\end{array}$ & $\begin{array}{l}\text { Peak cladding } \\
\text { temperature (K) }\end{array}$ & $\begin{array}{c}\text { Peak fuel } \\
\text { temperature (K) }\end{array}$ & $\begin{array}{c}\text { Average cladding } \\
\text { internal } \\
\text { temperature }(\mathbf{K})\end{array}$ & $\begin{array}{l}\text { Peak cumulative } \\
\text { damage factor }\end{array}$ & $\begin{array}{l}\text { Cladding interior } \\
\text { volume }\left(\mathrm{m}^{3}\right)\end{array}$ & $\begin{array}{l}\text { Fission gas } \\
\text { release (\%) }\end{array}$ & $\begin{array}{l}\text { Fission gas } \\
\text { produced } \\
\text { (moles) }\end{array}$ & $\begin{array}{l}\text { Fission gas } \\
\text { released (moles) }\end{array}$ & $\begin{array}{l}\text { Fuel volume } \\
\left(\mathrm{m}^{3}\right)\end{array}$ & $\begin{array}{l}\text { Plenum gas } \\
\text { volume }\left(\mathrm{m}^{3}\right)\end{array}$ & $\begin{array}{l}\text { Peak cladding } \\
\text { radial growth } \\
(\mathrm{m})\end{array}$ & $\begin{array}{l}\text { Peak fuel axial } \\
\text { growth }(m)\end{array}$ & $\begin{array}{c}\text { Peak burnup } \\
\text { (at\%) }\end{array}$ & $\begin{array}{l}\text { Peak cladding } \\
\text { hoop strain }\end{array}$ & $\begin{array}{c}\text { Plenum pressure } \\
(\mathrm{Pa})\end{array}$ \\
\hline $\begin{array}{l}78045984.3 \\
\end{array}$ & 732.517917 & 758.082537 & 927.792376 & 727.910247 & 0.00655964 & $2.55 \mathrm{E}-05$ & 75.5227073 & $\begin{array}{l}0.01585783 \\
\end{array}$ & 0.01197626 & $1.32 \mathrm{E}-05$ & $1.23 \mathrm{E}-05$ & 0.00012067 & 0.02830955 & 0.10840562 & 0.03787321 & 6191248.39 \\
\hline 78257084.5 & 732.517917 & 758.06226 & $\begin{array}{l}927.78372 \\
\end{array}$ & \begin{tabular}{|l|l|}
727.907099 \\
\end{tabular} & 0.00680471 & $2.55 \mathrm{E}-05$ & 75.5280186 & 0.01589661 & 0.01200639 & $1.32 \mathrm{E}-05$ & $1.23 \mathrm{E}-05$ & 0.00012119 & 0.02832531 & 0.10867071 & 0.03803851 & 6203337.27 \\
\hline $\begin{array}{l}78520959.7 \\
\end{array}$ & 732.517917 & 758.039322 & 927.77287 & 727.903176 & 0.00710835 & \begin{tabular}{l|l|}
$2.55 E-05$ \\
\end{tabular} & 75.5346213 & 0.01594508 & 0.01204406 & $1.32 E-05$ & $1.23 \mathrm{E}-05$ & 0.00012184 & 0.02834527 & 0.10900208 & 0.03824476 & 6218456.19 \\
\hline 78850803.8 & 732.517917 & 758.01469 & \begin{tabular}{|l|}
927.759314 \\
\end{tabular} & 727.898296 & 0.00748406 & $2.55 \mathrm{E}-05$ & 75.5428185 & 0.01600567 & \begin{tabular}{|l|l|}
0.01209114 \\
\end{tabular} & $1.32 \mathrm{E}-05$ & $1.23 E-05$ & 0.00012265 & 0.02837046 & 0.10941628 & \begin{tabular}{|l|l|}
0.03850218 \\
\end{tabular} & 6237355.68 \\
\hline 79263108.9 & 732.517917 & 757.990108 & 927.742433 & 727.892234 & 0.00794861 & $2.55 \mathrm{E}-05$ & 75.5529782 & 0.01608141 & 0.01214998 & $1.32 \mathrm{E}-05$ & $1.23 \mathrm{E}-05$ & 0.00012366 & 0.02840259 & 0.10993403 & 0.03882348 & 6260986.64 \\
\hline 79675414 & 732.517917 & 757.972644 & \begin{tabular}{|l|l|}
927.725667 \\
\end{tabular} & 727.886213 & 0.00843752 & $2.55 \mathrm{E}-05$ & 75.5630425 & 0.01615715 & 0.01220883 & $1.33 \mathrm{E}-05$ & $1.23 \mathrm{E}-05$ & 0.00012467 & 0.02843494 & 0.11045179 & \begin{tabular}{l|l|}
0.03914439 \\
\end{tabular} & 6290434.93 \\
\hline 80087719.1 & 732.517917 & \begin{tabular}{|l|l|}
757.962314 \\
\end{tabular} & 927.709396 & 727.880242 & 0.00893907 & $2.55 \mathrm{E}-05$ & 75.573013 & 0.01623289 & 0.01226768 & $1.33 \mathrm{E}-05$ & $1.22 \mathrm{E}-05$ & 0.00012568 & 0.02846739 & 0.11096954 & \begin{tabular}{ll|}
0.03946495 \\
\end{tabular} & 6319888.9 \\
\hline 80444447 & 732.517917 & \begin{tabular}{|l|l|}
757.959181 \\
\end{tabular} & 927.695986 & 727.875112 & 0.00937104 & $2.55 \mathrm{E}-05$ & 75.5815647 & 0.01629842 & 0.0123186 & $1.33 \mathrm{E}-05$ & $1.22 \mathrm{E}-05$ & 0.00012656 & 0.02849552 & 0.1114175 & \begin{tabular}{ll|}
0.03974207 \\
\end{tabular} & 6349292.21 \\
\hline 80444694.4 & 726.404853 & 750.066418 & 910.138081 & \begin{tabular}{|l|l|}
722.084287 \\
\end{tabular} & 0.00937107 & $2.55 \mathrm{E}-05$ & 75.5815704 & 0.01629846 & 0.01231863 & $1.33 \mathrm{E}-05$ & $1.22 \mathrm{E}-05$ & 0.00012594 & 0.02844417 & 0.1114178 & \begin{tabular}{ll|}
0.03955469 \\
\end{tabular} & 6325180.01 \\
\hline 80444941.8 & 720.297227 & 742.184182 & \begin{tabular}{|l|l|}
892.341577 \\
\end{tabular} & 716.298846 & 0.00937107 & $2.55 \mathrm{E}-05$ & 75.5815757 & 0.0162985 & 0.01231866 & $1.33 \mathrm{E}-05$ & $1.22 \mathrm{E}-05$ & 0.00012534 & 0.028393 & 0.11141808 & 0.03936902 & 6275805.41 \\
\hline 80445189.2 & \begin{tabular}{|l|l|}
714.19517 \\
\end{tabular} & 734.313447 & \begin{tabular}{|l|}
874.301151 \\
\end{tabular} & 710.51904 & 0.00937107 & $2.55 \mathrm{E}-05$ & 75.5815806 & 0.01629854 & 0.01231869 & $1.32 \mathrm{E}-05$ & $1.22 \mathrm{E}-05$ & 0.00012472 & 0.02834233 & 0.11141834 & $\begin{array}{l}0.03917739 \\
\end{array}$ & 6226408.18 \\
\hline 80445498.4 & 706.575632 & 724.491076 & 851.390364 & 703.302259 & 0.00937107 & $2.55 \mathrm{E}-05$ & 75.5815861 & 0.01629858 & 0.01231873 & $1.32 \mathrm{E}-05$ & $1.22 \mathrm{E}-05$ & 0.0001239 & 0.02827941 & 0.11141863 & \begin{tabular}{|l|l|}
0.03892774 \\
\end{tabular} & 6164770.45 \\
\hline 80445885 & \begin{tabular}{|l|l|}
697.064121 \\
\end{tabular} & 712.237626 & 822.158542 & 694.293679 & 0.00937107 & $2.55 \mathrm{E}-05$ & 75.5815922 & 0.01629863 & 0.01231876 & $1.32 \mathrm{E}-05$ & $1.22 \mathrm{E}-05$ & 0.00012285 & 0.02819941 & 0.11141895 & \begin{tabular}{|l|l|}
0.03861044 \\
\end{tabular} & 6087677.99 \\
\hline 80446132.3 & 690.984489 & 704.409342 & 803.085066 & 688.53533 & 0.00937107 & $2.55 \mathrm{E}-05$ & 75.5815955 & 0.01629865 & 0.01231878 & $1.32 \mathrm{E}-05$ & $1.22 \mathrm{E}-05$ & 0.00012216 & 0.02814635 & 0.11141912 & 0.0384032 & 6038371.06 \\
\hline 80446379.7 & 684.911043 & 696.591667 & \begin{tabular}{|l|l|}
783.711002 \\
\end{tabular} & 682.782506 & 0.00937107 & $2.54 \mathrm{E}-05$ & 75.5815985 & 0.01629867 & $\begin{array}{l}0.0123188 \\
\end{array}$ & $1.32 \mathrm{E}-05$ & $1.22 \mathrm{E}-05$ & 0.00012147 & 0.02809127 & 0.11141928 & $\begin{array}{l}0.03819656 \\
\end{array}$ & 5989016.6 \\
\hline 80446627.1 & 678.843905 & 688.784417 & 764.022918 & 677.03514 & 0.00937107 & $2.54 \mathrm{E}-05$ & 75.581601 & 0.01629869 & 0.01231881 & $1.32 \mathrm{E}-05$ & $1.22 \mathrm{E}-05$ & 0.00012078 & 0.02803356 & 0.11141944 & 0.03798877 & 5939639.55 \\
\hline 80446660.3 & 678.030075 & 687.737332 & 761.355753 & 676.264152 & 0.00937107 & $2.54 \mathrm{E}-05$ & 75.5816013 & 0.0162987 & 0.01231882 & $1.32 \mathrm{E}-05$ & $1.22 \mathrm{E}-05$ & 0.00012069 & 0.02802564 & 0.11141943 & 0.03796051 & 5933018.01 \\
\hline 80446693.5 & 677.21636 & 686.690434 & 758.682606 & 675.493263 & 0.00937107 & $2.54 \mathrm{E}-05$ & 75.5816016 & 0.0162987 & 0.01231882 & $1.32 \mathrm{E}-05$ & $1.22 \mathrm{E}-05$ & 0.00012059 & 0.02801765 & 0.11141944 & 0.03793254 & 5926384.48 \\
\hline 80446714.8 & 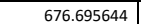 & 686.020518 & 756.968653 & 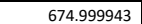 & 0.00937107 & $\begin{array}{l}2.54 \mathrm{E}-05 \\
\end{array}$ & 75.5816018 & 0.0162987 & 0.01231882 & $\begin{array}{ll}1.32 E-05 \\
\end{array}$ & $1.22 \mathrm{E}-05$ & 0.00012053 & 0.02801251 & 0.11141945 & \begin{tabular}{|l|}
0.03791447 \\
\end{tabular} & 5922142 \\
\hline 80446736 & 676.174975 & 685.350677 & 755.252225 & 674.506666 & 0.00937107 & $2.54 \mathrm{E}-05$ & 75.581602 & 0.0162987 & 0.01231882 & $1.32 \mathrm{E}-05$ & $1.22 \mathrm{E}-05$ & 0.00012047 & 0.02800733 & 0.11141946 & 0.0378966 & 5917894.06 \\
\hline 80446749.6 & 675.841772 & 684.922022 & 754.15242 & 674.190987 & 0.00937107 & $2.54 \mathrm{E}-05$ & 75.5816021 & 0.0162987 & 0.01231882 & $1.32 \mathrm{E}-05$ & $1.22 \mathrm{E}-05$ & 0.00012043 & 0.02800401 & 0.11141947 & 0.037885 & 5915178.96 \\
\hline 80446763.2 & 675.508589 & 684.493394 & 753.051593 & 673.875326 & 0.00937107 & $2.54 \mathrm{E}-05$ & 75.5816022 & 0.0162987 & 0.01231882 & $1.32 E-05$ & $1.22 E-05$ & 0.00012039 & 0.02800068 & 0.11141947 & $\begin{array}{l}0.03787359 \\
\end{array}$ & 5912458.79 \\
\hline 80446776.8 & 675.175426 & $\begin{array}{l}684.0648 \\
\end{array}$ & 751.949753 & 673.559682 & 0.00937107 & $2.54 \mathrm{E}-05$ & 75.5816023 & 0.0162987 & 0.01231882 & $1.32 \mathrm{E}-05$ & $1.22 \mathrm{E}-05$ & 0.00012036 & 0.02799731 & 0.11141948 & $\begin{array}{l}0.0378621 \\
\end{array}$ & 5909739.78 \\
\hline 80446793.8 & 674.758999 & 683.529102 & 750.57102 & 673.165148 & 0.00937107 & $2.54 \mathrm{E}-05$ & 75.5816025 & 0.01629871 & 0.01231882 & $1.32 \mathrm{E}-05$ & $1.22 E-05$ & 0.00012031 & 0.02799312 & 0.11141949 & 0.03784771 & 5906341.81 \\
\hline 80446810.8 & 674.342603 & 682.993457 & 749.190689 & 672.770641 & 0.00937107 & $2.54 \mathrm{E}-05$ & 75.5816026 & 0.01629871 & 0.01231882 & $1.32 \mathrm{E}-05$ & $1.22 \mathrm{E}-05$ & 0.00012026 & 0.02798887 & 0.1114195 & 0.03783347 & 5902939.01 \\
\hline 80446821.7 & 674.076126 & 682.650666 & 748.306442 & 672.518169 & 0.00937107 & $2.54 \mathrm{E}-05$ & 75.5816027 & 0.01629871 & 0.01231882 & $1.32 \mathrm{E}-05$ & $1.22 E-05$ & 0.00012023 & 0.02798617 & 0.1114195 & 0.03782413 & 5900766.92 \\
\hline 80446827.3 & 673.939694 & 682.475167 & 747.853454 & 672.388907 & 0.00937107 & $\begin{array}{ll}2.54 \mathrm{E}-05 \\
\end{array}$ & 75.5816027 & 0.01629871 & 0.01231882 & $\begin{array}{ll}1.32 \mathrm{E}-05 \\
\end{array}$ & $1.22 \mathrm{E}-05$ & 0.00012021 & 0.02798477 & 0.1114195 & 0.03781941 & 5899652.78 \\
\hline 80446830.1 & 673.869843 & 682.385305 & 747.621457 & 672.322726 & 0.00937107 & $2.54 \mathrm{E}-05$ & 75.5816028 & 0.01629871 & 0.01231882 & $1.32 E-05$ & $1.22 \mathrm{E}-05$ & 0.00012021 & 0.02798409 & 0.1114195 & 0.03781701 & 5899085.06 \\
\hline 80446833 & 673.799992 & 682.295461 & 747.38941 & 672.256547 & 0.00937107 & $2.54 \mathrm{E}-05$ & 75.5816028 & 0.01629871 & 0.01231882 & $1.32 \mathrm{E}-05$ & $1.22 E-05$ & 0.0001202 & 0.02798334 & 0.1114195 & \begin{tabular}{l|l}
0.03781459 \\
\end{tabular} & 5898511.5 \\
\hline 80446836.6 & 673.712679 & 682.183149 & 747.099289 & 672.173823 & 0.00937107 & $2.54 \mathrm{E}-05$ & 75.5816028 & 0.01629871 & 0.01231883 & $1.32 \mathrm{E}-05$ & $1.22 \mathrm{E}-05$ & 0.00012019 & 0.02798244 & 0.11141955 & 0.03781157 & 5897798.04 \\
\hline 80446837.7 & \begin{tabular}{|c|}
673.684069 \\
\end{tabular} & 682.146342 & 747.004205 & 672.146716 & 0.00937107 & $2.54 \mathrm{E}-05$ & 75.5816028 & 0.01629871 & 0.01231883 & $1.32 \mathrm{E}-05$ & $1.22 \mathrm{E}-05$ & 0.00012018 & 0.02798217 & 0.11141951 & 0.03781059 & 5897566.05 \\
\hline
\end{tabular}


PIN T654 FULL RESULTS

\begin{tabular}{|c|c|c|c|c|c|c|c|c|c|c|c|c|c|c|c|c|}
\hline Time (s) & $\begin{array}{l}\text { Coolant outlet } \\
\text { temperature (K) }\end{array}$ & $\begin{array}{l}\text { Peak Cladding } \\
\text { Temperature (K) }\end{array}$ & $\begin{array}{c}\text { Peak fuel } \\
\text { temperature (K) }\end{array}$ & $\begin{array}{c}\text { Average cladding } \\
\text { internal } \\
\text { temperature (K) }\end{array}$ & $\begin{array}{c}\text { Peak cumulative } \\
\text { damage factor }\end{array}$ & $\begin{array}{l}\text { Cladding interior } \\
\text { volume }\left(\mathrm{m}^{3}\right)\end{array}$ & $\begin{array}{l}\text { Fission Gas } \\
\text { Release (\%) }\end{array}$ & $\begin{array}{l}\text { Fission gas } \\
\text { produced } \\
\text { (moles) }\end{array}$ & $\begin{array}{c}\text { Fission gas } \\
\text { released (moles) }\end{array}$ & Fuel volume $\left(\mathrm{m}^{3}\right)$ & $\begin{array}{l}\text { Plenum gas } \\
\text { volume }\left(m^{3}\right)\end{array}$ & $\begin{array}{l}\text { Peak cladding } \\
\text { radial growth } \\
\text { (m) }\end{array}$ & $\begin{array}{l}\text { Peak fuel axial } \\
\text { growth }(m)\end{array}$ & $\begin{array}{c}\text { Peak burnup } \\
\text { (at\%) }\end{array}$ & $\begin{array}{l}\text { Peak cladding } \\
\text { hoop strain }\end{array}$ & $\begin{array}{c}\text { Plenum pressure } \\
(\mathrm{Pa})\end{array}$ \\
\hline 0 & & 295 & 295 & 295 & 0 & $2.45 \mathrm{E}-05$ & & 0 & 0 & $8.80 E-06$ & $1.57 \mathrm{E}-05$ & 0 & 0 & 0 & 0 & \\
\hline 100 & 309.333714 & 310.330309 & 322.569249 & 308.870699 & 0 & $2.45 \mathrm{E}-05$ & 0 & $3.30 \mathrm{E}-10$ & 0 & $8.81 E-06$ & $1.57 \mathrm{E}-05$ & $6.67 \mathrm{E}-07$ & 0.00011878 & $2.36 \mathrm{E}-09$ & \begin{tabular}{|l|}
0.00018117 \\
\end{tabular} & 95462.2265 \\
\hline 225 & 327.292835 & 329.527787 & 354.264964 & 326.243437 & 0 & $2.45 \mathrm{E}-05$ & 0 & $1.67 E-09$ & 0 & $8.82 \mathrm{E}-06$ & $1.57 \mathrm{E}-05$ & $1.54 \mathrm{E}-06$ & 0.00026069 & $1.20 \mathrm{E}-08$ & 0.00041832 & 100798.676 \\
\hline 381.25 & 349.81139 & 353.558426 & 390.975152 & 348.007682 & 0 & $2.45 \mathrm{E}-05$ & 0 & $4.79 E-09$ & 0 & $8.83 E-06$ & $1.57 \mathrm{E}-05$ & $2.65 E-06$ & 0.0004306 & $3.43 \mathrm{E}-08$ & 0.00071767 & 107474.772 \\
\hline 576.5625 & $\begin{array}{ll}378.073328 \\
\end{array}$ & \begin{tabular}{|l|}
383.671394 \\
\end{tabular} & 433.910347 & \begin{tabular}{|l|l|}
375.289788 \\
\end{tabular} & $1.30 \mathrm{E}-65$ & $2.45 \mathrm{E}-05$ & 0 & $1.10 \mathrm{E}-08$ & 0 & $8.85 \mathrm{E}-06$ & $1.57 \mathrm{E}-05$ & $4.03 E-06$ & 0.00063487 & $\begin{array}{ll}7.85 \mathrm{E}-08 \\
\end{array}$ & 0.00109336 & 115830.056 \\
\hline 820.703125 & 413.582692 & 421.457765 & 484.495689 & 409.513858 & $9.62 E-56$ & $2.46 \mathrm{E}-05$ & 0 & $2.22 \mathrm{E}-08$ & 0 & $8.86 \mathrm{E}-06$ & $1.57 \mathrm{E}-05$ & $5.77 \mathrm{E}-06$ & 0.0008816 & $1.59 \mathrm{E}-07$ & 0.00156539 & 126291.038 \\
\hline 1125.87891 & 458.252644 & 468.921381 & 544.629676 & 452.484989 & $4.04 E-46$ & $2.46 \mathrm{E}-05$ & 0 & $4.18 \mathrm{E}-08$ & 0 & $8.89 \mathrm{E}-06$ & $1.57 \mathrm{E}-05$ & $7.96 \mathrm{E}-06$ & 0.00118125 & $2.99 \mathrm{E}-07$ & 0.00215902 & 139394.405 \\
\hline 1507.34863 & 514.514727 & 528.717646 & 616.822925 & 506.499013 & $7.97 E-37$ & $2.47 \mathrm{E}-05$ & 0 & $7.49 \mathrm{E}-08$ & 0 & $8.92 E-06$ & $1.58 \mathrm{E}-05$ & $1.07 \mathrm{E}-05$ & 0.00154746 & $5.37 \mathrm{E}-07$ & 0.002907 & 155816.901 \\
\hline 1984.18579 & 585.443117 & 604.178281 & 704.694678 & 574.481649 & $6.63 \mathrm{E}-28$ & $2.47 \mathrm{E}-05$ & 0 & $1.30 \mathrm{E}-07$ & 0 & $8.95 E-06$ & $1.58 \mathrm{E}-05$ & $1.42 \mathrm{E}-05$ & 0.00199792 & $9.30 \mathrm{E}-07$ & 0.00385112 & 176411.582 \\
\hline 2580.23224 & 674.870048 & 699.796883 & 812.777778 & 660.160349 & $1.70 \mathrm{E}-19$ & $2.48 \mathrm{E}-05$ & 0 & $2.19 \mathrm{E}-07$ & 0 & $8.99 \mathrm{E}-06$ & $1.58 \mathrm{E}-05$ & $1.86 \mathrm{E}-05$ & 0.00254924 & $1.57 \mathrm{E}-06$ & $\begin{array}{ll}0.00504493 \\
\end{array}$ & 202251.071 \\
\hline 3325.2903 & 787.424599 & 820.130515 & 947.637561 & 768.222791 & $1.05 E-11$ & $2.49 \mathrm{E}-05$ & 0 & $3.65 \mathrm{E}-07$ & 0 & $9.05 \mathrm{E}-06$ & $1.59 \mathrm{E}-05$ & $2.42 \mathrm{E}-05$ & 0.0032325 & $2.61 \mathrm{E}-06$ & 0.00655167 & 234662.44 \\
\hline 3600 & 829.01858 & 864.981118 & \begin{tabular}{|l|l|}
997.786367 \\
\end{tabular} & 808.288223 & $8.24 E-10$ & $2.50 \mathrm{E}-05$ & 0 & $4.27 \mathrm{E}-07$ & 0 & $9.07 \mathrm{E}-06$ & $1.59 \mathrm{E}-05$ & $2.63 \mathrm{E}-05$ & 0.00347441 & $\begin{array}{ll}.06 \mathrm{E}-06 \\
\end{array}$ & 0.00711122 & 246629.517 \\
\hline 4345.05806 & 829.01858 & 864.986553 & \begin{tabular}{|l|l|}
997.792253 \\
\end{tabular} & \begin{tabular}{|l|}
808.294376 \\
\end{tabular} & $3.03 E-09$ & $2.50 \mathrm{E}-05$ & 0 & $\begin{array}{ll}6.04 \mathrm{E}-07 \\
\end{array}$ & 0 & $9.07 \mathrm{E}-06$ & $1.59 \mathrm{E}-05$ & $2.63 \mathrm{E}-05$ & $\begin{array}{ll}0.00347067 \\
\end{array}$ & $\begin{array}{l}4.33 \mathrm{E}-06 \\
\end{array}$ & 0.0071113 & 246632.002 \\
\hline 5276.38063 & 829.01858 & 864.986701 & 997.79236 & 808.295114 & $5.79 \mathrm{E}-09$ & $2.50 \mathrm{E}-05$ & 0 & $8.25 \mathrm{E}-07$ & 0 & $\begin{array}{l}9.07 E-06 \\
\end{array}$ & $1.59 \mathrm{E}-05$ & $2.63 \mathrm{E}-05$ & 0.00346938 & $5.91 \mathrm{E}-06$ & 0.00711132 & 246632.789 \\
\hline 6440.53385 & 829.01858 & 864.987076 & 997.792893 & 808.295678 & $9.24 \mathrm{E}-09$ & $2.50 \mathrm{E}-05$ & 0 & \begin{tabular}{ll|}
$1.10 \mathrm{E}-06$ \\
\end{tabular} & 0 & \begin{tabular}{|l|l|}
$9.07 E-06$ \\
\end{tabular} & $1.59 \mathrm{E}-05$ & $2.63 \mathrm{E}-05$ & 0.00346883 & $\begin{array}{l}7.89 \mathrm{E}-06 \\
\end{array}$ & 0.00711133 & 246633.629 \\
\hline 7895.72538 & 829.01858 & 864.987616 & 997.793998 & 808.296214 & \begin{tabular}{|l|l|}
$1.35 E-08$ \\
\end{tabular} & $2.50 \mathrm{E}-05$ & 0 & $1.45 \mathrm{E}-06$ & 0 & $9.07 \mathrm{E}-06$ & $1.59 \mathrm{E}-05$ & $2.63 \mathrm{E}-05$ & 0.00346871 & $1.04 \mathrm{E}-05$ & 0.00711135 & 246634.635 \\
\hline 9714.71478 & 829.01858 & 864.988338 & 997.795893 & 808.296788 & $1.89 E-08$ & $2.50 \mathrm{E}-05$ & 0 & $1.88 \mathrm{E}-06$ & 0 & $9.07 E-06$ & $1.59 \mathrm{E}-05$ & $2.63 \mathrm{E}-05$ & 0.00346895 & $1.35 \mathrm{E}-05$ & 0.00711136 & 246635.895 \\
\hline 11988.4515 & 829.01858 & 864.989047 & 997.799894 & \begin{tabular}{|l|}
808.29749 \\
\end{tabular} & $2.56 \mathrm{E}-08$ & $2.50 \mathrm{E}-05$ & 0 & $2.42 E-06$ & 0 & $9.07 \mathrm{E}-06$ & $1.59 \mathrm{E}-05$ & $2.63 \mathrm{E}-05$ & 0.00346957 & $1.73 \mathrm{E}-05$ & 0.00711139 & 246636.099 \\
\hline 14830.6225 & 829.01858 & 864.990186 & 997.804261 & 808.29834 & $3.40 \mathrm{E}-08$ & $2.50 \mathrm{E}-05$ & 0 & $3.09 \mathrm{E}-06$ & 0 & $9.07 \mathrm{E}-06$ & $1.59 \mathrm{E}-05$ & $2.63 E-05$ & 0.00347051 & $2.22 \mathrm{E}-05$ & 0.00711141 & 246637.788 \\
\hline 18383.3362 & 829.01858 & 864.991714 & 997.810777 & 808.299447 & 4.44E- 08 & $2.50 \mathrm{E}-05$ & 0 & $3.94 \mathrm{E}-06$ & 0 & $9.07 \mathrm{E}-06$ & $1.59 \mathrm{E}-05$ & $2.63 \mathrm{E}-05$ & 0.00347182 & $2.82 \mathrm{E}-05$ & 0.00711145 & 246639.998 \\
\hline $\begin{array}{r}22824.2283 \\
\end{array}$ & 829.01858 & 864.993782 & 997.8202 & 808.300919 & $5.75 E-08$ & $2.50 \mathrm{E}-05$ & 0 & $4.99 \mathrm{E}-06$ & 0 & $9.07 \mathrm{E}-06$ & $1.59 \mathrm{E}-05$ & $2.63 \mathrm{E}-05$ & 0.00347358 & $3.58 \mathrm{E}-05$ & 0.00711149 & 246642.938 \\
\hline 28375.3434 & 829.01858 & 864.996595 & 997.833571 & \begin{tabular}{|r|}
808.3029 \\
\end{tabular} & \begin{tabular}{ll|}
$7.37 E-08$ \\
\end{tabular} & $2.50 \mathrm{E}-05$ & 0 & $6.31 \mathrm{E}-06$ & 0 & $9.07 \mathrm{E}-06$ & $1.59 \mathrm{E}-05$ & $2.63 \mathrm{E}-05$ & 0.00347591 & $4.52 \mathrm{E}-05$ & 0.00711155 & 246646.867 \\
\hline $\begin{array}{l}35314.2373 \\
\end{array}$ & 829.01858 & 865.000442 & 997.852353 & 808.305578 & \begin{tabular}{|c|}
$9.39 E-08$ \\
\end{tabular} & $2.50 \mathrm{E}-05$ & 0 & $7.96 \mathrm{E}-06$ & 0 & $9.07 \mathrm{E}-06$ & $\begin{array}{l}1.59 \mathrm{E}-05 \\
\end{array}$ & $2.63 \mathrm{E}-05$ & 0.00347899 & $5.70 \mathrm{E}-05$ & 0.00711163 & 246652.142 \\
\hline 43987.8547 & 829.01858 & 865.008149 & 997.876473 & 808.308957 & $1.19 \mathrm{E}-07$ & $2.50 \mathrm{E}-05$ & 0 & $1.00 \mathrm{E}-05$ & 0 & $9.07 \mathrm{E}-06$ & $1.59 \mathrm{E}-05$ & $2.63 E-05$ & 0.0034831 & $7.18 \mathrm{E}-05$ & 0.00711173 & 246667.24 \\
\hline 54829.8764 & 829.01858 & 865.016799 & 997.912275 & 808.313787 & $1.50 \mathrm{E}-07$ & $2.50 \mathrm{E}-05$ & 0 & $1.26 \mathrm{E}-05$ & 0 & $9.07 \mathrm{E}-06$ & $\begin{array}{l}1.59 \mathrm{E}-05 \\
\end{array}$ & $2.63 \mathrm{E}-05$ & 0.00348859 & $\begin{array}{l}9.02 \mathrm{E}-05 \\
\end{array}$ & 0.00711185 & 246679.625 \\
\hline 68382.4035 & 829.01858 & 865.029221 & 997.962171 & 808.32036 & $1.89 \mathrm{E}-07$ & $2.50 \mathrm{E}-05$ & 0 & $1.58 \mathrm{E}-05$ & 0 & $9.07 E-06$ & $1.59 \mathrm{E}-05$ & $2.63 \mathrm{E}-05$ & 0.00349592 & 0.00011325 & 0.00711202 & 246696.41 \\
\hline 85323.0625 & 829.01858 & 865.047813 & 998.031719 & 808.329335 & $2.37 E-07$ & $2.50 \mathrm{E}-05$ & 0 & $1.98 \mathrm{E}-05$ & 0 & $\begin{array}{l}9.07 E-06 \\
\end{array}$ & $1.59 \mathrm{E}-05$ & $2.63 \mathrm{E}-05$ & 0.00350575 & 0.00014206 & 0.00711224 & 246719.215 \\
\hline 106498.886 & 829.01858 & 865.075215 & 998.128679 & 808.34162 & $2.96 E-07$ & $2.50 \mathrm{E}-05$ & 0 & $2.49 \mathrm{E}-05$ & 0 & $9.08 \mathrm{E}-06$ & $1.59 \mathrm{E}-05$ & $2.63 \mathrm{E}-05$ & 0.00351895 & 0.00017808 & 0.00711253 & 246750.27 \\
\hline 132968.666 & 829.01858 & 865.116358 & 998.263893 & 808.358478 & $3.70 \mathrm{E}-07$ & $2.50 \mathrm{E}-05$ & 0 & $3.11 \mathrm{E}-05$ & 0 & $9.08 \mathrm{E}-06$ & $1.59 \mathrm{E}-05$ & $2.63 E-05$ & 0.00353674 & 0.0002231 & 0.00711293 & 246792.657 \\
\hline 166055.89 & 829.01858 & 865.180108 & 998.452481 & \begin{tabular}{|l|}
808.381679 \\
\end{tabular} & $4.60 \mathrm{E}-07$ & $2.50 \mathrm{E}-05$ & 0 & $3.90 \mathrm{E}-05$ & 0 & $9.08 \mathrm{E}-06$ & $1.59 \mathrm{E}-05$ & $2.63 \mathrm{E}-05$ & 0.00356076 & 0.00027938 & 0.00711348 & 246850.58 \\
\hline 207414.921 & 829.01858 & 865.2809 & 998.715998 & 808.413703 & $5.711-07$ & $2.50 \mathrm{E}-05$ & 0 & $4.88 \mathrm{E}-05$ & 0 & $9.09 \mathrm{E}-06$ & $1.59 \mathrm{E}-05$ & $2.63 \mathrm{E}-05$ & 0.00359328 & 0.00034972 & 0.00711425 & 246930.07 \\
\hline 259113.709 & 829.01858 & 865.446434 & \begin{tabular}{|c|}
99.084431 \\
\end{tabular} & 808.458091 & \begin{tabular}{|l|}
$7.07 E-07$ \\
\end{tabular} & $2.50 \mathrm{E}-05$ & 0 & $6.111-05$ & 0 & \begin{tabular}{|l|l|}
$9.09 E-06$ \\
\end{tabular} & $1.59 \mathrm{E}-05$ & $2.63 \mathrm{E}-05$ & 0.00363741 & 0.00043766 & 0.00711535 & 247039.334 \\
\hline 323737.195 & 829.01858 & 865.532418 & 999.599108 & 808.518836 & $8.72 E-07$ & $2.50 \mathrm{E}-05$ & 0 & $7.64 E-05$ & 0 & $\begin{array}{l}9.10 \mathrm{E}-06 \\
\end{array}$ & \begin{tabular}{ll|}
$1.59 E-05$ \\
\end{tabular} & $2.63 \mathrm{E}-05$ & 0.00369742 & 0.00054757 & \begin{tabular}{|l|}
0.00711647 \\
\end{tabular} & 247189.573 \\
\hline 404516.551 & 829.01858 & 865.390529 & 1000.3205 & 808.601891 & $1.07 E-06$ & $2.50 \mathrm{E}-05$ & 0 & $9.56 \mathrm{E}-05$ & 0 & $9.11 \mathrm{E}-06$ & $1.58 \mathrm{E}-05$ & $2.63 \mathrm{E}-05$ & 0.00377919 & 0.00068497 & 0.00711724 & 247396.552 \\
\hline 505490.747 & 829.01858 & 865.276303 & 1001.33357 & 808.718356 & $1.30 \mathrm{E}-06$ & $2.50 \mathrm{E}-05$ & 0 & 0.00011956 & 0 & $9.13 \mathrm{E}-06$ & $1.58 \mathrm{E}-05$ & $2.63 \mathrm{E}-05$ & 0.00389087 & 0.00085671 & 0.00711853 & 247683.25 \\
\hline 631708.492 & 829.01858 & 865.327954 & 1002.76783 & 808.883035 & $1.58 E-06$ & $2.50 \mathrm{E}-05$ & 0 & 0.00014952 & 0 & $9.15 \mathrm{E}-06$ & $1.58 \mathrm{E}-05$ & $2.63 \mathrm{E}-05$ & 0.00404373 & 0.00107139 & 0.00712083 & 248081.805 \\
\hline 789480.673 & 829.01858 & 865.829276 & 1004.80111 & 809.184517 & $1.84 E-06$ & $2.50 \mathrm{E}-05$ & 0 & 0.00018697 & 0 & $\begin{array}{l}9.18 E-06 \\
\end{array}$ & $1.58 \mathrm{E}-05$ & $2.63 \mathrm{E}-05$ & 0.00425367 & 0.00133974 & 0.00711522 & 248658.235 \\
\hline 986695.899 & 829.01858 & 864.921167 & 1006.88736 & 809.213926 & $2.14 \mathrm{E}-06$ & $2.50 \mathrm{E}-05$ & 0 & 0.00023378 & 0 & $9.23 E-06$ & $1.57 \mathrm{E}-05$ & $2.63 \mathrm{E}-05$ & 0.00453714 & 0.00167518 & 0.0071183 & 249339.879 \\
\hline 1233214.93 & 829.01858 & 865.192919 & 1009.87159 & 809.146906 & $2.49 \mathrm{E}-06$ & $2.50 \mathrm{E}-05$ & 0 & 0.00029229 & 0 & $\begin{array}{l}9.28 E-06 \\
\end{array}$ & $1.57 \mathrm{E}-05$ & $2.63 \mathrm{E}-05$ & 0.00492437 & 0.00209448 & 0.00712558 & 250258.835 \\
\hline 1541363.72 & 829.01858 & 865.433121 & 1014.09233 & 809.02708 & $2.86 \mathrm{E}-06$ & $2.50 \mathrm{E}-05$ & 0 & 0.00036544 & 0 & $9.37 \mathrm{E}-06$ & $1.56 \mathrm{E}-05$ & $2.64 \mathrm{E}-05$ & 0.00545496 & 0.0026186 & 0.00712785 & 251542.152 \\
\hline 1926549.71 & 829.01858 & 865.228091 & 1020.04807 & 808.917446 & $3.20 \mathrm{E}-06$ & $2.50 \mathrm{E}-05$ & 0 & 0.00045687 & 0 & $9.48 E-06$ & $1.55 E-05$ & $2.63 \mathrm{E}-05$ & 0.00618288 & 0.00327375 & 0.00712809 & 253376.685 \\
\hline 2408032.2 & 829.01858 & 864.357416 & 1028.4575 & 808.745014 & $3.52 \mathrm{E}-06$ & $2.50 \mathrm{E}-05$ & 0 & 0.00057115 & 0 & $9.64 \mathrm{E}-06$ & $1.53 \mathrm{E}-05$ & $2.64 \mathrm{E}-05$ & 0.00717979 & 0.00409269 & 0.00713195 & 255991.221 \\
\hline 3009885.31 & 829.01858 & 866.817134 & 1040.38775 & 808.536663 & $4.17 \mathrm{E}-06$ & $2.50 \mathrm{E}-05$ & 0 & 0.00071401 & 0 & $9.87 \mathrm{E}-06$ & $1.51 \mathrm{E}-05$ & $2.64 \mathrm{E}-05$ & 0.00854232 & 0.00511637 & 0.00714044 & 259782.226 \\
\hline
\end{tabular}




\begin{tabular}{|c|c|c|c|c|c|c|c|c|c|c|c|c|c|c|c|c|}
\hline Time (s) & $\begin{array}{c}\text { Coolant outlet } \\
\text { temperature }(K)\end{array}$ & $\begin{array}{l}\text { Peak Cladding } \\
\text { Temperature (K) }\end{array}$ & $\begin{array}{c}\text { Peak fuel } \\
\text { temperature (K) }\end{array}$ & $\begin{array}{c}\text { Average cladding } \\
\text { internal } \\
\text { temperature (K) }\end{array}$ & $\begin{array}{l}\text { Peak cumulative } \\
\text { damage factor }\end{array}$ & $\begin{array}{l}\text { Cladding interior } \\
\text { volume }\left(\mathrm{m}^{3}\right)\end{array}$ & $\begin{array}{l}\text { Fission Gas } \\
\text { Release (\%) }\end{array}$ & $\begin{array}{c}\text { Fission gas } \\
\text { produced } \\
\text { (moles) }\end{array}$ & $\begin{array}{c}\text { Fission gas } \\
\text { released (moles) }\end{array}$ & Fuel volume $\left(\mathrm{m}^{3}\right)$ & $\begin{array}{l}\text { Plenum gas } \\
\text { volume }\left(m^{3}\right)\end{array}$ & $\begin{array}{l}\text { Peak cladding } \\
\text { radial growth } \\
\text { (m) }\end{array}$ & $\begin{array}{l}\text { Peak fuel axial } \\
\text { growth }(m)\end{array}$ & $\begin{array}{c}\text { Peak burnup } \\
\text { (at\%) }\end{array}$ & $\begin{array}{l}\text { Peak cladding } \\
\text { hoop strain }\end{array}$ & $\begin{array}{l}\text { Plenum pressure } \\
(\mathrm{Pa})\end{array}$ \\
\hline 3762201.69 & 829.01858 & 865.330163 & 1057.40318 & \begin{tabular}{|l|l|}
808.210596 \\
\end{tabular} & $5.37 \mathrm{E}-06$ & $2.50 \mathrm{E}-05$ & 0 & 0.00089258 & 0 & $1.02 E-05$ & $1.48 \mathrm{E}-05$ & $2.64 \mathrm{E}-05$ & 0.01040058 & 0.00639596 & 0.00714374 & 265349.599 \\
\hline 4702597.17 & 829.01858 & 861.885934 & 1081.29123 & 805.673291 & $5.37 \mathrm{E}-06$ & $2.50 \mathrm{E}-05$ & 6.0147857 & 0.0011158 & $6.71 E-05$ & $1.06 \mathrm{E}-05$ & $1.43 E-05$ & $2.61 \mathrm{E}-05$ & 0.01284855 & 0.00799545 & 0.00703064 & 272776.6 \\
\hline 5702597.17 & 829.01858 & 865.844456 & 1100.78783 & 806.014066 & \begin{tabular}{ll|l|}
$5.38 E-06$ \\
\end{tabular} & $2.50 \mathrm{E}-05$ & 30.8542347 & 0.00135316 & 0.00041751 & $1.11 \mathrm{E}-05$ & $1.39 \mathrm{E}-05$ & $2.63 E-05$ & 0.01532219 & 0.00969632 & 0.00707936 & 313917.696 \\
\hline 6702597.17 & 829.01858 & 862.875704 & 1111.8551 & 807.066575 & $7.05 \mathrm{E}-06$ & $2.50 \mathrm{E}-05$ & 49.2070695 & 0.00159053 & 0.00078265 & $1.14 E-05$ & $1.36 \mathrm{E}-05$ & $2.65 \mathrm{E}-05$ & 0.01710555 & 0.01139719 & 0.00716469 & 494888.762 \\
\hline 7702597.17 & 829.01858 & 862.766648 & 1117.86239 & 806.910534 & $8.83 \mathrm{E}-06$ & $2.50 \mathrm{E}-05$ & 57.9624698 & 0.00182789 & 0.00105949 & $1.16 \mathrm{E}-05$ & $1.34 \mathrm{E}-05$ & $2.65 E-05$ & 0.01821914 & 0.01309807 & 0.0071786 & 685286.539 \\
\hline 8203212 & 829.01858 & 862.352796 & 1119.43175 & 806.838689 & $9.80 \mathrm{E}-06$ & $2.50 \mathrm{E}-05$ & 60.2729211 & 0.00194672 & 0.00117334 & $1.17 \mathrm{E}-05$ & $1.33 \mathrm{E}-05$ & $2.65 E-05$ & 0.01858686 & 0.01394955 & 0.00718836 & 828056.393 \\
\hline 8206812 & 822.624548 & 855.042225 & 1107.62737 & 801.386485 & $9.80 \mathrm{E}-06$ & $2.50 \mathrm{E}-05$ & 60.2799815 & 0.00194756 & 0.00117399 & $1.16 \mathrm{E}-05$ & $1.33 E-05$ & $2.62 E-05$ & 0.01854713 & 0.01395559 & 0.00710112 & 879491.447 \\
\hline 8707426.83 & 822.624548 & 856.324389 & 1107.69869 & 801.425666 & $1.02 E-05$ & $2.50 \mathrm{E}-05$ & 61.7644391 & 0.00206338 & 0.00127444 & $1.17 \mathrm{E}-05$ & $1.33 E-05$ & $2.62 E-05$ & 0.01876393 & 0.01478553 & 0.00710563 & 881786.319 \\
\hline 9333195.37 & 822.624548 & 854.757108 & 1108.68057 & 801.355701 & $1.07 \mathrm{E}-05$ & $2.50 \mathrm{E}-05$ & 63.0280362 & 0.00220816 & 0.00139176 & $1.17 \mathrm{E}-05$ & $1.32 E-05$ & $2.63 E-05$ & 0.01909591 & 0.01582294 & 0.00711311 & 936080.739 \\
\hline 10115406 & 822.624548 & 854.621139 & 1109.87629 & 801.289869 & $1.14 \mathrm{E}-05$ & $2.50 \mathrm{E}-05$ & 64.1393966 & 0.00238913 & 0.00153237 & $1.18 \mathrm{E}-05$ & $1.32 \mathrm{E}-05$ & $2.63 \mathrm{E}-05$ & 0.01947152 & 0.01711971 & 0.00712268 & 999865.503 \\
\hline 11093169.4 & 822.624548 & 854.51427 & 1110.88041 & 801.221016 & $1.22 E-05$ & $2.50 \mathrm{E}-05$ & 65.3123127 & 0.00261534 & 0.00170814 & $1.19 \mathrm{E}-05$ & $1.31 E-05$ & $2.63 \mathrm{E}-05$ & 0.01986708 & 0.01874068 & 0.00713508 & 1076145.13 \\
\hline 12093169.4 & 822.624548 & 854.382726 & 1111.39349 & $\begin{array}{l}801.175918 \\
\end{array}$ & $1.31 \mathrm{E}-05$ & $2.50 \mathrm{E}-05$ & 66.3190851 & 0.0028467 & 0.0018879 & $1.19 \mathrm{E}-05$ & $1.30 \mathrm{E}-05$ & $2.64 E-05$ & 0.02019783 & 0.02039851 & 0.00714898 & 1169961.88 \\
\hline 13093169.4 & 822.624548 & 854.614524 & 1111.58759 & 801.227266 & $1.46 \mathrm{E}-05$ & $2.50 \mathrm{E}-05$ & 67.1745128 & 0.00307805 & 0.00206767 & $1.20 \mathrm{E}-05$ & $1.30 \mathrm{E}-05$ & $2.64 \mathrm{E}-05$ & 0.0204814 & 0.02205633 & 0.00716373 & 1265772.54 \\
\hline 13814423 & 822.624548 & 854.562001 & 1111.60426 & 801.199867 & $1.58 \mathrm{E}-05$ & 2.50 E- 05 & 67.715776 & 0.00324492 & 0.00219732 & $1.20 \mathrm{E}-05$ & $1.30 \mathrm{E}-05$ & $2.65 E-05$ & 0.02066194 & 0.02325205 & 0.00717598 & 1360395.88 \\
\hline 13818023 & 817.2221601 & 847.79938 & 1096.01786 & 796.247806 & $1.58 \mathrm{E}-05$ & $2.50 \mathrm{E}-05$ & 67.7182874 & 0.00324574 & 0.00219796 & $1.20 \mathrm{E}-05$ & $1.30 \mathrm{E}-05$ & $2.62 E-05$ & 0.02060891 & 0.0232579 & 0.00709856 & 1417923.53 \\
\hline 13860007.3 & 817.221601 & 847.799457 & 1096.01536 & 796.24721 & $1.59 \mathrm{E}-05$ & $2.50 \mathrm{E}-05$ & 67.7468972 & 0.00325507 & 0.00220521 & $1.20 \mathrm{E}-05$ & $1.30 \mathrm{E}-05$ & $2.62 E-05$ & 0.02069768 & 0.02332475 & 0.00709905 & 1418350.5 \\
\hline 13901991.6 & 817.221601 & 847.802902 & 1096.00851 & 796.246352 & $1.59 \mathrm{E}-05$ & $2.50 \mathrm{E}-05$ & 67.7753434 & 0.0032644 & 0.00221246 & $1.20 \mathrm{E}-05$ & $1.30 \mathrm{E}-05$ & $2.62 E-05$ & 0.0207387 & 0.02339161 & 0.00709969 & 1422155.69 \\
\hline 13954472 & 817.221601 & 847.812137 & 1095.99957 & 796.245187 & $1.60 \mathrm{E}-05$ & $2.50 \mathrm{E}-05$ & 67.8106733 & 0.00327606 & 0.00222152 & $1.20 \mathrm{E}-05$ & $1.30 \mathrm{E}-05$ & $2.62 E-05$ & 0.02076564 & 0.02347517 & 0.00710044 & 1425988.81 \\
\hline 14020072.5 & 817.221601 & 847.826964 & 1095.98835 & 796.243701 & $1.60 \mathrm{E}-05$ & 2.50 E- 05 & 67.8544835 & 0.00329064 & 0.00223284 & $1.20 \mathrm{E}-05$ & $1.30 \mathrm{E}-05$ & $2.62 E-05$ & 0.02078909 & 0.02357963 & 0.00710139 & 1430781.21 \\
\hline 14102073 & 817.221601 & 847.850527 & 1095.97432 & 796.24185 & $1.61 E-05$ & $2.50 \mathrm{E}-05$ & 67.9087034 & 0.00330886 & 0.002247 & $1.20 \mathrm{E}-05$ & $1.30 \mathrm{E}-05$ & $2.62 E-05$ & 0.02081334 & 0.02371021 & 0.00710257 & 1436773.32 \\
\hline 14204573.8 & 817.221601 & 847.886624 & 1095.95679 & 796.239559 & $1.62 \mathrm{E}-05$ & $2.50 \mathrm{E}-05$ & 67.9756443 & 0.00333164 & 0.0022647 & $1.20 \mathrm{E}-05$ & $1.30 \mathrm{E}-05$ & $2.62 E-05$ & 0.02084012 & 0.02387342 & 0.00710406 & 1444266.13 \\
\hline 14332699.7 & 817.221601 & 847.940765 & 1095.93496 & 796.236749 & $1.63 \mathrm{E}-05$ & $2.50 \mathrm{E}-05$ & 68.0580441 & 0.00336011 & 0.00228682 & $1.20 \mathrm{E}-05$ & $1.30 \mathrm{E}-05$ & $2.62 E-05$ & 0.02087094 & 0.02407744 & 0.00710592 & 1453637.63 \\
\hline 14428975 & 817.221601 & 847.987897 & 1095.9187 & 796.234683 & $1.64 \mathrm{E}-05$ & $2.50 \mathrm{E}-05$ & 68.1190472 & 0.0033815 & 0.00230345 & $1.20 \mathrm{E}-05$ & $1.30 \mathrm{E}-05$ & $2.62 E-05$ & 0.02089319 & 0.02423075 & 0.00710752 & 1465187.29 \\
\hline 14432575 & 810.789189 & 841.227361 & 1090.04484 & $\begin{array}{r}791.14738 \\
\end{array}$ & $1.64 \mathrm{E}-05$ & $2.49 \mathrm{E}-05$ & 68.1213056 & 0.0033823 & 0.00230407 & $1.20 \mathrm{E}-05$ & $1.30 \mathrm{E}-05$ & $2.59 \mathrm{E}-05$ & 0.020866 & 0.02423646 & 0.00702289 & 1464495.4 \\
\hline 14528850.3 & 810.789189 & 841.276518 & 1090.02953 & 791.145318 & $1.64 \mathrm{E}-05$ & $2.49 \mathrm{E}-05$ & 68.1811034 & 0.00340355 & 0.00232058 & $1.20 \mathrm{E}-05$ & $1.30 \mathrm{E}-05$ & $2.59 \mathrm{E}-05$ & 0.02088681 & 0.02438871 & $\begin{array}{l}0.007024 \\
\end{array}$ & 1465068.55 \\
\hline 14649194.4 & 810.789189 & 841.293911 & 1090.00944 & 791.142575 & $1.65 \mathrm{E}-05$ & $2.49 \mathrm{E}-05$ & 68.2548088 & 0.00343011 & 0.00234121 & $1.20 \mathrm{E}-05$ & $1.30 \mathrm{E}-05$ & $2.59 E-05$ & 0.02091259 & 0.02457903 & 0.00702572 & 1473767.82 \\
\hline 14799624.6 & 810.789189 & 841.235707 & 1089.98439 & 791.13871 & $1.65 \mathrm{E}-05$ & $2.49 \mathrm{E}-05$ & 68.3453509 & 0.00346331 & 0.00236701 & $1.20 \mathrm{E}-05$ & $1.30 \mathrm{E}-05$ & $2.59 \mathrm{E}-05$ & 0.0209443 & 0.02481693 & 0.00702788 & 1484648.5 \\
\hline 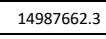 & 810.789189 & 841.168059 & 1089.95305 & 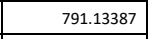 & $1.66 \mathrm{E}-05$ & $2.49 \mathrm{E}-05$ & 688.4561163 & 0.0035048 & 0.00239925 & $1.20 \mathrm{E}-05$ & $1.30 \mathrm{E}-05$ & $2.59 \mathrm{E}-05$ & 0.02098335 & 0.0251143 & 0.00703059 & 1498260.13 \\
\hline 15222709.4 & 810.789189 & 841.068488 & 1089.91397 & 791.127707 & $1.67 \mathrm{E}-05$ & $2.49 \mathrm{E}-05$ & 68.5909381 & 0.00355668 & 0.00243956 & $1.20 \mathrm{E}-05$ & $1.29 \mathrm{E}-05$ & $2.60 \mathrm{E}-05$ & 0.02103157 & 0.02548601 & 0.00703399 & 1515290.29 \\
\hline 15516518.3 & 810.789189 & 840.958476 & 1089.86531 & 791.119948 & $1.68 \mathrm{E}-05$ & $2.49 \mathrm{E}-05$ & 68.754034 & 0.00362152 & 0.00248994 & $1.20 \mathrm{E}-05$ & $1.29 \mathrm{E}-05$ & $2.60 \mathrm{E}-05$ & 0.02109121 & 0.02595065 & 0.00703823 & 1536605.1 \\
\hline 15883779.4 & 810.789189 & 840.842622 & 1089.80473 & 791.110196 & $1.70 \mathrm{E}-05$ & $2.49 \mathrm{E}-05$ & 68.9498707 & 0.00370257 & 0.00255292 & $1.20 \mathrm{E}-05$ & $1.29 \mathrm{E}-05$ & $2.60 \mathrm{E}-05$ & 0.02116529 & 0.02653145 & 0.00704357 & 1563295.71 \\
\hline 16342855.8 & 810.789189 & 840.826471 & 1089.7293 & 791.098135 & $1.72 E-05$ & $2.49 \mathrm{E}-05$ & 69.1829302 & 0.00380389 & 0.00263164 & $1.20 \mathrm{E}-05$ & $1.29 \mathrm{E}-05$ & $2.60 \mathrm{E}-05$ & 0.02125749 & 0.02725746 & 0.00705032 & 1596732.5 \\
\hline 16916701.4 & 810.789189 & 840.816199 & 1089.63506 & 791.082745 & $1.75 E-05$ & $2.49 \mathrm{E}-05$ & 69.4573585 & 0.00393054 & 0.00273005 & $1.20 \mathrm{E}-05$ & $1.29 \mathrm{E}-05$ & $2.60 \mathrm{E}-05$ & 0.02137245 & 0.02816496 & 0.00705886 & 1638636.17 \\
\hline 17634008.2 & 810.789189 & 840.815574 & 1089.5173 & 791.063976 & $1.78 E-05$ & $2.49 \mathrm{E}-05$ & 69.7764876 & 0.00408884 & 0.00285305 & $1.21 E-05$ & $1.29 \mathrm{E}-05$ & $2.61 E-05$ & 0.02151553 & 0.02929934 & 0.00706973 & 1691173.61 \\
\hline 18530641.9 & 810.789189 & 840.794705 & 1089.37079 & 791.040138 & $1.83 \mathrm{E}-05$ & $2.50 \mathrm{E}-05$ & 70.1422528 & 0.00428673 & 0.00300681 & $1.21 \mathrm{E}-05$ & $1.29 \mathrm{E}-05$ & $2.61 \mathrm{E}-05$ & 0.02169419 & 0.03071731 & 0.00708355 & 1757067.52 \\
\hline 19530641.9 & 810.789189 & 841.024022 & 1089.20737 & 791.01384 & $1.88 E-05$ & $2.50 \mathrm{E}-05$ & 70.5123019 & 0.00450742 & 0.00317829 & $1.21 E-05$ & $1.28 \mathrm{E}-05$ & $2.62 E-05$ & 0.02189249 & 0.03229875 & 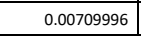 & 1839310.34 \\
\hline 20530641.9 & 810.789189 & 840.725746 & 1089.04182 & 790.984851 & $1.94 E-05$ & $2.50 \mathrm{E}-05$ & 70.8478052 & 0.00472812 & 0.00334977 & $1.21 E-05$ & $1.28 \mathrm{E}-05$ & $2.62 E-05$ & 0.02208633 & 0.03388019 & 0.00711737 & 1930921.61 \\
\hline 21312419 & 810.789189 & 840.720042 & 1088.91177 & 790.966361 & $1.99 \mathrm{E}-05$ & $2.50 \mathrm{E}-05$ & 71.0890478 & 0.00490066 & 0.00348383 & $1.22 E-05$ & $1.28 \mathrm{E}-05$ & $2.63 E-05$ & 0.02223781 & 0.03511652 & 0.00713229 & 2022000.92 \\
\hline 21312902.2 & 810.043394 & 839.749178 & 1086.28276 & 790.254248 & $1.99 \mathrm{E}-05$ & $2.50 \mathrm{E}-05$ & 71.0891911 & 0.00490076 & 0.00348391 & $1.22 \mathrm{E}-05$ & $1.28 \mathrm{E}-05$ & $2.63 E-05$ & 0.02222823 & 0.03511728 & 0.00712384 & 2088956.52 \\
\hline 21313385.4 & 809.293313 & 838.774098 & 1083.64931 & 789.538816 & $1.99 \mathrm{E}-05$ & $2.50 \mathrm{E}-05$ & 71.0893334 & 0.00490087 & 0.00348399 & $1.22 E-05$ & $1.28 \mathrm{E}-05$ & $2.62 E-05$ & 0.02221902 & 0.03511804 & 0.00711232 & 2087047.03 \\
\hline 21313989.3 & 808.34963 & 837.549368 & 1080.35086 & 788.639883 & $1.99 \mathrm{E}-05$ & $2.50 \mathrm{E}-05$ & 71.0895097 & $\begin{array}{l}0.004901 \\
\end{array}$ & 0.0034841 & $1.22 \mathrm{E}-05$ & $1.28 \mathrm{E}-05$ & $2.62 E-05$ & 0.02220796 & 0.03511898 & 0.00709784 & 2084636.5 \\
\hline 21314744.3 & 807.160416 & 836.009062 & 1076.21709 & 787.508878 & $1.99 \mathrm{E}-05$ & $2.49 \mathrm{E}-05$ & 71.0897278 & 0.00490116 & 0.00348422 & $1.22 \mathrm{E}-05$ & $1.28 \mathrm{E}-05$ & $2.61 \mathrm{E}-05$ & 0.02219451 & 0.03512014 & 0.00707961 & 2081603.44 \\
\hline 21315688 & 805.658678 & 834.067363 & 1071.03271 & 786.083486 & $1.99 \mathrm{E}-05$ & $2.49 \mathrm{E}-05$ & 71.0899968 & 0.00490136 & 0.00348438 & $1.22 E-05$ & $1.28 E-05$ & $2.60 \mathrm{E}-05$ & 0.02217797 & 0.03512156 & 0.00705664 & 2077780.97 \\
\hline 21316019 & 805.127969 & 833.382559 & 1069.20961 & 785.580509 & $1.99 \mathrm{E}-05$ & $2.49 \mathrm{E}-05$ & 71.0900902 & 0.00490143 & 0.00348443 & $1.22 \mathrm{E}-05$ & $1.28 \mathrm{E}-05$ & $2.60 \mathrm{E}-05$ & 0.02217219 & 0.03512206 & 0.00704852 & 2076488.54 \\
\hline 21316962.7 & $\begin{array}{l}805.127969 \\
\end{array}$ & 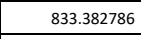 & 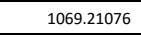 & 785.580453 & $1.99 \mathrm{E}-05$ & $\begin{array}{l}2.49 \mathrm{E}-05 \\
\end{array}$ & $\begin{array}{l}71.0903558 \\
\end{array}$ & 0.00490163 & 0.00348458 & $1.22 E-05$ & $1.28 \mathrm{E}-05$ & $2.60 \mathrm{E}-05$ & 0.02217344 & 0.03512347 & 0.00704854 & 2076516.96 \\
\hline 21318142.4 & 805.127969 & 833.382976 & 1069.21166 & 785.580438 & $1.99 \mathrm{E}-05$ & $2.49 \mathrm{E}-05$ & 71.0906877 & 0.00490187 & 0.00348477 & $1.22 \mathrm{E}-05$ & $1.28 \mathrm{E}-05$ & $2.60 \mathrm{E}-05$ & 0.02217428 & 0.03512524 & $\begin{array}{c}0.00704856 \\
\end{array}$ & 2076597.8 \\
\hline
\end{tabular}




\begin{tabular}{|c|c|c|c|c|c|c|c|c|c|c|c|c|c|c|c|c|}
\hline Time (s) & $\begin{array}{c}\text { Coolant outlet } \\
\text { temperature }(K)\end{array}$ & $\begin{array}{l}\text { Peak Cladding } \\
\text { Temperature (K) }\end{array}$ & $\begin{array}{c}\text { Peak fuel } \\
\text { temperature (K) }\end{array}$ & $\begin{array}{c}\text { Average cladding } \\
\text { internal } \\
\text { temperature (K) }\end{array}$ & \begin{tabular}{|l|}
$\begin{array}{c}\text { Peak cumulative } \\
\text { damage factor }\end{array}$ \\
\end{tabular} & $\begin{array}{l}\text { Cladding interior } \\
\text { volume }\left(\mathrm{m}^{3}\right)\end{array}$ & $\begin{array}{l}\text { Fission Gas } \\
\text { Release (\%) }\end{array}$ & $\begin{array}{c}\text { Fission gas } \\
\text { produced } \\
\text { (moles) }\end{array}$ & $\begin{array}{c}\text { Fission gas } \\
\text { released (moles) }\end{array}$ & Fuel volume $\left(\mathrm{m}^{3}\right)$ & $\begin{array}{l}\text { Plenum gas } \\
\text { volume }\left(m^{3}\right)\end{array}$ & $\begin{array}{l}\text { Peak cladding } \\
\text { radial growth } \\
\text { (m) }\end{array}$ & $\begin{array}{l}\text { Peak fuel axial } \\
\text { growth }(m)\end{array}$ & $\begin{array}{c}\text { Peak burnup } \\
\text { (at\%) }\end{array}$ & $\begin{array}{l}\text { Peak cladding } \\
\text { hoop strain }\end{array}$ & $\begin{array}{c}\text { Plenum pressure } \\
(\mathrm{Pa})\end{array}$ \\
\hline 21319616.9 & 805.127969 & 833.383109 & 1069.21232 & \begin{tabular}{ll|l|}
785.580414 \\
\end{tabular} & $1.99 \mathrm{E}-05$ & $2.49 \mathrm{E}-05$ & 71.0911026 & 0.00490218 & 0.00348501 & $1.22 \mathrm{E}-05$ & $1.28 \mathrm{E}-05$ & $2.60 \mathrm{E}-05$ & 0.02217492 & 0.03512744 & 0.00704858 & 2076699.65 \\
\hline 21321460.1 & 805.127969 & 833.383203 & 1069.21278 & 785.580382 & $1.99 \mathrm{E}-05$ & $2.49 \mathrm{E}-05$ & 71.0916211 & 0.00490256 & 0.00348531 & $1.22 \mathrm{E}-05$ & $1.28 \mathrm{E}-05$ & $2.60 \mathrm{E}-05$ & 0.02217545 & 0.0351302 & 0.00704861 & 2076827.53 \\
\hline 21323764.1 & 805.127969 & 833.383271 & 1069.21303 & 785.58034 & $1.99 \mathrm{E}-05$ & $2.49 \mathrm{E}-05$ & 71.0922692 & 0.00490305 & 0.00348569 & $1.22 E-05$ & $1.28 \mathrm{E}-05$ & $2.60 \mathrm{E}-05$ & 0.02217594 & 0.03513364 & 0.00704865 & 2076987.81 \\
\hline 21326644.1 & 805.127969 & 833.383326 & 1069.21303 & 785.580286 & $1.99 \mathrm{E}-05$ & $2.49 \mathrm{E}-05$ & 71.093079 & 0.00490365 & 0.00348615 & $1.22 E-05$ & $1.28 \mathrm{E}-05$ & $2.60 \mathrm{E}-05$ & 0.02217647 & 0.03513795 & 0.0070487 & 2077188.46 \\
\hline 21330244.1 & 805.127969 & 833.383379 & 1069.21278 & 785.580217 & $1.99 \mathrm{E}-05$ & $2.49 \mathrm{E}-05$ & 71.0940911 & $\begin{array}{l}0.0049044 \\
\end{array}$ & 0.00348674 & $1.22 E-05$ & $1.28 \mathrm{E}-05$ & $2.60 \mathrm{E}-05$ & 0.02217709 & 0.03514333 & 0.00704876 & 2077439.52 \\
\hline 21334744.1 & 805.127969 & 833.38344 & 1069.21227 & 785.58013 & $1.99 \mathrm{E}-05$ & $2.49 E-05$ & 71.0953557 & 0.00490534 & 0.00348747 & $1.22 E-05$ & $1.28 \mathrm{E}-05$ & $2.60 \mathrm{E}-05$ & 0.02217787 & 0.03515006 & 0.00704883 & 2077753.49 \\
\hline 21340369.1 & 805.127969 & 833.383516 & 1069.21151 & 785.58002 & $1.99 \mathrm{E}-05$ & $2.49 \mathrm{E}-05$ & 71.0969358 & 0.00490651 & 0.00348838 & $1.22 \mathrm{E}-05$ & $1.28 \mathrm{E}-05$ & $2.60 \mathrm{E}-05$ & 0.02217885 & 0.03515847 & 0.00704892 & 2078146.07 \\
\hline 21347400.4 & 805.127969 & 833.383614 & 1069.21049 & 785.579883 & $1.99 \mathrm{E}-05$ & $2.49 \mathrm{E}-05$ & 71.0989099 & 0.00490798 & 0.00348952 & $1.22 \mathrm{E}-05$ & $1.28 \mathrm{E}-05$ & $2.60 \mathrm{E}-05$ & 0.02218009 & 0.03516899 & 0.00704904 & 2078636.86 \\
\hline 21356189.4 & 805.127969 & 833.383739 & 1069.20917 & \begin{tabular}{|l}
785.579711 \\
\end{tabular} & $1.99 \mathrm{E}-05$ & $2.49 \mathrm{E}-05$ & 71.1013758 & 0.00490981 & 0.00349094 & $1.22 \mathrm{E}-05$ & $1.28 \mathrm{E}-05$ & $2.60 \mathrm{E}-05$ & 0.02218164 & 0.03518213 & 0.00704918 & 2079250.39 \\
\hline 21367175.8 & 805.127969 & 833.383899 & 1069.20751 & 785.579497 & $1.99 \mathrm{E}-05$ & $2.49 \mathrm{E}-05$ & 71.1044556 & 0.0049121 & 0.00349273 & $1.22 \mathrm{E}-05$ & $1.28 \mathrm{E}-05$ & $2.60 \mathrm{E}-05$ & 0.02218358 & 0.03519856 & 0.00704936 & 2080017.34 \\
\hline 21380908.7 & 805.127969 & 833.384099 & 1069.20543 & 785.57923 & $1.99 \mathrm{E}-05$ & $2.49 E-05$ & 71.1083013 & 0.00491497 & 0.00349495 & $1.22 E-05$ & $1.28 \mathrm{E}-05$ & $2.60 \mathrm{E}-05$ & 0.02218601 & 0.03521909 & 0.00704959 & 2080976.08 \\
\hline 21398074.8 & 805.127969 & 833.383964 & 1069.20282 & \begin{tabular}{|l|l|}
785.578894 \\
\end{tabular} & $1.99 \mathrm{E}-05$ & $2.49 \mathrm{E}-05$ & 71.1131022 & 0.00491855 & 0.00349774 & $1.22 E-05$ & $1.28 \mathrm{E}-05$ & $2.60 \mathrm{E}-05$ & 0.02218906 & 0.03524476 & 0.00704987 & 2082174.56 \\
\hline 21419532.5 & 805.127969 & 833.383804 & 1069.19961 & 785.578478 & $1.99 \mathrm{E}-05$ & $2.49 \mathrm{E}-05$ & 71.1190934 & 0.00492303 & 0.00350121 & $1.22 \mathrm{E}-05$ & $1.28 \mathrm{E}-05$ & $2.60 \mathrm{E}-05$ & 0.02219283 & 0.03527685 & 0.00705022 & 2083672.62 \\
\hline 21446354.6 & 805.127969 & 833.383621 & 1069.19556 & 785.577958 & $1.99 \mathrm{E}-05$ & $2.49 E-05$ & 71.1265671 & 0.00492863 & 0.00350556 & $1.22 \mathrm{E}-05$ & $1.28 \mathrm{E}-05$ & $2.60 \mathrm{E}-05$ & 0.02219754 & 0.03531696 & 0.00705066 & 2085545.5 \\
\hline 21479882.2 & 805.127969 & 833.383415 & 1069.19049 & 785.577309 & $1.99 \mathrm{E}-05$ & $2.49 \mathrm{E}-05$ & 71.1358855 & 0.00493562 & 0.003511 & $1.22 E-05$ & $1.28 \mathrm{E}-05$ & $2.60 \mathrm{E}-05$ & 0.02220343 & 0.03536709 & 0.00705121 & 2087886.85 \\
\hline 21521791.7 & 805.127969 & 833.383191 & 1069.18415 & 785.576503 & $1.99 \mathrm{E}-05$ & $2.49 \mathrm{E}-05$ & 71.1474963 & 0.00494437 & 0.0035178 & $1.22 \mathrm{E}-05$ & $1.28 \mathrm{E}-05$ & $2.60 \mathrm{E}-05$ & 0.0222108 & 0.03542976 & 0.0070519 & 2090813.92 \\
\hline 21574178.6 & 805.127969 & 833.382963 & 1069.17623 & 785.575501 & $2.00 \mathrm{E}-05$ & $2.49 \mathrm{E}-05$ & 71.1619522 & 0.0049553 & 0.00352629 & $1.22 \mathrm{E}-05$ & $1.28 \mathrm{E}-05$ & $2.60 \mathrm{E}-05$ & 0.02222002 & 0.0355081 & 0.00705277 & 2094473.37 \\
\hline 21639662.2 & 805.127969 & 833.382756 & 1069.16634 & 785.574259 & $2.00 \mathrm{E}-05$ & $2.49 \mathrm{E}-05$ & 71.1799326 & 0.00496897 & 0.00353691 & $1.22 E-05$ & $1.28 \mathrm{E}-05$ & $2.60 \mathrm{E}-05$ & 0.02223155 & 0.03560602 & 0.00705385 & 2099048.63 \\
\hline 21721516.7 & 805.127969 & 833.382662 & 1069.15397 & 785.572717 & $2.00 \mathrm{E}-05$ & $2.49 \mathrm{E}-05$ & 71.2022695 & 0.00498605 & 0.00355018 & $1.22 \mathrm{E}-05$ & $1.28 \mathrm{E}-05$ & $2.60 \mathrm{E}-05$ & 0.02224597 & 0.03572842 & 0.0070552 & 2104769.19 \\
\hline 21823834.9 & 805.127969 & 833.382776 & 1069.13852 & 785.570809 & $2.00 \mathrm{E}-05$ & $2.49 \mathrm{E}-05$ & 71.2299764 & 0.0050074 & 0.00356677 & $1.22 E-05$ & $1.28 \mathrm{E}-05$ & $2.60 \mathrm{E}-05$ & 0.022264 & 0.03588142 & 0.00705689 & 2111922.19 \\
\hline 21951732.6 & 805.127969 & 833.383165 & 1069.11923 & 785.5684677 & $2.01 \mathrm{E}-05$ & $2.49 \mathrm{E}-05$ & 71.2642795 & 0.00503409 & 0.00358751 & $1.22 E-05$ & $1.28 \mathrm{E}-05$ & $2.60 \mathrm{E}-05$ & 0.02228655 & 0.03607267 & 0.00705902 & 2120867.08 \\
\hline 22111604.7 & 805.127969 & 833.385292 & 1069.09512 & 785.565604 & $2.01 \mathrm{E}-05$ & $2.49 \mathrm{E}-05$ & 71.3066501 & 0.00506745 & 0.00361343 & $1.22 E-05$ & $1.28 \mathrm{E}-05$ & $2.60 \mathrm{E}-05$ & 0.02231475 & 0.03631174 & 0.00706169 & 2132053.88 \\
\hline 22311444.8 & 805.127969 & 833.489992 & 1069.06503 & 785.562101 & $2.02 E-05$ & $2.49 \mathrm{E}-05$ & 71.3588353 & 0.00510916 & 0.00364583 & $1.22 E-05$ & $1.28 \mathrm{E}-05$ & $2.60 \mathrm{E}-05$ & 0.02235002 & 0.03661057 & 0.00706504 & 2146046.22 \\
\hline 22561245 & 805.127969 & 833.503262 & 1069.02749 & 785.55712 & $2.02 \mathrm{E}-05$ & $2.49 \mathrm{E}-05$ & 71.4228809 & 0.00516129 & 0.00368634 & $1.22 \mathrm{E}-05$ & $1.28 \mathrm{E}-05$ & $2.61 \mathrm{E}-05$ & 0.0223941 & 0.03698411 & 0.00706925 & 2163548.38 \\
\hline 22873495.3 & 805.127969 & 833.411048 & 1068.98077 & 785.550352 & $2.03 \mathrm{E}-05$ & $2.49 \mathrm{E}-05$ & 71.5011413 & 0.00522645 & 0.00373697 & $1.22 \mathrm{E}-05$ & $1.28 \mathrm{E}-05$ & $2.61 \mathrm{E}-05$ & 0.02244922 & 0.03745103 & 0.00707455 & 2185445.56 \\
\hline 23263808 & 805.127969 & 833.400037 & 1068.92251 & 785.541741 & $2.05 E-05$ & $2.49 \mathrm{E}-05$ & 71.5962647 & 0.0053079 & 0.00380026 & $1.22 E-05$ & $1.28 \mathrm{E}-05$ & $2.61 E-05$ & 0.02251982 & 0.03803469 & 0.00708132 & 2212849.47 \\
\hline 23751699 & 805.127969 & 833.403553 & 1068.84993 & 785.610036 & $2.06 E-05$ & $2.49 \mathrm{E}-05$ & 71.7111408 & 0.00540971 & 0.00387937 & $1.22 \mathrm{E}-05$ & $1.27 \mathrm{E}-05$ & $2.61 \mathrm{E}-05$ & 0.02260777 & 0.03876425 & 0.00708992 & 2247406.14 \\
\hline 24361562.7 & 805.127969 & 833.385582 & 1068.75955 & \begin{tabular}{|l}
785.599519 \\
\end{tabular} & $2.08 E-05$ & $2.50 \mathrm{E}-05$ & 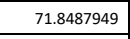 & 0.00553698 & 0.00397825 & $1.22 E-05$ & $1.27 E-05$ & $2.62 E-05$ & 0.02271629 & 0.03967621 & 0.00710076 & 2290387.4 \\
\hline 25123892.4 & 805.127969 & 833.374139 & 1068.64615 & 785.585855 & $2.11 \mathrm{E}-05$ & $2.50 \mathrm{E}-05$ & 72.0122125 & 0.00569606 & 0.00410186 & $1.22 E-05$ & $1.27 \mathrm{E}-05$ & $2.62 E-05$ & 0.02285143 & 0.04081616 & 0.00711459 & 2344239.2 \\
\hline 25596874 & 805.127969 & 833.373075 & 1068.575 & 785.576171 & $2.12 E-05$ & $2.50 \mathrm{E}-05$ & 72.1090931 & 0.00579477 & 0.00417855 & $1.23 \mathrm{E}-05$ & $1.27 E-05$ & $2.62 E-05$ & 0.02293512 & 0.04152343 & 0.00712481 & 2409725.38 \\
\hline 25597817.7 & 804.927561 & 833.071358 & 1067.44321 & 785.358455 & $2.12 \mathrm{E}-05$ & $2.50 \mathrm{E}-05$ & 72.1092828 & 0.00579496 & 0.00417871 & $1.23 \mathrm{E}-05$ & $1.27 \mathrm{E}-05$ & $2.62 E-05$ & 0.0229312 & 0.04152484 & 0.00712317 & 2448432.19 \\
\hline 25598761.4 & 804.726218 & 832.768716 & 1066.31064 & $\begin{array}{l}785.14 \\
\end{array}$ & $2.12 \mathrm{E}-05$ & $2.50 \mathrm{E}-05$ & 72.1094717 & 0.00579516 & 0.00417886 & $1.23 \mathrm{E}-05$ & $1.27 \mathrm{E}-05$ & $2.62 E-05$ & 0.02292736 & 0.04152625 & 0.00711975 & 2447786.85 \\
\hline 25599941.1 & 804.473214 & 832.389124 & 1064.89356 & 784.8659 & $2.12 \mathrm{E}-05$ & $2.50 \mathrm{E}-05$ & $\begin{array}{l}72.109707 \\
\end{array}$ & 0.0057954 & 0.00417905 & $1.23 \mathrm{E}-05$ & $1.27 \mathrm{E}-05$ & $2.62 E-05$ & 0.02292272 & 0.04152799 & 0.00711546 & 2446956.73 \\
\hline 25600474 & 804.358432 & 832.217175 & 1064.25279 & 784.741694 & $2.12 \mathrm{E}-05$ & $2.50 \mathrm{E}-05$ & 72.1098129 & 0.00579551 & 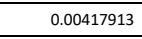 & $1.23 \mathrm{E}-05$ & $1.27 \mathrm{E}-05$ & $2.62 E-05$ & 0.02292065 & 0.04152878 & 0.00711352 & 2446642.43 \\
\hline 25601653.6 & 804.358432 & 832.217169 & 1064.25275 & $\begin{array}{l}784.741687 \\
\end{array}$ & $2.12 \mathrm{E}-05$ & $2.50 \mathrm{E}-05$ & 72.1100471 & 0.00579576 & 0.00417932 & $1.23 \mathrm{E}-05$ & $1.27 \mathrm{E}-05$ & $2.62 E-05$ & 0.02292094 & 0.04153052 & 0.00711354 & 2446686.22 \\
\hline 25603128.2 & 804.358432 & 832.2172 & 1064.25322 & 784.741664 & $2.12 E-05$ & $2.50 \mathrm{E}-05$ & 72.1103399 & 0.00579606 & 0.00417956 & $1.23 E-05$ & $1.27 E-05$ & $2.62 E-05$ & 0.0229212 & 0.0415327 & 0.00711357 & 2446787.34 \\
\hline 25604971.4 & 804.358432 & 832.217215 & 1064.25349 & 784.741635 & $2.12 E-05$ & $2.50 \mathrm{E}-05$ & 72.1107057 & 0.00579644 & 0.00417985 & $1.23 E-05$ & $1.27 E-05$ & $2.62 E-05$ & 0.02292148 & 0.04153541 & 0.0071136 & 2446914.18 \\
\hline 25607275.4 & 804.358432 & 832.217223 & $\begin{array}{l}1064.2536 \\
\end{array}$ & 784.741599 & $2.13 \mathrm{E}-05$ & $2.50 \mathrm{E}-05$ & 72.111163 & 0.00579691 & 0.00418022 & $1.23 \mathrm{E}-05$ & $1.27 \mathrm{E}-05$ & $2.62 E-05$ & 0.02292183 & 0.04153881 & 0.00711364 & 2447072.99 \\
\hline 25610155.4 & 804.358432 & 832.217226 & 1064.25356 & 784.741553 & $2.13 \mathrm{E}-05$ & $2.50 \mathrm{E}-05$ & 72.1117345 & 0.00579751 & 0.00418068 & $1.23 \mathrm{E}-05$ & $1.27 \mathrm{E}-05$ & $2.62 E-05$ & 0.02292229 & 0.04154306 & \begin{tabular}{|c|}
0.0071137 \\
\end{tabular} & 2447271.7 \\
\hline 25613755.4 & 804.358432 & 832.217227 & 1064.25334 & 784.741494 & $2.13 \mathrm{E}-05$ & $2.50 \mathrm{E}-05$ & 72.1124487 & 0.00579825 & 0.00418126 & $1.23 \mathrm{E}-05$ & $1.27 \mathrm{E}-05$ & $2.62 E-05$ & 0.02292288 & 0.04154837 & 0.00711376 & 2447520.23 \\
\hline 25618255.4 & 804.358432 & 832.21723 & 1064.25296 & 784.741419 & $2.13 \mathrm{E}-05$ & $2.50 \mathrm{E}-05$ & 72.1133413 & 0.00579917 & 0.00418198 & $1.23 \mathrm{E}-05$ & $1.27 \mathrm{E}-05$ & $2.62 E-05$ & 0.02292364 & 0.04155501 & 0.00711385 & 2447830.98 \\
\hline 25623880.4 & 804.358432 & 832.217233 & 1064.25238 & 784.741326 & $2.13 \mathrm{E}-05$ & 2.50 E- 05 & 72.1144565 & 0.00580033 & 0.00418288 & $1.23 \mathrm{E}-05$ & $1.27 E-05$ & $2.62 E-05$ & 0.02292461 & 0.04156331 & 0.00711395 & 2448219.5 \\
\hline 25630911.7 & 804.358432 & 832.217243 & 1064.2516 & 784.741208 & $2.13 \mathrm{E}-05$ & $2.50 \mathrm{E}-05$ & 72.1158499 & 0.00580178 & 0.004184 & $1.23 \mathrm{E}-05$ & $1.27 \mathrm{E}-05$ & $2.62 E-05$ & 0.02292582 & 0.04157368 & 0.00711408 & 2448705.2 \\
\hline 25639700.7 & 804.358432 & 832.21727 & 1064.24933 & 784.740876 & $2.13 \mathrm{E}-05$ & $2.50 \mathrm{E}-05$ & 72.1175907 & 0.00580359 & 0.00418541 & $1.23 \mathrm{E}-05$ & $1.27 E-05$ & $2.62 E-05$ & 0.02292735 & 0.04158664 & 0.00711424 & 2449349.06 \\
\hline 25650687 & 804.358432 & 832.217292 & 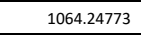 & \begin{tabular}{|l|l|}
784.740645 \\
\end{tabular} & $2.13 \mathrm{E}-05$ & $2.50 \mathrm{E}-05$ & $\begin{array}{l}72.1197652 \\
\end{array}$ & 0.00580585 & 0.00418717 & $1.23 \mathrm{E}-05$ & $1.27 \mathrm{E}-05$ & $2.62 E-05$ & 0.02292925 & 0.04160285 & 0.00711445 & 2450117.22 \\
\hline 25664420 & 804.358432 & 832.217322 & 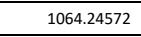 & 784.740355 & $2.13 \mathrm{E}-05$ & $2.50 \mathrm{E}-05$ & 72.1224809 & 0.00580868 & 0.00418936 & $1.23 \mathrm{E}-05$ & $1.27 \mathrm{E}-05$ & $2.62 E-05$ & 0.02293164 & 0.0416231 & 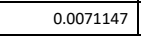 & 2451077.48 \\
\hline
\end{tabular}




\begin{tabular}{|c|c|c|c|c|c|c|c|c|c|c|c|c|c|c|c|c|}
\hline Time (s) & $\begin{array}{c}\text { Coolant outlet } \\
\text { temperature }(K)\end{array}$ & $\begin{array}{l}\text { Peak Cladding } \\
\text { Temperature (K) }\end{array}$ & $\begin{array}{c}\text { Peak fuel } \\
\text { temperature (K) }\end{array}$ & $\begin{array}{c}\text { Average cladding } \\
\text { internal } \\
\text { temperature }(\mathrm{K})\end{array}$ & $\begin{array}{l}\text { Peak cumulative } \\
\text { damage factor }\end{array}$ & $\begin{array}{l}\text { Cladding interior } \\
\text { volume }\left(\mathrm{m}^{3}\right)\end{array}$ & $\begin{array}{l}\text { Fission Gas } \\
\text { Release (\%) }\end{array}$ & 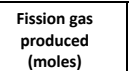 & $\begin{array}{c}\text { Fission gas } \\
\text { released (moles) }\end{array}$ & Fuel volume $\left(\mathrm{m}^{3}\right)$ & $\begin{array}{l}\text { Plenum gas } \\
\text { volume }\left(m^{3}\right)\end{array}$ & $\begin{array}{l}\text { Peak cladding } \\
\text { radial growth } \\
(\mathrm{m})\end{array}$ & $\begin{array}{l}\text { Peak fuel axial } \\
\text { growth }(m)\end{array}$ & $\begin{array}{c}\text { Peak burnup } \\
\text { (at\%) }\end{array}$ & $\begin{array}{l}\text { Peak cladding } \\
\text { hoop strain }\end{array}$ & $\begin{array}{l}\text { Plenum pressure } \\
(\mathrm{Pa})\end{array}$ \\
\hline 25681586.1 & 804.358432 & 832.217362 & 1064.24321 & \begin{tabular}{|l|l|}
784.739991 \\
\end{tabular} & $2.13 \mathrm{E}-05$ & $2.50 \mathrm{E}-05$ & 72.1258718 & 0.00581221 & 0.00419211 & $1.23 \mathrm{E}-05$ & $1.27 \mathrm{E}-05$ & $2.62 \mathrm{E}-05$ & 0.02293462 & 0.04164842 & 0.00711502 & 2452277.87 \\
\hline 25703043.8 & 804.358432 & 832.217415 & 1064.24008 & 784.739534 & $2.13 \mathrm{E}-05$ & $2.50 \mathrm{E}-05$ & 72.1301047 & 0.00581663 & 0.00419554 & $1.23 \mathrm{E}-05$ & $1.27 \mathrm{E}-05$ & $2.62 E-05$ & 0.02293835 & 0.04168007 & 0.00711542 & 2453778.44 \\
\hline 25729865.9 & 804.358432 & 832.217493 & 1064.23617 & $\begin{array}{l}784.738961 \\
\end{array}$ & $2.13 \mathrm{E}-05$ & $2.50 \mathrm{E}-05$ & 72.1353868 & 0.00582215 & 0.00419983 & $1.23 \mathrm{E}-05$ & $1.27 E-05$ & $2.62 E-05$ & 0.02294301 & 0.04171964 & 0.00711591 & 2455654.3 \\
\hline 25763393.5 & 804.358432 & 832.217602 & $\begin{array}{l}1064.23124 \\
\end{array}$ & $\begin{array}{ll}784.738228 \\
\end{array}$ & $2.13 \mathrm{E}-05$ & $2.50 \mathrm{E}-05$ & 72.1419752 & 0.00582905 & 0.00420519 & $1.23 E-05$ & $1.27 \mathrm{E}-05$ & $2.62 E-05$ & 0.02294883 & 0.04176909 & 0.00711653 & 2457999.32 \\
\hline 25805303 & 804.358432 & 832.217775 & 1064.22512 & \begin{tabular}{|l}
784.737313 \\
\end{tabular} & $2.13 \mathrm{E}-05$ & $2.50 \mathrm{E}-05$ & 72.150189 & 0.00583768 & 0.0042119 & $1.23 \mathrm{E}-05$ & $1.27 \mathrm{E}-05$ & $2.62 E-05$ & 0.0229561 & 0.04183091 & 0.00711731 & 2460930.97 \\
\hline 25857689.9 & 804.358432 & 832.218036 & 1064.21746 & 784.736156 & $2.13 \mathrm{E}-05$ & $2.50 \mathrm{E}-05$ & 72.160422 & 0.00584846 & 0.00422027 & $1.23 \mathrm{E}-05$ & $1.27 \mathrm{E}-05$ & $2.62 E-05$ & 0.02296519 & 0.04190818 & 0.00711828 & 2464596.07 \\
\hline 25923173.5 & 804.358432 & 832.218424 & 1064.20788 & \begin{tabular}{|l}
784.734837 \\
\end{tabular} & $2.14 \mathrm{E}-05$ & $2.50 \mathrm{E}-05$ & 72.1731604 & 0.00586194 & 0.00423075 & $1.23 \mathrm{E}-05$ & $1.27 \mathrm{E}-05$ & $2.62 E-05$ & 0.02297655 & 0.04200477 & $\begin{array}{l}0.0071195 \\
\end{array}$ & 2469178.76 \\
\hline 26005028 & 804.358432 & 832.219022 & 1064.19592 & 784.733201 & $2.14 \mathrm{E}-05$ & $2.50 \mathrm{E}-05$ & 72.1890012 & 0.00587879 & 0.00424384 & $1.23 \mathrm{E}-05$ & $1.27 \mathrm{E}-05$ & $2.62 E-05$ & 0.02299075 & 0.04212551 & 0.00712103 & 2474908.61 \\
\hline 26107346.2 & 804.358432 & 832.219951 & 1064.18097 & \begin{tabular}{|l|l|l|}
784.731119 \\
\end{tabular} & $2.14 \mathrm{E}-05$ & $2.50 \mathrm{E}-05$ & 72.208675 & 0.00589985 & 0.00426021 & $1.23 \mathrm{E}-05$ & $1.27 \mathrm{E}-05$ & $2.62 E-05$ & 0.0230085 & 0.04227644 & 0.00712294 & 2482073.04 \\
\hline 26235243.9 & 804.358432 & 832.2214 & 1064.1623 & 784.728466 & $2.15 E-05$ & $2.50 \mathrm{E}-05$ & 72.2330706 & 0.00592618 & 0.00428066 & $1.23 \mathrm{E}-05$ & $1.27 E-05$ & $2.62 E-05$ & 0.02303067 & 0.04246509 & 0.00712534 & 2491031.9 \\
\hline 26261755 & 804.358432 & 832.221733 & 1064.15846 & \begin{tabular}{|l|}
784.727919 \\
\end{tabular} & $2.15 \mathrm{E}-05$ & $2.50 \mathrm{E}-05$ & 72.2381003 & 0.00593164 & 0.0042849 & $1.23 \mathrm{E}-05$ & $1.27 \mathrm{E}-05$ & $2.62 \mathrm{E}-05$ & 0.02303527 & 0.0425042 & 0.00712625 & 2501661.11 \\
\hline 26265355 & 803.788828 & 832.438689 & 1071.99888 & $\begin{array}{l}784.800964 \\
\end{array}$ & $2.15 \mathrm{E}-05$ & $2.50 \mathrm{E}-05$ & 72.2387925 & 0.00593239 & 0.00428549 & $1.23 \mathrm{E}-05$ & $1.27 \mathrm{E}-05$ & $2.62 E-05$ & 0.02305882 & 0.04250958 & 0.00712566 & 2504671.37 \\
\hline 26291866.1 & 803.788828 & 832.439071 & 1071.99137 & 784.800344 & $2.15 E-05$ & $2.50 \mathrm{E}-05$ & 72.2439581 & 0.00593801 & 0.00428985 & $1.23 \mathrm{E}-05$ & $1.27 E-05$ & $2.62 \mathrm{E}-05$ & 0.0230636 & 0.04254983 & 0.0071261 & 2505093.05 \\
\hline 26325005 & 803.788828 & 832.439637 & 1071.98582 & 784.799588 & $2.15 \mathrm{E}-05$ & 2.50 E- 05 & 72.2504012 & 0.00594503 & 0.00429531 & $1.23 \mathrm{E}-05$ & $1.27 E-05$ & $2.62 E-05$ & 0.02306962 & 0.04260014 & 0.00712675 & 2507484.95 \\
\hline 26366428.7 & 803.788828 & 832.44038 & 1071.97934 & 784.798642 & $2.15 \mathrm{E}-05$ & $2.50 \mathrm{E}-05$ & 72.2584339 & 0.0059538 & 0.00430212 & $1.23 \mathrm{E}-05$ & $1.27 \mathrm{E}-05$ & $2.62 E-05$ & 0.02307709 & 0.04266302 & 0.00712755 & 2510474.79 \\
\hline 26418208.2 & 803.788828 & 832.441363 & 1071.97129 & 784.79745 & $2.15 E-05$ & $2.50 \mathrm{E}-05$ & 72.2684414 & 0.00596477 & 0.00431065 & $1.23 E-05$ & $1.27 \mathrm{E}-05$ & $2.62 E-05$ & 0.02308642 & 0.04274163 & 0.00712856 & 2514212.62 \\
\hline 26482932.7 & 803.788828 & 832.442678 & 1071.96123 & 784.795944 & $2.15 \mathrm{E}-05$ & $2.50 \mathrm{E}-05$ & 72.2808991 & 0.00597848 & 0.0043213 & $1.23 \mathrm{E}-05$ & $1.27 \mathrm{E}-05$ & $2.62 E-05$ & 0.02309808 & 0.04283988 & 0.00712983 & 2518885.8 \\
\hline 26563838.2 & 803.788828 & 832.444459 & 1071.94866 & 784.794038 & $2.16 \mathrm{E}-05$ & $2.50 \mathrm{E}-05$ & 72.2963912 & 0.00599562 & 0.00433462 & $1.23 E-05$ & $1.27 \mathrm{E}-05$ & $2.63 E-05$ & 0.02311264 & 0.04296271 & 0.00713141 & 2524728.68 \\
\hline 26664970.2 & 803.788828 & 832.44693 & 1071.93296 & 784.791808 & $2.16 \mathrm{E}-05$ & 2.50 E- 05 & 72.3156322 & 0.00601705 & 0.00435127 & $1.23 \mathrm{E}-05$ & $1.27 \mathrm{E}-05$ & $2.63 \mathrm{E}-05$ & 0.02313085 & 0.04311623 & 0.0071334 & 2532035.14 \\
\hline 26791385.1 & 803.788828 & 832.443454 & 1071.91333 & 784.788951 & $2.17 \mathrm{E}-05$ & $2.50 \mathrm{E}-05$ & 72.3394915 & 0.00604383 & 0.00437208 & $1.23 E-05$ & $1.27 E-05$ & $2.63 E-05$ & 0.0231536 & 0.04330814 & 0.00713587 & 2541171.77 \\
\hline 26949403.7 & 803.788828 & 832.439557 & 1071.88882 & 784.785305 & $2.17 \mathrm{E}-05$ & $2.50 \mathrm{E}-05$ & 72.36902 & 0.00607731 & 0.00439809 & $1.23 \mathrm{E}-05$ & $1.27 \mathrm{E}-05$ & $2.63 E-05$ & 0.02318205 & 0.04354803 & 0.00713897 & 2552598.46 \\
\hline 27146927 & 803.788828 & 832.434256 & 1071.85822 & 784.780635 & $2.18 \mathrm{E}-05$ & $2.50 \mathrm{E}-05$ & 72.4054763 & 0.00611915 & 0.0044306 & $1.23 \mathrm{E}-05$ & $1.27 \mathrm{E}-05$ & $2.63 E-05$ & 0.02321762 & 0.04384788 & 0.00714287 & 2566890.91 \\
\hline 27393831.2 & 803.788828 & 832.426824 & 1071.81999 & 784.774609 & $2.19 \mathrm{E}-05$ & $2.50 \mathrm{E}-05$ & 72.4503514 & 0.00617146 & 0.00447125 & $1.23 E-05$ & $1.27 \mathrm{E}-05$ & $2.63 E-05$ & 0.0232621 & 0.0442227 & 0.00714777 & 2584771.89 \\
\hline 27702461.3 & 803.788828 & 832.418248 & 1071.77231 & 784.766912 & $2.20 \mathrm{E}-05$ & $2.50 \mathrm{E}-05$ & 72.5053867 & 0.00623685 & 0.00452205 & $1.23 \mathrm{E}-05$ & $1.26 \mathrm{E}-05$ & $2.63 \mathrm{E}-05$ & 0.02331774 & 0.04469123 & 0.00715393 & 2607147.61 \\
\hline 28088249 & 803.788828 & 832.40934 & 1071.71299 & 784.757957 & $2.21 \mathrm{E}-05$ & $2.50 \mathrm{E}-05$ & 72.5725791 & 0.00631858 & 0.00458555 & $1.23 \mathrm{E}-05$ & $1.26 \mathrm{E}-05$ & $2.64 E-05$ & 0.02338734 & 0.04527689 & 0.00716171 & 2635159.72 \\
\hline 28570483.7 & 803.788828 & 832.401513 & 1071.64119 & 784.748058 & $2.23 \mathrm{E}-05$ & $2.50 \mathrm{E}-05$ & 72.6541641 & 0.00642074 & 0.00466494 & $1.23 \mathrm{E}-05$ & $1.26 \mathrm{E}-05$ & $2.64 E-05$ & 0.02347444 & 0.04600896 & 0.00717155 & 2670242.09 \\
\hline 29173277 & 803.788828 & 832.392473 & 1071.56709 & 784.733922 & $2.26 \mathrm{E}-05$ & $2.50 \mathrm{E}-05$ & 72.7525656 & 0.00654845 & 0.00476416 & $1.23 \mathrm{E}-05$ & $1.26 E-05$ & $2.64 \mathrm{E}-05$ & 0.02358353 & 0.04692405 & 0.00718401 & 2714188.02 \\
\hline 29926768.6 & 803.788828 & 832.385801 & 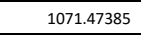 & 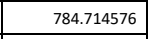 & $2.29 \mathrm{E}-05$ & $2.50 \mathrm{E}-05$ & 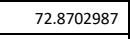 & 0.00670808 & 0.0048882 & $1.24 \mathrm{E}-05$ & $1.26 \mathrm{E}-05$ & $2.65 E-05$ & 0.02371981 & 0.04806791 & 0.00719985 & 2769254.99 \\
\hline 30868633.2 & 803.788828 & 832.383018 & 1071.35051 & 784.688773 & $2.33 \mathrm{E}-05$ & $2.50 \mathrm{E}-05$ & 73.009813 & 0.00690761 & 0.00504324 & $1.24 E-05$ & $1.26 \mathrm{E}-05$ & $2.65 E-05$ & 0.02389 & 0.04949774 & 0.00722009 & 2838301 \\
\hline 31868633.2 & 803.788828 & 832.388572 & 1071.21322 & 784.665542 & $2.38 E-05$ & $2.50 \mathrm{E}-05$ & 73.1493793 & 0.00711947 & 0.00520785 & $1.24 \mathrm{E}-05$ & $1.26 \mathrm{E}-05$ & 2.66 E- 05 & 0.02407329 & 0.05101582 & 0.00724272 & 2924027.77 \\
\hline 32714598 & 803.788828 & 832.379643 & 1071.13234 & 784.6495699 & $2.42 E-05$ & $2.50 \mathrm{E}-05$ & 73.2611212 & 0.00729869 & 0.0053471 & $1.24 \mathrm{E}-05$ & $1.25 \mathrm{E}-05$ & $2.67 E-05$ & 0.02415056 & 0.05230007 & 0.00726286 & 3014216.38 \\
\hline 32718198 & 644.15 & 644.175397 & 644.152564 & 644.150739 & $2.42 E-05$ & $2.48 \mathrm{E}-05$ & 73.2613531 & 0.00729907 & 0.0053474 & $1.23 \mathrm{E}-05$ & $1.26 \mathrm{E}-05$ & $1.88 \mathrm{E}-05$ & 0.02239831 & 0.0523028 & 0.00516244 & 2525579.1 \\
\hline 32721798 & 305 & 305.051386 & 305.006481 & 305.00218 & $2.42 E-05$ & $2.45 E-05$ & 73.2613531 & 0.00729907 & 0.0053474 & $1.20 \mathrm{E}-05$ & $1.25 \mathrm{E}-05$ & $2.54 \mathrm{E}-06$ & 0.02026117 & 0.0523028 & 0.00075586 & 1206544.39 \\
\hline 32725398 & 305 & 305.051386 & 305.006481 & 305.00218 & $2.42 E-05$ & $2.45 E-05$ & 73.2613531 & 0.00729907 & 0.0053474 & $1.20 \mathrm{E}-05$ & $1.25 E-05$ & $2.54 E-06$ & 0.02026117 & 0.0523028 & 0.00075586 & 1206544.39 \\
\hline 32728998 & 778.966356 & 802.973121 & 1012.40014 & 762.831162 & $2.42 E-05$ & $2.49 \mathrm{E}-05$ & 73.2615498 & $\begin{array}{l}0.0072994 \\
\end{array}$ & 0.00534765 & $1.24 \mathrm{E}-05$ & $1.25 E-05$ & $2.54 \mathrm{E}-05$ & 0.02383103 & 0.05230512 & 0.00690817 & 2999950.98 \\
\hline 32732598 & 778.966356 & 802.976508 & 1012.40386 & 762.835131 & $2.42 E-05$ & $2.49 E-05$ & 73.2619433 & 0.00730004 & 0.00534815 & $1.24 E-05$ & $1.25 E-05$ & $2.54 E-05$ & 0.02382429 & 0.05230975 & 0.00690827 & 3000114.74 \\
\hline 32737098 & 778.966356 & 802.975923 & 1012.40175 & 762.835108 & $2.42 E-05$ & $2.49 \mathrm{E}-05$ & 73.262435 & 0.00730085 & 0.00534878 & $1.24 E-05$ & $1.25 E-05$ & $2.54 E-05$ & 0.02382115 & 0.05231555 & 0.00690834 & 3000392.86 \\
\hline 32742723 & 778.966356 & 802.975637 & 1012.40007 & 762.835042 & $2.42 E-05$ & $2.49 \mathrm{E}-05$ & 73.2630494 & 0.00730186 & 0.00534957 & $1.24 \mathrm{E}-05$ & $1.25 \mathrm{E}-05$ & $2.54 \mathrm{E}-05$ & 0.02382013 & 0.05232279 & 0.00690845 & 3000737.97 \\
\hline 32749754.3 & 778.966356 & 802.975617 & 1012.40016 & 762.835045 & $2.42 E-05$ & $2.49 \mathrm{E}-05$ & 73.2638173 & 0.00730313 & 0.00535055 & $1.24 \mathrm{E}-05$ & $1.25 \mathrm{E}-05$ & $2.54 \mathrm{E}-05$ & 0.02382044 & 0.05233185 & 0.00690857 & 3001135.15 \\
\hline 32758543.3 & 778.966356 & 802.97551 & 1012.39865 & $\begin{array}{l}762.83494 \\
\end{array}$ & $2.42 \mathrm{E}-05$ & $2.49 \mathrm{E}-05$ & 73.2647767 & 0.00730471 & 0.00535178 & $1.24 E-05$ & $1.25 \mathrm{E}-05$ & $2.54 \mathrm{E}-05$ & 0.02382149 & 0.05234317 & 0.00690873 & 3001664.71 \\
\hline 32769529.6 & 778.966356 & 802.975471 & 1012.39731 & 762.834804 & $2.42 \mathrm{E}-05$ & $2.49 E-05$ & 73.2659754 & 0.00730668 & 0.00535331 & $1.24 \mathrm{E}-05$ & $1.25 \mathrm{E}-05$ & $2.54 \mathrm{E}-05$ & 0.0238231 & 0.05235732 & 0.00690893 & 3002325.67 \\
\hline 32783262.6 & 778.966356 & 802.975465 & 1012.39593 & 762.834629 & $2.42 E-05$ & $2.49 \mathrm{E}-05$ & 73.2674728 & 0.00730915 & 0.00535523 & $1.24 \mathrm{E}-05$ & $1.25 \mathrm{E}-05$ & $2.54 \mathrm{E}-05$ & 0.02382521 & 0.05237501 & 0.00690918 & 3003151.56 \\
\hline 32800428.7 & 778.966356 & 802.975474 & 1012.39436 & 762.834409 & $2.42 E-05$ & $2.49 \mathrm{E}-05$ & 73.2693433 & 0.00731224 & 0.00535763 & $1.24 E-05$ & $1.25 \mathrm{E}-05$ & $2.54 E-05$ & 0.02382788 & 0.05239712 & 0.0069095 & 3004183.84 \\
\hline 32821886.4 & 778.966356 & 802.975492 & 1012.39246 & 762.834133 & $2.42 \mathrm{E}-05$ & $2.49 \mathrm{E}-05$ & 73.271679 & 0.00731609 & 0.00536062 & $1.24 \mathrm{E}-05$ & $1.25 \mathrm{E}-05$ & $2.54 \mathrm{E}-05$ & 0.02383121 & 0.05242476 & 0.00690989 & 3005474.2 \\
\hline 32848708.5 & 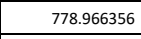 & \begin{tabular}{|l}
802.975519 \\
\end{tabular} & 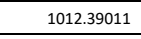 & 762.833785 & $2.42 E-05$ & $\begin{array}{l}2.49 \mathrm{E}-05 \\
\end{array}$ & $\begin{array}{l}73.2745953 \\
\end{array}$ & 0.00732091 & 0.00536437 & $1.24 \mathrm{E}-05$ & $1.25 \mathrm{E}-05$ & $2.54 \mathrm{E}-05$ & 0.02383538 & 0.0524593 & 0.00691038 & 3007087.23 \\
\hline 32882236.1 & 778.966356 & 802.975558 & \begin{tabular}{|l|l|}
12.38717 \\
\end{tabular} & \begin{tabular}{|l|l|}
762.833349 \\
\end{tabular} & $2.42 \mathrm{E}-05$ & $2.49 \mathrm{E}-05$ & 73.2782352 & 0.00732694 & 0.00536905 & $1.24 E-05$ & $1.25 \mathrm{E}-05$ & $2.54 E-05$ & 0.02384057 & 0.05250249 & 0.00691099 & 3009103.64 \\
\hline
\end{tabular}




\begin{tabular}{|c|c|c|c|c|c|c|c|c|c|c|c|c|c|c|c|c|}
\hline Time (s) & $\begin{array}{c}\text { Coolant outlet } \\
\text { temperature }(\mathrm{K})\end{array}$ & $\begin{array}{c}\text { Peak Cladding } \\
\text { Temperature (K) }\end{array}$ & $\begin{array}{c}\text { Peak fuel } \\
\text { temperature (K) }\end{array}$ & $\begin{array}{c}\text { Average cladding } \\
\text { internal } \\
\text { temperature (K) }\end{array}$ & $\begin{array}{l}\text { Peak cumulative } \\
\text { damage factor }\end{array}$ & $\begin{array}{l}\text { Cladding interior } \\
\text { volume }\left(\mathrm{m}^{3}\right)\end{array}$ & 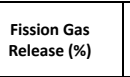 & $\begin{array}{l}\text { Fission gas } \\
\text { produced } \\
\text { (moles) }\end{array}$ & $\begin{array}{l}\text { Fission gas } \\
\text { released (moles) }\end{array}$ & Fuel volume $\left(\mathrm{m}^{3}\right)$ & $\begin{array}{l}\text { Plenum gas } \\
\text { volume }\left(\mathrm{m}^{3}\right)\end{array}$ & $\begin{array}{l}\text { Peak cladding } \\
\text { radial growth } \\
\text { (m) }\end{array}$ & $\begin{array}{l}\text { Peak fuel axial } \\
\text { growth }(m)\end{array}$ & $\begin{array}{c}\text { Peak burnup } \\
\text { (at\%) }\end{array}$ & $\begin{array}{l}\text { Peak cladding } \\
\text { hoop strain }\end{array}$ & $\begin{array}{l}\text { Plenum pressure } \\
(\mathrm{Pa})\end{array}$ \\
\hline 32896765 & 778.490606 & 802.493883 & 1012.099 & $\begin{array}{l}762.466483 \\
\end{array}$ & $2.42 \mathrm{E}-05$ & $2.49 \mathrm{E}-05$ & 73.2798107 & 0.00732955 & 0.00537108 & $1.24 \mathrm{E}-05$ & $1.25 \mathrm{E}-05$ & $2.54 \mathrm{E}-05$ & 0.0238412 & 0.0525212 & 0.00690516 & 3010288.37 \\
\hline 32900365 & \begin{tabular}{|l|l|}
792.161446 \\
\end{tabular} & 818.71412 & 1045.20885 & 774.512827 & $2.42 \mathrm{E}-05$ & $2.49 \mathrm{E}-05$ & 73.2802206 & 0.00733023 & 0.00537161 & $1.24 E-05$ & $1.25 E-05$ & $2.61 \mathrm{E}-05$ & 0.02395703 & 0.05252607 & 0.00710278 & 3059542.68 \\
\hline 32914893.9 & \begin{tabular}{|l|l|}
792.161446 \\
\end{tabular} & 818.713856 & 1045.20092 & 774.512687 & $2.42 \mathrm{E}-05$ & $2.49 \mathrm{E}-05$ & 73.2819531 & 0.00733311 & 0.00537384 & $1.24 \mathrm{E}-05$ & $1.25 \mathrm{E}-05$ & $2.61 \mathrm{E}-05$ & 0.02395712 & 0.05254667 & 0.00710315 & 3059899.97 \\
\hline 32933055.1 & 792.161446 & 818.713468 & 1045.19815 & 774.512373 & $2.42 \mathrm{E}-05$ & $2.49 \mathrm{E}-05$ & 73.2841169 & $\begin{array}{l}0.0073367 \\
\end{array}$ & 0.00537664 & $1.24 E-05$ & $1.25 E-05$ & $2.61 \mathrm{E}-05$ & 0.02395771 & 0.05257242 & 0.00710326 & 3061138.57 \\
\hline 32955756.6 & 792.161446 & 818.713058 & 1045.19679 & 774.511994 & $2.42 \mathrm{E}-05$ & $2.49 \mathrm{E}-05$ & \begin{tabular}{|l|}
73.2868187 \\
\end{tabular} & 0.00734119 & 0.00538013 & $1.24 \mathrm{E}-05$ & $1.25 \mathrm{E}-05$ & $2.61 \mathrm{E}-05$ & 0.02395811 & 0.0526046 & 0.00710364 & 3062687.97 \\
\hline 32984133.4 & 792.161446 & 818.712574 & 1045.19534 & 774.511524 & $2.42 \mathrm{E}-05$ & $2.49 \mathrm{E}-05$ & 73.2901912 & 0.00734681 & 0.00538449 & $1.24 E-05$ & $1.25 \mathrm{E}-05$ & $2.61 \mathrm{E}-05$ & 0.02395846 & 0.05264483 & 0.0071042 & 3064625.37 \\
\hline \begin{tabular}{|l|}
33019604.4 \\
\end{tabular} & \begin{tabular}{|l|l|}
792.161446 \\
\end{tabular} & \begin{tabular}{|l|l|}
818.71197 \\
\end{tabular} & 1045.19362 & 774.510936 & $2.43 \mathrm{E}-05$ & $2.49 \mathrm{E}-05$ & 73.2943997 & 0.00735382 & 0.00538994 & $1.24 \mathrm{E}-05$ & $1.25 E-05$ & $2.61 \mathrm{E}-05$ & 0.02395885 & 0.05269512 & 0.00710493 & 3067047.57 \\
\hline 33063943.2 & 792.161446 & 818.711215 & 1045.19148 & 774.510203 & $2.43 \mathrm{E}-05$ & $2.49 \mathrm{E}-05$ & 73.2996489 & 0.0073626 & 0.00539676 & $1.24 \mathrm{E}-05$ & $1.25 \mathrm{E}-05$ & $2.61 \mathrm{E}-05$ & 0.02395934 & 0.05275798 & 0.00710585 & 3070075.74 \\
\hline \begin{tabular}{|l|}
33119366.7 \\
\end{tabular} & \begin{tabular}{|l|l|}
792.161446 \\
\end{tabular} & 818.710281 & 1045.18897 & 774.509287 & $2.43 \mathrm{E}-05$ & $2.49 \mathrm{E}-05$ & $\begin{array}{l}73.306193 \\
\end{array}$ & 0.00737356 & 0.00540528 & $1.24 \mathrm{E}-05$ & $1.25 E-05$ & $2.61 \mathrm{E}-05$ & 0.02396193 & 0.05283656 & 0.00710713 & 3073861.07 \\
\hline 33188646.1 & 792.161446 & 818.709351 & 1045.17775 & 774.508054 & $2.43 \mathrm{E}-05$ & $2.49 \mathrm{E}-05$ & 73.3143457 & 0.00738727 & 0.00541593 & $1.24 \mathrm{E}-05$ & $1.25 \mathrm{E}-05$ & $2.61 \mathrm{E}-05$ & 0.02396495 & 0.05293478 & 0.00710858 & 3078590.34 \\
\hline 33275245.3 & 792.161446 & \begin{tabular}{|l|}
818.70877 \\
\end{tabular} & 1045.16971 & 774.506522 & $2.43 \mathrm{E}-05$ & $2.49 \mathrm{E}-05$ & 73.3244941 & 0.0074044 & 0.00542924 & $1.24 \mathrm{E}-05$ & $1.25 \mathrm{E}-05$ & $2.61 \mathrm{E}-05$ & 0.02397196 & 0.05305756 & 0.00711039 & 3084498.57 \\
\hline 33383494.3 & $\begin{array}{l}792.161446 \\
\end{array}$ & \begin{tabular}{l|l|}
818.708276 \\
\end{tabular} & 1045.16006 & 774.504602 & $2.43 \mathrm{E}-05$ & $2.49 \mathrm{E}-05$ & 73.3371138 & 0.00742582 & 0.00544588 & $1.24 E-05$ & $1.25 E-05$ & $2.61 \mathrm{E}-05$ & 0.02398188 & 0.05321103 & 0.00711266 & 3091872.99 \\
\hline 33518805.6 & $\begin{array}{l}92.161446 \\
\end{array}$ & 818.707926 & 1045.14841 & 774.502178 & $2.43 \mathrm{E}-05$ & $2.49 \mathrm{E}-05$ & 73.3527865 & 0.00745259 & 0.00546668 & $1.24 \mathrm{E}-05$ & $1.25 \mathrm{E}-05$ & $2.61 \mathrm{E}-05$ & 0.02399516 & 0.05340286 & 0.0071155 & 3101087.45 \\
\hline 33687944.7 & 792.161446 & \begin{tabular}{|l|l|}
818.707697 \\
\end{tabular} & 1045.13416 & 774.499126 & $2.43 \mathrm{E}-05$ & $2.49 \mathrm{E}-05$ & 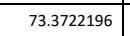 & 0.00748606 & 0.00549269 & $1.24 E-05$ & $1.25 \mathrm{E}-05$ & $2.61 \mathrm{E}-05$ & 0.02401235 & 0.05364266 & 0.00711907 & 3112598.99 \\
\hline 33899368.5 & 792.161446 & 818.707599 & 1045.11663 & 774.495342 & $2.43 \mathrm{E}-05$ & $2.49 \mathrm{E}-05$ & \begin{tabular}{|l}
7.3962681 \\
\end{tabular} & 0.00752789 & $\begin{array}{l}0.00552519 \\
\end{array}$ & $1.24 E-05$ & $1.25 E-05$ & $2.62 \mathrm{E}-05$ & $\begin{array}{l}0.02403453 \\
\end{array}$ & 0.05394241 & 0.00712355 & 3126962.24 \\
\hline 34163648.3 & 792.161446 & 818.707955 & 1045.09545 & 774.490574 & $2.44 \mathrm{E}-05$ & $2.49 \mathrm{E}-05$ & 73.4259554 & 0.00758018 & 0.00556582 & $1.24 \mathrm{E}-05$ & $1.25 \mathrm{E}-05$ & $2.62 \mathrm{E}-05$ & 0.02406377 & 0.05431709 & 0.00712917 & 3144881.8 \\
\hline 34427928.2 & 792.161446 & 818.708875 & 1045.07512 & 774.48669 & $2.44 \mathrm{E}-05$ & $2.50 \mathrm{E}-05$ & 73.455236 & 0.00763246 & 0.00560644 & $1.24 E-05$ & $1.25 E-05$ & $2.62 \mathrm{E}-05$ & 0.0240947 & 0.05469177 & 0.00713502 & 3166959.18 \\
\hline 34692208 & 792.161446 & 818.708905 & 1045.05544 & 774.482803 & $2.44 \mathrm{E}-05$ & $2.50 \mathrm{E}-05$ & 73.4841181 & 0.00768475 & 0.00564707 & $1.25 E-05$ & $1.25 \mathrm{E}-05$ & $2.62 \mathrm{E}-05$ & $\begin{array}{l}.02412684 \\
\end{array}$ & 0.05506645 & 0.0071409 & 3189022.57 \\
\hline 34956487.8 & 792.161446 & 818.709551 & 1045.03621 & 774.47887 & $2.45 \mathrm{E}-05$ & $2.50 \mathrm{E}-05$ & 73.5126098 & 0.00773704 & 0.0056877 & $1.25 \mathrm{E}-05$ & $1.25 \mathrm{E}-05$ & $2.62 \mathrm{E}-05$ & 0.02415974 & 0.05544113 & 0.00714682 & 3211066.16 \\
\hline 35220767.6 & $\begin{array}{l}792.161446 \\
\end{array}$ & 818.706714 & 1045.01737 & 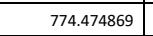 & $2.45 \mathrm{E}-05$ & $2.50 \mathrm{E}-05$ & 73.540719 & 0.00778933 & 0.00572833 & $1.25 E-05$ & $1.25 E-05$ & $2.63 \mathrm{E}-05$ & 0.02419324 & 0.05581581 & 0.00724817 & 3233088.13 \\
\hline 35485047.4 & 792.161446 & 818.70043 & 1044.99998 & 774.5114 & $2.45 \mathrm{E}-05$ & $2.50 \mathrm{E}-05$ & 73.5684534 & 0.00784162 & 0.00576896 & $1.25 E-05$ & $1.25 E-05$ & $2.64 \mathrm{E}-05$ & 0.02422615 & 0.0561905 & 0.00739463 & 3255246.23 \\
\hline 35749327.2 & 792.161446 & 818.694553 & 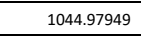 & 774.507567 & $2.46 \mathrm{E}-05$ & $2.50 \mathrm{E}-05$ & \begin{tabular}{|l}
73.5958203 \\
\end{tabular} & 0.00789391 & 0.00580958 & $1.25 E-05$ & $1.25 E-05$ & $2.69 \mathrm{E}-05$ & 0.02425983 & 0.05656518 & 0.00754674 & 3277211.85 \\
\hline 36013607.1 & 792.161446 & 818.689175 & 1044.92458 & 774.503816 & $2.46 \mathrm{E}-05$ & $2.50 \mathrm{E}-05$ & 73.622827 & 0.00794619 & 0.00585021 & $1.25 E-05$ & $1.25 E-05$ & $2.74 \mathrm{E}-05$ & 0.02429389 & 0.05693986 & 0.00770655 & 3299150.1 \\
\hline 36277886.9 & 792.161446 & 818.684547 & 1044.82896 & 774.500127 & $2.46 \mathrm{E}-05$ & $2.50 \mathrm{E}-05$ & 73.6494807 & 0.00799848 & 0.00589084 & $1.25 \mathrm{E}-05$ & $1.25 \mathrm{E}-05$ & $2.79 \mathrm{E}-05$ & 0.02432975 & 0.05731454 & 0.00787217 & 3321084.8 \\
\hline 36542166.7 & 792.161446 & 818.680709 & $\begin{array}{l}044.71598 \\
\end{array}$ & 774.496325 & $2.47 \mathrm{E}-05$ & $2.50 \mathrm{E}-05$ & 73.6757881 & 0.00805077 & 0.00593147 & $1.25 \mathrm{E}-05$ & $1.25 \mathrm{E}-05$ & $2.84 \mathrm{E}-05$ & 0.02436669 & 0.05768922 & 0.00804168 & 3343007.9 \\
\hline 36806446.5 & 792.161446 & 818.67769 & 1044.60034 & 774.492388 & $2.47 \mathrm{E}-05$ & $2.50 \mathrm{E}-05$ & 73.701756 & 0.00810306 & 0.0059721 & $1.25 E-05$ & $1.25 E-05$ & $2.89 \mathrm{E}-05$ & 0.0244041 & 0.0580639 & 0.00821455 & 3364920.71 \\
\hline 37070726.3 & 792.161446 & 818.675556 & 1044.48591 & 774.488338 & $2.47 \mathrm{E}-05$ & $2.50 \mathrm{E}-05$ & 73.727391 & 0.00815535 & 0.00601272 & $1.25 E-05$ & $1.25 E-05$ & $2.94 \mathrm{E}-05$ & 0.02444185 & 0.05843859 & 0.00839046 & 3386830.21 \\
\hline 37335006.1 & \begin{tabular}{|l}
792.161446 \\
\end{tabular} & 818.67411 & 1044.3731 & 774.484152 & $2.48 \mathrm{E}-05$ & $2.50 \mathrm{E}-05$ & 73.7526993 & 0.00820764 & 0.00605335 & $1.25 E-05$ & $1.25 \mathrm{E}-05$ & $3.00 \mathrm{E}-05$ & 0.02447992 & 0.05881327 & 0.00856914 & 3408716.76 \\
\hline 37599286 & 792.161446 & 818.673363 & 1044.26321 & 774.479848 & $2.48 \mathrm{E}-05$ & $2.50 \mathrm{E}-05$ & 73.7776872 & 0.00825992 & 0.00609398 & $1.25 E-05$ & $1.25 \mathrm{E}-05$ & $3.05 E-05$ & 0.02451814 & 0.05918795 & 0.00875075 & 3430577.46 \\
\hline 37929635.7 & $\begin{array}{l}792.161446 \\
\end{array}$ & 818.673656 & 1044.13185 & 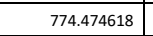 & $2.48 \mathrm{E}-05$ & $2.50 \mathrm{E}-05$ & 73.8084806 & 0.00832528 & 0.00614477 & $1.25 E-05$ & $1.25 E-05$ & $3.12 \mathrm{E}-05$ & 0.0245665 & 0.0596563 & 0.00898193 & 3452643.39 \\
\hline 38342572.9 & 792.161446 & 818.67569 & 1043.97852 & 774.467867 & $2.49 \mathrm{E}-05$ & $2.50 \mathrm{E}-05$ & 73.8462991 & 0.00840699 & 0.00620825 & $1.25 \mathrm{E}-05$ & $1.24 \mathrm{E}-05$ & $3.21 \mathrm{E}-05$ & 0.02462819 & 0.06024174 & 0.009277 & 3480121.62 \\
\hline 38755510.2 & 792.161446 & 818.67938 & 1043.85552 & \begin{tabular}{|l}
774.46086 \\
\end{tabular} & $2.50 \mathrm{E}-05$ & $2.50 \mathrm{E}-05$ & 73.8833896 & 0.00848869 & 0.00627173 & $1.25 \mathrm{E}-05$ & $1.24 \mathrm{E}-05$ & $3.31 \mathrm{E}-05$ & 0.02469021 & 0.06082718 & 0.00958112 & 3514040.64 \\
\hline 39271681.7 & 792.161446 & 818.685741 & 1043.70521 & 774.452138 & $2.50 \mathrm{E}-05$ & $2.50 \mathrm{E}-05$ & 73.9287607 & 0.00859081 & 0.00635108 & $1.25 E-05$ & $1.24 \mathrm{E}-05$ & $3.43 E-05$ & 0.02476913 & 0.06155898 & 0.00997162 & 3548245.21 \\
\hline 39574695 & 791.593043 & 818.103286 & 1043.29055 & $\begin{array}{l}774.009164 \\
\end{array}$ & $2.51 \mathrm{E}-05$ & $2.50 \mathrm{E}-05$ & \begin{tabular}{|l}
73.9548963 \\
\end{tabular} & 0.00865076 & 0.00639766 & $1.26 \mathrm{E}-05$ & $1.24 \mathrm{E}-05$ & $3.50 \mathrm{E}-05$ & \begin{tabular}{|l|}
0.02481101 \\
\end{tabular} & 0.06198858 & 0.01020039 & 3588129.47 \\
\hline 39578295 & 792.643859 & 819.350508 & 1045.82895 & 774.935415 & $2.51 \mathrm{E}-05$ & $2.50 \mathrm{E}-05$ & 73.9552057 & 0.00865148 & 0.00639822 & $1.26 \mathrm{E}-05$ & $1.24 \mathrm{E}-05$ & $3.51 \mathrm{E}-05$ & $\begin{array}{l}0.02481825 \\
\end{array}$ & 0.0619937 & 0.01022983 & 3616463.52 \\
\hline 39881308.3 & $\begin{array}{l}792.643859 \\
\end{array}$ & 819.344551 & 1045.75592 & 774.929799 & $2.51 \mathrm{E}-05$ & $2.50 \mathrm{E}-05$ & 73.9811579 & 0.00871185 & 0.00644513 & $1.26 \mathrm{E}-05$ & $1.24 E-05$ & $3.58 \mathrm{E}-05$ & 0.02486301 & 0.06242633 & 0.01046903 & 3617535.43 \\
\hline 40184321.7 & $\begin{array}{l}792.643859 \\
\end{array}$ & 819.337762 & 1045.67221 & 774.924136 & $2.52 \mathrm{E}-05$ & $2.50 \mathrm{E}-05$ & 74.0067528 & 0.00877223 & 0.00649204 & $1.26 \mathrm{E}-05$ & $1.24 E-05$ & $3.65 E-05$ & 0.02490916 & 0.06285895 & 0.01070931 & 3642627.4 \\
\hline 40487335 & 792.643859 & 819.331466 & 1045.59701 & 774.91842 & 3.15E-05 & $2.50 \mathrm{E}-05$ & 74.0319979 & 0.0088326 & 0.00653895 & $1.26 \mathrm{E}-05$ & $1.24 \mathrm{E}-05$ & $3.73 \mathrm{E}-05$ & 0.02495326 & 0.06329158 & 0.01095217 & 3667696.92 \\
\hline 40790348.3 & $\begin{array}{l}792.643859 \\
\end{array}$ & 819.325718 & 1045.51582 & 774.913422 & $4.46 \mathrm{E}-05$ & $2.50 \mathrm{E}-05$ & \begin{tabular}{|l|l}
74.0569001 \\
\end{tabular} & 0.00889298 & 0.00658586 & $1.26 \mathrm{E}-05$ & $1.24 E-05$ & $3.80 \mathrm{E}-05$ & 0.02499825 & 0.06372421 & 0.01119746 & $\begin{array}{l}6992792.37 \\
\end{array}$ \\
\hline 41093361.7 & 792.643859 & 819.320651 & 1045.44636 & 774.908355 & $6.15 \mathrm{E}-05$ & $2.50 \mathrm{E}-05$ & 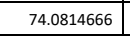 & 0.00895335 & 0.00663277 & $1.26 \mathrm{E}-05$ & $1.24 E-05$ & $3.88 \mathrm{E}-05$ & 0.02504266 & 0.06415683 & 0.01144502 & 3717864.17 \\
\hline 41396375 & 792.643859 & 819.316379 & 1045.37728 & 774.903046 & $8.34 \mathrm{E}-05$ & $2.50 \mathrm{E}-05$ & 74.1057039 & 0.00901373 & 0.00667969 & $1.26 \mathrm{E}-05$ & $1.24 \mathrm{E}-05$ & $3.966-05$ & 0.02508584 & 0.06458946 & 0.01169474 & 3742928.61 \\
\hline 41699388.3 & 792.643859 & 819.31303 & 1045.30604 & $\begin{array}{l}774.897525 \\
\end{array}$ & 0.00011102 & $2.50 \mathrm{E}-05$ & 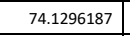 & 0.0090741 & 0.0067266 & $1.26 \mathrm{E}-05$ & $1.24 \mathrm{E}-05$ & $4.03 E-05$ & 0.02513031 & 0.06502209 & 0.01194647 & 3768009.51 \\
\hline 42002401.7 & 792.643859 & 820.742994 & 1045.23091 & 774.944852 & 0.00014554 & $2.50 \mathrm{E}-05$ & 74.1532173 & 0.00913448 & 0.00677351 & $1.26 \mathrm{E}-05$ & $1.24 \mathrm{E}-05$ & $4.11 \mathrm{E}-05$ & 0.02517427 & 0.06545472 & 0.01220008 & 3793360.87 \\
\hline 42194062 & \begin{tabular}{|l}
793.426091 \\
\end{tabular} & 821.618239 & 1045.65926 & 775.5439 & 0.00017216 & $2.50 \mathrm{E}-05$ & 74.1679834 & 0.00917267 & 0.00680318 & $1.26 E-05$ & $1.24 E-05$ & $4.16 \mathrm{E}-05$ & 0.02520205 & 0.06572836 & 0.01236606 & 3820997.45 \\
\hline 42197662 & 775.547815 & 800.14779 & \begin{tabular}{|l|l|}
002.55392 \\
\end{tabular} & 759.797226 & 0.00017216 & $2.50 \mathrm{E}-05$ & 74.1682432 & 0.00917334 & 0.00680371 & $1.26 \mathrm{E}-05$ & $1.24 E-05$ & $4.01 E-05$ & 0.02508516 & 0.06573319 & 0.01190698 & 3760017.57 \\
\hline 42389322.3 & 775.547815 & 800.26691 & $\begin{array}{l}1002.42951 \\
\end{array}$ & 759.794601 & 0.00017243 & $2.50 \mathrm{E}-05$ & 74.1811534 & 0.009207 & 0.00682986 & $1.26 \mathrm{E}-05$ & $1.24 \mathrm{E}-05$ & $4.05 E-05$ & 0.02513342 & 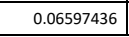 & 0.01201912 & 3760866.52 \\
\hline
\end{tabular}




\begin{tabular}{|c|c|c|c|c|c|c|c|c|c|c|c|c|c|c|c|c|}
\hline Time (s) & $\begin{array}{c}\text { Coolant outlet } \\
\text { temperature }(K)\end{array}$ & $\begin{array}{l}\text { Peak Cladding } \\
\text { Temperature (K) }\end{array}$ & $\begin{array}{c}\text { Peak fuel } \\
\text { temperature (K) }\end{array}$ & $\begin{array}{c}\text { Average cladding } \\
\text { internal } \\
\text { temperature (K) }\end{array}$ & \begin{tabular}{|l|}
$\begin{array}{l}\text { Peak cumulative } \\
\text { damage factor }\end{array}$ \\
\end{tabular} & $\begin{array}{l}\text { Cladding interior } \\
\text { volume }\left(\mathrm{m}^{3}\right)\end{array}$ & $\begin{array}{l}\text { Fission Gas } \\
\text { Release (\%) }\end{array}$ & $\begin{array}{c}\text { Fission gas } \\
\text { produced } \\
\text { (moles) }\end{array}$ & $\begin{array}{c}\text { Fission gas } \\
\text { released (moles) }\end{array}$ & Fuel volume $\left(\mathrm{m}^{3}\right)$ & $\begin{array}{l}\text { Plenum gas } \\
\text { volume }\left(m^{3}\right)\end{array}$ & $\begin{array}{l}\text { Peak cladding } \\
\text { radial growth } \\
\text { (m) }\end{array}$ & $\begin{array}{l}\text { Peak fuel axial } \\
\text { growth }(m)\end{array}$ & $\begin{array}{c}\text { Peak burnup } \\
(\text { (at\%) }\end{array}$ & $\begin{array}{l}\text { Peak cladding } \\
\text { hoop strain }\end{array}$ & $\begin{array}{l}\text { Plenum pressure } \\
(\mathrm{Pa})\end{array}$ \\
\hline 42580982.7 & 775.547815 & 800.296822 & 1002.38575 & $\begin{array}{l}759.792046 \\
\end{array}$ & 0.00017279 & $2.50 \mathrm{E}-05$ & 74.1939696 & 0.00924065 & 0.00685601 & $1.26 \mathrm{E}-05$ & $1.24 \mathrm{E}-05$ & 4.09E-05 & 0.0251571 & 0.06621553 & 0.01215452 & 3774630.39 \\
\hline 42772643 & 775.547815 & 800.134807 & 1002.38168 & 759.78873 & \begin{tabular}{|l|l|}
0.00017328 \\
\end{tabular} & $2.50 \mathrm{E}-05$ & 74.2066927 & 0.00927431 & 0.00688216 & $1.26 \mathrm{E}-05$ & $1.24 \mathrm{E}-05$ & $4.13 \mathrm{E}-05$ & 0.02517283 & 0.06645669 & 0.01229145 & 3788291.86 \\
\hline 43012218.5 & 775.547815 & 799.984823 & 1002.37785 & 759.784856 & 0.00017414 & $2.50 \mathrm{E}-05$ & 74.2224673 & 0.00931638 & 0.00691485 & $1.26 \mathrm{E}-05$ & $1.24 E-05$ & $4.18 \mathrm{E}-05$ & 0.02518985 & 0.06675815 & 0.01246403 & 3801957.16 \\
\hline 43311687.7 & 775.547815 & 799.738359 & 1002.36464 & 759.779651 & 0.00017573 & $2.50 \mathrm{E}-05$ & 74.2419864 & 0.00936896 & 0.00695571 & $1.26 \mathrm{E}-05$ & $1.24 E-05$ & $4.25 E-05$ & 0.0252167 & 0.06713497 & $\begin{array}{l}0.01268173 \\
\end{array}$ & 3819105.14 \\
\hline 43611157 & 775.547815 & 799.485643 & 1002.3416 & \begin{tabular}{|l|l|}
759.774378 \\
\end{tabular} & 0.00017805 & $2.50 \mathrm{E}-05$ & 74.2612876 & 0.00942155 & 0.00699657 & $1.26 \mathrm{E}-05$ & $1.24 E-05$ & $4.32 E-05$ & 0.02524652 & 0.0675118 & 0.01290151 & 3840454.76 \\
\hline 43820808 & 773.656685 & 797.41357 & 1001.19124 & \begin{tabular}{|l|}
758.315586 \\
\end{tabular} & 0.00017983 & $2.50 \mathrm{E}-05$ & 74.2746722 & 0.00945837 & 0.00702517 & $1.26 \mathrm{E}-05$ & $1.24 \mathrm{E}-05$ & $4.36 E-05$ & 0.02526082 & 0.0677756 & 0.01304585 & 3854496.26 \\
\hline 43824408 & 796.925405 & 824.923329 & 1057.73744 & \begin{tabular}{|l|l|l}
778.842331 \\
\end{tabular} & 0.00019353 & $2.51 \mathrm{E}-05$ & 74.2749215 & 0.00945905 & 0.00702571 & $1.26 \mathrm{E}-05$ & $1.24 E-05$ & $4.57 \mathrm{E}-05$ & 0.02540301 & 0.06778053 & 0.01366791 & 3971460.74 \\
\hline 43895709 & 796.952581 & 824.740554 & 1057.73454 & 778.860827 & 0.00057956 & $2.51 \mathrm{E}-05$ & 74.2802528 & 0.0094738 & 0.00703716 & $1.26 \mathrm{E}-05$ & $1.24 \mathrm{E}-05$ & $4.60 \mathrm{E}-05$ & 0.02541892 & 0.0678862 & 0.01376149 & 3972131.87 \\
\hline 43899309 & 796.541987 & 824.243174 & 1056.74606 & $\begin{array}{l}778.498338 \\
\end{array}$ & 0.00059795 & $2.51 \mathrm{E}-05$ & 74.2805212 & 0.00947455 & 0.00703774 & $1.26 \mathrm{E}-05$ & $1.24 \mathrm{E}-05$ & $4.59 E-05$ & 0.02541764 & 0.06789153 & 0.01375527 & 3976323.61 \\
\hline 43903809 & 796.541987 & 824.226705 & 1056.74511 & 778.498137 & 0.00062091 & $2.51 \mathrm{E}-05$ & 74.2808561 & 0.00947547 & 0.00703846 & $1.26 \mathrm{E}-05$ & $1.24 \mathrm{E}-05$ & $4.60 \mathrm{E}-05$ & 0.02541953 & 0.06789818 & 0.01375927 & 3976654.05 \\
\hline 43909434 & 796.541987 & 824.208363 & 1056.74394 & 778.497908 & 0.00064958 & $2.51 E-05$ & 74.2812748 & 0.00947663 & 0.00703936 & $1.26 \mathrm{E}-05$ & $1.24 \mathrm{E}-05$ & $4.60 \mathrm{E}-05$ & 0.02542184 & 0.0679065 & 0.01376437 & 3977061.17 \\
\hline 43916465.3 & 796.541987 & 824.187584 & 1056.74246 & 778.497635 & 0.00068673 & $2.51 \mathrm{E}-05$ & 74.2817979 & 0.00947808 & 0.00704049 & $1.26 \mathrm{E}-05$ & $1.24 E-05$ & $4.60 \mathrm{E}-05$ & 0.02542464 & 0.06791689 & 0.0137708 & 3977566.99 \\
\hline 43925254.3 & 796.541987 & 824.161496 & 1056.74056 & 778.497292 & 0.00073336 & $2.51 \mathrm{E}-05$ & 74.2824516 & 0.0094799 & 0.0070419 & $1.27 E-05$ & $1.24 \mathrm{E}-05$ & $4.60 \mathrm{E}-05$ & 0.0254279 & 0.06792988 & 0.01377882 & 3978202.52 \\
\hline 43936240.6 & 796.541987 & 824.161675 & 1056.73806 & 778.496891 & 0.00079168 & $2.51 E-05$ & 74.2832684 & 0.00948216 & 0.00704366 & $1.27 \mathrm{E}-05$ & $1.24 \mathrm{E}-05$ & $4.60 \mathrm{E}-05$ & 0.02543172 & 0.06794612 & 0.01378885 & 3978988.82 \\
\hline 43949973.6 & 796.541987 & 824.162224 & 1056.73469 & 778.496417 & 0.00086463 & $2.51 E-05$ & 74.2842888 & 0.009485 & 0.00704586 & $1.27 \mathrm{E}-05$ & $1.24 E-05$ & $4.61 \mathrm{E}-05$ & 0.025436 & 0.06796642 & 0.01380137 & 3979968.47 \\
\hline 43967139.7 & 796.541987 & 824.162821 & 1056.72996 & 778.495857 & 0.0009559 & $2.51 E-05$ & 74.2855634 & 0.00948854 & 0.00704861 & $1.27 \mathrm{E}-05$ & $1.24 \mathrm{E}-05$ & $4.61 E-05$ & 0.02544082 & 0.06799179 & 0.01381701 & 3981186.79 \\
\hline 43988597.4 & 796.541987 & 824.163417 & 1056.72323 & 778.495204 & 0.00107013 & $2.51 \mathrm{E}-05$ & 74.2871554 & 0.00949296 & 0.00705205 & $1.27 E-05$ & $1.24 E-05$ & $4.62 E-05$ & 0.0254462 & 0.0680235 & 0.01383657 & 3982697.91 \\
\hline 44015419.5 & 796.541987 & 824.164095 & 1056.71375 & 778.494418 & 0.00121315 & $2.51 E-05$ & 74.2891433 & 0.0094985 & 0.00705635 & $1.27 E-05$ & $1.24 E-05$ & $4.63 E-05$ & 0.02545231 & 0.06806315 & 0.01386106 & 3984583.5 \\
\hline 44048947.1 & 796.541987 & 824.164768 & 1056.70094 & 778.493492 & 0.00139228 & $2.51 E-05$ & 74.2916249 & 0.00950541 & 0.00706172 & $1.27 \mathrm{E}-05$ & $1.24 \mathrm{E}-05$ & $4.64 E-05$ & 0.02545901 & 0.06811271 & 0.01389175 & 3986930.42 \\
\hline 44090856.6 & 796.541987 & 824.165401 & 1056.6846 & 778.492398 & 0.00161659 & $2.51 \mathrm{E}-05$ & 74.2947218 & 0.00951406 & 0.00706844 & $1.27 \mathrm{E}-05$ & $1.24 E-05$ & $4.65 E-05$ & 0.02546615 & 0.06817465 & 0.01393023 & 3989850.39 \\
\hline 44143243.5 & 796.541987 & 824.165662 & 1056.6649 & 778.491029 & 0.00189721 & $2.51 E-05$ & 74.2985851 & 0.00952486 & 0.00707684 & $1.27 \mathrm{E}-05$ & $1.24 E-05$ & $4.66 E-05$ & 0.02547569 & 0.06825208 & 0.0139785 & 3993501.2 \\
\hline 44208727.1 & 796.541987 & 824.165529 & 1056.64158 & 778.489337 & 0.00224725 & $2.51 \mathrm{E}-05$ & $\begin{array}{l}74.3034019 \\
\end{array}$ & 0.00953837 & 0.00708733 & $1.27 E-05$ & $1.24 E-05$ & $4.68 E-05$ & 0.02548735 & 0.06834887 & 0.01403906 & 3998064.98 \\
\hline 44290581.6 & 796.541987 & 824.165487 & 1056.61419 & 778.487259 & 0.00268154 & $2.51 E-05$ & 74.3094037 & 0.00955525 & 0.00710045 & $1.27 \mathrm{E}-05$ & $1.24 E-05$ & 4.70 E- 05 & 0.02550174 & 0.06846986 & 0.014115 & 4003769.03 \\
\hline 44392899.8 & 795.27503 & 822.880061 & 1055.82698 & 777.508954 & 0.00315632 & $2.51 \mathrm{E}-05$ & 74.3168762 & 0.00957636 & 0.00711685 & $1.27 \mathrm{E}-05$ & $1.24 E-05$ & $4.73 E-05$ & 0.02551551 & 0.06862109 & 0.0142028 & 4006047.71 \\
\hline 44401212 & 794.715373 & 822.312242 & 1055.49074 & 777.077686 & 0.00319289 & $2.51 \mathrm{E}-05$ & 74.3174818 & 0.00957807 & 0.00711818 & $1.27 \mathrm{E}-05$ & $1.24 \mathrm{E}-05$ & $4.73 \mathrm{E}-05$ & 0.02551537 & 0.06863338 & 0.01420736 & 4012474.35 \\
\hline 44404812 & 788.198453 & 814.554418 & 1039.63074 & 771.319194 & 0.00319559 & $2.51 \mathrm{E}-05$ & 74.3177384 & 0.0095788 & 0.00711875 & $1.27 \mathrm{E}-05$ & $1.24 E-05$ & $4.68 E-05$ & 0.02547584 & 0.06863859 & 0.01404407 & 3984117.35 \\
\hline 44413124.2 & 788.198453 & 814.554753 & 1039.62934 & 771.318831 & 0.00320167 & $2.51 \mathrm{E}-05$ & 74.3183178 & 0.00958044 & 0.00712002 & $1.27 \mathrm{E}-05$ & $1.24 E-05$ & $4.68 \mathrm{E}-05$ & 0.02548212 & 0.06865034 & 0.01404557 & 3984462.82 \\
\hline 44423514.5 & \begin{tabular}{|l}
788.198453 \\
\end{tabular} & 814.55525 & 1039.62708 & 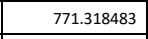 & 0.00320922 & $2.51 E-05$ & $\begin{array}{l}74.3190417 \\
\end{array}$ & 0.00958249 & 0.00712162 & $1.27 \mathrm{E}-05$ & $1.24 E-05$ & $4.68 \mathrm{E}-05$ & 0.02548749 & 0.06866504 & 0.01405288 & 3985190.43 \\
\hline 44436502.4 & 788.198453 & 814.555803 & 1039.62321 & 771.318076 & 0.00321867 & $2.51 E-05$ & 74.3199461 & 0.00958506 & 0.00712361 & $1.27 \mathrm{E}-05$ & $1.24 E-05$ & $4.68 E-05$ & 0.02549322 & 0.06868342 & 0.01406356 & 3986092.01 \\
\hline 44452737.2 & 788.198453 & 814.556346 & 1039.61676 & 771.317612 & 0.00323053 & $2.51 \mathrm{E}-05$ & 74.3210761 & 0.00958826 & 0.0071261 & $1.27 \mathrm{E}-05$ & $1.24 E-05$ & $4.69 E-05$ & 0.02549965 & 0.06870639 & 0.0140775 & 3987203.95 \\
\hline 44473030.7 & 788.198453 & 814.556935 & 1039.60585 & 771.317071 & 0.00324543 & $2.51 \mathrm{E}-05$ & 74.3224874 & 0.00959227 & 0.00712921 & $1.27 \mathrm{E}-05$ & $1.24 \mathrm{E}-05$ & $4.69 E-05$ & 0.02550591 & 0.0687351 & 0.01409513 & 3988587.92 \\
\hline 44498397.6 & 788.198453 & 814.557369 & 1039.59013 & $\begin{array}{l}771.316476 \\
\end{array}$ & 0.00326418 & $2.51 \mathrm{E}-05$ & 74.3242499 & 0.00959728 & 0.0071331 & $1.27 \mathrm{E}-05$ & $1.24 E-05$ & $4.70 \mathrm{E}-05$ & 0.02551197 & 0.06877098 & 0.01411722 & 3990293.24 \\
\hline 44530106.3 & 788.198453 & 814.55757 & 1039.57103 & 771.315804 & 0.0032878 & $2.51 E-05$ & 74.3264504 & 0.00960354 & 0.00713797 & $1.27 E-05$ & $1.24 E-05$ & $4.71 E-05$ & 0.02551753 & 0.06881584 & 0.01414488 & 3992401.2 \\
\hline 44569742.1 & 788.198453 & 814.557407 & 1039.55111 & 771.315032 & 0.00331756 & $2.51 \mathrm{E}-05$ & 74.3291971 & 0.00961136 & 0.00714405 & $1.27 \mathrm{E}-05$ & $1.24 E-05$ & $4.72 E-05$ & 0.02552202 & 0.06887191 & 0.0141795 & 3995004.23 \\
\hline 44619286.8 & 788.198453 & 814.556623 & 1039.53258 & 771.314164 & 0.00335507 & $2.51 E-05$ & $\begin{array}{r}74.3326241 \\
\end{array}$ & 0.00962114 & 0.00715165 & $1.27 \mathrm{E}-05$ & $1.24 E-05$ & $4.73 E-05$ & 0.02552467 & 0.06894201 & 0.01422283 & 3998214.04 \\
\hline 44650995.5 & 788.198453 & 814.556175 & 1039.5228 & 771.313605 & 0.00337918 & $2.51 E-05$ & 74.3348137 & 0.0096274 & 0.00715651 & $1.27 E-05$ & $1.24 E-05$ & $4.74 E-05$ & 0.02552664 & 0.06898686 & 0.01425061 & 4002187.34 \\
\hline 44682704.1 & 788.198453 & 814.555808 & 1039.51467 & 771.313035 & 0.00340336 & $2.51 \mathrm{E}-05$ & 74.3370005 & 0.00963366 & 0.00716138 & $1.27 \mathrm{E}-05$ & $1.24 E-05$ & $4.75 E-05$ & 0.02552889 & 0.06903172 & 0.01427839 & 4004751.39 \\
\hline 44722339.9 & 788.198453 & 814.555415 & 1039.50631 & 771.312313 & 0.00343366 & $2.51 E-05$ & 74.33973 & 0.00964149 & 0.00716746 & $1.27 \mathrm{E}-05$ & $1.24 E-05$ & $4.76 E-05$ & 0.02553227 & 0.0690878 & 0.01431313 & 4007330.39 \\
\hline 44771884.7 & 788.198453 & 814.555033 & 1039.49768 & 771.311397 & 0.00347159 & $2.51 E-05$ & 74.3431356 & 0.00965127 & 0.00717506 & $1.27 \mathrm{E}-05$ & $1.24 E-05$ & $4.77 E-05$ & 0.02553696 & 0.06915789 & $\begin{array}{l}0.0143566 \\
\end{array}$ & 4010562.04 \\
\hline 44833815.6 & 788.198453 & 814.554713 & 1039.48855 & 771.31023 & 0.00351907 & $2.51 E-05$ & 74.347383 & 0.0096635 & 0.00718456 & $1.27 \mathrm{E}-05$ & $1.24 E-05$ & $4.79 E-05$ & 0.02554358 & 0.0692455 & 0.01441097 & 4014608.18 \\
\hline 44911229.3 & 788.198453 & 814.554423 & 1039.47834 & 771.308763 & 0.00357861 & $2.51 E-05$ & 74.3526771 & 0.00967878 & 0.00719643 & $1.27 \mathrm{E}-05$ & $1.24 E-05$ & $4.81 E-05$ & 0.02555218 & 0.06935502 & 0.01447898 & 4019673.65 \\
\hline 45007996.4 & 788.198453 & 814.554171 & 1039.46609 & 771.306936 & 0.0036537 & $2.51 \mathrm{E}-05$ & 74.3592713 & 0.00969789 & 0.00721128 & $1.27 \mathrm{E}-05$ & $1.24 E-05$ & $4.84 \mathrm{E}-05$ & 0.02556329 & 0.06949192 & 0.01456404 & 4026007.69 \\
\hline 45128955.3 & 788.198453 & 814.554127 & 1039.45053 & 771.304639 & 0.00374944 & $2.51 E-05$ & 74.3674775 & 0.00972177 & 0.00722983 & $1.27 E-05$ & $1.24 E-05$ & $4.87 \mathrm{E}-05$ & 0.0255782 & 0.06966304 & 0.01467041 & 4033936.81 \\
\hline 45280153.9 & 788.198453 & 814.554343 & 1039.4298 & 771.301793 & $\begin{array}{l}0.0038737 \\
\end{array}$ & $2.51 \mathrm{E}-05$ & 74.3776789 & 0.00975162 & 0.00725303 & $1.27 E-05$ & $1.24 E-05$ & $4.91 E-05$ & 0.02559738 & 0.06987694 & 0.01480343 & 4043857.59 \\
\hline 45431352.4 & 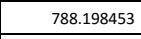 & 814.5548 & \begin{tabular}{|l|l|l}
1039.4079 \\
\end{tabular} & \begin{tabular}{|l}
771.298981 \\
\end{tabular} & 0.00400396 & $2.51 E-05$ & $\begin{array}{l}74.3878179 \\
\end{array}$ & 0.00978147 & 0.00727622 & $1.27 \mathrm{E}-05$ & $1.24 E-05$ & $4.95 E-05$ & 0.02561737 & 0.07009085 & 0.01493654 & 4056181.6 \\
\hline 45582551 & 788.198453 & 814.555284 & 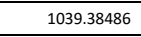 & 771.296213 & $\begin{array}{l}0.00414142 \\
\end{array}$ & $2.51 \mathrm{E}-05$ & 74.3978953 & 0.00981132 & 0.00729942 & $1.27 \mathrm{E}-05$ & $1.24 E-05$ & $4.99 E-05$ & 0.02563718 & 0.07030475 & 0.01506972 & 4068506.85 \\
\hline
\end{tabular}




\begin{tabular}{|c|c|c|c|c|c|c|c|c|c|c|c|c|c|c|c|c|}
\hline Time (s) & $\begin{array}{c}\text { Coolant outlet } \\
\text { temperature }(K)\end{array}$ & $\begin{array}{l}\text { Peak Cladding } \\
\text { Temperature (K) }\end{array}$ & $\begin{array}{c}\text { Peak fuel } \\
\text { temperature (K) }\end{array}$ & $\begin{array}{c}\text { Average cladding } \\
\text { internal } \\
\text { temperature (K) }\end{array}$ & $\begin{array}{l}\text { Peak cumulative } \\
\text { damage factor }\end{array}$ & $\begin{array}{l}\text { Cladding interior } \\
\text { volume }\left(\mathrm{m}^{3}\right)\end{array}$ & $\begin{array}{l}\text { Fission Gas } \\
\text { Release (\%) }\end{array}$ & $\begin{array}{c}\text { Fission gas } \\
\text { produced } \\
\text { (moles) }\end{array}$ & $\begin{array}{c}\text { Fission gas } \\
\text { released (moles) }\end{array}$ & Fuel volume $\left(\mathrm{m}^{3}\right)$ & $\begin{array}{l}\text { Plenum gas } \\
\text { volume }\left(m^{3}\right)\end{array}$ & $\begin{array}{l}\text { Peak cladding } \\
\text { radial growth } \\
\text { (m) }\end{array}$ & $\begin{array}{l}\text { Peak fuel axial } \\
\text { growth }(m)\end{array}$ & $\begin{array}{c}\text { Peak burnup } \\
(\text { (at\%) }\end{array}$ & $\begin{array}{l}\text { Peak cladding } \\
\text { hoop strain }\end{array}$ & $\begin{array}{l}\text { Plenum pressure } \\
(\mathrm{Pa})\end{array}$ \\
\hline 45733749.6 & 788.198453 & 814.556036 & 1039.36104 & $\begin{array}{l}771.293489 \\
\end{array}$ & 0.00428707 & $2.51 \mathrm{E}-05$ & 74.4079115 & 0.00984117 & 0.00732261 & $1.27 \mathrm{E}-05$ & $1.24 \mathrm{E}-05$ & 5.03E-05 & 0.02565712 & 0.07051865 & 0.01520301 & 4080839.18 \\
\hline 45884948.2 & 788.198453 & 814.556891 & 1039.33669 & 771.290803 & 0.00444172 & $2.51 \mathrm{E}-05$ & 74.4178671 & 0.00987102 & 0.0073458 & $1.27 E-05$ & $1.24 \mathrm{E}-05$ & $5.07 E-05$ & 0.02567711 & 0.07073256 & 0.01533641 & 4093168.92 \\
\hline 46036146.8 & 788.198453 & 814.557954 & 1039.31196 & $\begin{array}{l}771.288164 \\
\end{array}$ & 0.00460605 & $2.51 \mathrm{E}-05$ & 74.4277627 & 0.00990087 & 0.007369 & $1.27 E-05$ & $1.24 E-05$ & $5.11 E-05$ & 0.02569729 & 0.07094646 & 0.01546995 & 4105502.37 \\
\hline 46187345.4 & 788.198453 & 814.55931 & 1039.28676 & 771.285578 & 0.00478062 & $2.51 \mathrm{E}-05$ & 74.4375988 & 0.00993072 & 0.00739219 & $1.27 E-05$ & $1.24 E-05$ & $5.15 \mathrm{E}-05$ & 0.02571815 & 0.07116036 & 0.01560362 & 4117846.56 \\
\hline 46338544 & 788.198453 & 814.560923 & 1039.2609 & 771.283035 & 0.00496584 & $2.51 \mathrm{E}-05$ & 74.447376 & 0.00996058 & 0.00741539 & $1.27 E-05$ & $1.24 E-05$ & $5.19 \mathrm{E}-05$ & 0.02573953 & 0.07137427 & 0.01573744 & 4130199.67 \\
\hline 46489742.6 & 788.198453 & 814.562415 & 1039.23468 & 771.280529 & 0.00516205 & $2.51 \mathrm{E}-05$ & $\begin{array}{l}74.4570947 \\
\end{array}$ & 0.00999043 & 0.00743858 & $1.27 \mathrm{E}-05$ & $1.24 \mathrm{E}-05$ & $5.24 E-05$ & 0.02576011 & 0.07158817 & $\begin{array}{l}0.0158714 \\
\end{array}$ & 4142543.27 \\
\hline 46640941.1 & 788.198453 & 814.564037 & $\begin{array}{l}1039.2083 \\
\end{array}$ & \begin{tabular}{|l|l|}
771.277304 \\
\end{tabular} & 0.00536944 & $2.51 \mathrm{E}-05$ & 74.4667555 & 0.01002028 & 0.00746178 & $1.27 \mathrm{E}-05$ & $1.24 E-05$ & $5.28 \mathrm{E}-05$ & 0.02578082 & 0.07180207 & 0.0160055 & 4154885.73 \\
\hline 46792139.7 & 788.198453 & 814.564738 & 1039.18173 & 771.273598 & 0.00558815 & $2.51 \mathrm{E}-05$ & 74.476359 & 0.01005013 & 0.00748497 & $1.27 \mathrm{E}-05$ & $1.24 \mathrm{E}-05$ & $5.32 E-05$ & 0.02580207 & 0.07201597 & 0.01613974 & 4167233.73 \\
\hline 46943338.3 & 788.198453 & 814.565363 & 1039.15538 & 771.270097 & 0.00581827 & $2.51 \mathrm{E}-05$ & 74.4859055 & 0.01007998 & 0.00750816 & $1.27 \mathrm{E}-05$ & $1.24 \mathrm{E}-05$ & $5.36 \mathrm{E}-05$ & 0.02582176 & 0.07222988 & $\begin{array}{l}0.0162741 \\
\end{array}$ & 4179561.09 \\
\hline 47094536.9 & 788.198453 & 814.566429 & 1039.1295 & 771.266694 & 0.00605986 & $2.51 \mathrm{E}-05$ & 74.4953957 & 0.01010983 & 0.00753136 & $1.27 \mathrm{E}-05$ & $1.24 \mathrm{E}-05$ & $5.40 \mathrm{E}-05$ & 0.02584147 & 0.07244378 & 0.01640858 & 4191887.22 \\
\hline 47245735.5 & 788.198453 & 814.565372 & 1039.10414 & 771.263417 & 0.006313 & $2.51 \mathrm{E}-05$ & 74.50483 & 0.01013968 & 0.00755455 & $1.27 \mathrm{E}-05$ & $1.24 \mathrm{E}-05$ & $5.44 E-05$ & 0.02586093 & 0.07265768 & 0.01654317 & 4204212.67 \\
\hline 47385472 & 789.115457 & 815.491725 & 1039.62847 & 771.966445 & 0.00657925 & $2.51 \mathrm{E}-05$ & 74.5134998 & 0.01016727 & 0.00757599 & $1.27 E-05$ & $1.24 E-05$ & $5.48 \mathrm{E}-05$ & 0.0258824 & 0.07285537 & 0.01667308 & 4220243.93 \\
\hline 47389072 & 786.135636 & 811.949614 & 1032.39071 & 769.337046 & 0.00658231 & $2.51 \mathrm{E}-05$ & 74.5137203 & 0.01016797 & 0.00757654 & $1.27 E-05$ & $1.24 E-05$ & $5.46 E-05$ & 0.0258654 & 0.07286041 & 0.01660054 & 4217327.4 \\
\hline 47528808.5 & 786.135636 & 811.946256 & 1032.36931 & 769.334042 & 0.00670673 & $2.51 E-05$ & 74.522167 & $\begin{array}{l}0.010195 \\
\end{array}$ & 0.00759753 & $1.27 \mathrm{E}-05$ & $1.24 \mathrm{E}-05$ & 5.49E- -05 & 0.02588652 & 0.07305408 & 0.01671702 & 4217921.97 \\
\hline 47703479.1 & 786.135636 & 811.942553 & 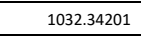 & 769.330588 & 0.0068755 & $2.51 E-05$ & 74.5326626 & 0.01022878 & 0.00762378 & $1.27 \mathrm{E}-05$ & $1.24 E-05$ & $5.54 \mathrm{E}-05$ & 0.02590566 & 0.07329615 & 0.01686798 & 4229092.23 \\
\hline 47878149.8 & 786.135636 & 811.939022 & 1032.3155 & $\begin{array}{ll}769.327088 \\
\end{array}$ & 0.007058 & $2.51 \mathrm{E}-05$ & 74.5430891 & 0.01026257 & 0.00765003 & $1.27 \mathrm{E}-05$ & $1.24 E-05$ & $5.59 \mathrm{E}-05$ & 0.02592632 & 0.07353823 & 0.01701933 & 4242993.6 \\
\hline 48052820.4 & 786.135636 & 811.935784 & 1032.28969 & 769.323567 & 0.00725466 & $2.51 \mathrm{E}-05$ & $\begin{array}{l}74.5534472 \\
\end{array}$ & 0.01029635 & 0.00767628 & $1.27 \mathrm{E}-05$ & $1.24 E-05$ & $5.63 E-05$ & 0.02594777 & 0.07378031 & 0.01717091 & 4256905.61 \\
\hline 48198548 & 787.622121 & 813.44014 & 1033.15663 & 770.465003 & 0.00745558 & $2.51 \mathrm{E}-05$ & $\begin{array}{l}74.562037 \\
\end{array}$ & 0.01032453 & 0.00769818 & $1.27 E-05$ & $1.24 E-05$ & $5.67 \mathrm{E}-05$ & 0.02597045 & 0.07398228 & 0.01730631 & 4276888.44 \\
\hline 48202148 & 644.15 & 644.173178 & 644.15362 & 644.150985 & 0.00745558 & 2.50 E- 05 & 74.5621428 & 0.01032488 & 0.00769845 & $1.26 \mathrm{E}-05$ & $1.24 \mathrm{E}-05$ & $4.68 E-05$ & 0.02444731 & 0.07398477 & 0.01435522 & 3585815.06 \\
\hline 48205748 & 305 & 305.051372 & 305.006476 & 305.002172 & 0.00745558 & $2.46 \mathrm{E}-05$ & 74.5621428 & 0.01032488 & 0.00769845 & $1.24 \mathrm{E}-05$ & $1.23 E-05$ & $3.00 E-05$ & 0.02229807 & 0.07398477 & 0.00984811 & 1713096.81 \\
\hline $\begin{array}{l}48209348 \\
\end{array}$ & 305 & 305.051372 & 305.006476 & 305.002172 & 0.00745558 & $2.46 \mathrm{E}-05$ & 74.5621428 & 0.01032488 & 0.00769845 & $1.24 \mathrm{E}-05$ & $1.23 E-05$ & $3.00 E-05$ & 0.02229807 & 0.07398477 & 0.00984811 & 1713096.81 \\
\hline 48210291.7 & 428.101999 & 434.55237 & 537.972754 & 424.311369 & 0.00745558 & $2.47 \mathrm{E}-05$ & 74.5621499 & 0.01032491 & 0.00769847 & $1.25 \mathrm{E}-05$ & $1.23 E-05$ & $3.52 E-05$ & 0.02339187 & 0.07398494 & 0.01124634 & 2382828.44 \\
\hline 48210539.1 & 460.719893 & 469.211658 & 587.731971 & 455.832723 & 0.00745558 & $2.48 E-05$ & 74.5621541 & 0.01032492 & 0.00769848 & $1.25 \mathrm{E}-05$ & $1.23 E-05$ & 3.66 E- 05 & 0.02364416 & 0.07398504 & 0.01161654 & 2558864.31 \\
\hline 48210665.8 & $\begin{array}{r}477.474612 \\
\end{array}$ & 486.876597 & 612.418898 & 472.002615 & 0.00745558 & $2.48 \mathrm{E}-05$ & 74.5621567 & 0.01032493 & 0.00769849 & $1.25 \mathrm{E}-05$ & $1.23 \mathrm{E}-05$ & $3.74 \mathrm{E}-05$ & 0.02376055 & 0.0739851 & 0.01182097 & 2648871.72 \\
\hline 48210792.4 & 494.265014 & 504.513607 & 636.681527 & 488.197447 & 0.00745558 & $2.48 \mathrm{E}-05$ & 74.5621595 & 0.01032494 & 0.0076985 & $1.25 \mathrm{E}-05$ & $1.23 \mathrm{E}-05$ & $3.85 \mathrm{E}-05$ & 0.02388025 & 0.07398517 & 0.01214374 & 2738561.65 \\
\hline 48210919.1 & 511.090063 & 522.059897 & 660.579604 & 504.41673 & 0.00745558 & $2.48 \mathrm{E}-05$ & 74.5621625 & 0.01032495 & 0.0076985 & $1.25 E-05$ & $1.23 E-05$ & $3.96 E-05$ & 0.02399786 & 0.07398524 & 0.01246368 & 2828409.44 \\
\hline 48211045.8 & 527.948602 & 539.655369 & 684.166792 & 520.661004 & 0.00745558 & $2.48 \mathrm{E}-05$ & 74.5621658 & 0.01032496 & 0.00769851 & $1.25 \mathrm{E}-05$ & $1.23 \mathrm{E}-05$ & $4.07 E-05$ & 0.02411374 & 0.07398531 & 0.01278162 & 2918245.08 \\
\hline 48211172.4 & 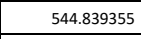 & 557.296008 & \begin{tabular}{|l}
707.634978 \\
\end{tabular} & 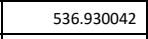 & 0.00745558 & $2.49 \mathrm{E}-05$ & 74.5621693 & 0.01032497 & 0.00769852 & $1.26 \mathrm{E}-05$ & $1.23 E-05$ & $4.18 \mathrm{E}-05$ & 0.02422815 & 0.0739854 & 0.01309786 & 3008074.49 \\
\hline 48211330.8 & 565.995987 & 579.406562 & 736.704743 & 557.300794 & 0.00745558 & $2.49 \mathrm{E}-05$ & 74.5621741 & 0.01032499 & 0.00769853 & $1.26 \mathrm{E}-05$ & $1.23 E-05$ & $4.32 E-05$ & 0.0243694 & 0.07398551 & 0.01349157 & 3120358.19 \\
\hline 48211489.1 & 587.19788 & 601.579109 & 765.496897 & 577.709227 & 0.00745558 & $2.49 \mathrm{E}-05$ & 74.5621793 & 0.010325 & 0.00769855 & $1.26 \mathrm{E}-05$ & $1.23 E-05$ & $4.45 E-05$ & 0.02450919 & 0.07398563 & 0.01388341 & 3232654.94 \\
\hline 48211647.4 & 608.441901 & 623.896087 & 794.067374 & 598.154599 & 0.00745558 & $2.49 \mathrm{E}-05$ & 74.5621849 & 0.01032502 & 0.00769856 & $1.26 \mathrm{E}-05$ & $1.23 \mathrm{E}-05$ & $4.59 E-05$ & 0.02464759 & 0.07398577 & 0.01427462 & 3344953.28 \\
\hline 48211805.7 & 629.724713 & 646.269364 & 822.457473 & 618.635992 & 0.00745558 & $2.49 \mathrm{E}-05$ & 74.5621909 & 0.01032504 & 0.00769858 & $1.26 \mathrm{E}-05$ & $1.23 \mathrm{E}-05$ & $4.73 \mathrm{E}-05$ & 0.02478453 & 0.07398591 & 0.01466673 & 3457257.61 \\
\hline 48211964.1 & 651.042791 & 668.696258 & 850.699009 & 639.152344 & 0.00745558 & $2.50 \mathrm{E}-05$ & 74.5621973 & 0.01032506 & 0.00769859 & $1.26 \mathrm{E}-05$ & $1.23 E-05$ & $4.87 \mathrm{E}-05$ & 0.02491925 & 0.07398606 & 0.01506476 & 3569562.28 \\
\hline 48212122.4 & 672.392437 & 6991.175787 & 878.827111 & 659.702424 & 0.00745558 & $2.50 \mathrm{E}-05$ & 74.5622041 & 0.01032508 & 0.00769861 & $1.27 \mathrm{E}-05$ & $1.23 E-05$ & $5.01 E-05$ & 0.02505051 & 0.07398622 & 0.01547189 & 3681863.47 \\
\hline 48212280.7 & 693.769792 & 713.706486 & 906.871015 & 680.284825 & 0.00745558 & $2.50 \mathrm{E}-05$ & 74.5622113 & 0.01032511 & 0.00769863 & $1.27 \mathrm{E}-05$ & $1.23 E-05$ & $5.16 \mathrm{E}-05$ & 0.02517708 & 0.07398639 & 0.01588934 & 3794157.03 \\
\hline 48212478.6 & 720.524345 & 741.938901 & 941.845088 & 706.055837 & 0.00745558 & $2.50 \mathrm{E}-05$ & 74.5622209 & 0.01032514 & 0.00769865 & $1.27 E-05$ & $1.24 E-05$ & $5.35 E-05$ & 0.02532666 & 0.07398661 & 0.01642901 & 3934490.14 \\
\hline 48212676.6 & 747.307834 & 770.243526 & 976.78261 & 731.871362 & 0.00745558 & $2.51 \mathrm{E}-05$ & 74.5622311 & 0.01032517 & 0.00769868 & $1.27 \mathrm{E}-05$ & $1.24 E-05$ & $5.54 E-05$ & 0.02546625 & 0.07398685 & 0.0169814 & 4074770.99 \\
\hline 48212874.5 & 774.111888 & 798.61424 & 1011.71033 & 757.727248 & 0.00745628 & $2.51 \mathrm{E}-05$ & 74.5622419 & 0.01032521 & 0.00769871 & $1.27 \mathrm{E}-05$ & $1.24 E-05$ & $5.73 E-05$ & 0.02559905 & 0.07398711 & 0.0175196 & 4214971.37 \\
\hline 48212948 & 784.07371 & 809.169778 & 1024.68942 & 767.342609 & 0.00745825 & $2.51 \mathrm{E}-05$ & $\begin{array}{l}74.562246 \\
\end{array}$ & 0.01032522 & 0.00769872 & $1.27 \mathrm{E}-05$ & $1.24 E-05$ & $5.80 \mathrm{E}-05$ & 0.02564737 & 0.07398721 & 0.01771783 & 4267028.01 \\
\hline 48213145.9 & 784.07371 & 809.174348 & 1024.69994 & 767.34683 & 0.00746427 & $2.51 \mathrm{E}-05$ & 74.5622574 & 0.01032526 & 0.00769875 & $1.27 \mathrm{E}-05$ & $1.24 E-05$ & $5.81 E-05$ & 0.0256353 & 0.07398747 & 0.01775164 & 4267045 \\
\hline 48213393.3 & $\begin{array}{l}784.07371 \\
\end{array}$ & 809.174374 & 1024.70285 & 767.346964 & 0.00747244 & $2.51 \mathrm{E}-05$ & 74.5622716 & 0.01032531 & 0.00769878 & $1.27 \mathrm{E}-05$ & $1.24 E-05$ & $5.82 \mathrm{E}-05$ & 0.02562693 & 0.07398781 & 0.01777433 & 4267061.65 \\
\hline 48213702.5 & 784.07371 & 809.174401 & 1024.7051 & 767.347065 & 0.00748327 & $2.51 \mathrm{E}-05$ & 74.5622893 & 0.01032536 & 0.00769883 & $1.27 \mathrm{E}-05$ & $1.24 E-05$ & $5.83 \mathrm{E}-05$ & 0.02562038 & 0.07398823 & 0.01779172 & 4267084.76 \\
\hline 48214089.1 & 784.07371 & 809.174426 & 1024.70695 & 767.347143 & 0.00749748 & $2.51 \mathrm{E}-05$ & 74.5623115 & 0.01032544 & 0.00769888 & $1.27 E-05$ & $1.24 E-05$ & $5.83 E-05$ & 0.02561495 & 0.07398875 & 0.01780629 & 4267115.11 \\
\hline 48214572.3 & $\begin{array}{r}784.07371 \\
\end{array}$ & 809.174448 & 1024.70848 & 767.347214 & 0.00751601 & $2.51 \mathrm{E}-05$ & 74.5623392 & 0.01032553 & 0.00769896 & $1.27 E-05$ & $1.24 E-05$ & $5.84 \mathrm{E}-05$ & 0.02561029 & 0.0739894 & 0.01781942 & 4267143.47 \\
\hline 48215176.3 & \begin{tabular}{|l}
784.07371 \\
\end{tabular} & 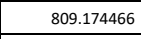 & 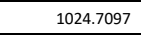 & 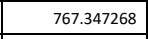 & $\begin{array}{l}0.00753999 \\
\end{array}$ & $2.51 \mathrm{E}-05$ & $\begin{array}{l}74.5623738 \\
\end{array}$ & 0.01032564 & 0.00769904 & $1.27 \mathrm{E}-05$ & $1.24 E-05$ & $5.84 \mathrm{E}-05$ & 0.02560622 & 0.07399022 & 0.01783079 & 4267185.04 \\
\hline 48215931.2 & 784.07371 & 809.174478 & 1024.71066 & 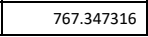 & $\begin{array}{c}0.00757096 \\
\end{array}$ & $2.51 \mathrm{E}-05$ & $\begin{array}{l}74.5624171 \\
\end{array}$ & 0.01032578 & 0.00769915 & $1.27 \mathrm{E}-05$ & $1.24 E-05$ & $5.85 \mathrm{E}-05$ & 0.02560262 & 0.07399124 & 0.01784163 & 4267232.93 \\
\hline
\end{tabular}




\begin{tabular}{|c|c|c|c|c|c|c|c|c|c|c|c|c|c|c|c|c|}
\hline Time (s) & $\begin{array}{c}\text { Coolant outlet } \\
\text { temperature }(\mathrm{K})\end{array}$ & $\begin{array}{l}\text { Peak Cladding } \\
\text { Temperature (K) }\end{array}$ & $\begin{array}{c}\text { Peak fuel } \\
\text { temperature (K) }\end{array}$ & $\begin{array}{c}\text { Average cladding } \\
\text { internal } \\
\text { temperature (K) }\end{array}$ & $\begin{array}{l}\text { Peak cumulative } \\
\text { damage factor }\end{array}$ & $\begin{array}{l}\text { Cladding interior } \\
\text { volume }\left(\mathrm{m}^{3}\right)\end{array}$ & $\begin{array}{l}\text { Fission Gas } \\
\text { Release (\%) }\end{array}$ & $\begin{array}{l}\text { Fission gas } \\
\text { produced } \\
\text { (moles) }\end{array}$ & $\begin{array}{c}\text { Fission gas } \\
\text { released (moles) }\end{array}$ & Fuel volume $\left(\mathrm{m}^{3}\right)$ & $\begin{array}{l}\text { Plenum gas } \\
\text { volume }\left(\mathrm{m}^{3}\right)\end{array}$ & $\begin{array}{l}\text { Peak cladding } \\
\text { radial growth } \\
\text { (m) }\end{array}$ & $\begin{array}{l}\text { Peak fuel axial } \\
\text { growth }(m)\end{array}$ & $\begin{array}{c}\text { Peak burnup } \\
\text { (at\%) }\end{array}$ & $\begin{array}{l}\text { Peak cladding } \\
\text { hoop strain }\end{array}$ & $\begin{array}{l}\text { Plenum pressure } \\
(\mathrm{Pa})\end{array}$ \\
\hline 48216874.9 & 784.07371 & 809.174482 & 1024.71134 & $\begin{array}{l}767.347353 \\
\end{array}$ & 0.00761077 & $2.51 \mathrm{E}-05$ & 74.5624712 & 0.01032596 & 0.00769929 & $1.27 \mathrm{E}-05$ & $1.24 \mathrm{E}-05$ & 5.85E-05 & 0.0255994 & 0.07399252 & 0.01785162 & 4267292.44 \\
\hline 48218054.6 & 784.07371 & 809.174477 & 1024.71178 & 767.347376 & 0.00766158 & $2.51 \mathrm{E}-05$ & 74.5625388 & 0.01032619 & 0.00769947 & $1.27 \mathrm{E}-05$ & $1.24 \mathrm{E}-05$ & $5.85 \mathrm{E}-05$ & 0.02559656 & 0.07399411 & 0.01786037 & 4267370.32 \\
\hline \begin{tabular}{|l|}
48219529.2 \\
\end{tabular} & \begin{tabular}{|l|l|}
784.07371 \\
\end{tabular} & 809.174464 & 1024.71196 & 767.34739 & 0.00772648 & $2.51 \mathrm{E}-05$ & 74.5626233 & 0.01032646 & 0.00769968 & $1.27 \mathrm{E}-05$ & $1.24 \mathrm{E}-05$ & $5.86 \mathrm{E}-05$ & 0.02559413 & 0.0739961 & 0.01786928 & 4267460.99 \\
\hline \begin{tabular}{|l|l|}
48221372.4 \\
\end{tabular} & 784.07371 & 809.174442 & $\begin{array}{l}1024.7119 \\
\end{array}$ & \begin{tabular}{|l|l|}
767.347391 \\
\end{tabular} & 0.0078088 & $2.51 \mathrm{E}-05$ & 74.562729 & 0.01032681 & 0.00769995 & $1.27 \mathrm{E}-05$ & $1.24 \mathrm{E}-05$ & $5.86 \mathrm{E}-05$ & 0.02559211 & 0.0739986 & 0.0178769 & 4267576.38 \\
\hline 48223676.4 & 784.07371 & 809.174415 & 1024.71162 & \begin{tabular}{|l|l|}
767.347374 \\
\end{tabular} & 0.00791274 & $2.51 \mathrm{E}-05$ & 74.5628611 & 0.01032725 & 0.00770029 & $1.27 \mathrm{E}-05$ & $1.24 \mathrm{E}-05$ & $5.86 \mathrm{E}-05$ & 0.02559058 & 0.07400171 & 0.01788371 & 4267720.73 \\
\hline 48226556.4 & 784.07371 & 809.174381 & 1024.71114 & \begin{tabular}{|l|l|}
767.347338 \\
\end{tabular} & 0.00804344 & $2.51 \mathrm{E}-05$ & 74.5630261 & 0.01032779 & 0.00770071 & $1.27 \mathrm{E}-05$ & $1.24 \mathrm{E}-05$ & $5.86 \mathrm{E}-05$ & 0.02558957 & 0.07400561 & 0.01788974 & 4267901.04 \\
\hline \begin{tabular}{|l|}
48230156.4 \\
\end{tabular} & 784.07371 & 809.174353 & 1024.71046 & \begin{tabular}{|l|l|}
767.347282 \\
\end{tabular} & 0.00820713 & $2.51 \mathrm{E}-05$ & 74.5632324 & 0.01032847 & 0.00770124 & $1.27 \mathrm{E}-05$ & $1.24 \mathrm{E}-05$ & $5.87 \mathrm{E}-05$ & 0.02558912 & $\begin{array}{l}0.07401047 \\
\end{array}$ & 0.01789523 & 4268125.88 \\
\hline 48234656.4 & 784.07371 & 809.174323 & 1024.70961 & 767.3472 & 0.0084115 & $2.51 \mathrm{E}-05$ & 74.5634902 & 0.01032932 & 0.0077019 & $1.27 \mathrm{E}-05$ & $1.24 \mathrm{E}-05$ & $5.87 \mathrm{E}-05$ & 0.02558916 & 0.07401656 & 0.01790054 & 4268407.57 \\
\hline \begin{tabular}{|l|l|}
48240281.4 \\
\end{tabular} & 784.07371 & 809.174301 & 1024.70856 & \begin{tabular}{|l|l|}
767.347091 \\
\end{tabular} & 0.00866596 & $2.51 \mathrm{E}-05$ & 74.5638125 & 0.01033038 & 0.00770272 & $1.27 \mathrm{E}-05$ & $1.24 \mathrm{E}-05$ & $5.87 \mathrm{E}-05$ & 0.02558966 & 0.07402416 & 0.01790615 & 4268759.34 \\
\hline \begin{tabular}{|l|l|}
48247312.6 \\
\end{tabular} & 784.07371 & 809.174292 & 1024.70728 & 767.346956 & 0.00898204 & $2.51 \mathrm{E}-05$ & 74.5642151 & 0.01033171 & 0.00770376 & $1.27 \mathrm{E}-05$ & $1.24 \mathrm{E}-05$ & $5.87 \mathrm{E}-05$ & 0.02559044 & 0.07403367 & 0.01791256 & 4269197.89 \\
\hline 48256101.7 & 784.07371 & 809.17428 & $\begin{array}{l}1024.7057 \\
\end{array}$ & 767.346788 & 0.0093737 & $2.51 \mathrm{E}-05$ & 74.5647184 & 0.01033336 & 0.00770504 & $1.27 \mathrm{E}-05$ & $1.24 \mathrm{E}-05$ & $5.87 \mathrm{E}-05$ & 0.02559159 & 0.07404555 & 0.01792025 & 4269745.66 \\
\hline \begin{tabular}{|l|}
48267088 \\
\end{tabular} & 784.07371 & 809.174277 & 1024.70376 & \begin{tabular}{|l|l|}
767.346577 \\
\end{tabular} & 0.00985773 & $2.51 \mathrm{E}-05$ & 74.5653472 & 0.01033544 & 0.00770665 & $1.27 \mathrm{E}-05$ & $1.24 \mathrm{E}-05$ & $5.88 \mathrm{E}-05$ & 0.02559303 & 0.0740604 & 0.01792976 & 4270431.29 \\
\hline 48280820.9 & \begin{tabular}{|l|l|}
784.07371 \\
\end{tabular} & 809.174297 & 1024.70136 & 767.34631 & 0.0104541 & $2.51 \mathrm{E}-05$ & 74.5661328 & 0.01033803 & 0.00770867 & $1.27 \mathrm{E}-05$ & $1.24 \mathrm{E}-05$ & $5.88 \mathrm{E}-05$ & 0.02559493 & 0.07407897 & 0.01794169 & 4271289.69 \\
\hline 48297987 & 784.07371 & 809.174313 & 1024.69839 & 767.345981 & 0.01118612 & $2.51 \mathrm{E}-05$ & 74.5671143 & 0.01034127 & 0.00771118 & $1.27 \mathrm{E}-05$ & $1.24 \mathrm{E}-05$ & $5.88 \mathrm{E}-05$ & 0.02559721 & 0.07410218 & 0.0179567 & 4272361.73 \\
\hline 48319444.7 & 784.07371 & 809.174323 & 1024.69473 & 767.345575 & 0.01208048 & $2.51 \mathrm{E}-05$ & 74.5683403 & 0.01034532 & 0.00771433 & $1.27 \mathrm{E}-05$ & $1.24 \mathrm{E}-05$ & $5.89 \mathrm{E}-05$ & 0.02559995 & 0.07413119 & 0.01797562 & 4273700.88 \\
\hline 48346266.8 & 784.07371 & 809.174326 & 1024.69022 & 767.345072 & 0.01316689 & $2.51 \mathrm{E}-05$ & 74.5698715 & 0.01035038 & 0.00771826 & $1.27 \mathrm{E}-05$ & $1.24 E-05$ & $5.90 \mathrm{E}-05$ & 0.02560325 & 0.07416745 & 0.0179994 & 4275373.85 \\
\hline 48379794.4 & 784.07371 & 809.174345 & 1024.6847 & 767.344442 & 0.01447732 & $2.51 \mathrm{E}-05$ & 74.5717833 & 0.0103567 & 0.00772318 & $1.27 \mathrm{E}-05$ & $1.24 \mathrm{E}-05$ & $5.91 \mathrm{E}-05$ & \begin{tabular}{|l|}
0.02560729 \\
\end{tabular} & 0.07421278 & 0.0180293 & 4277465.02 \\
\hline 48421703.9 & 784.07371 & 809.174359 & 1024.67797 & 767.343663 & 0.01604479 & $2.51 \mathrm{E}-05$ & 74.5741698 & 0.01036461 & 0.00772932 & $1.27 \mathrm{E}-05$ & $1.24 \mathrm{E}-05$ & $5.92 \mathrm{E}-05$ & 0.02561227 & 0.07426944 & 0.01806684 & 4280077.49 \\
\hline $\begin{array}{l}48474090.8 \\
\end{array}$ & 784.07371 & 809.174427 & 1024.66979 & 767.342694 & 0.01790053 & $2.51 \mathrm{E}-05$ & 74.5771479 & 0.01037449 & 0.007737 & $1.27 \mathrm{E}-05$ & $1.24 \mathrm{E}-05$ & $5.93 \mathrm{E}-05$ & 0.02561843 & 0.07434026 & 0.01811389 & 4283346.57 \\
\hline 48539574.4 & 784.07371 & 809.174519 & 1024.65991 & 767.341488 & 0.02007159 & $2.51 \mathrm{E}-05$ & 74.5808625 & 0.01038685 & 0.0077466 & $1.27 \mathrm{E}-05$ & $1.24 E-05$ & $5.95 E-05$ & 0.02562633 & 0.0744288 & 0.01817283 & 4287430.54 \\
\hline 48621429 & 784.07371 & 809.174536 & $\begin{array}{l}1024.64796 \\
\end{array}$ & 767.340019 & 0.02257656 & $2.51 \mathrm{E}-05$ & 74.5854933 & 0.01040229 & 0.0077586 & $1.27 \mathrm{E}-05$ & $1.24 \mathrm{E}-05$ & $5.97 \mathrm{E}-05$ & 0.02563558 & $\begin{array}{l}.07453946 \\
\end{array}$ & 0.01824656 & 4292526.17 \\
\hline 48703283.5 & 784.07371 & 809.174804 & 1024.63635 & 767.338542 & 0.02489553 & $2.51 \mathrm{E}-05$ & 74.5901104 & 0.01041774 & 0.0077706 & $1.27 \mathrm{E}-05$ & $1.24 \mathrm{E}-05$ & $6.00 \mathrm{E}-05$ & 0.02564561 & 0.07465013 & 0.01832031 & 4298871.46 \\
\hline 48745193 & 784.07371 & 809.174861 & 1024.63047 & 767.337798 & 0.02603791 & $2.51 \mathrm{E}-05$ & 74.592469 & 0.01042564 & 0.00777674 & $1.27 \mathrm{E}-05$ & $1.24 E-05$ & $6.01 \mathrm{E}-05$ & 0.02565004 & 0.07470679 & 0.01835808 & 4305131.73 \\
\hline 48797579.9 & $\begin{array}{r}784.07371 \\
\end{array}$ & 809.174822 & $\begin{array}{l}1024.62325 \\
\end{array}$ & 767.336882 & 0.02739987 & $2.51 \mathrm{E}-05$ & 74.5954123 & 0.01043553 & 0.00778442 & $1.27 \mathrm{E}-05$ & $1.24 \mathrm{E}-05$ & $6.02 \mathrm{E}-05$ & 0.02565529 & 0.07477761 & 0.01840525 & 4308385.58 \\
\hline 48863063.5 & 784.07371 & 809.174834 & 1024.61438 & 767.335736 & 0.02900758 & $2.51 \mathrm{E}-05$ & 74.5990836 & 0.01044788 & 0.00779402 & $1.27 \mathrm{E}-05$ & $1.24 \mathrm{E}-05$ & \begin{tabular}{ll|}
$6.04 \mathrm{E}-05$ \\
\end{tabular} & 0.02566256 & 0.07486614 & 0.01846417 & 431245.2 \\
\hline 48944918 & 784.07371 & 809.175236 & 1024.60351 & 767.334287 & 0.03088393 & $2.51 \mathrm{E}-05$ & 74.6036605 & 0.01046332 & 0.00780602 & $1.27 \mathrm{E}-05$ & $1.24 \mathrm{E}-05$ & $6.06 \mathrm{E}-05$ & 0.02567261 & 0.07497681 & 0.01853776 & 4317566.78 \\
\hline 49026772.6 & 784.07371 & 809.175503 & 1024.59282 & 767.332865 & 0.03264102 & $2.51 \mathrm{E}-05$ & 74.6082239 & 0.01047877 & 0.00781802 & $1.27 \mathrm{E}-05$ & $1.24 \mathrm{E}-05$ & $6.08 \mathrm{E}-05$ & 0.02568206 & 0.07508747 & 0.01861129 & 4323908.97 \\
\hline 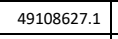 & \begin{tabular}{|l}
784.07371 \\
\end{tabular} & 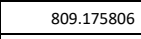 & \begin{tabular}{|l|l|}
1024.58227 \\
\end{tabular} & 767.331454 & \begin{tabular}{|l|l|}
0.03429113 \\
\end{tabular} & $2.51 E-05$ & 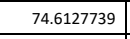 & 0.01049421 & 0.00783002 & $1.27 \mathrm{E}-05$ & $1.24 \mathrm{E}-05$ & $6.11 \mathrm{E}-05$ & 0.0256919 & 0.07519814 & 0.01868474 & 4330243.12 \\
\hline 49210945.2 & 784.07371 & 809.176314 & 1024.56926 & 767.329707 & 0.03620589 & $2.51 \mathrm{E}-05$ & 74.6184426 & 0.01051352 & 0.00784502 & $1.27 \mathrm{E}-05$ & $1.24 \mathrm{E}-05$ & $6.14 \mathrm{E}-05$ & 0.0257041 & 0.07533647 & 0.01877641 & 4336627.79 \\
\hline 49237767.3 & 784.07371 & 809.176454 & 1024.56584 & 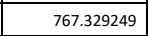 & 0.03669846 & $2.51 \mathrm{E}-05$ & 74.6199252 & 0.01051858 & 0.00784895 & $1.28 \mathrm{E}-05$ & $1.24 E-05$ & $6.14 \mathrm{E}-05$ & 0.0257072 & 0.07537273 & 0.01880048 & 4344407.67 \\
\hline 49264589.4 & 784.07371 & 809.176579 & 1024.56245 & 767.328794 & 0.03718182 & $2.51 \mathrm{E}-05$ & 74.6214063 & 0.01052364 & 0.00785289 & $1.28 \mathrm{E}-05$ & $1.24 \mathrm{E}-05$ & $6.15 \mathrm{E}-05$ & 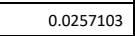 & 0.07540899 & 0.0188245 & 4346484.57 \\
\hline 49298117 & 784.07371 & 809.176726 & 1024.55821 & 767.328228 & 0.03777215 & $2.51 \mathrm{E}-05$ & 74.6232558 & 0.01052996 & 0.0078578 & $1.28 \mathrm{E}-05$ & $1.24 \mathrm{E}-05$ & $\begin{array}{ll}6.16 \mathrm{E}-05 \\
\end{array}$ & $\begin{array}{l}0.02571416 \\
\end{array}$ & 0.07545432 & 0.01885451 & 4348573.92 \\
\hline 49340026.6 & 784.07371 & 809.176956 & 1024.55294 & 767.327521 & 0.03848942 & $2.51 \mathrm{E}-05$ & 74.6255644 & 0.01053787 & 0.00786395 & $1.28 \mathrm{E}-05$ & $1.24 \mathrm{E}-05$ & $6.17 \mathrm{E}-05$ & 0.02571915 & 0.07551098 & 0.01889199 & 4351184.01 \\
\hline 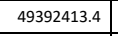 & $\begin{array}{r}784.07371 \\
\end{array}$ & 809.177231 & 1024.54638 & 767.326651 & 0.03935561 & $2.51 \mathrm{E}-05$ & 74.6284454 & 0.01054776 & 0.00787163 & $1.28 \mathrm{E}-05$ & $1.24 \mathrm{E}-05$ & $6.18 \mathrm{E}-05$ & \begin{tabular}{|l|}
0.02572476 \\
\end{tabular} & 0.07558181 & 0.0189388 & 4354450.05 \\
\hline 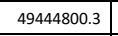 & $\begin{array}{l}784.07371 \\
\end{array}$ & 809.177372 & 1024.53984 & 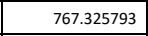 & 0.04019339 & $2.51 \mathrm{E}-05$ & $\begin{array}{l}74.631321 \\
\end{array}$ & 0.01055764 & 0.00787931 & $1.28 \mathrm{E}-05$ & $1.24 \mathrm{E}-05$ & $6.20 \mathrm{E}-05$ & $\begin{array}{l}0.02573037 \\
\end{array}$ & 0.07565263 & 0.01898559 & 4358497.14 \\
\hline 49510284 & 784.07371 & 809.177826 & $\begin{array}{l}1024.5317 \\
\end{array}$ & 767.324725 & 0.04119925 & $2.51 \mathrm{E}-05$ & \begin{tabular}{ll|}
74.6349079 \\
\end{tabular} & 0.01056999 & 0.00788891 & $1.28 \mathrm{E}-05$ & $1.24 E-05$ & $6.22 \mathrm{E}-05$ & 0.02573828 & 0.07574117 & 0.01904401 & 4362579.57 \\
\hline 49552193.5 & 784.07371 & 809.178137 & 1024.52651 & 767.324041 & 0.04182708 & $2.51 \mathrm{E}-05$ & 74.6371991 & 0.0105779 & 0.00789505 & $1.28 \mathrm{E}-05$ & $1.24 E-05$ & $6.23 \mathrm{E}-05$ & 0.02574329 & 0.07579783 & 0.0190814 & 4367607.92 \\
\hline 49594103 & 784.07371 & 809.178302 & 1024.52134 & 767.32337 & 0.04243972 & $2.51 \mathrm{E}-05$ & 74.6394869 & 0.01058581 & 0.00790119 & $1.28 \mathrm{E}-05$ & $1.24 \mathrm{E}-05$ & $6.24 \mathrm{E}-05$ & 0.02574774 & $\begin{array}{l}0.07585449 \\
\end{array}$ & 0.01911876 & 4370846.77 \\
\hline 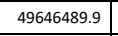 & $\begin{array}{l}784.07371 \\
\end{array}$ & 809.17859 & $\begin{array}{l}1024.5149 \\
\end{array}$ & 767.322529 & 0.04318298 & $2.51 \mathrm{E}-05$ & 74.6423418 & 0.01059569 & 0.00790887 & $1.28 \mathrm{E}-05$ & $1.24 \mathrm{E}-05$ & $6.25 \mathrm{E}-05$ & $\begin{array}{l}0.02575353 \\
\end{array}$ & $\begin{array}{l}0.07592531 \\
\end{array}$ & 0.01916541 & 4374107.46 \\
\hline 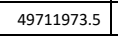 & 784.07371 & 809.178992 & 1024.5069 & 767.321484 & 0.04407922 & $2.51 \mathrm{E}-05$ & 74.645903 & 0.01060805 & 0.00791847 & $1.28 \mathrm{E}-05$ & $1.24 \mathrm{E}-05$ & $6.27 \mathrm{E}-05$ & 0.02576102 & 0.07601384 & 0.01922367 & 4378186.08 \\
\hline 49793828 & $\begin{array}{r}784.07371 \\
\end{array}$ & 809.179719 & 1024.49697 & 767.320185 & 0.04515243 & $2.51 \mathrm{E}-05$ & 74.6503428 & 0.01062349 & 0.00793047 & $1.28 \mathrm{E}-05$ & $1.24 \mathrm{E}-05$ & $6.29 \mathrm{E}-05$ & 0.02577104 & 0.07612451 & 0.01929643 & 4383291.88 \\
\hline 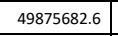 & 784.07371 & 809.180337 & 1024.48708 & 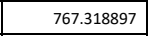 & 0.04618256 & $2.51 \mathrm{E}-05$ & 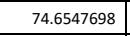 & 0.01063893 & 0.00794247 & $1.28 \mathrm{E}-05$ & $1.24 E-05$ & $6.32 \mathrm{E}-05$ & 0.02578059 & 0.07623517 & 0.01936911 & 4389631.8 \\
\hline 49957537.1 & 784.07371 & 809.180994 & 1024.47725 & 767.317613 & 0.04717314 & $2.51 \mathrm{E}-05$ & 74.6591839 & 0.01065438 & 0.00795447 & $1.28 \mathrm{E}-05$ & $1.24 \mathrm{E}-05$ & $6.34 \mathrm{E}-05$ & 0.02579031 & 0.07634584 & 0.01944172 & 4395969 \\
\hline 50039391.6 & 784.07371 & 809.181631 & 1024.46745 & 767.316257 & 0.04812733 & $2.51 \mathrm{E}-05$ & 74.6635853 & 0.01066982 & 0.00796647 & $1.28 \mathrm{E}-05$ & $1.24 \mathrm{E}-05$ & $6.36 \mathrm{E}-05$ & 0.02579968 & 0.0764565 & 0.01951426 & 4402305.2 \\
\hline 50121246.1 & 784.07371 & 809.182348 & 1024.45768 & 767.314395 & 0.04904796 & $2.51 \mathrm{E}-05$ & 74.6679739 & 0.01068527 & 0.00797847 & $1.28 \mathrm{E}-05$ & $1.24 \mathrm{E}-05$ & $6.38 \mathrm{E}-05$ & 0.02580943 & 0.07656717 & 0.01958673 & 4408641.41 \\
\hline \begin{tabular}{l|l}
50203100.6 \\
\end{tabular} & $\begin{array}{l}784.07371 \\
\end{array}$ & 809.18305 & \begin{tabular}{|l|l|}
1024.44793 \\
\end{tabular} & 767.312546 & 0.0499376 & $2.51 \mathrm{E}-05$ & $\begin{array}{ll}7.6723498 \\
\end{array}$ & 0.01070071 & 0.00799047 & $1.28 \mathrm{E}-05$ & $1.24 \mathrm{E}-05$ & $6.41 \mathrm{E}-05$ & 0.02581935 & 0.07667783 & 0.01965913 & 4414980.93 \\
\hline
\end{tabular}




\begin{tabular}{|c|c|c|c|c|c|c|c|c|c|c|c|c|c|c|c|c|}
\hline Time (s) & $\begin{array}{c}\text { Coolant outlet } \\
\text { temperature }(K)\end{array}$ & $\begin{array}{l}\text { Peak Cladding } \\
\text { Temperature (K) }\end{array}$ & $\begin{array}{c}\text { Peak fuel } \\
\text { temperature (K) }\end{array}$ & $\begin{array}{c}\text { Average cladding } \\
\text { internal } \\
\text { temperature (K) }\end{array}$ & \begin{tabular}{|l|}
$\begin{array}{c}\text { Peak cumulative } \\
\text { damage factor }\end{array}$ \\
\end{tabular} & $\begin{array}{l}\text { Cladding interior } \\
\text { volume }\left(\mathrm{m}^{3}\right)\end{array}$ & $\begin{array}{l}\text { Fission Gas } \\
\text { Release (\%) }\end{array}$ & $\begin{array}{c}\text { Fission gas } \\
\text { produced } \\
\text { (moles) }\end{array}$ & $\begin{array}{c}\text { Fission gas } \\
\text { released (moles) }\end{array}$ & Fuel volume $\left(\mathrm{m}^{3}\right)$ & $\begin{array}{l}\text { Plenum gas } \\
\text { volume }\left(m^{3}\right)\end{array}$ & $\begin{array}{l}\text { Peak cladding } \\
\text { radial growth } \\
\text { (m) }\end{array}$ & $\begin{array}{l}\text { Peak fuel axial } \\
\text { growth }(m)\end{array}$ & $\begin{array}{c}\text { Peak burnup } \\
(\text { (at\%) }\end{array}$ & $\begin{array}{l}\text { Peak cladding } \\
\text { hoop strain }\end{array}$ & $\begin{array}{c}\text { Plenum pressure } \\
(\mathrm{Pa})\end{array}$ \\
\hline 50284955.2 & 784.07371 & 809.183217 & 1024.4382 & 767.31071 & 0.05079857 & $2.51 \mathrm{E}-05$ & 74.6767132 & 0.01071615 & 0.00800247 & $1.28 \mathrm{E}-05$ & $1.24 \mathrm{E}-05$ & 6.43E-05 & 0.02582941 & 0.0767885 & 0.01973146 & 4421323.04 \\
\hline 50366809.7 & 784.07371 & 809.183278 & 1024.42848 & 767.308962 & 0.05163295 & $2.51 \mathrm{E}-05$ & 74.681064 & 0.0107316 & 0.00801447 & $1.28 \mathrm{E}-05$ & $1.24 \mathrm{E}-05$ & $6.45 E-05$ & 0.02583831 & 0.07689916 & 0.01980373 & 4427653.66 \\
\hline 50469127.8 & 784.07371 & 809.183634 & 1024.41636 & 767.306766 & 0.05263782 & $2.51 \mathrm{E}-05$ & 74.6864849 & 0.0107509 & 0.00802947 & $1.28 \mathrm{E}-05$ & $1.24 E-05$ & $6.48 \mathrm{E}-05$ & 0.02585034 & 0.07703749 & 0.01989397 & 4434024 \\
\hline 50597025.5 & 784.07371 & 809.184363 & 1024.40126 & 767.304055 & 0.05384035 & $2.51 \mathrm{E}-05$ & 74.6932337 & 0.01077503 & 0.00804822 & $1.28 \mathrm{E}-05$ & $1.24 E-05$ & $6.51 \mathrm{E}-05$ & 0.02586546 & 0.07721041 & 0.02000662 & 4441992.03 \\
\hline 50678880.1 & 784.07371 & 809.184745 & 1024.39162 & \begin{tabular}{ll|}
767.302404 \\
\end{tabular} & 0.05458985 & $2.51 \mathrm{E}-05$ & 74.6975371 & 0.01079048 & 0.00806022 & $1.28 \mathrm{E}-05$ & $1.24 \mathrm{E}-05$ & $6.53 \mathrm{E}-05$ & 0.02587425 & 0.07732107 & 0.02007869 & 4451795.41 \\
\hline 50760734.6 & 784.07371 & 809.185099 & 1024.38201 & 767.300821 & 0.05532042 & $2.52 \mathrm{E}-05$ & 74.7018282 & 0.01080592 & 0.00807222 & $1.28 \mathrm{E}-05$ & $1.24 \mathrm{E}-05$ & $6.56 \mathrm{E}-05$ & 0.02588255 & 0.07743174 & 0.02015069 & 4458112.01 \\
\hline 50802644.1 & 784.07371 & 809.184736 & $\begin{array}{l}1024.3771 \\
\end{array}$ & 767.300015 & 0.05568976 & $2.52 \mathrm{E}-05$ & 74.7040205 & 0.01081383 & 0.00807836 & $1.28 \mathrm{E}-05$ & $1.24 E-05$ & $6.57 \mathrm{E}-05$ & 0.02588653 & 0.0774884 & 0.02018756 & 4464361.82 \\
\hline 50844553.6 & 784.07371 & 809.183806 & 1024.37221 & 767.299205 & 0.0560545 & $2.52 \mathrm{E}-05$ & 74.7062095 & 0.01082173 & 0.00808451 & $1.28 \mathrm{E}-05$ & $1.24 \mathrm{E}-05$ & $6.58 \mathrm{E}-05$ & 0.02589071 & 0.07754506 & 0.0202244 & 4467592.62 \\
\hline 50896940.5 & 784.07371 & 809.182642 & 1024.36613 & \begin{tabular}{|l|l|}
767.298164 \\
\end{tabular} & 0.05650345 & $2.52 \mathrm{E}-05$ & 74.7089414 & 0.01083162 & 0.00809219 & $1.28 \mathrm{E}-05$ & $1.24 \mathrm{E}-05$ & $6.59 \mathrm{E}-05$ & 0.02589631 & 0.07761588 & 0.02027042 & 4470847.32 \\
\hline 50923762.6 & 784.07371 & 809.182051 & 1024.36302 & 767.297632 & 0.05673157 & $2.52 \mathrm{E}-05$ & 74.7103382 & 0.01083668 & 0.00809612 & $1.28 \mathrm{E}-05$ & $1.24 \mathrm{E}-05$ & $6.60 \mathrm{E}-05$ & 0.0258993 & 0.07765214 & 0.02029399 & 4474851.27 \\
\hline 50957290.2 & 784.07371 & 809.181319 & 1024.35915 & 767.296964 & 0.05701402 & $2.52 \mathrm{E}-05$ & 74.7120824 & 0.01084301 & 0.00810103 & $1.28 \mathrm{E}-05$ & $1.24 \mathrm{E}-05$ & $6.61 \mathrm{E}-05$ & 0.02590299 & 0.07769747 & 0.02032343 & 4476935.64 \\
\hline 50999199.7 & 784.07371 & 809.180421 & 1024.35433 & 767.296132 & 0.05736298 & $2.52 \mathrm{E}-05$ & 74.7142597 & 0.01085091 & 0.00810718 & $1.28 \mathrm{E}-05$ & $1.24 E-05$ & $6.62 E-05$ & 0.0259077 & 0.07775413 & 0.02036022 & 4479538.26 \\
\hline 51051586.6 & 784.07371 & 809.179306 & 1024.34835 & 767.295076 & 0.05779301 & $2.52 \mathrm{E}-05$ & 74.7169769 & 0.0108608 & 0.00811486 & $1.28 \mathrm{E}-05$ & $1.24 \mathrm{E}-05$ & $6.64 \mathrm{E}-05$ & 0.02591369 & 0.07782496 & 0.02040617 & 4482799.05 \\
\hline 51103973.5 & 784.07371 & 809.178214 & 1024.34241 & 767.294012 & 0.05821715 & $2.52 E-05$ & 74.7196892 & 0.01087068 & 0.00812254 & $1.28 \mathrm{E}-05$ & $1.24 \mathrm{E}-05$ & $6.65 \mathrm{E}-05$ & 0.02591979 & 0.07789578 & 0.02045212 & 4486855.38 \\
\hline 51130795.6 & 784.07371 & 809.17766 & 1024.33938 & 767.29348 & 0.05843283 & $2.52 \mathrm{E}-05$ & 74.7210759 & 0.01087574 & 0.00812647 & $1.28 \mathrm{E}-05$ & $1.24 E-05$ & $6.66 \mathrm{E}-05$ & 0.02592295 & 0.07793205 & 0.02047566 & 4490859.01 \\
\hline 51164323.2 & 784.07371 & 809.176975 & 1024.3356 & 767.292793 & 0.05870012 & $2.52 E-05$ & 74.7228076 & 0.01088207 & 0.00813139 & $1.28 \mathrm{E}-05$ & $1.24 E-05$ & $6.67 E-05$ & 0.02592699 & 0.07797738 & 0.02050505 & 4492950.05 \\
\hline 51206232.7 & 784.07371 & 809.17614 & 1024.33091 & 767.291954 & 0.05903075 & $2.52 E-05$ & 74.7249693 & 0.01088997 & 0.00813753 & $1.28 \mathrm{E}-05$ & $1.24 E-05$ & $6.68 \mathrm{E}-05$ & 0.02593177 & 0.07803404 & 0.02054178 & 4495557.72 \\
\hline 51233054.8 & 784.07371 & 809.175613 & 1024.32791 & 767.29141 & 0.05924096 & $2.52 \mathrm{E}-05$ & 74.7263512 & 0.01089503 & 0.00814146 & $1.28 E-05$ & $1.24 E-05$ & $6.68 \mathrm{E}-05$ & 0.02593505 & 0.0780703 & 0.02056529 & 4498774.52 \\
\hline 51259876.9 & 784.07371 & 809.175094 & 1024.32493 & 767.290866 & 0.05944982 & $2.52 E-05$ & 74.7277318 & 0.01090009 & 0.00814539 & $1.28 \mathrm{E}-05$ & $1.24 \mathrm{E}-05$ & $6.69 \mathrm{E}-05$ & 0.02593826 & 0.07810656 & 0.0205888 & 4500851.21 \\
\hline 51293404.5 & 784.07371 & 809.174531 & 1024.3212 & 767.290221 & 0.05970879 & $2.52 E-05$ & 74.7294557 & 0.01090642 & 0.00815031 & $1.28 \mathrm{E}-05$ & $1.24 E-05$ & $6.70 \mathrm{E}-05$ & 0.02594163 & 0.07815189 & 0.02061816 & 4502930.72 \\
\hline 51335314 & \begin{tabular}{|l}
784.07371 \\
\end{tabular} & 809.173842 & 1024.31657 & 767.289419 & 0.06002934 & $2.52 \mathrm{E}-05$ & 74.7316078 & 0.01091433 & 0.00815645 & $1.28 \mathrm{E}-05$ & $1.24 E-05$ & $6.71 E-05$ & 0.02594585 & 0.07820855 & 0.02065486 & 4505528.54 \\
\hline 51387700.9 & 784.07371 & 809.172975 & 1024.3108 & 767.2884 & 0.06042527 & $2.52 \mathrm{E}-05$ & 74.7342935 & 0.01092421 & 0.00816413 & $1.28 \mathrm{E}-05$ & $1.24 E-05$ & $6.73 E-05$ & 0.02595119 & 0.07827938 & 0.02070071 & 4508783.26 \\
\hline 51414523 & 784.07371 & 809.172526 & 1024.30785 & 767.287896 & 0.0606268 & $2.52 E-05$ & 74.7356667 & 0.01092927 & 0.00816806 & $1.28 \mathrm{E}-05$ & $1.24 E-05$ & $6.73 E-05$ & 0.02595388 & 0.07831564 & 0.0207242 & 4512780.53 \\
\hline 51448050.6 & $\begin{array}{r}784.07371 \\
\end{array}$ & 809.171944 & 1024.30417 & 767.287255 & 0.06087685 & $2.52 \mathrm{E}-05$ & 74.7373815 & 0.0109356 & 0.00817298 & $1.28 \mathrm{E}-05$ & $1.24 E-05$ & $6.74 \mathrm{E}-05$ & 0.02595722 & 0.07836097 & 0.02075354 & 4514857.9 \\
\hline 51489960.2 & 784.07371 & 809.171227 & 1024.29959 & 767.286463 & 0.0611866 & $2.52 \mathrm{E}-05$ & 74.7395221 & 0.01094351 & 0.00817912 & $1.28 \mathrm{E}-05$ & $1.24 \mathrm{E}-05$ & $6.75 E-05$ & 0.02596116 & 0.07841763 & 0.02079019 & 4517454.39 \\
\hline 51542347.1 & 784.07371 & 809.170337 & 1024.29388 & 767.285465 & 0.06156956 & $2.52 \mathrm{E}-05$ & 74.7421935 & 0.01095339 & 0.0081868 & $1.28 \mathrm{E}-05$ & $1.24 E-05$ & $6.77 E-05$ & 0.02596653 & 0.07848845 & 0.02083601 & 4520701.46 \\
\hline 51607830.7 & 784.07371 & 809.169264 & 1024.28678 & 767.284193 & 0.06204195 & $2.52 \mathrm{E}-05$ & 74.7455261 & 0.01096574 & 0.0081964 & $1.28 \mathrm{E}-05$ & $1.24 \mathrm{E}-05$ & $6.79 \mathrm{E}-05$ & 0.02597364 & 0.07857698 & 0.02089325 & 4524769.82 \\
\hline 51673314.3 & \begin{tabular}{|l}
784.07371 \\
\end{tabular} & 809.168231 & 1024.27973 & \begin{tabular}{|l}
767.282921 \\
\end{tabular} & 0.06250838 & $2.52 \mathrm{E}-05$ & 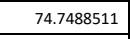 & 0.0109781 & 0.008206 & $1.28 \mathrm{E}-05$ & $1.24 E-05$ & $6.80 \mathrm{E}-05$ & 0.02598091 & 0.07866552 & 0.02095048 & 4529827.35 \\
\hline 51755168.8 & 784.07371 & 809.167138 & 1024.27098 & 767.281332 & 0.06308259 & $2.52 \mathrm{E}-05$ & 74.7529969 & 0.01099354 & 0.008218 & $1.28 \mathrm{E}-05$ & $1.24 E-05$ & $6.83 E-05$ & 0.02599015 & 0.07877618 & 0.02102198 & 4534913.97 \\
\hline 51857487 & 784.07371 & 809.16591 & 1024.26015 & 767.27936 & 0.06378757 & $2.52 \mathrm{E}-05$ & 74.7581627 & 0.01101285 & 0.008233 & $1.28 \mathrm{E}-05$ & $1.24 E-05$ & $6.85 \mathrm{E}-05$ & 0.02600143 & 0.07891451 & 0.0211113 & 4541268.01 \\
\hline 51985384.7 & 784.07371 & 809.164501 & 1024.24676 & 767.276882 & 0.0646506 & $2.52 \mathrm{E}-05$ & 74.7645947 & 0.01103698 & 0.00825175 & $1.28 \mathrm{E}-05$ & $1.24 \mathrm{E}-05$ & $6.89 \mathrm{E}-05$ & 0.02601598 & 0.07908743 & 0.0212229 & 4549218.25 \\
\hline 52079977 & 784.07371 & 809.163551 & 1024.23692 & 767.27504 & 0.06527978 & $2.52 \mathrm{E}-05$ & 74.7693336 & 0.01105483 & 0.00826562 & $1.28 \mathrm{E}-05$ & $1.24 E-05$ & $6.91 \mathrm{E}-05$ & 0.02602704 & 0.07921531 & 0.02130543 & 4559053.26 \\
\hline 52083577 & 784.07371 & 809.16351 & 1024.23654 & 767.274971 & 0.06530372 & $2.52 E-05$ & 74.7695137 & 0.0110555 & 0.00826615 & $1.28 \mathrm{E}-05$ & $1.24 E-05$ & $6.91 E-05$ & 0.02602747 & 0.07922018 & 0.02130862 & 4566211.14 \\
\hline 52178169.3 & 784.07371 & $\begin{array}{l}809.162616 \\
\end{array}$ & $\begin{array}{l}1024.22677 \\
\end{array}$ & 767.273144 & $\begin{array}{c}0.06592396 \\
\end{array}$ & $2.52 E-05$ & $\begin{array}{l}74.7742367 \\
\end{array}$ & 0.01107335 & 0.00828001 & $1.28 \mathrm{E}-05$ & $1.24 E-05$ & $6.94 \mathrm{E}-05$ & 0.02603846 & 0.07934806 & 0.02139105 & 4566645.85 \\
\hline 52272761.7 & 784.07371 & 809.161783 & 1024.21703 & 767.271322 & 0.06653619 & $2.52 E-05$ & 74.7789446 & $\begin{array}{l}0.0110912 \\
\end{array}$ & 0.00829388 & $1.28 \mathrm{E}-05$ & $1.24 E-05$ & $6.96 \mathrm{E}-05$ & 0.02604971 & 0.07947595 & 0.02147349 & $\begin{array}{l}4573960.7 \\
\end{array}$ \\
\hline 52367354 & 784.07371 & 809.161035 & 1024.20732 & 767.269495 & 0.06714093 & $2.52 E-05$ & 74.7836374 & 0.01110905 & 0.00830775 & $1.28 \mathrm{E}-05$ & $1.24 E-05$ & $6.99 E-05$ & 0.02606098 & 0.07960384 & 0.02155591 & 4581280.97 \\
\hline 52461946.4 & 784.07371 & 809.160356 & 1024.19763 & 767.267671 & 0.06773869 & $2.52 \mathrm{E}-05$ & 74.7883151 & 0.01112689 & 0.00832162 & $1.28 \mathrm{E}-05$ & $1.24 E-05$ & $7.02 E-05$ & 0.02607223 & 0.07973172 & 0.02163829 & 4588603.27 \\
\hline 52556538.7 & 784.07371 & 809.159498 & 1024.18794 & 767.265885 & 0.06841363 & $2.52 \mathrm{E}-05$ & 74.7929778 & 0.01114474 & 0.00833548 & $1.28 \mathrm{E}-05$ & $1.24 E-05$ & $7.04 E-05$ & 0.0260832 & 0.07985961 & 0.02172065 & 4595914.14 \\
\hline 52674779.1 & 784.07371 & 809.157873 & 1024.17584 & 767.26369 & 0.06927034 & $2.52 E-05$ & 74.7987853 & 0.01116705 & 0.00835282 & $1.28 \mathrm{E}-05$ & $1.24 E-05$ & $7.07 E-05$ & 0.02609582 & 0.08001947 & 0.02182355 & 4603253.84 \\
\hline 52793019.5 & 784.07371 & 809.155912 & 1024.16376 & 767.261543 & 0.07012018 & $2.52 \mathrm{E}-05$ & 74.8045696 & 0.01118936 & 0.00837015 & $1.28 \mathrm{E}-05$ & $1.24 E-05$ & $7.10 \mathrm{E}-05$ & 0.02610727 & 0.08017932 & 0.02192642 & 4612363.78 \\
\hline 52911260 & $\begin{array}{l}784.07371 \\
\end{array}$ & 809.153773 & 1024.15172 & 767.259437 & 0.07096372 & $2.52 \mathrm{E}-05$ & 74.8103308 & 0.01121167 & 0.00838748 & $1.28 \mathrm{E}-05$ & $1.24 E-05$ & $7.14 \mathrm{E}-05$ & 0.02611808 & 0.08033918 & 0.02202926 & 4621454.28 \\
\hline 53029500.4 & 784.07371 & 809.151764 & 1024.13976 & 767.257291 & 0.07180147 & $2.52 \mathrm{E}-05$ & 74.8160692 & 0.01123398 & 0.00840482 & $1.28 \mathrm{E}-05$ & $1.24 E-05$ & $7.17 \mathrm{E}-05$ & 0.02612987 & 0.08049904 & 0.02213206 & 4630561.45 \\
\hline 53177300.9 & 784.07371 & 809.148754 & 1024.12501 & 767.254613 & 0.07284041 & $2.52 \mathrm{E}-05$ & 74.8232103 & 0.01126186 & 0.00842649 & $1.28 \mathrm{E}-05$ & $1.24 E-05$ & $7.21 E-05$ & 0.02614488 & 0.08069886 & 0.0222605 & 4639712.89 \\
\hline 53362051.6 & $\begin{array}{r}784.07371 \\
\end{array}$ & 809.144687 & 1024.10691 & 767.251298 & 0.07412799 & $2.52 \mathrm{E}-05$ & 74.832087 & 0.01129672 & 0.00845357 & $1.28 \mathrm{E}-05$ & $1.24 E-05$ & $7.266-05$ & 0.02616279 & 0.08094864 & 0.02242097 & 4651141.74 \\
\hline 53546802.2 & \begin{tabular}{|l}
784.07371 \\
\end{tabular} & 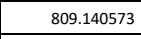 & 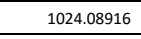 & $\begin{array}{l}767.247955 \\
\end{array}$ & 0.07540643 & $2.52 \mathrm{E}-05$ & \begin{tabular}{|l}
74.840909 \\
\end{tabular} & 0.01133158 & 0.00848065 & $1.28 \mathrm{E}-05$ & $1.24 E-05$ & $7.31 E-05$ & 0.02618304 & 0.08119841 & 0.02258139 & 4665389.31 \\
\hline 53731552.9 & 784.07371 & 809.136517 & 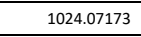 & 767.244669 & 0.07667733 & $2.52 \mathrm{E}-05$ & 74.849677 & 0.01136643 & 0.00850774 & $1.28 \mathrm{E}-05$ & $1.24 E-05$ & $7.36 E-05$ & 0.02620283 & $\begin{array}{c}0.08144819 \\
\end{array}$ & 0.02274175 & 4679632.28 \\
\hline
\end{tabular}




\begin{tabular}{|c|c|c|c|c|c|c|c|c|c|c|c|c|c|c|c|c|}
\hline Time (s) & $\begin{array}{c}\text { Coolant outlet } \\
\text { temperature }(K)\end{array}$ & $\begin{array}{l}\text { Peak Cladding } \\
\text { Temperature (K) }\end{array}$ & $\begin{array}{c}\text { Peak fuel } \\
\text { temperature (K) }\end{array}$ & $\begin{array}{c}\text { Average cladding } \\
\text { internal } \\
\text { temperature (K) }\end{array}$ & \begin{tabular}{|l|}
$\begin{array}{l}\text { Peak cumulative } \\
\text { damage factor }\end{array}$ \\
\end{tabular} & $\begin{array}{l}\text { Cladding interior } \\
\text { volume }\left(\mathrm{m}^{3}\right)\end{array}$ & $\begin{array}{l}\text { Fission Gas } \\
\text { Release (\%) }\end{array}$ & $\begin{array}{c}\text { Fission gas } \\
\text { produced } \\
\text { (moles) }\end{array}$ & $\begin{array}{c}\text { Fission gas } \\
\text { released (moles) }\end{array}$ & Fuel volume $\left(\mathrm{m}^{3}\right)$ & $\begin{array}{l}\text { Plenum gas } \\
\text { volume }\left(m^{3}\right)\end{array}$ & $\begin{array}{l}\text { Peak cladding } \\
\text { radial growth } \\
\text { (m) }\end{array}$ & $\begin{array}{l}\text { Peak fuel axial } \\
\text { growth }(m)\end{array}$ & $\begin{array}{c}\text { Peak burnup } \\
(\text { (at\%) }\end{array}$ & $\begin{array}{l}\text { Peak cladding } \\
\text { hoop strain }\end{array}$ & $\begin{array}{l}\text { Plenum pressure } \\
(\mathrm{Pa})\end{array}$ \\
\hline 53874489 & 785.999361 & 811.084169 & 1025.20964 & \begin{tabular}{ll|}
768.724221 \\
\end{tabular} & 0.07794518 & $2.52 \mathrm{E}-05$ & 74.8564237 & 0.0113934 & 0.00852869 & $1.28 \mathrm{E}-05$ & $1.24 \mathrm{E}-05$ & $7.40 \mathrm{E}-05$ & 0.02622307 & 0.08164144 & 0.02287721 & 4702540.49 \\
\hline 53878089 & 786.481015 & 811.653931 & 1026.36094 & 769.147462 & 0.07798661 & $2.52 \mathrm{E}-05$ & 74.8565935 & 0.01139408 & 0.00852922 & $1.28 \mathrm{E}-05$ & $1.24 \mathrm{E}-05$ & $7.40 \mathrm{E}-05$ & 0.02622611 & 0.08164631 & 0.02289246 & 4715890.28 \\
\hline 54021025.1 & 786.481015 & 811.650813 & 1026.34753 & 769.144605 & 0.07962529 & $2.52 E-05$ & 74.8633301 & 0.01142114 & 0.00855025 & $1.29 \mathrm{E}-05$ & $1.24 E-05$ & $7.44 E-05$ & 0.02624173 & 0.08184021 & 0.0230177 & 4716379.65 \\
\hline 54163961.2 & 786.481015 & 811.647969 & 1026.33427 & 769.141779 & $\begin{array}{ll}0.08125508 \\
\end{array}$ & $2.52 \mathrm{E}-05$ & 74.8700348 & 0.0114482 & 0.00857127 & $1.29 E-05$ & $1.24 E-05$ & $7.48 E-05$ & 0.0262577 & 0.0820341 & 0.02314228 & 4727467.86 \\
\hline 54306897.3 & 786.481015 & 811.644794 & 1026.32113 & 769.139051 & \begin{tabular}{|l|l|}
0.08287724 \\
\end{tabular} & $2.52 \mathrm{E}-05$ & 74.8767079 & 0.01147526 & 0.0085923 & $1.29 \mathrm{E}-05$ & $1.24 E-05$ & $7.52 E-05$ & 0.02627258 & 0.082228 & 0.0232668 & 4738539.06 \\
\hline 54449833.4 & 786.481015 & 811.641612 & 1026.30811 & \begin{tabular}{|l|}
769.136384 \\
\end{tabular} & 0.08449302 & $2.52 \mathrm{E}-05$ & 74.8833496 & 0.01150232 & 0.00861332 & $1.29 \mathrm{E}-05$ & $1.24 \mathrm{E}-05$ & $7.56 \mathrm{E}-05$ & 0.02628678 & 0.08242189 & 0.02339127 & 4749594.05 \\
\hline 54592769.4 & 786.481015 & 811.638947 & 1026.2952 & 769.133668 & 0.08610353 & $2.52 \mathrm{E}-05$ & 74.8899602 & 0.01152938 & 0.00863435 & $1.29 \mathrm{E}-05$ & $1.24 E-05$ & $7.60 \mathrm{E}-05$ & 0.02630237 & 0.08261579 & 0.02351569 & 4760680.72 \\
\hline 54735705.5 & 786.481015 & 811.636373 & 1026.28239 & 769.131007 & 0.08770976 & $2.52 \mathrm{E}-05$ & 74.8965397 & 0.01155643 & 0.00865537 & $1.29 \mathrm{E}-05$ & $1.24 \mathrm{E}-05$ & $7.63 \mathrm{E}-05$ & 0.02631806 & 0.08280968 & 0.02364007 & 4771761.31 \\
\hline 54808888.8 & 786.481015 & 811.634941 & 1026.27586 & 769.12968 & 0.0885314 & $2.52 \mathrm{E}-05$ & 74.8998966 & 0.01157029 & 0.00866613 & $1.29 \mathrm{E}-05$ & $1.24 \mathrm{E}-05$ & $7.65 E-05$ & 0.02632516 & 0.08290896 & 0.02370379 & 4782732.01 \\
\hline 54900367.9 & 786.481015 & 811.633203 & 1026.26773 & 769.128044 & 0.08955695 & $2.52 \mathrm{E}-05$ & 74.9040813 & 0.01158761 & 0.00867959 & $1.29 \mathrm{E}-05$ & $1.24 \mathrm{E}-05$ & $7.68 \mathrm{E}-05$ & 0.02633374 & 0.08303305 & 0.02378334 & 4788406.85 \\
\hline 55014716.8 & 786.481015 & 811.631093 & 1026.25765 & 769.126029 & 0.09083698 & $2.52 \mathrm{E}-05$ & 74.9092947 & 0.01160925 & 0.00869641 & $1.29 \mathrm{E}-05$ & $1.24 \mathrm{E}-05$ & $7.71 E-05$ & 0.02634395 & 0.08318816 & 0.02388276 & 4795488.47 \\
\hline 55157652.9 & 786.481015 & 811.62847 & 1026.24515 & 769.123506 & 0.09243482 & $2.52 \mathrm{E}-05$ & 74.9157842 & 0.01163631 & 0.00871743 & $1.29 \mathrm{E}-05$ & $1.24 E-05$ & $7.75 E-05$ & 0.0263576 & 0.08338206 & 0.024007 & 4804352.85 \\
\hline 55195122.7 & 786.481015 & 811.627413 & 1026.24186 & 769.122837 & 0.09285378 & $2.52 E-05$ & 74.9174803 & 0.01164341 & 0.00872295 & $1.29 \mathrm{E}-05$ & $1.24 E-05$ & $7.76 E-05$ & 0.02636152 & 0.08343289 & 0.02403963 & 4815278.4 \\
\hline 55232592.5 & 786.481015 & 811.626664 & 1026.2386 & 769.122173 & 0.0932726 & $2.52 E-05$ & 74.9191744 & 0.0116505 & 0.00872846 & $1.29 \mathrm{E}-05$ & $1.24 \mathrm{E}-05$ & $7.77 E-05$ & 0.02636549 & 0.08348372 & 0.02407219 & 4818176 \\
\hline 55279429.8 & 786.481015 & 811.625893 & 1026.23453 & 769.121341 & 0.09379592 & $2.52 \mathrm{E}-05$ & 74.9212892 & 0.01165937 & 0.00873535 & $1.29 \mathrm{E}-05$ & $1.24 E-05$ & $7.78 E-05$ & 0.02637026 & 0.08354725 & 0.02411289 & 4821089.9 \\
\hline 55337976.4 & 786.481015 & 811.624814 & 1026.22947 & 769.120314 & 0.09444981 & $2.52 \mathrm{E}-05$ & 74.9239281 & 0.01167045 & 0.00874396 & $1.29 \mathrm{E}-05$ & $1.24 \mathrm{E}-05$ & $7.80 \mathrm{E}-05$ & 0.0263765 & 0.08362667 & 0.02416375 & 4824728.64 \\
\hline 55411159.7 & 786.481015 & 811.623272 & 1026.22318 & 769.11905 & 0.09526683 & $2.52 E-05$ & 74.9272197 & 0.0116843 & 0.00875472 & $1.29 \mathrm{E}-05$ & $1.24 E-05$ & $7.82 E-05$ & 0.02638438 & 0.08372595 & 0.02422732 & 4829278.94 \\
\hline 55484343 & 786.481015 & 811.622041 & 1026.21692 & 769.117798 & 0.09608366 & $2.52 \mathrm{E}-05$ & 74.9305035 & 0.01169816 & 0.00876549 & $1.29 E-05$ & $1.23 \mathrm{E}-05$ & $7.84 E-05$ & 0.02639243 & 0.08382522 & 0.02429089 & 4834948.6 \\
\hline 55557526.3 & 786.481015 & 811.620609 & 1026.21069 & 769.116546 & 0.09690038 & $2.52 E-05$ & 74.9337795 & 0.01171201 & 0.00877625 & $1.29 \mathrm{E}-05$ & $1.23 \mathrm{E}-05$ & 7.86 E-05 & 0.02640046 & 0.08392449 & 0.02435444 & 4840619.55 \\
\hline 55630709.6 & 786.481015 & 811.619275 & 1026.20449 & 769.115308 & 0.09792934 & $2.52 E-05$ & 74.9370478 & 0.01172587 & 0.00878702 & $1.29 \mathrm{E}-05$ & $1.23 E-05$ & $7.88 E-05$ & 0.02640858 & 0.08402377 & 0.02441799 & 4846290.18 \\
\hline 55703892.8 & 786.481015 & 811.617948 & 1026.19833 & 769.114076 & 0.1000323 & $2.52 \mathrm{E}-05$ & 74.9403084 & 0.01173972 & 0.00879778 & $1.29 \mathrm{E}-05$ & $1.23 E-05$ & $7.90 E-05$ & 0.02641687 & 0.08412304 & 0.02448153 & 4851961.89 \\
\hline 55795371.9 & 786.481015 & 811.616349 & 1026.19067 & 769.11255 & 0.10270146 & $2.52 \mathrm{E}-05$ & 74.9443733 & 0.01175704 & 0.00881124 & $1.29 \mathrm{E}-05$ & $1.23 E-05$ & $7.92 E-05$ & 0.02642702 & 0.08424713 & 0.02456092 & 4857658.96 \\
\hline 55886851 & 786.481015 & 811.614803 & 1026.18306 & 769.111042 & 0.10541186 & $2.52 E-05$ & 74.9484263 & 0.01177436 & 0.00882469 & $1.29 \mathrm{E}-05$ & $1.23 E-05$ & $7.95 E-05$ & 0.02643695 & 0.08437123 & 0.02464031 & 4864743.02 \\
\hline 55978330.1 & 786.481015 & 811.613271 & 1026.17548 & 769.109554 & 0.10816388 & $2.52 \mathrm{E}-05$ & 74.9524674 & 0.01179167 & 0.00883815 & $1.29 \mathrm{E}-05$ & $1.23 \mathrm{E}-05$ & $7.97 E-05$ & 0.02644646 & 0.08449532 & 0.02471969 & 4871820.89 \\
\hline 56069809.2 & 786.481015 & 811.611607 & 1026.16794 & 769.108047 & 0.11095779 & $2.52 \mathrm{E}-05$ & 74.9564966 & 0.01180899 & 0.00885161 & $1.29 \mathrm{E}-05$ & $1.23 \mathrm{E}-05$ & $7.99 E-05$ & 0.02645697 & 0.08461941 & 0.02479905 & 4878910.9 \\
\hline 56161288.3 & 786.481015 & 811.610018 & 1026.16042 & 769.106381 & 0.11379371 & $2.52 \mathrm{E}-05$ & 74.960514 & 0.01182631 & 0.00886506 & $1.29 \mathrm{E}-05$ & $1.23 E-05$ & $8.02 E-05$ & 0.02646732 & 0.08474351 & 0.0248784 & 4885996.8 \\
\hline 56252767.4 & 786.481015 & 811.60845 & 1026.15293 & 769.104708 & 0.11667164 & $2.52 \mathrm{E}-05$ & 74.9645196 & 0.01184363 & 0.00887852 & $1.29 \mathrm{E}-05$ & $1.23 \mathrm{E}-05$ & $8.04 E-05$ & 0.02647778 & 0.0848676 & 0.02495773 & 4893090.25 \\
\hline 56344246.5 & \begin{tabular}{|l}
786.481015 \\
\end{tabular} & 811.607009 & $\begin{array}{l}1026.14547 \\
\end{array}$ & 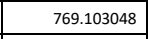 & 0.11959145 & $2.52 \mathrm{E}-05$ & 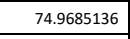 & 0.01186094 & 0.00889197 & $1.29 \mathrm{E}-05$ & $1.23 E-05$ & $8.07 E-05$ & 0.02648826 & 0.08499169 & 0.02503705 & 4900182.88 \\
\hline 56435725.6 & 786.481015 & 811.605601 & 1026.13802 & 769.101396 & 0.1225529 & $2.52 \mathrm{E}-05$ & 74.9724959 & 0.01187826 & 0.00890543 & $1.29 \mathrm{E}-05$ & $1.23 E-05$ & $8.09 E-05$ & 0.02649875 & 0.08511578 & 0.02511635 & 4907277.37 \\
\hline 56482562.9 & 786.481015 & 811.604939 & 1026.13421 & 769.100562 & 0.12408041 & $2.52 \mathrm{E}-05$ & 74.9745304 & 0.01188713 & 0.00891232 & $1.29 \mathrm{E}-05$ & $1.23 E-05$ & $8.11 E-05$ & 0.02650389 & 0.08517932 & 0.02515698 & 4914304.99 \\
\hline 56529400.2 & 786.481015 & 811.603972 & 1026.13041 & 769.099725 & 0.12561874 & $2.52 \mathrm{E}-05$ & 74.9765618 & 0.01189599 & 0.00891921 & $1.29 \mathrm{E}-05$ & $1.23 \mathrm{E}-05$ & $8.12 E-05$ & 0.02650909 & 0.08524286 & 0.02519758 & 4917932.23 \\
\hline 56587946.8 & 786.481015 & 811.60321 & 1026.12566 & 769.09868 & 0.12755834 & $2.52 \mathrm{E}-05$ & 74.9790968 & 0.01190708 & 0.00892782 & $1.29 \mathrm{E}-05$ & $1.23 \mathrm{E}-05$ & $8.13 \mathrm{E}-05$ & 0.02651571 & 0.08532227 & 0.02524831 & 4921581.86 \\
\hline 56661130.1 & 786.481015 & 811.602056 & 1026.11974 & 769.097376 & 0.13000863 & $2.53 E-05$ & 74.9822589 & 0.01192093 & 0.00893858 & $1.29 \mathrm{E}-05$ & $1.23 E-05$ & $8.15 E-05$ & 0.02652404 & 0.08542155 & 0.02531171 & 4926141.28 \\
\hline 56752609.2 & $\begin{array}{l}786.481015 \\
\end{array}$ & 811.60067 & 1026.11234 & 769.095756 & $\begin{array}{l}0.13311116 \\
\end{array}$ & $2.53 E-05$ & 74.9862013 & 0.01193825 & 0.00895204 & $1.29 \mathrm{E}-05$ & $1.23 E-05$ & $8.18 \mathrm{E}-05$ & 0.02653448 & 0.08554564 & 0.02539096 & 4931838.93 \\
\hline 56844088.3 & 786.481015 & 811.599193 & 1026.10495 & 769.094119 & 0.13625296 & $2.53 E-05$ & 74.9901322 & 0.01195557 & 0.0089655 & $1.29 \mathrm{E}-05$ & $1.23 E-05$ & $8.20 \mathrm{E}-05$ & 0.02654552 & 0.08566973 & 0.0254702 & 4938942.85 \\
\hline 56935567.4 & 786.481015 & 811.597997 & 1026.09757 & 769.092504 & 0.13943324 & $2.53 \mathrm{E}-05$ & 74.9940518 & 0.01197288 & 0.00897895 & $1.29 \mathrm{E}-05$ & $1.23 E-05$ & $8.23 E-05$ & 0.02655621 & 0.08579383 & 0.02554943 & 4946042.64 \\
\hline 57027046.5 & 786.481015 & 811.596607 & 1026.09019 & 769.090917 & 0.14265113 & $2.53 \mathrm{E}-05$ & 74.9979601 & 0.0119902 & 0.00899241 & $1.29 \mathrm{E}-05$ & $1.23 E-05$ & $8.25 E-05$ & 0.02656687 & 0.08591792 & 0.02562864 & 4953135.78 \\
\hline 57118525.6 & 786.481015 & 811.595492 & 1026.08281 & 769.089331 & 0.14590568 & $2.53 \mathrm{E}-05$ & 75.001857 & 0.01200752 & 0.00900586 & $1.29 \mathrm{E}-05$ & $1.23 E-05$ & $8.28 E-05$ & 0.02657732 & 0.08604201 & 0.02570785 & 4960235.98 \\
\hline 57210004.7 & 786.481015 & 811.594132 & 1026.07544 & 769.087741 & 0.14949899 & $2.53 E-05$ & 75.0057428 & 0.01202484 & 0.00901932 & $1.29 \mathrm{E}-05$ & $1.23 E-05$ & $8.30 \mathrm{E}-05$ & 0.02658822 & 0.08616611 & 0.02578704 & 4967336.41 \\
\hline 57301483.8 & 786.481015 & 811.591596 & 1026.06806 & 769.086163 & 0.15335983 & $2.53 E-05$ & 75.0096174 & 0.01204216 & 0.00903277 & $1.29 \mathrm{E}-05$ & $1.23 E-05$ & $8.33 E-05$ & 0.0265987 & 0.0862902 & 0.02586621 & 4974429.32 \\
\hline 57415832.6 & 786.481015 & 811.587727 & 1026.05885 & 769.084174 & 0.15825865 & $2.53 \mathrm{E}-05$ & 75.0144449 & 0.0120638 & 0.00904959 & $1.29 \mathrm{E}-05$ & $1.23 \mathrm{E}-05$ & $8.36 \mathrm{E}-05$ & 0.02661212 & 0.08644531 & 0.02596515 & 4981568.24 \\
\hline 57530181.5 & 786.481015 & 811.58395 & 1026.04962 & 769.08219 & 0.16322767 & $2.53 \mathrm{E}-05$ & 75.0192552 & 0.01208545 & 0.00906641 & $1.29 \mathrm{E}-05$ & $1.23 \mathrm{E}-05$ & $8.39 E-05$ & 0.02662565 & 0.08660043 & 0.02606408 & 4990445.63 \\
\hline 57588728.1 & 786.481015 & 811.582293 & 1026.04489 & 769.081208 & 0.16579051 & $2.53 \mathrm{E}-05$ & 75.0217114 & 0.01209653 & 0.00907503 & $1.29 \mathrm{E}-05$ & $1.23 E-05$ & $8.40 \mathrm{E}-05$ & 0.02663224 & 0.08667985 & 0.02611475 & 4999237.61 \\
\hline 57661911.4 & 786.481015 & 811.580098 & 1026.03899 & 769.079986 & 0.16902098 & $2.53 \mathrm{E}-05$ & 75.0247754 & 0.01211039 & 0.00908579 & $1.29 \mathrm{E}-05$ & $1.23 \mathrm{E}-05$ & $8.42 E-05$ & 0.02664022 & 0.08677912 & 0.02617804 & 5003799.18 \\
\hline 57735094.7 & \begin{tabular}{|l}
786.481015 \\
\end{tabular} & 811.577543 & 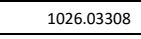 & $\begin{array}{ll}769.078767 \\
\end{array}$ & 0.17227774 & $2.53 E-05$ & 755.0278323 & 0.01212424 & 0.00909656 & $1.29 \mathrm{E}-05$ & $1.23 E-05$ & $8.44 E-05$ & 0.0266488 & 0.0868784 & 0.02624133 & 5009472.16 \\
\hline 57826573.8 & $\begin{array}{l}786.481015 \\
\end{array}$ & 811.575062 & \begin{tabular}{l|l}
1026.02569 \\
\end{tabular} & 769.077243 & $\begin{array}{l}0.1763877 \\
\end{array}$ & $2.53 \mathrm{E}-05$ & 75.0316436 & 0.01214156 & 0.00911001 & $1.29 \mathrm{E}-05$ & $1.23 \mathrm{E}-05$ & $8.47 E-05$ & 0.02665933 & 0.08700249 & 0.02632042 & 5015180.58 \\
\hline
\end{tabular}




\begin{tabular}{|c|c|c|c|c|c|c|c|c|c|c|c|c|c|c|c|c|}
\hline Time (s) & $\begin{array}{c}\text { Coolant outlet } \\
\text { temperature }(\mathrm{K})\end{array}$ & $\begin{array}{l}\text { Peak Cladding } \\
\text { Temperature (K) }\end{array}$ & $\begin{array}{l}\text { Peak fuel } \\
\text { temperature (K) }\end{array}$ & $\begin{array}{c}\text { Average cladding } \\
\text { internal } \\
\text { temperature (K) }\end{array}$ & $\begin{array}{l}\text { Peak cumulative } \\
\text { damage factor }\end{array}$ & $\begin{array}{l}\text { Cladding interior } \\
\text { volume }\left(\mathrm{m}^{3}\right)\end{array}$ & $\begin{array}{l}\text { Fission Gas } \\
\text { Release (\%) }\end{array}$ & $\begin{array}{l}\text { Fission gas } \\
\text { produced } \\
\text { (moles) }\end{array}$ & $\begin{array}{c}\text { Fission gas } \\
\text { released (moles) }\end{array}$ & Fuel volume $\left(\mathrm{m}^{3}\right)$ & $\begin{array}{l}\text { Plenum gas } \\
\text { volume }\left(\mathrm{m}^{3}\right)\end{array}$ & $\begin{array}{l}\text { Peak cladding } \\
\text { radial growth } \\
\text { (m) }\end{array}$ & $\begin{array}{l}\text { Peak fuel axial } \\
\text { growth }(m)\end{array}$ & $\begin{array}{c}\text { Peak burnup } \\
\text { (at\%) }\end{array}$ & $\begin{array}{l}\text { Peak cladding } \\
\text { hoop strain }\end{array}$ & $\begin{array}{l}\text { Plenum pressure } \\
(\mathrm{Pa})\end{array}$ \\
\hline 57873411.1 & 786.481015 & 811.573311 & 1026.0219 & $\begin{array}{l}769.076456 \\
\end{array}$ & 0.17850258 & $2.53 \mathrm{E}-05$ & 75.0335908 & 0.01215043 & 0.0091169 & $1.29 \mathrm{E}-05$ & $1.23 \mathrm{E}-05$ & $8.48 \mathrm{E}-05$ & 0.02666483 & 0.08706603 & 0.02636094 & 5022220.5 \\
\hline 57920248.4 & \begin{tabular}{|l|l|}
786.481015 \\
\end{tabular} & 811.571871 & 1026.01811 & 769.075675 & 0.18062732 & $2.53 \mathrm{E}-05$ & 75.0355352 & 0.01215929 & 0.00912379 & $1.29 \mathrm{E}-05$ & $1.23 \mathrm{E}-05$ & $8.49 \mathrm{E}-05$ & 0.02667035 & 0.08712956 & 0.02640143 & 5025857.53 \\
\hline 57978795 & 786.481015 & 811.57016 & 1026.01338 & 769.074702 & 0.18329804 & $2.53 \mathrm{E}-05$ & 75.0379617 & 0.01217038 & 0.0091324 & $1.29 \mathrm{E}-05$ & $1.23 \mathrm{E}-05$ & $8.51 \mathrm{E}-05$ & 0.02667716 & 0.08720898 & 0.02645203 & 5029510.7 \\
\hline \begin{tabular}{|l|}
58051978.3 \\
\end{tabular} & 786.481015 & 811.567844 & 1026.00746 & 769.073495 & 0.18665875 & $2.53 \mathrm{E}-05$ & 75.0409886 & 0.01218423 & 0.00914317 & $1.29 \mathrm{E}-05$ & $1.23 \mathrm{E}-05$ & $8.53 \mathrm{E}-05$ & 0.0266856 & 0.08730826 & 0.02651527 & 5034075.58 \\
\hline 58143457.4 & 786.481015 & 811.565423 & 1026.00005 & \begin{tabular}{|l|l|}
769.072004 \\
\end{tabular} & 0.19089296 & $2.53 \mathrm{E}-05$ & 75.0447625 & 0.01220155 & 0.00915662 & $1.29 \mathrm{E}-05$ & $1.23 \mathrm{E}-05$ & $8.55 \mathrm{E}-05$ & 0.02669624 & 0.08743235 & 0.02659431 & 5039785.32 \\
\hline 58234936.5 & 786.481015 & 811.562713 & 1025.99264 & \begin{tabular}{|l|l|}
769.070511 \\
\end{tabular} & 0.19515938 & $2.53 \mathrm{E}-05$ & 75.0485257 & 0.01221886 & 0.00917008 & $1.29 \mathrm{E}-05$ & $1.23 \mathrm{E}-05$ & $8.58 \mathrm{E}-05$ & 0.02670694 & 0.08755644 & 0.02667335 & 5046889.28 \\
\hline 58326415.6 & 786.481015 & 811.560059 & 1025.98523 & \begin{tabular}{|l|l|}
769.069018 \\
\end{tabular} & 0.19945648 & $2.53 \mathrm{E}-05$ & 75.0522783 & 0.01223618 & 0.00918353 & $1.29 \mathrm{E}-05$ & $1.23 \mathrm{E}-05$ & $8.60 \mathrm{E}-05$ & 0.02671761 & 0.08768053 & 0.02675237 & 5053993.49 \\
\hline 58373252.9 & 786.481015 & 811.558439 & 1025.98143 & 769.068253 & 0.20166494 & $2.53 \mathrm{E}-05$ & 75.0541955 & 0.01224505 & 0.00919042 & $1.29 \mathrm{E}-05$ & $1.23 \mathrm{E}-05$ & $8.61 \mathrm{E}-05$ & 0.02672307 & 0.08774407 & 0.02679285 & 5061031.85 \\
\hline \begin{tabular}{|l|l|}
58431799.5 \\
\end{tabular} & 786.481015 & 811.556787 & 1025.97669 & \begin{tabular}{|l|l|}
769.067298 \\
\end{tabular} & 0.20443718 & $2.53 \mathrm{E}-05$ & 75.0565881 & 0.01225613 & 0.00919903 & $1.29 \mathrm{E}-05$ & $1.23 \mathrm{E}-05$ & $8.63 \mathrm{E}-05$ & 0.02672989 & 0.08782349 & 0.02684341 & 5064686.43 \\
\hline \begin{tabular}{|l|}
58504982.8 \\
\end{tabular} & 786.481015 & 811.554467 & 1025.97076 & \begin{tabular}{|l|}
769.066104 \\
\end{tabular} & 0.20791997 & $2.53 \mathrm{E}-05$ & 75.0595728 & 0.01226999 & 0.0092098 & $1.29 \mathrm{E}-05$ & $1.23 \mathrm{E}-05$ & $8.65 \mathrm{E}-05$ & 0.02673835 & 0.08792276 & 0.02690659 & 5069252.28 \\
\hline 58596461.8 & 786.481015 & 811.551317 & 1025.96335 & 769.064599 & 0.21229943 & $2.53 \mathrm{E}-05$ & 75.0632942 & 0.0122873 & 0.00922326 & $1.29 \mathrm{E}-05$ & $1.23 \mathrm{E}-05$ & $8.67 \mathrm{E}-05$ & 0.02674954 & 0.08804686 & 0.02698556 & 5074973.96 \\
\hline \begin{tabular}{|l|}
58687940.9 \\
\end{tabular} & 786.481015 & 811.548351 & 1025.95593 & \begin{tabular}{|l|}
769.063098 \\
\end{tabular} & 0.21670385 & $2.53 \mathrm{E}-05$ & 75.0670052 & 0.01230462 & 0.00923671 & $1.29 \mathrm{E}-05$ & $1.23 \mathrm{E}-05$ & $8.70 \mathrm{E}-05$ & 0.02676043 & 0.08817095 & 0.02706453 & 5082084.64 \\
\hline 58779420 & 786.481015 & 811.545274 & 1025.9485 & \begin{tabular}{|l|}
769.061598 \\
\end{tabular} & 0.22113179 & $2.53 \mathrm{E}-05$ & 75.0707057 & 0.01232194 & 0.00925017 & $1.29 \mathrm{E}-05$ & $1.23 \mathrm{E}-05$ & $8.72 \mathrm{E}-05$ & 0.02677132 & 0.08829504 & 0.02714348 & 5089186.82 \\
\hline 58870899.1 & 786.481015 & 811.542171 & 1025.94108 & 769.060089 & 0.22558183 & $2.53 \mathrm{E}-05$ & 75.0743958 & 0.01233926 & 0.00926362 & $1.30 \mathrm{E}-05$ & $1.23 \mathrm{E}-05$ & $8.75 \mathrm{E}-05$ & 0.02678219 & 0.08841913 & 0.02722241 & 5096300.81 \\
\hline 58962378.2 & 786.481015 & 811.539383 & 1025.93365 & 769.058586 & 0.23005248 & $2.53 \mathrm{E}-05$ & 75.0780756 & 0.01235658 & 0.00927708 & $1.30 \mathrm{E}-05$ & $1.23 \mathrm{E}-05$ & $8.77 \mathrm{E}-05$ & 0.02679273 & 0.08854323 & 0.02730133 & 5103401.33 \\
\hline 59076727.1 & 786.481015 & 811.535621 & 1025.92436 & 769.056731 & 0.23566963 & $2.53 \mathrm{E}-05$ & 75.0826608 & 0.01237822 & 0.0092939 & $1.30 \mathrm{E}-05$ & $1.23 \mathrm{E}-05$ & $8.80 \mathrm{E}-05$ & 0.02680613 & 0.08869834 & 0.02739995 & 5110543.35 \\
\hline 59191076 & 786.481015 & 811.531742 & 1025.91509 & 769.055026 & 0.24131353 & $2.53 \mathrm{E}-05$ & 75.08723 & 0.01239987 & 0.00931072 & $1.30 \mathrm{E}-05$ & $1.23 \mathrm{E}-05$ & $8.83 E-05$ & 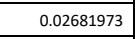 & 0.08885346 & 0.02749856 & 5119433.51 \\
\hline 59305424.8 & 786.481015 & 811.52812 & 1025.90585 & 769.053344 & 0.24698148 & $2.53 \mathrm{E}-05$ & 75.0917833 & 0.01242152 & 0.00932754 & $1.30 \mathrm{E}-05$ & $1.23 \mathrm{E}-05$ & $8.86 \mathrm{E}-05$ & 0.0268333 & 0.08900857 & 0.02759714 & 5128326.77 \\
\hline 59419773.7 & 786.481015 & 811.524686 & 1025.8966 & 769.051682 & 0.25267087 & $2.53 \mathrm{E}-05$ & 75.0963208 & 0.01244316 & 0.00934436 & $1.30 \mathrm{E}-05$ & $1.23 \mathrm{E}-05$ & $8.90 \mathrm{E}-05$ & 0.02684628 & 0.08916369 & 0.02769569 & 5137203.12 \\
\hline 59478320.3 & $\begin{array}{l}786.481015 \\
\end{array}$ & 811.522675 & 1025.89185 & 769.050848 & 0.2555899 & $2.53 \mathrm{E}-05$ & 75.0986378 & 0.01245425 & 0.00935297 & $1.30 \mathrm{E}-05$ & $1.23 \mathrm{E}-05$ & $8.91 E-05$ & 0.02685316 & 0.08924311 & 0.02774618 & 5146009.75 \\
\hline 59551503.6 & 786.481015 & 811.5203 & 1025.88593 & 769.049811 & 0.2592459 & $2.53 \mathrm{E}-05$ & 75.1015284 & 0.0124681 & 0.00936373 & $1.30 \mathrm{E}-05$ & $1.23 \mathrm{E}-05$ & $8.93 \mathrm{E}-05$ & 0.02686159 & 0.08934238 & 0.02780923 & 5150579.92 \\
\hline 59642982.7 & 786.481015 & 811.517588 & 1025.87853 & 769.048528 & 0.26382603 & $2.53 \mathrm{E}-05$ & 75.1051325 & 0.01248542 & 0.00937719 & $1.30 \mathrm{E}-05$ & $1.23 \mathrm{E}-05$ & $8.96 \mathrm{E}-05$ & 0.02687217 & 0.08946648 & 0.02788802 & 5156299.01 \\
\hline 59689820 & 786.481015 & 811.515969 & 1025.87473 & 769.047883 & 0.26617419 & $2.53 \mathrm{E}-05$ & 75.106974 & 0.01249429 & 0.00938408 & $1.30 \mathrm{E}-05$ & $1.23 \mathrm{E}-05$ & $8.97 \mathrm{E}-05$ & 0.02687792 & 0.08953001 & 0.02792839 & 5163345.2 \\
\hline 59736657.3 & 786.481015 & 811.655446 & 1025.87093 & 769.118448 & 0.26852485 & $2.53 \mathrm{E}-05$ & 75.1088128 & 0.01250315 & 0.00939097 & $1.30 \mathrm{E}-05$ & $1.23 \mathrm{E}-05$ & $8.98 \mathrm{E}-05$ & 0.02688357 & 0.08959355 & 0.02796874 & 5167455.42 \\
\hline 59795203.9 & 786.481015 & 811.648843 & 1025.86619 & 769.117473 & 0.27146652 & $2.53 \mathrm{E}-05$ & 75.1111077 & 0.01251424 & 0.00939958 & $1.30 \mathrm{E}-05$ & $1.23 \mathrm{E}-05$ & $9.00 \mathrm{E}-05$ & 0.0268903 & 0.08967297 & 0.02801915 & 5171110.61 \\
\hline 59868387.2 & 786.481015 & 811.635139 & 1025.86026 & 769.11622 & 0.27514833 & $2.53 \mathrm{E}-05$ & 75.1139707 & 0.01252809 & 0.00941035 & $1.30 \mathrm{E}-05$ & $1.23 \mathrm{E}-05$ & $9.02 E-05$ & 0.02689909 & 0.08977224 & 0.02808215 & 5175689.25 \\
\hline 59959866.3 & 786.481015 & 811.619341 & 1025.85284 & 769.114662 & 0.27975715 & $2.53 \mathrm{E}-05$ & 75.1175404 & 0.01254541 & 0.0094238 & $1.30 \mathrm{E}-05$ & $1.23 \mathrm{E}-05$ & $9.04 E-05$ & 0.02691 & 0.08989633 & 0.02816089 & 5181405.75 \\
\hline 60006703.6 & \begin{tabular}{|l|l|}
786.41015 \\
\end{tabular} & 811.611335 & 1025.84904 & 769.113858 & 0.28211908 & $2.53 E-05$ & 75.1193643 & 0.01255427 & 0.00943069 & $1.30 \mathrm{E}-05$ & $1.23 \mathrm{E}-05$ & $9.05 E-05$ & 0.02691581 & 0.08995987 & 0.02820123 & 5188455.85 \\
\hline 60053540.9 & 786.481015 & 811.604318 & 1025.84524 & 769.113068 & 0.28448257 & $2.53 \mathrm{E}-05$ & 75.1211856 & 0.01256314 & 0.00943758 & $1.30 \mathrm{E}-05$ & $1.23 \mathrm{E}-05$ & $9.07 \mathrm{E}-05$ & 0.0269212 & 0.09002341 & 0.02824154 & 5192094.7 \\
\hline 60112087.5 & 786.481015 & 811.595499 & 1025.8405 & 769.112072 & 0.28743894 & $2.53 \mathrm{E}-05$ & 75.1234587 & 0.01257422 & 0.00944619 & $1.30 \mathrm{E}-05$ & $1.23 \mathrm{E}-05$ & $9.08 \mathrm{E}-05$ & 0.02692819 & 0.09010282 & 0.02829192 & 5195758.74 \\
\hline 60185270.8 & 786.481015 & 811.585591 & 1025.83456 & 769.110839 & 0.29113717 & $2.53 \mathrm{E}-05$ & 75.1262944 & 0.01258808 & 0.00945696 & $1.30 \mathrm{E}-05$ & $1.23 \mathrm{E}-05$ & $9.10 \mathrm{E}-05$ & 0.02693678 & 0.0902021 & 0.02835488 & 5200331.62 \\
\hline 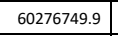 & 786.481015 & 811.573716 & 1025.82715 & 769.109294 & 0.29576354 & $\begin{array}{l}2.53 \mathrm{E}-05 \\
\end{array}$ & 75.1298302 & 0.0126054 & 0.00947041 & $1.30 \mathrm{E}-05$ & $1.23 \mathrm{E}-05$ & $9.13 \mathrm{E}-05$ & 0.02694789 & 0.09032619 & 0.02843356 & 5206051.42 \\
\hline 60368229 & 786.481015 & 811.562868 & 1025.81973 & 769.10775 & 0.30039312 & $2.53 \mathrm{E}-05$ & 75.1333563 & 0.01262271 & 0.00948387 & $1.30 \mathrm{E}-05$ & $1.23 \mathrm{E}-05$ & $9.15 \mathrm{E}-05$ & 0.0269589 & 0.09045028 & 0.02851223 & 5213172.53 \\
\hline 60459708.1 & 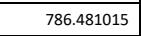 & 811.553184 & 1025.81231 & 769.106212 & 0.30502508 & $2.53 \mathrm{E}-05$ & 75.1368728 & 0.01264003 & 0.00949732 & $1.30 \mathrm{E}-05$ & $1.23 \mathrm{E}-05$ & $9.17 \mathrm{E}-05$ & 0.0269699 & 0.09057438 & 0.02859089 & 5220290.85 \\
\hline 60551187.2 & 786.481015 & 811.545269 & 1025.80488 & 769.104696 & 0.31003869 & $2.53 \mathrm{E}-05$ & $\begin{array}{ll}75.1403796 \\
\end{array}$ & 0.01265735 & 0.00951078 & $1.30 \mathrm{E}-05$ & $1.23 \mathrm{E}-05$ & $9.20 \mathrm{E}-05$ & 0.02698029 & 0.09069847 & 0.02866954 & 5227396.34 \\
\hline 60642666.3 & 786.481015 & 811.537795 & 1025.79746 & 769.103176 & 0.31511973 & $2.53 \mathrm{E}-05$ & $\begin{array}{ll}75.1438769 \\
\end{array}$ & 0.01267467 & 0.00952424 & $1.30 \mathrm{E}-05$ & $1.23 \mathrm{E}-05$ & $9.22 \mathrm{E}-05$ & 0.02699105 & 0.09082256 & 0.02874816 & 5234509.57 \\
\hline 60734145.4 & 786.481015 & 811.531011 & 1025.79003 & 769.101647 & 0.32020343 & $2.53 \mathrm{E}-05$ & 75.1473646 & 0.01269198 & 0.00953769 & $1.30 \mathrm{E}-05$ & $1.23 \mathrm{E}-05$ & $9.25 \mathrm{E}-05$ & 0.02700232 & 0.09094666 & 0.02882677 & 5241631.55 \\
\hline 60825624.5 & 786.481015 & 811.525331 & 1025.7826 & 769.100128 & 0.32528878 & $2.53 \mathrm{E}-05$ & 75.1508428 & 0.0127093 & 0.00955115 & $1.30 \mathrm{E}-05$ & $1.23 \mathrm{E}-05$ & $9.27 \mathrm{E}-05$ & 0.02701337 & 0.09107075 & 0.02890536 & 5248750.64 \\
\hline 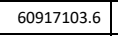 & 786.481015 & 811.52081 & 1025.77517 & 769.098647 & 0.33037486 & $2.53 \mathrm{E}-05$ & 75.1543116 & 0.01272662 & 0.0095646 & $1.30 \mathrm{E}-05$ & $1.23 \mathrm{E}-05$ & $9.30 \mathrm{E}-05$ & 0.02702381 & 0.09119484 & 0.02898394 & 5255859.23 \\
\hline 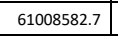 & 786.481015 & 811.516977 & 1025.76774 & 769.097172 & 0.33546077 & $2.53 \mathrm{E}-05$ & $\begin{array}{ll}75.1577709 \\
\end{array}$ & 0.01274394 & 0.00957806 & $1.30 \mathrm{E}-05$ & $1.23 \mathrm{E}-05$ & $9.32 \mathrm{E}-05$ & 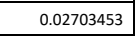 & 0.09131893 & 0.0290625 & 5262976.42 \\
\hline 61100061.7 & 786.481015 & 811.513988 & 1025.7603 & 769.095703 & $\begin{array}{l}0.3405457 \\
\end{array}$ & $2.53 \mathrm{E}-05$ & 75.1612208 & 0.01276125 & 0.00959151 & $1.30 \mathrm{E}-05$ & $1.23 \mathrm{E}-05$ & $9.35 \mathrm{E}-05$ & 0.02704528 & 0.09144303 & 0.02914104 & 5270094.14 \\
\hline 61191540.8 & 786.481015 & 811.511815 & 1025.75287 & 769.094239 & 0.34562891 & $\begin{array}{l}2.53 \mathrm{E}-05 \\
\end{array}$ & $\begin{array}{l}5.1646614 \\
\end{array}$ & 0.01277857 & 0.00960497 & $1.30 \mathrm{E}-05$ & $1.23 \mathrm{E}-05$ & $9.37 \mathrm{E}-05$ & 0.02705633 & 0.09156712 & 0.02921956 & 5277210.57 \\
\hline 61283019.9 & 786.481015 & 811.510459 & 1025.74543 & 769.092776 & 0.35070974 & $2.53 \mathrm{E}-05$ & 75.1680927 & 0.01279589 & 0.00961843 & $1.30 \mathrm{E}-05$ & $1.23 \mathrm{E}-05$ & $9.40 \mathrm{E}-05$ & 0.02706713 & 0.09169121 & 0.02929807 & 5284326.22 \\
\hline 61374499 & 786.481015 & 811.509897 & 1025.73799 & 769.091313 & 0.3557876 & $2.53 \mathrm{E}-05$ & 75.1715147 & 0.01281321 & 0.00963188 & $1.30 \mathrm{E}-05$ & $1.23 \mathrm{E}-05$ & $9.42 \mathrm{E}-05$ & 0.02707798 & 0.09181531 & 0.02937656 & 5291444.43 \\
\hline 61465978.1 & \begin{tabular}{|l}
786.481015 \\
\end{tabular} & 811.510155 & 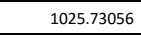 & 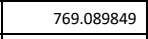 & 0.36102067 & $\begin{array}{l}2.53 \mathrm{E}-05 \\
\end{array}$ & 75.1749274 & 0.01283052 & 0.00964534 & $1.30 \mathrm{E}-05$ & $1.23 \mathrm{E}-05$ & $9.44 \mathrm{E}-05$ & 0.02708882 & 0.0919394 & 0.02945503 & 5298566.84 \\
\hline 61557457.2 & 786.481015 & 811.511135 & 1025.72312 & 769.088393 & 0.36650371 & $2.53 \mathrm{E}-05$ & 75.178331 & 0.01284784 & 0.00965879 & $1.30 \mathrm{E}-05$ & $1.23 \mathrm{E}-05$ & $9.47 E-05$ & 0.02709973 & 0.09206349 & 0.02953348 & 5305682.17 \\
\hline
\end{tabular}




\begin{tabular}{|c|c|c|c|c|c|c|c|c|c|c|c|c|c|c|c|c|}
\hline Time (s) & $\begin{array}{c}\text { Coolant outlet } \\
\text { temperature }(K)\end{array}$ & $\begin{array}{l}\text { Peak Cladding } \\
\text { Temperature (K) }\end{array}$ & $\begin{array}{c}\text { Peak fuel } \\
\text { temperature (K) }\end{array}$ & $\begin{array}{c}\text { Average cladding } \\
\text { internal } \\
\text { temperature (K) }\end{array}$ & $\begin{array}{l}\text { Peak cumulative } \\
\text { damage factor }\end{array}$ & $\begin{array}{l}\text { Cladding interior } \\
\text { volume }\left(\mathrm{m}^{3}\right)\end{array}$ & $\begin{array}{l}\text { Fission Gas } \\
\text { Release (\%) }\end{array}$ & $\begin{array}{c}\text { Fission gas } \\
\text { produced } \\
\text { (moles) }\end{array}$ & $\begin{array}{c}\text { Fission gas } \\
\text { released (moles) }\end{array}$ & Fuel volume $\left(\mathrm{m}^{3}\right)$ & $\begin{array}{l}\text { Plenum gas } \\
\text { volume }\left(m^{3}\right)\end{array}$ & $\begin{array}{l}\text { Peak cladding } \\
\text { radial growth } \\
\text { (m) }\end{array}$ & $\begin{array}{l}\text { Peak fuel axial } \\
\text { growth }(m)\end{array}$ & $\begin{array}{c}\text { Peak burnup } \\
(\text { (at\%) }\end{array}$ & $\begin{array}{l}\text { Peak cladding } \\
\text { hoop strain }\end{array}$ & $\begin{array}{l}\text { Plenum pressure } \\
\text { (Pa) }\end{array}$ \\
\hline 61648936.3 & 786.481015 & 811.513134 & 1025.71568 & $\begin{array}{l}769.086935 \\
\end{array}$ & 0.37198501 & $2.53 \mathrm{E}-05$ & 75.1817254 & 0.01286516 & 0.00967225 & $1.30 \mathrm{E}-05$ & $1.23 \mathrm{E}-05$ & 9.49E- 05 & 0.02711093 & 0.09218758 & 0.02961192 & 5312811.12 \\
\hline 61740415.4 & 786.481015 & 811.515577 & 1025.70825 & 769.085492 & 0.37746397 & $2.53 \mathrm{E}-05$ & 75.1851107 & 0.01288248 & 0.00968571 & $1.30 \mathrm{E}-05$ & $1.23 \mathrm{E}-05$ & $9.52 \mathrm{E}-05$ & 0.02712174 & 0.09231168 & 0.02969033 & 5319929.33 \\
\hline 61831894.5 & 786.481015 & 811.519072 & 1025.70081 & 769.084051 & 0.38293998 & $2.53 \mathrm{E}-05$ & 75.1884868 & 0.0128998 & 0.00969916 & $1.30 \mathrm{E}-05$ & $1.23 E-05$ & $9.54 E-05$ & 0.02713295 & 0.09243577 & 0.02976873 & 5327050.72 \\
\hline 61923373.6 & 786.481015 & 811.522923 & 1025.69337 & 769.082616 & 0.38841239 & $2.53 \mathrm{E}-05$ & 75.191854 & 0.01291711 & 0.00971262 & $1.30 \mathrm{E}-05$ & $1.23 \mathrm{E}-05$ & $9.57 \mathrm{E}-05$ & 0.02714358 & 0.09255986 & 0.02984712 & 5334168.07 \\
\hline 62014852.7 & 786.481015 & 811.527764 & 1025.68594 & 769.081178 & 0.39388067 & $2.53 \mathrm{E}-05$ & 75.1952121 & 0.01293443 & 0.00972607 & $1.30 \mathrm{E}-05$ & $1.23 \mathrm{E}-05$ & $9.59 \mathrm{E}-05$ & 0.02715448 & 0.09268395 & 0.02992548 & 5341291.34 \\
\hline 62106331.8 & 786.782841 & 811.83972 & 1025.85866 & 769.312052 & 0.39956671 & $2.53 \mathrm{E}-05$ & 75.1985612 & 0.01295175 & 0.00973953 & $1.30 \mathrm{E}-05$ & $1.23 \mathrm{E}-05$ & $9.62 \mathrm{E}-05$ & 0.02716598 & 0.09280805 & 0.03000565 & 5349961.42 \\
\hline 62125235 & 786.884213 & 811.94417 & 1025.91762 & $\begin{array}{l}769.38977 \\
\end{array}$ & \begin{tabular}{ll|}
0.40075784 \\
\end{tabular} & $2.53 \mathrm{E}-05$ & 75.1992521 & 0.01295533 & 0.00974231 & $1.30 \mathrm{E}-05$ & $1.23 \mathrm{E}-05$ & $9.62 E-05$ & 0.02716862 & 0.09283369 & 0.03002249 & 5357497.5 \\
\hline 62128835 & 780.31567 & 804.184104 & 1010.22864 & 763.622501 & 0.40079059 & $2.53 \mathrm{E}-05$ & 75.1993807 & 0.01295599 & 0.00974283 & $1.30 \mathrm{E}-05$ & $1.23 \mathrm{E}-05$ & $9.57 \mathrm{E}-05$ & 0.0271333 & 0.09283846 & 0.02985988 & 5320021.15 \\
\hline 62147738.2 & 780.31567 & 804.189 & 1010.22726 & \begin{tabular}{|l|l|}
763.622111 \\
\end{tabular} & 0.40095824 & $2.53 E-05$ & 75.2000397 & 0.01295941 & 0.00974548 & $1.30 \mathrm{E}-05$ & $1.23 \mathrm{E}-05$ & $9.57 \mathrm{E}-05$ & 0.02714349 & 0.09286294 & \begin{tabular}{|l|l|}
0.02986681 \\
\end{tabular} & 5320384.45 \\
\hline 62171367.2 & 780.31567 & 804.195672 & 1010.22557 & 763.621741 & 0.40116853 & $2.53 \mathrm{E}-05$ & 75.200863 & 0.01296368 & 0.0097488 & $1.30 \mathrm{E}-05$ & $1.23 \mathrm{E}-05$ & $9.58 \mathrm{E}-05$ & 0.02715171 & 0.09289353 & 0.02988409 & 5321881.05 \\
\hline 62200903.4 & 780.31567 & 804.202021 & 1010.22347 & 763.621313 & 0.40143446 & $2.53 \mathrm{E}-05$ & 75.2018914 & 0.01296901 & 0.00975294 & $1.30 \mathrm{E}-05$ & $1.23 \mathrm{E}-05$ & $9.58 \mathrm{E}-05$ & 0.02715906 & 0.09293177 & 0.02990749 & 5323700.59 \\
\hline 62237823.7 & 780.723644 & 804.622429 & 1010.46462 & $\begin{array}{l}763.934702 \\
\end{array}$ & 0.40179121 & $2.53 \mathrm{E}-05$ & 75.2031756 & 0.01297568 & 0.00975813 & $1.30 \mathrm{E}-05$ & $1.23 \mathrm{E}-05$ & $9.59 \mathrm{E}-05$ & 0.02716697 & 0.09297957 & 0.02993949 & 5328051.64 \\
\hline 62256058 & 780.752132 & 804.653545 & 1010.48035 & 763.956361 & 0.40196947 & $2.53 \mathrm{E}-05$ & 75.2038094 & 0.01297898 & 0.00976069 & $1.30 \mathrm{E}-05$ & $1.23 \mathrm{E}-05$ & $9.60 \mathrm{E}-05$ & 0.02716966 & 0.09300317 & 0.0299543 & 5330911.66 \\
\hline 62259658 & 784.014229 & 808.50624 & 1018.26468 & 766.819148 & 0.40206209 & $2.53 \mathrm{E}-05$ & 75.203936 & 0.01297964 & 0.0097612 & $1.30 \mathrm{E}-05$ & $1.23 \mathrm{E}-05$ & $9.63 \mathrm{E}-05$ & 0.02718787 & 0.09300789 & 0.03003976 & 5351580.78 \\
\hline 62277892.3 & 784.014229 & 808.506738 & 1018.26328 & 766.818943 & 0.40254084 & $2.53 E-05$ & 75.2045843 & 0.01298301 & 0.00976382 & $1.30 \mathrm{E}-05$ & $1.23 E-05$ & $9.63 \mathrm{E}-05$ & 0.0271871 & 0.09303206 & 0.03005892 & 5351857.46 \\
\hline 62300685.1 & 784.014229 & 808.507221 & 1018.26152 & 766.818624 & 0.40314343 & $2.53 \mathrm{E}-05$ & 75.2053943 & 0.01298723 & 0.00976709 & $1.30 \mathrm{E}-05$ & $1.23 \mathrm{E}-05$ & $9.64 E-05$ & 0.02718819 & 0.09306226 & 0.03007846 & 5353226.82 \\
\hline 62329176.1 & 784.014229 & 808.507963 & 1018.25933 & 766.818218 & 0.40390081 & $2.53 \mathrm{E}-05$ & 75.2064061 & $\begin{array}{l}0.01299249 \\
\end{array}$ & 0.00977119 & $1.30 \mathrm{E}-05$ & $1.23 E-05$ & $9.65 \mathrm{E}-05$ & 0.02718999 & 0.09310002 & 0.03010217 & 5354939.46 \\
\hline 62364789.9 & 784.014229 & 808.509105 & 1018.25661 & 766.817708 & 0.40485332 & $2.53 \mathrm{E}-05$ & 75.2076696 & 0.01299908 & 0.00977631 & $1.30 \mathrm{E}-05$ & $1.23 \mathrm{E}-05$ & $9.65 \mathrm{E}-05$ & 0.02719249 & 0.09314722 & 0.0301317 & 5357084.4 \\
\hline 62409307.2 & 784.014229 & 808.510802 & 1018.25319 & 766.817066 & 0.40605234 & $2.53 \mathrm{E}-05$ & 75.2092472 & 0.01300732 & 0.0097827 & $1.30 \mathrm{E}-05$ & $1.23 \mathrm{E}-05$ & $9.67 E-05$ & 0.02719588 & 0.09320622 & 0.0301686 & 5359766.26 \\
\hline 62464953.8 & 784.014229 & 808.513912 & 1018.24891 & 766.816261 & 0.4075629 & $2.53 \mathrm{E}-05$ & 75.2112164 & 0.01301761 & 0.0097907 & $1.30 \mathrm{E}-05$ & $1.23 E-05$ & $9.68 \mathrm{E}-05$ & 0.02720067 & 0.09327997 & 0.03021473 & 5363134.05 \\
\hline 62534512 & 784.014229 & 808.518339 & 1018.24352 & 766.815254 & 0.40946688 & $2.53 \mathrm{E}-05$ & 75.2136736 & 0.01303047 & 0.0098007 & $1.30 \mathrm{E}-05$ & $1.23 E-05$ & $9.70 \mathrm{E}-05$ & 0.02720699 & 0.09337215 & 0.03027239 & 5367344.12 \\
\hline 62620357 & 783.521737 & 808.024409 & 1017.94268 & 766.435016 & 0.41168631 & $2.53 \mathrm{E}-05$ & 75.2166994 & 0.01304635 & 0.00981303 & $1.30 \mathrm{E}-05$ & $1.23 E-05$ & $9.72 E-05$ & 0.02721398 & 0.09348592 & 0.03034057 & 5370061.52 \\
\hline 62623957 & 784.261754 & 808.899083 & 1019.70956 & 767.084692 & 0.41180203 & $2.53 \mathrm{E}-05$ & 75.2168264 & 0.01304702 & 0.00981355 & $1.30 \mathrm{E}-05$ & $1.23 E-05$ & $9.73 E-05$ & 0.02721833 & 0.09349071 & 0.03036224 & 5380854.95 \\
\hline 62709802 & 784.261754 & 808.906394 & 1019.70273 & 767.083441 & 0.41458465 & $2.54 \mathrm{E}-05$ & 75.2198604 & 0.01306298 & 0.00982595 & $1.30 \mathrm{E}-05$ & $1.23 \mathrm{E}-05$ & $9.75 \mathrm{E}-05$ & 0.02722643 & 0.09360507 & 0.03043501 & 5381228.32 \\
\hline 62817108.3 & 784.261754 & 808.917975 & 1019.69413 & 767.081853 & 0.41807818 & $2.54 \mathrm{E}-05$ & 75.2236425 & 0.01308293 & 0.00984145 & $1.30 \mathrm{E}-05$ & $1.23 \mathrm{E}-05$ & $9.78 \mathrm{E}-05$ & 0.02723836 & 0.09374803 & 0.03052466 & 5387799.08 \\
\hline 62951241.1 & 784.261754 & 808.934035 & 1019.68335 & 767.079862 & 0.42247039 & $2.54 \mathrm{E}-05$ & 75.2283539 & 0.01310787 & 0.00986083 & $1.30 \mathrm{E}-05$ & $1.23 E-05$ & $9.81 E-05$ & 0.02725337 & 0.09392673 & 0.03063665 & 5396017.99 \\
\hline 63085374 & 784.261754 & 808.951119 & 1019.67257 & 767.077881 & 0.42689482 & $2.54 \mathrm{E}-05$ & 75.2330474 & 0.0131328 & 0.00988021 & $1.30 \mathrm{E}-05$ & $1.23 E-05$ & $9.85 \mathrm{E}-05$ & 0.02726839 & 0.09410544 & 0.03074864 & 5406236.19 \\
\hline 63219506.8 & 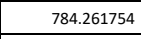 & 808.970642 & 1019.66183 & 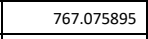 & 0.43135874 & $2.54 \mathrm{E}-05$ & 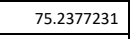 & 0.01315774 & 0.00989959 & $1.30 \mathrm{E}-05$ & $1.23 E-05$ & $9.88 \mathrm{E}-05$ & 0.02728391 & 0.09428414 & 0.03086062 & 5416461.16 \\
\hline 63353639.6 & 784.261754 & 808.991086 & 1019.65114 & 767.0739 & 0.4358688 & $2.54 E-05$ & 75.2423811 & 0.01318268 & 0.00991896 & $1.30 \mathrm{E}-05$ & $1.23 E-05$ & $9.92 E-05$ & 0.02729891 & 0.09446284 & 0.03097259 & 5426689.07 \\
\hline 63487772.5 & 784.261754 & 809.013229 & 1019.64051 & 767.071912 & 0.44043041 & $2.54 \mathrm{E}-05$ & 75.2470215 & 0.01320762 & 0.00993834 & $1.30 \mathrm{E}-05$ & $1.23 E-05$ & $9.95 E-05$ & 0.02731408 & 0.09464154 & 0.03108454 & 5436914.12 \\
\hline 63621905.3 & 784.261754 & $\begin{array}{l}809.03816 \\
\end{array}$ & 1019.62994 & 767.069925 & 0.44504743 & $2.54 \mathrm{E}-05$ & 75.2516444 & 0.01323256 & 0.00995772 & $1.30 \mathrm{E}-05$ & $1.23 \mathrm{E}-05$ & $9.99 E-05$ & 0.02732971 & 0.09482024 & 0.03119649 & 5447147.74 \\
\hline 63756038.1 & 784.261754 & 809.063821 & 1019.61943 & 767.067938 & 0.44972216 & $2.54 \mathrm{E}-05$ & 75.25625 & 0.0132575 & 0.00997709 & $1.31 E-05$ & $1.23 \mathrm{E}-05$ & 0.00010024 & 0.02734504 & 0.09499894 & 0.03130842 & 5457380.94 \\
\hline 63890171 & 784.261754 & 809.089062 & 1019.60896 & 767.065942 & 0.45445572 & $2.54 E-05$ & 75.2608382 & 0.01328243 & 0.00999647 & $1.31 E-05$ & $1.23 E-05$ & 0.00010059 & 0.02736034 & 0.09517764 & 0.03142034 & 5467607.34 \\
\hline 64024303.8 & 784.261754 & 809.115821 & 1019.59853 & 767.063943 & $\begin{array}{c}0.45924814 \\
\end{array}$ & $2.54 \mathrm{E}-05$ & 755.2654093 & 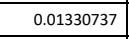 & 0.01001585 & $1.31 E-05$ & $1.23 E-05$ & 0.00010094 & 0.02737561 & 0.09535634 & 0.03153224 & 5477838.41 \\
\hline 64158436.6 & 784.261754 & 809.145527 & 1019.58814 & 767.061941 & 0.46409861 & $2.54 E-05$ & 75.2699632 & 0.01333231 & 0.01003523 & $1.31 \mathrm{E}-05$ & $1.23 E-05$ & 0.0001013 & 0.02739143 & 0.09553504 & 0.03164412 & 5488079.55 \\
\hline 64292569.5 & 784.261754 & 809.176193 & 1019.57778 & 767.059948 & 0.46900574 & $2.54 E-05$ & 75.2745002 & 0.01335725 & 0.0100546 & $1.31 E-05$ & $1.23 \mathrm{E}-05$ & 0.00010165 & 0.02740697 & 0.09571374 & 0.03175599 & 5498312.8 \\
\hline 64460235.5 & 784.261754 & 809.215816 & 1019.56486 & 767.057449 & 0.47522312 & $2.54 \mathrm{E}-05$ & 75.2801476 & 0.01338842 & 0.01007882 & $1.31 E-05$ & $1.23 \mathrm{E}-05$ & 0.00010209 & 0.02742595 & 0.09593711 & 0.03189578 & 5508599.18 \\
\hline 64516928 & 782.474049 & 807.413382 & 1018.49294 & 765.680771 & 0.47687687 & $2.54 \mathrm{E}-05$ & 75.2820512 & 0.01339896 & 0.01008701 & $1.31 \mathrm{E}-05$ & $1.23 E-05$ & 0.0001022 & 0.02742833 & 0.09601264 & 0.03193229 & 5511696.59 \\
\hline 64520528 & 781.616134 & 806.398784 & 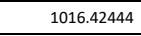 & 764.926481 & 0.47695827 & $2.54 \mathrm{E}-05$ & 75.2821716 & 0.01339963 & 0.01008753 & $1.31 \mathrm{E}-05$ & $1.23 E-05$ & 0.00010214 & 0.02742458 & 0.09601742 & 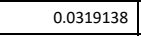 & 5510683.92 \\
\hline 64577220.5 & 781.616134 & 806.412465 & 1016.42013 & 764.925641 & 0.47824226 & $2.54 E-05$ & 75.2840604 & 0.01341011 & 0.01009567 & $1.31 E-05$ & $1.23 E-05$ & 0.00010228 & 0.02743158 & 0.09609249 & 0.03195888 & 5511035.54 \\
\hline 64648086.1 & 781.616134 & 806.403011 & 1016.4148 & 764.924491 & 0.47986526 & $2.54 \mathrm{E}-05$ & 75.2864172 & 0.0134232 & 0.01010585 & $1.31 \mathrm{E}-05$ & $1.23 \mathrm{E}-05$ & 0.00010246 & 0.02743848 & 0.09618632 & 0.03201725 & 5515321.48 \\
\hline 64736668.1 & 781.616134 & 806.379685 & 1016.40814 & 764.922978 & 0.48192313 & $2.54 \mathrm{E}-05$ & 75.2893567 & 0.01343957 & 0.01011856 & $1.31 E-05$ & $1.23 \mathrm{E}-05$ & 0.00010269 & 0.02744755 & 0.09630361 & 0.03209037 & 5520689.07 \\
\hline 64766586 & 782.095695 & 806.859665 & 1016.69226 & 765.291545 & 0.48266967 & $2.54 \mathrm{E}-05$ & 75.2903479 & 0.0134451 & 0.01012286 & $1.31 E-05$ & $1.23 E-05$ & 0.00010278 & 0.02745193 & 0.09634322 & 0.03211799 & 5529860.28 \\
\hline 64770186 & 780.46995 & 804.929584 & 1012.77861 & 763.862886 & 0.48272476 & $2.54 \mathrm{E}-05$ & 75.2904664 & 0.01344576 & 0.01012337 & $1.31 \mathrm{E}-05$ & $1.23 \mathrm{E}-05$ & 0.00010265 & 0.02744411 & 0.09634796 & 0.03207978 & 5522106.25 \\
\hline 64800103.9 & 780.46995 & \begin{tabular}{|l}
804.91658 \\
\end{tabular} & 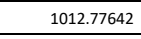 & $\begin{array}{l}763.862283 \\
\end{array}$ & $\begin{array}{l}0.48318161 \\
\end{array}$ & $2.54 \mathrm{E}-05$ & 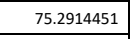 & 0.01345122 & 0.01012762 & $1.31 E-05$ & $1.23 E-05$ & 0.00010272 & 0.02745096 & 0.09638711 & 0.03210167 & 5522444.32 \\
\hline 64837501.2 & 780.46995 & 804.908519 & 1012.77367 & 763.861667 & 0.4837573 & $2.54 \mathrm{E}-05$ & 75.2926674 & 0.01345805 & 0.01013293 & $1.31 E-05$ & $1.23 \mathrm{E}-05$ & 0.00010281 & 0.02745464 & 0.09643605 & 0.03213167 & 5524683.37 \\
\hline
\end{tabular}




\begin{tabular}{|c|c|c|c|c|c|c|c|c|c|c|c|c|c|c|c|c|}
\hline Time (s) & $\begin{array}{c}\text { Coolant outlet } \\
\text { temperature }(K)\end{array}$ & $\begin{array}{l}\text { Peak Cladding } \\
\text { Temperature (K) }\end{array}$ & $\begin{array}{c}\text { Peak fuel } \\
\text { temperature (K) }\end{array}$ & $\begin{array}{c}\text { Average cladding } \\
\text { internal } \\
\text { temperature (K) }\end{array}$ & $\begin{array}{l}\text { Peak cumulative } \\
\text { damage factor }\end{array}$ & $\begin{array}{l}\text { Cladding interior } \\
\text { volume }\left(\mathrm{m}^{3}\right)\end{array}$ & $\begin{array}{l}\text { Fission Gas } \\
\text { Release (\%) }\end{array}$ & $\begin{array}{c}\text { Fission gas } \\
\text { produced } \\
\text { (moles) }\end{array}$ & $\begin{array}{c}\text { Fission gas } \\
\text { released (moles) }\end{array}$ & Fuel volume $\left(\mathrm{m}^{3}\right)$ & $\begin{array}{l}\text { Plenum gas } \\
\text { volume }\left(m^{3}\right)\end{array}$ & $\begin{array}{l}\text { Peak cladding } \\
\text { radial growth } \\
\text { (m) }\end{array}$ & $\begin{array}{l}\text { Peak fuel axial } \\
\text { growth }(m)\end{array}$ & $\begin{array}{c}\text { Peak burnup } \\
(\text { (at\%) }\end{array}$ & $\begin{array}{l}\text { Peak cladding } \\
\text { hoop strain }\end{array}$ & $\begin{array}{l}\text { Plenum pressure } \\
(\mathrm{Pa})\end{array}$ \\
\hline 64884247.9 & 780.46995 & 804.900941 & 1012.77024 & $\begin{array}{l}763.860938 \\
\end{array}$ & 0.4844854 & $2.54 \mathrm{E}-05$ & 75.2941935 & 0.01346659 & 0.01013956 & $1.31 \mathrm{E}-05$ & $1.23 \mathrm{E}-05$ & 0.00010293 & 0.02745824 & 0.09649723 & 0.03216959 & 5527460.7 \\
\hline 64942681.3 & 780.46995 & 804.890845 & 1012.76596 & 763.860016 & 0.48540891 & $2.54 \mathrm{E}-05$ & 75.2960984 & 0.01347726 & 0.01014785 & $1.31 \mathrm{E}-05$ & $1.23 \mathrm{E}-05$ & 0.00010308 & 0.0274631 & 0.09657369 & 0.03221703 & 5530937.65 \\
\hline 65015723 & 780.46995 & 804.879646 & 1012.76061 & 763.858868 & 0.486584 & $2.54 \mathrm{E}-05$ & 75.2984753 & 0.0134906 & 0.01015822 & $1.31 E-05$ & $1.23 E-05$ & 0.00010327 & 0.02746921 & 0.09666928 & 0.03227635 & 5535281.71 \\
\hline 65107025.2 & 780.46995 & 804.864312 & 1012.75393 & $\begin{array}{ll}763.857404 \\
\end{array}$ & 0.4880848 & $2.54 \mathrm{E}-05$ & 75.3014399 & 0.01350727 & 0.01017117 & $1.31 E-05$ & $1.23 \mathrm{E}-05$ & 0.0001035 & 0.02747791 & 0.09678876 & 0.03235051 & 5540727.65 \\
\hline 65221152.9 & 780.46995 & 804.845056 & 1012.74558 & 763.855539 & 0.49001005 & $2.54 \mathrm{E}-05$ & 75.3051353 & 0.01352812 & 0.01018737 & $1.31 E-05$ & $1.23 \mathrm{E}-05$ & 0.00010379 & 0.02748952 & 0.09693811 & 0.03244322 & 5547553.76 \\
\hline 65363812.4 & 780.46995 & 804.821998 & 1012.73515 & 763.853209 & 0.49249291 & $2.54 \mathrm{E}-05$ & 75.3097386 & 0.01355417 & 0.01020761 & $1.31 E-05$ & $1.23 \mathrm{E}-05$ & 0.00010415 & 0.02750442 & 0.09712479 & 0.03255912 & 5556087.09 \\
\hline 65506472 & 780.46995 & 804.798499 & 1012.72475 & \begin{tabular}{|l|}
763.850874 \\
\end{tabular} & 0.4950526 & $2.54 \mathrm{E}-05$ & 75.3143242 & 0.01358022 & 0.01022785 & $1.31 E-05$ & $1.23 \mathrm{E}-05$ & 0.00010452 & 0.02751952 & 0.09731148 & 0.03267505 & 5566714.05 \\
\hline 65649131.6 & 780.46995 & 804.773994 & 1012.71438 & 763.848516 & 0.49768969 & $2.54 \mathrm{E}-05$ & 75.3188922 & 0.01360627 & 0.0102481 & $1.31 E-05$ & $1.23 \mathrm{E}-05$ & 0.00010488 & 0.02753541 & 0.09749817 & 0.03279099 & 5577352.43 \\
\hline 65791791.2 & 780.46995 & 804.750779 & 1012.70404 & $\begin{array}{ll}763.846168 \\
\end{array}$ & 0.50040485 & $2.54 \mathrm{E}-05$ & 75.3234428 & 0.01363233 & 0.01026834 & $1.31 \mathrm{E}-05$ & $1.23 \mathrm{E}-05$ & 0.00010525 & 0.02755078 & 0.09768486 & $\begin{array}{ll}0.03290694 \\
\end{array}$ & 5587984.64 \\
\hline 65934450.8 & 780.46995 & 804.727116 & 1012.69374 & 763.843808 & 0.50319863 & $2.54 \mathrm{E}-05$ & 75.327976 & 0.01365838 & 0.01028858 & $1.31 E-05$ & $1.23 \mathrm{E}-05$ & 0.00010561 & 0.02756642 & 0.09787154 & 0.0330229 & 5598620.67 \\
\hline 66007492.6 & 780.46995 & 804.715175 & 1012.68847 & 763.842598 & 0.50465032 & $2.54 \mathrm{E}-05$ & 75.3302903 & 0.01367172 & 0.01029895 & $1.31 E-05$ & $1.23 \mathrm{E}-05$ & 0.0001058 & 0.0275745 & 0.09796713 & 0.03308231 & 5609160.91 \\
\hline 66080534.3 & 780.46995 & 804.703145 & 1012.68321 & 763.841382 & 0.50612276 & $2.54 \mathrm{E}-05$ & 75.3326001 & 0.01368506 & 0.01030931 & $1.31 E-05$ & $1.23 \mathrm{E}-05$ & 0.00010599 & 0.02758276 & 0.09806271 & 0.03314168 & 5614610.9 \\
\hline 66171836.4 & 780.46995 & 804.688357 & 1012.67666 & 763.839864 & 0.50799555 & $2.54 \mathrm{E}-05$ & 75.3354811 & 0.01370173 & 0.01032227 & $1.31 E-05$ & $1.23 \mathrm{E}-05$ & 0.00010622 & 0.02759294 & 0.09818219 & 0.03321589 & 5620085.14 \\
\hline 66285964.1 & 780.46995 & 804.670167 & 1012.6685 & 763.837962 & 0.51038666 & $2.54 E-05$ & 75.3390724 & 0.01372258 & 0.01033846 & $1.31 E-05$ & $1.23 \mathrm{E}-05$ & 0.00010651 & 0.02760565 & 0.09833154 & 0.03330865 & 5626929.53 \\
\hline 66428623.7 & 780.46995 & 804.648177 & 1012.65832 & 763.835587 & 0.51345288 & $2.54 E-05$ & 75.3435463 & 0.01374863 & 0.0103587 & $1.31 \mathrm{E}-05$ & $1.23 E-05$ & 0.00010688 & 0.02762149 & 0.09851823 & 0.03342461 & 5635482.64 \\
\hline 66571283.3 & 780.46995 & 804.626336 & 1012.64816 & 763.833196 & 0.5165955 & $2.54 \mathrm{E}-05$ & 75.3480032 & 0.01377468 & 0.01037895 & $1.31 E-05$ & $1.23 \mathrm{E}-05$ & 0.00010724 & 0.02763764 & 0.09870492 & 0.03354058 & 5646131.89 \\
\hline 66713942.9 & 780.46995 & 804.605355 & 1012.63802 & 763.830815 & 0.51981282 & $2.54 \mathrm{E}-05$ & 75.3524433 & 0.01380073 & 0.01039919 & $1.31 \mathrm{E}-05$ & $1.23 E-05$ & 0.00010761 & 0.02765373 & 0.0988916 & 0.03365655 & 5656776.85 \\
\hline 66856602.5 & 780.46995 & 804.58522 & $\begin{array}{l}1012.6279 \\
\end{array}$ & 763.82843 & 0.52310273 & $2.54 \mathrm{E}-05$ & 75.3568666 & 0.01382679 & 0.01041943 & $1.31 E-05$ & $1.23 \mathrm{E}-05$ & 0.00010797 & 0.02766974 & 0.09907829 & 0.03377252 & 5667423.39 \\
\hline 66999262.1 & 780.46995 & 804.565913 & 1012.61779 & 763.826038 & 0.52646286 & $2.54 E-05$ & 75.3612734 & 0.01385284 & 0.01043968 & $1.31 E-05$ & $1.23 \mathrm{E}-05$ & 0.00010834 & 0.02768586 & 0.09926498 & 0.03388849 & 5678071.65 \\
\hline 67141921.7 & 780.46995 & 804.54742 & 1012.60769 & 763.823644 & 0.5298906 & $2.54 \mathrm{E}-05$ & 75.3656635 & 0.01387889 & 0.01045992 & $1.31 E-05$ & $1.23 E-05$ & 0.0001087 & 0.02770202 & 0.09945167 & 0.03400445 & 5688722.8 \\
\hline 67284581.3 & 780.46995 & 804.53006 & 1012.59759 & 763.821257 & 0.53338322 & $2.54 \mathrm{E}-05$ & 75.3700373 & 0.01390495 & 0.01048016 & $1.31 E-05$ & $1.23 E-05$ & 0.00010907 & 0.02771825 & 0.09963835 & 0.03412063 & 5699369.63 \\
\hline 67427240.9 & 780.46995 & 804.513645 & 1012.5875 & 763.818865 & 0.53693784 & $2.54 \mathrm{E}-05$ & 75.3743946 & 0.013931 & 0.01050041 & $1.31 E-05$ & $1.23 E-05$ & 0.00010943 & 0.02773435 & 0.09982504 & 0.03423685 & 5710023.52 \\
\hline 67535546 & 781.971694 & 806.026304 & 1013.47737 & 764.972642 & 0.54032584 & $2.54 E-05$ & 75.3776918 & 0.01395078 & 0.01051577 & $1.31 E-05$ & $1.23 E-05$ & 0.00010974 & 0.02775064 & 0.09996677 & 0.03433481 & 5728957.64 \\
\hline 67539146 & 780.327827 & $\begin{array}{l}804.0857 \\
\end{array}$ & 1009.54872 & 763.530609 & 0.5403943 & $2.54 \mathrm{E}-05$ & 75.3778006 & 0.01395143 & 0.01051628 & $1.31 \mathrm{E}-05$ & $1.23 \mathrm{E}-05$ & 0.00010962 & 0.02774291 & 0.09997146 & 0.03429624 & $\begin{array}{r}726442.1 \\
\end{array}$ \\
\hline 67647451.1 & 780.327827 & 804.074884 & 1009.54113 & 763.528799 & 0.54246697 & $2.54 \mathrm{E}-05$ & 75.3810494 & 0.01397098 & 0.01053147 & $1.31 E-05$ & $1.23 \mathrm{E}-05$ & 0.00010988 & 0.02775695 & 0.10011151 & 0.0343798 & 5726877.93 \\
\hline 67782832.5 & 780.327827 & 804.063804 & 1009.53175 & 763.526651 & 0.54512136 & $2.54 \mathrm{E}-05$ & 75.3850976 & 0.01399541 & 0.01055045 & $1.31 E-05$ & $1.23 E-05$ & 0.00011022 & 0.02777042 & 0.10028658 & 0.03448816 & 5734880.55 \\
\hline 67952059.2 & 780.327827 & 804.050588 & 1009.52003 & 763.523937 & 0.54853678 & $2.54 \mathrm{E}-05$ & 75.390138 & 0.01402595 & 0.01057418 & $1.31 \mathrm{E}-05$ & $1.23 \mathrm{E}-05$ & 0.00011065 & 0.02778838 & 0.10050542 & $\begin{array}{l}\mid 0.03462379 \\
\end{array}$ & 5744898.22 \\
\hline 68121286 & \begin{tabular}{|l}
780.327827 \\
\end{tabular} & \begin{tabular}{|l}
804.038841 \\
\end{tabular} & 1009.50832 & 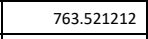 & $\begin{array}{l}0.55204618 \\
\end{array}$ & $2.54 \mathrm{E}-05$ & 75.3951565 & 0.01405649 & 0.01059791 & $1.31 E-05$ & $1.23 E-05$ & 0.00011107 & 0.02780685 & 0.10072426 & 0.03475946 & 5757372.26 \\
\hline 68332819.4 & 780.327827 & 804.026692 & 1009.49368 & 763.517811 & 0.55657146 & $2.54 E-05$ & 75.401399 & 0.01409466 & 0.01062757 & $1.31 E-05$ & $1.23 \mathrm{E}-05$ & 0.00011161 & 0.02783053 & 0.1009978 & 0.03492903 & 5769917.98 \\
\hline 68544352.8 & 780.327827 & 804.018046 & 1009.47904 & 763.514421 & 0.56122959 & $2.54 \mathrm{E}-05$ & 75.4076078 & 0.01413284 & 0.01065723 & $1.31 E-05$ & $1.23 E-05$ & 0.00011214 & 0.02785424 & 0.10127135 & 0.03509863 & 5785523.59 \\
\hline 68755886.2 & 780.327827 & 804.013257 & 1009.46442 & 763.511037 & 0.56601408 & $2.54 \mathrm{E}-05$ & 75.4137832 & 0.01417101 & 0.0106869 & $1.31 E-05$ & $1.23 \mathrm{E}-05$ & 0.00011267 & 0.02787802 & 0.1015449 & 0.03526824 & 5801132.37 \\
\hline 689967419.6 & 780.327827 & 803.979695 & 1009.44981 & 763.507509 & 0.57093646 & $2.55 \mathrm{E}-05$ & 75.4199254 & 0.01420919 & 0.01071656 & $1.32 E-05$ & $1.23 \mathrm{E}-05$ & 0.00011321 & 0.02790187 & 0.10181844 & 0.03543784 & 5816742.46 \\
\hline 69178953 & 780.327827 & 803.944307 & 1009.43521 & 763.503964 & 0.57650247 & $2.55 E-05$ & 75.4260346 & 0.01424736 & 0.01074622 & $1.32 E-05$ & $1.23 E-05$ & 0.00011374 & 0.02792588 & 0.10209199 & 0.03560743 & 5832354.52 \\
\hline 69390486.5 & 780.327827 & 803.915249 & 1009.42061 & 763.500435 & 0.58219159 & $2.55 \mathrm{E}-05$ & $\begin{array}{l}75.4321112 \\
\end{array}$ & 0.01428554 & 0.01077588 & $1.32 \mathrm{E}-05$ & $1.23 E-05$ & 0.00011427 & 0.02794977 & 0.10236554 & 0.03577701 & 5847970.25 \\
\hline 69602019.9 & 780.327827 & 803.910239 & 1009.40603 & 763.496931 & 0.58799538 & $2.55 \mathrm{E}-05$ & 75.4381555 & 0.01432371 & 0.01080554 & $1.32 E-05$ & $1.23 E-05$ & 0.00011481 & 0.02797383 & 0.10263908 & 0.03594658 & 5863586.38 \\
\hline 69813553.3 & 780.327827 & 803.905547 & 1009.39145 & 763.49344 & 0.59390498 & $2.55 E-05$ & 75.4441676 & 0.01436188 & 0.0108352 & $1.32 E-05$ & $1.23 \mathrm{E}-05$ & 0.00011534 & 0.02799784 & 0.10291263 & 0.03611612 & 5879210.22 \\
\hline 70025086.7 & 780.327827 & 803.900887 & 1009.37687 & 763.489995 & 0.59991213 & $2.55 \mathrm{E}-05$ & 75.4501478 & 0.01440000 & 0.01086487 & $1.32 E-05$ & $1.23 E-05$ & 0.00011588 & 0.02802186 & 0.10318618 & 0.03628564 & 5894823.96 \\
\hline 70236620.1 & 780.327827 & 803.896266 & 1009.36229 & 763.486556 & 0.60600862 & $2.55 \mathrm{E}-05$ & 75.4560964 & 0.01443823 & 0.01089453 & $1.32 E-05$ & $1.23 E-05$ & 0.00011641 & 0.02804589 & 0.10345972 & 0.03645513 & 5910449.74 \\
\hline 70448153.5 & 780.327827 & 803.89169 & 1009.34772 & 763.483149 & 0.61218655 & $2.55 \mathrm{E}-05$ & 75.4620136 & $\begin{array}{l}0.01447641 \\
\end{array}$ & $\begin{array}{l}0.01092419 \\
\end{array}$ & $1.32 E-05$ & $1.23 E-05$ & 0.00011694 & 0.02807004 & 0.10373327 & 0.03662459 & 5926073.98 \\
\hline 70659687 & 780.327827 & 803.887163 & 1009.33316 & 763.479767 & 0.61843846 & $2.55 \mathrm{E}-05$ & 75.4678997 & 0.01451458 & 0.01095385 & $1.32 E-05$ & $1.23 E-05$ & 0.00011748 & 0.0280941 & 0.10400682 & 0.03679402 & 5941701.4 \\
\hline 70871220.4 & 780.327827 & 803.889441 & 1009.31859 & 763.47644 & 0.62475773 & $2.55 \mathrm{E}-05$ & 75.4737549 & 0.01455276 & 0.01098351 & $1.32 \mathrm{E}-05$ & $1.23 \mathrm{E}-05$ & 0.00011801 & 0.02811728 & 0.10428036 & 0.03696341 & 5957312.37 \\
\hline 71082753.8 & 780.327827 & 803.887874 & 1009.30403 & 763.472981 & 0.63113863 & $2.55 \mathrm{E}-05$ & 75.4795795 & 0.01459093 & 0.01101317 & $1.32 E-05$ & $1.23 \mathrm{E}-05$ & 0.00011855 & 0.02814189 & 0.10455391 & 0.03713276 & 5972944.87 \\
\hline 71191058.9 & 780.327827 & 803.880184 & 1009.29657 & 763.471182 & 0.63442241 & $2.55 \mathrm{E}-05$ & 75.4825499 & 0.01461048 & 0.01102836 & $1.32 E-05$ & $1.23 E-05$ & 0.00011882 & 0.02815392 & 0.10469397 & 0.03721951 & 5988419.34 \\
\hline 71299364 & 780.327827 & 803.873654 & $\begin{array}{r}1009.28912 \\
\end{array}$ & 763.469372 & 0.63772054 & $2.55 \mathrm{E}-05$ & 75.4855123 & 0.01463002 & 0.01104355 & $1.32 E-05$ & $1.23 \mathrm{E}-05$ & 0.00011909 & 0.02816656 & 0.10483402 & 0.03730619 & 5996424.33 \\
\hline 71434745.4 & $\begin{array}{l}780.327827 \\
\end{array}$ & $\begin{array}{l}803.871007 \\
\end{array}$ & 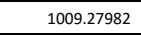 & 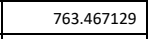 & 0.64186364 & $2.55 \mathrm{E}-05$ & $\begin{array}{l}75.4892043 \\
\end{array}$ & 0.01465446 & 0.01106253 & $1.32 E-05$ & $1.23 E-05$ & 0.00011943 & 0.02818146 & 0.10500909 & $\begin{array}{l}0.03741451 \\
\end{array}$ & 6004467.26 \\
\hline 71570126.8 & 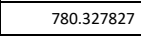 & 803.868148 & \begin{tabular}{|l|l|}
1009.27051 \\
\end{tabular} & $\begin{array}{l}763.464893 \\
\end{array}$ & 0.64602633 & $2.55 \mathrm{E}-05$ & 75.492884 & $\begin{array}{l}0.01467889 \\
\end{array}$ & 0.01108151 & $1.32 E-05$ & $1.23 \mathrm{E}-05$ & 0.00011978 & 0.02819693 & 0.10518416 & 0.03752282 & 6014469.05 \\
\hline
\end{tabular}




\begin{tabular}{|c|c|c|c|c|c|c|c|c|c|c|c|c|c|c|c|c|}
\hline Time (s) & $\begin{array}{c}\text { Coolant outlet } \\
\text { temperature }(K)\end{array}$ & $\begin{array}{l}\text { Peak Cladding } \\
\text { Temperature (K) }\end{array}$ & $\begin{array}{c}\text { Peak fuel } \\
\text { temperature (K) }\end{array}$ & $\begin{array}{c}\text { Average cladding } \\
\text { internal } \\
\text { temperature (K) }\end{array}$ & $\begin{array}{l}\text { Peak cumulative } \\
\text { damage factor }\end{array}$ & $\begin{array}{l}\text { Cladding interior } \\
\text { volume }\left(\mathrm{m}^{3}\right)\end{array}$ & $\begin{array}{l}\text { Fission Gas } \\
\text { Release (\%) }\end{array}$ & $\begin{array}{c}\text { Fission gas } \\
\text { produced } \\
\text { (moles) }\end{array}$ & $\begin{array}{c}\text { Fission gas } \\
\text { released (moles) }\end{array}$ & Fuel volume $\left(\mathrm{m}^{3}\right)$ & $\begin{array}{l}\text { Plenum gas } \\
\text { volume }\left(m^{3}\right)\end{array}$ & $\begin{array}{l}\text { Peak cladding } \\
\text { radial growth } \\
\text { (m) }\end{array}$ & $\begin{array}{l}\text { Peak fuel axial } \\
\text { growth }(m)\end{array}$ & $\begin{array}{c}\text { Peak burnup } \\
(\text { (at\%) }\end{array}$ & $\begin{array}{l}\text { Peak cladding } \\
\text { hoop strain }\end{array}$ & $\begin{array}{l}\text { Plenum pressure } \\
(\mathrm{Pa})\end{array}$ \\
\hline 71705508.2 & 780.327827 & 803.8654 & 1009.26121 & $\begin{array}{l}763.462658 \\
\end{array}$ & 0.65020725 & $2.55 \mathrm{E}-05$ & 75.4965514 & 0.01470332 & 0.0111005 & $1.32 \mathrm{E}-05$ & $1.23 \mathrm{E}-05$ & 0.00012012 & 0.02821246 & 0.10535923 & 0.03763112 & 6024476.16 \\
\hline 71840889.6 & 780.327827 & 803.862562 & 1009.25191 & 763.460425 & 0.65440511 & $2.55 E-05$ & 75.5002067 & 0.01472775 & 0.01111948 & $1.32 E-05$ & $1.23 \mathrm{E}-05$ & 0.00012046 & 0.02822788 & 0.1055343 & 0.0377394 & 6034480.88 \\
\hline 72010116.3 & 780.327827 & 803.859194 & 1009.24029 & 763.457647 & 0.6596756 & $2.55 E-05$ & 75.5047588 & 0.01475829 & 0.01114321 & $1.32 E-05$ & $1.23 \mathrm{E}-05$ & 0.00012089 & 0.02824707 & 0.10575314 & 0.03787469 & 6044539.44 \\
\hline 72155534 & 780.327827 & 803.861585 & 1009.23031 & 763.455275 & 0.66422143 & $2.55 \mathrm{E}-05$ & 75.5086554 & 0.01478453 & 0.0111636 & $1.32 E-05$ & $1.23 \mathrm{E}-05$ & 0.00012125 & 0.02826329 & 0.10594119 & 0.03799096 & 6057002.45 \\
\hline 72159134 & 780.327827 & 803.861547 & 1009.23005 & 763.455221 & 0.66433404 & $2.55 \mathrm{E}-05$ & 75.5087517 & 0.01478518 & 0.01116411 & $1.32 E-05$ & $1.23 \mathrm{E}-05$ & 0.00012126 & 0.02826348 & 0.10594584 & 0.03799389 & 6067531.16 \\
\hline 72185697 & 781.864795 & 805.421669 & 1010.14781 & 764.637232 & 0.66539787 & $2.55 \mathrm{E}-05$ & 75.5094619 & 0.01478998 & 0.01116783 & $1.32 E-05$ & $1.23 \mathrm{E}-05$ & 0.00012136 & 0.02827035 & 0.10598019 & 0.038025 & 6076879.13 \\
\hline 72189297 & 782.255999 & 805.881906 & $\begin{array}{l}1011.07439 \\
\end{array}$ & 764.979637 & $\begin{array}{ll}0.6655674 \\
\end{array}$ & $2.55 \mathrm{E}-05$ & 75.5095583 & 0.01479063 & 0.01116834 & $1.32 \mathrm{E}-05$ & $1.23 \mathrm{E}-05$ & 0.0001214 & 0.02827268 & 0.10598486 & 0.03803807 & 6081439.96 \\
\hline 72193797 & 782.255999 & 805.881998 & 1011.07415 & 764.97957 & 0.66577824 & $2.55 \mathrm{E}-05$ & 75.5096789 & 0.01479144 & 0.01116897 & $1.32 E-05$ & $1.23 \mathrm{E}-05$ & 0.00012141 & 0.02827292 & 0.10599069 & 0.03804195 & 6081711.54 \\
\hline 72199422 & 782.255999 & 805.882161 & 1011.07379 & $\begin{array}{ll}764.979478 \\
\end{array}$ & 0.66604102 & $2.55 \mathrm{E}-05$ & 75.5098296 & 0.01479246 & 0.01116976 & $1.32 \mathrm{E}-05$ & $1.23 \mathrm{E}-05$ & 0.00012143 & 0.02827346 & 0.10599799 & 0.03804671 & 6082047.64 \\
\hline 72206453.3 & 782.255999 & 805.882376 & 1011.07332 & 764.979362 & 0.66636838 & $2.55 \mathrm{E}-05$ & 75.510018 & 0.01479373 & 0.01117075 & $1.32 E-05$ & $1.23 \mathrm{E}-05$ & 0.00012145 & 0.02827429 & 0.10600711 & 0.03805247 & 6082467.95 \\
\hline 72215242.3 & 782.255999 & 805.882752 & 1011.07272 & 764.97921 & 0.66677609 & $2.55 \mathrm{E}-05$ & 75.5102534 & 0.01479532 & 0.01117199 & $1.32 E-05$ & $1.23 \mathrm{E}-05$ & 0.00012147 & 0.0282754 & 0.1060185 & 0.03805957 & 6082996.87 \\
\hline 72226228.6 & 782.255999 & 805.883192 & $\begin{array}{l}1011.07197 \\
\end{array}$ & $\begin{array}{l}764.979023 \\
\end{array}$ & 0.66728389 & $2.55 \mathrm{E}-05$ & 75.5105477 & 0.01479731 & 0.01117353 & $1.32 E-05$ & $1.23 \mathrm{E}-05$ & 0.0001215 & 0.02827684 & 0.10603275 & 0.03806842 & 6083655.07 \\
\hline 72239961.6 & 782.255999 & 805.883803 & 1011.07103 & 764.978786 & 0.66791644 & $2.55 \mathrm{E}-05$ & 75.5109153 & 0.0147998 & 0.01117546 & $1.32 E-05$ & $1.23 \mathrm{E}-05$ & 0.00012153 & 0.02827864 & 0.10605056 & 0.03807946 & 6084479.69 \\
\hline 72257127.7 & 782.255999 & 805.884556 & 1011.06986 & 764.978494 & 0.66870454 & $2.55 \mathrm{E}-05$ & 75.5113748 & 0.0148029 & 0.01117788 & $1.32 E-05$ & $1.23 \mathrm{E}-05$ & 0.00012158 & 0.02828088 & 0.10607282 & 0.03809325 & 6085509.65 \\
\hline 72278585.4 & 782.255999 & 805.885487 & 1011.06838 & 764.978131 & 0.66968661 & $2.55 \mathrm{E}-05$ & 75.5119487 & 0.01480679 & 0.01118089 & $1.32 E-05$ & $1.23 E-05$ & 0.00012163 & 0.0282837 & 0.10610065 & 0.03811049 & 6086795.3 \\
\hline 72305407.5 & 782.255999 & 805.886686 & 1011.06654 & 764.97768 & 0.67091059 & $2.55 \mathrm{E}-05$ & 75.5126658 & 0.01481164 & 0.01118466 & $1.32 E-05$ & $1.23 \mathrm{E}-05$ & 0.0001217 & 0.02828708 & 0.10613543 & 0.03813203 & 6088402.11 \\
\hline 72338935.1 & 782.255999 & 805.888328 & 1011.06424 & 764.977114 & 0.67243612 & $2.55 \mathrm{E}-05$ & 75.5135615 & 0.01481771 & 0.01118938 & $1.32 \mathrm{E}-05$ & $1.23 E-05$ & 0.00012178 & 0.02829127 & 0.10617891 & 0.03815896 & 6090412.46 \\
\hline 72380844.6 & 782.255999 & 805.890262 & 1011.06135 & 764.976418 & 0.67433738 & $2.55 \mathrm{E}-05$ & 75.5146801 & 0.01482529 & 0.01119527 & $1.32 E-05$ & $1.23 \mathrm{E}-05$ & 0.00012189 & 0.02829634 & 0.10623326 & 0.03819261 & 6092917.96 \\
\hline 72433231.5 & 782.255999 & 805.893186 & 1011.05773 & 764.975536 & 0.67670644 & $2.55 \mathrm{E}-05$ & 75.5160767 & 0.01483477 & 0.01120264 & $1.32 E-05$ & $1.23 \mathrm{E}-05$ & 0.00012202 & 0.02830275 & 0.10630119 & 0.03823467 & 6096059.75 \\
\hline 72498715.1 & 782.255999 & 805.896879 & 1011.0532 & 764.974443 & 0.67965909 & $2.55 \mathrm{E}-05$ & 75.5178199 & 0.01484662 & 0.01121185 & $1.32 E-05$ & $1.23 E-05$ & 0.00012219 & 0.02831047 & 0.10638611 & 0.03828724 & 6099979.09 \\
\hline 72580569.6 & 782.255999 & 805.901502 & 1011.04754 & 764.973105 & 0.68333926 & $2.55 \mathrm{E}-05$ & 75.5199951 & 0.01486144 & 0.01122336 & $1.32 E-05$ & $1.23 E-05$ & $\begin{array}{l}0.0001224 \\
\end{array}$ & 0.02831998 & 0.10649226 & 0.03835294 & 6104863.36 \\
\hline 72682887.8 & 782.255999 & 805.908595 & 1011.04046 & 764.971393 & 0.68792146 & $2.55 \mathrm{E}-05$ & 75.5227079 & 0.01487995 & 0.01123774 & $1.32 E-05$ & $1.23 E-05$ & 0.00012266 & 0.02833184 & 0.10662495 & 0.03843505 & 6110990.37 \\
\hline 72785205.9 & 782.255999 & 805.916406 & 1011.03337 & 764.969703 & 0.69248532 & $2.55 \mathrm{E}-05$ & 75.525414 & 0.01489847 & 0.01125213 & $1.32 E-05$ & $1.23 \mathrm{E}-05$ & 0.00012291 & 0.028344 & 0.10675763 & 0.03851715 & 6118598.41 \\
\hline 72913103.6 & 782.255999 & 805.926916 & 1011.02451 & 764.967599 & 0.69815978 & $2.55 \mathrm{E}-05$ & 75.5287872 & 0.01492162 & 0.01127012 & $1.32 \mathrm{E}-05$ & $1.23 \mathrm{E}-05$ & 0.00012324 & 0.028359 & 0.10692349 & 0.03861974 & 6126242.53 \\
\hline 73041001.3 & 782.255999 & 805.936967 & 1011.01565 & 764.965539 & 0.70380638 & $2.55 \mathrm{E}-05$ & 75.5321499 & 0.01494476 & 0.0112881 & $1.32 E-05$ & $1.23 \mathrm{E}-05$ & 0.00012356 & 0.0283726 & 0.10708935 & 0.03872234 & 6135730.28 \\
\hline 73200873.4 & 782.255999 & 805.954306 & 1011.00457 & 764.962883 & 0.71081834 & $2.55 \mathrm{E}-05$ & 75.5363387 & 0.0149737 & 0.01131058 & $1.32 E-05$ & $1.23 E-05$ & 0.00012397 & 0.02839217 & 0.10729667 & 0.03885055 & 6145306.08 \\
\hline 73360745.5 & 782.255999 & 805.971726 & 1010.99349 & 764.960311 & 0.71778648 & $2.55 \mathrm{E}-05$ & 75.5405113 & 0.01500263 & 0.01133306 & $1.32 E-05$ & $1.23 E-05$ & 0.00012437 & 0.02841085 & 0.10750399 & 0.03897871 & 6157194.49 \\
\hline \begin{tabular}{|l}
73520617.7 \\
\end{tabular} & \begin{tabular}{|l}
782.255999 \\
\end{tabular} & 805.989252 & 1010.98241 & 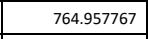 & $\begin{array}{l}0.72470848 \\
\end{array}$ & $2.55 \mathrm{E}-05$ & $\begin{array}{l}755.5446679 \\
\end{array}$ & 0.01503156 & 0.01135554 & $1.32 E-05$ & $1.23 E-05$ & 0.00012478 & 0.02842887 & 0.10771131 & 0.03910687 & 6169066.61 \\
\hline 73680489.8 & 782.255999 & 806.00769 & 1010.97133 & 764.955225 & 0.73158748 & $2.55 E-05$ & 75.5488085 & 0.01506049 & 0.01137802 & $1.32 E-05$ & $1.23 E-05$ & 0.00012518 & 0.02844678 & 0.10791863 & 0.039235 & 6180940.26 \\
\hline 73840361.9 & 782.255999 & 806.029945 & 1010.96026 & 764.952638 & 0.73842648 & $2.55 \mathrm{E}-05$ & 75.5529332 & 0.01508943 & 0.0114005 & $1.32 E-05$ & $1.23 E-05$ & 0.00012558 & 0.02846579 & 0.10812596 & 0.03936313 & 6192841.2 \\
\hline 74000234 & 782.255999 & 806.052018 & 1010.9492 & 764.950068 & 0.74522722 & $2.55 \mathrm{E}-05$ & 75.5570421 & 0.01511836 & 0.01142298 & $1.33 E-05$ & $1.23 \mathrm{E}-05$ & 0.00012599 & 0.02848445 & 0.10833328 & 0.03949126 & 6204728.16 \\
\hline 74160106.1 & 782.255999 & 806.07371 & 1010.93814 & 764.947516 & 0.75199309 & $2.55 \mathrm{E}-05$ & 75.5611354 & 0.01514729 & 0.01144547 & $1.33 E-05$ & $1.23 \mathrm{E}-05$ & 0.00012639 & 0.02850218 & 0.1085406 & 0.03961932 & 6216606.89 \\
\hline 74319978.2 & 782.255999 & 806.096575 & 1010.9271 & 764.944961 & 0.7587246 & $2.55 \mathrm{E}-05$ & 75.565213 & 0.01517622 & 0.01146795 & $1.33 E-05$ & $1.23 E-05$ & 0.0001268 & 0.02852059 & 0.10874792 & 0.03974731 & 6228487.48 \\
\hline 74479850.3 & 782.255999 & 806.118036 & 1010.91607 & 764.942336 & $\begin{array}{l}0.7654236 \\
\end{array}$ & $2.55 \mathrm{E}-05$ & 755.5692751 & $\begin{array}{l}0.01520516 \\
\end{array}$ & 0.01149043 & $1.33 \mathrm{E}-05$ & $1.23 E-05$ & 0.0001272 & 0.02853975 & 0.10895525 & $\begin{array}{l}0.0398753 \\
\end{array}$ & 6240393.85 \\
\hline 74639722.5 & 782.255999 & 806.138678 & 1010.90505 & 764.939733 & 0.77208969 & $2.55 E-05$ & 75.5733218 & 0.01523409 & 0.01151291 & $1.33 \mathrm{E}-05$ & $1.23 E-05$ & 0.00012761 & 0.02855854 & 0.10916257 & 0.04000321 & 6252285.65 \\
\hline 74799594.6 & 782.255999 & 806.165849 & 1010.89404 & 764.937159 & 0.77872415 & $2.55 E-05$ & 75.5773532 & 0.01526302 & 0.01153539 & $1.33 E-05$ & $1.23 \mathrm{E}-05$ & 0.00012801 & 0.02857721 & 0.10936989 & 0.04013109 & 6264180.13 \\
\hline 74959466.7 & 782.255999 & 806.199642 & 1010.88304 & 764.934608 & 0.785328 & $2.56 \mathrm{E}-05$ & 75.5813692 & 0.01529195 & 0.01155787 & $1.33 E-05$ & $1.23 E-05$ & 0.00012841 & 0.02859587 & 0.10957721 & 0.04025894 & 6276079.83 \\
\hline 75119338.8 & 782.255999 & 806.149616 & 1010.87204 & 764.93165 & 0.79189979 & $2.56 \mathrm{E}-05$ & 75.5853702 & 0.01532089 & 0.01158035 & $1.33 E-05$ & $1.23 E-05$ & 0.00012882 & 0.02861451 & 0.10978453 & 0.0403867 & 6287971.49 \\
\hline 75279210.9 & 782.255999 & 806.083119 & 1010.86105 & 764.928612 & 0.79844021 & $2.56 \mathrm{E}-05$ & 75.589356 & 0.01534982 & 0.01160283 & $1.33 \mathrm{E}-05$ & $1.23 E-05$ & 0.00012922 & 0.02863307 & 0.10999186 & 0.04051449 & 6299859.64 \\
\hline 75439083 & 782.255999 & 806.03646 & 1010.85007 & 764.926121 & 0.80494949 & $2.56 \mathrm{E}-05$ & 75.5933268 & 0.01537875 & 0.01162531 & $1.33 E-05$ & $1.23 E-05$ & 0.00012963 & 0.02865112 & 0.11019918 & 0.04064221 & 6311744.9 \\
\hline 75598955.2 & 782.255999 & 805.991669 & 1010.8391 & 764.923655 & $\begin{array}{l}0.8114287 \\
\end{array}$ & $2.56 \mathrm{E}-05$ & 75.5972828 & 0.01540769 & $\begin{array}{l}0.01164779 \\
\end{array}$ & $1.33 E-05$ & $1.23 \mathrm{E}-05$ & 0.00013003 & 0.02866997 & 0.1104065 & 0.04076992 & 6323653.27 \\
\hline 75758827.3 & 782.255999 & 805.952088 & 1010.82813 & 764.92123 & 0.81787754 & $2.56 \mathrm{E}-05$ & 75.6012238 & 0.01543662 & 0.01167027 & $1.33 E-05$ & $1.23 \mathrm{E}-05$ & 0.00013043 & 0.02868856 & 0.11061382 & 0.04089759 & 6335549.39 \\
\hline 75918699.4 & 782.255999 & 805.91765 & 1010.81716 & 764.918831 & 0.82429634 & $2.56 \mathrm{E}-05$ & 75.6051502 & 0.01546555 & 0.01169275 & $1.33 E-05$ & $1.23 E-05$ & 0.00013084 & 0.02870663 & 0.11082114 & 0.0410252 & 6347439.04 \\
\hline 76078571.5 & 782.255999 & 805.884649 & 1010.8062 & 764.916428 & 0.83068695 & $2.56 \mathrm{E}-05$ & 75.6090619 & 0.01549448 & 0.01171523 & $1.33 E-05$ & $1.23 \mathrm{E}-05$ & 0.00013124 & 0.02872544 & 0.11102847 & 0.04115273 & 6359349.34 \\
\hline 76238443.6 & $\begin{array}{l}782.255999 \\
\end{array}$ & 805.856163 & 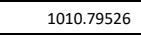 & 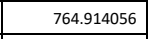 & $\begin{array}{l}0.83705122 \\
\end{array}$ & $2.56 \mathrm{E}-05$ & 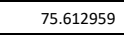 & 0.01552342 & 0.01173771 & $1.33 E-05$ & $1.23 E-05$ & 0.00013164 & 0.02874402 & 0.11123579 & 0.04128025 & 6371248.78 \\
\hline 76398315.7 & 782.255999 & 805.830974 & 1010.78432 & 764.911695 & 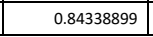 & $2.56 \mathrm{E}-05$ & 75.6168416 & 0.01555235 & 0.01176019 & $1.33 E-05$ & $1.23 \mathrm{E}-05$ & 0.00013205 & 0.02876258 & 0.11144311 & 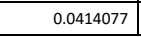 & 6383155.54 \\
\hline
\end{tabular}




\begin{tabular}{|c|c|c|c|c|c|c|c|c|c|c|c|c|c|c|c|c|}
\hline Time (s) & $\begin{array}{l}\text { Coolant outlet } \\
\text { temperature }(\mathrm{K})\end{array}$ & $\begin{array}{l}\text { Peak Cladding } \\
\text { Temperature (K) }\end{array}$ & $\begin{array}{c}\text { Peak fuel } \\
\text { temperature (K) }\end{array}$ & \begin{tabular}{|c} 
Average cladding \\
internal \\
temperature (K)
\end{tabular} & $\begin{array}{l}\text { Peak cumulative } \\
\text { damage factor }\end{array}$ & $\begin{array}{c}\text { Cladding interior } \\
\text { volume }\left(\mathrm{m}^{3}\right)\end{array}$ & $\begin{array}{l}\text { Fission Gas } \\
\text { Release (\%) }\end{array}$ & 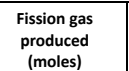 & $\begin{array}{c}\text { Fission gas } \\
\text { released (moles) }\end{array}$ & Fuel volume $\left(\mathrm{m}^{3}\right)$ & $\begin{array}{l}\text { Plenum gas } \\
\text { volume }\left(m^{3}\right)\end{array}$ & $\begin{array}{l}\text { Peak cladding } \\
\text { radial growth } \\
(\mathrm{m})\end{array}$ & $\begin{array}{l}\text { Peak fuel axial } \\
\text { growth (m) }\end{array}$ & $\begin{array}{l}\text { Peak burnup } \\
\text { (at\%) }\end{array}$ & $\begin{array}{l}\text { Peak cladding } \\
\text { hoop strain }\end{array}$ & $\begin{array}{c}\text { Plenum pressure } \\
(\mathrm{Pa})\end{array}$ \\
\hline 76558187.9 & 782.255999 & 805.810026 & 1010.77339 & 764.909367 & \begin{tabular}{ll|}
0.84970084 \\
\end{tabular} & $2.56 \mathrm{E}-05$ & 75.6207098 & 0.01558128 & 0.01178268 & $1.33 E-05$ & $1.23 \mathrm{E}-05$ & 0.00013245 & 0.02878067 & 0.11165043 & 0.04153512 & 6395045.63 \\
\hline 76718060 & 782.255999 & 805.79114 & 1010.76246 & $\begin{array}{l}764.90704 \\
\end{array}$ & \begin{tabular}{|l|l|}
0.85598797 \\
\end{tabular} & $2.56 \mathrm{E}-05$ & 75.6245636 & 0.01561021 & 0.01180516 & $1.33 \mathrm{E}-05$ & $1.23 \mathrm{E}-05$ & 0.00013286 & 0.02879941 & 0.11185775 & 0.04166251 & 6406955.46 \\
\hline 76833647 & 781.272965 & 804.788629 & 1010.16605 & 764.149111 & 0.85982523 & $2.56 \mathrm{E}-05$ & 75.6273411 & 0.01563113 & 0.01182141 & $1.33 E-05$ & $1.23 E-05$ & 0.00013313 & 0.02881025 & 0.11200765 & 0.04174762 & 6412677.15 \\
\hline 76837247 & 782.66454 & 806.427973 & 1013.47491 & 765.367531 & 0.86004882 & $2.56 \mathrm{E}-05$ & 75.6274279 & 0.01563179 & 0.01182192 & $1.33 E-05$ & $1.23 \mathrm{E}-05$ & 0.00013325 & 0.02881786 & 0.11201234 & 0.04180857 & 6431001.97 \\
\hline 76952834 & 782.66454 & 806.425453 & 1013.46808 & 765.365862 & 0.86677264 & $2.56 \mathrm{E}-05$ & 75.6302255 & 0.01565292 & 0.01183834 & $1.33 \mathrm{E}-05$ & $1.23 \mathrm{E}-05$ & 0.00013357 & 0.0288298 & 0.11216374 & 0.04189476 & 6431441.01 \\
\hline 77097317.8 & 782.66454 & 806.421758 & 1013.45858 & 765.363725 & 0.87477111 & $2.56 \mathrm{E}-05$ & 75.6337118 & 0.01567933 & 0.01185886 & $1.33 \mathrm{E}-05$ & $1.23 \mathrm{E}-05$ & 0.00013394 & 0.02884798 & 0.11235299 & 0.04200682 & 6440218.35 \\
\hline 77241801.6 & 782.66454 & 806.418677 & 1013.44873 & 765.361614 & 0.88253495 & $2.56 \mathrm{E}-05$ & 75.6371865 & 0.01570574 & 0.01187938 & $1.33 E-05$ & $1.23 \mathrm{E}-05$ & 0.00013431 & 0.02886567 & 0.11254224 & 0.04212153 & 6451105.59 \\
\hline 77340548 & 782.66454 & 806.416557 & 1013.44191 & 765.360175 & 0.88775789 & $2.56 \mathrm{E}-05$ & 75.6395545 & 0.01572379 & 0.0118934 & $1.33 \mathrm{E}-05$ & $1.23 \mathrm{E}-05$ & 0.00013456 & 0.0288778 & 0.11267158 & 0.04220048 & 6461919.48 \\
\hline 77344148 & 782.66454 & 806.416547 & 1013.44165 & $\begin{array}{ll}765.360118 \\
\end{array}$ & 0.88791093 & $2.56 \mathrm{E}-05$ & 75.6396407 & 0.01572444 & 0.01189391 & $1.33 E-05$ & $1.23 \mathrm{E}-05$ & 0.00013457 & 0.02887829 & 0.1126763 & 0.04220341 & 6469197.8 \\
\hline 77742894.4 & 782.66454 & 806.414687 & 1013.43481 & 765.358699 & 0.89204988 & $2.56 \mathrm{E}-05$ & 75.6420031 & 0.0157425 & 0.01190794 & $1.33 \mathrm{E}-05$ & $1.23 \mathrm{E}-05$ & 0.00013482 & 0.02888982 & 0.11280564 & 0.04228251 & 6469626.29 \\
\hline 77566327.4 & 782.66454 & 806.412253 & 1013.42622 & 765.356926 & 0.89714472 & $2.56 \mathrm{E}-05$ & 75.6449485 & 0.01576506 & 0.01192547 & $1.33 \mathrm{E}-05$ & $1.23 \mathrm{E}-05$ & 0.00013513 & 0.02890516 & 0.11296731 & 0.04238158 & 6477109.31 \\
\hline 77720618.6 & 782.100518 & 805.838747 & 1013.07791 & $\begin{array}{l}764.920774 \\
\end{array}$ & 0.90283479 & $2.56 \mathrm{E}-05$ & 75.6486183 & 0.01579326 & 0.01194738 & $1.33 \mathrm{E}-05$ & $1.23 \mathrm{E}-05$ & 0.00013551 & 0.02892289 & 0.11316941 & 0.04250156 & 6482922.4 \\
\hline 77738400 & 782.100518 & 805.83827 & 1013.07664 & 764.92052 & 0.90348975 & $2.56 \mathrm{E}-05$ & 75.6490404 & 0.01579651 & 0.01194991 & $1.33 \mathrm{E}-05$ & $1.23 \mathrm{E}-05$ & 0.00013556 & 0.02892525 & 0.1131927 & 0.04251588 & 6494304.44 \\
\hline 77742000 & 782.745069 & 806.598028 & 1014.61215 & 765.485059 & 0.90365458 & $2.56 \mathrm{E}-05$ & 75.6491261 & 0.01579717 & 0.01195042 & $1.33 E-05$ & $1.23 \mathrm{E}-05$ & 0.00013562 & 0.02892896 & 0.11319743 & 0.04253803 & 6500259.13 \\
\hline 77759781.4 & 782.745069 & 806.597888 & 1014.61112 & 765.484821 & 0.90447599 & $2.56 \mathrm{E}-05$ & 75.6495499 & 0.01580044 & 0.01195296 & $1.33 \mathrm{E}-05$ & $1.23 \mathrm{E}-05$ & 0.00013567 & 0.02893024 & 0.11322082 & 0.04255407 & 6500542.65 \\
\hline 77782008.2 & 782.745069 & 806.597351 & $\begin{array}{l}1014.60972 \\
\end{array}$ & $\begin{array}{l}765.484505 \\
\end{array}$ & 0.90550442 & $2.56 \mathrm{E}-05$ & 755.6500794 & 0.01580452 & 0.01195613 & $1.33 \mathrm{E}-05$ & $1.23 \mathrm{E}-05$ & 0.00013573 & 0.02893288 & 0.11325007 & 0.0425724 & 6501893.23 \\
\hline 77809791.7 & 782.745069 & 806.596572 & 1014.6079 & 765.484106 & 0.90678693 & $2.56 \mathrm{E}-05$ & 75.650741 & 0.01580962 & 0.01196009 & $1.33 E-05$ & $1.23 E-05$ & 0.0001358 & 0.02893655 & 0.11328663 & 0.04259475 & 6503588.23 \\
\hline 77844521 & 782.745069 & 806.595699 & 1014.60558 & 765.483607 & 0.90838206 & $2.56 \mathrm{E}-05$ & 75.6515673 & 0.015816 & 0.01196505 & $1.33 E-05$ & $1.23 E-05$ & 0.00013589 & 0.02894131 & 0.11333233 & 0.04262257 & 6505711.18 \\
\hline 77887932.7 & 782.745069 & 806.594849 & 1014.60263 & 765.482982 & 0.91036207 & $2.56 \mathrm{E}-05$ & 75.6525993 & 0.01582397 & 0.01197124 & $1.33 \mathrm{E}-05$ & $1.23 \mathrm{E}-05$ & 0.00013601 & 0.02894718 & 0.11338946 & 0.04265738 & 6508366.91 \\
\hline 77942197.3 & 782.745069 & 806.593825 & 1014.59888 & 765.482209 & 0.91281575 & $2.56 \mathrm{E}-05$ & 75.6538879 & 0.01583393 & 0.01197899 & $1.33 E-05$ & $1.23 E-05$ & 0.00013614 & 0.02895418 & 0.11346086 & 0.04270098 & 6511675.51 \\
\hline 78010028 & 782.745069 & 806.592578 & 1014.59415 & 765.481246 & 0.91585135 & $2.56 \mathrm{E}-05$ & 75.6554963 & 0.01584639 & 0.01198867 & $1.33 \mathrm{E}-05$ & $1.23 \mathrm{E}-05$ & 0.00013632 & 0.02896273 & 0.11355012 & 0.04275559 & 6515806.39 \\
\hline 78094816.4 & \begin{tabular}{|l}
782.745069 \\
\end{tabular} & 806.59098 & 1014.58822 & $\begin{array}{l}765.480049 \\
\end{array}$ & 0.91959965 & $2.56 \mathrm{E}-05$ & 755.6575032 & 0.01586196 & 0.01200076 & $1.33 \mathrm{E}-05$ & $1.23 E-05$ & 0.00013654 & 0.02897362 & 0.1136617 & 0.04282395 & 6520975.25 \\
\hline 78200801.9 & 782.745069 & 806.597601 & 1014.58078 & 765.478557 & 0.92421712 & $2.56 \mathrm{E}-05$ & 75.6600063 & 0.01588142 & 0.01201589 & $1.33 E-05$ & $1.23 E-05$ & 0.00013681 & 0.02898668 & 0.11380116 & 0.04290948 & 6527426.64 \\
\hline 78333283.9 & 782.745069 & 806.609583 & 1014.57149 & 765.476701 & 0.92988895 & $2.56 \mathrm{E}-05$ & 75.6631266 & 0.01590575 & 0.01203479 & $1.33 E-05$ & $1.23 E-05$ & 0.00013715 & 0.02900306 & 0.11397549 & 0.04301643 & 6535501.78 \\
\hline 78465765.8 & 782.745069 & 806.623282 & 1014.5622 & 765.474863 & 0.9354676 & $2.56 \mathrm{E}-05$ & 75.6662374 & 0.01593008 & 0.01205369 & $1.33 E-05$ & $1.23 \mathrm{E}-05$ & 0.00013749 & 0.02901973 & 0.11414983 & 0.04312343 & 6545523.86 \\
\hline 78631368.2 & 782.745069 & 806.645368 & 1014.5506 & 765.472572 & 0.94230337 & $2.56 \mathrm{E}-05$ & 75.6701125 & 0.01596049 & 0.01207732 & $1.33 E-05$ & $1.23 E-05$ & 0.00013791 & 0.02904095 & 0.11436774 & 0.04325713 & 6555632.81 \\
\hline 78796970.5 & 782.745069 & 806.669393 & 1014.539 & 765.470297 & 0.94616303 & 2.56 E- 05 & 75.6739728 & 0.0159909 & 0.01210095 & $1.34 E-05$ & $1.23 E-05$ & 0.00013833 & 0.02906117 & 0.11458566 & 0.04339081 & 6568168.43 \\
\hline 78962572.9 & 782.745069 & 806.697186 & 1014.52741 & 765.468039 & 0.94995397 & 2.56 E- 05 & 75.6778186 & 0.01602132 & 0.01212458 & $1.34 E-05$ & $1.23 \mathrm{E}-05$ & 0.00013876 & 0.0290819 & 0.11480357 & 0.04352444 & 6580706.66 \\
\hline 79128175.3 & 782.745069 & 806.728618 & 1014.51582 & 765.465803 & 0.95368083 & $2.56 \mathrm{E}-05$ & 75.6816497 & 0.01605173 & 0.01214821 & $1.34 E-05$ & $1.23 E-05$ & 0.00013918 & 0.02910241 & 0.11502149 & 0.04365802 & 6593242.18 \\
\hline 79335178.3 & 782.745069 & 806.775088 & 1014.50137 & 765.463034 & 0.95824604 & $2.56 E-05$ & 75.6864183 & 0.01608974 & 0.01217775 & $1.34 E-05$ & $1.23 E-05$ & 0.00013971 & 0.02912872 & 0.11529388 & 0.04382491 & 6605868.26 \\
\hline 79542181.3 & 782.745069 & 806.827036 & 1014.48691 & 765.460296 & 0.96272529 & $2.56 \mathrm{E}-05$ & 75.6911644 & 0.01612775 & 0.01220728 & $1.34 E-05$ & $1.23 \mathrm{E}-05$ & 0.00014024 & 0.02915465 & 0.11556627 & 0.04399175 & 6621547.17 \\
\hline 79749184.3 & 782.745069 & 806.883769 & 1014.47247 & 765.457567 & 0.96712495 & $2.56 \mathrm{E}-05$ & 75.6958881 & 0.01616577 & 0.01223682 & $1.34 \mathrm{E}-05$ & $1.23 E-05$ & 0.00014077 & 0.02917989 & 0.11583867 & 0.0441585 & 6637221.26 \\
\hline 79956187.2 & 782.745069 & 806.9494966 & 1014.45804 & $\begin{array}{ll}765.454882 \\
\end{array}$ & 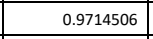 & $2.56 \mathrm{E}-05$ & 75.7005897 & 0.01620378 & 0.01226636 & $1.34 \mathrm{E}-05$ & $1.23 E-05$ & 0.0001413 & 0.02920613 & 0.11611106 & 0.04432515 & 6652919.87 \\
\hline 80163190.2 & 782.745069 & 807.015593 & 1014.44362 & 765.452259 & 0.97570799 & $2.56 E-05$ & 75.7052693 & 0.01624179 & 0.01229589 & $1.34 E-05$ & $1.23 E-05$ & 0.00014183 & 0.02923165 & 0.11638346 & 0.04449172 & 6668590.99 \\
\hline 80370193.2 & 782.745069 & 807.019866 & 1014.42923 & 765.449288 & 0.97990213 & 2.56 E- 05 & 75.7099271 & 0.01627981 & 0.01232543 & $1.34 \mathrm{E}-05$ & $1.23 \mathrm{E}-05$ & 0.00014236 & 0.02925702 & 0.11665585 & 0.04465821 & 6684274.9 \\
\hline 80444447 & 782.745069 & 807.001861 & 1014.42405 & 765.44811 & 0.98139964 & $2.56 \mathrm{E}-05$ & 75.7115925 & 0.01629344 & 0.01233603 & 1.34 E- 05 & $1.23 \mathrm{E}-05$ & 0.00014255 & 0.02926631 & 0.11675356 & 0.04471799 & 6699718.53 \\
\hline 80448047 & 644.15 & 644.172403 & 644.15337 & 644.150942 & 0.98139964 & $2.55 \mathrm{E}-05$ & 75.7116329 & 0.01629377 & 0.01233628 & $1.32 E-05$ & $1.23 \mathrm{E}-05$ & 0.00013356 & 0.0278167 & 0.11675593 & 0.04211477 & 5642757.09 \\
\hline 80451647 & 305 & 305.051392 & 305.006402 & 305.002155 & 0.98139964 & $2.51 E-05$ & 75.7116329 & 0.01629377 & 0.01233628 & $1.30 \mathrm{E}-05$ & $1.21 \mathrm{E}-05$ & 0.00011122 & 0.02565196 & 0.11675593 & 0.03620498 & 2702908.27 \\
\hline 80455247 & 305 & 305.051392 & 305.006402 & 305.002155 & 0.98139964 & $2.51 \mathrm{E}-05$ & 75.7116329 & 0.01629377 & 0.01233628 & $1.30 \mathrm{E}-05$ & $1.21 \mathrm{E}-05$ & 0.00011122 & 0.02565196 & 0.11675593 & 0.03620498 & 2702908.27 \\
\hline
\end{tabular}


TUNED PIN T654 FULL RESULTS

\begin{tabular}{|c|c|c|c|c|c|c|c|c|c|c|c|c|c|c|c|c|}
\hline Time (s) & $\begin{array}{l}\text { Coolant outlet } \\
\text { temperature (K) }\end{array}$ & $\begin{array}{c}\text { Peak cladding } \\
\text { temperature }(\mathrm{K})\end{array}$ & $\begin{array}{c}\text { Peak fuel } \\
\text { temperature (K) }\end{array}$ & $\begin{array}{c}\text { Average cladding } \\
\text { internal } \\
\text { temperature (K) }\end{array}$ & $\begin{array}{c}\text { Peak cumulative } \\
\text { damage factor }\end{array}$ & $\begin{array}{l}\text { Cladding interior } \\
\text { volume }\left(\mathrm{m}^{3}\right)\end{array}$ & $\begin{array}{l}\text { Fission gas } \\
\text { release (\%) }\end{array}$ & $\begin{array}{l}\text { Fission gas } \\
\text { produced } \\
\text { (moles) }\end{array}$ & $\begin{array}{c}\text { Fission gas } \\
\text { released (moles) }\end{array}$ & Fuel volume $\left(\mathrm{m}^{3}\right)$ & $\begin{array}{l}\text { Plenum gas } \\
\text { volume }\left(m^{3}\right)\end{array}$ & $\begin{array}{l}\text { Peak cladding } \\
\text { radial growth } \\
\text { (m) }\end{array}$ & $\begin{array}{l}\text { Peak fuel axial } \\
\text { growth }(m)\end{array}$ & $\begin{array}{c}\text { Peak burnup } \\
\text { (at\%) }\end{array}$ & $\begin{array}{l}\text { Peak cladding } \\
\text { hoop strain }\end{array}$ & $\begin{array}{c}\text { Plenum pressure } \\
(\mathrm{Pa})\end{array}$ \\
\hline 0 & & 295 & 295 & 295 & 0 & $2.45 \mathrm{E}-05$ & & 0 & 0 & $8.80 E-06$ & $1.57 \mathrm{E}-05$ & 0 & 0 & 0 & 0 & \\
\hline 100 & 309.333714 & 310.330309 & 322.569249 & 308.870699 & 0 & $2.45 \mathrm{E}-05$ & 0 & $3.30 \mathrm{E}-10$ & 0 & $8.81 E-06$ & $1.57 \mathrm{E}-05$ & $6.67 \mathrm{E}-07$ & 0.00011878 & $2.36 \mathrm{E}-09$ & \begin{tabular}{|l|}
0.00018117 \\
\end{tabular} & 95462.2273 \\
\hline 225 & 327.292835 & 329.527786 & 354.264971 & 326.243436 & 0 & $2.45 \mathrm{E}-05$ & 0 & $1.67 \mathrm{E}-09$ & 0 & $8.82 \mathrm{E}-06$ & $1.57 \mathrm{E}-05$ & $1.54 \mathrm{E}-06$ & 0.00026069 & $1.20 \mathrm{E}-08$ & 0.00041832 & 100798.673 \\
\hline 381.25 & 349.81139 & 353.558427 & 390.975152 & 348.007682 & 0 & $2.45 \mathrm{E}-05$ & 0 & $4.79 E-09$ & 0 & $8.83 E-06$ & $1.57 \mathrm{E}-05$ & $2.65 \mathrm{E}-06$ & 0.0004306 & $3.43 \mathrm{E}-08$ & 0.00071767 & 107474.771 \\
\hline 576.5625 & $\begin{array}{ll}378.073328 \\
\end{array}$ & 383.671396 & 433.910346 & \begin{tabular}{|l|l|}
375.289787 \\
\end{tabular} & $1.30 \mathrm{E}-65$ & $2.45 \mathrm{E}-05$ & 0 & $1.10 \mathrm{E}-08$ & 0 & $8.85 \mathrm{E}-06$ & $1.57 \mathrm{E}-05$ & $4.03 E-06$ & 0.00063486 & $\begin{array}{ll}7.85 \mathrm{E}-08 \\
\end{array}$ & 0.00109336 & 115830.056 \\
\hline 820.703125 & 413.582692 & 421.457767 & 484.495688 & 409.513857 & $9.62 E-56$ & $2.46 \mathrm{E}-05$ & 0 & $2.22 \mathrm{E}-08$ & 0 & $8.86 \mathrm{E}-06$ & $1.57 \mathrm{E}-05$ & $5.77 \mathrm{E}-06$ & 0.00088158 & $1.59 \mathrm{E}-07$ & 0.00156539 & 126291.027 \\
\hline 1125.87891 & 458.252644 & 468.921383 & 544.629671 & 452.484985 & $4.04 E-46$ & $2.46 \mathrm{E}-05$ & 0 & $4.18 \mathrm{E}-08$ & 0 & $8.89 \mathrm{E}-06$ & $1.57 \mathrm{E}-05$ & $7.96 \mathrm{E}-06$ & 0.00118122 & $2.99 \mathrm{E}-07$ & 0.00215902 & 139394.381 \\
\hline 1507.34863 & 514.514727 & 528.717635 & 616.822905 & 506.499004 & $7.97 E-37$ & $2.47 \mathrm{E}-05$ & 0 & $7.49 \mathrm{E}-08$ & 0 & $8.92 E-06$ & $1.58 \mathrm{E}-05$ & $1.07 \mathrm{E}-05$ & 0.0015474 & $5.37 \mathrm{E}-07$ & 0.002907 & 155816.852 \\
\hline 1984.18579 & 585.443117 & 604.178198 & 704.694652 & 574.481626 & $6.63 \mathrm{E}-28$ & $2.47 \mathrm{E}-05$ & 0 & $1.30 \mathrm{E}-07$ & 0 & $8.95 \mathrm{E}-06$ & $1.58 \mathrm{E}-05$ & $1.42 \mathrm{E}-05$ & 0.00199782 & $9.30 \mathrm{E}-07$ & 0.00385112 & 176411.644 \\
\hline 2580.23224 & 674.870048 & 699.796488 & 812.777605 & 660.160291 & $1.70 \mathrm{E}-19$ & $2.48 \mathrm{E}-05$ & 0 & $2.19 \mathrm{E}-07$ & 0 & $8.99 \mathrm{E}-06$ & $1.58 \mathrm{E}-05$ & $1.86 \mathrm{E}-05$ & 0.00254906 & $1.57 \mathrm{E}-06$ & $\begin{array}{ll}0.00504493 \\
\end{array}$ & 202250.864 \\
\hline 3325.2903 & 787.424599 & 820.130514 & 947.637065 & 768.222657 & $1.05 E-11$ & $2.49 \mathrm{E}-05$ & 0 & $3.65 \mathrm{E}-07$ & 0 & $9.05 \mathrm{E}-06$ & $1.59 \mathrm{E}-05$ & $2.42 \mathrm{E}-05$ & 0.00323218 & $2.61 \mathrm{E}-06$ & 0.00655168 & 234662.009 \\
\hline 3600 & 829.01858 & 864.98067 & 997.785682 & 808.288043 & $8.24 E-10$ & $2.50 \mathrm{E}-05$ & 0 & $4.27 \mathrm{E}-07$ & 0 & $9.07 E-06$ & $1.59 \mathrm{E}-05$ & $2.63 \mathrm{E}-05$ & 0.00347403 & $3.06 \mathrm{E}-06$ & 0.00711122 & 246628.966 \\
\hline 4345.05806 & 829.01858 & 864.985998 & \begin{tabular}{|l|l|}
997.791167 \\
\end{tabular} & \begin{tabular}{|l|l|}
808.294108 \\
\end{tabular} & $3.03 E-09$ & $2.50 \mathrm{E}-05$ & 0 & $\begin{array}{ll}6.04 \mathrm{E}-07 \\
\end{array}$ & 0 & $9.07 \mathrm{E}-06$ & $1.59 \mathrm{E}-05$ & $2.63 \mathrm{E}-05$ & $\begin{array}{ll}0.00347011 \\
\end{array}$ & $\begin{array}{l}4.33 \mathrm{E}-06 \\
\end{array}$ & 0.0071113 & 246631.186 \\
\hline 5276.38063 & 829.01858 & 864.985936 & 997.790687 & 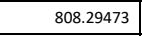 & $5.79 \mathrm{E}-09$ & $2.50 \mathrm{E}-05$ & 0 & $8.25 \mathrm{E}-07$ & 0 & $\begin{array}{l}9.07 E-06 \\
\end{array}$ & $1.59 \mathrm{E}-05$ & $2.63 \mathrm{E}-05$ & 0.00346859 & $5.91 \mathrm{E}-06$ & 0.00711132 & 246631.627 \\
\hline 6440.53385 & 829.01858 & 864.986029 & \begin{tabular}{|l|l}
997.79039 \\
\end{tabular} & 808.29514 & $9.23 \mathrm{E}-09$ & $2.50 \mathrm{E}-05$ & 0 & \begin{tabular}{ll|}
$1.10 \mathrm{E}-06$ \\
\end{tabular} & 0 & $9.07 \mathrm{E}-06$ & $1.59 \mathrm{E}-05$ & $2.63 \mathrm{E}-05$ & 0.00346775 & $\begin{array}{l}7.89 \mathrm{E}-06 \\
\end{array}$ & 0.00711133 & 246632.01 \\
\hline 7895.72538 & 829.01858 & 864.986191 & 997.790326 & 808.295471 & \begin{tabular}{|l|l|}
$1.35 E-08$ \\
\end{tabular} & $2.50 \mathrm{E}-05$ & 0 & $1.45 \mathrm{E}-06$ & 0 & $9.07 \mathrm{E}-06$ & $1.59 \mathrm{E}-05$ & $2.63 \mathrm{E}-05$ & 0.00346724 & $1.04 \mathrm{E}-05$ & 0.00711134 & 246632.409 \\
\hline 9714.71478 & 829.01858 & 864.986409 & \begin{tabular}{|l}
997.79058 \\
\end{tabular} & 808.295771 & $1.89 E-08$ & $2.50 \mathrm{E}-05$ & 0 & $1.88 \mathrm{E}-06$ & 0 & $9.07 E-06$ & $1.59 \mathrm{E}-05$ & $2.63 \mathrm{E}-05$ & 0.00346697 & $1.35 \mathrm{E}-05$ & 0.00711136 & 246632.866 \\
\hline 11988.4515 & 829.01858 & 864.986708 & 997.792035 & 808.296073 & $2.56 \mathrm{E}-08$ & $2.50 \mathrm{E}-05$ & 0 & $2.42 E-06$ & 0 & $9.07 \mathrm{E}-06$ & $1.59 \mathrm{E}-05$ & $2.63 \mathrm{E}-05$ & 0.00346691 & $1.73 \mathrm{E}-05$ & 0.00711138 & 246632.948 \\
\hline 14830.6225 & 829.01858 & 864.987027 & 997.793118 & 808.296423 & $3.40 \mathrm{E}-08$ & $2.50 \mathrm{E}-05$ & 0 & $3.09 \mathrm{E}-06$ & 0 & $9.07 \mathrm{E}-06$ & $1.59 \mathrm{E}-05$ & $2.63 E-05$ & 0.00346695 & $2.22 \mathrm{E}-05$ & 0.00711141 & 246633.528 \\
\hline 18383.3362 & 829.01858 & 864.987449 & 997.795045 & 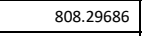 & 4.44E- 08 & $2.50 \mathrm{E}-05$ & 0 & $3.94 \mathrm{E}-06$ & 0 & $9.07 \mathrm{E}-06$ & $1.59 \mathrm{E}-05$ & $2.63 \mathrm{E}-05$ & 0.00346708 & $2.82 \mathrm{E}-05$ & 0.00711144 & 246634.262 \\
\hline $\begin{array}{r}22824.2283 \\
\end{array}$ & 829.01858 & 864.988018 & 997.798066 & 808.297431 & $5.74 \mathrm{E}-08$ & $2.50 \mathrm{E}-05$ & 0 & $4.99 \mathrm{E}-06$ & 0 & $9.07 \mathrm{E}-06$ & $1.59 \mathrm{E}-05$ & $2.63 \mathrm{E}-05$ & 0.00346729 & $3.58 \mathrm{E}-05$ & 0.00711148 & 246635.229 \\
\hline 28375.3434 & 829.01858 & 864.988784 & 997.802515 & 808.298196 & $7.35 E-08$ & $2.50 \mathrm{E}-05$ & 0 & $6.31 \mathrm{E}-06$ & 0 & $9.07 \mathrm{E}-06$ & $1.59 \mathrm{E}-05$ & $2.63 \mathrm{E}-05$ & 0.00346756 & $4.52 \mathrm{E}-05$ & 0.00711153 & 246636.52 \\
\hline $\begin{array}{l}35314.2373 \\
\end{array}$ & 829.01858 & 864.989816 & $\begin{array}{l}997.808869 \\
\end{array}$ & 808.299231 & \begin{tabular}{|c|}
$9.37 E-08$ \\
\end{tabular} & $2.50 \mathrm{E}-05$ & 0 & $7.96 \mathrm{E}-06$ & 0 & $9.07 \mathrm{E}-06$ & $\begin{array}{l}1.59 \mathrm{E}-05 \\
\end{array}$ & $2.63 \mathrm{E}-05$ & 0.00346791 & $5.70 \mathrm{E}-05$ & 0.0071116 & 246638.252 \\
\hline 43987.8547 & 829.01858 & 864.991216 & 997.817813 & 808.300635 & $1.19 \mathrm{E}-07$ & $2.50 \mathrm{E}-05$ & 0 & $1.00 \mathrm{E}-05$ & 0 & $9.07 \mathrm{E}-06$ & $1.59 \mathrm{E}-05$ & $2.63 E-05$ & 0.00346834 & $7.18 \mathrm{E}-05$ & 0.00711168 & 246640.587 \\
\hline 54829.8764 & 829.01858 & 864.993121 & 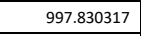 & 808.302545 & $1.50 \mathrm{E}-07$ & $2.50 \mathrm{E}-05$ & 0 & $1.26 \mathrm{E}-05$ & 0 & $9.07 \mathrm{E}-06$ & $\begin{array}{l}1.59 \mathrm{E}-05 \\
\end{array}$ & $2.63 \mathrm{E}-05$ & 0.00346889 & $\begin{array}{l}9.02 \mathrm{E}-05 \\
\end{array}$ & 0.00711179 & 246643.742 \\
\hline 68382.4035 & 829.01858 & 864.99572 & 997.847767 & 808.305148 & $1.88 E-07$ & $2.50 \mathrm{E}-05$ & 0 & $1.58 \mathrm{E}-05$ & 0 & $9.07 E-06$ & $1.59 \mathrm{E}-05$ & $2.63 \mathrm{E}-05$ & 0.00346959 & 0.00011325 & 0.00711192 & 246648.015 \\
\hline 85323.0625 & 829.01858 & 864.999272 & \begin{tabular}{|l|l|}
977211 \\
\end{tabular} & \begin{tabular}{|l|l|}
808.308705 \\
\end{tabular} & $2.35 E-07$ & $2.50 \mathrm{E}-05$ & 0 & $1.98 \mathrm{E}-05$ & 0 & \begin{tabular}{|l|l|}
$9.07 E-06$ \\
\end{tabular} & $1.59 \mathrm{E}-05$ & $2.63 \mathrm{E}-05$ & 0.00347046 & 0.00014206 & 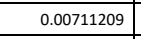 & 246653.819 \\
\hline 106498.886 & 829.01858 & 865.004377 & 997.904402 & 808.313431 & $2.94 E-07$ & $2.50 \mathrm{E}-05$ & 0 & $2.49 \mathrm{E}-05$ & 0 & $9.07 \mathrm{E}-06$ & $1.59 \mathrm{E}-05$ & $2.63 \mathrm{E}-05$ & 0.00347172 & 0.00017808 & 0.0071123 & 246670.406 \\
\hline 132968.666 & 829.01858 & 865.011129 & 997.950941 & 808.320012 & $3.66 \mathrm{E}-07$ & $2.50 \mathrm{E}-05$ & 0 & $3.11 \mathrm{E}-05$ & 0 & $9.07 \mathrm{E}-06$ & $1.59 \mathrm{E}-05$ & $2.63 E-05$ & 0.00347334 & 0.0002231 & 0.00711258 & 246684.332 \\
\hline 166055.89 & 829.01858 & 865.020411 & 998.015855 & 808.329045 & $4.53 \mathrm{E}-07$ & $2.50 \mathrm{E}-05$ & 0 & $3.90 \mathrm{E}-05$ & 0 & $9.07 \mathrm{E}-06$ & $1.59 \mathrm{E}-05$ & $2.63 \mathrm{E}-05$ & 0.00347545 & 0.00027938 & 0.00711293 & 246703.388 \\
\hline 207414.921 & 829.01858 & 865.033196 & 998.106423 & 808.341467 & $5.60 \mathrm{E}-07$ & $2.50 \mathrm{E}-05$ & 0 & $4.88 \mathrm{E}-05$ & 0 & $9.08 E-06$ & $1.59 \mathrm{E}-05$ & $2.63 \mathrm{E}-05$ & 0.0034782 & 0.00034972 & 0.00711337 & 246729.511 \\
\hline 259113.709 & 829.01858 & 865.050854 & 998.232825 & 808.358585 & \begin{tabular}{|c|}
$6.88 E-07$ \\
\end{tabular} & $2.50 \mathrm{E}-05$ & 0 & $6.111-05$ & 0 & $\begin{array}{l}9.08 E-06 \\
\end{array}$ & $1.59 \mathrm{E}-05$ & $2.63 \mathrm{E}-05$ & 0.00348178 & 0.00043766 & 0.00711394 & 246765.391 \\
\hline 323737.195 & 829.01858 & 865.075343 & 998.409283 & 808.382211 & $8.42 E-07$ & $2.50 \mathrm{E}-05$ & 0 & $7.64 \mathrm{E}-05$ & 0 & \begin{tabular}{|c|}
$9.08 E-06$ \\
\end{tabular} & \begin{tabular}{ll|}
$1.59 E-05$ \\
\end{tabular} & $2.63 \mathrm{E}-05$ & $\begin{array}{l}0.00348647 \\
\end{array}$ & 0.00054757 & \begin{tabular}{|l|}
0.00711468 \\
\end{tabular} & 246814.76 \\
\hline 404516.551 & 829.01858 & 865.109387 & 998.656009 & 808.414896 & $1.02 E-06$ & $2.50 \mathrm{E}-05$ & 0 & $9.56 \mathrm{E}-05$ & 0 & $9.08 E-06$ & $1.59 \mathrm{E}-05$ & $2.63 \mathrm{E}-05$ & 0.00349262 & 0.00068497 & 0.00711562 & 246882.813 \\
\hline 505490.747 & 829.01858 & 865.156876 & 999.001199 & 808.460212 & $1.24 E-06$ & $2.50 \mathrm{E}-05$ & 0 & 0.00011956 & 0 & $9.09 \mathrm{E}-06$ & $1.59 \mathrm{E}-05$ & $2.63 \mathrm{E}-05$ & 0.00350073 & 0.00085671 & 0.00711684 & 246976.792 \\
\hline 631708.492 & 829.01858 & 865.228682 & 999.478816 & 808.522947 & $1.48 E-06$ & $2.50 \mathrm{E}-05$ & 0 & 0.00014952 & 0 & $9.10 \mathrm{E}-06$ & $1.59 \mathrm{E}-05$ & $2.63 \mathrm{E}-05$ & 0.00351156 & 0.00107139 & 0.00711845 & 247106.674 \\
\hline 789480.673 & 829.01858 & 865.326301 & 1000.15348 & 808.610689 & $1.76 \mathrm{E}-06$ & $2.50 \mathrm{E}-05$ & 0 & 0.00018697 & 0 & $9.11 \mathrm{E}-06$ & $1.58 \mathrm{E}-05$ & $2.63 \mathrm{E}-05$ & 0.00352593 & 0.00133974 & 0.00712053 & 247286.924 \\
\hline 986695.899 & 829.01858 & 865.465593 & 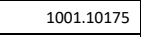 & 808.73357 & $2.07 E-06$ & $2.50 \mathrm{E}-05$ & 0 & 0.00023378 & 0 & $\begin{array}{l}9.12 E-06 \\
\end{array}$ & $1.58 \mathrm{E}-05$ & $2.63 \mathrm{E}-05$ & 0.00354505 & 0.00167518 & 0.00712326 & 247537.465 \\
\hline 1233214.93 & 829.01858 & 865.666472 & 1002.44572 & 808.906565 & $2.41 E-06$ & $2.50 \mathrm{E}-05$ & 0 & 0.00029229 & 0 & $\begin{array}{l}9.14 \mathrm{E}-06 \\
\end{array}$ & $1.58 \mathrm{E}-05$ & $2.63 \mathrm{E}-05$ & 0.00357056 & 0.00209448 & 0.00712685 & 247886.71 \\
\hline 1541363.72 & 829.01858 & 865.959835 & 1004.35519 & 809.104736 & $2.76 \mathrm{E}-06$ & $2.50 \mathrm{E}-05$ & 0 & 0.00036544 & 0 & $9.17 \mathrm{E}-06$ & $1.58 \mathrm{E}-05$ & $2.63 \mathrm{E}-05$ & 0.00360414 & 0.0026186 & 0.00713162 & 248360.196 \\
\hline 1926549.71 & 829.01858 & 866.392577 & 1006.25391 & 809.155607 & $3.11 \mathrm{E}-06$ & $2.50 \mathrm{E}-05$ & 0 & 0.00045687 & 0 & $9.20 \mathrm{E}-06$ & $1.58 \mathrm{E}-05$ & $2.64 \mathrm{E}-05$ & 0.0036463 & 0.00327375 & 0.00713798 & 248950.677 \\
\hline 2408032.2 & 829.01858 & 866.936532 & 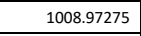 & 809.108971 & $3.46 \mathrm{E}-06$ & $2.50 \mathrm{E}-05$ & 0 & 0.00057115 & 0 & $9.25 \mathrm{E}-06$ & $1.57 \mathrm{E}-05$ & $2.64 \mathrm{E}-05$ & 0.00370113 & 0.00409269 & 0.00714612 & 249740.65 \\
\hline 3009885.31 & 829.01858 & 866.709504 & 1012.8157 & 809.008524 & $3.72 E-06$ & $2.50 \mathrm{E}-05$ & 0 & 0.00071401 & 0 & $9.32 \mathrm{E}-06$ & $1.56 \mathrm{E}-05$ & $2.64 \mathrm{E}-05$ & 0.00377365 & 0.00511637 & 0.00715007 & 250840.818 \\
\hline
\end{tabular}




\begin{tabular}{|c|c|c|c|c|c|c|c|c|c|c|c|c|c|c|c|c|}
\hline Time (s) & $\begin{array}{c}\text { Coolant outlet } \\
\text { temperature }(K)\end{array}$ & $\begin{array}{l}\text { Peak cladding } \\
\text { temperature (K) }\end{array}$ & $\begin{array}{c}\text { Peak fuel } \\
\text { temperature (K) }\end{array}$ & $\begin{array}{c}\text { Average cladding } \\
\text { internal } \\
\text { temperature (K) }\end{array}$ & \begin{tabular}{|l|}
$\begin{array}{c}\text { Peak cumulative } \\
\text { damage factor }\end{array}$ \\
\end{tabular} & $\begin{array}{l}\text { Cladding interior } \\
\text { volume }\left(\mathrm{m}^{3}\right)\end{array}$ & $\begin{array}{l}\text { Fission gas } \\
\text { release (\%) }\end{array}$ & $\begin{array}{c}\text { Fission gas } \\
\text { produced } \\
\text { (moles) }\end{array}$ & $\begin{array}{c}\text { Fission gas } \\
\text { released (moles) }\end{array}$ & Fuel volume $\left(\mathrm{m}^{3}\right)$ & $\begin{array}{l}\text { Plenum gas } \\
\text { volume }\left(m^{3}\right)\end{array}$ & $\begin{array}{l}\text { Peak cladding } \\
\text { radial growth } \\
\text { (m) }\end{array}$ & $\begin{array}{l}\text { Peak fuel axial } \\
\text { growth }(m)\end{array}$ & $\begin{array}{c}\text { Peak burnup } \\
\text { (at\%) }\end{array}$ & $\begin{array}{l}\text { Peak cladding } \\
\text { hoop strain }\end{array}$ & $\begin{array}{l}\text { Plenum pressure } \\
\text { (Pa) }\end{array}$ \\
\hline 3762201.69 & 829.01858 & 866.362031 & 1018.23623 & \begin{tabular}{|l|l|}
808.869379 \\
\end{tabular} & $3.90 \mathrm{E}-06$ & $2.50 \mathrm{E}-05$ & 0 & 0.00089258 & 0 & $9.42 \mathrm{E}-06$ & $1.55 \mathrm{E}-05$ & $2.64 \mathrm{E}-05$ & 0.00386981 & 0.00639596 & 0.00715484 & 252398.212 \\
\hline 4702597.17 & 829.01858 & 866.027605 & 1025.89488 & 808.678753 & $4.00 \mathrm{E}-06$ & $2.50 \mathrm{E}-05$ & 0 & 0.0011158 & 0 & $9.56 \mathrm{E}-06$ & $1.54 \mathrm{E}-05$ & $2.65 E-05$ & 0.00399652 & 0.00799545 & 0.007161 & 254620.47 \\
\hline 5702597.17 & 829.01858 & 866.824777 & 1035.04676 & 808.576555 & $4.02 E-06$ & $2.50 \mathrm{E}-05$ & 0 & 0.00135316 & 0 & $9.73 \mathrm{E}-06$ & $1.52 \mathrm{E}-05$ & $2.64 E-05$ & 0.00413863 & 0.00969632 & 0.00715815 & 257356.652 \\
\hline 6702597.17 & 829.01858 & 865.928052 & 1045.29938 & 808.335765 & $4.03 \mathrm{E}-06$ & $2.50 \mathrm{E}-05$ & 0 & 0.00159053 & 0 & $9.91 \mathrm{E}-06$ & $1.50 \mathrm{E}-05$ & $2.64 \mathrm{E}-05$ & 0.00428369 & 0.01139719 & 0.00716446 & 260404.381 \\
\hline 7702597.17 & 829.01858 & 865.079629 & 1056.65464 & $\begin{array}{l}808.080981 \\
\end{array}$ & $4.03 \mathrm{E}-06$ & $2.50 \mathrm{E}-05$ & 0 & 0.00182789 & 0 & $1.01 E-05$ & $1.48 \mathrm{E}-05$ & $2.65 E-05$ & 0.00443168 & 0.01309807 & 0.00717031 & 263817.795 \\
\hline 8203212 & 829.01858 & 864.677904 & 1062.67296 & 807.948882 & $4.03 E-06$ & $2.50 \mathrm{E}-05$ & 0 & 0.00194672 & 0 & $1.02 E-05$ & $1.47 \mathrm{E}-05$ & $2.65 E-05$ & 0.00450739 & 0.01394955 & 0.00717282 & 265663.512 \\
\hline 8206812 & 822.624548 & 857.371637 & 1052.08118 & \begin{tabular}{|l|}
802.467559 \\
\end{tabular} & $4.03 \mathrm{E}-06$ & $2.50 \mathrm{E}-05$ & 0 & 0.00194756 & 0 & $1.02 E-05$ & $1.47 \mathrm{E}-05$ & $2.62 E-05$ & 0.00447599 & 0.01395559 & 0.00708335 & 263883.775 \\
\hline 8707426.83 & 822.624548 & 857.060527 & 1057.05195 & 802.358543 & 4.03E- -06 & $2.50 \mathrm{E}-05$ & 0 & 0.00206338 & 0 & $1.03 E-05$ & $1.46 E-05$ & $2.62 E-05$ & 0.00456248 & 0.01478553 & 0.00708594 & 265482.04 \\
\hline 9333195.37 & 822.624548 & 856.639799 & 1064.20967 & 802.204828 & $4.03 \mathrm{E}-06$ & $2.50 \mathrm{E}-05$ & 0 & 0.00220816 & 0 & $1.04 \mathrm{E}-05$ & $1.45 \mathrm{E}-05$ & $2.62 E-05$ & 0.00465721 & 0.01582294 & 0.00708891 & 267818.725 \\
\hline 10115406 & 822.624548 & 856.274189 & 1072.62574 & 802.024089 & $4.03 \mathrm{E}-06$ & $2.50 \mathrm{E}-05$ & 2.18726569 & 0.00238913 & $5.23 \mathrm{E}-05$ & $1.06 \mathrm{E}-05$ & $1.44 \mathrm{E}-05$ & $2.62 \mathrm{E}-05$ & 0.00477098 & 0.01711971 & 0.00709442 & 270746.896 \\
\hline 11093169.4 & 822.624548 & 856.150562 & 1081.83544 & 801.817104 & $4.03 E-06$ & $2.50 \mathrm{E}-05$ & 12.73612 & 0.00261534 & 0.00033309 & $1.08 E-05$ & $1.42 E-05$ & $2.62 \mathrm{E}-05$ & 0.0049044 & 0.01874068 & 0.00710168 & 298864.073 \\
\hline 12093169.4 & 822.624548 & 855.756481 & 1089.57258 & 801.633539 & $4.03 \mathrm{E}-06$ & $2.50 \mathrm{E}-05$ & 24.050955 & 0.0028467 & 0.00068466 & $1.10 \mathrm{E}-05$ & $1.40 \mathrm{E}-05$ & $2.63 E-05$ & 0.00502787 & 0.02039851 & 0.00711302 & 436154.947 \\
\hline 13093169.4 & 822.624548 & 855.196653 & 1095.82198 & 801.478903 & $4.03 \mathrm{E}-06$ & $2.50 \mathrm{E}-05$ & 36.8645772 & 0.00307805 & 0.00113471 & $1.11 \mathrm{E}-05$ & $1.39 \mathrm{E}-05$ & $2.63 E-05$ & 0.00513821 & 0.02205633 & 0.00712608 & 609831.439 \\
\hline 13814423 & 822.624548 & 854.842435 & 1099.42122 & 801.386799 & $4.03 E-06$ & 2.50 E- 05 & 43.102821 & 0.00324492 & 0.00139865 & $1.12 E-05$ & $1.38 \mathrm{E}-05$ & $2.64 \mathrm{E}-05$ & 0.005209 & 0.02325205 & 0.00714031 & 831602.233 \\
\hline 13815366.7 & 821.218269 & 853.067492 & 1095.44436 & 800.094437 & $4.03 \mathrm{E}-06$ & $2.50 \mathrm{E}-05$ & 43.103786 & 0.00324514 & 0.00139878 & $1.12 E-05$ & $1.38 E-05$ & $2.63 E-05$ & 0.00519501 & 0.0232536 & 0.00712475 & 957801.512 \\
\hline 13816310.4 & 819.804866 & 851.295969 & 1091.45975 & 798.796681 & $4.03 \mathrm{E}-06$ & $2.50 \mathrm{E}-05$ & 43.1047408 & 0.00324535 & 0.0013989 & $1.12 \mathrm{E}-05$ & $1.38 E-05$ & $2.62 E-05$ & 0.00518164 & 0.02325514 & 0.00710378 & 956290.286 \\
\hline 13817490.1 & 818.028013 & 849.131466 & 1086.46704 & 797.167132 & $4.03 \mathrm{E}-06$ & $2.50 \mathrm{E}-05$ & 43.10592 & 0.00324562 & 0.00139905 & $1.12 \mathrm{E}-05$ & $1.38 E-05$ & $2.61 \mathrm{E}-05$ & 0.00516548 & 0.02325705 & 0.00707757 & 954376.353 \\
\hline 13818023 & 817.221601 & 848.146387 & 1084.20674 & 796.428143 & $4.03 \mathrm{E}-06$ & $2.50 \mathrm{E}-05$ & 43.1064475 & 0.00324574 & 0.00139912 & $1.12 E-05$ & $1.38 E-05$ & $2.61 E-05$ & 0.00515824 & 0.0232579 & 0.00706568 & 953554.763 \\
\hline 13819202.6 & 817.221601 & 848.145648 & 1084.20658 & 796.428094 & $4.03 E-06$ & 2.50 E- 05 & 43.1076115 & 0.003246 & 0.00139927 & $1.12 \mathrm{E}-05$ & $1.38 \mathrm{E}-05$ & $2.61 \mathrm{E}-05$ & 0.00515882 & 0.02325978 & 0.00706569 & 953587.53 \\
\hline 13820677.2 & 817.221601 & 848.143151 & 1084.20792 & 796.428077 & $4.03 E-06$ & $2.50 \mathrm{E}-05$ & 43.1090662 & 0.00324633 & 0.00139946 & $1.12 E-05$ & $1.38 E-05$ & $2.61 E-05$ & 0.00515911 & 0.02326212 & 0.0070657 & 953659.772 \\
\hline 13822520.4 & 817.221601 & 848.141904 & 1084.20883 & 796.428064 & $4.03 \mathrm{E}-06$ & $2.50 \mathrm{E}-05$ & 43.1108842 & 0.00324674 & 0.0013997 & $1.12 E-05$ & $1.38 E-05$ & $2.61 \mathrm{E}-05$ & 0.00515924 & 0.02326506 & 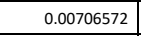 & 953750.605 \\
\hline 13824824.4 & 817.221601 & 848.14135 & 1084.20949 & 796.428051 & $4.03 \mathrm{E}-06$ & $2.50 \mathrm{E}-05$ & 43.1131561 & 0.00324725 & 0.00139999 & $1.12 E-05$ & $1.38 E-05$ & $2.61 \mathrm{E}-05$ & 0.00515929 & 0.02326873 & 0.00706574 & 953864.463 \\
\hline 13827704.4 & 817.221601 & 848.141142 & 1084.20992 & 796.428037 & $4.03 E-06$ & $2.50 \mathrm{E}-05$ & 43.1159949 & 0.00324789 & 0.00140036 & $1.12 E-05$ & $1.38 E-05$ & $2.61 E-05$ & 0.00515932 & 0.02327331 & 0.00706578 & 954007.003 \\
\hline 13831304.4 & 817.221601 & 848.141031 & 1084.21017 & 796.428017 & $4.03 \mathrm{E}-06$ & $2.50 \mathrm{E}-05$ & 43.1195418 & 0.00324869 & 0.00140082 & $1.12 \mathrm{E}-05$ & $1.38 E-05$ & $2.61 \mathrm{E}-05$ & 0.00515937 & 0.02327905 & 0.00706582 & 954185.331 \\
\hline 13835804.4 & 817.221601 & 848.140845 & 1084.21023 & 796.427991 & $4.03 E-06$ & $2.50 \mathrm{E}-05$ & 43.1239731 & 0.00324969 & 0.00140139 & $1.12 \mathrm{E}-05$ & $1.38 \mathrm{E}-05$ & $2.61 \mathrm{E}-05$ & 0.00515945 & 0.02328621 & 0.00706587 & 954408.344 \\
\hline 13841429.4 & 817.221601 & 848.14049 & 1084.21014 & 796.427956 & $4.03 E-06$ & $2.50 \mathrm{E}-05$ & 43.1295083 & 0.00325094 & 0.00140211 & $1.12 E-05$ & $1.38 E-05$ & $2.61 E-05$ & 0.00515959 & 0.02329517 & 0.00706594 & 954687.177 \\
\hline 13848460.7 & 817.221601 & 848.139932 & 1084.20993 & 796.427911 & $4.03 E-06$ & $2.50 \mathrm{E}-05$ & 43.1364213 & 0.0032525 & 0.00140301 & $1.12 \mathrm{E}-05$ & $1.38 E-05$ & $2.61 \mathrm{E}-05$ & 0.00515978 & 0.02330637 & 0.00706602 & 955035.76 \\
\hline \begin{tabular}{|l|l|l} 
\\
\end{tabular} & 817.221601 & 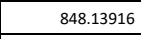 & 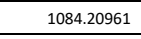 & 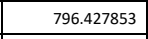 & $4.03 E-06$ & $2.50 \mathrm{E}-05$ & 43.1450532 & 0.00325445 & 0.00140414 & $1.12 E-05$ & $1.38 E-05$ & $2.61 E-05$ & 0.00516002 & 0.02332036 & 0.00706612 & 955471.51 \\
\hline 13868236 & 817.221601 & 848.138154 & 1084.20918 & 796.427777 & $4.03 \mathrm{E}-06$ & $2.50 \mathrm{E}-05$ & 43.1558286 & 0.00325689 & 0.00140554 & $1.12 E-05$ & $1.38 E-05$ & $2.61 \mathrm{E}-05$ & 0.00516033 & 0.02333785 & 0.00706625 & 956016.216 \\
\hline 13881969 & 817.221601 & 848.136868 & 1084.20863 & 796.427683 & $4.03 E-06$ & $2.50 \mathrm{E}-05$ & 43.1692751 & 0.00325995 & 0.0014073 & $1.12 E-05$ & $1.38 E-05$ & $2.61 E-05$ & 0.00516073 & 0.02335972 & 0.0070664 & 956697.129 \\
\hline 13899135.1 & 817.221601 & 848.133219 & 1084.20709 & 796.427398 & $4.03 E-06$ & $2.50 \mathrm{E}-05$ & 43.1860479 & 0.00326376 & 0.00140949 & $1.12 \mathrm{E}-05$ & $1.38 \mathrm{E}-05$ & $2.61 \mathrm{E}-05$ & 0.00516121 & 0.02338706 & 0.0070666 & 957558.207 \\
\hline 13920592.8 & 817.221601 & 848.130496 & 1084.20605 & 796.427163 & $4.03 \mathrm{E}-06$ & $2.50 \mathrm{E}-05$ & 43.2069589 & 0.00326853 & 0.00141223 & $1.12 E-05$ & $1.38 E-05$ & $2.61 \mathrm{E}-05$ & 0.00516186 & 0.02342122 & 0.00706684 & 958627.887 \\
\hline 13947414.9 & 817.221601 & 848.126892 & 1084.20479 & 796.426809 & $4.03 \mathrm{E}-06$ & $2.50 \mathrm{E}-05$ & 43.2330119 & 0.00327449 & 0.00141566 & $1.12 E-05$ & $1.38 E-05$ & $2.61 \mathrm{E}-05$ & 0.00516273 & 0.02346394 & 0.00706715 & 959969.359 \\
\hline 13980942.5 & 817.221601 & 848.118609 & 1084.20326 & 796.42619 & $4.03 \mathrm{E}-06$ & $2.50 \mathrm{E}-05$ & 43.2728413 & 0.00328194 & $\begin{array}{l}0.00142019 \\
\end{array}$ & $1.12 \mathrm{E}-05$ & $1.38 E-05$ & $2.61 \mathrm{E}-05$ & $\begin{array}{l}0.00516397 \\
\end{array}$ & 0.02351732 & 0.00706751 & 961659.04 \\
\hline 14022852 & 817.221601 & 848.103766 & $\begin{array}{l}1084.20146 \\
\end{array}$ & 796.425059 & $4.03 \mathrm{E}-06$ & $2.50 \mathrm{E}-05$ & 43.3429163 & 0.00329125 & 0.00142653 & $1.12 \mathrm{E}-05$ & $1.38 E-05$ & $2.61 \mathrm{E}-05$ & 0.00516592 & 0.02358406 & 0.00706794 & 963915.636 \\
\hline 14075238.9 & 817.221601 & 848.079942 & 1084.20952 & 796.422954 & $4.03 E-06$ & $2.50 \mathrm{E}-05$ & 43.5033755 & 0.00330289 & 0.00143687 & $1.12 E-05$ & $1.38 E-05$ & $2.61 E-05$ & 0.005169 & 0.02366748 & 0.00706846 & 967113.266 \\
\hline 14140722.5 & 817.221601 & 848.047146 & 1084.25594 & 796.419374 & $4.03 E-06$ & $2.50 \mathrm{E}-05$ & 43.878397 & 0.00331745 & 0.00145564 & $1.12 E-05$ & $1.38 E-05$ & $2.61 E-05$ & 0.00517344 & 0.02377175 & 0.00706914 & 972347.236 \\
\hline 14222577 & 817.221601 & 848.0262 & 1084.39005 & 796.413899 & $4.03 \mathrm{E}-06$ & $2.50 \mathrm{E}-05$ & 44.4099367 & 0.00333564 & 0.00148135 & $1.12 E-05$ & $1.37 E-05$ & $2.61 E-05$ & 0.00517958 & 0.02390209 & 0.00707018 & 981785.024 \\
\hline 14324895.2 & 817.221601 & 848.018237 & 1084.62762 & 796.406085 & $4.03 \mathrm{E}-06$ & $2.50 \mathrm{E}-05$ & 45.0600521 & 0.00335837 & 0.00151328 & $1.12 E-05$ & $1.37 \mathrm{E}-05$ & $2.61 \mathrm{E}-05$ & 0.00518738 & 0.02406502 & 0.00707157 & 994752.944 \\
\hline 14428975 & 817.221601 & 848.010036 & 1084.92973 & 796.397399 & $4.03 \mathrm{E}-06$ & $2.50 \mathrm{E}-05$ & 45.7347598 & 0.0033815 & 0.00154652 & $1.12 E-05$ & $1.37 \mathrm{E}-05$ & $2.61 \mathrm{E}-05$ & 0.00519591 & 0.02423075 & 0.00707311 & 1010797.6 \\
\hline 14429918.7 & 815.49668 & 846.19939 & 1083.42715 & 795.033943 & $4.03 \mathrm{E}-06$ & $2.50 \mathrm{E}-05$ & 45.7356661 & 0.00338171 & 0.00154665 & $1.12 \mathrm{E}-05$ & $1.37 \mathrm{E}-05$ & $2.60 \mathrm{E}-05$ & 0.00518899 & 0.02423225 & 0.00705103 & 1025110.41 \\
\hline 14430862.4 & 813.799662 & 844.417007 & 1081.94042 & 793.691883 & $4.03 E-06$ & $2.49 \mathrm{E}-05$ & 45.7365707 & 0.00338192 & 0.00154677 & $1.12 \mathrm{E}-05$ & $1.37 E-05$ & $2.59 \mathrm{E}-05$ & 0.00518215 & 0.02423375 & 0.00702865 & 1023486.87 \\
\hline 14432042.1 & 811.716609 & 842.22773 & 1080.10374 & 792.043684 & $4.03 \mathrm{E}-06$ & $2.49 \mathrm{E}-05$ & 45.7376991 & 0.00338218 & 0.00154693 & $1.12 E-05$ & $1.37 \mathrm{E}-05$ & $2.58 E-05$ & 0.00517372 & 0.02423562 & 0.00700116 & 1021478.23 \\
\hline 14432575 & 810.789189 & 841.252536 & 1079.28171 & 791.309561 & $4.03 E-06$ & $2.49 \mathrm{E}-05$ & 45.7382079 & 0.0033823 & 0.001547 & $1.12 \mathrm{E}-05$ & $1.37 E-05$ & $2.58 \mathrm{E}-05$ & 0.00516996 & 0.02423646 & 0.00698893 & 1020631.81 \\
\hline 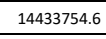 & $\begin{array}{l}810.789189 \\
\end{array}$ & 8441.252482 & \begin{tabular}{|l|l|l|}
1079.28166 \\
\end{tabular} & \begin{tabular}{|l}
791.309518 \\
\end{tabular} & $4.03 \mathrm{E}-06$ & $\begin{array}{l}2.49 \mathrm{E}-05 \\
\end{array}$ & 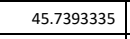 & 0.00338256 & 0.00154716 & $1.12 E-05$ & $1.37 E-05$ & $2.58 \mathrm{E}-05$ & 0.00516996 & 0.02423833 & 0.00698894 & 1020665.79 \\
\hline 14435229.2 & 810.789189 & 841.252457 & \begin{tabular}{l|l}
1079.28189 \\
\end{tabular} & 791.309511 & $4.03 \mathrm{E}-06$ & $2.49 \mathrm{E}-05$ & 45.7407403 & 0.00338288 & 0.00154736 & $1.12 E-05$ & $1.37 \mathrm{E}-05$ & $2.58 E-05$ & 0.00516996 & 0.02424066 & $\begin{array}{c}0.00698896 \\
\end{array}$ & 1020741.59 \\
\hline
\end{tabular}




\begin{tabular}{|c|c|c|c|c|c|c|c|c|c|c|c|c|c|c|c|c|}
\hline Time (s) & $\begin{array}{l}\text { coolant outlet } \\
\text { temperature }(K)\end{array}$ & $\begin{array}{l}\text { Peak cladding } \\
\text { temperature (K) }\end{array}$ & $\begin{array}{l}\text { Peak fuel } \\
\text { temperature (K) }\end{array}$ & $\begin{array}{c}\text { Average cladding } \\
\text { internal } \\
\text { temperature (K) }\end{array}$ & $\begin{array}{l}\text { Peak cumulative } \\
\text { damage factor }\end{array}$ & $\begin{array}{l}\text { Cladding interior } \\
\text { volume }\left(\mathrm{m}^{3}\right)\end{array}$ & $\begin{array}{l}\text { Fission gas } \\
\text { release (\%) }\end{array}$ & $\begin{array}{c}\text { Fission gas } \\
\text { produced } \\
\text { (moles) }\end{array}$ & $\begin{array}{c}\text { Fission gas } \\
\text { released (moles) }\end{array}$ & Fuel volume $\left(\mathrm{m}^{3}\right)$ & $\begin{array}{l}\text { Plenum gas } \\
\text { volume }\left(m^{3}\right)\end{array}$ & $\begin{array}{l}\text { Peak cladding } \\
\text { radial growth } \\
\text { (m) }\end{array}$ & $\begin{array}{l}\text { Peak fuel axial } \\
\text { growth }(m)\end{array}$ & $\begin{array}{c}\text { Peak burnup } \\
\text { (at\%) }\end{array}$ & $\begin{array}{l}\text { Peak cladding } \\
\text { hoop strain }\end{array}$ & \begin{tabular}{|c} 
Plenum pressure \\
$(\mathrm{Pa})$
\end{tabular} \\
\hline 14437072.4 & 810.789189 & 841.252427 & 1079.28204 & $\begin{array}{l}791.309502 \\
\end{array}$ & $4.03 E-06$ & $2.49 \mathrm{E}-05$ & 45.7424985 & 0.00338329 & 0.0015476 & $1.12 \mathrm{E}-05$ & $1.37 E-05$ & $2.58 \mathrm{E}-05$ & 0.00516996 & 0.02424357 & 0.00698898 & 1020836.41 \\
\hline 14439376.4 & 810.789189 & 841.252413 & 1079.28212 & 791.309489 & $4.03 E-06$ & $2.49 \mathrm{E}-05$ & 45.7446955 & 0.0033838 & 0.00154791 & $1.12 \mathrm{E}-05$ & $1.37 E-05$ & $2.58 \mathrm{E}-05$ & 0.00516999 & 0.02424722 & 0.00698901 & 1020954.97 \\
\hline 14442256.4 & 810.789189 & 841.25243 & 1079.28213 & 791.309472 & $4.03 E-06$ & $2.49 \mathrm{E}-05$ & 45.747441 & 0.00338444 & 0.00154829 & $1.12 E-05$ & $1.37 E-05$ & $2.58 \mathrm{E}-05$ & 0.00517004 & 0.02425177 & 0.00698904 & 1021103.19 \\
\hline 14445856.4 & 810.789189 & 841.25249 & 1079.28208 & 791.30945 & $4.03 E-06$ & $2.49 \mathrm{E}-05$ & 45.7508713 & 0.00338523 & 0.00154877 & $1.12 \mathrm{E}-05$ & $1.37 E-05$ & $2.58 \mathrm{E}-05$ & 0.00517012 & 0.02425746 & 0.00698908 & 1021288.5 \\
\hline 14450356.4 & 810.789189 & 841.252768 & 1079.28178 & 791.309387 & $4.03 E-06$ & $2.49 \mathrm{E}-05$ & 45.7551569 & 0.00338622 & 0.00154937 & $1.12 \mathrm{E}-05$ & $1.37 E-05$ & $2.58 \mathrm{E}-05$ & 0.00517023 & 0.02426458 & 0.00698914 & 1021522.67 \\
\hline $\begin{array}{l}14455981.4 \\
\end{array}$ & 810.789189 & 841.253111 & 1079.28141 & 791.309316 & $4.03 E-06$ & $2.49 \mathrm{E}-05$ & 445.7605104 & 0.00338746 & 0.00155012 & $1.12 \mathrm{E}-05$ & $1.37 E-05$ & $2.58 \mathrm{E}-05$ & 0.00517038 & 0.02427348 & 0.0069892 & 1021813.36 \\
\hline $\begin{array}{l}14463012.7 \\
\end{array}$ & 810.789189 & 841.253326 & 1079.28119 & 791.309265 & $4.03 E-06$ & $2.49 \mathrm{E}-05$ & $\begin{array}{l}45.7671968 \\
\end{array}$ & 0.00338902 & 0.00155106 & $1.12 \mathrm{E}-05$ & $1.37 \mathrm{E}-05$ & $2.58 \mathrm{E}-05$ & 0.00517059 & 0.0242846 & 0.00698929 & 1022177.52 \\
\hline 14471801.7 & 810.789189 & 841.253771 & 1079.28077 & 791.309157 & $4.03 E-06$ & $2.49 \mathrm{E}-05$ & 45.7755461 & 0.00339096 & 0.00155223 & $1.12 \mathrm{E}-05$ & $1.37 E-05$ & $2.58 \mathrm{E}-05$ & 0.00517086 & 0.0242985 & 0.0069894 & 1022634.08 \\
\hline $\begin{array}{l}14482788 \\
\end{array}$ & 810.789189 & \begin{tabular}{|l|l|}
841.254394 \\
\end{tabular} & 1079.28027 & 791.308992 & $4.03 E-06$ & $2.49 \mathrm{E}-05$ & $\begin{array}{ll}45.7859694 \\
\end{array}$ & 0.00339338 & 0.00155369 & $1.12 E-05$ & $1.37 E-05$ & $2.58 \mathrm{E}-05$ & 0.00517124 & 0.02431587 & 0.00698953 & 1023207.21 \\
\hline 14496521 & 810.789189 & 841.255261 & 1079.27965 & 791.308738 & $4.03 E-06$ & $2.49 \mathrm{E}-05$ & 45.8186116 & 0.00339641 & 0.00155619 & $1.12 \mathrm{E}-05$ & $1.37 E-05$ & $2.58 \mathrm{E}-05$ & 0.00517175 & 0.02433759 & 0.00698969 & 1023927.28 \\
\hline 14513687.1 & 810.789189 & 841.256439 & 1079.27891 & 791.308358 & $4.03 E-06$ & $2.49 \mathrm{E}-05$ & 45.8730385 & 0.0034002 & 0.00155978 & $1.12 \mathrm{E}-05$ & $1.37 E-05$ & $2.58 \mathrm{E}-05$ & 0.00517244 & 0.02436473 & 0.00698992 & 1025151.81 \\
\hline $\begin{array}{l}14535144.8 \\
\end{array}$ & 810.789189 & 841.258009 & 1079.27801 & 791.307798 & $4.03 E-06$ & $2.49 \mathrm{E}-05$ & 45.9550978 & 0.00340494 & 0.00156474 & $1.12 E-05$ & $1.37 E-05$ & $2.58 \mathrm{E}-05$ & 0.00517336 & 0.02439867 & 0.0069902 & 1026912.22 \\
\hline 14561966.9 & 810.789189 & 841.259672 & 1079.27691 & 791.306977 & $4.03 E-06$ & $2.49 \mathrm{E}-05$ & 46.056673 & 0.00341086 & 0.00157093 & $1.12 \mathrm{E}-05$ & $1.37 E-05$ & $2.58 \mathrm{E}-05$ & 0.00517456 & 0.02444109 & 0.00699057 & 1029353.84 \\
\hline 14595494.5 & 810.789189 & 841.25603 & 1079.27559 & 791.305638 & $4.03 E-06$ & $2.49 \mathrm{E}-05$ & 46.171488 & 0.00341825 & 0.00157826 & $1.12 \mathrm{E}-05$ & $1.37 E-05$ & $2.58 \mathrm{E}-05$ & 0.0051764 & 0.02449411 & 0.00699098 & 1032422.06 \\
\hline 14637404 & 810.789189 & 841.251532 & 1079.27986 & 791.303334 & $4.03 E-06$ & $2.49 \mathrm{E}-05$ & 46.386093 & 0.0034275 & 0.00158989 & $1.12 E-05$ & $1.37 E-05$ & $2.58 \mathrm{E}-05$ & 0.00517913 & 0.02456039 & 0.00699148 & 1036119.08 \\
\hline 14689790.9 & 810.789189 & 841.246646 & 1079.36371 & 791.299504 & $4.03 E-06$ & $2.49 \mathrm{E}-05$ & 46.7205011 & 0.00343907 & 0.00160675 & $1.12 E-05$ & $1.37 E-05$ & $2.58 \mathrm{E}-05$ & 0.00518303 & 0.02464323 & 0.00699216 & 1041996.58 \\
\hline 14755274.5 & 810.789189 & 841.240658 & 1079.55576 & 791.29408 & $4.03 E-06$ & $2.49 \mathrm{E}-05$ & 47.1035958 & 0.00345352 & 0.00162673 & $1.12 E-05$ & $1.37 \mathrm{E}-05$ & $2.58 \mathrm{E}-05$ & 0.00518823 & 0.02474679 & 0.00699307 & 1050515.56 \\
\hline 14837129 & 810.789189 & 841.232122 & 1079.82059 & 791.287132 & $4.03 E-06$ & $2.49 \mathrm{E}-05$ & 47.6161193 & 0.00347158 & 0.00165303 & $1.12 E-05$ & $1.37 \mathrm{E}-05$ & $2.58 \mathrm{E}-05$ & 0.00519456 & 0.02487624 & 0.00699417 & 1060660.44 \\
\hline 14939447.2 & 810.789189 & 841.223296 & 1080.16722 & 791.278362 & $4.03 E-06$ & $2.49 \mathrm{E}-05$ & 48.2215364 & 0.00349416 & 0.00168494 & $1.13 \mathrm{E}-05$ & $1.37 E-05$ & $2.58 \mathrm{E}-05$ & 0.00520252 & 0.02503805 & 0.0069956 & 1073999.96 \\
\hline 15067344.9 & 810.789189 & 841.22836 & 1080.61249 & 791.267421 & $4.03 E-06$ & $2.49 \mathrm{E}-05$ & 48.9220686 & 0.00352239 & 0.00172323 & $1.13 E-05$ & $1.37 E-05$ & $2.58 \mathrm{E}-05$ & 0.00521251 & 0.02524031 & 0.00699742 & 1090238.44 \\
\hline 15227217 & 810.789189 & 841.235155 & 1081.16763 & 791.253787 & $4.03 E-06$ & $2.49 \mathrm{E}-05$ & 49.7997409 & 0.00355767 & 0.00177171 & $1.13 E-05$ & $1.37 E-05$ & $2.58 \mathrm{E}-05$ & 0.00522505 & 0.02549314 & 0.0069997 & 1109805.17 \\
\hline 15427057.1 & 810.789189 & 841.241629 & 1081.83384 & 791.237009 & $4.03 E-06$ & $2.49 \mathrm{E}-05$ & 50.8141212 & 0.00360178 & 0.00183021 & $1.13 \mathrm{E}-05$ & $1.37 E-05$ & $2.58 \mathrm{E}-05$ & 0.0052402 & 0.02580917 & 0.00700257 & 1134601.98 \\
\hline 15676857.3 & 810.789189 & 841.247441 & 1082.63438 & 791.216698 & $4.03 E-06$ & $2.49 \mathrm{E}-05$ & 51.9912337 & 0.00365691 & 0.00190127 & $1.13 \mathrm{E}-05$ & $1.36 \mathrm{E}-05$ & $2.59 \mathrm{E}-05$ & 0.0052585 & 0.02620422 & 0.00700615 & 1164616.26 \\
\hline 15989107.5 & 810.789189 & 841.2523 & 1083.58687 & 791.192347 & $4.03 E-06$ & $2.49 \mathrm{E}-05$ & 53.3138586 & 0.00372582 & 0.00198638 & $1.13 \mathrm{E}-05$ & $1.36 \mathrm{E}-05$ & $2.59 \mathrm{E}-05$ & 0.00528079 & 0.02669802 & 0.00701064 & 1201180.38 \\
\hline 16379420.3 & 810.789189 & 841.254676 & 1084.70537 & 791.163463 & $4.03 E-06$ & $2.49 \mathrm{E}-05$ & 54.8623129 & 0.00381196 & 0.00209133 & $1.14 E-05$ & 1.36 E- 05 & $2.59 \mathrm{E}-05$ & 0.00530777 & 0.02731528 & 0.00701628 & 1245133.84 \\
\hline 16867311.3 & 810.789189 & 841.251662 & 1085.99689 & 791.129643 & $4.03 E-06$ & $2.49 \mathrm{E}-05$ & 56.6141733 & 0.00391964 & 0.00221907 & $1.14 E-05$ & $1.35 E-05$ & $2.59 \mathrm{E}-05$ & 0.00534021 & 0.02808685 & 0.00702342 & 1299449.09 \\
\hline 17477175 & 810.789189 & 841.238945 & 1087.45613 & 791.09071 & $4.03 E-06$ & $2.49 \mathrm{E}-05$ & 58.3930014 & 0.00405423 & 0.00236739 & $1.15 \mathrm{E}-05$ & $1.35 \mathrm{E}-05$ & $2.60 \mathrm{E}-05$ & 0.00537892 & 0.02905131 & 0.00703248 & 1365732.66 \\
\hline 18239504.7 & 810.789189 & 841.209749 & 1089.05885 & 791.046817 & $4.03 E-06$ & $2.49 \mathrm{E}-05$ & 60.2655069 & 0.00422247 & 0.0025447 & $1.15 E-05$ & $1.34 E-05$ & $2.60 \mathrm{E}-05$ & 0.00542471 & 0.03025689 & 0.00704404 & 1443091.27 \\
\hline 19192416.7 & 810.789189 & 841.153704 & 1090.75497 & 790.998599 & $4.03 E-06$ & $2.50 \mathrm{E}-05$ & 61.8898175 & 0.00443278 & 0.00274344 & $1.16 E-05$ & $1.34 E-05$ & $2.60 \mathrm{E}-05$ & 0.00547834 & 0.03176387 & 0.00705889 & 1535800.96 \\
\hline 20192416.7 & 810.789189 & 841.073985 & 1092.18922 & 790.955533 & $4.03 E-06$ & $2.50 \mathrm{E}-05$ & 62.9669631 & 0.00465348 & 0.00293015 & $1.16 \mathrm{E}-05$ & $1.33 E-05$ & $2.61 \mathrm{E}-05$ & 0.00553047 & 0.03334531 & 0.00707536 & 1639145.4 \\
\hline 21192416.7 & 810.789189 & 841.005561 & 1093.3267 & 790.918864 & $4.03 E-06$ & $2.50 \mathrm{E}-05$ & 63.6974939 & 0.00487417 & 0.00310473 & $1.16 \mathrm{E}-05$ & $1.33 \mathrm{E}-05$ & $2.62 E-05$ & 0.00557897 & 0.03492675 & 0.00709228 & 1736363.48 \\
\hline 21312419 & 810.789189 & 841.001457 & 1093.43426 & 790.915093 & $4.03 E-06$ & $2.50 \mathrm{E}-05$ & 63.7731661 & 0.00490066 & 0.0031253 & $1.17 \mathrm{E}-05$ & $1.33 E-05$ & $2.62 \mathrm{E}-05$ & 0.00558437 & 0.03511652 & 0.00709765 & 1823177.07 \\
\hline 21312666.4 & 810.407875 & 840.498473 & 1092.06842 & 790.55121 & $4.03 E-06$ & $2.50 \mathrm{E}-05$ & 63.773321 & 0.00490071 & 0.00312535 & $1.17 E-05$ & $1.33 E-05$ & $2.62 E-05$ & 0.00557958 & 0.03511691 & 0.00709223 & 1832479.64 \\
\hline 21312913.8 & 810.025442 & 839.99435 & $\begin{array}{l}1090.70129 \\
\end{array}$ & 790.186422 & $4.03 E-06$ & $2.50 \mathrm{E}-05$ & $\begin{array}{l}63.7734753 \\
\end{array}$ & 0.00490077 & 0.00312539 & $1.17 \mathrm{E}-05$ & $1.33 E-05$ & $2.61 \mathrm{E}-05$ & 0.00557486 & 0.0351173 & 0.00708637 & 1831631.05 \\
\hline 21313223 & 809.545821 & 839.362572 & 1088.99087 & 789.729228 & $4.03 E-06$ & $2.50 \mathrm{E}-05$ & 63.7736673 & 0.00490083 & 0.00312544 & $1.16 \mathrm{E}-05$ & $1.33 E-05$ & $2.61 \mathrm{E}-05$ & 0.00556912 & 0.03511779 & 0.00707902 & 1830561.99 \\
\hline 21313609.6 & 808.94381 & 838.570423 & 1086.85025 & 789.155831 & $4.03 E-06$ & $2.50 \mathrm{E}-05$ & 63.773906 & 0.00490092 & 0.00312551 & $1.16 \mathrm{E}-05$ & $1.33 E-05$ & $2.61 \mathrm{E}-05$ & 0.0055622 & 0.03511839 & 0.0070698 & 1829220.97 \\
\hline 21314092.7 & 808.187389 & 837.577453 & 1084.17026 & 788.436097 & $4.03 E-06$ & $2.49 \mathrm{E}-05$ & 63.7742025 & 0.00490102 & 0.00312559 & $1.16 \mathrm{E}-05$ & $1.33 E-05$ & $2.60 \mathrm{E}-05$ & 0.00555377 & 0.03511914 & 0.00705822 & 1827537.48 \\
\hline 21314696.7 & 807.235702 & 836.34252 & 1080.81353 & 787.531692 & $4.03 E-06$ & $2.49 \mathrm{E}-05$ & 63.7745699 & 0.00490115 & 0.00312569 & $1.16 \mathrm{E}-05$ & $1.33 \mathrm{E}-05$ & $2.60 \mathrm{E}-05$ & 0.00554343 & 0.03512006 & 0.00704373 & 1825421.86 \\
\hline 21315451.7 & 806.036363 & 834.787625 & 1076.60685 & 786.393712 & $4.03 E-06$ & $2.49 \mathrm{E}-05$ & 63.7750243 & 0.00490131 & 0.00312581 & $1.16 \mathrm{E}-05$ & $1.33 E-05$ & $2.59 \mathrm{E}-05$ & 0.00553069 & 0.03512121 & 0.00702548 & 1822759.7 \\
\hline 21316019 & 805.127969 & 833.611578 & 1073.43774 & 785.5331115 & $4.03 E-06$ & $2.49 \mathrm{E}-05$ & 63.7753622 & 0.00490143 & 0.0031259 & $1.16 E-05$ & $1.33 E-05$ & $2.59 \mathrm{E}-05$ & 0.00552117 & 0.03512206 & 0.00701168 & 1820770.47 \\
\hline 21316774 & 805.127969 & 833.611653 & 1073.43748 & 785.533069 & $4.03 E-06$ & $2.49 \mathrm{E}-05$ & 63.7758097 & 0.00490159 & 0.00312603 & $1.16 \mathrm{E}-05$ & $1.33 E-05$ & $2.59 \mathrm{E}-05$ & 0.00552211 & 0.03512319 & 0.00701169 & 1820815.53 \\
\hline 21317717.7 & 805.127969 & 833.612116 & 1073.43873 & 785.533074 & $4.03 E-06$ & $2.49 \mathrm{E}-05$ & 63.7763692 & 0.00490178 & 0.00312618 & $1.16 \mathrm{E}-05$ & $1.33 E-05$ & $2.59 \mathrm{E}-05$ & 0.00552276 & 0.0351246 & 0.0070117 & 1820874.56 \\
\hline 21318897.3 & 805.127969 & 833.612422 & 1073.43971 & 785.533075 & $4.03 E-06$ & $2.49 \mathrm{E}-05$ & 63.7770684 & 0.00490203 & 0.00312637 & $1.16 E-05$ & $1.33 E-05$ & $2.59 \mathrm{E}-05$ & 0.00552321 & 0.03512636 & 0.00701172 & 1820949.25 \\
\hline 21320371.9 & 805.127969 & 833.612624 & 1073.44053 & 785.533074 & $4.03 E-06$ & $2.49 \mathrm{E}-05$ & 63.7779423 & 0.00490234 & 0.00312661 & $1.16 \mathrm{E}-05$ & $1.33 E-05$ & $2.59 \mathrm{E}-05$ & 0.00552352 & 0.03512857 & 0.00701175 & 1821043.21 \\
\hline 21322215.1 & 805.127969 & 833.612752 & 1073.44121 & 785.533069 & $4.03 E-06$ & $2.49 \mathrm{E}-05$ & 63.7790346 & 0.00490272 & 0.00312691 & $1.16 \mathrm{E}-05$ & $1.33 E-05$ & $2.59 \mathrm{E}-05$ & 0.00552371 & 0.03513133 & 0.00701177 & 1821161.13 \\
\hline 21324519.1 & 805.127969 & 833.612821 & 1073.44177 & 785.533062 & $4.03 E-06$ & $2.49 \mathrm{E}-05$ & 63.7803996 & 0.0049032 & 0.00312728 & $1.16 \mathrm{E}-05$ & $1.33 E-05$ & $2.59 \mathrm{E}-05$ & 0.00552382 & 0.03513477 & 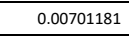 & 1821308.91 \\
\hline
\end{tabular}




\begin{tabular}{|c|c|c|c|c|c|c|c|c|c|c|c|c|c|c|c|c|}
\hline Time (s) & $\begin{array}{c}\text { Coolant outlet } \\
\text { temperature }(K)\end{array}$ & $\begin{array}{l}\text { Peak cladding } \\
\text { temperature (K) }\end{array}$ & $\begin{array}{c}\text { Peak fuel } \\
\text { temperature (K) }\end{array}$ & $\begin{array}{c}\text { Average cladding } \\
\text { internal } \\
\text { temperature (K) }\end{array}$ & $\begin{array}{l}\text { Peak cumulative } \\
\text { damage factor }\end{array}$ & $\begin{array}{l}\text { Cladding interior } \\
\text { volume }\left(\mathrm{m}^{3}\right)\end{array}$ & $\begin{array}{l}\text { Fission gas } \\
\text { release (\%) }\end{array}$ & $\begin{array}{c}\text { Fission gas } \\
\text { produced } \\
\text { (moles) }\end{array}$ & $\begin{array}{c}\text { Fission gas } \\
\text { released (moles) }\end{array}$ & Fuel volume $\left(\mathrm{m}^{3}\right)$ & $\begin{array}{l}\text { Plenum gas } \\
\text { volume }\left(m^{3}\right)\end{array}$ & $\begin{array}{l}\text { Peak cladding } \\
\text { radial growth } \\
\text { (m) }\end{array}$ & $\begin{array}{l}\text { Peak fuel axial } \\
\text { growth }(m)\end{array}$ & $\begin{array}{c}\text { Peak burnup } \\
\text { (at\%) }\end{array}$ & $\begin{array}{l}\text { Peak cladding } \\
\text { hoop strain }\end{array}$ & $\begin{array}{c}\text { Plenum pressure } \\
(\mathrm{Pa})\end{array}$ \\
\hline 21327399.1 & 805.127969 & 833.612847 & 1073.44219 & $\begin{array}{l}785.53305 \\
\end{array}$ & $4.03 \mathrm{E}-06$ & $2.49 \mathrm{E}-05$ & 63.7821056 & $\begin{array}{l}0.0049038 \\
\end{array}$ & 0.00312775 & $1.16 \mathrm{E}-05$ & $1.33 \mathrm{E}-05$ & $2.59 \mathrm{E}-05$ & 0.00552389 & 0.03513908 & 0.00701186 & 1821493.95 \\
\hline 21330999.1 & 805.127969 & 833.612845 & 1073.44245 & 785.533033 & $4.03 E-06$ & $2.49 \mathrm{E}-05$ & 63.7842375 & 0.00490456 & 0.00312833 & $1.16 \mathrm{E}-05$ & $1.33 E-05$ & $2.59 \mathrm{E}-05$ & 0.00552395 & 0.03514446 & 0.00701191 & 1821725.51 \\
\hline 21335499.1 & 805.127969 & 833.612832 & 1073.44255 & 785.533011 & $4.03 \mathrm{E}-06$ & $2.49 \mathrm{E}-05$ & 63.7869014 & 0.00490549 & 0.00312906 & $1.16 \mathrm{E}-05$ & $1.33 E-05$ & $2.59 \mathrm{E}-05$ & 0.00552404 & 0.03515119 & 0.00701198 & 1822015.13 \\
\hline 21341124.1 & 805.127969 & 833.612818 & 1073.44251 & 785.532983 & $\begin{array}{l}4.03 E-06 \\
\end{array}$ & $2.49 \mathrm{E}-05$ & 63.7902299 & 0.00490667 & 0.00312998 & $1.16 \mathrm{E}-05$ & $1.33 E-05$ & $2.59 \mathrm{E}-05$ & 0.00552416 & 0.0351596 & 0.00701207 & 1822377.3 \\
\hline 21348155.4 & 805.127969 & 833.612807 & 1073.44233 & 785.532947 & $4.03 \mathrm{E}-06$ & $2.49 \mathrm{E}-05$ & 63.7943882 & 0.00490814 & 0.00313112 & $1.16 \mathrm{E}-05$ & $1.33 E-05$ & $2.59 \mathrm{E}-05$ & 0.00552433 & 0.03517012 & 0.00701219 & 1822830.09 \\
\hline 21356944.4 & 805.127969 & 833.6128 & 1073.44205 & 785.532902 & $4.03 E-06$ & $2.49 \mathrm{E}-05$ & 63.7995827 & 0.00490997 & 0.00313254 & $1.16 \mathrm{E}-05$ & $1.33 \mathrm{E}-05$ & $2.59 \mathrm{E}-05$ & 0.00552454 & 0.03518326 & 0.00701232 & 1823396.14 \\
\hline 21367930.7 & 805.127969 & 833.612796 & 1073.44166 & $\begin{array}{l}785.532846 \\
\end{array}$ & $4.03 \mathrm{E}-06$ & $2.49 \mathrm{E}-05$ & 63.8060703 & 0.00491226 & 0.00313432 & $1.16 \mathrm{E}-05$ & $1.33 E-05$ & $2.59 \mathrm{E}-05$ & 0.00552482 & 0.03519969 & 0.0070125 & 1824103.73 \\
\hline 21381663.7 & 805.127969 & 833.612793 & 1073.44116 & 785.532775 & 4.03E- -06 & $2.49 \mathrm{E}-05$ & 63.8141713 & 0.00491513 & 0.00313655 & $1.16 \mathrm{E}-05$ & $1.33 E-05$ & $2.59 \mathrm{E}-05$ & 0.00552517 & 0.03522022 & 0.00701272 & 1824988.24 \\
\hline 21398829.8 & 805.127969 & 833.612791 & 1073.44053 & $\begin{array}{l}785.532687 \\
\end{array}$ & $4.03 \mathrm{E}-06$ & $2.49 \mathrm{E}-05$ & 63.8242842 & 0.00491871 & 0.00313933 & $1.16 \mathrm{E}-05$ & $1.33 E-05$ & $2.59 \mathrm{E}-05$ & 0.0055256 & 0.03524589 & 0.00701299 & 1826093.89 \\
\hline 21420287.5 & 805.127969 & 833.612789 & 1073.43974 & 785.532576 & $4.03 \mathrm{E}-06$ & $2.49 \mathrm{E}-05$ & 63.8369048 & 0.00492319 & 0.00314281 & $1.16 \mathrm{E}-05$ & $1.33 E-05$ & $2.59 \mathrm{E}-05$ & 0.00552615 & 0.03527798 & 0.00701333 & 1827475.98 \\
\hline 21447109.6 & 805.127969 & 833.612784 & 1073.43874 & 785.532439 & $4.03 E-06$ & $2.49 E-05$ & 63.8526481 & 0.00492879 & 0.00314716 & $1.16 \mathrm{E}-05$ & $1.33 \mathrm{E}-05$ & $2.59 \mathrm{E}-05$ & 0.00552684 & 0.03531809 & 0.00701375 & 1829203.62 \\
\hline 21480637.2 & 805.127969 & 833.612775 & 1073.4375 & 785.532266 & $4.03 \mathrm{E}-06$ & $2.49 \mathrm{E}-05$ & 63.8722772 & 0.00493578 & 0.0031526 & $1.16 \mathrm{E}-05$ & $1.33 E-05$ & $2.59 \mathrm{E}-05$ & 0.0055277 & 0.03536822 & 0.00701429 & 1831363.23 \\
\hline 21522546.7 & 805.127969 & 833.612757 & 1073.43595 & 785.532051 & $4.03 E-06$ & $2.49 \mathrm{E}-05$ & 63.8967353 & 0.00494453 & 0.00315939 & $1.16 \mathrm{E}-05$ & $1.33 \mathrm{E}-05$ & $2.59 \mathrm{E}-05$ & 0.00552877 & 0.03543089 & 0.00701495 & 1834062.82 \\
\hline 21574933.6 & 805.127969 & 833.612723 & 1073.434 & 785.531775 & $4.03 E-06$ & $2.49 E-05$ & 63.9271866 & 0.00495546 & 0.00316789 & $1.16 \mathrm{E}-05$ & $1.33 \mathrm{E}-05$ & $2.59 \mathrm{E}-05$ & 0.00553012 & 0.03550923 & 0.00701578 & 1837437.41 \\
\hline 21640417.2 & 805.127969 & 833.612525 & 1073.42858 & 785.531004 & $4.03 \mathrm{E}-06$ & $2.49 \mathrm{E}-05$ & 63.9650623 & 0.00496913 & 0.0031785 & $1.16 \mathrm{E}-05$ & $1.33 E-05$ & $2.59 E-05$ & 0.00553172 & 0.03560715 & 0.00701682 & 1841717.57 \\
\hline 21722271.7 & 805.127969 & 833.612414 & 1073.42482 & 785.530443 & $4.03 \mathrm{E}-06$ & $2.49 \mathrm{E}-05$ & 64.012115 & 0.00498621 & 0.00319178 & $1.16 \mathrm{E}-05$ & $1.33 E-05$ & $2.59 E-05$ & 0.00553375 & 0.03572955 & 0.00701813 & 1847010.91 \\
\hline 21824589.9 & 805.127969 & 833.611991 & \begin{tabular}{|l}
1073.4201 \\
\end{tabular} & 785.529695 & $4.03 \mathrm{E}-06$ & $2.49 \mathrm{E}-05$ & 64.0704795 & 0.00500756 & 0.00320837 & $1.16 \mathrm{E}-05$ & $1.33 E-05$ & $2.59 \mathrm{E}-05$ & 0.00553631 & 0.03588255 & 0.00701976 & 1853634.12 \\
\hline 21952487.6 & 805.127969 & 833.609848 & $\begin{array}{l}1073.4144 \\
\end{array}$ & 785.528695 & $4.03 \mathrm{E}-06$ & $2.49 \mathrm{E}-05$ & 64.1427388 & 0.00503425 & 0.00322911 & $1.16 \mathrm{E}-05$ & $1.33 E-05$ & $2.59 \mathrm{E}-05$ & 0.00553962 & 0.0360738 & 0.0070218 & 1861932.11 \\
\hline 22112359.7 & 805.127969 & 833.603296 & 1073.40724 & 785.527198 & $4.03 E-06$ & $2.49 \mathrm{E}-05$ & 64.2319926 & 0.00506761 & 0.00325503 & $1.17 \mathrm{E}-05$ & $1.33 \mathrm{E}-05$ & $2.59 \mathrm{E}-05$ & 0.00554407 & 0.03631287 & 0.00702434 & 1872345.16 \\
\hline 22312199.8 & 805.127969 & 833.599433 & 1073.39838 & 785.524972 & $4.03 E-06$ & $2.49 \mathrm{E}-05$ & 64.3419207 & 0.00510931 & 0.00328743 & $1.17 \mathrm{E}-05$ & $1.33 E-05$ & $2.59 E-05$ & 0.00554992 & 0.0366117 & 0.00702753 & 1885430.85 \\
\hline 22562000 & 805.127969 & 833.592957 & 1073.38741 & 785.521651 & $4.03 \mathrm{E}-06$ & $2.49 \mathrm{E}-05$ & 64.4768328 & 0.00516144 & 0.00332794 & $1.17 \mathrm{E}-05$ & $1.33 E-05$ & $2.59 E-05$ & 0.00555796 & 0.03698524 & $\begin{array}{l}0.0070316 \\
\end{array}$ & 1901895.41 \\
\hline 22874250.2 & 805.127969 & 833.583654 & 1073.42298 & 785.516698 & $4.03 \mathrm{E}-06$ & $2.49 \mathrm{E}-05$ & 64.6416886 & 0.0052266 & 0.00337857 & $1.17 \mathrm{E}-05$ & $1.33 E-05$ & $2.59 E-05$ & 0.00556866 & 0.03745216 & 0.0070367 & 1922627.17 \\
\hline 23264563 & 805.127969 & 833.570815 & 1073.47972 & 785.509818 & $4.03 E-06$ & $2.49 \mathrm{E}-05$ & 64.8420664 & 0.00530806 & 0.00344185 & $1.17 \mathrm{E}-05$ & $1.33 E-05$ & $2.60 \mathrm{E}-05$ & 0.00558242 & 0.03803581 & 0.00704314 & 1948686.63 \\
\hline 23752454 & 805.127969 & 833.552185 & 1073.64669 & 785.500345 & $4.03 \mathrm{E}-06$ & $2.49 \mathrm{E}-05$ & 65.0840537 & 0.00540987 & 0.00352096 & $1.17 \mathrm{E}-05$ & $1.33 E-05$ & $2.60 \mathrm{E}-05$ & 0.00560002 & 0.03876538 & 0.00705129 & 1981449.41 \\
\hline 24362317.7 & 805.127969 & 833.524476 & 1073.87676 & 785.488163 & $4.03 E-06$ & $2.49 \mathrm{E}-05$ & 65.3740234 & 0.00553714 & 0.00361985 & $1.17 \mathrm{E}-05$ & $1.33 E-05$ & $2.60 \mathrm{E}-05$ & 0.00562176 & 0.03967734 & 0.00706164 & 2022538.94 \\
\hline 25124647.4 & 805.127969 & 833.482654 & 1074.21402 & 785.47285 & $4.03 E-06$ & $2.49 \mathrm{E}-05$ & 65.7182644 & 0.00569622 & 0.00374346 & $1.17 \mathrm{E}-05$ & $1.32 E-05$ & $2.61 E-05$ & 0.005648 & 0.04081729 & 0.00707482 & 2074029.06 \\
\hline 25596874 & 805.127969 & 833.453637 & 1074.41802 & 785.463779 & $4.03 E-06$ & $2.50 \mathrm{E}-05$ & 65.9220247 & 0.00579477 & 0.00382003 & $1.17 \mathrm{E}-05$ & $1.32 E-05$ & $2.61 \mathrm{E}-05$ & 0.00566378 & 0.04152343 & 0.00708453 & 2136688.39 \\
\hline 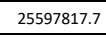 & \begin{tabular}{|l}
804.927561 \\
\end{tabular} & 833.15266 & 1073.25742 & \begin{tabular}{|l}
785.246476 \\
\end{tabular} & $4.03 E-06$ & $2.49 \mathrm{E}-05$ & 65.9224243 & 0.00579496 & 0.00382018 & $1.17 \mathrm{E}-05$ & $1.32 E-05$ & $2.61 E-05$ & 0.00565985 & 0.04152484 & 0.00708281 & 2173849.97 \\
\hline 25598761.4 & 804.726218 & 832.850484 & 1072.09603 & 785.028437 & $4.03 \mathrm{E}-06$ & $2.49 \mathrm{E}-05$ & 65.9228224 & 0.00579516 & 0.00382033 & $1.17 \mathrm{E}-05$ & $1.32 E-05$ & $2.61 \mathrm{E}-05$ & 0.005656 & 0.04152625 & 0.00707941 & 2173284.64 \\
\hline 25599941.1 & 804.473214 & 832.471173 & 1070.64298 & 784.754861 & $4.03 E-06$ & $2.49 \mathrm{E}-05$ & 65.9233179 & 0.0057954 & 0.00382052 & $1.17 \mathrm{E}-05$ & $1.32 E-05$ & $2.61 E-05$ & 0.00565136 & 0.04152799 & 0.00707515 & 2172555.68 \\
\hline 25600474 & 804.358432 & 832.299244 & 1069.98595 & 784.630893 & $4.03 E-06$ & $2.49 \mathrm{E}-05$ & 65.9235411 & 0.00579551 & 0.00382061 & $1.17 \mathrm{E}-05$ & $1.32 E-05$ & $2.61 \mathrm{E}-05$ & 0.00564929 & 0.04152878 & 0.00707322 & 2172284.78 \\
\hline 25601653.6 & 804.358432 & 832.299235 & 1069.98591 & 784.630885 & $4.03 E-06$ & $2.49 \mathrm{E}-05$ & 65.9240345 & 0.00579576 & 0.0038208 & $1.17 \mathrm{E}-05$ & $1.32 E-05$ & $2.61 \mathrm{E}-05$ & 0.00564941 & 0.04153052 & 0.00707324 & 2172326.84 \\
\hline 25603128.2 & 804.358432 & 832.299234 & 1069.98652 & 784.63088 & $4.03 \mathrm{E}-06$ & $2.49 \mathrm{E}-05$ & 65.9246512 & 0.00579606 & 0.00382103 & $1.17 \mathrm{E}-05$ & $1.32 E-05$ & $2.61 \mathrm{E}-05$ & 0.00564946 & 0.0415327 & 0.00707327 & 2172420.5 \\
\hline 25604971.4 & 804.358432 & 832.299226 & 1069.98696 & 784.630873 & $4.03 \mathrm{E}-06$ & $2.49 \mathrm{E}-05$ & 65.925422 & $\begin{array}{c}0.00579644 \\
\end{array}$ & 0.00382133 & $1.17 \mathrm{E}-05$ & $1.32 E-05$ & $2.61 \mathrm{E}-05$ & 0.00564947 & 0.04153541 & $\begin{array}{l}0.0070733 \\
\end{array}$ & 2172537.96 \\
\hline 25607275.4 & 804.358432 & 832.299213 & 1069.98728 & 784.630863 & $4.03 \mathrm{E}-06$ & $2.49 \mathrm{E}-05$ & 65.9263853 & 0.00579691 & 0.0038217 & $1.17 \mathrm{E}-05$ & $1.32 E-05$ & $2.61 \mathrm{E}-05$ & 0.00564947 & 0.04153881 & 0.00707334 & 2172685.04 \\
\hline 25610155.4 & 804.358432 & 832.299194 & 1069.98748 & 784.63085 & $4.03 E-06$ & $2.49 E-05$ & 65.9275892 & 0.00579751 & 0.00382216 & $1.17 \mathrm{E}-05$ & $1.32 E-05$ & $2.61 E-05$ & 0.00564949 & 0.04154306 & 0.00707339 & 2172869.05 \\
\hline 25613755.4 & 804.358432 & 832.29917 & 1069.98757 & 784.630833 & $4.03 E-06$ & $2.49 \mathrm{E}-05$ & 65.9290938 & 0.00579825 & 0.00382273 & $1.17 \mathrm{E}-05$ & $1.32 E-05$ & $2.61 E-05$ & 0.00564953 & 0.04154837 & 0.00707346 & 2173099.2 \\
\hline 25618255.4 & 804.358432 & 832.29914 & 1069.98755 & 784.630811 & $4.03 \mathrm{E}-06$ & $2.49 \mathrm{E}-05$ & 65.930974 & 0.00579917 & 0.00382345 & $1.17 \mathrm{E}-05$ & $1.32 E-05$ & $2.61 E-05$ & 0.00564961 & 0.04155501 & 0.00707354 & 2173386.98 \\
\hline 25623880.4 & 804.358432 & 832.299102 & 1069.98742 & 784.630783 & $4.03 E-06$ & $2.49 \mathrm{E}-05$ & 65.9333234 & 0.00580033 & 0.00382435 & $1.17 \mathrm{E}-05$ & $1.32 E-05$ & $2.61 \mathrm{E}-05$ & 0.00564973 & 0.04156331 & 0.00707364 & 2173746.77 \\
\hline 25630911.7 & 804.358432 & 832.299056 & 1069.9872 & 784.630747 & $4.03 \mathrm{E}-06$ & $2.49 \mathrm{E}-05$ & 65.9362588 & 0.00580178 & 0.00382548 & $1.17 \mathrm{E}-05$ & $1.32 E-05$ & $2.61 \mathrm{E}-05$ & 0.00564989 & 0.04157368 & 0.00707377 & 2174196.56 \\
\hline 25639700.7 & 804.358432 & 832.298998 & 1069.98689 & 784.630701 & $4.03 \mathrm{E}-06$ & $2.49 E-05$ & 65.9399261 & 0.00580359 & 0.00382688 & $1.17 \mathrm{E}-05$ & $1.32 E-05$ & $2.61 \mathrm{E}-05$ & 0.00565009 & 0.04158664 & 0.00707392 & 2174758.82 \\
\hline 25650687 & 804.358432 & 832.298834 & 1069.98594 & 784.630574 & $4.03 E-06$ & $2.49 \mathrm{E}-05$ & 65.9445069 & 0.00580585 & 0.00382864 & $1.17 \mathrm{E}-05$ & $1.32 E-05$ & $2.61 \mathrm{E}-05$ & 0.00565034 & 0.04160285 & 0.00707412 & 2175473.37 \\
\hline 25664420 & 804.358432 & 832.298718 & 1069.98529 & 784.630485 & $4.03 \mathrm{E}-06$ & $2.49 \mathrm{E}-05$ & 65.9502279 & 0.00580868 & 0.00383084 & $1.17 \mathrm{E}-05$ & $1.32 E-05$ & $2.61 \mathrm{E}-05$ & 0.00565065 & 0.0416231 & 0.00707437 & 2176354.87 \\
\hline 25681586.1 & 804.358432 & 832.298572 & 1069.98447 & 784.630374 & $4.03 E-06$ & $2.49 \mathrm{E}-05$ & 65.9573713 & 0.00581221 & 0.00383358 & $1.17 \mathrm{E}-05$ & $1.32 E-05$ & $2.61 \mathrm{E}-05$ & 0.00565104 & 0.04164842 & 0.00707468 & 2177456.77 \\
\hline 25703043.8 & 804.358432 & $\begin{array}{l}832.298415 \\
\end{array}$ & \begin{tabular}{|l|l|}
1069.98351 \\
\end{tabular} & \begin{tabular}{|l}
784.630235 \\
\end{tabular} & $4.03 \mathrm{E}-06$ & $\begin{array}{l}2.49 \mathrm{E}-05 \\
\end{array}$ & 65.9662884 & 0.00581663 & 0.00383701 & $1.17 \mathrm{E}-05$ & $1.32 E-05$ & $2.61 \mathrm{E}-05$ & 0.00565153 & 0.04168007 & 0.00707506 & 2178834.23 \\
\hline 25729865.9 & 804.358432 & 832.298212 & 1069.98226 & 784.630056 & $4.03 \mathrm{E}-06$ & $2.49 \mathrm{E}-05$ & 65.9774157 & 0.00582215 & 0.0038413 & $1.17 \mathrm{E}-05$ & $1.32 E-05$ & $2.61 E-05$ & 0.00565214 & 0.04171964 & 0.00707554 & 2180556.75 \\
\hline
\end{tabular}




\begin{tabular}{|c|c|c|c|c|c|c|c|c|c|c|c|c|c|c|c|c|}
\hline Time (s) & $\begin{array}{c}\text { Coolant outlet } \\
\text { temperature }(\mathrm{K})\end{array}$ & $\begin{array}{l}\text { Peak cladding } \\
\text { temperature (K) }\end{array}$ & $\begin{array}{l}\text { Peak fuel } \\
\text { temperature (K) }\end{array}$ & $\begin{array}{c}\text { Average cladding } \\
\text { internal } \\
\text { temperature (K) }\end{array}$ & $\begin{array}{l}\text { Peak cumulative } \\
\text { damage factor }\end{array}$ & $\begin{array}{l}\text { Cladding interior } \\
\text { volume }\left(\mathrm{m}^{3}\right)\end{array}$ & $\begin{array}{l}\text { Fission gas } \\
\text { release (\%) }\end{array}$ & $\begin{array}{l}\text { Fission gas } \\
\text { produced } \\
\text { (moles) }\end{array}$ & $\begin{array}{c}\text { Fission gas } \\
\text { released (moles) }\end{array}$ & Fuel volume $\left(\mathrm{m}^{3}\right)$ & $\begin{array}{l}\text { Plenum gas } \\
\text { volume }\left(\mathrm{m}^{3}\right)\end{array}$ & $\begin{array}{l}\text { Peak cladding } \\
\text { radial growth } \\
\text { (m) }\end{array}$ & $\begin{array}{l}\text { Peak fuel axial } \\
\text { growth }(m)\end{array}$ & $\begin{array}{c}\text { Peak burnup } \\
\text { (at\%) }\end{array}$ & $\begin{array}{l}\text { Peak cladding } \\
\text { hoop strain }\end{array}$ & $\begin{array}{l}\text { Plenum pressure } \\
(\mathrm{Pa})\end{array}$ \\
\hline 25763393.5 & 804.358432 & 832.297837 & 1069.98069 & \begin{tabular}{|l}
784.629829 \\
\end{tabular} & $4.03 E-06$ & $2.49 \mathrm{E}-05$ & 65.9912953 & 0.00582905 & 0.00384667 & $1.17 \mathrm{E}-05$ & $1.32 \mathrm{E}-05$ & $2.61 \mathrm{E}-05$ & 0.00565291 & 0.04176909 & 0.00707615 & 2182711.75 \\
\hline 25805303 & 804.358432 & 832.296837 & 1069.97873 & 784.629522 & $4.03 \mathrm{E}-06$ & $2.49 \mathrm{E}-05$ & 66.0085985 & 0.00583768 & 0.00385337 & $1.17 \mathrm{E}-05$ & $1.32 \mathrm{E}-05$ & $2.61 \mathrm{E}-05$ & 0.0056539 & 0.04183091 & 0.0070769 & 2185409.51 \\
\hline 25857689.9 & 804.358432 & 832.295165 & 1069.97631 & \begin{tabular}{|l|l|}
784.629104 \\
\end{tabular} & $4.03 \mathrm{E}-06$ & $2.49 \mathrm{E}-05$ & 66.0301558 & 0.00584846 & 0.00386175 & $1.17 \mathrm{E}-05$ & $1.32 \mathrm{E}-05$ & $2.61 \mathrm{E}-05$ & 0.00565518 & 0.04190818 & 0.00707783 & 2188790.12 \\
\hline 25923173.5 & 804.358432 & 832.292358 & 1069.97336 & \begin{tabular}{|l|l|}
784.628527 \\
\end{tabular} & $4.03 \mathrm{E}-06$ & $2.49 \mathrm{E}-05$ & 66.0569908 & 0.00586194 & 0.00387222 & $1.17 \mathrm{E}-05$ & $1.32 \mathrm{E}-05$ & $2.61 E-05$ & 0.00565684 & 0.04200477 & 0.00707901 & 2193029.87 \\
\hline 26005028 & 804.358432 & 832.28805 & 1069.96967 & \begin{tabular}{|l|l|}
784.627703 \\
\end{tabular} & $4.03 \mathrm{E}-06$ & $2.49 \mathrm{E}-05$ & 66.0903616 & 0.00587879 & 0.00388531 & $1.17 \mathrm{E}-05$ & $1.32 E-05$ & $2.61 \mathrm{E}-05$ & 0.00565906 & 0.04212551 & 0.00708047 & 2198351.81 \\
\hline 26107346.2 & 804.358432 & 832.282055 & 1069.97522 & \begin{tabular}{|l|l|}
784.626511 \\
\end{tabular} & $4.03 E-06$ & $2.49 \mathrm{E}-05$ & 66.131807 & 0.00589985 & 0.00390168 & $1.17 \mathrm{E}-05$ & $1.32 \mathrm{E}-05$ & $2.61 \mathrm{E}-05$ & 0.00566199 & 0.04227644 & 0.00708229 & 2205038.18 \\
\hline 26235243.9 & $\begin{array}{l}804.358432 \\
\end{array}$ & 832.274406 & 1069.98531 & \begin{tabular}{|l|l|}
784.624857 \\
\end{tabular} & $4.03 \mathrm{E}-06$ & $2.49 \mathrm{E}-05$ & 66.1831995 & 0.00592618 & 0.00392214 & $1.17 \mathrm{E}-05$ & $1.32 \mathrm{E}-05$ & $2.61 \mathrm{E}-05$ & 0.00566577 & 0.04246509 & 0.00708457 & 2213431.66 \\
\hline 26261755 & 804.358432 & 832.27287 & 1069.99057 & $\begin{array}{l}784.62451 \\
\end{array}$ & 4.03E- -06 & $2.49 \mathrm{E}-05$ & 66.1937953 & 0.00593164 & 0.00392638 & $1.17 \mathrm{E}-05$ & $1.32 E-05$ & $2.61 \mathrm{E}-05$ & 0.00566657 & 0.0425042 & 0.00708544 & 2223593.07 \\
\hline 26265355 & 803.788828 & 832.455452 & $\begin{array}{l}1078.62767 \\
\end{array}$ & \begin{tabular}{|l|l|}
784.684348 \\
\end{tabular} & $4.03 \mathrm{E}-06$ & $2.49 \mathrm{E}-05$ & 66.1952535 & 0.00593239 & 0.00392696 & $1.17 \mathrm{E}-05$ & $1.32 \mathrm{E}-05$ & $2.61 \mathrm{E}-05$ & 0.00569937 & 0.04250958 & 0.00708461 & 2228465.83 \\
\hline 26291866.1 & 803.788828 & 832.455349 & 1078.63473 & 784.68389 & $4.03 E-06$ & $2.49 \mathrm{E}-05$ & 66.2061354 & 0.00593801 & 0.00393132 & $1.17 \mathrm{E}-05$ & $1.32 \mathrm{E}-05$ & $2.61 \mathrm{E}-05$ & 0.00569805 & 0.04254983 & 0.00708503 & 2228850.03 \\
\hline 26325005 & \begin{tabular}{ll|}
803.788828 \\
\end{tabular} & 832.453008 & 1078.64807 & 784.683311 & $4.03 E-06$ & $2.49 \mathrm{E}-05$ & 66.2197088 & 0.00594503 & 0.00393678 & $1.17 \mathrm{E}-05$ & $1.32 \mathrm{E}-05$ & $2.61 \mathrm{E}-05$ & 0.00569892 & 0.04260014 & 0.00708564 & 2231115.24 \\
\hline \begin{tabular}{|l|}
26366428.7 \\
\end{tabular} & 803.788828 & 832.449699 & 1078.66543 & \begin{tabular}{|l|l|}
784.682546 \\
\end{tabular} & $4.03 \mathrm{E}-06$ & $2.49 \mathrm{E}-05$ & 66.2366305 & 0.0059538 & 0.0039436 & $1.18 \mathrm{E}-05$ & $1.32 \mathrm{E}-05$ & $2.61 \mathrm{E}-05$ & 0.00570025 & 0.04266302 & 0.00708641 & 2233956.25 \\
\hline 26418208.2 & 803.788828 & 832.445537 & 1078.68706 & \begin{tabular}{|l|l|}
784.681614 \\
\end{tabular} & $4.03 \mathrm{E}-06$ & $2.49 \mathrm{E}-05$ & 66.2577127 & 0.00596477 & 0.00395212 & $1.18 \mathrm{E}-05$ & $1.32 E-05$ & $2.61 \mathrm{E}-05$ & 0.00570195 & 0.04274163 & 0.0070874 & 2237501.92 \\
\hline 26482932.7 & 803.788828 & 832.440268 & 1078.7138 & 784.680453 & $4.03 \mathrm{E}-06$ & $2.50 \mathrm{E}-05$ & 66.2839566 & 0.00597848 & 0.00396278 & $1.18 \mathrm{E}-05$ & $1.32 E-05$ & $2.61 \mathrm{E}-05$ & 0.00570408 & 0.04283988 & 0.00708863 & 2241933.81 \\
\hline 26563838.2 & 803.788828 & 832.433598 & 1078.74676 & 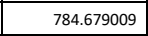 & $4.03 \mathrm{E}-06$ & $2.50 \mathrm{E}-05$ & 66.3165926 & 0.00599562 & 0.00397609 & $1.18 \mathrm{E}-05$ & $1.32 \mathrm{E}-05$ & $2.61 \mathrm{E}-05$ & 0.00570673 & 0.04296271 & 0.00709017 & 2247473.31 \\
\hline 26664970.2 & 803.788828 & 832.425145 & 1078.78729 & 784.677214 & $4.03 \mathrm{E}-06$ & $2.50 \mathrm{E}-05$ & 66.3571262 & 0.00601705 & 0.00399274 & $1.18 \mathrm{E}-05$ & $1.32 \mathrm{E}-05$ & $2.61 \mathrm{E}-05$ & 0.00571005 & 0.04311623 & 0.00709209 & 2254397.14 \\
\hline 26791385.1 & 803.788828 & 832.414401 & 1078.83705 & 784.674985 & $4.03 \mathrm{E}-06$ & $2.50 \mathrm{E}-05$ & 66.4073891 & 0.00604383 & 0.00401355 & $1.18 \mathrm{E}-05$ & $1.32 \mathrm{E}-05$ & $2.61 \mathrm{E}-05$ & $\begin{array}{l}0.00571417 \\
\end{array}$ & 0.04330814 & 0.00709451 & 2263051.83 \\
\hline 26949403.7 & 803.788828 & 832.400704 & 1078.8991 & 784.672224 & $4.03 \mathrm{E}-06$ & $2.50 \mathrm{E}-05$ & 66.4695947 & 0.00607731 & 0.00403956 & $1.18 \mathrm{E}-05$ & $1.32 \mathrm{E}-05$ & $2.61 \mathrm{E}-05$ & 0.00571931 & 0.04354803 & 0.00709755 & 2273868.96 \\
\hline 27146927 & 803.788828 & 832.383154 & 1078.97798 & 784.668817 & $4.03 E-06$ & $2.50 \mathrm{E}-05$ & 66.5463946 & 0.00611915 & 0.00407208 & $1.18 \mathrm{E}-05$ & $1.32 \mathrm{E}-05$ & $2.62 E-05$ & 0.00572571 & 0.04384788 & 0.00710135 & 2287387.73 \\
\hline 27393831.2 & 803.788828 & 832.360547 & 1079.07265 & $\begin{array}{l}784.664626 \\
\end{array}$ & $4.03 E-06$ & $2.50 \mathrm{E}-05$ & 66.6409298 & 0.00617146 & 0.00411272 & $1.18 \mathrm{E}-05$ & $1.32 E-05$ & $2.62 E-05$ & 0.00573367 & 0.0442227 & 0.00710614 & 2304282.13 \\
\hline 27702461.3 & 803.788828 & 832.331247 & 1079.18461 & 784.65949 & $4.04 \mathrm{E}-06$ & $2.50 \mathrm{E}-05$ & 66.7568689 & 0.00623685 & 0.00416352 & $1.18 \mathrm{E}-05$ & $1.32 \mathrm{E}-05$ & $2.62 \mathrm{E}-05$ & 0.00574355 & 0.04469123 & 0.00711233 & 2325393.63 \\
\hline 28088249 & 803.788828 & 832.292956 & 1079.31775 & 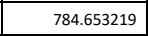 & $4.04 \mathrm{E}-06$ & $2.50 \mathrm{E}-05$ & 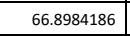 & 0.00631858 & 0.00422703 & $1.18 \mathrm{E}-05$ & $1.32 \mathrm{E}-05$ & $2.62 \mathrm{E}-05$ & 0.00575584 & 0.04527689 & 0.00712014 & 2351774.25 \\
\hline 28570483.7 & 803.788828 & 832.772847 & 1079.47356 & 784.695902 & $4.04 \mathrm{E}-06$ & $2.50 \mathrm{E}-05$ & 67.070288 & 0.00642074 & 0.00430641 & $1.18 \mathrm{E}-05$ & $1.32 E-05$ & $2.62 \mathrm{E}-05$ & 0.00577197 & 0.04600896 & 0.00713012 & 2384921.56 \\
\hline 29173277 & 803.788828 & 832.735614 & 1079.64982 & 784.686791 & $4.04 \mathrm{E}-06$ & $2.50 \mathrm{E}-05$ & 67.2775835 & 0.00654845 & 0.00440564 & $1.18 \mathrm{E}-05$ & $1.31 \mathrm{E}-05$ & $2.63 \mathrm{E}-05$ & 0.0057908 & 0.04692405 & 0.00714262 & 2426095.04 \\
\hline 29926768.6 & 803.788828 & 832.692105 & 1079.84394 & 784.675958 & $4.04 \mathrm{E}-06$ & $2.50 \mathrm{E}-05$ & 67.5256036 & 0.00670808 & 0.00452967 & $1.18 \mathrm{E}-05$ & $1.31 E-05$ & $2.63 \mathrm{E}-05$ & 0.00581397 & 0.04806791 & 0.00715851 & 2477520.86 \\
\hline 30868633.2 & 803.788828 & 832.641922 & 1080.04354 & 784.663231 & $4.05 E-06$ & $2.50 \mathrm{E}-05$ & 67.8195087 & 0.00690761 & 0.00468471 & $1.18 \mathrm{E}-05$ & $1.31 E-05$ & $2.64 \mathrm{E}-05$ & 0.0058424 & 0.04949774 & 0.00717881 & 2541739.93 \\
\hline 31868633.2 & 803.788828 & 832.593239 & 1080.2045 & 784.650692 & $4.05 E-06$ & $2.50 \mathrm{E}-05$ & 68.1135234 & 0.00711947 & 0.00484932 & $1.18 \mathrm{E}-05$ & $1.31 \mathrm{E}-05$ & $2.65 \mathrm{E}-05$ & 0.00587216 & 0.05101582 & 0.00720145 & 2621445.88 \\
\hline 32714598 & 803.788828 & 832.127532 & 1080.12197 & 784.597237 & $4.06 E-06$ & $2.50 \mathrm{E}-05$ & 68.3489223 & 0.00729869 & 0.00498858 & $1.19 \mathrm{E}-05$ & $1.31 E-05$ & $2.65 E-05$ & 0.00559329 & 0.05230007 & 0.00722123 & 2704761.78 \\
\hline 32718198 & 6444.15 & 644.175799 & 644.154096 & 644.151101 & $4.06 \mathrm{E}-06$ & $2.48 \mathrm{E}-05$ & 68.3494109 & 0.00729907 & 0.00498887 & $1.17 \mathrm{E}-05$ & $1.31 \mathrm{E}-05$ & $1.89 \mathrm{E}-05$ & 0.00390501 & 0.0523028 & 0.005186 & 2271498.62 \\
\hline 32721798 & 305 & 305.051386 & 305.006658 & 305.002179 & $4.06 E-06$ & $2.45 \mathrm{E}-05$ & 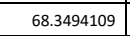 & 0.00729907 & 0.00498887 & $1.15 \mathrm{E}-05$ & $1.30 \mathrm{E}-05$ & $2.62 \mathrm{E}-06$ & 0.0018842 & 0.0523028 & 0.00077549 & 1085561.5 \\
\hline 32725398 & 305 & 305.051386 & 305.006658 & 305.002179 & $4.06 E-06$ & $2.45 \mathrm{E}-05$ & 68.3494109 & 0.00729907 & 0.00498887 & $1.15 \mathrm{E}-05$ & $1.30 \mathrm{E}-05$ & $2.62 \mathrm{E}-06$ & 0.0018842 & 0.0523028 & 0.00077549 & 1085561.5 \\
\hline 32728998 & 778.966356 & 802.498979 & 1020.37279 & 762.788336 & $4.06 \mathrm{E}-06$ & $2.49 \mathrm{E}-05$ & 68.3498253 & 0.0072994 & 0.00498912 & $1.18 \mathrm{E}-05$ & $1.31 \mathrm{E}-05$ & $\begin{array}{l}2.52 \mathrm{E}-05 \\
\end{array}$ & 0.00528456 & 0.05230512 & 0.00687112 & 2696734.64 \\
\hline 32732598 & 778.966356 & 802.49097 & 1020.37705 & 762.792204 & $4.06 \mathrm{E}-06$ & $2.49 \mathrm{E}-05$ & 68.3506541 & 0.00730004 & 0.00498963 & $1.18 \mathrm{E}-05$ & $1.31 \mathrm{E}-05$ & $2.52 \mathrm{E}-05$ & 0.00527904 & 0.05230975 & 0.00687122 & 2696879.46 \\
\hline 32737098 & 778.966356 & 802.483394 & $\begin{array}{l}1020.37587 \\
\end{array}$ & 762.792195 & $4.06 \mathrm{E}-06$ & $2.49 E-05$ & 68.3516899 & 0.00730085 & 0.00499026 & $1.18 \mathrm{E}-05$ & $1.31 \mathrm{E}-05$ & $2.52 \mathrm{E}-05$ & 0.0052772 & 0.05231555 & 0.0068713 & 2697069.73 \\
\hline 32742723 & 778.966356 & 802.480857 & 1020.37467 & \begin{tabular}{|l}
762.79215 \\
\end{tabular} & $4.06 \mathrm{E}-06$ & $2.49 \mathrm{E}-05$ & 68.3529844 & 0.00730186 & 0.00499104 & $1.18 \mathrm{E}-05$ & $1.31 \mathrm{E}-05$ & $2.52 \mathrm{E}-05$ & 0.00527682 & 0.05232279 & 0.00687141 & 2697372.31 \\
\hline 32749754.3 & 778.966356 & 802.4805 & 1020.37392 & 762.792098 & $4.06 E-06$ & $2.49 \mathrm{E}-05$ & 68.3546019 & 0.00730313 & 0.00499202 & $1.18 \mathrm{E}-05$ & $1.31 E-05$ & $2.52 \mathrm{E}-05$ & 0.00527712 & 0.05233185 & 0.00687154 & 2697763.21 \\
\hline 32758543.3 & 778.966356 & 802.481212 & 1020.37344 & 762.792048 & $4.06 E-06$ & $2.49 \mathrm{E}-05$ & 68.3566231 & 0.00730471 & 0.00499325 & $1.18 \mathrm{E}-05$ & $1.31 E-05$ & $2.52 \mathrm{E}-05$ & 0.00527769 & 0.05234317 & 0.0068717 & 2698250.02 \\
\hline 32769529.6 & 778.966356 & 802.482497 & 1020.37311 & 762.791994 & $4.06 \mathrm{E}-06$ & $2.49 \mathrm{E}-05$ & 68.3591483 & 0.00730668 & 0.00499479 & $1.18 \mathrm{E}-05$ & $1.31 \mathrm{E}-05$ & $2.52 \mathrm{E}-05$ & 0.00527835 & 0.05235732 & 0.00687191 & 2698855.93 \\
\hline 32783262.6 & 778.966356 & 802.484096 & 1020.37283 & 762.791932 & $4.06 \mathrm{E}-06$ & $2.49 \mathrm{E}-05$ & 68.3623029 & 0.00730915 & $\begin{array}{l}0.0049967 \\
\end{array}$ & $1.18 \mathrm{E}-05$ & $1.31 \mathrm{E}-05$ & $2.52 \mathrm{E}-05$ & 0.00527907 & 0.05237501 & 0.00687216 & 2699613 \\
\hline 32800428.7 & 778.966356 & 802.486116 & 1020.37253 & 762.791855 & $4.06 \mathrm{E}-06$ & $2.49 \mathrm{E}-05$ & 68.3662431 & 0.00731224 & 0.0049991 & $1.18 \mathrm{E}-05$ & $1.31 \mathrm{E}-05$ & $2.52 \mathrm{E}-05$ & 0.00527988 & 0.05239712 & 0.00687248 & 2700559.86 \\
\hline 32821886.4 & 778.966356 & 802.488196 & 1020.3721 & 762.791759 & $4.06 \mathrm{E}-06$ & $2.49 \mathrm{E}-05$ & 68.3711638 & 0.00731609 & 0.0050021 & $1.18 \mathrm{E}-05$ & $1.31 \mathrm{E}-05$ & $2.52 \mathrm{E}-05$ & 0.00528078 & 0.05242476 & 0.00687289 & 2701741 \\
\hline 32848708.5 & 778.966356 & 802.490805 & 1020.37158 & 762.791638 & $4.06 \mathrm{E}-06$ & $2.49 \mathrm{E}-05$ & 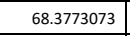 & 0.00732091 & 0.00500584 & $1.18 \mathrm{E}-05$ & $1.31 \mathrm{E}-05$ & $2.52 \mathrm{E}-05$ & 0.00528183 & 0.0524593 & 0.00687339 & 2703217.64 \\
\hline 32882236.1 & 778.966356 & 802.493687 & 1020.37088 & 762.791486 & $4.06 \mathrm{E}-06$ & $2.49 \mathrm{E}-05$ & 68.3849753 & 0.00732694 & 0.00501053 & $1.18 \mathrm{E}-05$ & $1.31 E-05$ & $2.52 \mathrm{E}-05$ & 0.00528306 & 0.05250249 & 0.00687401 & 2705061.8 \\
\hline 32896765 & 778.490606 & 802.01294 & 1020.10636 & 762.425415 & $4.06 E-06$ & $2.49 \mathrm{E}-05$ & 68.3882942 & 0.00732955 & 0.00501256 & $1.18 \mathrm{E}-05$ & $1.31 \mathrm{E}-05$ & $2.52 \mathrm{E}-05$ & 0.00528214 & 0.0525212 & 0.00686825 & 2706085.22 \\
\hline 32898608.2 & \begin{tabular}{|l}
785.487562 \\
\end{tabular} & $\begin{array}{l}810.340536 \\
\end{array}$ & 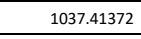 & 768.587866 & $4.06 \mathrm{E}-06$ & $2.49 E-05$ & 68.388726 & 0.00732989 & 0.00501282 & $1.18 \mathrm{E}-05$ & $1.31 E-05$ & $2.56 \mathrm{E}-05$ & 0.00533557 & 0.05252364 & 0.00696803 & 2728880.16 \\
\hline 32900365 & 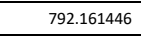 & $\begin{array}{l}818.28 \\
\end{array}$ & 1053.81334 & 774.466995 & $4.06 \mathrm{E}-06$ & $2.49 \mathrm{E}-05$ & 68.3891576 & 0.00733023 & 0.00501308 & $1.18 \mathrm{E}-05$ & $1.31 \mathrm{E}-05$ & $2.59 E-05$ & 0.00538111 & 0.05252607 & 0.00706323 & 2749816.65 \\
\hline
\end{tabular}




\begin{tabular}{|c|c|c|c|c|c|c|c|c|c|c|c|c|c|c|c|c|}
\hline Time (s) & $\begin{array}{c}\text { Coolant outlet } \\
\text { temperature }(\mathrm{K})\end{array}$ & $\begin{array}{l}\text { Peak cladding } \\
\text { temperature (K) }\end{array}$ & $\begin{array}{c}\text { Peak fuel } \\
\text { temperature (K) }\end{array}$ & $\begin{array}{c}\text { Average cladding } \\
\text { internal } \\
\text { temperature (K) }\end{array}$ & $\begin{array}{l}\text { Peak cumulative } \\
\text { damage factor }\end{array}$ & $\begin{array}{l}\text { Cladding interior } \\
\text { volume }\left(\mathrm{m}^{3}\right)\end{array}$ & $\begin{array}{l}\text { Fission gas } \\
\text { release (\%) }\end{array}$ & $\begin{array}{l}\text { Fission gas } \\
\text { produced } \\
\text { (moles) }\end{array}$ & $\begin{array}{c}\text { Fission gas } \\
\text { released (moles) }\end{array}$ & Fuel volume $\left(\mathrm{m}^{3}\right)$ & $\begin{array}{l}\text { Plenum gas } \\
\text { volume }\left(\mathrm{m}^{3}\right)\end{array}$ & $\begin{array}{l}\text { Peak cladding } \\
\text { radial growth } \\
\text { (m) }\end{array}$ & $\begin{array}{l}\text { Peak fuel axial } \\
\text { growth }(m)\end{array}$ & $\begin{array}{c}\text { Peak burnup } \\
\text { (at\%) }\end{array}$ & $\begin{array}{l}\text { Peak cladding } \\
\text { hoop strain }\end{array}$ & $\begin{array}{l}\text { Plenum pressure } \\
(\mathrm{Pa})\end{array}$ \\
\hline 32902208.2 & 792.161446 & 818.275009 & 1053.81086 & \begin{tabular}{|l|l|}
774.467117 \\
\end{tabular} & 4.06E-06 & $2.49 \mathrm{E}-05$ & 68.3896208 & 0.0073306 & 0.00501337 & $1.18 \mathrm{E}-05$ & $1.31 \mathrm{E}-05$ & $2.59 \mathrm{E}-05$ & 0.00537839 & 0.05252868 & 0.00706327 & 2749916.3 \\
\hline 32904512.2 & \begin{tabular}{|l|l|}
792.161446 \\
\end{tabular} & 818.273401 & 1053.80973 & 774.467135 & $4.06 \mathrm{E}-06$ & $2.49 \mathrm{E}-05$ & \begin{tabular}{|l|l|}
68.3901998 \\
\end{tabular} & 0.00733105 & 0.00501372 & $1.18 \mathrm{E}-05$ & $1.31 \mathrm{E}-05$ & $2.59 \mathrm{E}-05$ & 0.0053772 & 0.05253195 & 0.00706332 & 2750033.43 \\
\hline 32907392.2 & \begin{tabular}{|l|l|}
792.161446 \\
\end{tabular} & 818.273059 & 1053.80879 & 774.467126 & $4.06 \mathrm{E}-06$ & $2.49 \mathrm{E}-05$ & 68.3909233 & 0.00733162 & 0.00501416 & $1.18 \mathrm{E}-05$ & $1.31 \mathrm{E}-05$ & $2.59 \mathrm{E}-05$ & 0.00537668 & 0.05253603 & 0.00706338 & 2750205.02 \\
\hline 32910992.2 & \begin{tabular}{|l|l|}
792.161446 \\
\end{tabular} & \begin{tabular}{|l|l|}
818.273283 \\
\end{tabular} & 1053.80809 & \begin{tabular}{|l|l|}
774.467107 \\
\end{tabular} & $4.06 \mathrm{E}-06$ & $2.49 \mathrm{E}-05$ & 68.3918276 & 0.00733233 & 0.00501472 & $1.18 \mathrm{E}-05$ & $1.31 \mathrm{E}-05$ & $2.59 \mathrm{E}-05$ & 0.0053766 & 0.05254114 & 0.00706345 & 2750425.5 \\
\hline 32915492.2 & \begin{tabular}{|l|l|}
792.161446 \\
\end{tabular} & 818.273857 & 1053.80757 & \begin{tabular}{|l|l|}
774.467082 \\
\end{tabular} & $4.06 \mathrm{E}-06$ & $2.49 \mathrm{E}-05$ & 68.3929577 & 0.00733322 & 0.00501541 & $1.18 \mathrm{E}-05$ & $1.31 E-05$ & $2.60 \mathrm{E}-05$ & 0.00537681 & 0.05254752 & 0.00706355 & 2750701.56 \\
\hline 32921117.2 & 792.161446 & \begin{tabular}{|l|}
818.274671 \\
\end{tabular} & 1053.8072 & \begin{tabular}{|l|l|}
774.467051 \\
\end{tabular} & $4.06 E-06$ & $2.49 \mathrm{E}-05$ & \begin{tabular}{|l|l|}
68.39437 \\
\end{tabular} & 0.00733434 & 0.00501627 & $1.18 \mathrm{E}-05$ & $1.31 E-05$ & $2.60 \mathrm{E}-05$ & 0.0053772 & 0.05255549 & 0.00706367 & 2751046.84 \\
\hline \begin{tabular}{|l|}
32928148.5 \\
\end{tabular} & \begin{tabular}{|l|l|}
792.161446 \\
\end{tabular} & 818.275705 & 1053.80691 & \begin{tabular}{|l|l|}
774.467013 \\
\end{tabular} & $4.06 \mathrm{E}-06$ & $2.49 \mathrm{E}-05$ & 68.3961347 & 0.00733573 & 0.00501735 & $1.18 \mathrm{E}-05$ & $1.31 \mathrm{E}-05$ & $2.60 \mathrm{E}-05$ & 0.00537771 & 0.05256546 & 0.00706382 & 2751478.76 \\
\hline 32936937.5 & 792.161446 & 818.276922 & 1053.80665 & 774.466965 & $4.06 \mathrm{E}-06$ & $2.49 \mathrm{E}-05$ & 68.3983397 & 0.00733747 & 0.00501871 & $1.18 \mathrm{E}-05$ & $1.31 E-05$ & $2.60 \mathrm{E}-05$ & 0.00537834 & 0.05257792 & 0.007064 & 2752018.7 \\
\hline \begin{tabular}{|l|l|}
32947923.8 \\
\end{tabular} & \begin{tabular}{|l|l|}
792.161446 \\
\end{tabular} & 818.278408 & 1053.80639 & \begin{tabular}{|l|l|}
774.466906 \\
\end{tabular} & $4.06 \mathrm{E}-06$ & $2.49 \mathrm{E}-05$ & 68.4010944 & 0.00733964 & 0.00502039 & $1.18 \mathrm{E}-05$ & $1.31 \mathrm{E}-05$ & $2.60 \mathrm{E}-05$ & 0.00537911 & 0.0525935 & 0.00706423 & 2752693.49 \\
\hline 32961656.8 & 792.161446 & 818.280238 & 1053.80607 & 774.466833 & $4.06 \mathrm{E}-06$ & $2.49 \mathrm{E}-05$ & 68.4045355 & 0.00734236 & 0.00502251 & $1.18 \mathrm{E}-05$ & $1.31 \mathrm{E}-05$ & $2.60 \mathrm{E}-05$ & 0.00538007 & 0.05261297 & 0.00706452 & 2753536.88 \\
\hline 32978822.9 & 792.161446 & $\begin{array}{l}818.282571 \\
\end{array}$ & 1053.8057 & 774.466741 & $4.06 \mathrm{E}-06$ & $2.49 \mathrm{E}-05$ & 68.4088334 & 0.00734575 & 0.00502514 & $1.18 \mathrm{E}-05$ & $1.31 \mathrm{E}-05$ & $2.60 \mathrm{E}-05$ & 0.00538125 & 0.05263731 & 0.00706489 & 2754591.23 \\
\hline 33000280.6 & \begin{tabular}{|l|}
792.161446 \\
\end{tabular} & 818.285246 & 1053.8052 & \begin{tabular}{ll|}
774.466627 \\
\end{tabular} & $4.06 \mathrm{E}-06$ & $2.50 \mathrm{E}-05$ & $\begin{aligned} 68.4142 \\
\end{aligned}$ & 0.00735 & 0.00502844 & $1.18 \mathrm{E}-05$ & $1.31 \mathrm{E}-05$ & $2.60 \mathrm{E}-05$ & 0.00538267 & 0.05266773 & 0.00706534 & 2755908.3 \\
\hline 33027102.7 & \begin{tabular}{|l|l|}
792.161446 \\
\end{tabular} & 818.288444 & 1053.80455 & \begin{tabular}{|l|l|}
774.466483 \\
\end{tabular} & $4.06 \mathrm{E}-06$ & $2.50 \mathrm{E}-05$ & 68.4208997 & 0.00735531 & 0.00503257 & $1.18 \mathrm{E}-05$ & $1.31 E-05$ & $2.60 \mathrm{E}-05$ & 0.00538434 & 0.05270575 & 0.00706591 & 2757554.34 \\
\hline 33060630.3 & $\begin{array}{l}792.161446 \\
\end{array}$ & 818.292145 & 1053.80368 & 774.466303 & $4.06 \mathrm{E}-06$ & $2.50 \mathrm{E}-05$ & 68.4292607 & 0.00736194 & 0.00503772 & $1.18 \mathrm{E}-05$ & $1.31 E-05$ & $2.60 \mathrm{E}-05$ & 0.00538634 & 0.05275329 & 0.00706662 & 2759611.08 \\
\hline 33102539.8 & 792.161446 & 818.29656 & 1053.80256 & $\begin{array}{l}774.466078 \\
\end{array}$ & $4.06 \mathrm{E}-06$ & $2.50 \mathrm{E}-05$ & 68.4396908 & 0.00737023 & 0.00504416 & $1.18 \mathrm{E}-05$ & $1.31 \mathrm{E}-05$ & $2.60 \mathrm{E}-05$ & 0.00538875 & 0.0528127 & 0.00706751 & 2762181.39 \\
\hline 33154926.7 & 792.161446 & 818.301735 & 1053.80111 & 7774.4658 & $4.06 \mathrm{E}-06$ & $2.50 \mathrm{E}-05$ & 68.4526954 & 0.0073806 & 0.00505222 & $1.18 \mathrm{E}-05$ & $1.31 E-05$ & $2.60 \mathrm{E}-05$ & 0.00539163 & 0.05288698 & 0.00706862 & 2765393.05 \\
\hline 33220410.3 & 792.161446 & 818.308046 & 1053.79928 & 774.465452 & $4.06 \mathrm{E}-06$ & $2.50 \mathrm{E}-05$ & 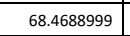 & 0.00739355 & 0.00506228 & $1.18 \mathrm{E}-05$ & $1.31 E-05$ & $2.60 \mathrm{E}-05$ & 0.0053952 & 0.05297981 & 0.00707001 & 2769407.39 \\
\hline 33302264.8 & \begin{tabular}{|l}
792.161446 \\
\end{tabular} & 818.315701 & 1053.79697 & 774.465015 & $4.06 \mathrm{E}-06$ & $2.50 \mathrm{E}-05$ & 68.4890758 & 0.00740975 & 0.00507487 & $1.18 \mathrm{E}-05$ & $1.31 \mathrm{E}-05$ & $2.60 \mathrm{E}-05$ & 0.0053996 & 0.05309586 & 0.00707175 & 2774424.87 \\
\hline 33404583 & 792.161446 & 818.324975 & 1053.79407 & 774.464467 & $4.06 E-06$ & $2.50 \mathrm{E}-05$ & 68.5141721 & 0.00742999 & 0.0050906 & $1.18 \mathrm{E}-05$ & $1.31 \mathrm{E}-05$ & $2.60 \mathrm{E}-05$ & 0.00540505 & 0.05324092 & 0.00707394 & 2780696.21 \\
\hline 33532480.7 & $\begin{array}{l}792.161446 \\
\end{array}$ & 818.336139 & 1053.79042 & 774.463783 & $4.06 E-06$ & $2.50 \mathrm{E}-05$ & 68.5453507 & 0.0074553 & 0.00511026 & $1.19 \mathrm{E}-05$ & $1.31 E-05$ & $2.60 \mathrm{E}-05$ & 0.00541179 & 0.05342225 & 0.00707667 & 2788534.85 \\
\hline 33692352.8 & $\begin{array}{l}792.161446 \\
\end{array}$ & 818.349415 & 1053.78582 & 774.462923 & $4.06 \mathrm{E}-06$ & $2.50 \mathrm{E}-05$ & 68.5840277 & 0.00748693 & 0.00513484 & $1.19 \mathrm{E}-05$ & $1.31 \mathrm{E}-05$ & $2.60 \mathrm{E}-05$ & 0.00542014 & 0.05364891 & $\begin{array}{l}.00708009 \\
\end{array}$ & 2798331.41 \\
\hline 33892192.9 & $\begin{array}{l}792.161446 \\
\end{array}$ & 818.364971 & 1053.78001 & 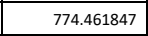 & $4.06 \mathrm{E}-06$ & $2.50 \mathrm{E}-05$ & $\begin{array}{l}68.6319167 \\
\end{array}$ & 0.00752647 & 0.00516556 & $1.19 \mathrm{E}-05$ & $1.31 \mathrm{E}-05$ & $2.60 \mathrm{E}-05$ & 0.0054304 & 0.05393223 & 0.00708439 & 2810574.91 \\
\hline 34141993.1 & 792.161446 & 818.383016 & 1053.77271 & 774.460488 & $4.06 \mathrm{E}-06$ & $2.50 \mathrm{E}-05$ & 68.691075 & 0.00757589 & 0.00520396 & $1.19 \mathrm{E}-05$ & $1.31 E-05$ & $2.60 \mathrm{E}-05$ & 0.00544315 & 0.05428639 & 0.00708979 & 2825875.92 \\
\hline 34391793.3 & 792.161446 & 818.400289 & 1053.76557 & 774.459127 & $4.06 \mathrm{E}-06$ & $2.50 \mathrm{E}-05$ & 68.7494664 & 0.00762531 & 0.00524236 & $1.19 \mathrm{E}-05$ & $1.31 \mathrm{E}-05$ & $2.61 \mathrm{E}-05$ & 0.00545622 & 0.05464054 & 0.00709538 & 2844953.75 \\
\hline 34641593.5 & $\begin{array}{l}792.161446 \\
\end{array}$ & 818.416328 & 1053.75858 & 774.457753 & $4.06 E-06$ & $2.50 \mathrm{E}-05$ & 68.8071058 & 0.00767474 & 0.00528076 & $1.19 \mathrm{E}-05$ & $1.31 E-05$ & $2.61 \mathrm{E}-05$ & 0.00546963 & 0.05499469 & 0.00710101 & 2864033.17 \\
\hline 34891393.6 & 792.161446 & 818.430872 & 1053.75167 & 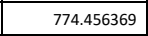 & $4.06 E-06$ & $2.50 \mathrm{E}-05$ & 68.8640076 & 0.00772416 & 0.00531917 & $1.19 \mathrm{E}-05$ & $1.31 E-05$ & $2.61 \mathrm{E}-05$ & 0.00548316 & 0.05534884 & 0.00710667 & 2883113.67 \\
\hline 35141193.8 & 792.161446 & 818.443729 & 1053.74478 & $\begin{array}{l}774.454969 \\
\end{array}$ & $4.06 \mathrm{E}-06$ & $2.50 \mathrm{E}-05$ & 68.9201858 & 0.00777358 & 0.00535757 & $1.19 \mathrm{E}-05$ & $1.31 \mathrm{E}-05$ & $2.61 \mathrm{E}-05$ & 0.00549673 & 0.055703 & 0.00711236 & 2902194.8 \\
\hline \begin{tabular}{|l|l}
35390994 \\
\end{tabular} & 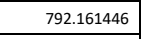 & 818.454766 & \begin{tabular}{|l|l|}
1053.73789 \\
\end{tabular} & 774.453563 & $4.06 E-06$ & $2.50 \mathrm{E}-05$ & 68.9756542 & 0.00782301 & 0.00539597 & $1.19 \mathrm{E}-05$ & $1.31 E-05$ & $2.61 \mathrm{E}-05$ & 0.00551028 & 0.05605715 & 0.00711808 & $\begin{array}{l}2921276.1 \\
\end{array}$ \\
\hline 35640794.2 & $\begin{array}{l}792.161446 \\
\end{array}$ & 818.463943 & 1053.73103 & 774.452141 & $4.06 \mathrm{E}-06$ & $2.50 \mathrm{E}-05$ & 69.0304261 & 0.00787243 & 0.00543437 & $1.19 \mathrm{E}-05$ & $1.31 \mathrm{E}-05$ & $2.61 \mathrm{E}-05$ & 0.00552387 & 0.0564113 & 0.00712383 & 2940358.08 \\
\hline 35890594.4 & $\begin{array}{l}792.161446 \\
\end{array}$ & 818.470947 & 1053.72421 & 774.450703 & $4.06 E-06$ & $2.50 \mathrm{E}-05$ & $\begin{array}{l}69.0845146 \\
\end{array}$ & 0.00792186 & 0.00547278 & $1.19 \mathrm{E}-05$ & $1.31 E-05$ & $2.62 \mathrm{E}-05$ & 0.00553743 & 0.05676546 & 0.00712961 & 2959440.07 \\
\hline 36140394.5 & $\begin{array}{l}792.161446 \\
\end{array}$ & 818.475588 & 1053.71736 & 774.449231 & $4.06 E-06$ & $2.50 \mathrm{E}-05$ & 69.1379323 & 0.00797128 & 0.00551118 & $1.19 \mathrm{E}-05$ & $1.31 E-05$ & $2.62 \mathrm{E}-05$ & 0.005551 & 0.05711961 & 0.00714913 & 2978522.43 \\
\hline 36390194.7 & 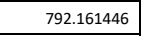 & 818.476729 & $\begin{array}{l}1053.71053 \\
\end{array}$ & 774.447704 & $4.06 \mathrm{E}-06$ & $2.50 \mathrm{E}-05$ & $\begin{array}{ll}6.1906918 \\
\end{array}$ & 0.0080207 & 0.00554958 & $1.19 \mathrm{E}-05$ & $1.31 \mathrm{E}-05$ & $2.62 \mathrm{E}-05$ & 0.00556457 & $\begin{array}{l}0.05747376 \\
\end{array}$ & 0.00721224 & 2997606.49 \\
\hline 36639994.9 & 792.161446 & 818.479372 & 1053.70361 & 774.446052 & $4.07 \mathrm{E}-06$ & $2.50 \mathrm{E}-05$ & 69.242805 & 0.00807013 & 0.00558798 & $1.19 \mathrm{E}-05$ & $1.31 \mathrm{E}-05$ & $2.62 \mathrm{E}-05$ & 0.00557807 & 0.05782792 & 0.00727607 & 3016690.79 \\
\hline 36889795.1 & $\begin{array}{l}792.161446 \\
\end{array}$ & 818.498165 & 1053.69668 & $\begin{array}{l}774.444476 \\
\end{array}$ & $4.07 E-06$ & $2.50 \mathrm{E}-05$ & 69.2942838 & 0.00811955 & 0.00562638 & $1.19 \mathrm{E}-05$ & $1.31 E-05$ & $2.62 \mathrm{E}-05$ & 0.00559167 & 0.05818207 & 0.00734151 & 3035778.69 \\
\hline 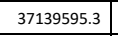 & \begin{tabular}{|l}
792.161446 \\
\end{tabular} & 818.51192 & 1053.68935 & 774.442891 & $4.07 \mathrm{E}-06$ & $2.50 \mathrm{E}-05$ & 69.3451397 & 0.00816897 & 0.00566479 & $1.19 \mathrm{E}-05$ & $1.31 \mathrm{E}-05$ & $2.63 \mathrm{E}-05$ & 0.0056049 & 0.05853622 & 0.00740768 & 3054865.77 \\
\hline 37389395.4 & 792.161446 & 818.523105 & 1053.68178 & 774.441268 & $4.07 E-06$ & $2.50 \mathrm{E}-05$ & 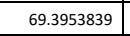 & 0.0082184 & 0.00570319 & $1.19 \mathrm{E}-05$ & $1.31 E-05$ & $2.65 \mathrm{E}-05$ & 0.00561858 & 0.05889038 & 0.00747457 & 3073959.49 \\
\hline 37639195.6 & $\begin{array}{l}792.161446 \\
\end{array}$ & 818.532292 & 1053.67211 & 774.43964 & $4.07 E-06$ & $2.50 \mathrm{E}-05$ & 69.4450274 & 0.00826782 & 0.00574159 & $1.19 \mathrm{E}-05$ & $1.31 E-05$ & $2.67 \mathrm{E}-05$ & 0.00563204 & 0.05924453 & 0.00754219 & 3093054.48 \\
\hline 37888995.8 & \begin{tabular}{|l}
792.161446 \\
\end{tabular} & 818.537418 & 1053.65537 & 774.437983 & $4.07 E-06$ & $2.50 \mathrm{E}-05$ & 69.4940809 & 0.00831724 & 0.00577999 & $1.19 \mathrm{E}-05$ & $1.31 \mathrm{E}-05$ & $2.69 \mathrm{E}-05$ & 0.00564543 & 0.05959868 & 0.00761051 & 3112151.7 \\
\hline 38138796 & \begin{tabular}{|l}
792.161446 \\
\end{tabular} & 818.540129 & 1053.62985 & 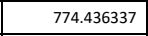 & $4.07 \mathrm{E}-06$ & $2.50 \mathrm{E}-05$ & 69.5425549 & 0.00836667 & 0.00581839 & $1.19 \mathrm{E}-05$ & $1.31 \mathrm{E}-05$ & $2.71 \mathrm{E}-05$ & 0.00565864 & 0.05995284 & 0.00767954 & 3131250.93 \\
\hline 38388596.2 & 792.161446 & 818.540179 & 1053.59783 & 774.434665 & $4.07 \mathrm{E}-06$ & $2.50 \mathrm{E}-05$ & 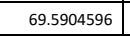 & 0.00841609 & 0.0058568 & $1.19 \mathrm{E}-05$ & $1.31 \mathrm{E}-05$ & $2.73 \mathrm{E}-05$ & 0.00567243 & 0.06030699 & 0.00774926 & 3150357.24 \\
\hline 38638396.4 & 792.161446 & 818.537551 & 1053.56354 & $\begin{array}{r}774.43297 \\
\end{array}$ & $4.07 \mathrm{E}-06$ & $2.50 \mathrm{E}-05$ & 69.6378049 & 0.00846551 & 0.0058952 & $1.19 \mathrm{E}-05$ & $1.31 \mathrm{E}-05$ & $2.75 \mathrm{E}-05$ & 0.00568626 & 0.06066114 & 0.00781966 & 3169467.84 \\
\hline 38888196.5 & \begin{tabular}{|l}
792.161446 \\
\end{tabular} & 818.532358 & 1053.5282 & $\begin{array}{ll}774.431259 \\
\end{array}$ & $4.07 \mathrm{E}-06$ & $2.50 \mathrm{E}-05$ & 69.6846006 & 0.00851494 & 0.0059336 & $1.19 \mathrm{E}-05$ & $1.31 \mathrm{E}-05$ & $2.78 \mathrm{E}-05$ & 0.00570021 & 0.0610153 & 0.00789161 & 3188582.83 \\
\hline 39137996.7 & 792.161446 & 818.523515 & 1053.49241 & 774.429513 & $4.07 E-06$ & $2.50 \mathrm{E}-05$ & 69.7308561 & 0.00856436 & 0.005972 & $1.19 \mathrm{E}-05$ & $1.31 E-05$ & $2.80 \mathrm{E}-05$ & 0.00571414 & 0.06136945 & 0.00796454 & 3207701.13 \\
\hline 39387796.9 & 792.161446 & 818.511769 & 1053.45653 & 774.427745 & $4.08 E-06$ & $2.50 \mathrm{E}-05$ & 69.7765809 & 0.00861379 & 0.0060104 & $1.19 \mathrm{E}-05$ & $1.31 \mathrm{E}-05$ & $2.82 \mathrm{E}-05$ & 0.00572799 & 0.0617236 & 0.0080382 & 3226822.55 \\
\hline 39574695 & 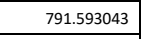 & $\begin{array}{l}817.927281 \\
\end{array}$ & 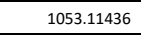 & \begin{tabular}{|l}
773.989017 \\
\end{tabular} & $4.08 E-06$ & $2.50 \mathrm{E}-05$ & 69.8104501 & 0.00865076 & 0.00603914 & $1.19 \mathrm{E}-05$ & $1.31 E-05$ & $2.83 \mathrm{E}-05$ & 0.00573661 & 0.06198858 & 0.00809053 & 3244095.51 \\
\hline 39578295 & 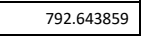 & 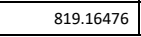 & 1055.6924 & 774.914581 & $4.08 \mathrm{E}-06$ & $2.50 \mathrm{E}-05$ & 69.8111019 & 0.00865148 & 0.00603969 & $1.19 \mathrm{E}-05$ & $1.31 \mathrm{E}-05$ & $2.84 \mathrm{E}-05$ & 0.00574444 & 0.0619937 & 0.00812624 & 3262142.38 \\
\hline
\end{tabular}




\begin{tabular}{|c|c|c|c|c|c|c|c|c|c|c|c|c|c|c|c|c|}
\hline Time (s) & $\begin{array}{c}\text { Coolant outlet } \\
\text { temperature }(\mathrm{K})\end{array}$ & $\begin{array}{l}\text { Peak cladding } \\
\text { temperature (K) }\end{array}$ & $\begin{array}{c}\text { Peak fuel } \\
\text { temperature (K) }\end{array}$ & $\begin{array}{c}\text { Average cladding } \\
\text { internal } \\
\text { temperature (K) }\end{array}$ & $\begin{array}{l}\text { Peak cumulative } \\
\text { damage factor }\end{array}$ & $\begin{array}{l}\text { Cladding interior } \\
\text { volume }\left(\mathrm{m}^{3}\right)\end{array}$ & $\begin{array}{l}\text { Fission gas } \\
\text { release (\%) }\end{array}$ & $\begin{array}{l}\text { Fission gas } \\
\text { produced } \\
\text { (moles) }\end{array}$ & $\begin{array}{c}\text { Fission gas } \\
\text { released (moles) }\end{array}$ & Fuel volume $\left(\mathrm{m}^{3}\right)$ & $\begin{array}{l}\text { Plenum gas } \\
\text { volume }\left(\mathrm{m}^{3}\right)\end{array}$ & $\begin{array}{l}\text { Peak cladding } \\
\text { radial growth } \\
\text { (m) }\end{array}$ & $\begin{array}{l}\text { Peak fuel axial } \\
\text { growth }(m)\end{array}$ & $\begin{array}{c}\text { Peak burnup } \\
\text { (at\%) }\end{array}$ & $\begin{array}{l}\text { Peak cladding } \\
\text { hoop strain }\end{array}$ & $\begin{array}{l}\text { Plenum pressure } \\
(\mathrm{Pa})\end{array}$ \\
\hline 39765193.1 & 792.643859 & $\begin{array}{ll}819.150448 \\
\end{array}$ & 1055.65489 & $\begin{array}{l}774.913172 \\
\end{array}$ & $4.08 E-06$ & $2.50 \mathrm{E}-05$ & 69.8449131 & 0.00868872 & 0.00606863 & $1.19 \mathrm{E}-05$ & $1.31 \mathrm{E}-05$ & $2.86 \mathrm{E}-05$ & 0.00575541 & 0.06226054 & 0.0081838 & 3262595.07 \\
\hline 39952091.2 & 792.643859 & 819.134568 & 1055.6266 & 774.911748 & $4.08 \mathrm{E}-06$ & $2.50 \mathrm{E}-05$ & 69.8784357 & 0.00872596 & 0.00609756 & $1.19 \mathrm{E}-05$ & $1.31 \mathrm{E}-05$ & $2.88 \mathrm{E}-05$ & 0.00576637 & 0.06252739 & 0.00824118 & 3277026.39 \\
\hline \begin{tabular}{|l|l|}
40138989.3 \\
\end{tabular} & 792.643859 & 819.117057 & 1055.59944 & 774.910316 & $4.08 \mathrm{E}-06$ & $2.50 \mathrm{E}-05$ & 69.9116734 & 0.0087632 & 0.0061265 & $1.19 \mathrm{E}-05$ & $1.31 \mathrm{E}-05$ & $2.90 \mathrm{E}-05$ & 0.00577708 & 0.06279423 & 0.00829892 & 3291457.29 \\
\hline \begin{tabular}{|l|}
40325887.4 \\
\end{tabular} & 792.643859 & 819.097802 & 1055.57272 & \begin{tabular}{|l|l|}
774.908875 \\
\end{tabular} & $4.08 \mathrm{E}-06$ & $2.50 \mathrm{E}-05$ & 69.9446297 & 0.00880043 & 0.00615543 & $1.19 \mathrm{E}-05$ & $1.31 \mathrm{E}-05$ & $2.91 \mathrm{E}-05$ & 0.0057876 & 0.06306107 & 0.00835691 & 3305888.34 \\
\hline 40512785.5 & 792.643859 & 819.541739 & 1055.54641 & 774.953596 & $4.08 \mathrm{E}-06$ & $2.50 \mathrm{E}-05$ & 69.9773084 & 0.00883767 & 0.00618437 & $1.19 \mathrm{E}-05$ & $1.31 \mathrm{E}-05$ & $2.93 \mathrm{E}-05$ & 0.00579854 & 0.06332792 & 0.00841507 & 3320560.03 \\
\hline 40699683.6 & 792.643859 & 819.533101 & 1055.51998 & \begin{tabular}{|l|l|}
774.952174 \\
\end{tabular} & $4.08 \mathrm{E}-06$ & $2.50 \mathrm{E}-05$ & $\begin{array}{ll}70.0097128 \\
\end{array}$ & 0.00887491 & 0.0062133 & $1.19 \mathrm{E}-05$ & $1.31 E-05$ & $2.95 \mathrm{E}-05$ & 0.00580901 & 0.06359476 & 0.00847345 & 3334994.43 \\
\hline \begin{tabular}{|l|}
40886581.7 \\
\end{tabular} & $\begin{array}{l}792.643859 \\
\end{array}$ & 819.524405 & 1055.49378 & \begin{tabular}{|l|l|}
774.950737 \\
\end{tabular} & $4.08 \mathrm{E}-06$ & $2.50 \mathrm{E}-05$ & \begin{tabular}{|l|l|}
70.0418464 \\
\end{tabular} & 0.00891215 & 0.00624224 & $1.19 \mathrm{E}-05$ & $1.31 \mathrm{E}-05$ & $2.97 \mathrm{E}-05$ & 0.00581947 & 0.0638616 & 0.00853248 & 3349430.23 \\
\hline 41073479.8 & 792.643859 & 819.515891 & 1055.46739 & \begin{tabular}{|l|l|}
774.94929 \\
\end{tabular} & 4.09E- -06 & $2.50 \mathrm{E}-05$ & 70.0737126 & 0.00894939 & 0.00627117 & $1.19 \mathrm{E}-05$ & $1.31 E-05$ & $2.99 \mathrm{E}-05$ & 0.00582999 & 0.06412845 & 0.00859163 & 3363868.49 \\
\hline \begin{tabular}{|l|}
41260378 \\
\end{tabular} & 792.643859 & 819.507417 & 1055.44151 & \begin{tabular}{|l|l|}
774.947844 \\
\end{tabular} & $4.09 \mathrm{E}-06$ & $2.50 \mathrm{E}-05$ & 70.1053147 & 0.00898663 & 0.00630011 & $1.19 \mathrm{E}-05$ & $1.31 \mathrm{E}-05$ & $3.00 \mathrm{E}-05$ & 0.00584046 & 0.06439529 & 0.00865122 & 3378307.7 \\
\hline \begin{tabular}{|l|l|}
41447276.1 \\
\end{tabular} & 792.643859 & 819.499049 & 1055.4152 & 774.946384 & $4.09 \mathrm{E}-06$ & $2.50 \mathrm{E}-05$ & 70.136656 & 0.00902387 & 0.00632904 & $1.19 \mathrm{E}-05$ & $1.31 \mathrm{E}-05$ & $3.02 \mathrm{E}-05$ & 0.00585104 & 0.06466214 & 0.00871134 & 3392749.71 \\
\hline 41634174.2 & 792.643859 & 819.490902 & 1055.38985 & 774.944932 & $4.09 \mathrm{E}-06$ & $2.50 \mathrm{E}-05$ & 70.1677397 & 0.00906111 & 0.00635798 & $1.19 \mathrm{E}-05$ & $1.31 \mathrm{E}-05$ & $3.04 \mathrm{E}-05$ & 0.00586147 & 0.06492898 & 0.00877157 & 3407191.57 \\
\hline \begin{tabular}{|l|l|}
41821072.3 \\
\end{tabular} & 792.643859 & 819.482862 & 1055.36415 & \begin{tabular}{|l|l|}
774.943468 \\
\end{tabular} & $4.09 \mathrm{E}-06$ & $2.50 \mathrm{E}-05$ & 70.1985689 & 0.00909835 & 0.00638691 & $1.19 \mathrm{E}-05$ & $1.31 \mathrm{E}-05$ & $3.06 \mathrm{E}-05$ & $\begin{array}{ll}0.00587197 \\
\end{array}$ & 0.06519582 & 0.00883191 & 3421635.65 \\
\hline \begin{tabular}{|l|l|}
42007970.4 \\
\end{tabular} & 792.643859 & 819.47507 & 1055.33925 & \begin{tabular}{|l|l|}
774.941999 \\
\end{tabular} & $4.09 \mathrm{E}-06$ & $2.50 \mathrm{E}-05$ & 70.2291468 & 0.00913559 & 0.00641584 & $1.19 \mathrm{E}-05$ & $1.31 \mathrm{E}-05$ & $3.07 \mathrm{E}-05$ & 0.00588238 & 0.06546267 & 0.00889235 & 3436080.09 \\
\hline 42194062 & 793.426091 & 820.256581 & 1055.7491 & 775.542347 & $4.11 \mathrm{E}-06$ & $2.50 \mathrm{E}-05$ & 70.259346 & 0.00917267 & 0.00644465 & $1.19 \mathrm{E}-05$ & $1.31 E-05$ & $3.09 \mathrm{E}-05$ & 0.0058954 & 0.06572836 & 0.00895993 & 3453210.04 \\
\hline 42194188.7 & 792.796477 & 819.510232 & 1054.21052 & 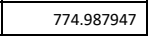 & $4.11 \mathrm{E}-06$ & $2.50 \mathrm{E}-05$ & 70.2593664 & 0.00917269 & 0.00644467 & $1.19 \mathrm{E}-05$ & $1.31 \mathrm{E}-05$ & $3.09 \mathrm{E}-05$ & 0.00589088 & 0.06572854 & 0.00894656 & 3464985.28 \\
\hline 42194315.3 & 792.166904 & 818.763863 & 1052.67224 & 774.433461 & $4.11 \mathrm{E}-06$ & $2.50 \mathrm{E}-05$ & 70.2593868 & 0.00917272 & 0.00644469 & $1.19 \mathrm{E}-05$ & $1.31 \mathrm{E}-05$ & $3.09 \mathrm{E}-05$ & 0.00588636 & 0.06572872 & 0.00893277 & 3462558.42 \\
\hline 42194473.7 & 791.379995 & 817.831041 & 1050.74907 & 773.740422 & $4.11 \mathrm{E}-06$ & $2.50 \mathrm{E}-05$ & 70.2594121 & 0.00917275 & 0.00644472 & $1.19 \mathrm{E}-05$ & $1.31 \mathrm{E}-05$ & $3.08 E-05$ & 0.00588082 & 0.06572894 & 0.00891531 & 3459518.61 \\
\hline 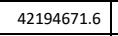 & 790.39645 & 816.665212 & 1048.34344 & 772.874221 & $4.11 \mathrm{E}-06$ & $2.50 \mathrm{E}-05$ & 70.2594435 & 0.00917279 & 0.00644475 & $1.19 \mathrm{E}-05$ & $1.31 \mathrm{E}-05$ & $3.07 \mathrm{E}-05$ & 0.00587406 & 0.06572922 & 0.00889266 & 3455721.36 \\
\hline 42194919 & 789.16716 & 815.208238 & 1045.33367 & 771.791634 & $4.11 \mathrm{E}-06$ & $2.50 \mathrm{E}-05$ & 70.2594826 & 0.00917283 & 0.00644479 & $1.19 \mathrm{E}-05$ & $1.31 \mathrm{E}-05$ & $3.06 E-05$ & 0.00586575 & 0.06572956 & 0.00886424 & 3450969.17 \\
\hline 42195228.2 & 787.630771 & 813.387519 & 1041.56719 & 770.438652 & $4.11 E-06$ & $2.50 \mathrm{E}-05$ & 70.2595309 & 0.00917289 & 0.00644483 & $1.19 \mathrm{E}-05$ & $1.31 E-05$ & $3.05 E-05$ & 0.00585551 & 0.06572999 & 0.00882825 & 3445026.93 \\
\hline 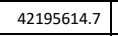 & 785.710638 & 811.112449 & 1036.8523 & 768.74782 & $4.11 \mathrm{E}-06$ & $2.50 \mathrm{E}-05$ & 70.2595905 & 0.00917297 & 0.00644489 & $1.19 \mathrm{E}-05$ & $1.31 \mathrm{E}-05$ & $3.04 \mathrm{E}-05$ & 0.00584278 & 0.06573052 & 0.00878261 & 3437596.82 \\
\hline 42195862.1 & 784.481961 & 809.656897 & 1033.83076 & 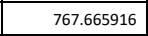 & $4.11 \mathrm{E}-06$ & $2.50 \mathrm{E}-05$ & 70.2596283 & 0.00917301 & 0.00644493 & $1.19 \mathrm{E}-05$ & $1.31 \mathrm{E}-05$ & $3.03 E-05$ & 0.00583463 & 0.06573085 & 0.00875201 & 3432862.47 \\
\hline 42196109.5 & 783.253447 & 808.20173 & 1030.80604 & 766.584205 & $4.11 \mathrm{E}-06$ & $2.50 \mathrm{E}-05$ & 70.2596658 & 0.00917306 & 0.00644496 & $1.19 \mathrm{E}-05$ & $1.31 E-05$ & $3.02 E-05$ & 0.00582649 & 0.06573118 & 0.00872355 & 3428096.63 \\
\hline 42196418.8 & 781.718036 & 806.383325 & 1027.02058 & 765.232323 & $4.11 \mathrm{E}-06$ & $2.50 \mathrm{E}-05$ & 70.2597121 & 0.00917312 & 0.00644501 & $1.19 \mathrm{E}-05$ & $1.31 \mathrm{E}-05$ & $3.01 \mathrm{E}-05$ & 0.00581628 & 0.06573159 & 0.00868746 & 3422135.09 \\
\hline 42196805.3 & 779.799137 & 804.11122 & 1022.28148 & 763.542857 & $4.11 \mathrm{E}-06$ & $2.50 \mathrm{E}-05$ & 70.2597693 & 0.00917319 & 0.00644506 & $1.19 \mathrm{E}-05$ & $1.31 E-05$ & $2.99 \mathrm{E}-05$ & 0.00580344 & 0.0657321 & 0.00863964 & 3414700.16 \\
\hline 42197288.5 & 777.401091 & 801.272478 & 1016.34601 & 761.431667 & $4.11 E-06$ & $2.50 \mathrm{E}-05$ & 70.2598397 & 0.00917327 & 0.00644513 & $1.19 \mathrm{E}-05$ & $1.31 E-05$ & $2.97 \mathrm{E}-05$ & 0.00578724 & 0.06573272 & 0.00858074 & 3405386.33 \\
\hline 42197662 & 775.547815 & 799.079181 & 1011.74874 & 759.800175 & $4.11 \mathrm{E}-06$ & $2.50 \mathrm{E}-05$ & 70.2598933 & 0.00917334 & 0.00644518 & $1.19 \mathrm{E}-05$ & $1.31 \mathrm{E}-05$ & $2.96 \mathrm{E}-05$ & 0.00577456 & 0.06573319 & 0.00853486 & 3398192.64 \\
\hline 42198145.2 & 775.547815 & 799.078505 & 1011.7493 & 759.800008 & $4.11 \mathrm{E}-06$ & $2.50 \mathrm{E}-05$ & 70.2599621 & 0.00917342 & 0.00644524 & $1.19 \mathrm{E}-05$ & $1.31 \mathrm{E}-05$ & $2.96 \mathrm{E}-05$ & 0.00577653 & 0.0657338 & 0.00853102 & 3398212.41 \\
\hline 42198749.2 & 775.547815 & 799.078091 & 1011.75013 & 759.799976 & $4.11 \mathrm{E}-06$ & $2.50 \mathrm{E}-05$ & 70.2600481 & 0.00917353 & 0.00644533 & $1.19 \mathrm{E}-05$ & $1.31 \mathrm{E}-05$ & $2.96 \mathrm{E}-05$ & 0.00577821 & 0.06573456 & 0.00852688 & 3398246.28 \\
\hline 42199504.1 & 775.547815 & 799.077714 & 1011.75089 & 759.799947 & $4.11 \mathrm{E}-06$ & $2.50 \mathrm{E}-05$ & 70.2601556 & 0.00917366 & 0.00644543 & $1.19 \mathrm{E}-05$ & $1.31 E-05$ & $2.95 \mathrm{E}-05$ & 0.00577974 & 0.06573551 & 0.00852341 & 3398285.75 \\
\hline 42200447.9 & 775.547815 & 799.077368 & 1011.75158 & 759.79992 & $4.11 \mathrm{E}-06$ & $2.50 \mathrm{E}-05$ & 70.26029 & 0.00917383 & 0.00644556 & $1.19 \mathrm{E}-05$ & $1.31 E-05$ & $2.95 \mathrm{E}-05$ & 0.00578113 & 0.0657367 & 0.00852036 & 3398334.52 \\
\hline 42201627.5 & 775.547815 & 799.07705 & 1011.75221 & 759.799894 & $4.11 \mathrm{E}-06$ & $2.50 \mathrm{E}-05$ & 70.260458 & 0.00917404 & 0.00644572 & $1.19 \mathrm{E}-05$ & $1.31 \mathrm{E}-05$ & $2.95 \mathrm{E}-05$ & 0.00578239 & 0.06573818 & 0.00851745 & 3398397.48 \\
\hline 42203102.1 & 775.547815 & 799.07676 & 1011.75278 & 759.799869 & $4.11 \mathrm{E}-06$ & $2.50 \mathrm{E}-05$ & 70.260668 & 0.0091743 & 0.00644592 & $1.19 \mathrm{E}-05$ & $1.31 \mathrm{E}-05$ & $2.95 \mathrm{E}-05$ & 0.00578353 & 0.06574004 & 0.00851497 & 3398476.19 \\
\hline 42204945.3 & 775.547815 & 799.076493 & 1011.75328 & 759.799846 & $4.11 \mathrm{E}-06$ & $2.50 \mathrm{E}-05$ & $\begin{array}{ll}7.2609304 \\
\end{array}$ & 0.00917462 & $\begin{array}{l}0.00644617 \\
\end{array}$ & $1.19 \mathrm{E}-05$ & $1.31 \mathrm{E}-05$ & $2.95 E-05$ & 0.00578452 & 0.06574236 & 0.00851307 & 3398574.49 \\
\hline 42207249.3 & 775.547815 & $\begin{array}{l}799.076241 \\
\end{array}$ & 1011.75373 & 759.799822 & $4.11 \mathrm{E}-06$ & $2.50 \mathrm{E}-05$ & 70.2612585 & 0.00917502 & 0.00644649 & $1.19 \mathrm{E}-05$ & $1.31 \mathrm{E}-05$ & $2.95 \mathrm{E}-05$ & 0.00578535 & 0.06574526 & 0.00851167 & 3398698.72 \\
\hline 42210129.3 & 775.547815 & 799.076029 & 1011.75409 & 759.799799 & $4.11 E-06$ & $2.50 \mathrm{E}-05$ & 70.2616685 & 0.00917553 & 0.00644688 & $1.19 \mathrm{E}-05$ & $1.31 E-05$ & $2.95 \mathrm{E}-05$ & 0.00578601 & 0.06574888 & 0.00851098 & 3398853.41 \\
\hline 42213729.3 & $\begin{array}{l}775.547815 \\
\end{array}$ & 799.075835 & 1011.75437 & 759.799776 & $4.11 \mathrm{E}-06$ & $2.50 \mathrm{E}-05$ & 70.2621809 & 0.00917616 & 0.00644737 & $1.19 \mathrm{E}-05$ & $1.31 E-05$ & $2.95 \mathrm{E}-05$ & 0.00578655 & 0.06575341 & 0.00851093 & 3399046.71 \\
\hline 42218229.3 & 775.547815 & 799.075642 & 1011.75458 & 759.79975 & $4.11 \mathrm{E}-06$ & $2.50 \mathrm{E}-05$ & 70.2628214 & 0.00917695 & 0.00644799 & $1.19 \mathrm{E}-05$ & $1.31 \mathrm{E}-05$ & $2.95 \mathrm{E}-05$ & 0.00578699 & 0.06575907 & 0.00851148 & 3399288.73 \\
\hline 42223854.3 & 775.547815 & $\begin{array}{l}799.075431 \\
\end{array}$ & 1011.75469 & 759.799719 & $4.11 \mathrm{E}-06$ & $2.50 \mathrm{E}-05$ & 70.2636218 & 0.00917794 & 0.00644875 & $1.19 \mathrm{E}-05$ & $1.31 \mathrm{E}-05$ & $2.95 \mathrm{E}-05$ & 0.00578742 & 0.06576615 & 0.00851259 & 3399591.07 \\
\hline 42230885.5 & 775.547815 & 799.075197 & 1011.75471 & 759.799681 & $4.11 \mathrm{E}-06$ & $2.50 \mathrm{E}-05$ & 70.2646221 & 0.00917917 & 0.00644971 & $1.19 \mathrm{E}-05$ & $1.31 \mathrm{E}-05$ & $2.95 \mathrm{E}-05$ & 0.00578787 & 0.065775 & 0.00851418 & 3399969.01 \\
\hline $\begin{array}{l}22239674.6 \\
\end{array}$ & 775.547815 & 799.074918 & 1011.75465 & 759.799635 & $4.11 \mathrm{E}-06$ & $2.50 \mathrm{E}-05$ & 70.265872 & 0.00918072 & 0.00645091 & $1.19 \mathrm{E}-05$ & $1.31 \mathrm{E}-05$ & $2.95 E-05$ & 0.00578839 & 0.06578606 & 0.00851626 & 3400441.25 \\
\hline 42250660.9 & 775.547815 & $\begin{array}{l}799.074577 \\
\end{array}$ & 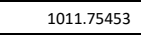 & 759.799578 & $4.11 \mathrm{E}-06$ & $2.50 \mathrm{E}-05$ & $\begin{array}{ll}70.2674339 \\
\end{array}$ & 0.00918265 & 0.00645241 & $1.19 \mathrm{E}-05$ & $1.31 \mathrm{E}-05$ & $2.95 \mathrm{E}-05$ & 0.00578903 & 0.06579988 & 0.00851886 & 3401031.63 \\
\hline 42264393.8 & 775.547815 & 799.074186 & 1011.75432 & 759.799508 & $4.11 \mathrm{E}-06$ & $2.50 \mathrm{E}-05$ & 70.2693853 & 0.00918506 & 0.00645428 & $1.19 \mathrm{E}-05$ & $1.31 E-05$ & $2.95 E-05$ & 0.00578981 & 0.06581716 & 0.00852209 & 3401768.87 \\
\hline 42281560 & 775.547815 & 799.073699 & 1011.75405 & 759.79942 & 4.11E-06 & $2.50 \mathrm{E}-05$ & 70.2718231 & 0.00918807 & 0.00645663 & $1.19 \mathrm{E}-05$ & $1.31 \mathrm{E}-05$ & $2.95 \mathrm{E}-05$ & 0.00579075 & 0.06583876 & 0.00852609 & 3402690.71 \\
\hline 42303017.6 & 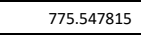 & \begin{tabular}{|l}
799.073125 \\
\end{tabular} & 1011.75369 & 759.799311 & $4.11 \mathrm{E}-06$ & $2.50 \mathrm{E}-05$ & 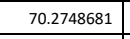 & 0.00919184 & 0.00645955 & $1.19 \mathrm{E}-05$ & $1.31 \mathrm{E}-05$ & $2.96 \mathrm{E}-05$ & 0.00579187 & 0.06586576 & 0.00853107 & 3403842.43 \\
\hline 42329839.7 & 775.547815 & 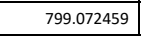 & 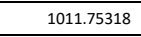 & 759.799178 & $4.11 \mathrm{E}-06$ & $2.50 \mathrm{E}-05$ & $\begin{array}{ll}70.2786709 \\
\end{array}$ & 0.00919655 & 0.00646321 & $1.19 \mathrm{E}-05$ & $1.31 \mathrm{E}-05$ & $2.96 E-05$ & $\begin{array}{l}0.00579319 \\
\end{array}$ & $\begin{array}{l}0.06589951 \\
\end{array}$ & 0.00853727 & 3405280.92 \\
\hline
\end{tabular}




\begin{tabular}{|c|c|c|c|c|c|c|c|c|c|c|c|c|c|c|c|c|}
\hline Time (s) & $\begin{array}{c}\text { Coolant outlet } \\
\text { temperature }(K)\end{array}$ & $\begin{array}{l}\text { Peak cladding } \\
\text { temperature (K) }\end{array}$ & $\begin{array}{c}\text { Peak fuel } \\
\text { temperature (K) }\end{array}$ & $\begin{array}{c}\text { Average cladding } \\
\text { internal } \\
\text { temperature (K) }\end{array}$ & \begin{tabular}{|l|}
$\begin{array}{l}\text { Peak cumulative } \\
\text { damage factor }\end{array}$ \\
\end{tabular} & $\begin{array}{l}\text { Cladding interior } \\
\text { volume }\left(\mathrm{m}^{3}\right)\end{array}$ & $\begin{array}{l}\text { Fission gas } \\
\text { release (\%) }\end{array}$ & $\begin{array}{c}\text { Fission gas } \\
\text { produced } \\
\text { (moles) }\end{array}$ & $\begin{array}{c}\text { Fission gas } \\
\text { released (moles) }\end{array}$ & Fuel volume $\left(\mathrm{m}^{3}\right)$ & $\begin{array}{l}\text { Plenum gas } \\
\text { volume }\left(m^{3}\right)\end{array}$ & $\begin{array}{l}\text { Peak cladding } \\
\text { radial growth } \\
\text { (m) }\end{array}$ & $\begin{array}{l}\text { Peak fuel axial } \\
\text { growth }(m)\end{array}$ & $\begin{array}{c}\text { Peak burnup } \\
\text { (at\%) }\end{array}$ & $\begin{array}{l}\text { Peak cladding } \\
\text { hoop strain }\end{array}$ & $\begin{array}{l}\text { Plenum pressure } \\
\text { (Pa) }\end{array}$ \\
\hline 42363367.3 & 775.547815 & 799.07167 & 1011.7525 & \begin{tabular}{|l|l|}
759.799013 \\
\end{tabular} & $4.11 \mathrm{E}-06$ & $2.50 \mathrm{E}-05$ & 70.2834189 & 0.00920244 & 0.00646779 & $1.19 \mathrm{E}-05$ & $1.31 \mathrm{E}-05$ & $2.96 \mathrm{E}-05$ & 0.00579477 & 0.0659417 & 0.008545 & 3407077.69 \\
\hline 42405276.8 & 775.547815 & 799.070704 & 1011.75163 & 759.798808 & $4.11 E-06$ & $2.50 \mathrm{E}-05$ & 70.2893453 & 0.0092098 & 0.00647351 & $1.19 \mathrm{E}-05$ & $1.31 E-05$ & $2.96 \mathrm{E}-05$ & 0.00579667 & 0.06599444 & 0.00855465 & 3409323.59 \\
\hline 42457663.7 & 775.547815 & 799.069528 & 1011.75052 & 759.798553 & $4.11 E-06$ & $2.50 \mathrm{E}-05$ & 70.2967401 & 0.009219 & 0.00648065 & $1.19 \mathrm{E}-05$ & $1.31 E-05$ & $2.97 E-05$ & 0.005799 & 0.06606035 & 0.00856671 & 3412129.75 \\
\hline 42523147.4 & 775.547815 & 799.06808 & 1011.74912 & \begin{tabular}{|l|l}
759.798234 \\
\end{tabular} & $4.11 \mathrm{E}-06$ & $2.50 \mathrm{E}-05$ & 70.3059628 & 0.0092305 & 0.00648959 & $1.19 \mathrm{E}-05$ & $1.31 E-05$ & $2.97 E-05$ & 0.00580188 & 0.06614275 & 0.00858179 & 3415636.89 \\
\hline 42605001.9 & 775.547815 & 799.066351 & 1011.74729 & \begin{tabular}{|l|l|}
759.79784 \\
\end{tabular} & $4.11 \mathrm{E}-06$ & $2.50 \mathrm{E}-05$ & 70.3174589 & 0.00924487 & 0.00650076 & $1.19 \mathrm{E}-05$ & $1.31 E-05$ & $2.98 \mathrm{E}-05$ & 0.00580535 & 0.06624575 & 0.00860065 & 3420018.57 \\
\hline 42707320 & 775.547815 & 799.06414 & 1011.74508 & 759.797342 & $4.11 \mathrm{E}-06$ & $2.50 \mathrm{E}-05$ & 70.3317788 & 0.00926284 & 0.00651472 & $1.19 \mathrm{E}-05$ & $1.31 \mathrm{E}-05$ & $2.98 \mathrm{E}-05$ & 0.00580983 & 0.0663745 & 0.00862426 & 3425496.46 \\
\hline 42835217.7 & 775.547815 & 799.061446 & 1011.74228 & $\begin{array}{l}759.796723 \\
\end{array}$ & $4.11 \mathrm{E}-06$ & $2.50 \mathrm{E}-05$ & 70.3496009 & 0.0092853 & 0.00653217 & $1.19 \mathrm{E}-05$ & $1.31 E-05$ & $2.99 \mathrm{E}-05$ & 0.00581538 & 0.06653543 & 0.00865385 & 3432342.15 \\
\hline 42995089.8 & 775.547815 & 799.058099 & 1011.73881 & 759.795947 & 4.11E- -06 & $2.50 \mathrm{E}-05$ & 70.3717575 & 0.00931337 & 0.00655398 & $1.19 \mathrm{E}-05$ & $1.31 E-05$ & $3.00 E-05$ & 0.00582233 & 0.0667366 & 0.00869091 & 3440898.2 \\
\hline 43194930 & 775.547815 & 799.053909 & 1011.73457 & $\begin{array}{l}759.79497 \\
\end{array}$ & $4.11 \mathrm{E}-06$ & $2.50 \mathrm{E}-05$ & 70.3992662 & 0.00934846 & 0.00658125 & $1.19 \mathrm{E}-05$ & $1.31 E-05$ & $3.02 E-05$ & 0.00583126 & 0.06698806 & 0.00873742 & 3451592.85 \\
\hline 43444730.2 & 775.547815 & 799.04879 & 1011.72927 & 759.793746 & $4.11 \mathrm{E}-06$ & $2.50 \mathrm{E}-05$ & 70.433363 & 0.00939233 & 0.00661533 & $1.19 \mathrm{E}-05$ & $1.31 \mathrm{E}-05$ & $3.04 \mathrm{E}-05$ & 0.00584243 & 0.06730238 & 0.00879583 & 3464958.39 \\
\hline 43694530.3 & 775.547815 & 799.043837 & 1011.72392 & \begin{tabular}{|l|}
759.792519 \\
\end{tabular} & $4.11 \mathrm{E}-06$ & $2.50 \mathrm{E}-05$ & 70.4671428 & 0.00943619 & 0.00664941 & $1.19 \mathrm{E}-05$ & $1.31 E-05$ & $3.06 E-05$ & 0.00585353 & 0.06761671 & 0.00885457 & 3481615.6 \\
\hline 43820808 & 773.656685 & 797.127484 & 1010.66643 & 758.337023 & $4.11 \mathrm{E}-06$ & $2.50 \mathrm{E}-05$ & 70.4840998 & 0.00945837 & 0.00666664 & $1.19 \mathrm{E}-05$ & $1.31 E-05$ & $3.06 E-05$ & 0.00585358 & 0.0677756 & 0.00887755 & 3491709.56 \\
\hline 43821751.7 & 779.750737 & 804.355764 & 1025.93326 & 763.711291 & $4.11 E-06$ & $2.50 \mathrm{E}-05$ & 70.4842292 & 0.00945854 & 0.00666678 & $1.19 \mathrm{E}-05$ & $1.31 E-05$ & $3.11 E-05$ & 0.00589536 & 0.06777682 & 0.00902467 & 3524397.36 \\
\hline 43822695.4 & 785.848929 & 811.594569 & 1041.12148 & 769.090351 & $4.11 \mathrm{E}-06$ & 2.50 E- 05 & 70.4843644 & 0.00945871 & 0.00666691 & $1.19 \mathrm{E}-05$ & $1.31 \mathrm{E}-05$ & $3.16 \mathrm{E}-05$ & 0.00593377 & 0.06777809 & 0.00917008 & 3548845.83 \\
\hline 43823875.1 & 793.47727 & 820.634474 & 1060.01619 & 775.818016 & $4.11 \mathrm{E}-06$ & $2.50 \mathrm{E}-05$ & 70.4845418 & 0.00945895 & 0.00666709 & $1.19 \mathrm{E}-05$ & $1.31 E-05$ & $3.25 E-05$ & 0.00598386 & 0.06777975 & 0.00946408 & 3580015 \\
\hline 43824408 & 796.925405 & 824.70244 & 1068.67751 & 778.855066 & $4.11 \mathrm{E}-06$ & $2.50 \mathrm{E}-05$ & 70.4846249 & 0.00945905 & 0.00666718 & $1.19 \mathrm{E}-05$ & $1.31 \mathrm{E}-05$ & $3.39 E-05$ & 0.00601357 & 0.06778053 & 0.00985933 & 3595291.22 \\
\hline 43825587.6 & 796.925405 & 824.702933 & 1068.67933 & 778.855301 & $4.11 \mathrm{E}-06$ & $2.50 \mathrm{E}-05$ & 70.484811 & 0.0094593 & 0.00666737 & $1.19 \mathrm{E}-05$ & $1.31 E-05$ & 3.40 E- 05 & 0.00601355 & 0.06778228 & 0.00987305 & 3595241.67 \\
\hline 43827062.2 & 796.925405 & 824.702695 & 1068.68042 & 778.855249 & $4.11 \mathrm{E}-06$ & $2.50 \mathrm{E}-05$ & 70.4850436 & 0.0094596 & 0.00666761 & $1.19 E-05$ & $1.31 E-05$ & $3.40 \mathrm{E}-05$ & 0.00601441 & 0.06778447 & 0.00987126 & 3595345.5 \\
\hline 43828905.4 & 796.925405 & 824.702249 & 1068.68136 & 778.855181 & $4.11 \mathrm{E}-06$ & 2.50 E- 05 & 70.4853344 & 0.00945999 & 0.0066679 & $1.19 \mathrm{E}-05$ & $1.31 E-05$ & 3.40 E- 05 & 0.00601574 & 0.0677872 & 0.00986871 & 3595475.18 \\
\hline 43831209.4 & 796.925405 & 824.70168 & 1068.68196 & 778.855107 & $4.11 E-06$ & $2.50 \mathrm{E}-05$ & 70.4856978 & 0.00946046 & 0.00666827 & $1.19 \mathrm{E}-05$ & $1.31 E-05$ & 3.40 E- 05 & 0.00601737 & 0.06779061 & 0.0098652 & 3595635.97 \\
\hline 43834089.4 & 796.925405 & 824.700969 & 1068.68214 & 778.855022 & $4.11 \mathrm{E}-06$ & $2.50 \mathrm{E}-05$ & 70.4861519 & 0.00946106 & 0.00666874 & $1.19 \mathrm{E}-05$ & $1.31 E-05$ & $3.40 E-05$ & 0.00601927 & 0.06779488 & 0.00986074 & 3595836.74 \\
\hline 43837689.4 & 796.925405 & 824.700116 & 1068.68176 & 778.854924 & $4.12 \mathrm{E}-06$ & $2.50 \mathrm{E}-05$ & 70.4867196 & 0.0094618 & 0.00666931 & $1.19 \mathrm{E}-05$ & $1.31 E-05$ & $3.40 \mathrm{E}-05$ & 0.00602136 & 0.06780022 & 0.00985552 & 3596085.58 \\
\hline 43842189.4 & 796.925405 & 824.699194 & 1068.68044 & 778.854816 & $4.12 E-06$ & $2.50 \mathrm{E}-05$ & 70.4874291 & 0.00946273 & 0.00667004 & $1.19 \mathrm{E}-05$ & $1.31 E-05$ & 3.40 E- 05 & 0.00602374 & 0.06780689 & 0.00984991 & 3596393.42 \\
\hline 43847814.4 & 796.925405 & 824.697985 & 1068.6767 & 778.854681 & $4.12 \mathrm{E}-06$ & $2.50 \mathrm{E}-05$ & 70.4883157 & 0.0094639 & 0.00667094 & $1.19 \mathrm{E}-05$ & $1.31 E-05$ & $3.39 E-05$ & 0.00602639 & 0.06781522 & 0.00984438 & 3596780.98 \\
\hline 43854845.7 & 796.925405 & 824.696668 & 1068.66903 & 778.85453 & $4.12 \mathrm{E}-06$ & $2.50 \mathrm{E}-05$ & 70.4894236 & 0.00946535 & 0.00667207 & $1.19 \mathrm{E}-05$ & $1.31 E-05$ & $3.39 E-05$ & 0.00602934 & 0.06782564 & 0.00983929 & 3597260.09 \\
\hline 43863634.7 & 796.925405 & 824.695112 & 1068.65422 & 778.854344 & $4.12 E-06$ & $2.50 \mathrm{E}-05$ & 70.4908081 & 0.00946717 & 0.00667348 & $1.19 \mathrm{E}-05$ & $1.31 E-05$ & $3.39 E-05$ & 0.00603265 & 0.06783867 & 0.00983499 & 3597858.95 \\
\hline 43874621 & 796.925405 & 824.69344 & 1068.6319 & 778.854136 & $4.12 \mathrm{E}-06$ & $2.50 \mathrm{E}-05$ & 70.492538 & 0.00946944 & 0.00667525 & $1.19 \mathrm{E}-05$ & $1.31 E-05$ & $3.39 \mathrm{E}-05$ & 0.00603633 & 0.06785495 & 0.00983219 & 3598599.54 \\
\hline 43888354 & $\begin{array}{l}796.95141 \\
\end{array}$ & 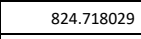 & 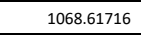 & \begin{tabular}{|l}
778.873896 \\
\end{tabular} & $4.12 \mathrm{E}-06$ & $2.50 \mathrm{E}-05$ & \begin{tabular}{|l}
70.4946991 \\
\end{tabular} & 0.00947228 & 0.00667746 & $1.19 \mathrm{E}-05$ & $1.31 E-05$ & $3.39 E-05$ & 0.00604036 & 0.0678753 & 0.00983139 & 3599614.38 \\
\hline 43895709 & 796.952581 & 824.718265 & 1068.599966 & 778.874668 & $4.12 \mathrm{E}-06$ & $2.50 \mathrm{E}-05$ & 70.4958561 & 0.0094738 & 0.00667864 & $1.19 \mathrm{E}-05$ & $1.31 E-05$ & $3.39 E-05$ & 0.00604238 & 0.0678862 & 0.00983155 & 3600737.08 \\
\hline 43899309 & 796.541987 & 824.229825 & 1067.57757 & 778.51231 & $4.12 E-06$ & $2.50 \mathrm{E}-05$ & 70.4964214 & 0.00947455 & 0.00667922 & $1.19 \mathrm{E}-05$ & $1.31 E-05$ & $3.39 E-05$ & 0.00604094 & 0.06789153 & 0.00982408 & 3599696.82 \\
\hline 43906664 & 796.541987 & 824.228814 & 1067.55415 & 778.512178 & $4.12 \mathrm{E}-06$ & $2.50 \mathrm{E}-05$ & 70.4975747 & 0.00947606 & 0.00668039 & $1.19 \mathrm{E}-05$ & $1.31 E-05$ & $3.39 E-05$ & 0.00604329 & 0.0679024 & 0.00982441 & 3600007.64 \\
\hline 43915857.8 & 796.541987 & 824.22768 & 1067.52679 & 778.512028 & $4.13 \mathrm{E}-06$ & $2.50 \mathrm{E}-05$ & 70.4990159 & 0.00947796 & 0.00668187 & $1.19 \mathrm{E}-05$ & $1.31 E-05$ & $3.39 E-05$ & 0.00604582 & 0.06791599 & 0.00982577 & 3600619.48 \\
\hline 43927350.1 & 796.541987 & 824.226514 & 1067.50093 & 778.511863 & $4.13 \mathrm{E}-06$ & $2.50 \mathrm{E}-05$ & 70.5008164 & 0.00948033 & 0.00668371 & $1.19 \mathrm{E}-05$ & $1.31 E-05$ & $3.39 E-05$ & 0.00604846 & 0.06793298 & 0.00982824 & 3601377.63 \\
\hline 43941715.4 & 796.541987 & 824.225227 & 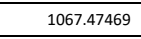 & 778.511669 & $4.13 \mathrm{E}-06$ & $2.50 \mathrm{E}-05$ & $\begin{array}{l}70.5030659 \\
\end{array}$ & $\begin{array}{l}0.00948329 \\
\end{array}$ & 0.00668601 & $1.19 \mathrm{E}-05$ & $1.31 E-05$ & $3.39 \mathrm{E}-05$ & 0.00605121 & 0.06795421 & 0.00983196 & 3602322.33 \\
\hline 43959672.1 & 796.541987 & 824.223921 & $\begin{array}{l}1067.4539 \\
\end{array}$ & 778.51145 & $4.13 \mathrm{E}-06$ & $2.50 \mathrm{E}-05$ & 70.5058757 & 0.009487 & 0.00668889 & $1.19 \mathrm{E}-05$ & $1.31 E-05$ & $3.39 E-05$ & 0.00605395 & 0.06798075 & 0.00983721 & 3603496.42 \\
\hline 43982117.9 & 796.541987 & 824.222606 & 1067.43915 & 778.511201 & $4.13 \mathrm{E}-06$ & $2.50 \mathrm{E}-05$ & 70.5093849 & 0.00949163 & 0.00669249 & $1.19 \mathrm{E}-05$ & $1.31 E-05$ & $3.39 E-05$ & 0.00605673 & 0.06801393 & 0.00984432 & 3604956.58 \\
\hline 44010175.1 & 796.541987 & 824.221184 & 1067.42766 & 778.510908 & $4.14 \mathrm{E}-06$ & $2.50 \mathrm{E}-05$ & 70.5137666 & 0.00949741 & 0.00669698 & $1.19 \mathrm{E}-05$ & $1.31 E-05$ & $3.40 E-05$ & 0.00605966 & 0.0680554 & 0.00985349 & 3606775.59 \\
\hline 44045246.7 & 796.541987 & 824.21959 & 1067.41787 & 778.510555 & $4.14 \mathrm{E}-06$ & $2.50 \mathrm{E}-05$ & 70.5192363 & 0.00950465 & 0.0067026 & $1.19 \mathrm{E}-05$ & $1.31 E-05$ & $3.40 E-05$ & 0.00606286 & 0.06810724 & 0.00986468 & 3609046.47 \\
\hline 44089086.1 & 796.541987 & 824.217856 & 1067.41153 & 778.510134 & $4.15 \mathrm{E}-06$ & $2.50 \mathrm{E}-05$ & 70.5260616 & 0.00951369 & 0.00670963 & $1.19 \mathrm{E}-05$ & $1.31 E-05$ & $3.40 \mathrm{E}-05$ & 0.00606629 & 0.06817203 & 0.00987831 & 3611879.1 \\
\hline 44143885.5 & 796.541987 & 824.215805 & 1067.40464 & 778.509617 & $4.16 \mathrm{E}-06$ & $2.50 \mathrm{E}-05$ & 70.5345751 & 0.00952499 & 0.00671841 & $1.19 \mathrm{E}-05$ & $1.31 E-05$ & $3.41 E-05$ & 0.00607033 & 0.06825303 & 0.00989499 & 3615416.75 \\
\hline 44212384.6 & 796.541987 & 824.213585 & 1067.40122 & 778.509001 & $4.16 \mathrm{E}-06$ & $2.50 \mathrm{E}-05$ & 70.5451885 & 0.00953912 & 0.00672939 & $1.19 \mathrm{E}-05$ & $1.31 \mathrm{E}-05$ & $3.42 E-05$ & 0.00607463 & 0.06835428 & 0.00991598 & 3619830.47 \\
\hline 44298008.6 & 796.541987 & 824.210569 & 1067.38912 & 778.508216 & $4.17 \mathrm{E}-06$ & 2.50 E- 05 & 70.5584112 & 0.00955679 & 0.00674312 & $1.19 \mathrm{E}-05$ & $1.31 E-05$ & $3.42 E-05$ & 0.00608058 & 0.06848084 & 0.00994202 & 3625353.15 \\
\hline 44401212 & 794.715373 & 822.354627 & 1066.36148 & 777.100871 & $4.19 \mathrm{E}-06$ & $2.50 \mathrm{E}-05$ & 70.5742838 & 0.00957807 & 0.00675966 & $1.19 \mathrm{E}-05$ & $1.31 E-05$ & $3.43 E-05$ & 0.00608184 & 0.06863338 & 0.00997243 & 3625917.01 \\
\hline 44404812 & 788.198453 & 814.60224 & 1050.13521 & 771.342895 & $4.19 \mathrm{E}-06$ & $2.50 \mathrm{E}-05$ & 70.5748244 & 0.0095788 & 0.00676022 & $1.19 \mathrm{E}-05$ & $1.31 E-05$ & $3.39 E-05$ & 0.00604068 & 0.06863859 & 0.00985026 & 3607645.24 \\
\hline 44508015.4 & 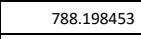 & $\begin{array}{l}814.596577 \\
\end{array}$ & 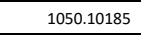 & \begin{tabular}{|l}
771.341814 \\
\end{tabular} & $4.19 \mathrm{E}-06$ & $2.50 \mathrm{E}-05$ & $\begin{array}{l}70.5899484 \\
\end{array}$ & 0.00959917 & 0.00677605 & $1.19 \mathrm{E}-05$ & $1.31 E-05$ & $3.40 \mathrm{E}-05$ & 0.00605541 & 0.06878459 & 0.0098731 & 3608091.6 \\
\hline 44637019.7 & 788.198453 & $\begin{array}{l}814.591212 \\
\end{array}$ & \begin{tabular}{|l|l|}
1050.08318 \\
\end{tabular} & 771.340672 & $4.20 \mathrm{E}-06$ & $2.50 \mathrm{E}-05$ & 70.6087634 & 0.00962464 & 0.00679584 & $1.19 \mathrm{E}-05$ & $1.31 E-05$ & $3.41 E-05$ & 0.00606777 & $\begin{array}{l}0.06896709 \\
\end{array}$ & 0.00991004 & 3616038.31 \\
\hline
\end{tabular}




\begin{tabular}{|c|c|c|c|c|c|c|c|c|c|c|c|c|c|c|c|c|}
\hline Time (s) & $\begin{array}{c}\text { Coolant outlet } \\
\text { temperature }(\mathrm{K})\end{array}$ & $\begin{array}{l}\text { Peak cladding } \\
\text { temperature (K) }\end{array}$ & $\begin{array}{c}\text { Peak fuel } \\
\text { temperature (K) }\end{array}$ & $\begin{array}{c}\text { Average cladding } \\
\text { internal } \\
\text { temperature (K) }\end{array}$ & $\begin{array}{l}\text { Peak cumulative } \\
\text { damage factor }\end{array}$ & $\begin{array}{l}\text { Cladding interior } \\
\text { volume }\left(\mathrm{m}^{3}\right)\end{array}$ & $\begin{array}{l}\text { Fission gas } \\
\text { release (\%) }\end{array}$ & $\begin{array}{l}\text { Fission gas } \\
\text { produced } \\
\text { (moles) }\end{array}$ & $\begin{array}{c}\text { Fission gas } \\
\text { released (moles) }\end{array}$ & Fuel volume $\left(\mathrm{m}^{3}\right)$ & $\begin{array}{l}\text { Plenum gas } \\
\text { volume }\left(\mathrm{m}^{3}\right)\end{array}$ & $\begin{array}{l}\text { Peak cladding } \\
\text { radial growth } \\
\text { (m) }\end{array}$ & $\begin{array}{l}\text { Peak fuel axial } \\
\text { growth }(m)\end{array}$ & $\begin{array}{c}\text { Peak burnup } \\
\text { (at\%) }\end{array}$ & $\begin{array}{l}\text { Peak cladding } \\
\text { hoop strain }\end{array}$ & $\begin{array}{l}\text { Plenum pressure } \\
(\mathrm{Pa})\end{array}$ \\
\hline 44766024 & 788.198453 & 814.587255 & 1050.06678 & 771.339648 & $4.20 \mathrm{E}-06$ & $2.50 \mathrm{E}-05$ & 70.6274791 & 0.00965011 & 0.00681563 & $1.19 \mathrm{E}-05$ & $1.31 \mathrm{E}-05$ & $3.42 \mathrm{E}-05$ & 0.00607658 & 0.0691496 & 0.00994603 & 3625890.32 \\
\hline 44895028.2 & 788.198453 & 814.584341 & 1050.05298 & 771.338713 & $4.21 E-06$ & $2.50 \mathrm{E}-05$ & 70.6460962 & 0.00967558 & 0.00683542 & $1.19 \mathrm{E}-05$ & $1.31 \mathrm{E}-05$ & $3.43 E-05$ & 0.00608267 & 0.0693321 & 0.00998169 & 3635713.27 \\
\hline 45056283.6 & 788.198453 & 814.581093 & 1050.04117 & 771.337582 & $4.21 \mathrm{E}-06$ & $2.50 \mathrm{E}-05$ & 70.6692303 & 0.00970742 & 0.00686016 & $1.19 \mathrm{E}-05$ & $1.31 \mathrm{E}-05$ & $3.45 \mathrm{E}-05$ & 0.0060893 & 0.06956023 & 0.01002611 & 3645548.05 \\
\hline \begin{tabular}{|l|l|}
45257852.8 \\
\end{tabular} & 788.198453 & \begin{tabular}{|l|l|}
814.577667 \\
\end{tabular} & 1050.05357 & 771.336235 & $4.22 \mathrm{E}-06$ & $2.50 \mathrm{E}-05$ & 70.6979353 & 0.00974721 & 0.00689108 & $1.19 \mathrm{E}-05$ & $1.30 \mathrm{E}-05$ & $3.46 \mathrm{E}-05$ & 0.00609609 & 0.0698454 & 0.01008158 & 3657813.41 \\
\hline 45509814.2 & 788.198453 & 814.572633 & 1050.0482 & 771.334484 & $4.23 \mathrm{E}-06$ & $2.50 \mathrm{E}-05$ & 70.7334887 & 0.00979696 & 0.00692973 & $1.19 \mathrm{E}-05$ & $1.30 \mathrm{E}-05$ & $3.49 \mathrm{E}-05$ & $\begin{array}{ll}0.0061067 \\
\end{array}$ & 0.07020185 & 0.0101506 & 3673165.75 \\
\hline \begin{tabular}{|l|}
45761775.7 \\
\end{tabular} & \begin{tabular}{|l|l|}
788.198453 \\
\end{tabular} & 814.567265 & 1050.03277 & 771.332699 & $4.24 \mathrm{E}-06$ & $2.50 \mathrm{E}-05$ & 70.7686828 & 0.0098467 & 0.00696838 & $1.20 \mathrm{E}-05$ & $1.30 \mathrm{E}-05$ & $3.51 \mathrm{E}-05$ & 0.00611837 & 0.0705583 & 0.01022166 & 3692326 \\
\hline \begin{tabular}{|l|}
46013737.2 \\
\end{tabular} & 788.198453 & \begin{tabular}{|l|l|}
814.561797 \\
\end{tabular} & 1050.01421 & 771..330905 & $4.25 \mathrm{E}-06$ & $2.50 \mathrm{E}-05$ & 70.8035232 & 0.00989645 & 0.00700703 & $1.20 \mathrm{E}-05$ & $1.30 \mathrm{E}-05$ & $3.53 \mathrm{E}-05$ & 0.00613046 & 0.07091476 & 0.01029856 & 3711490.3 \\
\hline 46265698.7 & 788.198453 & 814.556235 & 1049.9936 & 771.329104 & $4.26 \mathrm{E}-06$ & $2.50 \mathrm{E}-05$ & 70.838015 & 0.00994619 & 0.00704569 & $1.20 \mathrm{E}-05$ & $1.30 \mathrm{E}-05$ & $3.55 \mathrm{E}-05$ & 0.00614289 & 0.07127121 & 0.01037491 & 3730658.29 \\
\hline \begin{tabular}{|l|}
46517660.1 \\
\end{tabular} & 788.198453 & 814.550629 & 1049.97215 & \begin{tabular}{|l|l|}
771.327311 \\
\end{tabular} & $4.27 \mathrm{E}-06$ & $2.50 \mathrm{E}-05$ & 70.8721636 & 0.00999594 & 0.00708434 & $1.20 \mathrm{E}-05$ & $1.30 \mathrm{E}-05$ & $3.57 \mathrm{E}-05$ & 0.00615552 & 0.07162766 & 0.01045077 & 3749828.39 \\
\hline \begin{tabular}{ll|}
46769621.6 \\
\end{tabular} & 788.198453 & 814.545123 & 1049.9511 & 771.325524 & 4.28E-06 & $2.50 \mathrm{E}-05$ & 70.9059739 & 0.01004568 & 0.00712299 & $1.20 \mathrm{E}-05$ & $1.30 \mathrm{E}-05$ & $3.59 \mathrm{E}-05$ & 0.00616815 & 0.07198412 & 0.01052619 & 3768998.63 \\
\hline \begin{tabular}{|l|}
47021583.1 \\
\end{tabular} & 788.198453 & 814.53981 & 1049.92913 & 771.323748 & $4.30 \mathrm{E}-06$ & $2.50 \mathrm{E}-05$ & 70.9394511 & 0.01009543 & 0.00716164 & $1.20 \mathrm{E}-05$ & $1.30 \mathrm{E}-05$ & $3.61 \mathrm{E}-05$ & 0.00618104 & 0.07234057 & 0.01060123 & 3788171.77 \\
\hline \begin{tabular}{ll|}
47273544.6 \\
\end{tabular} & 788.198453 & 814.534294 & 1049.90752 & 771.321962 & $4.31 \mathrm{E}-06$ & $2.50 \mathrm{E}-05$ & 70.9725999 & 0.01014517 & 0.00720029 & $1.20 \mathrm{E}-05$ & $1.30 \mathrm{E}-05$ & $3.64 \mathrm{E}-05$ & 0.00619389 & 0.07269703 & 0.01067593 & 3807344.69 \\
\hline 47385472 & 789.115457 & 815.461201 & 1050.40951 & 772.027086 & $4.31 E-06$ & $2.50 \mathrm{E}-05$ & 70.9872214 & 0.01016727 & 0.00721746 & $1.20 \mathrm{E}-05$ & $1.30 \mathrm{E}-05$ & $3.65 E-05$ & 0.00620197 & 0.07285537 & 0.01071163 & 3829793.96 \\
\hline 47389072 & 786.135636 & 811.922688 & 1042.9978 & 769.397194 & $4.31 E-06$ & $2.50 \mathrm{E}-05$ & 70.9876859 & 0.01016797 & 0.00721801 & $1.20 \mathrm{E}-05$ & $1.30 \mathrm{E}-05$ & $3.63 \mathrm{E}-05$ & 0.00618342 & 0.07286041 & 0.01064495 & 3825418.18 \\
\hline 47500999.4 & 786.135636 & 811.918687 & 1042.93089 & 769.396272 & $4.32 \mathrm{E}-06$ & $2.50 \mathrm{E}-05$ & 71.0019462 & 0.01018962 & 0.00723483 & $1.20 \mathrm{E}-05$ & $1.30 \mathrm{E}-05$ & $3.63 \mathrm{E}-05$ & 0.00619433 & 0.07301553 & 0.01067222 & 3825822.35 \\
\hline 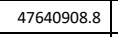 & 786.135636 & 811.914881 & 1042.91003 & 769.395363 & $4.32 \mathrm{E}-06$ & $2.50 \mathrm{E}-05$ & 71.0196867 & 0.01021668 & 0.00725585 & $1.20 \mathrm{E}-05$ & $1.30 \mathrm{E}-05$ & $3.65 \mathrm{E}-05$ & 0.00620263 & 0.07320944 & 0.0107115 & 3834187.31 \\
\hline 47780818.1 & 786.135636 & 811.912354 & 1042.92552 & 769.394592 & $4.33 \mathrm{E}-06$ & $2.50 \mathrm{E}-05$ & 71.0373335 & 0.01024374 & 0.00727688 & $1.20 \mathrm{E}-05$ & $1.30 \mathrm{E}-05$ & $3.66 \mathrm{E}-05$ & 0.00620706 & 0.07340334 & 0.01075089 & 3844568.45 \\
\hline 47955704.7 & 786.135636 & 811.908643 & 1042.93115 & 769.393618 & $4.33 \mathrm{E}-06$ & $2.50 \mathrm{E}-05$ & 71.0592613 & 0.01027757 & 0.00730316 & $1.20 \mathrm{E}-05$ & $1.30 \mathrm{E}-05$ & $3.67 \mathrm{E}-05$ & 0.00621385 & 0.07364572 & 0.0108001 & 3854976.94 \\
\hline 48174313 & 786.135636 & 811.903613 & 1042.9273 & 769.392385 & $4.34 \mathrm{E}-06$ & $2.50 \mathrm{E}-05$ & 71.0864688 & 0.01031985 & 0.00733601 & $1.20 \mathrm{E}-05$ & $1.30 \mathrm{E}-05$ & $3.69 \mathrm{E}-05$ & 0.00622356 & 0.07394869 & 0.01086153 & 3867998.56 \\
\hline 48198548 & 787.622121 & 813.409046 & 1043.75548 & 770.536352 & $4.34 E-06$ & $2.50 \mathrm{E}-05$ & 71.0894714 & 0.01032453 & 0.00733966 & $1.20 \mathrm{E}-05$ & $1.30 \mathrm{E}-05$ & $3.69 \mathrm{E}-05$ & 0.00622856 & 0.07398228 & 0.01087247 & 3889689.53 \\
\hline 48202148 & 644.15 & 644.172793 & 644.152336 & 644.150643 & $4.34 \mathrm{E}-06$ & $2.49 \mathrm{E}-05$ & 71.0896942 & 0.01032488 & 0.00733993 & $1.18 \mathrm{E}-05$ & $1.31 \mathrm{E}-05$ & $3.28 \mathrm{E}-05$ & 0.00470087 & 0.07398477 & 0.00960645 & 3246621.41 \\
\hline 48205748 & 305 & 305.051372 & 305.006678 & 305.002174 & $4.34 \mathrm{E}-06$ & $2.46 \mathrm{E}-05$ & 71.0896942 & 0.01032488 & 0.00733993 & $1.16 \mathrm{E}-05$ & $1.29 \mathrm{E}-05$ & $1.63 \mathrm{E}-05$ & 0.00267544 & 0.07398477 & 0.0051416 & 1552224.73 \\
\hline 48209348 & 305 & 305.051372 & 305.006678 & 305.002174 & $4.34 \mathrm{E}-06$ & $2.46 \mathrm{E}-05$ & 71.0896942 & 0.01032488 & 0.00733993 & $1.16 \mathrm{E}-05$ & $1.29 \mathrm{E}-05$ & $1.63 \mathrm{E}-05$ & 0.00267544 & 0.07398477 & 0.0051416 & 1552224.73 \\
\hline 48212948 & $\begin{array}{r}784.07371 \\
\end{array}$ & 809.158648 & 1035.05625 & 767.404845 & $4.34 \mathrm{E}-06$ & $2.50 \mathrm{E}-05$ & 71.0899117 & 0.01032522 & 0.00734019 & $1.20 \mathrm{E}-05$ & $1.30 \mathrm{E}-05$ & $3.67 \mathrm{E}-05$ & 0.006288 & 0.07398721 & 0.01074533 & 3876908.91 \\
\hline 48216548 & 784.07371 & 809.16367 & 1035.04574 & 767.408458 & $4.34 \mathrm{E}-06$ & $2.50 \mathrm{E}-05$ & 71.0903465 & 0.0103259 & 0.00734072 & $1.20 \mathrm{E}-05$ & $1.30 \mathrm{E}-05$ & $3.65 \mathrm{E}-05$ & 0.0063049 & 0.07399207 & 0.01069961 & 3877006.29 \\
\hline 48221048 & 784.07371 & 809.163889 & $\begin{array}{l}1035.0369 \\
\end{array}$ & 767.407865 & $4.34 E-06$ & $2.50 \mathrm{E}-05$ & $\begin{array}{ll}71.0908899 \\
\end{array}$ & 0.01032675 & 0.00734138 & $1.20 \mathrm{E}-05$ & $1.30 \mathrm{E}-05$ & $3.60 \mathrm{E}-05$ & 0.00631192 & 0.07399816 & 0.01054415 & 3877819.91 \\
\hline 48226673 & 784.07371 & 809.163882 & 1035.03242 & 767.407634 & $4.34 \mathrm{E}-06$ & $2.50 \mathrm{E}-05$ & 71.091569 & 0.01032781 & 0.0073422 & $1.20 \mathrm{E}-05$ & $1.30 \mathrm{E}-05$ & $3.58 \mathrm{E}-05$ & 0.0063162 & 0.07400576 & 0.01049797 & 3878336.78 \\
\hline 48233704.3 & 784.07371 & 809.163769 & 1035.03005 & 767.407548 & $4.34 \mathrm{E}-06$ & $2.50 \mathrm{E}-05$ & $\begin{array}{ll}1.0924178 \\
\end{array}$ & 0.01032914 & 0.00734323 & $1.20 \mathrm{E}-05$ & $1.30 \mathrm{E}-05$ & $3.57 \mathrm{E}-05$ & 0.00631926 & 0.07401527 & 0.01048768 & 3878755.34 \\
\hline 48242493.3 & 784.07371 & 809.163591 & 1035.02881 & $\begin{array}{l}767.407476 \\
\end{array}$ & $4.34 \mathrm{E}-06$ & $2.50 \mathrm{E}-05$ & 71.0934784 & 0.0103308 & 0.00734452 & $1.20 \mathrm{E}-05$ & $1.30 \mathrm{E}-05$ & $3.56 \mathrm{E}-05$ & 0.00632127 & 0.07402715 & 0.01048297 & 3879271.56 \\
\hline 48253479.6 & 784.07371 & 809.163369 & 1035.02811 & 767.407405 & $4.34 \mathrm{E}-06$ & $2.50 \mathrm{E}-05$ & 71.0948037 & 0.01033287 & 0.00734613 & $1.20 \mathrm{E}-05$ & $1.30 \mathrm{E}-05$ & $3.56 \mathrm{E}-05$ & 0.00632263 & 0.074042 & 0.01048239 & 3879911.4 \\
\hline 48267212.6 & 784.07371 & 809.163097 & 1035.02761 & 767.407324 & $4.34 \mathrm{E}-06$ & $2.50 \mathrm{E}-05$ & 71.0964596 & 0.01033546 & 0.00734815 & $1.20 \mathrm{E}-05$ & $1.30 \mathrm{E}-05$ & $3.56 \mathrm{E}-05$ & 0.00632371 & 0.07406057 & 0.01048451 & 3880708.46 \\
\hline 48284378.7 & 784.07371 & 809.16277 & \begin{tabular}{l|l}
1035.02707 \\
\end{tabular} & 767.40723 & $4.34 \mathrm{E}-06$ & $2.50 \mathrm{E}-05$ & 71.0985283 & 0.0103387 & 0.00735066 & $1.20 \mathrm{E}-05$ & $1.30 \mathrm{E}-05$ & $3.56 \mathrm{E}-05$ & 0.00632474 & 0.07408378 & 0.01048824 & 3881703.68 \\
\hline 48305836.4 & 784.07371 & 809.162367 & 1035.02641 & 767.407116 & $4.34 \mathrm{E}-06$ & $2.50 \mathrm{E}-05$ & 71.1011123 & 0.01034275 & 0.00735381 & $1.20 \mathrm{E}-05$ & $1.30 \mathrm{E}-05$ & $3.56 \mathrm{E}-05$ & 0.00632587 & 0.07411279 & 0.0104933 & 3882947.99 \\
\hline 48332658.5 & $\begin{array}{r}784.07371 \\
\end{array}$ & 809.161874 & 1035.02553 & 767.406977 & $4.34 \mathrm{E}-06$ & $2.50 \mathrm{E}-05$ & 71.1043395 & 0.01034781 & 0.00735774 & $1.20 \mathrm{E}-05$ & $1.30 \mathrm{E}-05$ & $3.57 \mathrm{E}-05$ & \begin{tabular}{|l|}
0.0632719 \\
\end{tabular} & 0.07414905 & 0.01049966 & 3884502.96 \\
\hline 48366186.1 & $\begin{array}{l}784.07371 \\
\end{array}$ & 809.161207 & 1035.02433 & 767.406806 & $4.34 \mathrm{E}-06$ & $2.50 \mathrm{E}-05$ & 71.108369 & 0.01035413 & 0.00736266 & $1.20 \mathrm{E}-05$ & $1.30 \mathrm{E}-05$ & $3.57 \mathrm{E}-05$ & 0.00632875 & 0.07419438 & 0.01050762 & 3886446.18 \\
\hline 48408095.6 & 784.07371 & 809.160418 & 1035.0227 & 767.406596 & $4.34 E-06$ & $2.50 \mathrm{E}-05$ & 71.1133991 & 0.01036204 & 0.0073688 & $1.20 \mathrm{E}-05$ & $1.30 \mathrm{E}-05$ & $3.57 \mathrm{E}-05$ & 0.00633058 & 0.07425104 & 0.0105176 & 3888873.87 \\
\hline 48460482.5 & 784.07371 & 809.159406 & 1035.02045 & 767.406332 & $4.35 E-06$ & $2.50 \mathrm{E}-05$ & 71.1196758 & 0.01037193 & 0.00737648 & $1.20 \mathrm{E}-05$ & $1.30 \mathrm{E}-05$ & $3.58 \mathrm{E}-05$ & 0.0063329 & 0.07432187 & 0.01053011 & 3891909.07 \\
\hline 48525966.1 & 784.07371 & 809.15815 & 1035.0173 & 767.406004 & $4.35 \mathrm{E}-06$ & $2.50 \mathrm{E}-05$ & 71.1275049 & 0.01038428 & 0.00738608 & $1.20 \mathrm{E}-05$ & $1.30 \mathrm{E}-05$ & $3.58 \mathrm{E}-05$ & 0.00633569 & 0.0744104 & 0.0105458 & 3895703.02 \\
\hline 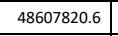 & $\begin{array}{l}784.07371 \\
\end{array}$ & 809.156543 & 1035.01302 & 767.405589 & $4.35 \mathrm{E}-06$ & $2.50 \mathrm{E}-05$ & 71.1372652 & 0.01039972 & 0.00739808 & $1.20 \mathrm{E}-05$ & $1.30 \mathrm{E}-05$ & $3.59 \mathrm{E}-05$ & 0.00633936 & $\begin{array}{l}0.07452106 \\
\end{array}$ & 0.01056546 & 3900446.29 \\
\hline 48710138.8 & 784.07371 & 809.154505 & 1035.00715 & 767.405069 & $4.35 \mathrm{E}-06$ & $2.50 \mathrm{E}-05$ & 71.1494248 & 0.01041903 & 0.00741308 & $1.20 \mathrm{E}-05$ & $1.30 \mathrm{E}-05$ & $3.60 \mathrm{E}-05$ & 0.00634396 & 0.07465939 & 0.01059009 & 3906376.26 \\
\hline 48838036.5 & $\begin{array}{r}784.07371 \\
\end{array}$ & 809.151958 & 1034.99938 & $\begin{array}{l}767.404419 \\
\end{array}$ & $4.36 \mathrm{E}-06$ & $2.50 \mathrm{E}-05$ & 71.1645611 & 0.01044316 & 0.00743183 & $1.20 \mathrm{E}-05$ & $1.30 \mathrm{E}-05$ & $3.61 \mathrm{E}-05$ & 0.00634966 & 0.07483231 & 0.01062097 & 3913788.17 \\
\hline \begin{tabular}{|l}
8997908.6 \\
\end{tabular} & 784.07371 & 809.14871 & $\begin{array}{l}1034.98923 \\
\end{array}$ & 767.403602 & $4.36 \mathrm{E}-06$ & $2.50 \mathrm{E}-05$ & 71.1833834 & 0.01047332 & 0.00745527 & $1.20 \mathrm{E}-05$ & $1.30 \mathrm{E}-05$ & $3.62 \mathrm{E}-05$ & 0.00635775 & $\begin{array}{l}0.07504845 \\
\end{array}$ & 0.01065969 & 3923054.72 \\
\hline 49197748.7 & 784.07371 & 809.144573 & 1034.97609 & 767.402571 & $4.36 \mathrm{E}-06$ & $2.50 \mathrm{E}-05$ & 71.2067593 & 0.01051103 & 0.00748456 & $1.20 \mathrm{E}-05$ & $1.30 \mathrm{E}-05$ & $3.63 \mathrm{E}-05$ & 0.00636835 & 0.07531863 & 0.01070832 & 3934639.32 \\
\hline 49397588.9 & 784.07371 & 809.140374 & 1034.96272 & 767.401528 & $4.37 \mathrm{E}-06$ & $2.50 \mathrm{E}-05$ & 71.2299682 & 0.01054873 & 0.00751386 & $1.20 \mathrm{E}-05$ & $1.30 \mathrm{E}-05$ & $3.65 \mathrm{E}-05$ & 0.00637904 & 0.07558881 & 0.01075721 & 3949092.06 \\
\hline 49597429 & $\begin{array}{l}784.07371 \\
\end{array}$ & 809.136136 & \begin{tabular}{|l|l|}
1034.94928 \\
\end{tabular} & 767.40048 & $4.37 \mathrm{E}-06$ & $2.50 \mathrm{E}-05$ & 71.2530117 & 0.01058644 & 0.00754315 & $1.20 \mathrm{E}-05$ & $1.30 \mathrm{E}-05$ & $3.67 \mathrm{E}-05$ & 0.00638975 & 0.07585898 & 0.01080695 & 3963545.25 \\
\hline 49797269.2 & 784.07371 & 809.131809 & 1034.93565 & 767.399423 & $4.38 \mathrm{E}-06$ & $2.50 \mathrm{E}-05$ & 71.2758917 & 0.01062414 & 0.00757245 & $1.20 \mathrm{E}-05$ & $1.30 \mathrm{E}-05$ & $3.68 E-05$ & 0.0064006 & 0.07612916 & 0.0108584 & 3978000.88 \\
\hline
\end{tabular}




\begin{tabular}{|c|c|c|c|c|c|c|c|c|c|c|c|c|c|c|c|c|}
\hline Time (s) & $\begin{array}{c}\text { Coolant outlet } \\
\text { temperature }(K)\end{array}$ & $\begin{array}{l}\text { Peak cladding } \\
\text { temperature (K) }\end{array}$ & $\begin{array}{c}\text { Peak fuel } \\
\text { temperature (K) }\end{array}$ & $\begin{array}{c}\text { Average cladding } \\
\text { internal } \\
\text { temperature }(\mathrm{K})\end{array}$ & $\begin{array}{l}\text { Peak cumulative } \\
\text { damage factor }\end{array}$ & $\begin{array}{l}\text { Cladding interior } \\
\text { volume }\left(\mathrm{m}^{3}\right)\end{array}$ & $\begin{array}{l}\text { Fission gas } \\
\text { release (\%) }\end{array}$ & 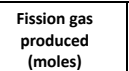 & $\begin{array}{c}\text { Fission gas } \\
\text { released (moles) }\end{array}$ & Fuel volume $\left(\mathrm{m}^{3}\right)$ & $\begin{array}{l}\text { Plenum gas } \\
\text { volume }\left(m^{3}\right)\end{array}$ & $\begin{array}{l}\text { Peak cladding } \\
\text { radial growth } \\
(\mathrm{m})\end{array}$ & $\begin{array}{l}\text { Peak fuel axial } \\
\text { growth }(m)\end{array}$ & $\begin{array}{c}\text { Peak burnup } \\
\text { (at\%) }\end{array}$ & $\begin{array}{l}\text { Peak cladding } \\
\text { hoop strain }\end{array}$ & $\begin{array}{l}\text { Plenum pressure } \\
(\mathrm{Pa})\end{array}$ \\
\hline 49997109.3 & 784.07371 & 809.127428 & 1034.92183 & $\begin{array}{l}767.398359 \\
\end{array}$ & $4.39 \mathrm{E}-06$ & $2.50 \mathrm{E}-05$ & 71.2986098 & 0.01066184 & 0.00760175 & $1.20 \mathrm{E}-05$ & $1.30 \mathrm{E}-05$ & $3.70 \mathrm{E}-05$ & 0.00641152 & 0.07639934 & 0.01091008 & 3992457.7 \\
\hline 50196949.4 & 784.07371 & 809.123024 & 1034.90802 & 767.397294 & $4.39 \mathrm{E}-06$ & $2.50 \mathrm{E}-05$ & 71.3211678 & 0.01069955 & 0.00763104 & $1.20 \mathrm{E}-05$ & $1.30 \mathrm{E}-05$ & $3.71 E-05$ & 0.00642244 & 0.07666952 & 0.01096198 & 4006914.94 \\
\hline 50396789.6 & 784.07371 & 809.118524 & 1034.89402 & 767.39622 & $4.40 \mathrm{E}-06$ & $2.50 \mathrm{E}-05$ & 71.3435674 & 0.01073725 & 0.00766034 & $1.20 \mathrm{E}-05$ & $1.30 \mathrm{E}-05$ & $3.73 E-05$ & 0.00643353 & 0.07693969 & 0.01101409 & 4021375.39 \\
\hline 50596629.7 & 784.07371 & 809.114076 & $\begin{array}{l}1034.8802 \\
\end{array}$ & \begin{tabular}{|l|l|}
767.395144 \\
\end{tabular} & $4.40 \mathrm{E}-06$ & $2.50 \mathrm{E}-05$ & 71.3658103 & 0.01077496 & 0.00768964 & $1.20 \mathrm{E}-05$ & $1.30 \mathrm{E}-05$ & $3.75 E-05$ & 0.00644447 & 0.07720987 & 0.0110664 & 4035833.53 \\
\hline 50796469.9 & 784.07371 & 809.109527 & 1034.86624 & 767.394056 & \begin{tabular}{ll|l|}
$4.41 E-06$ \\
\end{tabular} & $2.50 \mathrm{E}-05$ & $\begin{array}{l}71.387898 \\
\end{array}$ & 0.01081266 & 0.00771893 & $1.20 \mathrm{E}-05$ & $1.30 \mathrm{E}-05$ & $3.76 E-05$ & 0.00645557 & 0.07748005 & 0.01111889 & 4050295.17 \\
\hline 50996310 & 784.07371 & 809.104936 & 1034.85216 & 767.392961 & $4.41 E-06$ & $2.50 \mathrm{E}-05$ & 71.4098322 & 0.01085037 & 0.00774823 & $1.20 \mathrm{E}-05$ & $1.30 \mathrm{E}-05$ & $3.78 E-05$ & 0.00646676 & 0.07775023 & 0.01117156 & 4064758.36 \\
\hline 51196150.2 & 784.07371 & 809.100418 & 1034.83832 & 767.391863 & $4.42 \mathrm{E}-06$ & $2.50 \mathrm{E}-05$ & 71.4316145 & 0.01088807 & 0.00777753 & $1.20 \mathrm{E}-05$ & $1.30 \mathrm{E}-05$ & $3.80 \mathrm{E}-05$ & 0.00647774 & 0.0780204 & 0.01122439 & 4079219 \\
\hline 51395990.3 & 784.07371 & 809.095802 & 1034.82441 & 767.390755 & 4.42E-06 & $2.50 \mathrm{E}-05$ & 71.4532464 & 0.01092578 & 0.00780682 & $1.20 \mathrm{E}-05$ & $1.30 \mathrm{E}-05$ & $3.81 E-05$ & 0.00648892 & 0.07829058 & 0.01127736 & 4093682.9 \\
\hline 51595830.5 & 784.07371 & 809.091208 & 1034.81056 & 767.389646 & $4.43 \mathrm{E}-06$ & $2.50 \mathrm{E}-05$ & 71.4747296 & 0.01096348 & 0.00783612 & $1.20 \mathrm{E}-05$ & $1.30 \mathrm{E}-05$ & $3.83 E-05$ & 0.00650006 & 0.07856076 & 0.01133048 & 4108146.96 \\
\hline 51795670.6 & 784.07371 & 809.086634 & 1034.79683 & 767.388538 & $4.44 E-06$ & $2.50 \mathrm{E}-05$ & 71.4960655 & 0.01100118 & 0.00786541 & $1.20 \mathrm{E}-05$ & $1.30 \mathrm{E}-05$ & $3.85 E-05$ & 0.00651112 & 0.07883094 & 0.01138372 & 4122610.07 \\
\hline 51995510.7 & 784.07371 & 809.081981 & 1034.78296 & 767.387422 & $4.44 \mathrm{E}-06$ & $2.50 \mathrm{E}-05$ & 71.5172557 & 0.01103889 & 0.00789471 & $1.20 \mathrm{E}-05$ & $1.30 \mathrm{E}-05$ & $3.86 \mathrm{E}-05$ & 0.00652234 & 0.07910112 & 0.01143707 & 4137077.11 \\
\hline 52079977 & 784.07371 & 809.080012 & 1034.77708 & $\begin{array}{l}767.386947 \\
\end{array}$ & $4.44 \mathrm{E}-06$ & $2.50 \mathrm{E}-05$ & 71.5261686 & 0.01105483 & 0.00790709 & $1.20 \mathrm{E}-05$ & $1.30 \mathrm{E}-05$ & $3.87 \mathrm{E}-05$ & 0.00652714 & 0.07921531 & 0.01145971 & 4151469.96 \\
\hline 52083577 & 784.07371 & 809.079968 & 1034.77683 & 767.386928 & 4.44E-06 & $2.50 \mathrm{E}-05$ & 71.5265479 & 0.0110555 & 0.00790762 & $1.20 \mathrm{E}-05$ & $1.30 \mathrm{E}-05$ & $3.87 E-05$ & 0.00652727 & 0.07922018 & 0.01146077 & 4157531.99 \\
\hline 52168043.3 & 784.07371 & 809.078041 & 1034.77104 & 767.386458 & $4.45 E-06$ & 2.50 E- 05 & 71.5354342 & 0.01107144 & 0.00792 & $1.20 \mathrm{E}-05$ & $1.30 \mathrm{E}-05$ & $3.88 \mathrm{E}-05$ & 0.00653192 & 0.07933437 & 0.01148326 & 4157843.16 \\
\hline 52273626.1 & 784.07371 & 809.075642 & 1034.76387 & 767.38587 & $4.45 \mathrm{E}-06$ & $2.50 \mathrm{E}-05$ & 71.546506 & 0.01109136 & 0.00793548 & $1.20 \mathrm{E}-05$ & $1.30 \mathrm{E}-05$ & $3.89 \mathrm{E}-05$ & 0.00653771 & 0.07947712 & 0.01151152 & 4163969.83 \\
\hline 52405604.6 & $\begin{array}{r}784.07371 \\
\end{array}$ & 809.072612 & 1034.75503 & 767.385131 & $4.45 \mathrm{E}-06$ & $2.50 \mathrm{E}-05$ & 71.56029 & 0.01111626 & 0.00795483 & $1.20 \mathrm{E}-05$ & $1.30 \mathrm{E}-05$ & $3.90 E-05$ & 0.00654506 & 0.07965555 & 0.01154688 & $\begin{array}{r}4171629.5 \\
\end{array}$ \\
\hline 52570577.8 & 784.07371 & 809.06877 & 1034.74383 & 767.384202 & $4.46 E-06$ & $2.50 \mathrm{E}-05$ & 71.5774335 & $\begin{array}{l}0.01114739 \\
\end{array}$ & 0.00797901 & $1.20 \mathrm{E}-05$ & $1.30 \mathrm{E}-05$ & $3.91 \mathrm{E}-05$ & 0.00655437 & 0.07987859 & 0.01159115 & 4181206.16 \\
\hline 52735550.9 & 784.07371 & 809.064978 & 1034.7326 & 767.383271 & $4.46 \mathrm{E}-06$ & $2.50 \mathrm{E}-05$ & 71.5944814 & 0.01117851 & 0.0080032 & $1.20 \mathrm{E}-05$ & $1.30 \mathrm{E}-05$ & $3.92 E-05$ & 0.00656358 & 0.08010163 & 0.01163549 & 4193149.31 \\
\hline 52941767.4 & 784.07371 & 809.060292 & 1034.71872 & 767.382104 & $4.47 \mathrm{E}-06$ & 2.50 E- 05 & 71.6156583 & 0.01121742 & 0.00803343 & $1.20 \mathrm{E}-05$ & $1.30 \mathrm{E}-05$ & $3.94 E-05$ & 0.00657498 & 0.08038043 & 0.01169097 & 4205117.33 \\
\hline 53147983.8 & 784.07371 & 809.055552 & 1034.70482 & 767.380928 & $4.48 E-06$ & $2.50 \mathrm{E}-05$ & 71.6366888 & 0.01125633 & 0.00806366 & $1.20 \mathrm{E}-05$ & $1.30 \mathrm{E}-05$ & $3.96 E-05$ & 0.00658655 & 0.08065922 & 0.01174656 & 4220048.19 \\
\hline 53354200.2 & \begin{tabular}{|l}
784.07371 \\
\end{tabular} & 809.050845 & 1034.69091 & 767.379749 & $4.48 \mathrm{E}-06$ & $2.50 \mathrm{E}-05$ & 71.6575744 & 0.01129524 & 0.00809389 & $1.20 \mathrm{E}-05$ & $1.30 \mathrm{E}-05$ & $3.98 E-05$ & 0.00659809 & 0.08093802 & 0.01180221 & 4234978.99 \\
\hline 53560416.7 & 784.07371 & 809.04623 & 1034.6772 & 767.378574 & $4.49 \mathrm{E}-06$ & $2.50 \mathrm{E}-05$ & 71.6783167 & 0.01133414 & 0.00812412 & $1.20 \mathrm{E}-05$ & $1.30 \mathrm{E}-05$ & $3.99 E-05$ & 0.00660943 & 0.08121682 & 0.01185792 & 4249907.73 \\
\hline 53766633.1 & 784.07371 & 809.04154 & 1034.66344 & 767.377391 & $4.50 \mathrm{E}-06$ & $2.50 \mathrm{E}-05$ & 71.698917 & 0.01137305 & 0.00815436 & $1.20 \mathrm{E}-05$ & $1.30 \mathrm{E}-05$ & $4.01 E-05$ & 0.00662098 & 0.08149562 & 0.01191369 & 4264839.88 \\
\hline 53874489 & 785.999361 & 810.988625 & 1035.73281 & 768.8585 & $4.50 \mathrm{E}-06$ & $2.50 \mathrm{E}-05$ & 71.7096354 & 0.0113934 & 0.00817017 & $1.20 \mathrm{E}-05$ & $1.30 \mathrm{E}-05$ & $4.02 E-05$ & 0.00663185 & 0.08164144 & 0.01194997 & 4287677.79 \\
\hline 53878089 & 786.481015 & 811.557678 & 1036.91203 & 769.282124 & $4.50 \mathrm{E}-06$ & $2.50 \mathrm{E}-05$ & 71.7099931 & 0.01139408 & 0.0081707 & $1.20 \mathrm{E}-05$ & $1.30 \mathrm{E}-05$ & $4.03 E-05$ & 0.00663495 & 0.08164631 & 0.01196231 & 4297746.72 \\
\hline 53985944.9 & 786.481015 & 811.555139 & 1036.90499 & 769.281497 & $4.50 \mathrm{E}-06$ & $2.50 \mathrm{E}-05$ & 71.7207078 & 0.0114145 & 0.00818656 & $1.20 \mathrm{E}-05$ & $1.30 \mathrm{E}-05$ & $4.04 E-05$ & 0.00664088 & 0.08179262 & 0.01199268 & 4298077.01 \\
\hline 54120764.7 & 786.481015 & 811.551868 & 1036.89592 & 769.280693 & $4.51 \mathrm{E}-06$ & $2.50 \mathrm{E}-05$ & 71.7340474 & 0.01144002 & 0.00820639 & $1.20 \mathrm{E}-05$ & $1.30 \mathrm{E}-05$ & $4.05 E-05$ & 0.00664891 & 0.0819755 & 0.01202958 & 4305956.79 \\
\hline 54289289.5 & \begin{tabular}{|l}
786.481015 \\
\end{tabular} & 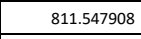 & 1036.88416 & 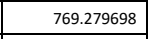 & $4.52 \mathrm{E}-06$ & $2.50 \mathrm{E}-05$ & $\begin{array}{l}71.7506385 \\
\end{array}$ & 0.01147193 & 0.00823118 & $1.20 \mathrm{E}-05$ & $1.30 \mathrm{E}-05$ & $4.06 E-05$ & 0.00665865 & 0.08220411 & 0.01207568 & 4315803.95 \\
\hline 54457814.3 & 786.481015 & 811.544019 & 1036.8722 & 769.27871 & $4.53 \mathrm{E}-06$ & $2.50 \mathrm{E}-05$ & 71.7671376 & 0.01150383 & 0.00825597 & $1.20 \mathrm{E}-05$ & $1.30 \mathrm{E}-05$ & $4.08 E-05$ & 0.00666824 & 0.08243272 & 0.01212182 & 4328082.51 \\
\hline 54668470.3 & 786.481015 & 811.539374 & 1036.85757 & 769.277483 & $4.53 \mathrm{E}-06$ & $2.50 \mathrm{E}-05$ & 71.7876332 & 0.01154371 & 0.00828695 & $1.20 \mathrm{E}-05$ & $1.30 \mathrm{E}-05$ & $4.09 E-05$ & 0.00667977 & 0.08271848 & 0.01217948 & 4340383.22 \\
\hline 54879126.2 & 786.481015 & 811.534679 & 1036.84311 & 769.276246 & $4.54 \mathrm{E}-06$ & $2.50 \mathrm{E}-05$ & 71.8079876 & 0.01158359 & 0.00831794 & $1.20 \mathrm{E}-05$ & $1.30 \mathrm{E}-05$ & $4.11 \mathrm{E}-05$ & 0.00669148 & 0.08300423 & 0.01223718 & 4355728.89 \\
\hline 55089782.2 & 786.481015 & 811.530096 & 1036.82899 & 769.275012 & $4.55 \mathrm{E}-06$ & $2.50 \mathrm{E}-05$ & 71.8282024 & 0.01162346 & 0.00834893 & $1.20 \mathrm{E}-05$ & $1.30 \mathrm{E}-05$ & $4.13 \mathrm{E}-05$ & 0.00670305 & 0.08328999 & 0.01229489 & 4371072.22 \\
\hline 55300438.2 & 786.481015 & 811.525578 & 1036.81512 & 769.273776 & $4.56 \mathrm{E}-06$ & $2.50 \mathrm{E}-05$ & 71.848279 & 0.01166334 & 0.00837991 & $1.20 \mathrm{E}-05$ & $1.30 \mathrm{E}-05$ & $4.15 E-05$ & 0.00671453 & 0.08357575 & 0.01235262 & 4386414.99 \\
\hline 55511094.2 & $\begin{array}{l}786.481015 \\
\end{array}$ & 811.521035 & 1036.80135 & 769.272532 & $4.57 \mathrm{E}-06$ & $2.50 \mathrm{E}-05$ & 71.8682187 & 0.01170322 & 0.0084109 & $1.20 \mathrm{E}-05$ & $1.30 \mathrm{E}-05$ & $4.17 \mathrm{E}-05$ & 0.00672617 & 0.08386151 & 0.01241035 & 4401760.57 \\
\hline 55721750.2 & 786.481015 & 811.5167 & 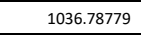 & 769.271296 & $4.58 \mathrm{E}-06$ & $2.50 \mathrm{E}-05$ & 71.888023 & 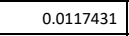 & 0.00844188 & $1.20 \mathrm{E}-05$ & $1.30 \mathrm{E}-05$ & $4.18 E-05$ & 0.00673757 & 0.08414727 & 0.01246808 & 4417103.18 \\
\hline 55932406.2 & 786.481015 & 811.512564 & 1036.77438 & 769.270061 & $4.59 \mathrm{E}-06$ & $2.50 \mathrm{E}-05$ & 71.9076933 & 0.01178298 & 0.00847287 & $1.20 \mathrm{E}-05$ & $1.30 \mathrm{E}-05$ & $4.20 \mathrm{E}-05$ & 0.00674903 & 0.08443302 & 0.01252582 & 4432447.24 \\
\hline 56143062.1 & 786.481015 & 811.507809 & 1036.76098 & 769.268817 & $4.60 \mathrm{E}-06$ & $2.50 \mathrm{E}-05$ & 71.9272308 & 0.01182286 & 0.00850385 & $1.20 \mathrm{E}-05$ & $1.30 \mathrm{E}-05$ & $4.22 E-05$ & 0.00676063 & 0.08471878 & 0.01258356 & 4447793.75 \\
\hline 56353718.1 & 786.481015 & 811.503339 & 1036.74767 & 769.267573 & $4.61 \mathrm{E}-06$ & $2.50 \mathrm{E}-05$ & 71.946637 & 0.01186274 & 0.00853484 & $1.20 \mathrm{E}-05$ & $1.30 \mathrm{E}-05$ & $4.24 E-05$ & 0.00677201 & 0.08500454 & 0.01264129 & 4463137.95 \\
\hline 56564374.1 & 786.481015 & 811.499415 & $\begin{array}{l}1036.7345 \\
\end{array}$ & 769.266331 & $4.62 \mathrm{E}-06$ & $2.50 \mathrm{E}-05$ & 71.9659132 & 0.01190262 & 0.00856583 & $1.20 \mathrm{E}-05$ & $1.30 \mathrm{E}-05$ & $4.26 E-05$ & 0.00678342 & 0.0852903 & 0.01269903 & 4478482.37 \\
\hline 56775030.1 & 786.481015 & 811.495577 & 1036.72137 & 769.265084 & $4.63 \mathrm{E}-06$ & $2.50 \mathrm{E}-05$ & 71.9850606 & 0.01194249 & 0.00859681 & $1.20 \mathrm{E}-05$ & $1.30 \mathrm{E}-05$ & $4.27 E-05$ & 0.00679495 & 0.08557606 & 0.01275675 & 4493829.32 \\
\hline 56985686.1 & 786.481015 & 813.771297 & $\begin{array}{c}1036.7084 \\
\end{array}$ & 769.320247 & $4.65 \mathrm{E}-06$ & $2.50 \mathrm{E}-05$ & 72.0040806 & 0.01198237 & 0.0086278 & $1.20 \mathrm{E}-05$ & $1.30 \mathrm{E}-05$ & $4.29 \mathrm{E}-05$ & 0.00680577 & 0.08586181 & 0.01281447 & 4509519.65 \\
\hline 57196342.1 & 786.481015 & 813.772818 & 1036.69559 & 769.319125 & $4.66 \mathrm{E}-06$ & $2.50 \mathrm{E}-05$ & 72.0229744 & 0.01202225 & 0.00865878 & $1.20 \mathrm{E}-05$ & $1.30 \mathrm{E}-05$ & $4.31 E-05$ & 0.00681674 & 0.08614757 & 0.01287218 & 4524867.89 \\
\hline 57406998.1 & 786.481015 & 813.780461 & 1036.6827 & 769.317973 & $4.68 \mathrm{E}-06$ & $2.50 \mathrm{E}-05$ & 72.0417433 & 0.01206213 & 0.00868977 & $1.20 \mathrm{E}-05$ & $1.30 \mathrm{E}-05$ & $4.33 E-05$ & 0.00682785 & 0.08643333 & 0.01292989 & 4540218.06 \\
\hline 57617654 & 786.481015 & 813.786755 & 1036.66981 & 769.316824 & $4.69 \mathrm{E}-06$ & $2.50 \mathrm{E}-05$ & 72.0603884 & 0.01210201 & 0.00872075 & $1.20 \mathrm{E}-05$ & $1.30 \mathrm{E}-05$ & $4.35 E-05$ & 0.00683879 & 0.08671909 & 0.01298757 & 4555567.26 \\
\hline 57828310 & \begin{tabular}{|l}
786.481015 \\
\end{tabular} & 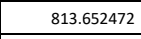 & $\begin{array}{l}1036.65716 \\
\end{array}$ & \begin{tabular}{|l|l|}
769.315024 \\
\end{tabular} & $4.71 \mathrm{E}-06$ & $2.50 \mathrm{E}-05$ & 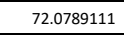 & 0.01214189 & 0.00875174 & $1.20 \mathrm{E}-05$ & $1.30 \mathrm{E}-05$ & $4.37 \mathrm{E}-05$ & 0.00684963 & 0.08700485 & 0.01304525 & 4570910.21 \\
\hline 58038966 & $\begin{array}{l}786.481015 \\
\end{array}$ & 813.518625 & 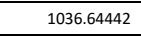 & 769.313225 & $4.73 \mathrm{E}-06$ & $2.50 \mathrm{E}-05$ & 72.0973125 & 0.01218177 & 0.00878273 & $1.20 \mathrm{E}-05$ & $1.30 \mathrm{E}-05$ & $4.38 E-05$ & 0.00686064 & 0.0872906 & 0.01310291 & 4586256.2 \\
\hline
\end{tabular}




\begin{tabular}{|c|c|c|c|c|c|c|c|c|c|c|c|c|c|c|c|c|}
\hline Time (s) & $\begin{array}{c}\text { Coolant outlet } \\
\text { temperature }(K)\end{array}$ & $\begin{array}{l}\text { Peak cladding } \\
\text { temperature (K) }\end{array}$ & $\begin{array}{c}\text { Peak fuel } \\
\text { temperature (K) }\end{array}$ & $\begin{array}{c}\text { Average cladding } \\
\text { internal } \\
\text { temperature (K) }\end{array}$ & $\begin{array}{l}\text { Peak cumulative } \\
\text { damage factor }\end{array}$ & $\begin{array}{l}\text { Cladding interior } \\
\text { volume }\left(\mathrm{m}^{3}\right)\end{array}$ & $\begin{array}{l}\text { Fission gas } \\
\text { release (\%) }\end{array}$ & $\begin{array}{c}\text { Fission gas } \\
\text { produced } \\
\text { (moles) }\end{array}$ & $\begin{array}{c}\text { Fission gas } \\
\text { released (moles) }\end{array}$ & Fuel volume $\left(\mathrm{m}^{3}\right)$ & $\begin{array}{l}\text { Plenum gas } \\
\text { volume }\left(m^{3}\right)\end{array}$ & $\begin{array}{l}\text { Peak cladding } \\
\text { radial growth } \\
\text { (m) }\end{array}$ & $\begin{array}{l}\text { Peak fuel axial } \\
\text { growth }(m)\end{array}$ & $\begin{array}{c}\text { Peak burnup } \\
\text { (at\%) }\end{array}$ & $\begin{array}{l}\text { Peak cladding } \\
\text { hoop strain }\end{array}$ & $\begin{array}{c}\text { Plenum pressure } \\
(\mathrm{Pa})\end{array}$ \\
\hline 58249622 & 786.481015 & 813.390716 & 1036.63166 & $\begin{array}{l}769.311453 \\
\end{array}$ & $4.74 \mathrm{E}-06$ & $2.51 \mathrm{E}-05$ & 72.1155939 & 0.01222165 & 0.00881371 & $1.20 \mathrm{E}-05$ & $1.30 \mathrm{E}-05$ & $4.40 \mathrm{E}-05$ & 0.00687175 & 0.08757636 & 0.01316056 & 4601604.72 \\
\hline 58460278 & 786.481015 & 813.271343 & 1036.61891 & 769.309711 & $4.76 \mathrm{E}-06$ & $2.51 \mathrm{E}-05$ & 72.1337563 & 0.01226152 & 0.0088447 & $1.20 \mathrm{E}-05$ & $1.30 \mathrm{E}-05$ & $4.42 E-05$ & 0.00688276 & 0.08786212 & 0.01321819 & 4616952.24 \\
\hline 58670934 & 786.481015 & 813.162807 & 1036.60635 & 769.308027 & \begin{tabular}{ll|l|l}
$4.77 E-06$ & \\
\end{tabular} & $2.51 \mathrm{E}-05$ & 72.151801 & 0.0123014 & 0.00887568 & $1.20 \mathrm{E}-05$ & $1.30 \mathrm{E}-05$ & $4.44 E-05$ & 0.00689356 & 0.08814788 & 0.01327581 & 4632297.21 \\
\hline 58881590 & 786.481015 & 813.054475 & 1036.59376 & 769.306342 & $4.79 \mathrm{E}-06$ & $2.51 \mathrm{E}-05$ & 72.169729 & 0.01234128 & 0.00890667 & $1.20 \mathrm{E}-05$ & $1.30 \mathrm{E}-05$ & $4.46 E-05$ & 0.00690458 & 0.08843364 & 0.01333341 & 4647645.88 \\
\hline 59092245.9 & 786.481015 & 812.952366 & 1036.58121 & \begin{tabular}{|l|l|}
769.304691 \\
\end{tabular} & \begin{tabular}{ll|l|}
$4.80 \mathrm{E}-06$ \\
\end{tabular} & $2.51 \mathrm{E}-05$ & 72.1875416 & 0.01238116 & 0.00893765 & $1.20 \mathrm{E}-05$ & $1.30 \mathrm{E}-05$ & $4.47 E-05$ & 0.00691566 & 0.08871939 & 0.01339099 & 4662995.71 \\
\hline 59302901.9 & 786.481015 & 812.859931 & 1036.56879 & $\begin{array}{l}769.30309 \\
\end{array}$ & $4.82 E-06$ & $2.51 E-05$ & 72.2052398 & 0.01242104 & 0.00896864 & $1.20 \mathrm{E}-05$ & $1.30 \mathrm{E}-05$ & $4.49 E-05$ & 0.00692641 & 0.08900515 & 0.01344855 & 4678341.52 \\
\hline 59513557.9 & 786.481015 & 812.7699 & 1036.55646 & $\begin{array}{l}769.301498 \\
\end{array}$ & $4.83 \mathrm{E}-06$ & $2.51 \mathrm{E}-05$ & 72.2228247 & 0.01246092 & 0.00899963 & $1.20 \mathrm{E}-05$ & $1.30 \mathrm{E}-05$ & $4.51 \mathrm{E}-05$ & 0.00693736 & 0.08929091 & 0.01350609 & 4693690.4 \\
\hline 59724213.9 & 786.481015 & 812.684726 & 1036.54419 & 769.299931 & $4.85 \mathrm{E}-06$ & $2.51 \mathrm{E}-05$ & 72.2402974 & 0.0125008 & 0.00903061 & $1.20 \mathrm{E}-05$ & $1.30 \mathrm{E}-05$ & $4.53 \mathrm{E}-05$ & 0.00694834 & 0.08957667 & 0.01356361 & 4709040.18 \\
\hline 59934869.9 & 786.481015 & 812.606919 & 1036.53206 & \begin{tabular}{|l|l|}
769.298411 \\
\end{tabular} & $4.87 \mathrm{E}-06$ & $2.51 \mathrm{E}-05$ & 72.2576589 & 0.01254067 & 0.0090616 & $1.20 \mathrm{E}-05$ & $1.30 \mathrm{E}-05$ & $4.55 \mathrm{E}-05$ & 0.00695905 & 0.08986243 & 0.01362111 & 4724386.34 \\
\hline 60145525.9 & 786.481015 & 812.530421 & 1036.51999 & 769.296889 & $4.88 \mathrm{E}-06$ & $2.51 \mathrm{E}-05$ & 72.2749105 & 0.01258055 & 0.00909258 & $1.20 \mathrm{E}-05$ & $1.30 \mathrm{E}-05$ & $4.56 \mathrm{E}-05$ & 0.00696999 & 0.09014818 & 0.01367859 & 4739736.44 \\
\hline 60356181.8 & 786.481015 & 812.458521 & 1036.50795 & 769.295393 & $4.90 \mathrm{E}-06$ & $2.51 \mathrm{E}-05$ & 72.2920529 & 0.01262043 & 0.00912357 & $1.20 \mathrm{E}-05$ & $1.30 \mathrm{E}-05$ & $4.58 \mathrm{E}-05$ & 0.00698094 & 0.09043394 & 0.01373604 & 4755087.06 \\
\hline 60566837.8 & 786.481015 & 812.393345 & 1036.49603 & 769.293939 & $4.91 \mathrm{E}-06$ & $2.51 E-05$ & $\begin{array}{l}72.3090874 \\
\end{array}$ & 0.01266031 & 0.00915456 & $1.20 \mathrm{E}-05$ & $1.30 \mathrm{E}-05$ & $4.60 \mathrm{E}-05$ & 0.00699162 & 0.0907197 & 0.01379348 & 4770434.44 \\
\hline 60777493.8 & 786.481015 & 812.329001 & 1036.48417 & 769.292485 & $4.93 \mathrm{E}-06$ & $2.51 \mathrm{E}-05$ & 72.326015 & 0.01270019 & 0.00918554 & $1.20 \mathrm{E}-05$ & $1.30 \mathrm{E}-05$ & 4.62E-05 & 0.00700253 & 0.09100546 & 0.01385089 & 4785785.51 \\
\hline 60988149.8 & 786.481015 & 812.269431 & 1036.47235 & 769.291057 & $4.95 E-06$ & $2.51 E-05$ & 72.3428365 & 0.01274007 & 0.00921653 & $1.21 E-05$ & $1.30 \mathrm{E}-05$ & $4.64 E-05$ & 0.00701335 & 0.09129122 & 0.01390827 & 4801135.79 \\
\hline 61198805.8 & 786.481015 & 812.21425 & 1036.46063 & 769.289655 & $4.96 \mathrm{E}-06$ & $2.51 E-05$ & 72.3595531 & 0.01277995 & $\begin{array}{l}0.00924751 \\
\end{array}$ & $1.21 \mathrm{E}-05$ & $1.30 \mathrm{E}-05$ & $4.66 E-05$ & 0.00702405 & 0.09157697 & 0.01396563 & 4816484.72 \\
\hline 61409461.8 & 786.481015 & 812.161026 & 1036.44896 & 769.288263 & $4.98 \mathrm{E}-06$ & $2.51 \mathrm{E}-05$ & 72.3761656 & 0.01281983 & 0.0092785 & $1.21 \mathrm{E}-05$ & $1.30 \mathrm{E}-05$ & $4.67 E-05$ & 0.00703492 & 0.09186273 & 0.01402297 & 4831836.7 \\
\hline 61620117.8 & 786.481015 & 812.113459 & 1036.43742 & 769.286907 & $5.00 \mathrm{E}-06$ & $2.51 \mathrm{E}-05$ & 72.3926752 & 0.0128597 & 0.00930948 & $1.21 \mathrm{E}-05$ & $1.30 \mathrm{E}-05$ & 4.69E-05 & 0.00704555 & 0.09214849 & 0.01408028 & 4847185.55 \\
\hline 61830773.7 & 786.481015 & 812.068122 & 1036.42596 & 769.285558 & $5.01 \mathrm{E}-06$ & $2.51 \mathrm{E}-05$ & 72.4090826 & 0.01289958 & 0.00934047 & $1.21 \mathrm{E}-05$ & $1.30 \mathrm{E}-05$ & $4.71 E-05$ & 0.00705625 & 0.09243425 & 0.01413757 & 4862535.63 \\
\hline 62041429.7 & 786.481015 & 812.024806 & 1036.41453 & 769.284217 & $5.03 E-06$ & $2.51 E-05$ & 72.4253889 & 0.01293946 & 0.00937146 & $1.21 \mathrm{E}-05$ & $1.30 \mathrm{E}-05$ & $4.73 E-05$ & 0.00706713 & 0.09272001 & 0.01419483 & 4877889.18 \\
\hline 62125235 & 786.884213 & 812.417844 & 1036.63573 & 769.593918 & $5.04 E-06$ & $2.51 \mathrm{E}-05$ & 72.4318482 & 0.01295533 & 0.00938378 & $1.21 E-05$ & $1.30 \mathrm{E}-05$ & $4.74 E-05$ & 0.00707229 & 0.09283369 & 0.01421918 & 4895073.85 \\
\hline 62126178.7 & 785.161856 & 810.386314 & 1032.42919 & 768.079596 & $5.04 \mathrm{E}-06$ & $2.51 \mathrm{E}-05$ & 72.4319204 & 0.0129555 & 0.00938392 & $1.21 \mathrm{E}-05$ & $1.30 \mathrm{E}-05$ & $4.72 E-05$ & 0.00706208 & 0.09283496 & 0.01418016 & 4891722.7 \\
\hline 62127122.4 & 783.439819 & 808.356997 & 1028.21651 & 766.565589 & $5.04 \mathrm{E}-06$ & $2.51 \mathrm{E}-05$ & 72.4319917 & 0.01295568 & 0.00938406 & $1.21 \mathrm{E}-05$ & $1.30 \mathrm{E}-05$ & $4.71 E-05$ & 0.00705241 & 0.09283622 & 0.01414022 & 4882351.1. \\
\hline 62128302.1 & 781.287727 & 805.822322 & 1022.94176 & 764.673574 & $5.04 \mathrm{E}-06$ & $2.51 \mathrm{E}-05$ & 72.4320797 & 0.0129559 & 0.00938423 & $1.21 E-05$ & $1.30 \mathrm{E}-05$ & 4.69E-05 & 0.00704065 & 0.09283777 & 0.01408764 & 4870636.6 \\
\hline 62128835 & 780.31567 & 804.678042 & 1020.55561 & 763.819031 & $5.04 \mathrm{E}-06$ & $2.51 \mathrm{E}-05$ & $\begin{aligned} 72.432119 \\
\end{aligned}$ & 0.01295599 & 0.0093843 & $1.21 \mathrm{E}-05$ & $1.30 \mathrm{E}-05$ & $4.69 \mathrm{E}-05$ & 0.00703535 & 0.09283846 & 0.01406385 & 4865388.16 \\
\hline 62130014.6 & 780.31567 & 804.67626 & 1020.55524 & 763.818943 & 5.04E-06 & $2.51 \mathrm{E}-05$ & 72.4322056 & 0.01295621 & 0.00938447 & $1.21 \mathrm{E}-05$ & $1.30 \mathrm{E}-05$ & $4.69 E-05$ & 0.00703699 & 0.09283999 & 0.01406095 & 4865423.1 \\
\hline 62131489.2 & 780.31567 & 804.674729 & 1020.55506 & 763.818911 & $5.04 E-06$ & $2.51 \mathrm{E}-05$ & 72.4323139 & 0.01295647 & 0.00938467 & $1.21 E-05$ & $1.30 \mathrm{E}-05$ & $4.68 E-05$ & 0.00703821 & 0.0928419 & 0.01405878 & 4865506.14 \\
\hline 62133332.4 & 780.31567 & 804.673252 & 1020.55491 & 763.818881 & $5.04 \mathrm{E}-06$ & $2.51 \mathrm{E}-05$ & 72.4324493 & 0.01295681 & 0.00938493 & $1.21 \mathrm{E}-05$ & $1.30 \mathrm{E}-05$ & $4.68 \mathrm{E}-05$ & 0.00703919 & 0.09284428 & 0.0140573 & 4865609.08 \\
\hline 62135636.4 & 780.31567 & \begin{tabular}{|l}
804.671811 \\
\end{tabular} & $\begin{array}{l}1020.55479 \\
\end{array}$ & 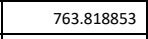 & $5.04 E-06$ & $2.51 \mathrm{E}-05$ & 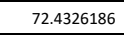 & 0.01295722 & 0.00938526 & $1.21 E-05$ & $1.30 \mathrm{E}-05$ & $4.68 \mathrm{E}-05$ & 0.00704001 & 0.09284727 & 0.01405636 & 4865738.23 \\
\hline 62138516.4 & 780.31567 & 804.670189 & 1020.55467 & 763.818824 & $5.04 \mathrm{E}-06$ & $2.51 \mathrm{E}-05$ & 72.4328301 & 0.01295774 & 0.00938566 & $1.21 E-05$ & $1.30 \mathrm{E}-05$ & $4.68 E-05$ & 0.00704068 & 0.092851 & 0.01405596 & 4865900.24 \\
\hline 62142116.4 & 780.31567 & 804.668655 & 1020.55454 & 763.818794 & $5.04 E-06$ & $2.51 \mathrm{E}-05$ & 72.4330945 & 0.01295839 & 0.00938616 & $1.21 E-05$ & $1.30 \mathrm{E}-05$ & $4.68 E-05$ & 0.00704127 & 0.09285566 & 0.01405611 & 4866101.92 \\
\hline 62146616.4 & 780.31567 & 804.666787 & 1020.55437 & 763.81876 & $5.04 \mathrm{E}-06$ & $2.51 \mathrm{E}-05$ & 72.4334249 & 0.01295921 & 0.0093868 & $1.21 E-05$ & $1.30 \mathrm{E}-05$ & $4.68 \mathrm{E}-05$ & 0.00704183 & 0.09286148 & 0.01405676 & 4866354.08 \\
\hline 62152241.4 & 780.31567 & 804.664762 & 1020.55414 & 763.81872 & $5.04 \mathrm{E}-06$ & $2.51 \mathrm{E}-05$ & 72.4338379 & 0.01296022 & 0.00938759 & $1.21 \mathrm{E}-05$ & $1.30 \mathrm{E}-05$ & $4.68 \mathrm{E}-05$ & 0.00704241 & 0.09286877 & 0.0140579 & 4866668.7 \\
\hline 62159272.7 & 780.31567 & 804.662084 & 1020.55383 & 763.818671 & $5.04 \mathrm{E}-06$ & $2.51 E-05$ & 72.4343541 & 0.01296149 & 0.00938857 & $1.21 \mathrm{E}-05$ & $1.30 \mathrm{E}-05$ & $4.68 E-05$ & 0.00704306 & 0.09287787 & 0.0140595 & 4867062.26 \\
\hline 62168061.7 & 780.31567 & 804.658782 & $\begin{array}{l}1020.55339 \\
\end{array}$ & 763.818609 & $5.04 \mathrm{E}-06$ & $2.51 \mathrm{E}-05$ & $\begin{array}{l}72.4349991 \\
\end{array}$ & 0.01296308 & 0.00938981 & $1.21 \mathrm{E}-05$ & $1.30 \mathrm{E}-05$ & $4.68 E-05$ & 0.00704383 & 0.09288925 & 0.01406161 & 4867554.56 \\
\hline 62179048 & 780.31567 & 804.655274 & 1020.55278 & 763.818538 & $5.04 \mathrm{E}-06$ & $2.51 E-05$ & 72.4358052 & 0.01296507 & 0.00939135 & $1.21 \mathrm{E}-05$ & $1.30 \mathrm{E}-05$ & $4.69 \mathrm{E}-05$ & 0.00704476 & 0.09290347 & 0.01406427 & 4868167.64 \\
\hline 62192781 & 780.31567 & 804.650422 & 1020.55183 & 763.818444 & $5.04 E-06$ & $2.51 E-05$ & 72.4368125 & 0.01296755 & 0.00939328 & $1.21 E-05$ & $1.30 \mathrm{E}-05$ & $4.69 E-05$ & 0.00704587 & 0.09292125 & 0.01406761 & 4868936.16 \\
\hline 62209947.1 & 780.31567 & 804.645439 & 1020.55044 & 763.818336 & $5.04 E-06$ & $2.51 \mathrm{E}-05$ & 72.438071 & 0.01297065 & 0.00939569 & $1.21 E-05$ & $1.30 \mathrm{E}-05$ & $4.69 E-05$ & 0.00704721 & 0.09294348 & 0.01407177 & 4869893.74 \\
\hline 62231404.8 & 780.31567 & 804.639535 & 1020.54826 & 763.818203 & $5.04 \mathrm{E}-06$ & $2.51 \mathrm{E}-05$ & 72.4396433 & 0.01297452 & 0.0093987 & $1.21 \mathrm{E}-05$ & $1.30 \mathrm{E}-05$ & $4.69 E-05$ & 0.00704876 & 0.09297126 & 0.01407698 & 4871090.43 \\
\hline 62256058 & 780.752132 & 805.076382 & 1020.79017 & 764.153811 & \begin{tabular}{|l|l|}
$5.04 E-06$ \\
\end{tabular} & $2.51 E-05$ & 72.4414486 & 0.01297898 & 0.00940216 & $1.21 \mathrm{E}-05$ & $1.30 \mathrm{E}-05$ & $4.69 \mathrm{E}-05$ & 0.00705131 & 0.09300317 & $\begin{array}{l}0.01408457 \\
\end{array}$ & 4874649.16 \\
\hline 62259658 & 784.014229 & 808.913878 & 1028.76769 & 767.020437 & $5.04 \mathrm{E}-06$ & $2.51 \mathrm{E}-05$ & 72.4417152 & 0.01297964 & 0.00940267 & $1.21 E-05$ & $1.30 \mathrm{E}-05$ & $4.72 E-05$ & 0.0070702 & 0.09300789 & 0.01416321 & 4894256.69 \\
\hline 62284311.2 & 784.014229 & 808.906934 & 1028.76514 & 767.020322 & $5.04 \mathrm{E}-06$ & $2.51 \mathrm{E}-05$ & 72.4435618 & 0.0129842 & 0.00940621 & $1.21 \mathrm{E}-05$ & $1.30 \mathrm{E}-05$ & $4.72 E-05$ & 0.00707045 & 0.09304056 & $\begin{array}{l}0.01417449 \\
\end{array}$ & 4894522.51 \\
\hline 62315127.8 & 784.014229 & 808.898979 & 1028.76202 & 767.020122 & 5.04E-06 & $2.51 \mathrm{E}-05$ & 72.4458681 & 0.0129899 & 0.00941064 & $1.21 \mathrm{E}-05$ & $1.30 \mathrm{E}-05$ & $4.72 E-05$ & 0.00707247 & 0.0930814 & 0.01418313 & 4896284.84 \\
\hline 62353648.4 & 784.014229 & 808.889388 & 1028.75839 & 767.019873 & $5.05 \mathrm{E}-06$ & $2.51 \mathrm{E}-05$ & 72.4487482 & 0.01299702 & 0.00941618 & $1.21 E-05$ & $1.30 \mathrm{E}-05$ & $4.73 E-05$ & 0.00707507 & 0.09313246 & 0.01419314 & 4898490.29 \\
\hline 62401799.3 & 784.014229 & 808.879541 & 1028.75417 & 767.01958 & $5.05 E-06$ & $2.51 \mathrm{E}-05$ & 72.4523439 & 0.01300593 & 0.0094231 & $1.21 \mathrm{E}-05$ & $1.30 \mathrm{E}-05$ & $4.73 E-05$ & 0.00707802 & 0.09319627 & 0.01420555 & 4901240.87 \\
\hline 62461987.8 & 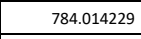 & 808.87012 & 1028.74922 & 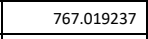 & $5.05 E-06$ & $2.51 \mathrm{E}-05$ & 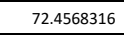 & 0.01301706 & 0.00943175 & $1.21 E-05$ & $1.30 \mathrm{E}-05$ & $4.74 E-05$ & 0.00708116 & 0.09327604 & 0.01422107 & 4904671.35 \\
\hline 62537223.5 & $\begin{array}{l}784.014229 \\
\end{array}$ & 808.860884 & 1028.74354 & 767.018831 & $5.06 \mathrm{E}-06$ & $2.51 \mathrm{E}-05$ & 72.4624304 & 0.01303097 & 0.00944256 & $1.21 E-05$ & $1.30 \mathrm{E}-05$ & $4.74 E-05$ & 0.00708461 & 0.09337575 & 0.01424047 & 4908951.53 \\
\hline
\end{tabular}




\begin{tabular}{|c|c|c|c|c|c|c|c|c|c|c|c|c|c|c|c|c|}
\hline Time (s) & $\begin{array}{c}\text { Coolant outlet } \\
\text { temperature }(K)\end{array}$ & $\begin{array}{l}\text { Peak cladding } \\
\text { temperature (K) }\end{array}$ & $\begin{array}{c}\text { Peak fuel } \\
\text { temperature (K) }\end{array}$ & $\begin{array}{c}\text { Average cladding } \\
\text { internal } \\
\text { temperature (K) }\end{array}$ & $\begin{array}{l}\text { Peak cumulative } \\
\text { damage factor }\end{array}$ & $\begin{array}{l}\text { Cladding interior } \\
\text { volume }\left(\mathrm{m}^{3}\right)\end{array}$ & $\begin{array}{l}\text { Fission gas } \\
\text { release (\%) }\end{array}$ & $\begin{array}{c}\text { Fission gas } \\
\text { produced } \\
\text { (moles) }\end{array}$ & $\begin{array}{c}\text { Fission gas } \\
\text { released (moles) }\end{array}$ & Fuel volume $\left(\mathrm{m}^{3}\right)$ & $\begin{array}{l}\text { Plenum gas } \\
\text { volume }\left(m^{3}\right)\end{array}$ & $\begin{array}{l}\text { Peak cladding } \\
\text { radial growth } \\
\text { (m) }\end{array}$ & $\begin{array}{l}\text { Peak fuel axial } \\
\text { growth }(m)\end{array}$ & $\begin{array}{c}\text { Peak burnup } \\
\text { (at\%) }\end{array}$ & $\begin{array}{l}\text { Peak cladding } \\
\text { hoop strain }\end{array}$ & $\begin{array}{c}\text { Plenum pressure } \\
(\mathrm{Pa})\end{array}$ \\
\hline 62620357 & 783.521737 & 808.353148 & 1028.46193 & $\begin{array}{l}766.639505 \\
\end{array}$ & $5.06 \mathrm{E}-06$ & $2.51 \mathrm{E}-05$ & 72.468603 & 0.01304635 & 0.00945451 & $1.21 \mathrm{E}-05$ & $1.30 \mathrm{E}-05$ & 4.75E- 05 & 0.00708699 & 0.09348592 & 0.01426009 & 4911947.75 \\
\hline 62623957 & 784.261754 & 809.226052 & 1030.27284 & 767.290103 & $5.06 \mathrm{E}-06$ & $2.51 \mathrm{E}-05$ & 72.4688707 & 0.01304702 & 0.00945503 & $1.21 \mathrm{E}-05$ & $1.30 \mathrm{E}-05$ & $4.75 E-05$ & 0.0070912 & 0.09349071 & 0.01427749 & 4921897.25 \\
\hline 62707090.5 & 784.261754 & 809.21876 & 1030.2678 & 767.289701 & 5.07E-06 & $2.51 \mathrm{E}-05$ & 72.4750605 & 0.01306247 & 0.00946704 & $1.21 E-05$ & $1.30 \mathrm{E}-05$ & $4.76 E-05$ & 0.00709402 & 0.09360146 & 0.01430159 & 4922154.46 \\
\hline 62811007.3 & 784.261754 & 809.208582 & 1030.26217 & 767.289154 & $5.07 \mathrm{E}-06$ & $2.51 \mathrm{E}-05$ & 72.4827772 & 0.01308179 & 0.00948205 & $1.21 \mathrm{E}-05$ & $1.30 \mathrm{E}-05$ & $4.77 E-05$ & 0.00709834 & 0.09373991 & 0.01432882 & 4928085.93 \\
\hline 62940903.3 & 784.261754 & 809.194558 & 1030.25597 & \begin{tabular}{|l|}
767.288454 \\
\end{tabular} & $5.08 \mathrm{E}-06$ & $2.51 \mathrm{E}-05$ & 72.4923912 & 0.01310594 & 0.00950081 & $1.21 E-05$ & $1.30 \mathrm{E}-05$ & $4.78 E-05$ & 0.00710412 & 0.09391296 & 0.01436279 & 4935507.32 \\
\hline 63103273.4 & 784.261754 & 809.176969 & 1030.24894 & 767.287573 & $5.09 \mathrm{E}-06$ & $2.51 E-05$ & 72.5043588 & 0.01313613 & 0.00952427 & $1.21 \mathrm{E}-05$ & $1.30 \mathrm{E}-05$ & $4.79 E-05$ & 0.0071116 & 0.09412928 & 0.01440526 & 4944787.08 \\
\hline 63306236 & 784.261754 & 809.155898 & 1030.24061 & $\begin{array}{l}767.28647 \\
\end{array}$ & $5.10 \mathrm{E}-06$ & $2.51 \mathrm{E}-05$ & 72.5192413 & 0.01317387 & 0.00955359 & $1.21 \mathrm{E}-05$ & $1.30 \mathrm{E}-05$ & $4.81 \mathrm{E}-05$ & 0.00712107 & 0.09439968 & 0.01445836 & 4956387.87 \\
\hline 63509198.5 & 784.261754 & 809.135533 & 1030.23236 & 767.285366 & $5.11 \mathrm{E}-06$ & $2.51 \mathrm{E}-05$ & 72.5340387 & 0.0132116 & 0.00958291 & $1.21 E-05$ & $1.30 \mathrm{E}-05$ & $4.83 E-05$ & 0.00713082 & 0.09467008 & 0.0145115 & 4970867.18 \\
\hline 63712161.1 & 784.261754 & 809.116213 & 1030.22399 & 767.28426 & $5.13 \mathrm{E}-06$ & $2.51 \mathrm{E}-05$ & 72.5487518 & 0.01324934 & 0.00961223 & $1.21 \mathrm{E}-05$ & $1.30 \mathrm{E}-05$ & $4.85 E-05$ & 0.00714078 & 0.09494048 & 0.01456465 & 4985350.35 \\
\hline 63915123.7 & 784.261754 & 809.099325 & 1030.21546 & 767.283169 & $5.14 \mathrm{E}-06$ & $2.51 \mathrm{E}-05$ & 72.5633814 & 0.01328707 & 0.00964155 & $1.21 \mathrm{E}-05$ & $1.30 \mathrm{E}-05$ & $4.86 \mathrm{E}-05$ & 0.00715052 & 0.09521088 & 0.01461781 & 4999829.96 \\
\hline 64118086.2 & 784.261754 & 809.083553 & 1030.20677 & 767.282076 & $5.15 \mathrm{E}-06$ & $2.51 \mathrm{E}-05$ & 72.5779281 & 0.01332481 & 0.00967087 & $1.21 E-05$ & $1.30 \mathrm{E}-05$ & $4.88 \mathrm{E}-05$ & 0.00716051 & 0.09548128 & 0.01467097 & 5014313.79 \\
\hline 64321048.8 & 784.261754 & 809.069363 & $\begin{array}{l}1030.1979 \\
\end{array}$ & 767.280989 & $5.16 \mathrm{E}-06$ & $2.51 E-05$ & 72.5923927 & 0.01336254 & 0.00970019 & $1.21 \mathrm{E}-05$ & $1.30 \mathrm{E}-05$ & $4.90 E-05$ & 0.00717061 & 0.09575168 & 0.01472414 & 5028799.88 \\
\hline 64516928 & 782.474049 & 807.243416 & 1029.18749 & 765.904459 & $5.17 \mathrm{E}-06$ & $2.51 \mathrm{E}-05$ & 72.6062752 & 0.01339896 & 0.00972849 & $1.21 \mathrm{E}-05$ & $1.30 \mathrm{E}-05$ & $4.91 E-05$ & 0.00717675 & 0.09601264 & 0.01476862 & 5034566.69 \\
\hline 64520528 & 781.616134 & 806.22733 & 1027.06657 & 765.149012 & $5.17 \mathrm{E}-06$ & $2.51 E-05$ & 72.6065288 & 0.01339963 & 0.00972901 & $1.21 E-05$ & $1.30 \mathrm{E}-05$ & $4.90 \mathrm{E}-05$ & 0.00717246 & 0.09601742 & 0.01474904 & 5043559.53 \\
\hline 64716407.2 & 781.616134 & 806.217645 & 1027.05775 & 765.147998 & $5.18 \mathrm{E}-06$ & $2.51 E-05$ & 72.6202501 & 0.01343582 & 0.00975713 & $1.21 \mathrm{E}-05$ & $1.30 \mathrm{E}-05$ & $4.92 E-05$ & 0.00718269 & 0.09627678 & 0.01479773 & 5043927.36 \\
\hline 64766586 & 782.095695 & 806.702907 & 1027.3242 & 765.516749 & $5.18 \mathrm{E}-06$ & $2.51 \mathrm{E}-05$ & 72.6237532 & 0.0134451 & 0.00976433 & $1.21 E-05$ & $1.30 \mathrm{E}-05$ & $4.92 E-05$ & 0.00718556 & 0.09634322 & 0.01481244 & 5060045.45 \\
\hline 64770186 & 780.46995 & 804.779263 & 1023.3114 & 764.085888 & $5.19 \mathrm{E}-06$ & $2.51 \mathrm{E}-05$ & 72.6240029 & 0.01344576 & 0.00976485 & $1.21 E-05$ & $1.30 \mathrm{E}-05$ & $4.91 E-05$ & 0.00717697 & 0.09634796 & 0.01477416 & 5054333.27 \\
\hline 64820364.8 & 780.46995 & 804.774741 & 1023.30899 & 764.085579 & $5.19 \mathrm{E}-06$ & $2.51 \mathrm{E}-05$ & 72.62746 & 0.01345492 & 0.00977197 & $1.21 \mathrm{E}-05$ & $1.30 \mathrm{E}-05$ & $4.91 E-05$ & 0.00718202 & 0.09641363 & 0.01478409 & 5054639.02 \\
\hline 64883088.3 & 780.46995 & 804.770824 & 1023.3059 & 764.085246 & $5.19 \mathrm{E}-06$ & $2.51 E-05$ & 72.6317749 & 0.01346638 & 0.00978087 & $1.21 \mathrm{E}-05$ & $1.30 \mathrm{E}-05$ & $4.92 E-05$ & 0.00718637 & 0.09649571 & 0.01479956 & 5058170.79 \\
\hline 64961492.7 & 780.46995 & 804.768312 & $\begin{array}{l}1023.3017 \\
\end{array}$ & 764.084871 & $5.19 \mathrm{E}-06$ & $2.51 \mathrm{E}-05$ & 72.6371581 & 0.0134807 & 0.00979199 & $1.21 E-05$ & $1.30 \mathrm{E}-05$ & $4.93 E-05$ & 0.00719005 & 0.09659831 & 0.01481917 & 5062559.92 \\
\hline 65059498.2 & 780.46995 & 804.766584 & 1023.29638 & 764.084431 & $5.20 \mathrm{E}-06$ & $2.51 \mathrm{E}-05$ & $\begin{array}{l}72.643871 \\
\end{array}$ & 0.01349859 & $\begin{array}{l}0.0098059 \\
\end{array}$ & $1.21 \mathrm{E}-05$ & $1.30 \mathrm{E}-05$ & $4.93 E-05$ & 0.00719351 & 0.09672656 & $\begin{array}{l}\mid 0.01484371 \\
\end{array}$ & 5068026.11 \\
\hline 65182005.1 & 780.46995 & 804.764314 & 1023.29012 & 764.083878 & $5.20 \mathrm{E}-06$ & $2.51 \mathrm{E}-05$ & 72.6522372 & 0.01352097 & 0.00982328 & $1.21 \mathrm{E}-05$ & $1.30 \mathrm{E}-05$ & $4.94 E-05$ & 0.00719807 & 0.09688688 & 0.01487442 & 5074857.68 \\
\hline 65335138.6 & 780.46995 & 804.760912 & 1023.28299 & 764.083164 & $5.21 E-06$ & $2.51 \mathrm{E}-05$ & 72.6626561 & 0.01354893 & 0.00984501 & $1.21 E-05$ & $1.30 \mathrm{E}-05$ & $4.96 E-05$ & 0.00720469 & 0.09708727 & 0.01491286 & 5083412.47 \\
\hline 65526555.6 & 780.46995 & 804.757073 & 1023.27488 & 764.082264 & $5.21 \mathrm{E}-06$ & $2.51 \mathrm{E}-05$ & 72.6756194 & 0.01358389 & 0.00987218 & $1.21 \mathrm{E}-05$ & $1.30 \mathrm{E}-05$ & $4.97 E-05$ & 0.00721343 & 0.09733776 & 0.01496098 & 5094113.41 \\
\hline 65765826.8 & 780.46995 & 804.753528 & 1023.26535 & 764.081133 & $5.22 \mathrm{E}-06$ & $2.51 \mathrm{E}-05$ & 72.6917299 & 0.01362759 & 0.00990613 & $1.21 \mathrm{E}-05$ & $1.30 \mathrm{E}-05$ & $4.99 E-05$ & 0.00722477 & 0.09765088 & 0.01502122 & 5107496.52 \\
\hline 66005098 & 780.46995 & 804.751783 & 1023.256 & 764.080007 & $5.23 \mathrm{E}-06$ & $2.51 \mathrm{E}-05$ & 72.7077375 & 0.01367128 & 0.00994008 & $1.21 E-05$ & $1.30 \mathrm{E}-05$ & $5.01 E-05$ & 0.00723617 & 0.09796399 & 0.01508158 & 5124196.28 \\
\hline 66244369.2 & 780.46995 & 804.751899 & 1023.24664 & 764.078887 & $5.24 \mathrm{E}-06$ & $2.51 \mathrm{E}-05$ & 72.7236431 & 0.01371498 & 0.00997403 & $1.21 \mathrm{E}-05$ & $1.30 \mathrm{E}-05$ & $5.03 E-05$ & 0.00724762 & 0.09827711 & 0.01514202 & 5140897.33 \\
\hline 66483640.4 & 780.46995 & 804.75405 & 1023.23715 & 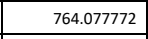 & $5.25 \mathrm{E}-06$ & $2.51 \mathrm{E}-05$ & 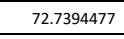 & 0.01375868 & 0.01000798 & $1.21 E-05$ & $1.30 \mathrm{E}-05$ & $5.05 E-05$ & 0.0072593 & 0.09859023 & 0.01520254 & 5157602.82 \\
\hline 66722911.7 & 780.46995 & 804.758074 & 1023.22752 & 764.076665 & $5.26 \mathrm{E}-06$ & $2.51 \mathrm{E}-05$ & 72.7551521 & 0.01380237 & 0.01004194 & $1.21 E-05$ & $1.30 \mathrm{E}-05$ & $5.07 E-05$ & 0.00727081 & 0.09890334 & 0.01526312 & 5174306.08 \\
\hline 66962182.9 & 780.46995 & 804.764156 & 1023.21777 & 764.075564 & $5.27 \mathrm{E}-06$ & $2.51 \mathrm{E}-05$ & 72.7707575 & 0.01384607 & 0.01007589 & $1.21 E-05$ & $1.30 \mathrm{E}-05$ & $5.09 E-05$ & 0.0072824 & 0.09921646 & 0.01532376 & 5191011.07 \\
\hline 67201454.1 & 780.46995 & 804.77252 & 1023.20788 & 764.074469 & $5.28 \mathrm{E}-06$ & $2.51 \mathrm{E}-05$ & 72.7862647 & 0.01388977 & 0.01010984 & $1.21 E-05$ & $1.30 \mathrm{E}-05$ & $5.11 \mathrm{E}-05$ & 0.00729416 & 0.09952957 & 0.01538445 & 5207720.02 \\
\hline 67440725.3 & 780.46995 & 804.782293 & 1023.19785 & 764.073376 & $5.29 \mathrm{E}-06$ & $2.51 \mathrm{E}-05$ & 72.8016746 & 0.01393346 & 0.01014379 & $1.21 \mathrm{E}-05$ & $1.30 \mathrm{E}-05$ & $5.13 \mathrm{E}-05$ & 0.00730601 & 0.09984269 & 0.01544519 & 5224431.21 \\
\hline 67535546 & 781.971694 & 806.310953 & 1024.03613 & 765.228283 & $5.29 \mathrm{E}-06$ & $2.51 E-05$ & 72.8077547 & 0.01395078 & 0.01015725 & $1.21 \mathrm{E}-05$ & $1.30 \mathrm{E}-05$ & $5.14 E-05$ & 0.00731352 & 0.09996677 & 0.01547503 & 5248701.48 \\
\hline 67539146 & 780.327827 & 804.364277 & $\begin{array}{l}1020.00766 \\
\end{array}$ & 763.783611 & $5.30 \mathrm{E}-06$ & $2.51 \mathrm{E}-05$ & $\begin{array}{l}72.8079839 \\
\end{array}$ & 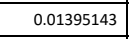 & 0.01015776 & $1.21 E-05$ & $1.30 \mathrm{E}-05$ & $5.13 \mathrm{E}-05$ & 0.00730527 & 0.09997146 & 0.0154366 & 5245610.18 \\
\hline 67633966.7 & 780.327827 & 804.373167 & 1020.00339 & 763.78314 & $5.30 \mathrm{E}-06$ & $2.51 E-05$ & 72.8139767 & 0.01396854 & 0.01017105 & $1.21 \mathrm{E}-05$ & $1.30 \mathrm{E}-05$ & $5.14 \mathrm{E}-05$ & 0.00731387 & 0.10009407 & 0.01545729 & 5245956.84 \\
\hline 67752492.6 & 780.327827 & 804.379979 & 1019.99786 & 763.78262 & $5.30 \mathrm{E}-06$ & $2.51 E-05$ & 72.8214472 & 0.01398993 & 0.01018767 & $1.21 E-05$ & $1.30 \mathrm{E}-05$ & $5.14 E-05$ & 0.00732016 & 0.10024735 & 0.01548654 & 5252527.75 \\
\hline 67871018.5 & 780.327827 & 804.383107 & 1019.99218 & 763.78212 & $5.31 E-06$ & $2.51 \mathrm{E}-05$ & 72.8288949 & 0.01401132 & 0.01020429 & $1.21 E-05$ & $1.30 \mathrm{E}-05$ & $5.15 E-05$ & 0.0073245 & 0.10040062 & 0.01551593 & 5260681.68 \\
\hline 68019175.9 & 780.327827 & 804.3884 & 1019.98517 & 763.781491 & $5.31 \mathrm{E}-06$ & $2.51 \mathrm{E}-05$ & 72.8381726 & 0.01403806 & 0.01022507 & $1.21 \mathrm{E}-05$ & $1.30 \mathrm{E}-05$ & $5.17 \mathrm{E}-05$ & 0.00733042 & 0.10059221 & 0.01555269 & 5268853.84 \\
\hline 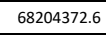 & 780.327827 & $\begin{array}{l}804.39736 \\
\end{array}$ & 1019.97683 & 763.780698 & $5.32 \mathrm{E}-06$ & $2.51 E-05$ & 72.8497201 & 0.01407148 & 0.01025104 & $1.21 \mathrm{E}-05$ & $1.30 \mathrm{E}-05$ & $5.18 \mathrm{E}-05$ & 0.00733862 & 0.1008317 & 0.01559869 & 5279082.02 \\
\hline 68435868.4 & 780.327827 & 804.411008 & 1019.966966 & 763.779708 & $5.33 \mathrm{E}-06$ & $2.51 \mathrm{E}-05$ & 72.8640775 & 0.01411326 & 0.0102835 & $1.21 E-05$ & $1.30 \mathrm{E}-05$ & $5.20 \mathrm{E}-05$ & 0.00734939 & 0.10113106 & 0.01565626 & 5291876.16 \\
\hline 68667364.3 & 780.327827 & 804.426735 & 1019.95741 & 763.778718 & $5.34 \mathrm{E}-06$ & $2.51 \mathrm{E}-05$ & 72.8783503 & 0.01415504 & 0.01031596 & $1.21 \mathrm{E}-05$ & $1.30 \mathrm{E}-05$ & $5.22 E-05$ & 0.00736037 & 0.10143042 & 0.01571393 & 5307844.31 \\
\hline 68898860.2 & 780.327827 & 804.444291 & 1019.948 & 763.777726 & $5.35 \mathrm{E}-06$ & $2.51 \mathrm{E}-05$ & 72.892539 & 0.01419681 & 0.01034842 & $1.21 \mathrm{E}-05$ & $1.30 \mathrm{E}-05$ & $5.24 E-05$ & 0.00737147 & 0.10172979 & 0.01577165 & 5323813.94 \\
\hline 69130356.1 & 780.327827 & 804.462619 & 1019.93865 & 763.776744 & $5.36 \mathrm{E}-06$ & $2.51 \mathrm{E}-05$ & 72.9066445 & 0.01423859 & 0.01038088 & $1.21 E-05$ & $1.30 \mathrm{E}-05$ & $5.26 E-05$ & 0.00738231 & 0.10202915 & 0.01582943 & 5339779.46 \\
\hline 69361852 & 780.327827 & 804.483326 & 1019.92929 & 763.775767 & $5.37 \mathrm{E}-06$ & $2.51 \mathrm{E}-05$ & 72.9206674 & 0.01428037 & 0.01041334 & $1.21 \mathrm{E}-05$ & $1.30 \mathrm{E}-05$ & $5.28 \mathrm{E}-05$ & 0.00739338 & 0.10232851 & 0.01588726 & 5355749.82 \\
\hline 69593347.9 & $\begin{array}{l}780.327827 \\
\end{array}$ & $\begin{array}{l}804.505842 \\
\end{array}$ & \begin{tabular}{|l|l|}
1019.91991 \\
\end{tabular} & \begin{tabular}{|l|l|}
763.774797 \\
\end{tabular} & $5.38 \mathrm{E}-06$ & $2.51 \mathrm{E}-05$ & $\begin{array}{l}72.9346085 \\
\end{array}$ & 0.01432215 & 0.0104458 & $1.21 E-05$ & $1.30 \mathrm{E}-05$ & $5.30 \mathrm{E}-05$ & 0.0074045 & 0.10262787 & 0.01594513 & 5371721.95 \\
\hline 69824843.7 & 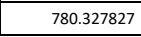 & 804.529237 & 1019.91047 & 763.773834 & $5.39 \mathrm{E}-06$ & $2.51 \mathrm{E}-05$ & 72.9484686 & 0.01436392 & 0.01047826 & $1.21 E-05$ & $1.30 \mathrm{E}-05$ & $5.31 E-05$ & 0.00741544 & 0.10292723 & 0.01600361 & 5387692.5 \\
\hline
\end{tabular}




\begin{tabular}{|c|c|c|c|c|c|c|c|c|c|c|c|c|c|c|c|c|}
\hline Time (s) & $\begin{array}{c}\text { Coolant outlet } \\
\text { temperature }(\mathrm{K})\end{array}$ & $\begin{array}{l}\text { Peak cladding } \\
\text { temperature (K) }\end{array}$ & $\begin{array}{c}\text { Peak fuel } \\
\text { temperature (K) }\end{array}$ & $\begin{array}{c}\text { Average cladding } \\
\text { internal } \\
\text { temperature (K) }\end{array}$ & $\begin{array}{l}\text { Peak cumulative } \\
\text { damage factor }\end{array}$ & $\begin{array}{l}\text { Cladding interior } \\
\text { volume }\left(\mathrm{m}^{3}\right)\end{array}$ & $\begin{array}{l}\text { Fission gas } \\
\text { release (\%) }\end{array}$ & $\begin{array}{l}\text { Fission gas } \\
\text { produced } \\
\text { (moles) }\end{array}$ & $\begin{array}{c}\text { Fission gas } \\
\text { released (moles) }\end{array}$ & Fuel volume $\left(\mathrm{m}^{3}\right)$ & $\begin{array}{l}\text { Plenum gas } \\
\text { volume }\left(\mathrm{m}^{3}\right)\end{array}$ & $\begin{array}{l}\text { Peak cladding } \\
\text { radial growth } \\
\text { (m) }\end{array}$ & $\begin{array}{l}\text { Peak fuel axial } \\
\text { growth }(m)\end{array}$ & $\begin{array}{c}\text { Peak burnup } \\
\text { (at\%) }\end{array}$ & $\begin{array}{l}\text { Peak cladding } \\
\text { hoop strain }\end{array}$ & $\begin{array}{l}\text { Plenum pressure } \\
(\mathrm{Pa})\end{array}$ \\
\hline 70056339.6 & 780.327827 & 804.555486 & 1019.90101 & $\begin{array}{l}763.772875 \\
\end{array}$ & $5.40 \mathrm{E}-06$ & $2.51 \mathrm{E}-05$ & 72.9622482 & 0.0144057 & 0.01051072 & $1.21 \mathrm{E}-05$ & $1.30 \mathrm{E}-05$ & $5.33 \mathrm{E}-05$ & 0.00742661 & 0.10322659 & 0.01606234 & 5403666.63 \\
\hline 70287835.5 & 780.327827 & 804.582516 & 1019.89151 & 763.771921 & $5.41 \mathrm{E}-06$ & $2.51 \mathrm{E}-05$ & 72.9759482 & 0.01444748 & 0.01054318 & $1.21 \mathrm{E}-05$ & $1.30 \mathrm{E}-05$ & $5.35 \mathrm{E}-05$ & 0.0074376 & 0.10352595 & 0.01612112 & 5419638.4 \\
\hline \begin{tabular}{|l|}
70519331.4 \\
\end{tabular} & \begin{tabular}{|l|l|}
780.327827 \\
\end{tabular} & 804.610326 & 1019.88197 & 763.770973 & $5.42 \mathrm{E}-06$ & $2.51 \mathrm{E}-05$ & 72.9895691 & 0.01448925 & 0.01057564 & $1.21 \mathrm{E}-05$ & $1.30 \mathrm{E}-05$ & $5.37 \mathrm{E}-05$ & 0.00744842 & 0.10382532 & 0.01617992 & 5435608.77 \\
\hline \begin{tabular}{|l|}
70750827.3 \\
\end{tabular} & 780.327827 & \begin{tabular}{|l|l|}
804.642797 \\
\end{tabular} & 1019.87243 & 763.770035 & $5.43 \mathrm{E}-06$ & $2.51 \mathrm{E}-05$ & 73.0031117 & 0.01453103 & 0.0106081 & $1.21 \mathrm{E}-05$ & $1.30 \mathrm{E}-05$ & $5.39 \mathrm{E}-05$ & 0.00745976 & 0.10412468 & 0.01623876 & 5451587.88 \\
\hline 70982323.2 & 780.327827 & 804.675874 & 1019.86283 & \begin{tabular}{ll|}
763.769104 \\
\end{tabular} & $5.44 \mathrm{E}-06$ & $2.51 \mathrm{E}-05$ & 73.0165767 & 0.01457281 & 0.01064057 & $1.21 E-05$ & $1.30 \mathrm{E}-05$ & $5.41 \mathrm{E}-05$ & 0.00747089 & 0.10442404 & 0.01629763 & 5467564.41 \\
\hline 71213819 & 780.327827 & 804.709125 & 1019.85322 & \begin{tabular}{|l|}
763.768179 \\
\end{tabular} & $5.45 \mathrm{E}-06$ & $2.51 \mathrm{E}-05$ & \begin{tabular}{|l|l|}
73.0299647 \\
\end{tabular} & 0.01461459 & 0.01067303 & $1.21 \mathrm{E}-05$ & $1.30 \mathrm{E}-05$ & $5.43 \mathrm{E}-05$ & 0.00748172 & 0.1047234 & 0.01635653 & 5483536.34 \\
\hline \begin{tabular}{|l|}
71445314.9 \\
\end{tabular} & \begin{tabular}{ll|}
780.327827 \\
\end{tabular} & $\begin{array}{l}804.745272 \\
\end{array}$ & 1019.84361 & \begin{tabular}{|l|l|}
763.767263 \\
\end{tabular} & $5.46 \mathrm{E}-06$ & $2.51 \mathrm{E}-05$ & \begin{tabular}{|l|l|}
73.0432764 \\
\end{tabular} & 0.01465636 & 0.01070549 & $1.21 \mathrm{E}-05$ & $1.30 \mathrm{E}-05$ & $5.45 \mathrm{E}-05$ & 0.00749274 & 0.10502276 & 0.01641545 & 5499512.44 \\
\hline 71676810.8 & 780.327827 & 804.783444 & 1019.834 & 763.766356 & $5.48 \mathrm{E}-06$ & $2.51 \mathrm{E}-05$ & 73.0565124 & 0.01469814 & 0.01073795 & $1.21 \mathrm{E}-05$ & $1.30 \mathrm{E}-05$ & $5.47 \mathrm{E}-05$ & 0.00750379 & 0.10532212 & 0.01647439 & 5515489.46 \\
\hline \begin{tabular}{|l|}
71908306.7 \\
\end{tabular} & \begin{tabular}{ll|}
780.327827 \\
\end{tabular} & \begin{tabular}{|l|}
804.8239 \\
\end{tabular} & 1019.82437 & \begin{tabular}{|l|l|}
763.765458 \\
\end{tabular} & $5.49 \mathrm{E}-06$ & $2.51 \mathrm{E}-05$ & 73.0696734 & 0.01473992 & 0.01077041 & $1.21 \mathrm{E}-05$ & $1.30 \mathrm{E}-05$ & $5.49 \mathrm{E}-05$ & 0.00751489 & 0.10562148 & 0.01653335 & 5531468.19 \\
\hline \begin{tabular}{|l|}
72139802.6 \\
\end{tabular} & 780.327827 & 804.863919 & 1019.81475 & 763.764561 & $5.50 \mathrm{E}-06$ & $2.51 \mathrm{E}-05$ & 73.0827599 & 0.01478169 & 0.01080287 & $1.21 \mathrm{E}-05$ & $1.30 \mathrm{E}-05$ & $5.51 E-05$ & 0.00752564 & 0.10592085 & 0.01659233 & 5547441.39 \\
\hline 72155534 & 780.327827 & 804.864591 & 1019.81408 & 763.764495 & $5.50 \mathrm{E}-06$ & $2.51 \mathrm{E}-05$ & 73.0836466 & 0.01478453 & 0.01080508 & $1.21 E-05$ & $1.30 \mathrm{E}-05$ & $5.51 \mathrm{E}-05$ & 0.00752611 & 0.10594119 & 0.01659655 & 5563295.93 \\
\hline 72159134 & \begin{tabular}{ll|}
780.327827 \\
\end{tabular} & 804.864759 & 1019.81393 & \begin{tabular}{|l|l|}
763.76448 \\
\end{tabular} & $5.50 \mathrm{E}-06$ & $2.51 \mathrm{E}-05$ & \begin{tabular}{|l|l|}
73.0838494 \\
\end{tabular} & 0.01478518 & 0.01080558 & $1.21 \mathrm{E}-05$ & $1.30 \mathrm{E}-05$ & $5.51 \mathrm{E}-05$ & 0.0075262 & 0.10594584 & 0.01659743 & 5564374.47 \\
\hline 72174865.4 & 781.864795 & 806.431747 & 1020.67627 & 764.946667 & $5.50 \mathrm{E}-06$ & $2.51 \mathrm{E}-05$ & 73.0847356 & 0.01478802 & 0.01080779 & $1.21 \mathrm{E}-05$ & $1.30 \mathrm{E}-05$ & $5.51 \mathrm{E}-05$ & 0.00752963 & 0.10596619 & 0.01660798 & 5572932.63 \\
\hline 72185697 & $\begin{array}{l}781.864795 \\
\end{array}$ & 806.433546 & 1020.67604 & 764.946629 & $5.50 \mathrm{E}-06$ & $2.51 \mathrm{E}-05$ & 73.0853456 & 0.01478998 & 0.01080931 & $1.21 \mathrm{E}-05$ & $1.30 \mathrm{E}-05$ & $5.51 \mathrm{E}-05$ & 0.00752998 & 0.10598019 & 0.01661091 & 5574016.92 \\
\hline 72189297 & 782.255999 & 806.899758 & 1021.62643 & 765.289882 & $5.50 \mathrm{E}-06$ & $2.51 \mathrm{E}-05$ & 73.0855486 & 0.01479063 & 0.01080981 & $1.21 \mathrm{E}-05$ & $1.30 \mathrm{E}-05$ & $5.51 \mathrm{E}-05$ & 0.00753201 & 0.10598486 & 0.01662137 & 5577199.39 \\
\hline 72200128.6 & 782.255999 & 806.901958 & 1021.62609 & 765.289847 & $5.50 \mathrm{E}-06$ & $2.51 \mathrm{E}-05$ & 73.0861601 & 0.01479259 & 0.01081133 & $1.21 \mathrm{E}-05$ & $1.30 \mathrm{E}-05$ & $5.52 \mathrm{E}-05$ & 0.00753225 & 0.1059989 & 0.01662481 & 5577451.25 \\
\hline 72213668 & 782.2559999 & 806.904949 & 1021.62554 & 765.289795 & $5.50 \mathrm{E}-06$ & $2.51 \mathrm{E}-05$ & 73.0869242 & 0.01479504 & 0.01081324 & $1.21 \mathrm{E}-05$ & $1.30 \mathrm{E}-05$ & $5.52 \mathrm{E}-05$ & 0.00753284 & 0.10601646 & 0.01662849 & 5578204.94 \\
\hline 72230592.3 & 782.255999 & 806.908795 & 1021.62482 & 765.28973 & $5.50 \mathrm{E}-06$ & $2.51 \mathrm{E}-05$ & \begin{tabular}{|l}
73.087879 \\
\end{tabular} & 0.0147981 & 0.01081562 & $1.21 \mathrm{E}-05$ & $1.30 \mathrm{E}-05$ & $5.52 \mathrm{E}-05$ & 0.00753372 & 0.10603841 & 0.01663291 & 5579147.06 \\
\hline 72251747.7 & 782.255999 & 806.914256 & 1021.62391 & 765.289648 & $5.50 \mathrm{E}-06$ & $2.51 \mathrm{E}-05$ & 73.0890719 & 0.01480193 & 0.01081859 & $1.21 \mathrm{E}-05$ & $1.30 \mathrm{E}-05$ & $5.52 \mathrm{E}-05$ & 0.00753494 & 0.10606584 & 0.01663838 & 5580326.47 \\
\hline 72278192 & 782.255999 & 806.921721 & 1021.62277 & 765.289547 & $5.51 \mathrm{E}-06$ & $2.51 \mathrm{E}-05$ & 73.0905622 & 0.01480671 & 0.01082231 & $1.21 \mathrm{E}-05$ & $1.30 \mathrm{E}-05$ & $5.52 E-05$ & 0.00753652 & 0.10610014 & 0.01664521 & 5581802.47 \\
\hline 72311247.3 & 782.255999 & 806.931068 & 1021.62135 & 765.289422 & $5.51 \mathrm{E}-06$ & $2.51 \mathrm{E}-05$ & 73.0924238 & 0.0148127 & 0.01082696 & $1.21 \mathrm{E}-05$ & $1.30 \mathrm{E}-05$ & $5.53 \mathrm{E}-05$ & 0.00753848 & 0.106143 & 0.01665374 & 5583647.64 \\
\hline 72352566.5 & 782.255999 & 806.941115 & 1021.61958 & 765.28926 & $5.51 \mathrm{E}-06$ & $2.51 \mathrm{E}-05$ & $\begin{array}{l}73.0947486 \\
\end{array}$ & 0.01482017 & 0.01083277 & $1.21 \mathrm{E}-05$ & $1.30 \mathrm{E}-05$ & $5.53 \mathrm{E}-05$ & 0.00754084 & 0.10619658 & 0.0166644 & 5585951.52 \\
\hline 72404215.4 & 782.255999 & 806.930896 & 1021.61738 & 765.288959 & $5.51 \mathrm{E}-06$ & $2.51 \mathrm{E}-05$ & 73.0976513 & 0.01482952 & 0.01084003 & $1.21 \mathrm{E}-05$ & $1.30 \mathrm{E}-05$ & $5.53 \mathrm{E}-05$ & 0.00754355 & 0.10626356 & 0.01667772 & 5588827.2 \\
\hline 72468776.5 & 782.255999 & 806.920334 & 1021.61463 & 765.288598 & $5.52 \mathrm{E}-06$ & $2.51 \mathrm{E}-05$ & 73.1012745 & 0.01484121 & 0.01084911 & $1.21 \mathrm{E}-05$ & $1.30 \mathrm{E}-05$ & $5.54 \mathrm{E}-05$ & 0.00754671 & 0.10634729 & 0.01669437 & 5592416.6 \\
\hline 72533337.7 & 782.255999 & 806.910439 & 1021.61188 & 765.288242 & $5.52 \mathrm{E}-06$ & $2.51 \mathrm{E}-05$ & 73.1048921 & 0.01485289 & 0.01085819 & $1.21 \mathrm{E}-05$ & $1.30 \mathrm{E}-05$ & $5.54 \mathrm{E}-05$ & 0.00754971 & 0.10643101 & 0.01671102 & 5596893.87 \\
\hline 72614039.2 & 782.255999 & 806.896015 & 1021.60844 & 765.287778 & $5.53 \mathrm{E}-06$ & $2.51 \mathrm{E}-05$ & 73.109406 & 0.01486749 & 0.01086954 & $1.21 \mathrm{E}-05$ & $1.30 \mathrm{E}-05$ & $5.55 \mathrm{E}-05$ & 0.00755385 & 0.10653566 & 0.01673183 & 5601386.74 \\
\hline 72694740.6 & 782.255999 & 806.883955 & 1021.60499 & 765.287334 & $5.53 \mathrm{E}-06$ & $2.51 \mathrm{E}-05$ & 73.1139111 & 0.0148821 & 0.01088088 & $1.21 \mathrm{E}-05$ & $1.30 \mathrm{E}-05$ & $5.56 \mathrm{E}-05$ & 0.00755773 & 0.10664032 & 0.01675264 & 5606984.4 \\
\hline 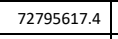 & \begin{tabular}{|l}
782.255999 \\
\end{tabular} & 806.868653 & 1021.60066 & 765.286772 & $5.54 \mathrm{E}-06$ & $2.51 E-05$ & 73.11953 & 0.01490035 & 0.01089507 & $1.21 \mathrm{E}-05$ & $1.30 \mathrm{E}-05$ & $5.57 \mathrm{E}-05$ & 0.00756273 & 0.10677113 & 0.01677863 & 5612596.11 \\
\hline 72921713.4 & 782.255999 & 806.852607 & 1021.59524 & 765.286094 & $5.55 \mathrm{E}-06$ & $2.51 \mathrm{E}-05$ & 73.1265343 & 0.01492318 & 0.0109128 & $1.21 \mathrm{E}-05$ & $1.30 \mathrm{E}-05$ & $5.58 \mathrm{E}-05$ & 0.00756853 & 0.10693465 & 0.01681118 & 5619603.29 \\
\hline 73079333.5 & 782.255999 & 806.832293 & 1021.58844 & 765.285237 & $5.56 \mathrm{E}-06$ & $2.51 \mathrm{E}-05$ & 73.1352596 & 0.0149517 & 0.01093496 & $1.21 \mathrm{E}-05$ & $1.30 \mathrm{E}-05$ & $5.59 \mathrm{E}-05$ & 0.00757612 & 0.10713906 & 0.01685209 & 5628368.71 \\
\hline 73236953.5 & 782.255999 & 806.814853 & 1021.58165 & 765.2844 & $5.57 \mathrm{E}-06$ & $2.51 \mathrm{E}-05$ & 73.1439517 & 0.01498023 & 0.01095713 & $1.21 \mathrm{E}-05$ & $1.30 \mathrm{E}-05$ & $5.60 \mathrm{E}-05$ & 0.0075834 & 0.10734346 & 0.016893 & 5639299.52 \\
\hline 73394573.5 & 782.255999 & 806.799474 & $\begin{array}{l}021.57488 \\
\end{array}$ & 765.283573 & $5.58 \mathrm{E}-06$ & $2.51 \mathrm{E}-05$ & 73.1526108 & 0.01500875 & 0.01097929 & $1.21 \mathrm{E}-05$ & $1.30 \mathrm{E}-05$ & $5.62 \mathrm{E}-05$ & 0.00759063 & 0.10754786 & 0.01693391 & 5650228.53 \\
\hline 73552193.5 & 782.255999 & 806.783929 & 1021.56814 & 765.282739 & $5.59 \mathrm{E}-06$ & $2.51 \mathrm{E}-05$ & 73.161237 & 0.01503728 & 0.01100146 & $1.21 \mathrm{E}-05$ & $1.30 \mathrm{E}-05$ & $5.63 \mathrm{E}-05$ & 0.0075983 & 0.10775226 & 0.01697481 & 5661165.02 \\
\hline 73749218.5 & 782.255999 & 806.743259 & 1021.55977 & 765.281621 & $5.60 \mathrm{E}-06$ & $2.51 \mathrm{E}-05$ & 73.1719738 & 0.01507293 & $\begin{array}{l}0.01102916 \\
\end{array}$ & $1.21 \mathrm{E}-05$ & $1.30 \mathrm{E}-05$ & $5.65 \mathrm{E}-05$ & 0.00760733 & 0.10800776 & 0.0170259 & 5672116.29 \\
\hline 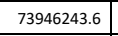 & 782.255999 & 806.70363 & $\begin{array}{l}1021.55146 \\
\end{array}$ & 765.280509 & $5.61 \mathrm{E}-06$ & $2.51 \mathrm{E}-05$ & 73.18266 & 0.01510859 & 0.01105687 & $1.21 \mathrm{E}-05$ & $1.30 \mathrm{E}-05$ & $5.66 \mathrm{E}-05$ & 0.00761627 & 0.10826326 & $\begin{array}{l}0.017077 \\
\end{array}$ & 5685777.55 \\
\hline 74143268.6 & 782.255999 & 806.663759 & 1021.54321 & 765.279392 & $5.63 \mathrm{E}-06$ & $2.51 \mathrm{E}-05$ & 73.1932959 & 0.01514424 & 0.01108457 & $1.21 \mathrm{E}-05$ & $1.30 \mathrm{E}-05$ & $5.68 \mathrm{E}-05$ & 0.00762529 & 0.10851877 & 0.0171281 & 5699439.67 \\
\hline 74340293.6 & 782.255999 & 806.62145 & 1021.53503 & 765.278255 & $5.64 \mathrm{E}-06$ & $2.51 \mathrm{E}-05$ & $\begin{array}{l}73.2038817 \\
\end{array}$ & 0.0151799 & 0.01111228 & $1.21 \mathrm{E}-05$ & $1.30 \mathrm{E}-05$ & $5.69 \mathrm{E}-05$ & 0.00763468 & 0.10877427 & 0.01717918 & 5713107.95 \\
\hline 74537318.6 & 782.255999 & 806.582273 & 1021.52688 & 765.277144 & $5.65 \mathrm{E}-06$ & $2.51 \mathrm{E}-05$ & \begin{tabular}{|l}
73.214418 \\
\end{tabular} & 0.01521556 & 0.01113998 & $1.21 \mathrm{E}-05$ & $1.30 \mathrm{E}-05$ & $5.71 E-05$ & 0.00764368 & 0.10902977 & 0.01723026 & 5726771.13 \\
\hline 74734343.7 & 782.255999 & 806.540442 & 1021.51877 & 765.276011 & $5.67 \mathrm{E}-06$ & $2.51 \mathrm{E}-05$ & 73.224905 & 0.01525121 & 0.01116769 & $1.21 \mathrm{E}-05$ & $1.30 \mathrm{E}-05$ & $5.73 \mathrm{E}-05$ & 0.00765312 & 0.10928527 & 0.01728132 & 5740441.02 \\
\hline 74931368.7 & 782.255999 & 806.498406 & 1021.51067 & 765.274878 & $5.68 \mathrm{E}-06$ & $2.51 \mathrm{E}-05$ & 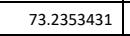 & 0.01528687 & 0.01119539 & $1.21 \mathrm{E}-05$ & $1.30 \mathrm{E}-05$ & $5.74 E-05$ & 0.00766267 & 0.10954077 & 0.01733238 & 5754110.78 \\
\hline 75128393.7 & 782.255999 & 806.460348 & 1021.50255 & 765.273771 & $5.70 \mathrm{E}-06$ & $2.51 \mathrm{E}-05$ & 73.2457326 & 0.01532253 & 0.0112231 & $1.21 \mathrm{E}-05$ & $1.30 \mathrm{E}-05$ & $5.76 \mathrm{E}-05$ & 0.00767151 & 0.10979628 & 0.01738343 & 5767776.53 \\
\hline 75325418.7 & 782.255999 & 806.423193 & 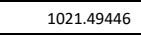 & 765.272673 & $5.71 \mathrm{E}-06$ & $2.51 \mathrm{E}-05$ & 73.2560738 & 0.01535818 & 0.0112508 & $1.21 \mathrm{E}-05$ & $1.30 \mathrm{E}-05$ & $5.78 \mathrm{E}-05$ & 0.00768052 & 0.11005178 & 0.01743448 & 5781440.08 \\
\hline 75522443.8 & 782.255999 & 806.385343 & 1021.4864 & 765.27156 & $5.72 E-06$ & $2.51 \mathrm{E}-05$ & 73.2663672 & 0.01539384 & 0.01127851 & $1.21 \mathrm{E}-05$ & $1.30 \mathrm{E}-05$ & $5.79 \mathrm{E}-05$ & 0.00768959 & 0.11030728 & 0.01748552 & 5795105.64 \\
\hline $\begin{array}{l}75719468.8 \\
\end{array}$ & 782.255999 & 806.347465 & 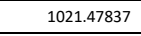 & 765.270443 & $5.74 E-06$ & $2.51 \mathrm{E}-05$ & 73.276613 & 0.0154295 & 0.01130621 & $1.21 \mathrm{E}-05$ & $1.30 \mathrm{E}-05$ & $5.81 \mathrm{E}-05$ & 0.00769871 & 0.11056278 & 0.01753655 & 5808772.57 \\
\hline \begin{tabular}{|l|l|}
7596933.8 \\
\end{tabular} & \begin{tabular}{|l}
782.255999 \\
\end{tabular} & 806.309853 & \begin{tabular}{|l|l|}
1021.47037 \\
\end{tabular} & 765.269328 & $5.75 \mathrm{E}-06$ & $2.51 E-05$ & 73.2868115 & 0.01546515 & 0.01133392 & $1.21 E-05$ & $1.30 \mathrm{E}-05$ & $5.83 \mathrm{E}-05$ & 0.00770786 & 0.11081828 & 0.01758758 & 5822440.34 \\
\hline 76113518.8 & 782.255999 & 806.272636 & 1021.46238 & 765.268216 & $5.77 \mathrm{E}-06$ & $2.51 \mathrm{E}-05$ & 73.2969631 & 0.01550081 & 0.01136162 & $1.21 \mathrm{E}-05$ & $1.30 \mathrm{E}-05$ & $5.84 \mathrm{E}-05$ & 0.00771701 & 0.11107379 & 0.0176386 & 5836108.72 \\
\hline
\end{tabular}




\begin{tabular}{|c|c|c|c|c|c|c|c|c|c|c|c|c|c|c|c|c|}
\hline Time (s) & $\begin{array}{c}\text { Coolant outlet } \\
\text { temperature (K) }\end{array}$ & $\begin{array}{l}\text { Peak cladding } \\
\text { temperature (K) }\end{array}$ & $\begin{array}{c}\text { Peak fuel } \\
\text { temperature (K) }\end{array}$ & $\begin{array}{c}\text { Average cladding } \\
\text { internal } \\
\text { temperature (K) } \\
\end{array}$ & $\begin{array}{l}\text { Peak cumulative } \\
\text { damage factor }\end{array}$ & $\begin{array}{l}\text { Cladding interior } \\
\text { volume }\left(\mathrm{m}^{3}\right)\end{array}$ & $\begin{array}{l}\text { Fission gas } \\
\text { release (\%) }\end{array}$ & $\begin{array}{c}\text { Fission gas } \\
\text { produced } \\
\text { (moles) }\end{array}$ & $\begin{array}{c}\text { Fission gas } \\
\text { released (moles) }\end{array}$ & Fuel volume $\left(\mathrm{m}^{3}\right)$ & $\begin{array}{l}\text { Plenum gas } \\
\text { volume }\left(m^{3}\right)\end{array}$ & $\begin{array}{l}\text { Peak cladding } \\
\text { radial growth } \\
(\mathrm{m})\end{array}$ & $\begin{array}{l}\text { Peak fuel axial } \\
\text { growth }(m)\end{array}$ & $\begin{array}{c}\text { Peak burnup } \\
\text { (at\%) }\end{array}$ & $\begin{array}{l}\text { Peak cladding } \\
\text { hoop strain }\end{array}$ & $\begin{array}{c}\text { Plenum pressure } \\
(\mathrm{Pa})\end{array}$ \\
\hline 76310543.9 & 782.255999 & 806.237461 & 1021.45442 & 765.26712 & $5.78 \mathrm{E}-06$ & $2.51 \mathrm{E}-05$ & 73.3070681 & 0.01553646 & 0.01138933 & $1.21 \mathrm{E}-05$ & $1.30 \mathrm{E}-05$ & $5.86 \mathrm{E}-05$ & 0.00772591 & 0.11132929 & 0.01768962 & 5849773.07 \\
\hline 76507568.9 & 782.255999 & 806.205288 & 1021.4465 & 765.266048 & $5.79 E-06$ & $2.51 \mathrm{E}-05$ & 73.3171269 & 0.01557212 & 0.01141703 & $1.21 \mathrm{E}-05$ & $1.30 \mathrm{E}-05$ & $5.87 \mathrm{E}-05$ & 0.00773444 & 0.11158479 & 0.01774063 & 5863431.28 \\
\hline 76704593.9 & 782.255999 & 806.167207 & 1021.43863 & 765.264932 & $5.81 \mathrm{E}-06$ & $2.51 \mathrm{E}-05$ & 73.3271397 & 0.01560778 & 0.01144474 & $1.21 E-05$ & $1.30 \mathrm{E}-05$ & $5.89 E-05$ & 0.00774388 & 0.11184029 & 0.01779163 & 5877104.9 \\
\hline 76833647 & 781.272965 & 805.148335 & 1020.88102 & 764.508101 & $5.82 \mathrm{E}-06$ & $2.51 \mathrm{E}-05$ & 73.3336734 & 0.01563113 & 0.01146288 & $1.21 \mathrm{E}-05$ & $1.30 \mathrm{E}-05$ & $5.90 \mathrm{E}-05$ & 0.00774857 & 0.11200765 & 0.01782022 & 5885127.04 \\
\hline 76837247 & $\begin{array}{l}782.66454 \\
\end{array}$ & 806.783956 & 1024.27665 & \begin{tabular}{ll|}
765.729843 \\
\end{tabular} & $5.82 E-06$ & $2.51 \mathrm{E}-05$ & 73.3338563 & 0.01563179 & 0.01146339 & $1.21 E-05$ & $1.30 \mathrm{E}-05$ & $5.91 E-05$ & 0.007755 & 0.11201234 & 0.01785601 & 5903192.53 \\
\hline 76966300.1 & 782.66454 & 806.758439 & 1024.2718 & $\begin{array}{l}765.729114 \\
\end{array}$ & $5.83 \mathrm{E}-06$ & $2.51 \mathrm{E}-05$ & 73.3404353 & 0.01565538 & 0.01148172 & $1.21 \mathrm{E}-05$ & $1.30 \mathrm{E}-05$ & $5.92 E-05$ & 0.00776006 & 0.11218138 & 0.0178929 & 5903515.91 \\
\hline 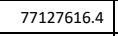 & 782.66454 & 806.722841 & 1024.26517 & 765.728143 & $5.84 \mathrm{E}-06$ & $2.51 \mathrm{E}-05$ & 73.3486312 & 0.01568486 & 0.01150463 & $1.22 \mathrm{E}-05$ & $1.30 \mathrm{E}-05$ & $5.94 \mathrm{E}-05$ & 0.00776844 & 0.11239267 & 0.01793567 & 5912599.43 \\
\hline 77288932.8 & 782.66454 & 806.690844 & 1024.25855 & 765.727197 & $5.85 \mathrm{E}-06$ & $2.51 \mathrm{E}-05$ & 73.3567964 & 0.01571435 & 0.01152755 & $1.22 \mathrm{E}-05$ & $1.30 \mathrm{E}-05$ & $5.95 E-05$ & 0.00777647 & 0.11260397 & 0.01797834 & 5923920.78 \\
\hline 77340548 & 782.66454 & 806.681003 & 1024.25641 & 765.726897 & $5.86 \mathrm{E}-06$ & $2.51 \mathrm{E}-05$ & 73.3594025 & 0.01572379 & 0.01153488 & $1.22 \mathrm{E}-05$ & $1.30 \mathrm{E}-05$ & $5.96 \mathrm{E}-05$ & 0.00777899 & 0.11267158 & 0.01799205 & 5935173.59 \\
\hline 77344148 & 782.66454 & 806.68081 & 1024.25626 & $\begin{array}{l}765.726879 \\
\end{array}$ & $5.86 \mathrm{E}-06$ & $2.51 \mathrm{E}-05$ & 73.3595842 & 0.01572444 & 0.01153539 & $1.22 \mathrm{E}-05$ & $1.30 \mathrm{E}-05$ & $5.96 \mathrm{E}-05$ & 0.0077791 & 0.1126763 & 0.0179931 & 5938764.77 \\
\hline 77395763.2 & 782.66454 & 806.671261 & 1024.25415 & 765.726583 & $5.86 \mathrm{E}-06$ & $2.51 E-05$ & 73.3621869 & 0.01573388 & 0.01154272 & $1.22 \mathrm{E}-05$ & $1.30 \mathrm{E}-05$ & $5.96 E-05$ & 0.00778157 & 0.1127439 & 0.01800665 & 5939045.45 \\
\hline \begin{tabular}{|l|l|}
77460282.3 \\
\end{tabular} & 782.66454 & 806.660921 & $\begin{array}{l}1024.25149 \\
\end{array}$ & 765.726223 & $5.87 \mathrm{E}-06$ & $2.51 \mathrm{E}-05$ & 73.365436 & 0.01574567 & 0.01155188 & $1.22 E-05$ & $1.30 \mathrm{E}-05$ & $5.97 E-05$ & 0.00778435 & 0.11282841 & 0.0180237 & 5942670.01 \\
\hline 77540931.1 & 782.66454 & 806.645825 & 1024.24818 & 765.725757 & $5.88 \mathrm{E}-06$ & $2.51 \mathrm{E}-05$ & 73.3694905 & 0.01576042 & 0.01156334 & $1.22 E-05$ & $1.30 \mathrm{E}-05$ & $5.97 E-05$ & 0.0077883 & 0.11293405 & 0.018045 & 5947206.33 \\
\hline 77621579.9 & 782.66454 & 806.630867 & 1024.24485 & 765.725292 & $5.88 \mathrm{E}-06$ & $2.51 \mathrm{E}-05$ & 73.3735374 & 0.01577516 & 0.01157479 & $1.22 E-05$ & $1.30 \mathrm{E}-05$ & $5.98 E-05$ & 0.00779214 & 0.11303968 & 0.01806631 & 5952863.41 \\
\hline 77702228.7 & 782.66454 & 806.616908 & 1024.24152 & 765.724834 & $5.89 \mathrm{E}-06$ & $2.51 \mathrm{E}-05$ & 73.3775767 & 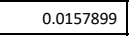 & 0.01158625 & $1.22 \mathrm{E}-05$ & $1.30 \mathrm{E}-05$ & $5.99 E-05$ & 0.00779595 & 0.11314532 & 0.01808762 & 5958520.36 \\
\hline 777738400 & 782.100518 & 806.037035 & 1023.92315 & 765.290744 & $5.89 \mathrm{E}-06$ & $2.51 \mathrm{E}-05$ & 73.3793859 & 0.01579651 & 0.01159138 & $1.22 \mathrm{E}-05$ & $1.30 \mathrm{E}-05$ & $5.99 E-05$ & 0.00779741 & 0.1131927 & 0.01809451 & 5960902.29 \\
\hline 77742000 & 782.745069 & 806.793456 & $\begin{array}{l}1025.49916 \\
\end{array}$ & 765.856887 & $5.89 \mathrm{E}-06$ & $2.51 \mathrm{E}-05$ & 73.3795663 & 0.01579717 & 0.0115919 & $1.22 \mathrm{E}-05$ & $1.30 \mathrm{E}-05$ & $5.99 E-05$ & 0.0078005 & 0.11319743 & 0.01811144 & 5967723.32 \\
\hline 77778171.3 & 782.745069 & 806.788731 & 1025.49784 & 765.856702 & $5.89 \mathrm{E}-06$ & $2.51 \mathrm{E}-05$ & 73.3813822 & 0.01580381 & 0.01159706 & $1.22 E-05$ & $1.30 \mathrm{E}-05$ & $6.00 E-05$ & 0.00780144 & 0.11324502 & 0.01812238 & 5967988.83 \\
\hline 77823385.3 & 782.745069 & 806.781541 & 1025.49597 & 765.856447 & $5.90 \mathrm{E}-06$ & $2.51 \mathrm{E}-05$ & 73.3836499 & 0.01581212 & 0.01160351 & $1.22 \mathrm{E}-05$ & $1.30 \mathrm{E}-05$ & $6.00 E-05$ & 0.00780338 & 0.11330452 & 0.01813458 & 5970540.9 \\
\hline 77879902.9 & 782.745069 & 806.771636 & 1025.49359 & 765.85612 & $5.90 \mathrm{E}-06$ & $2.51 \mathrm{E}-05$ & 73.3864813 & 0.0158225 & 0.01161157 & $1.22 E-05$ & $1.30 \mathrm{E}-05$ & $6.01 E-05$ & 0.00780609 & 0.11337889 & 0.01814967 & 5973733.9 \\
\hline 77950549.9 & 782.745069 & 806.758804 & 1025.49062 & 765.855707 & $5.91 \mathrm{E}-06$ & $2.51 \mathrm{E}-05$ & 73.3900152 & 0.01583547 & 0.01162165 & $1.22 \mathrm{E}-05$ & $1.30 \mathrm{E}-05$ & $6.01 \mathrm{E}-05$ & 0.00780942 & 0.11347186 & 0.0181685 & 5977728.21 \\
\hline \begin{tabular}{|l|l|}
7821196.9 \\
\end{tabular} & \begin{tabular}{|l}
782.745069 \\
\end{tabular} & 806.747645 & 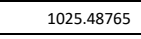 & $\begin{array}{l}765.855308 \\
\end{array}$ & $5.92 \mathrm{E}-06$ & $2.51 \mathrm{E}-05$ & $\begin{array}{l}73.3935433 \\
\end{array}$ & 0.01584844 & 0.01163173 & $1.22 E-05$ & $1.30 \mathrm{E}-05$ & $6.02 E-05$ & 0.00781267 & 0.11356482 & 0.01818733 & 5982704.22 \\
\hline 78109505.7 & 782.745069 & 806.733435 & 1025.48395 & 765.854806 & $5.92 \mathrm{E}-06$ & $2.51 \mathrm{E}-05$ & 73.3979454 & 0.01586466 & 0.01164433 & $1.22 E-05$ & $1.30 \mathrm{E}-05$ & $6.03 E-05$ & 0.00781676 & 0.11368102 & 0.01821086 & 5987692.33 \\
\hline 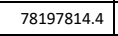 & 782.745069 & 806.717203 & 1025.48025 & 765.854288 & $5.93 \mathrm{E}-06$ & $2.51 \mathrm{E}-05$ & 73.4023385 & 0.01588088 & 0.01165693 & $1.22 E-05$ & $1.30 \mathrm{E}-05$ & $6.03 E-05$ & 0.00782117 & 0.11379723 & 0.01823438 & 5993920.11 \\
\hline 78286123.2 & 782.745069 & 806.704026 & 1025.47656 & 765.853794 & $5.94 \mathrm{E}-06$ & $2.51 \mathrm{E}-05$ & 73.4067226 & 0.01589709 & 0.01166953 & $1.22 E-05$ & $1.30 \mathrm{E}-05$ & $6.04 E-05$ & 0.00782511 & 0.11391343 & 0.0182579 & 6000140 \\
\hline 78396509.1 & 782.745069 & 806.685696 & 1025.47197 & 765.853163 & $5.95 E-06$ & $2.51 \mathrm{E}-05$ & 73.4121902 & 0.01591736 & 0.01168529 & $1.22 \mathrm{E}-05$ & $1.30 \mathrm{E}-05$ & $6.05 E-05$ & 0.00783039 & 0.11405869 & 0.01828727 & 6006377.23 \\
\hline 78506895.1 & 782.745069 & 806.667804 & 1025.46738 & 765.852534 & $5.96 \mathrm{E}-06$ & $2.51 E-05$ & 73.4176439 & 0.01593764 & 0.01170104 & $1.22 E-05$ & $1.30 \mathrm{E}-05$ & $6.06 E-05$ & 0.00783564 & 0.11420395 & 0.01831665 & 6014158.52 \\
\hline 78617281 & 782.745069 & 806.651835 & 1025.46279 & 765.85192 & $5.97 \mathrm{E}-06$ & $2.52 E-05$ & 73.4230837 & 0.01595791 & 0.01171679 & $1.22 E-05$ & $1.30 \mathrm{E}-05$ & $6.07 E-05$ & 0.00784057 & 0.1143492 & 0.01834601 & 6021934.24 \\
\hline 78755263.4 & 782.745069 & 806.632252 & 1025.45709 & 765.851157 & $5.98 \mathrm{E}-06$ & $2.52 E-05$ & 73.4298641 & 0.01598325 & 0.01173648 & $1.22 E-05$ & $1.30 \mathrm{E}-05$ & $6.08 E-05$ & 0.00784672 & 0.11453077 & 0.01838268 & 6029724.12 \\
\hline 78893245.9 & 782.745069 & 806.612891 & 1025.4514 & 765.850396 & $5.99 \mathrm{E}-06$ & $2.52 \mathrm{E}-05$ & 73.436623 & 0.01600858 & 0.01175616 & $1.22 \mathrm{E}-05$ & $1.30 \mathrm{E}-05$ & $6.09 E-05$ & 0.00785283 & 0.11471234 & 0.01841934 & 6039442.99 \\
\hline 79031228.3 & 782.745069 & 806.595105 & 1025.44575 & 765.849645 & $6.00 \mathrm{E}-06$ & $2.52 \mathrm{E}-05$ & 73.4433605 & 0.01603392 & 0.01177585 & $1.22 E-05$ & $1.30 \mathrm{E}-05$ & $6.10 \mathrm{E}-05$ & 0.00785885 & 0.11489391 & 0.0184561 & 6049159.32 \\
\hline $\begin{array}{l}79203706.3 \\
\end{array}$ & 782.745069 & 806.568365 & 1025.43873 & 765.848671 & $6.02 \mathrm{E}-06$ & $2.52 \mathrm{E}-05$ & 73.4517525 & 0.0160656 & 0.01180046 & $1.22 \mathrm{E}-05$ & $1.30 \mathrm{E}-05$ & $6.12 \mathrm{E}-05$ & 0.00786718 & 0.11512088 & 0.0185022 & 6058907.68 \\
\hline 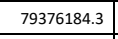 & \begin{tabular}{|l|l|}
782.745069 \\
\end{tabular} & 806.545107 & 1025.43174 & 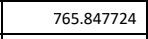 & $6.03 \mathrm{E}-06$ & $2.52 \mathrm{E}-05$ & 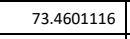 & 0.01609727 & 0.01182507 & $1.22 E-05$ & $1.30 \mathrm{E}-05$ & $6.13 \mathrm{E}-05$ & 0.007875 & 0.11534784 & 0.01854828 & 6071059.86 \\
\hline 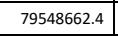 & 782.745069 & 806.521172 & 1025.42478 & 765.84677 & $6.05 \mathrm{E}-06$ & $2.52 \mathrm{E}-05$ & 73.4684377 & 0.01612894 & 0.01184968 & $1.22 E-05$ & $1.30 \mathrm{E}-05$ & $6.15 \mathrm{E}-05$ & 0.00788313 & 0.1155748 & 0.01859435 & 6083216.54 \\
\hline 79721140.4 & 782.745069 & 806.49986 & 1025.41785 & 765.845835 & $6.06 \mathrm{E}-06$ & $2.52 E-05$ & 73.4767313 & 0.01616062 & 0.01187429 & $1.22 E-05$ & $1.30 \mathrm{E}-05$ & $6.16 \mathrm{E}-05$ & 0.00789081 & 0.11580177 & 0.01864039 & 6095365.62 \\
\hline 79893618.4 & 782.745069 & 806.478072 & 1025.41093 & 765.844893 & $6.08 \mathrm{E}-06$ & $2.52 \mathrm{E}-05$ & 73.4849924 & 0.01619229 & 0.0118989 & $1.22 E-05$ & $1.30 \mathrm{E}-05$ & $6.18 \mathrm{E}-05$ & 0.00789862 & 0.11602873 & 0.0186864 & 6107517.34 \\
\hline 80066096.4 & 782.745069 & 806.457964 & 1025.40405 & 765.843965 & $6.10 \mathrm{E}-06$ & $2.52 \mathrm{E}-05$ & 73.4932212 & 0.01622396 & 0.01192351 & $1.22 E-05$ & $1.30 \mathrm{E}-05$ & $6.19 \mathrm{E}-05$ & 0.00790619 & 0.11625569 & 0.01873239 & 6119666.82 \\
\hline 80238574.5 & 782.745069 & 806.436954 & 1025.39719 & 765.843026 & $6.11 \mathrm{E}-06$ & $2.52 E-05$ & 73.501418 & 0.01625564 & 0.01194812 & $1.22 E-05$ & $1.30 \mathrm{E}-05$ & $6.21 E-05$ & 0.00791422 & 0.11648265 & 0.01877836 & 6131821.44 \\
\hline 80444447 & 782.745069 & 806.412916 & 1025.38904 & 765.841913 & $6.13 \mathrm{E}-06$ & $2.52 E-05$ & 73.5111601 & 0.01629344 & 0.0119775 & $1.22 E-05$ & $1.30 \mathrm{E}-05$ & $6.22 E-05$ & 0.00792366 & 0.11675356 & 0.01883317 & 6143994.53 \\
\hline $\begin{array}{l}80448047 \\
\end{array}$ & $\begin{array}{r}644.15 \\
\end{array}$ & 644.172401 & 644.153569 & 644.150952 & $6.13 \mathrm{E}-06$ & $2.51 \mathrm{E}-05$ & 73.5112451 & 0.01629377 & 0.01197776 & $1.20 \mathrm{E}-05$ & $1.30 \mathrm{E}-05$ & $5.80 \mathrm{E}-05$ & 0.00645238 & 0.11675593 & 0.01764659 & 5163920.2 \\
\hline 80451647 & 305 & 305.051392 & 305.006649 & 305.002174 & $6.13 \mathrm{E}-06$ & $2.47 \mathrm{E}-05$ & 73.5112451 & 0.01629377 & 0.01197776 & $1.18 \mathrm{E}-05$ & $1.28 \mathrm{E}-05$ & $3.52 E-05$ & 0.00440858 & 0.11675593 & 0.01115273 & 2479257.78 \\
\hline 80455247 & 305 & 305.051392 & 305.006649 & 305.002174 & $6.13 \mathrm{E}-06$ & $2.47 \mathrm{E}-05$ & 73.5112451 & 0.01629377 & 0.01197776 & $1.18 \mathrm{E}-05$ & $1.28 \mathrm{E}-05$ & $3.52 \mathrm{E}-05$ & 0.00440858 & 0.11675593 & 0.01115273 & 2479257.78 \\
\hline
\end{tabular}




\section{APPENDIX B. BISON INPUT FILES}





\section{INPUT FILE FOR PIN T651}

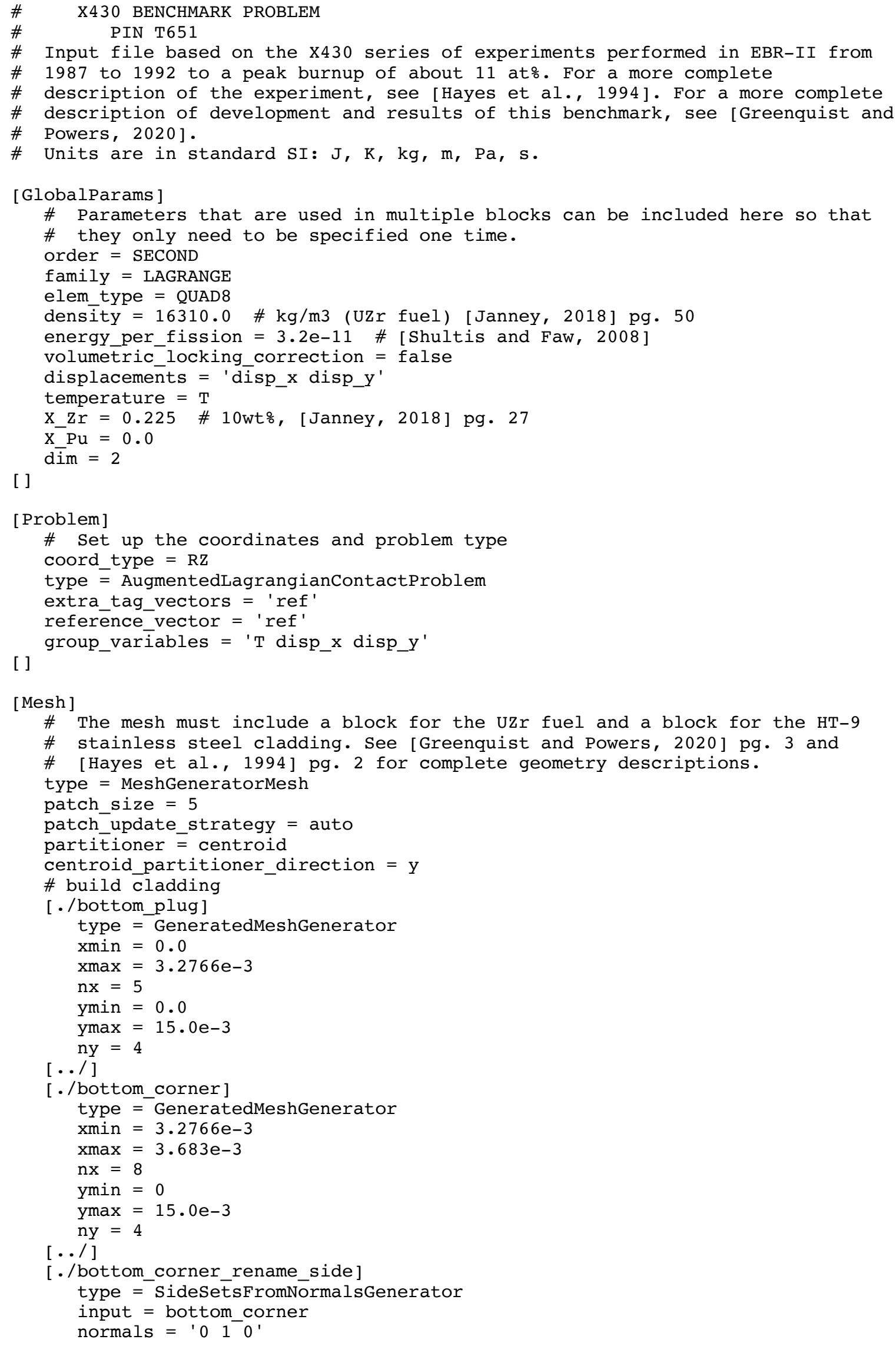




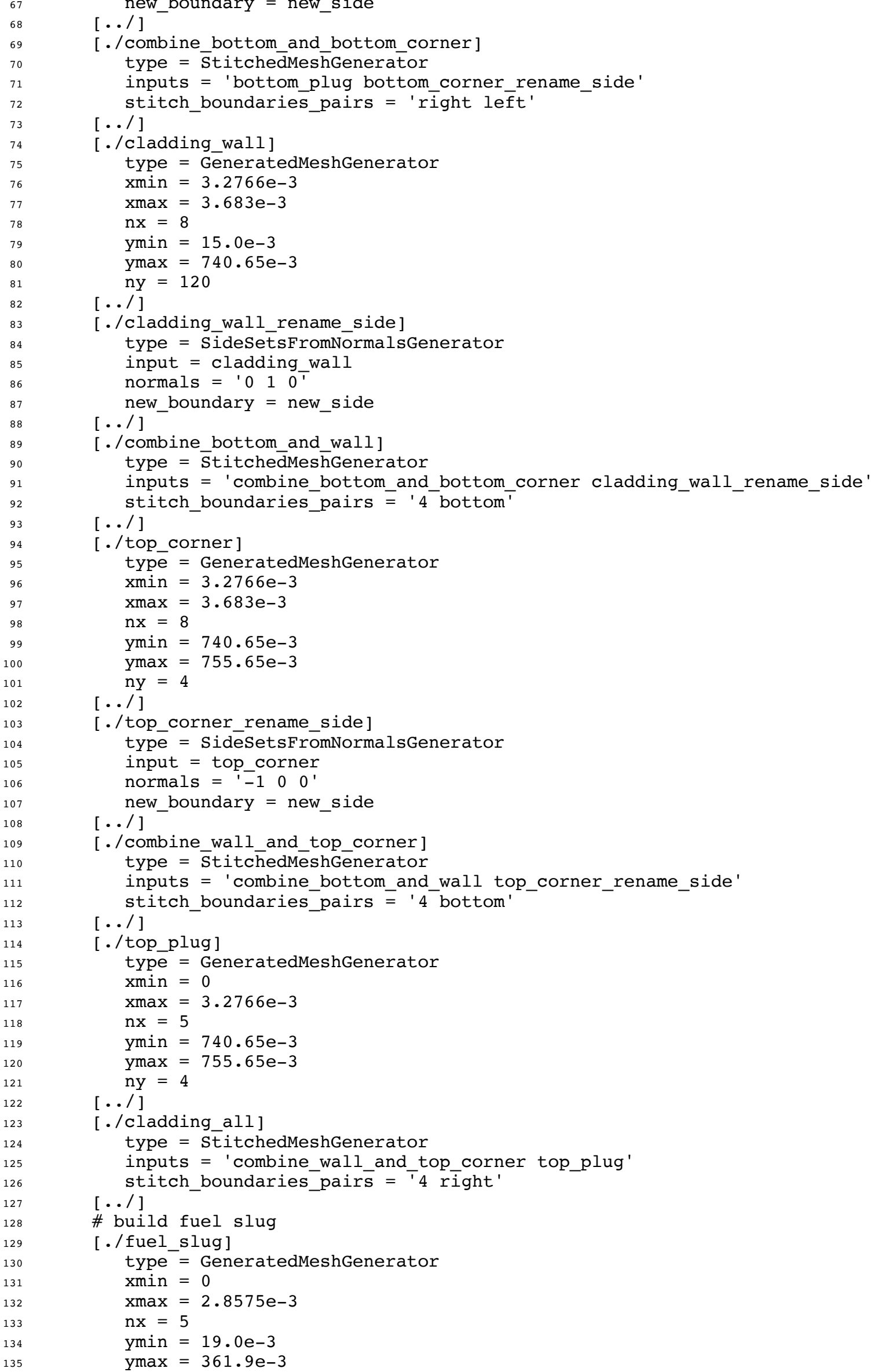




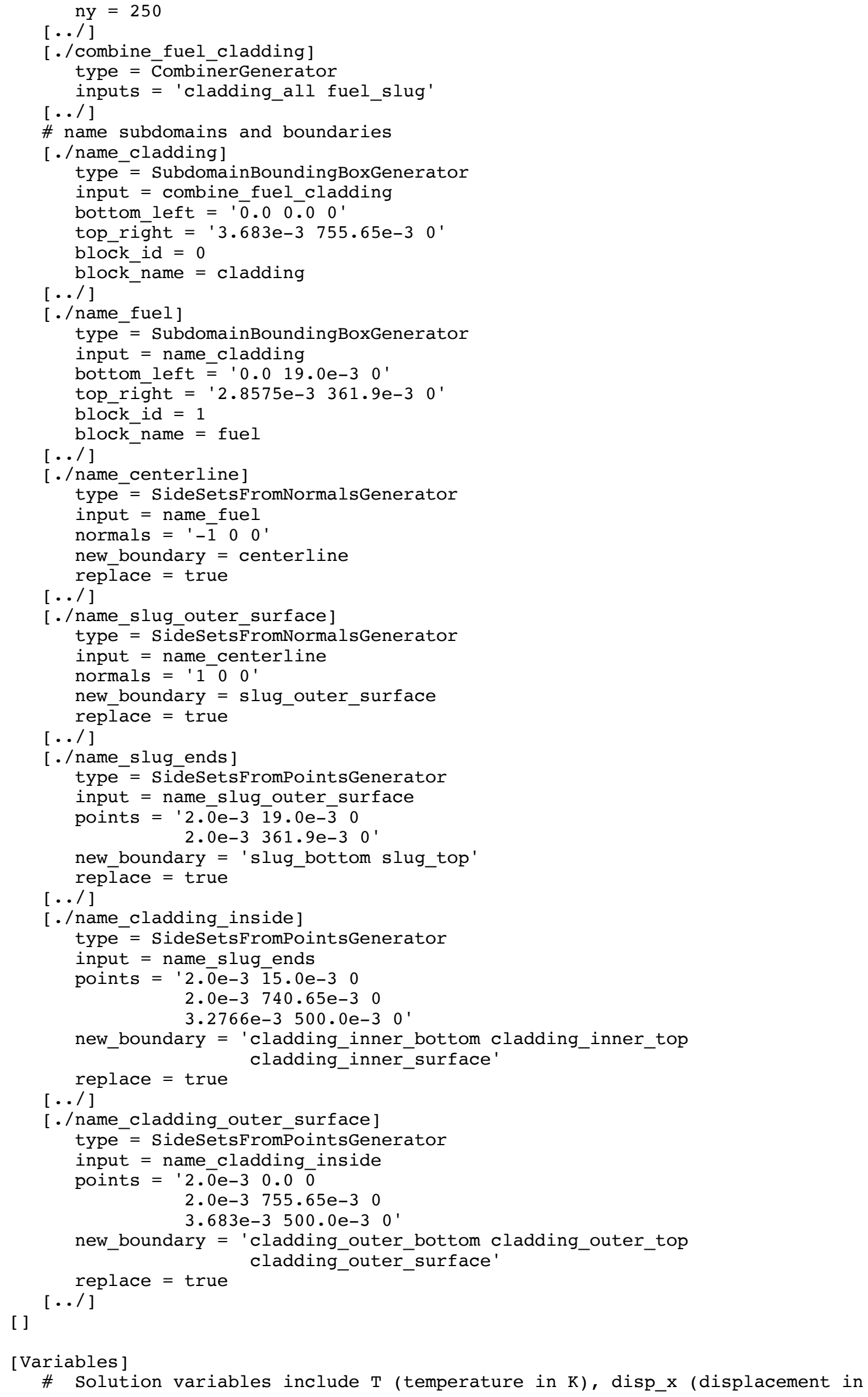


[ ]

\# radial direction), and disp_y (displacement in axial direction). Only $T$ is

\# specified here. The others are generated by the [Modules/TensorMechanics]

\# block.

$[\cdot / \mathrm{T}]$

initial_condition $=295$ \#[Greenquist and Powers, 2020] pg.9

$[\cdot$. / ]

[AuxVariables ]

\# Variables that are not part of the system of coupled PDEs are specified

\# here.

[./creep strain mag]

order $=$ CONSTANT

family $=$ MONOMIAL

$[\ldots /]$

[./conductance]

order $=$ CONSTANT

family $=$ MONOMIAL

$[\ldots /]$

[ - /element_failed]

order $=$ CONSTANT

[.../ ]

family = MONOMIAL

[./volumetric strain]

block = fuèl

order $=$ CONSTANT

family $=$ MONOMIAL

$[\ldots /$ ]

[ . /local power]

block = fuel

order = CONSTANT

family = MONOMIAL

$[\ldots /]$

[./coolant_T]

block $=$ cladding

order $=$ CONSTANT

family $=$ MONOMIAL

[../]

[./linear_heat_rate]

order = CONSTANT

family $=$ MONOMIAL

[../]

[./fast_fluence]

orde $\bar{r}=$ CONSTANT

family $=$ MONOMIAL

[ ]

[../]

[Functions ]

\# Any parameter that is a pre-defined function of space and time is defined

\# here.

[./power_history]

\# $\mathrm{x}$ : time, $\mathrm{y}$ : Average LHGR $(\mathrm{W} / \mathrm{m})$. [Greenquist and Powers, 2020] pgs. 4

$\#$ and 5 .

type $=$ PiecewiseLinear

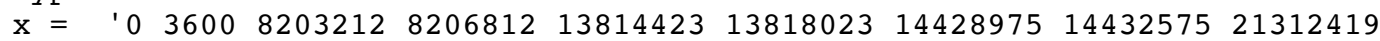

$\begin{array}{lllllllll}21316019 & 25596874 & 25600474 & 26261755 & 26265355 & 32714598 & 32718198 & 32721798\end{array}$

$\begin{array}{lllllllll}32725398 & 32728998 & 32896765 & 32900365 & 39574695 & 39578295 & 42194062 & 42197662\end{array}$

$\begin{array}{llllllllll}43820808 & 43824408 & 43895709 & 43899309 & 44401212 & 44404812 & 47385472 & 47389072\end{array}$

$\begin{array}{lllllllll}48198548 & 48202148 & 48205748 & 48209348 & 48212948 & 52079977 & 52083577 & 53874489\end{array}$

$\begin{array}{llllllllll}53878089 & 62125235 & 62128835 & 62256058 & 62259658 & 62620357 & 62623957 & 64516928\end{array}$

$\begin{array}{llllllllll}64520528 & 64766586 & 64770186 & 67535546 & 67539146 & 72155534 & 72159134 & 72185697\end{array}$

$\begin{array}{llllllll}72189297 & 76833647 & 76837247 & 77340548 & 77344148 & 77738400 & 77742000 & 80444447\end{array}$ $804480478045164780455247^{\prime}$

$\mathrm{y}=' 0.0 \quad 44225.3 \quad 44225.3 \quad 43106.143106 .1 \quad 41403.6 \quad 41403.6 \quad 41119.941119 .9$

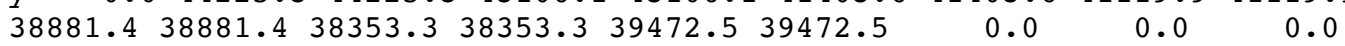

$\begin{array}{llllllllll}33490.2 & 33490.2 & 36863.6 & 36863.6 & 37123.7 & 37123.7 & 32717.8 & 32717.8 & 38534.6\end{array}$

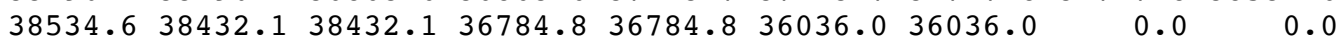
$0.035153 .3 \quad 35153.3 \quad 35153.3 \quad 35153.3 \quad 35271.5 \quad 35271.533663 .6 \quad 33663.6$ 


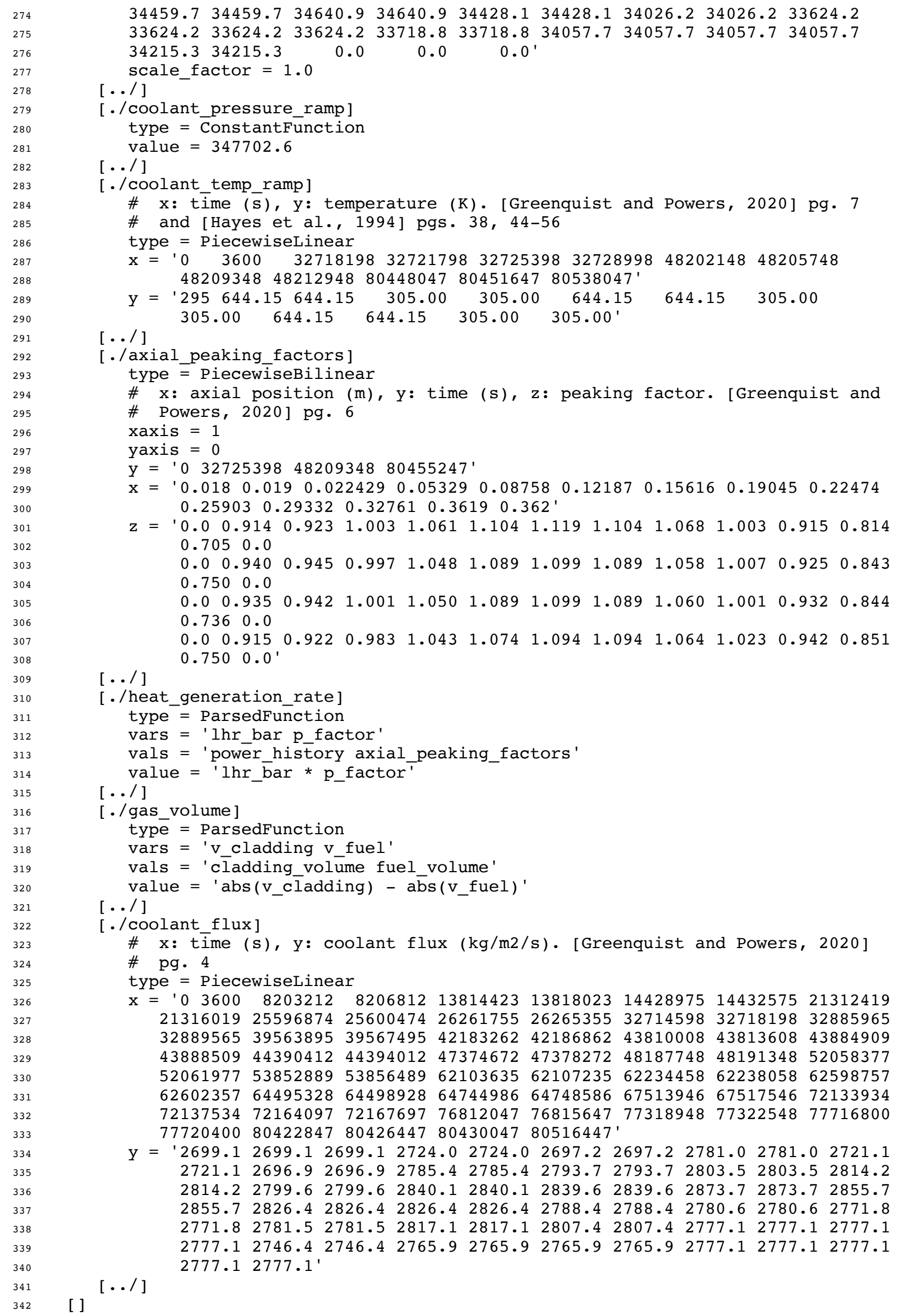




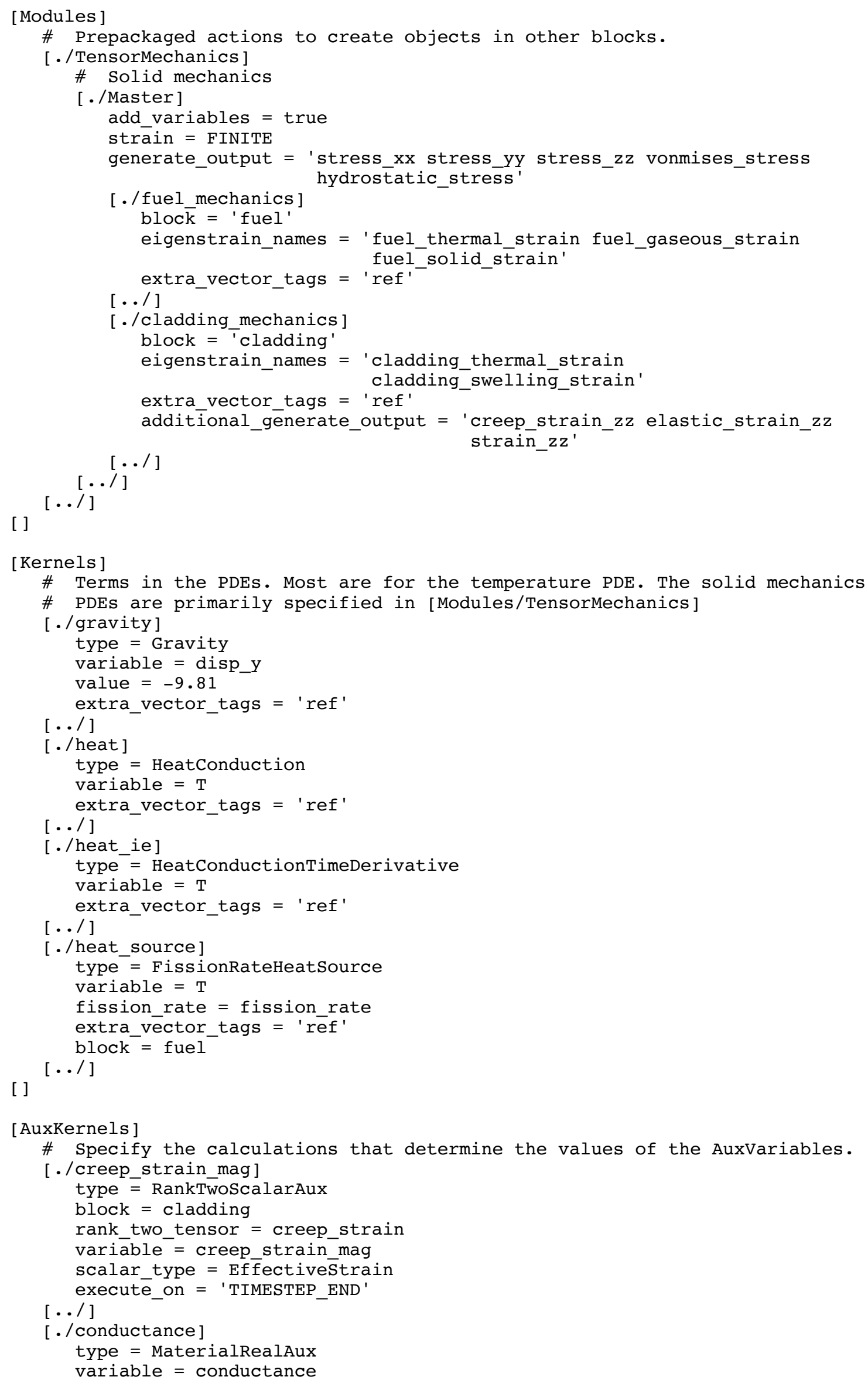




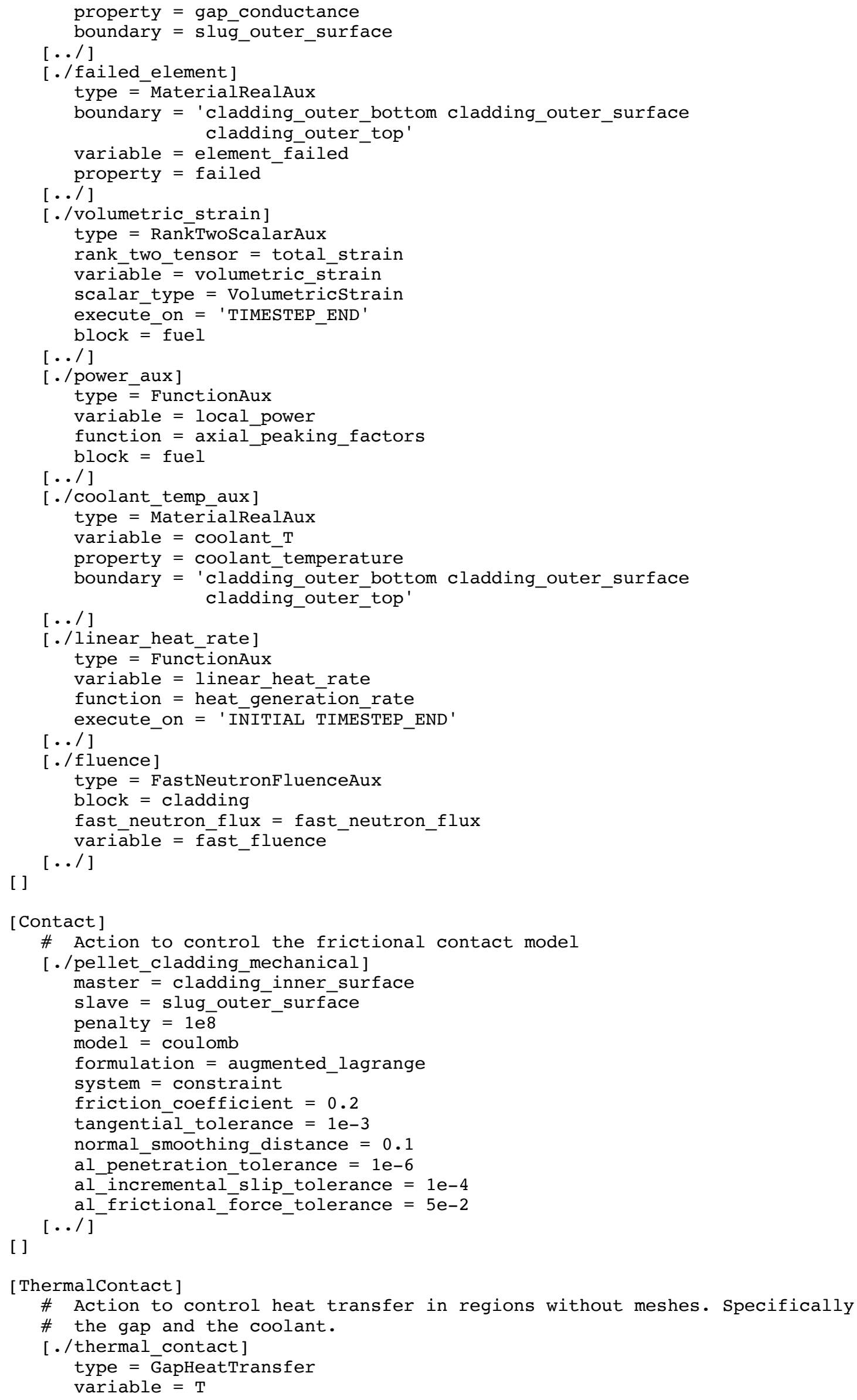




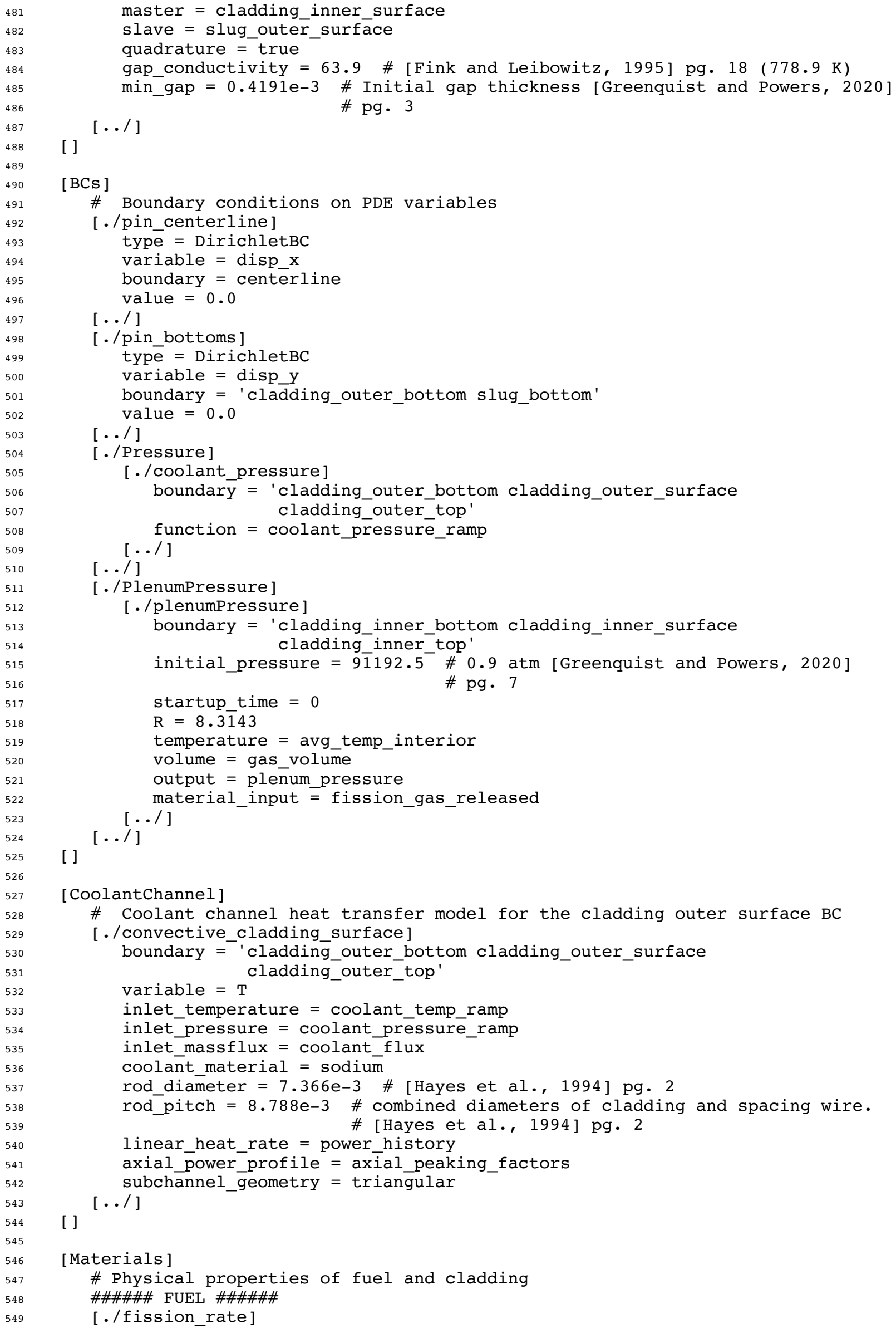




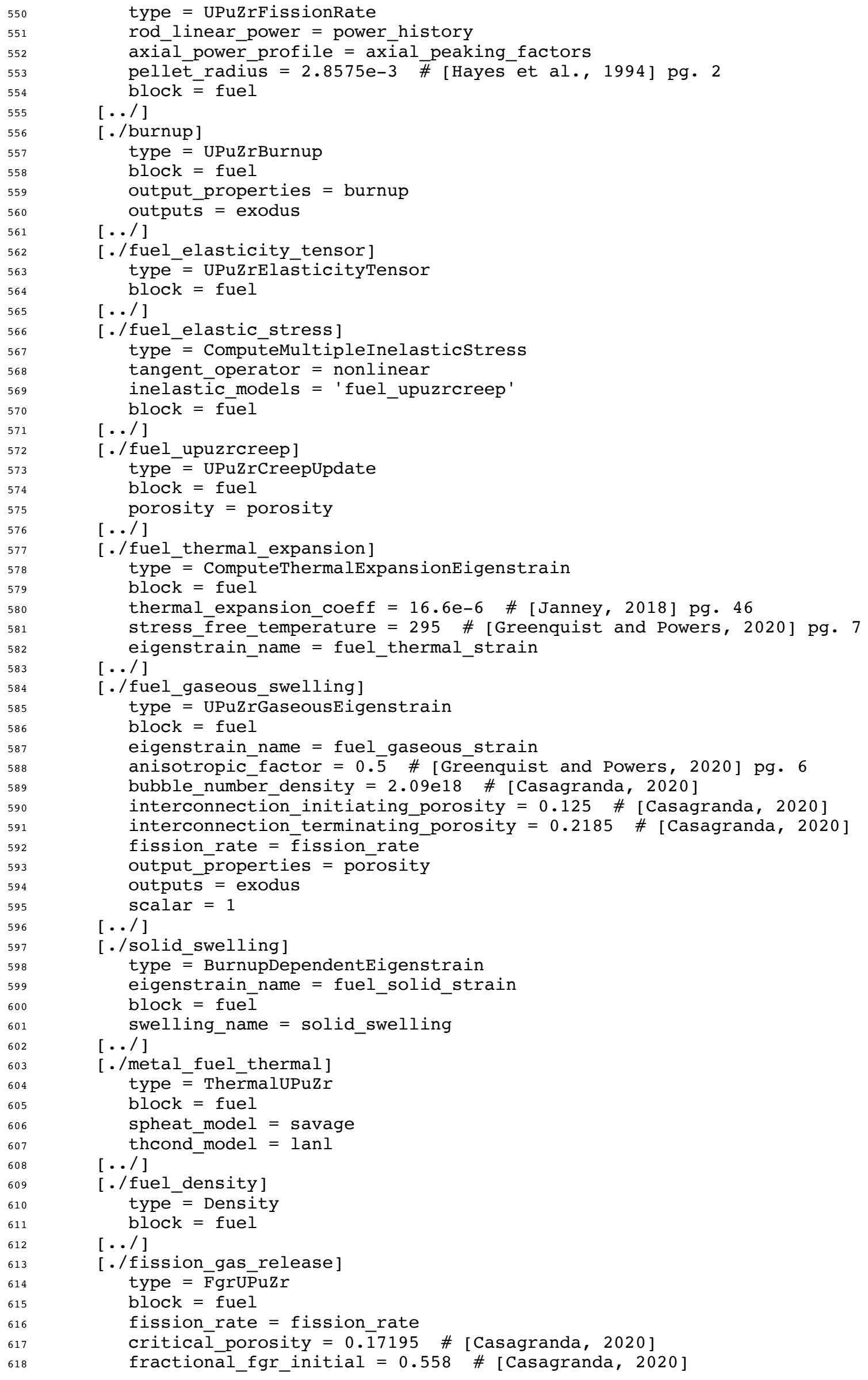




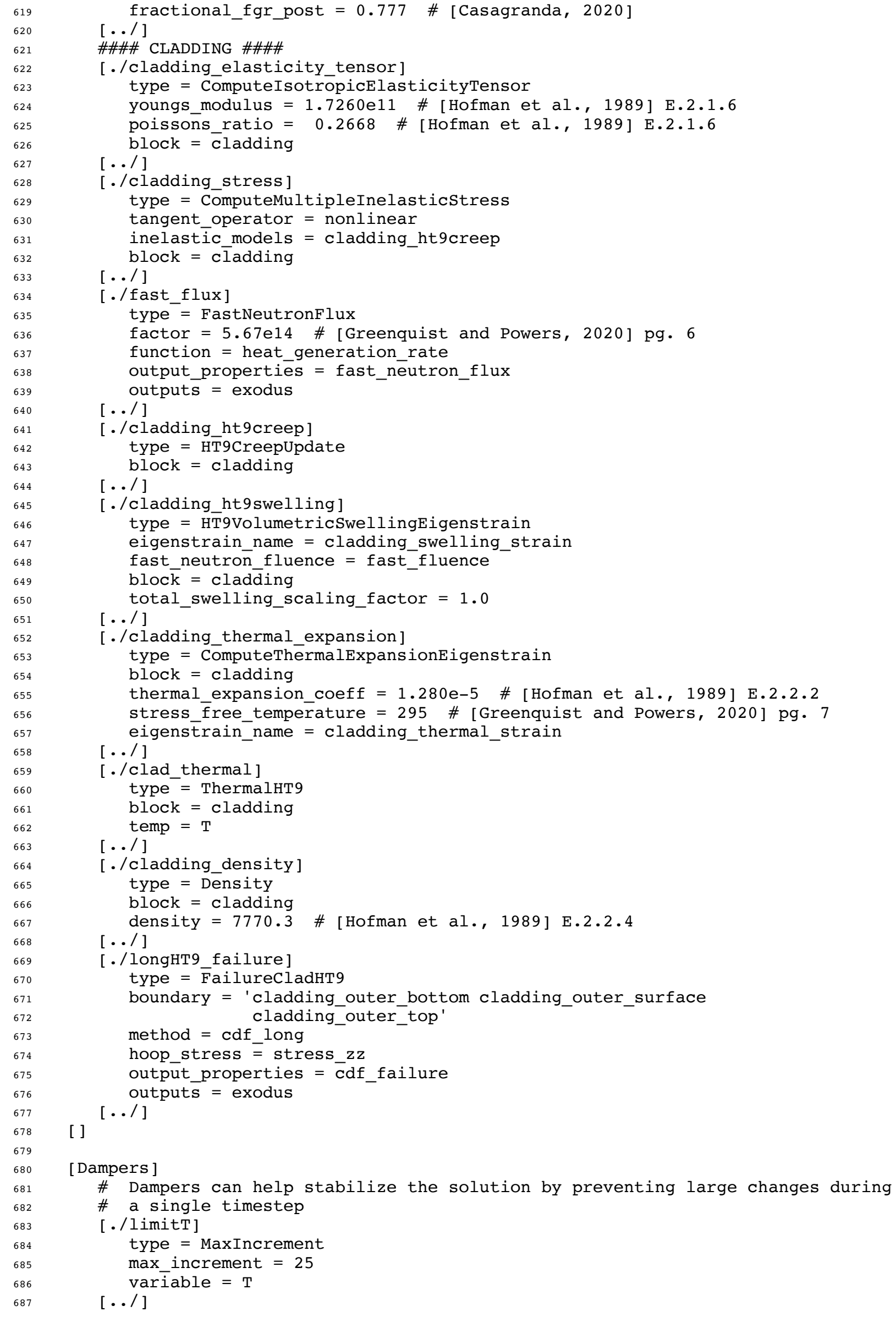




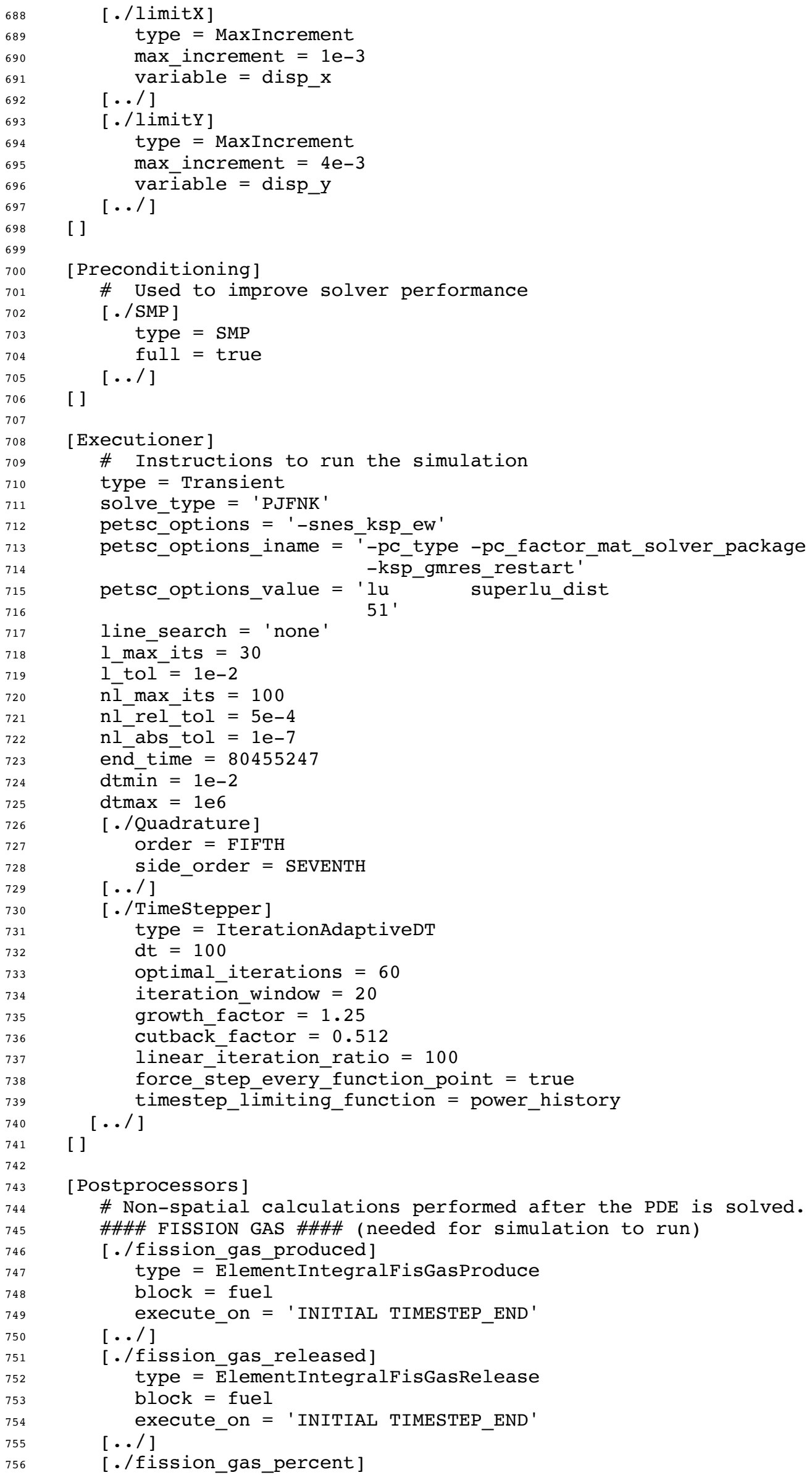




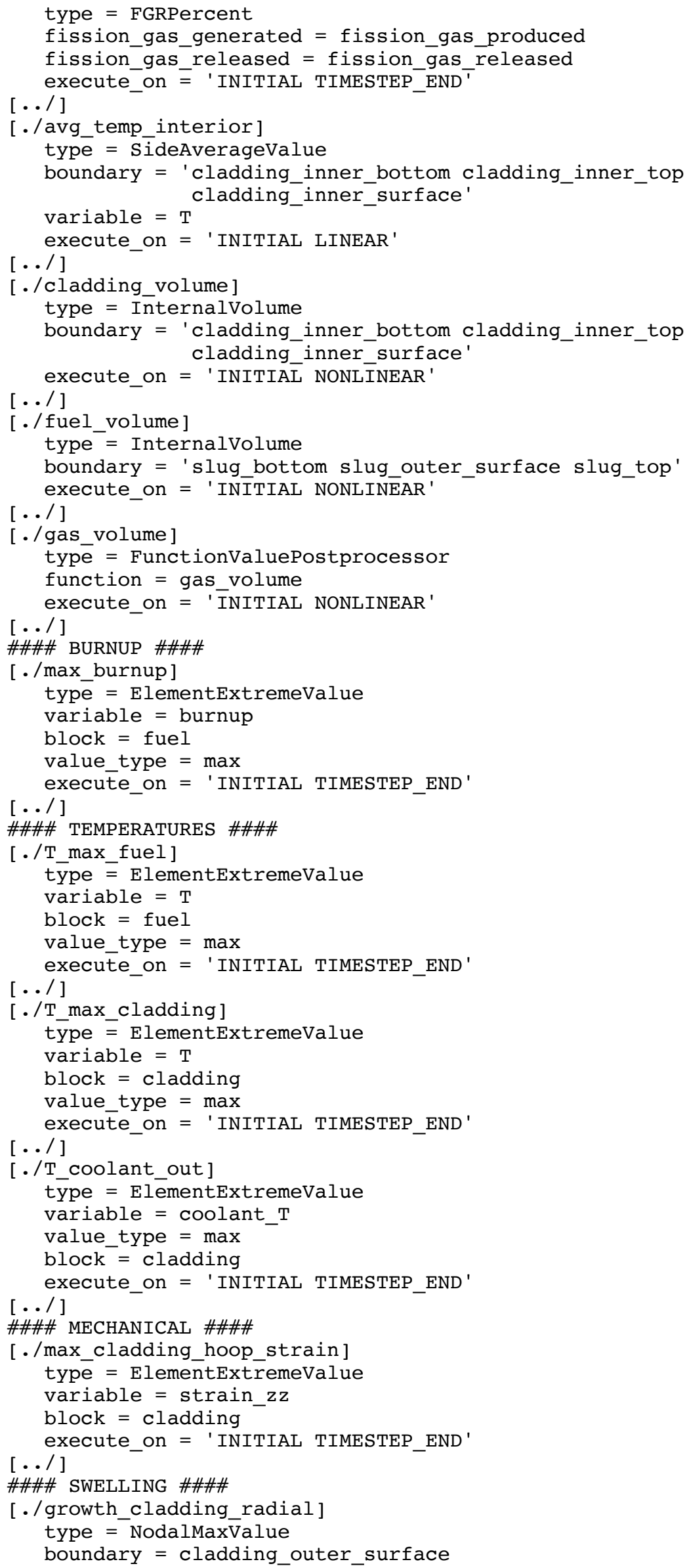




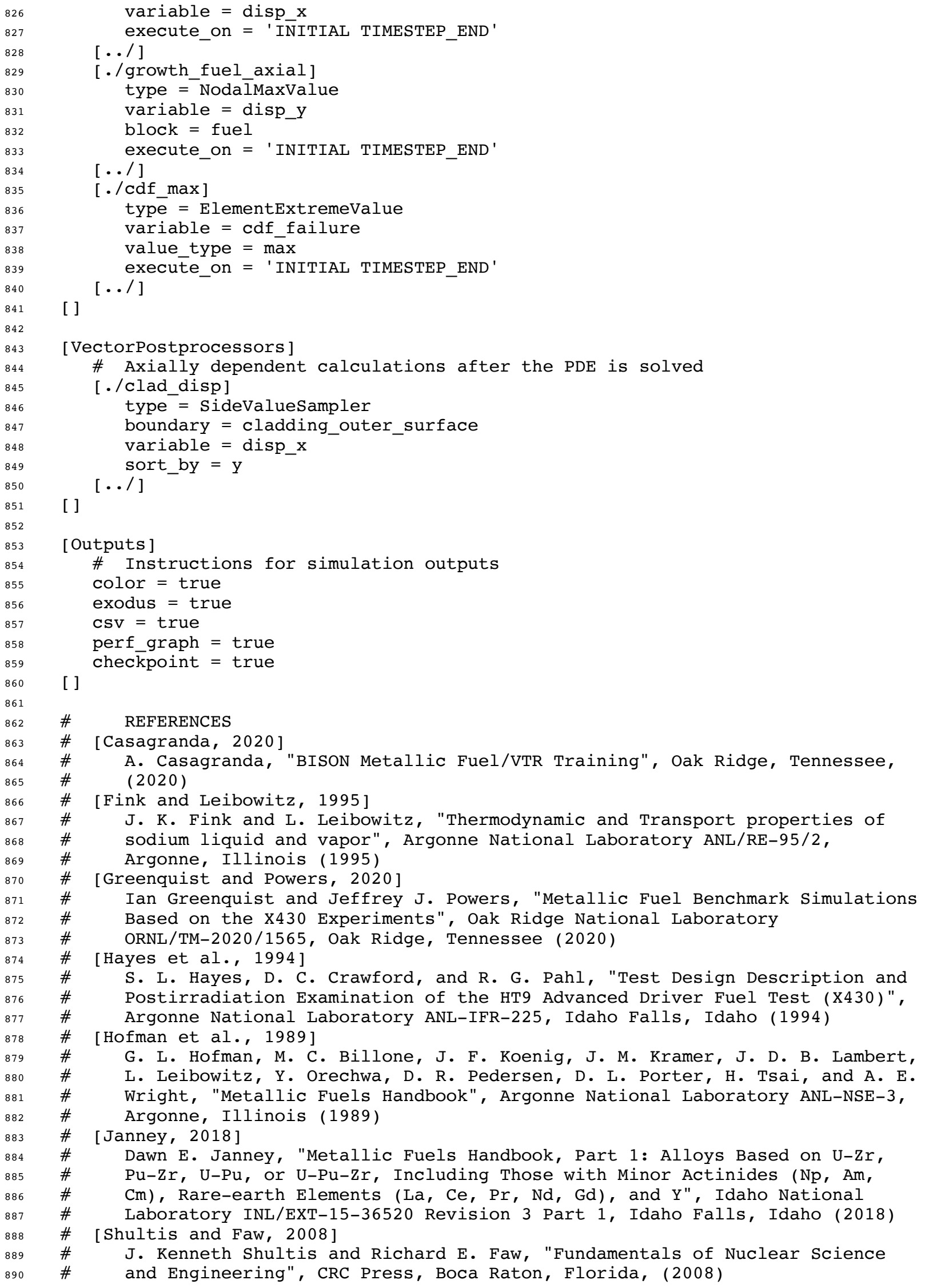




\section{INPUT FILE FOR T654}

The values that were changed in section 3.4 to tune the simulation are on lines 588 and 595 . The values are highlighted in red.

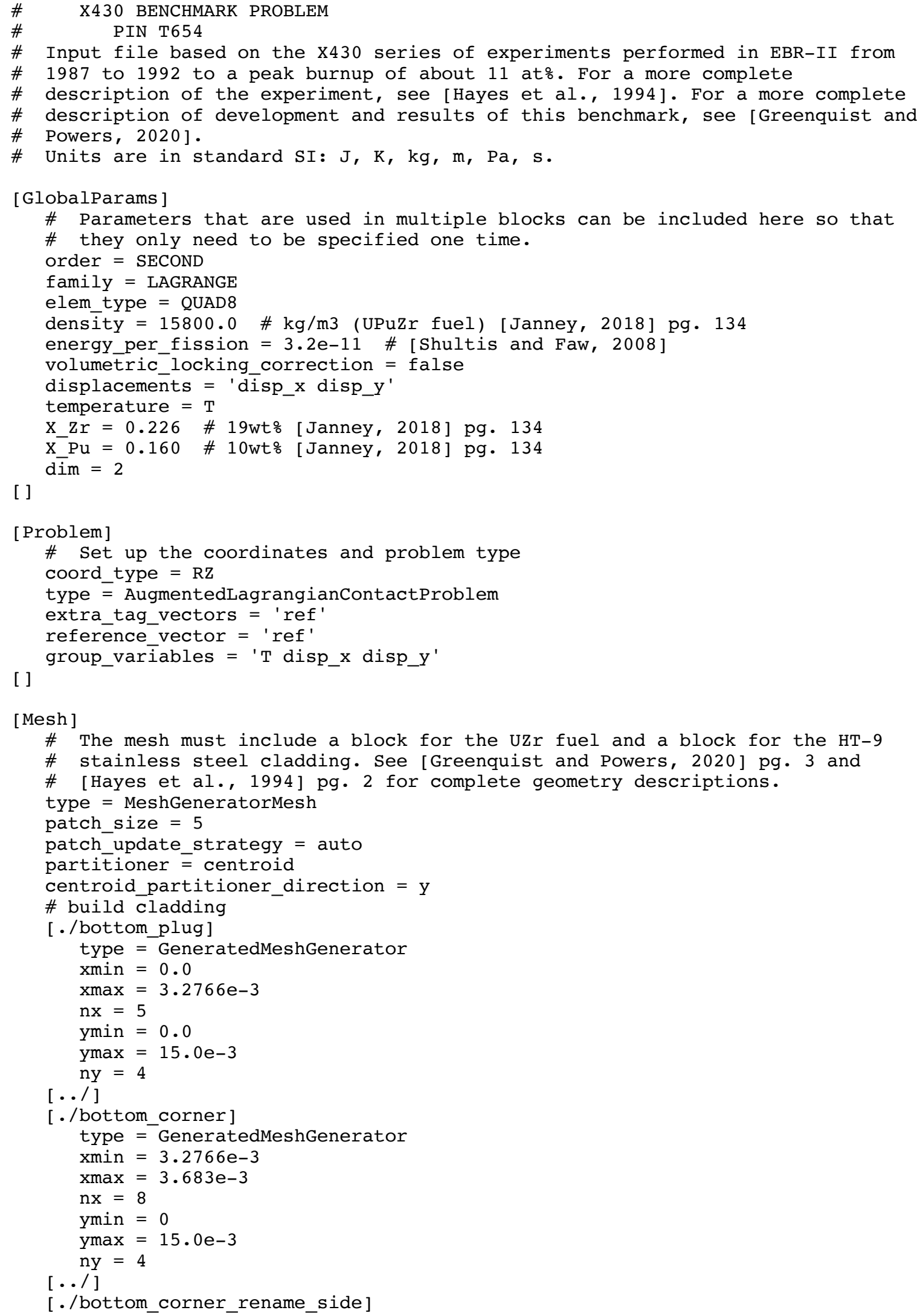




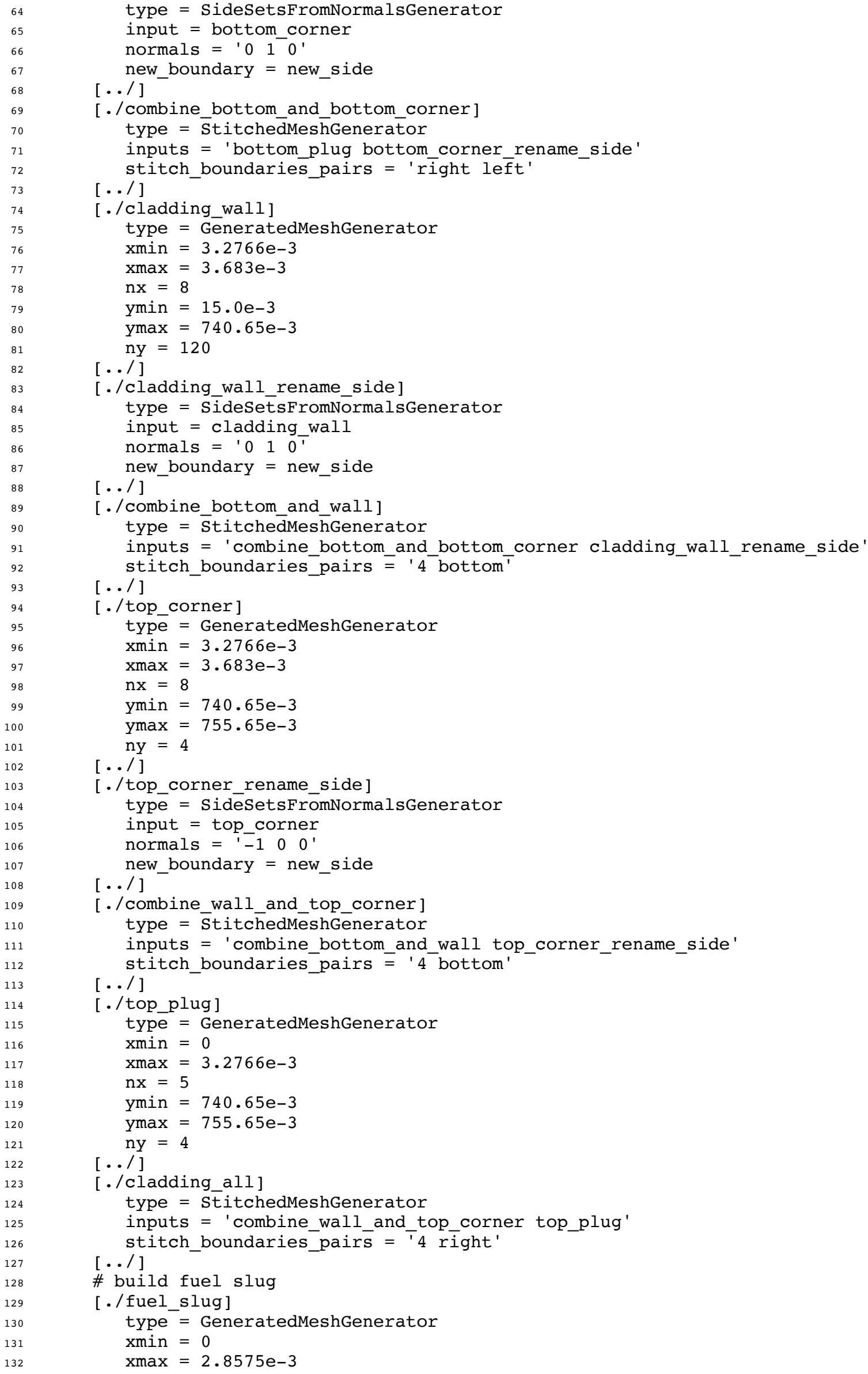




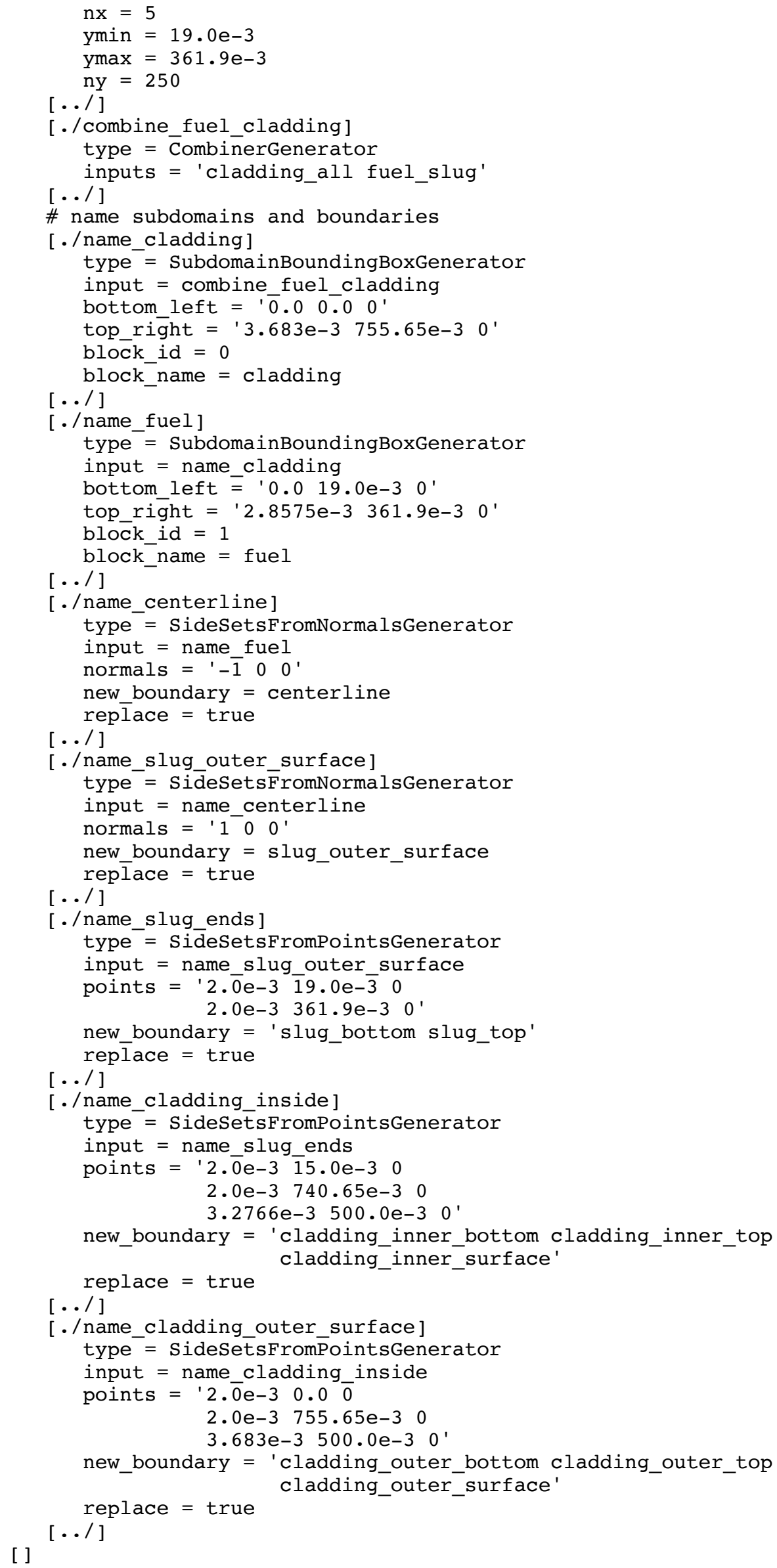


[Variables ]

\# Solution variables include $T$ (temperature in $\mathrm{K}$ ), disp_x (displacement in

\# radial direction), and disp_y (displacement in axial direction). Only $T$ is

\# specified here. The others are generated by the [Modules/TensorMechanics]

\# block.

$[. / \mathrm{T}]$

] $[\cdot /]$

initial_condition $=295$ \# [Greenquist and Powers, 2020] pg. 9

[AuxVariables ]

\# Variables that are not part of the system of coupled PDEs are specified

\# here.

[./creep_strain_mag]

order $=$ CONSTANT

[... ]

family $=$ MONOMIAL

[./conductance]

order $=$ CONSTANT

$[\ldots /]$

family = MONOMIAL

[ . /element_failed]

order $=$ CONSTANT

family $=$ MONOMIAL

$[\ldots /]$

[./volumetric strain]

block = fuel

order $=$ CONSTANT

family = MONOMIAL

[../]

[ / local_power]

block = fuel

order $=$ CONSTANT

family = MONOMIAL

[../]

[./coolant_T]

block = cladding

order $=$ CONSTANT

family $=$ MONOMIAL

[../]

[./linear_heat_rate]

order = CONSTANT

[../]

family $=$ MONOMIAL

[./fast fluence]

orde $\bar{r}=$ CONSTANT

[../]

family $=$ MONOMIAL

[ ]

[Functions ]

\# Any parameter that is a pre-defined function of space and time is defined

\# here.

[./power_history]

\# $x$ : time, y: Average LHGR (W/m). [Greenquist and Powers, 2020] pgs. 4

\# and 5 .

type $=$ PiecewiseLinear

$\mathrm{x}=$ ' $0 \quad 3600 \quad 8203212 \quad 8206812 \quad 13814423 \quad 13818023 \quad 14428975 \quad 14432575 \quad 21312419$

$\begin{array}{lllllllll}21316019 & 25596874 & 25600474 & 26261755 & 26265355 & 32714598 & 32718198 & 32721798\end{array}$

$\begin{array}{lllllllll}32725398 & 32728998 & 32896765 & 32900365 & 39574695 & 39578295 & 42194062 & 42197662\end{array}$

$\begin{array}{llllllllll}43820808 & 43824408 & 43895709 & 43899309 & 44401212 & 44404812 & 47385472 & 47389072\end{array}$

$\begin{array}{llllllllll}48198548 & 48202148 & 48205748 & 48209348 & 48212948 & 52079977 & 52083577 & 53874489\end{array}$

$\begin{array}{llllllllll}53878089 & 62125235 & 62128835 & 62256058 & 62259658 & 62620357 & 62623957 & 64516928\end{array}$

$\begin{array}{lllllllllll}64520528 & 64766586 & 64770186 & 67535546 & 67539146 & 72155534 & 72159134 & 72185697\end{array}$

$\begin{array}{llllllllll}72189297 & 76833647 & 76837247 & 77340548 & 77344148 & 77738400 & 77742000 & 80444447\end{array}$ $804480478045164780455247^{\prime}$

$y=10.0 \quad 44225.3 \quad 44225.3 \quad 43106.1 \quad 43106.1 \quad 41403.6 \quad 41403.6 \quad 41119.941119 .9$

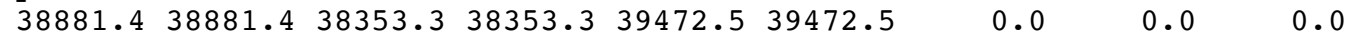

B-6 


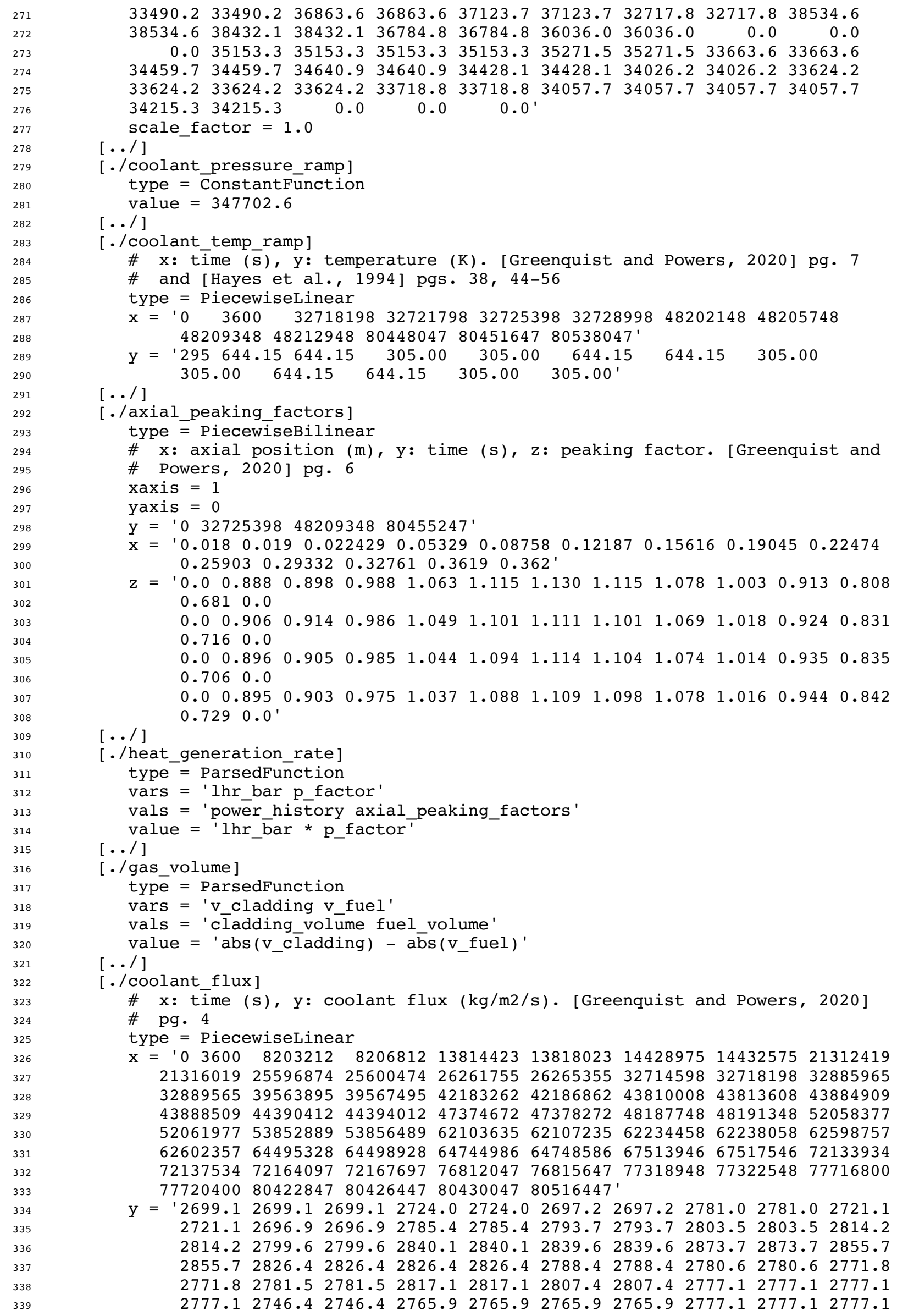




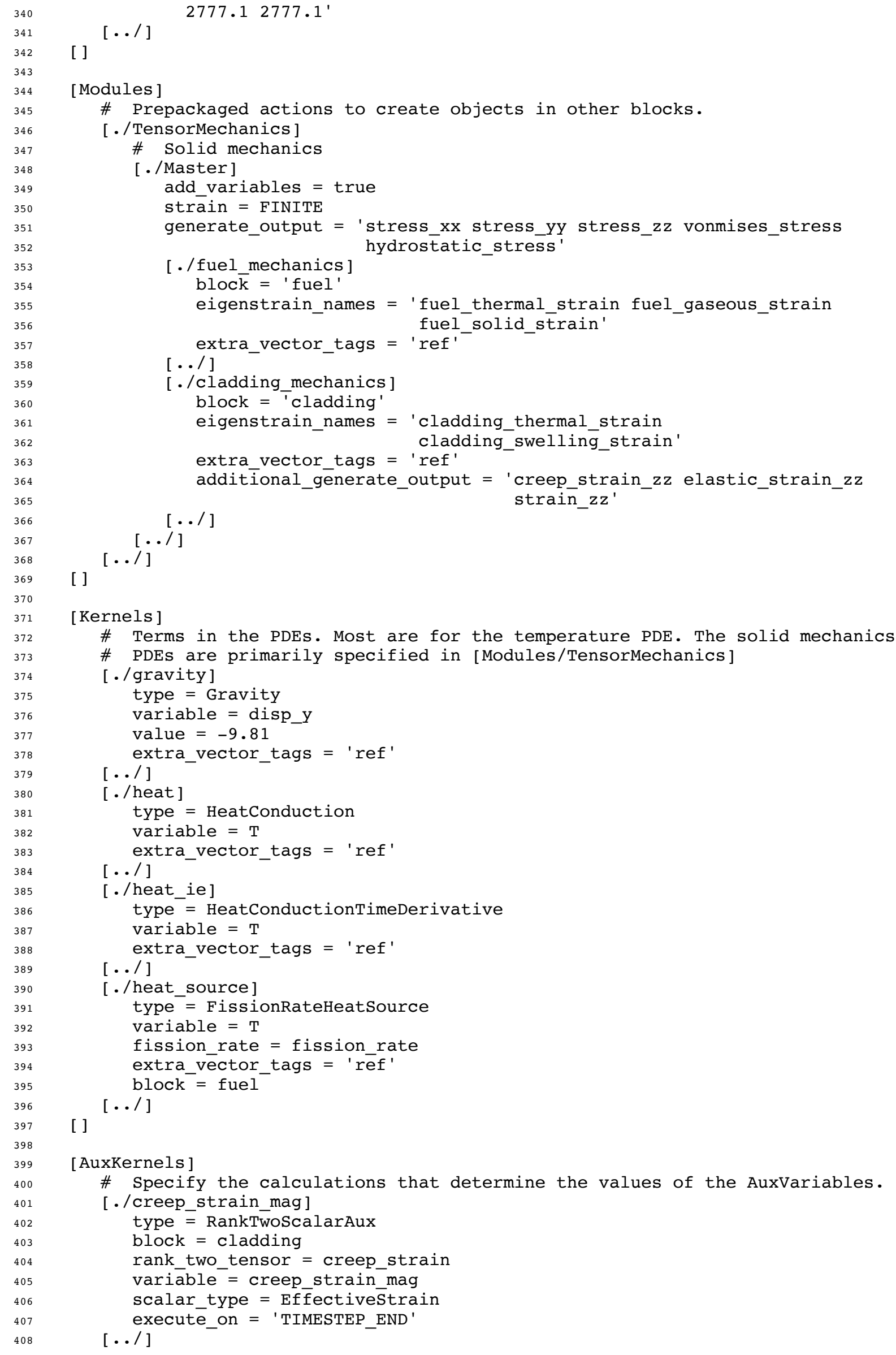




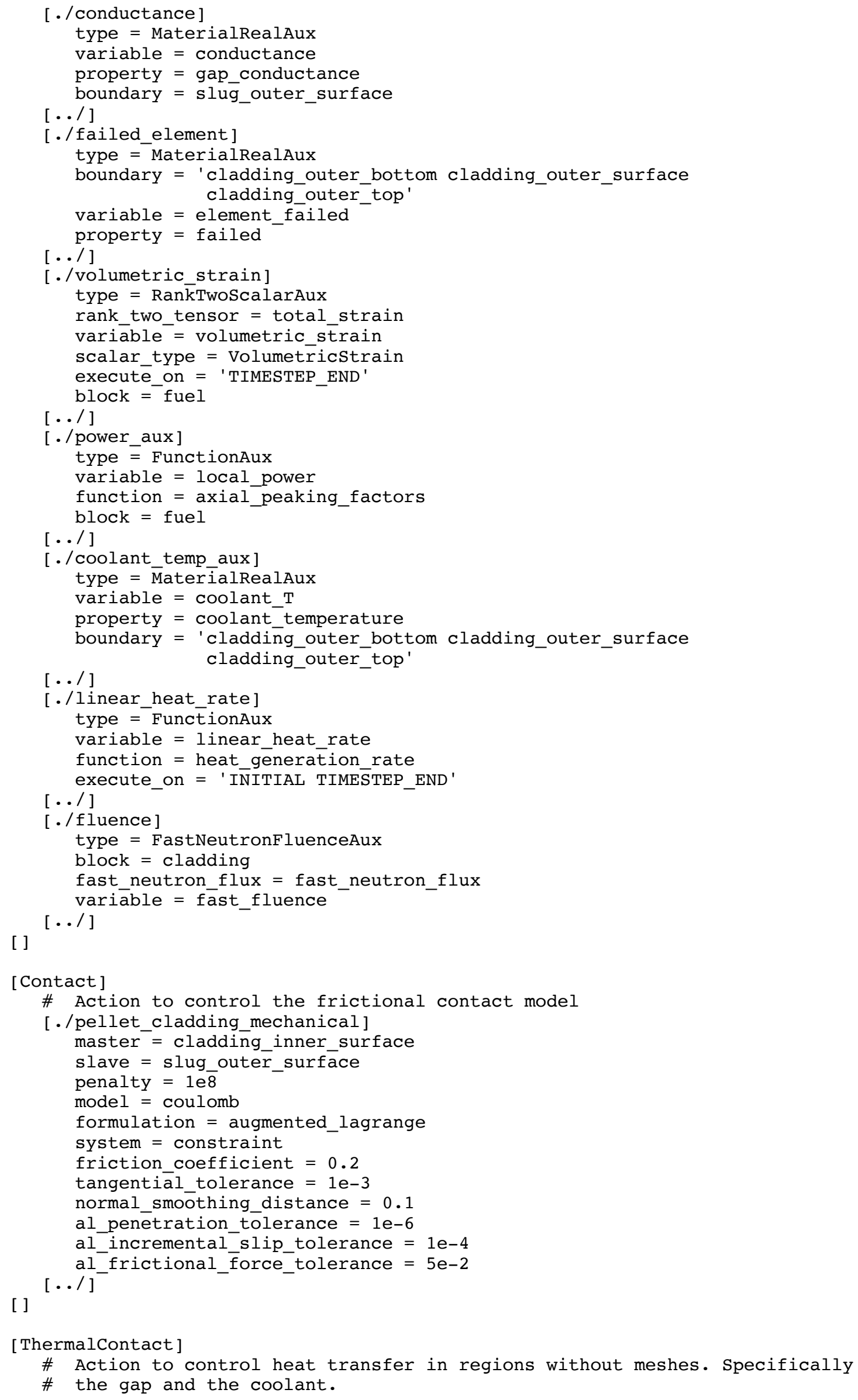




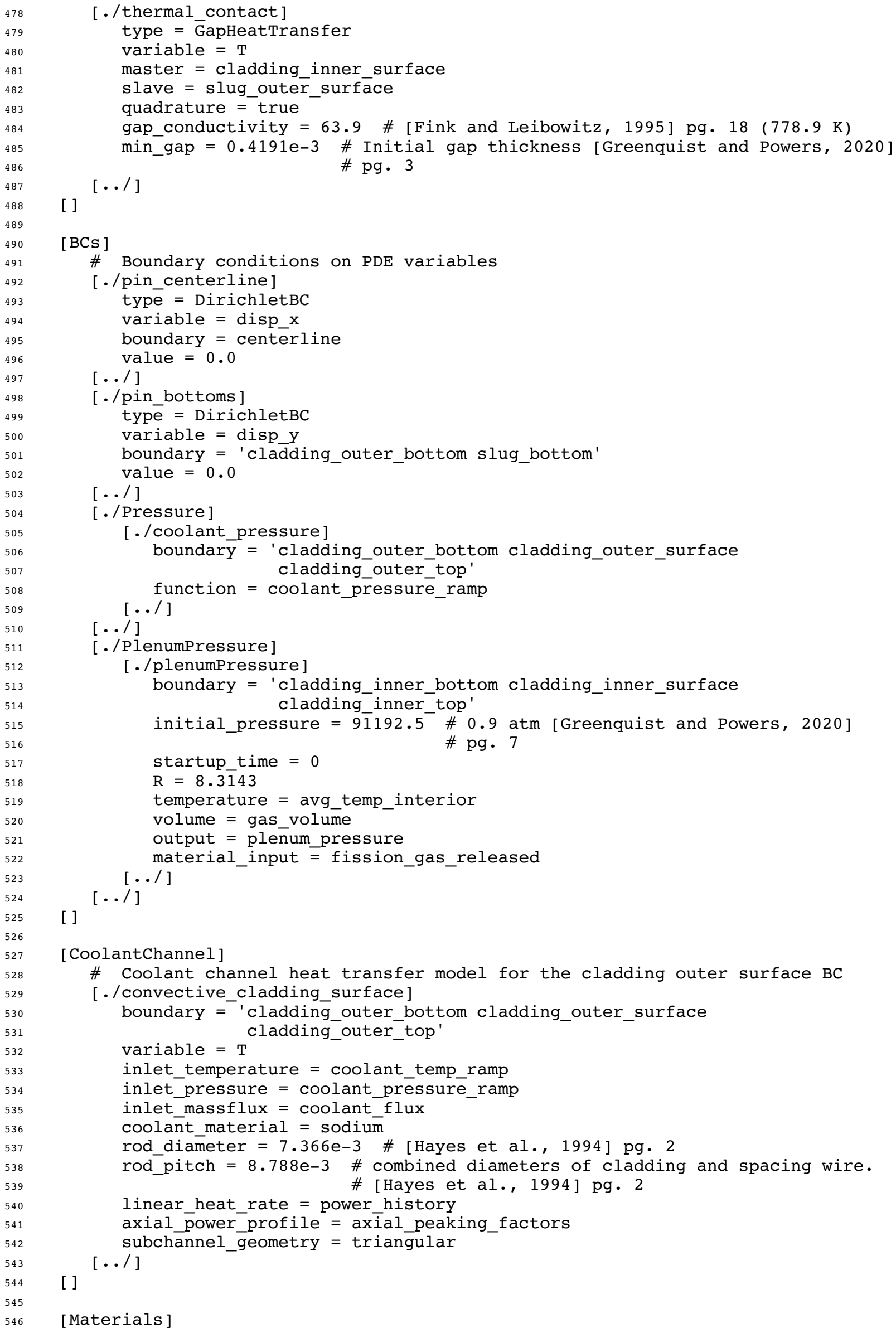




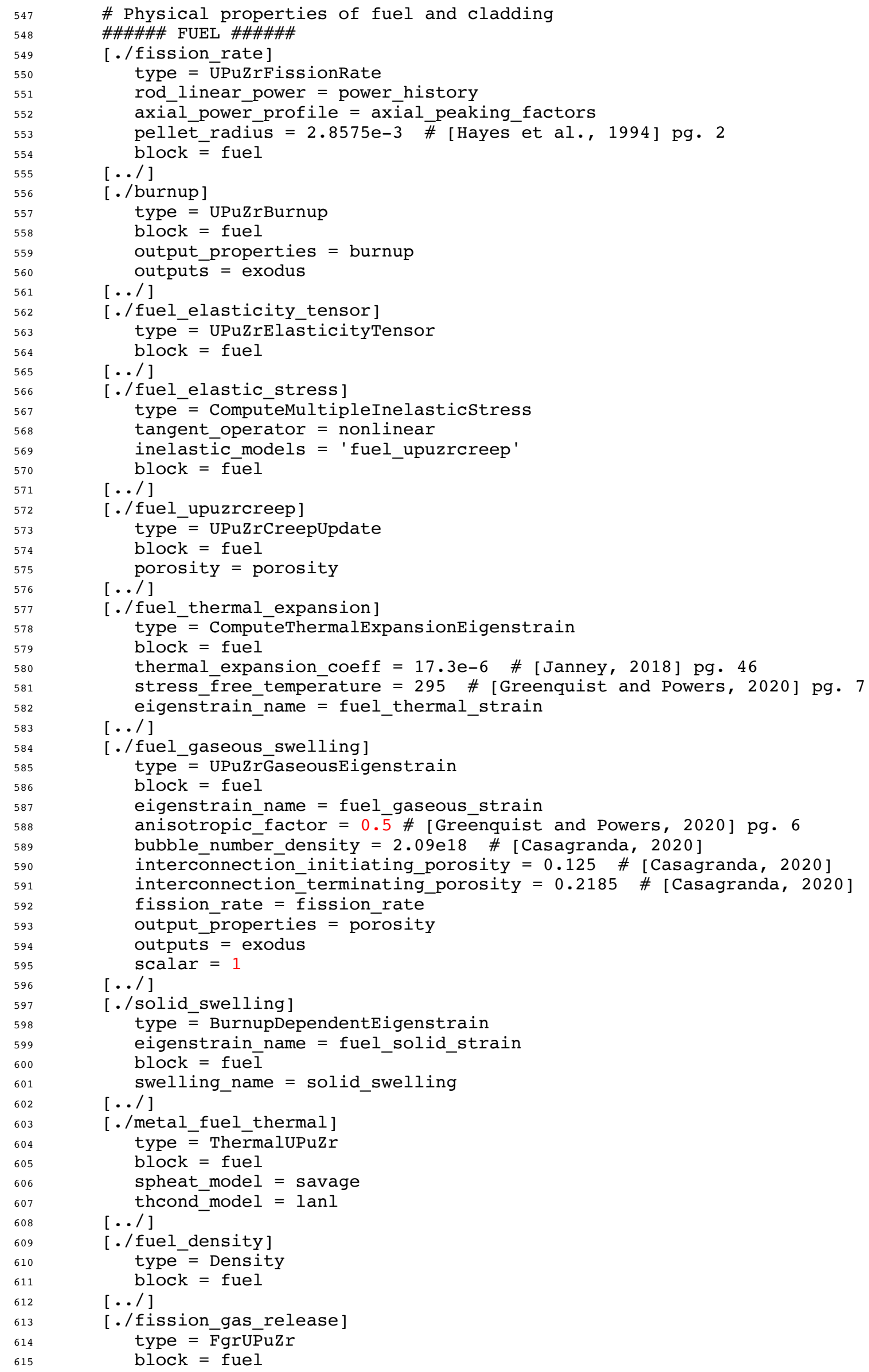




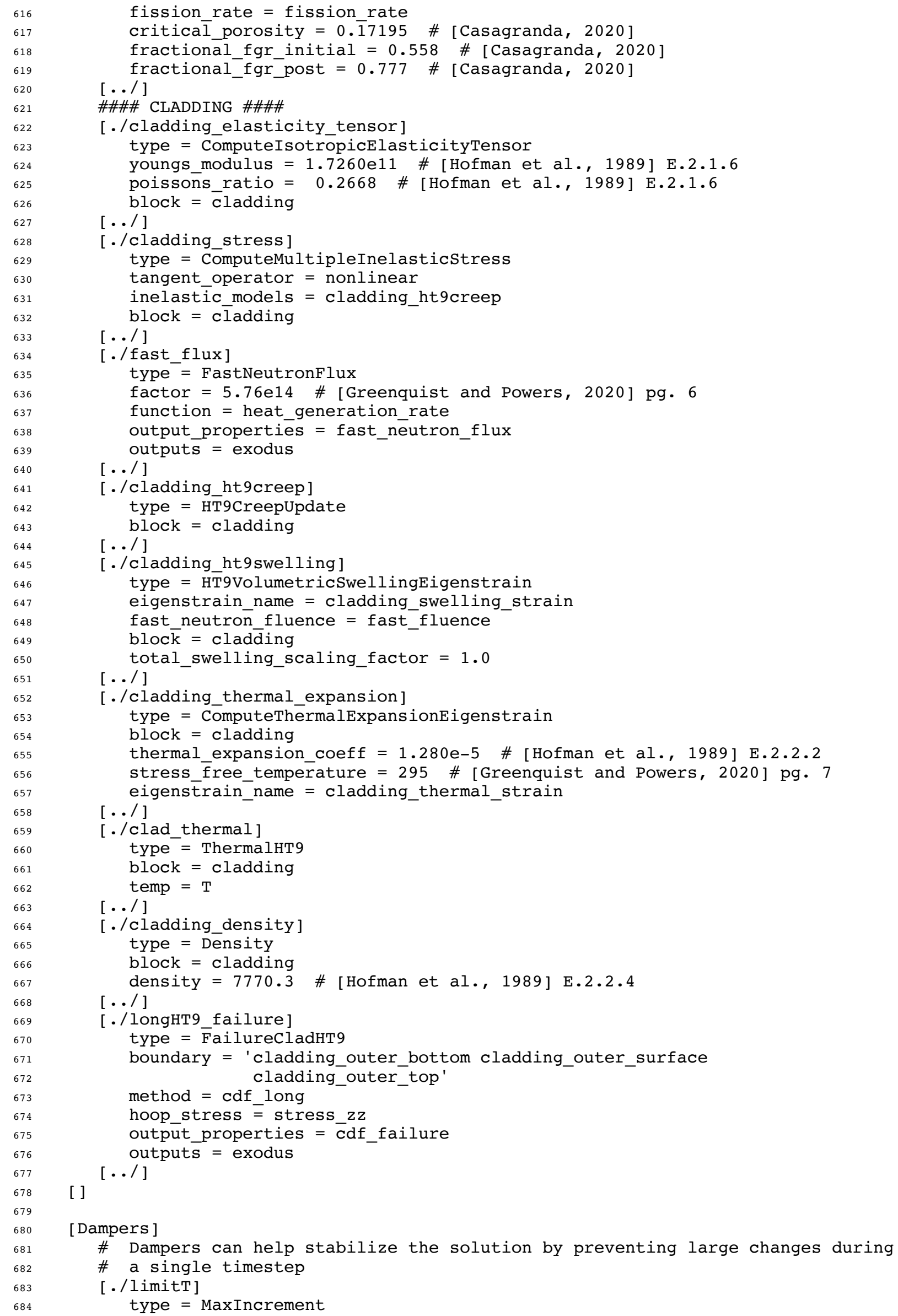




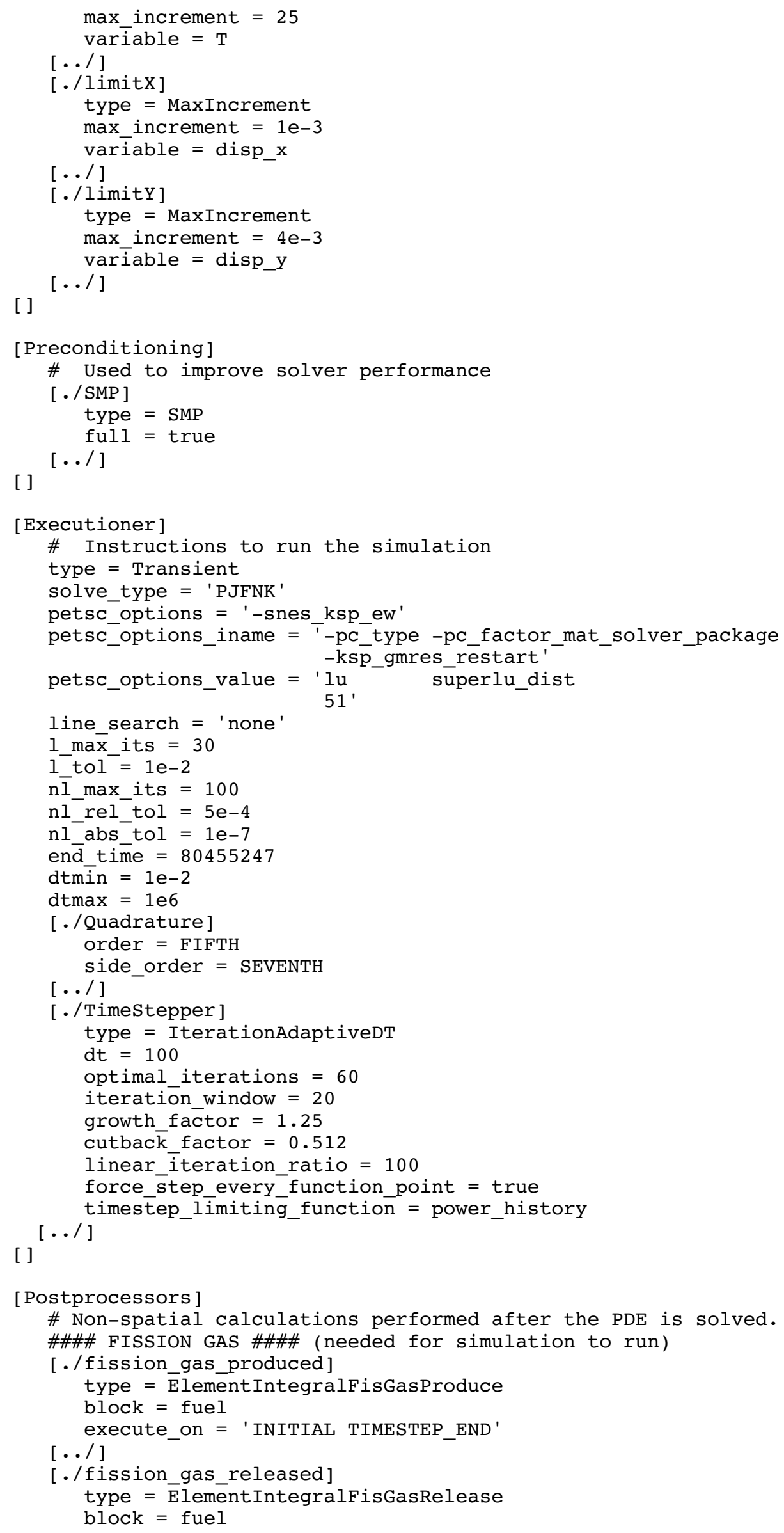




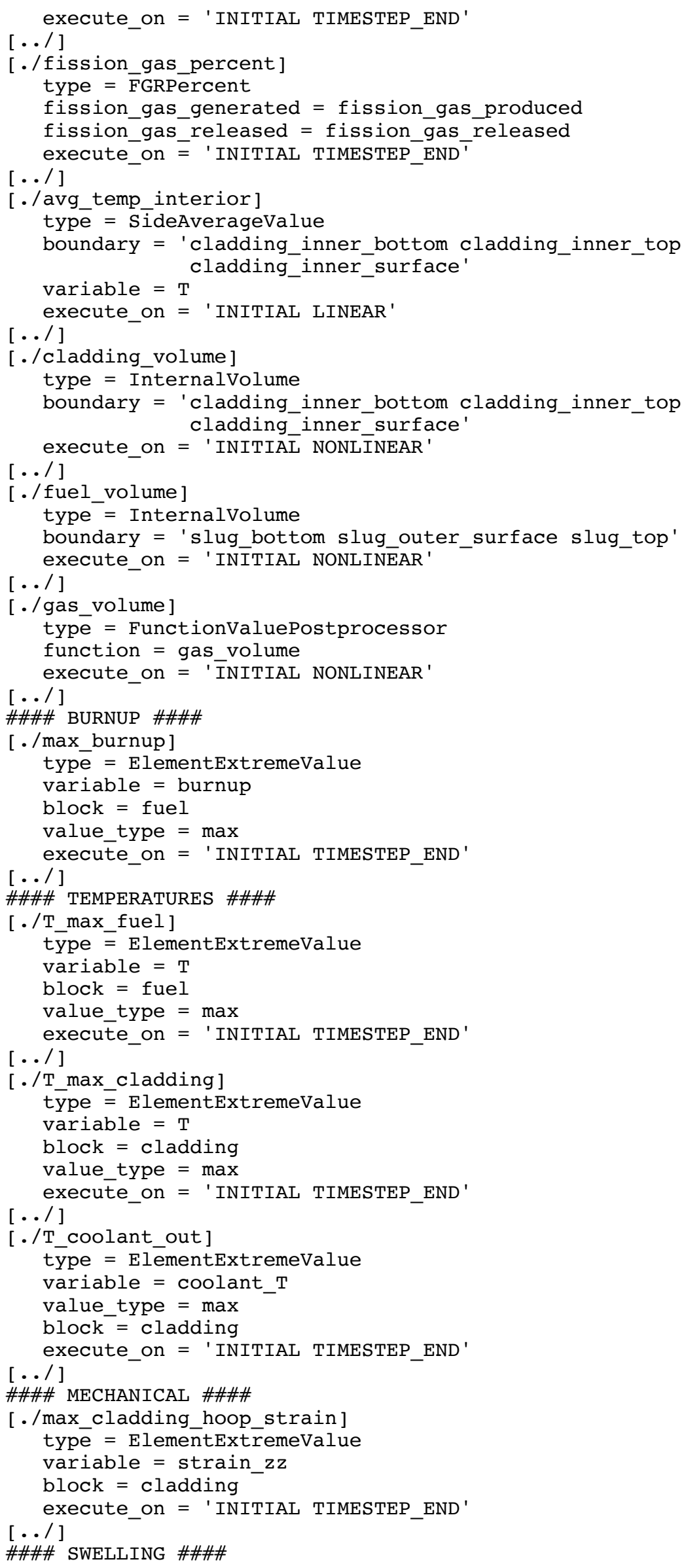




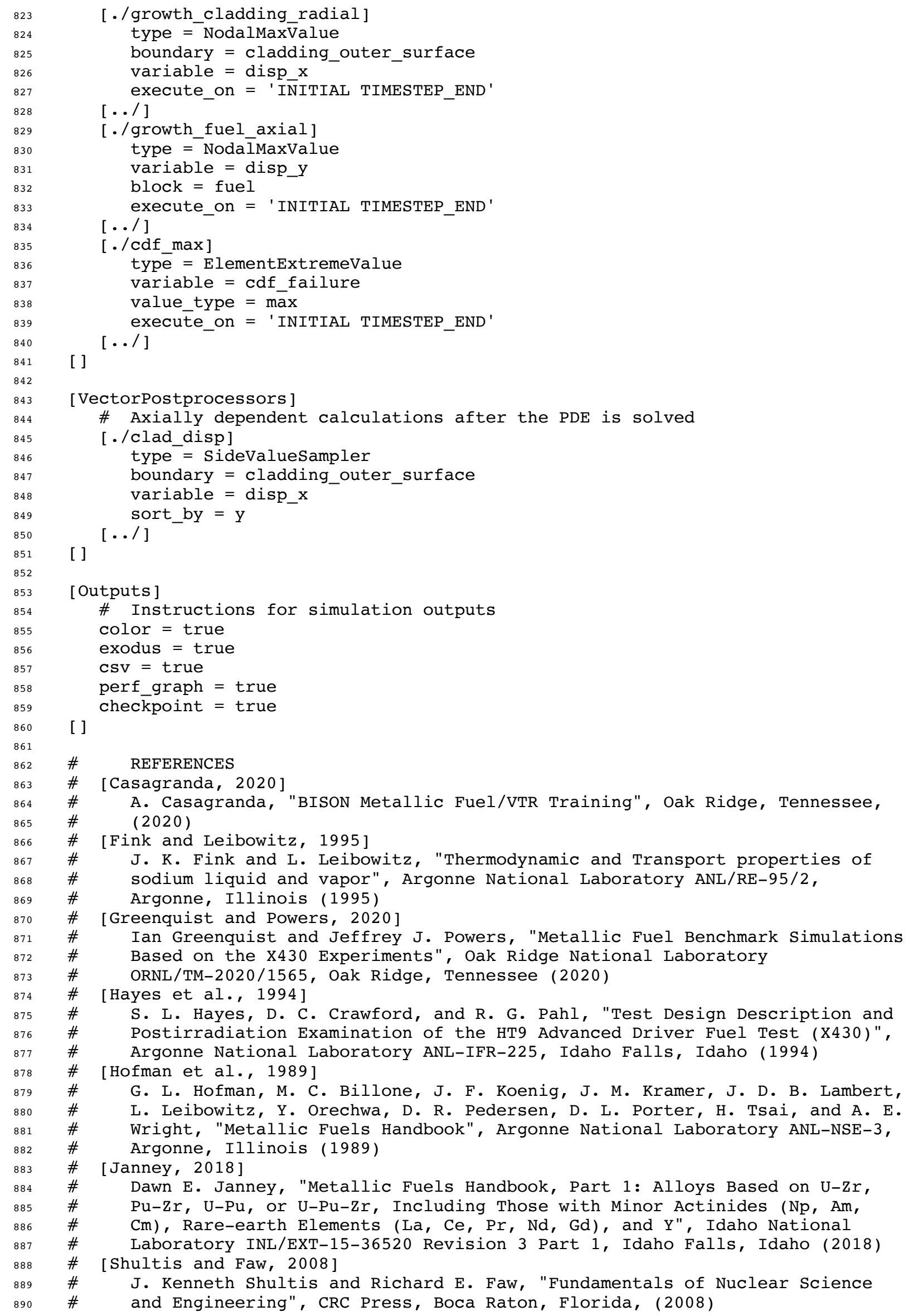

